\title{
ROYAL BOTANIC GARDEN, EDINBURGH
}

\section{LIST OF SEEDS}

COLLECTED DURING 1890.

PRINTWD FOR HRR MAIHSTY'S STATHONERY OFHCE. Br NEIBL \& COMPANT, Rombriza. 1891 . 



\section{ROYAL BOTANIC GARDEN, EDINBURGH.}

\section{LIST OF SEEDS}

\section{COLLECTED DURING 1890.}

\section{RANUNCULACEÆ.}

Aconitum

anthora, Linn.

eminens, Koch.

longigaleatum, Hort.

Lycoctonum, Linn.

moldavicum, Bes.

Napellus, Linn.

\section{- var.}

paniculatum, Lamk.

Actæa septentrionale, Baumg.

Adonis

spicata, Linn.

- var. rubra, Michx.

æstivalis, Linn.

pyrenaica, DC.

vernalis, Willd.

Anemone

alpina, Linn.

decapetala, Linn.

narcissiflora, Linn.

Pulsatilla, Linn.

rivularis, Buch.

vernalis, Linn.

Aquilegia

californica, var. alba, Hort.

canadensis, Linn.

chrysantha, Gray.

- var. truncata, Hort.

cærulea, James.

flavescens, Watson.

glandulosa, Fisch.

pyrenaica, DC.

Skinnerii, Hort.

viridiflora, Pall.

vulgaris, Linn.

Clematis erecta, All. angustifolia, Jacq.

Delphinium

altissimum, Wall.

Brunonianum, Royle. cashmirianum, Royle. crassifolium, Schrd.
Delphinium-contd.

formosum, Boiss. et Reut.

hybridum, Steph.

Nahamah, Hort.

Staphisagria, Linn.

velutinum, Bertol.

Helleborus

colchicus, Regel.

niger, Linn.

orientalis, Lamk.

Isopyrum fumarioides, Linn.

Myosurus minimus, Linn.

Ranunculus

acris, Linn.

amplexicaulis, Linn.

bulbosus, Linn.

cortusæfolius, Willd.

Friesianus, Jord.

propinquus, Meyer.

pyrenæus, Gouan.

sceleratus, Linn.

Thalictrum

appendiculatum, Ledeb.

aquilegiæfolium, Linn.

- var. roseum.

Cornuti, Linn.

elatum, Murr.

glaucum, Desf.

lucidum, Linn.

minus, Linn.

- sub.-sp. majus, Jacq.

Schweiggeri, Spreng.

simplex, Linn., var. Jordani,

Hort.

Trollius speciosum, Mil.

aconitifolius, Hort. asiaticus, Linn.

- var. hybridus, Hort.

caucasicus, Stev.

intermedius, Hort.

japonicus, Hort.

laxus, Salisb. 


\section{BERBERIDEÆE.}

Podophyllum emodi, Wall. $\quad$ Mahonia glumacea, DC.

\section{PAPAVERACEA.}

Catheartia villosa, Hook, f.

Chelidonium majus, Linn.

Corydalis

- var. laciniatum, DC.

africana (Cysticapnos africanus, Gærtn.)

glauca, Pursh.

- var. rosea.

nobilis, Pers.

Eschscholtzia

californica, Cham.

- var. Mandarin, Hort.

Fumaria capreolata, Linn.

Glaucium

corniculatum, Curt.

luteum, Scop.

Meconopsis

cambrica, Vig.

- var. aurantiaca, Hort.
Meconopsis-contd.

nipalensis, DC.

simplicifolia, Hook, f. et Thoms.

Wallichii, Hook.

Papaver

- var. fusco-purpurea, Hook. f.

aculeatum, Thunb.

alpinum, Linn.

- varieties.

Argemone, Linn.

bracteatum, Lindl.

- pink var.

Heldreichii, Boiss.

nudicaule, Linn.

pilosum, Sibth.

orientale, Linn.

Rhoeas, Linn.

rupifragum, Boiss. et Reut.

somniferum, Linn.

Platystemon californicus, Benth.

\section{CRUCIFERA.}

Alyssum

podolicum, Benth. et Hook.

repens, Baumg.

saxatile, Linn.

Wiersbeckii, Heuffl.

Arabis

albida, Stev.

alpina, Linn.

lucida, Linn. fil.

petræa, Lamk.

rosea, DC.

Aubrietia

deltoidea, DC.

- var. grandiflora.

- var. Campbelli, Hort.

- var. cælestis, Hort.

- var, compacta, Hort.

- var. spathulata, Hort.

- var. Mooreana, Hort.

- var. Pinardii, Hort.

erubescens, Griseb.

Brassica
alba, Boiss.
campestris, Linn.
oleracea, Linn.

Braya alpina, Sternb.

Bunias orientalis, Linn.

Camelina sativa, Crantz.

Capsella Bursa-pastoris, Muench.

Cheiranthus

Allionii, Hort.

Marshalli, Hort.

Cochlearia officinalis, Linn.

saxatilis, Benth. et Hook.

Crambe maritima, Linn.

Draba

aizoides, Linn.

Aizoon, Wahl.

altaica, Bunge.

bruniæfolia, Stev.

cuspidata, Bbrst.

incana, Linn.

frigida, Saut.

lasiocarpa, Reichb.

rupestris, R. Br.

tomentosa, Clair.

Erysimum

latifolium, Hort.

rupestre, DC. 
Hesperis

matronalis, Linn.

fragrans, Fisch.

Iberis

corifolia, Sweet.

gibraltarica, Linn.

Garrexiana, All.

sempervirens, Linn.

superba, Hort.

umbellata, Linn.

Isatis tinctoria, Linn.

Lepidium

erassifolium, Wald. et Kit.

ruderale, Linn.
Lepidium-contd.

sativum, Linn.

Smithii, Hook.

Matthiola incana, R. Br.

Raphanus caudatus, Linn.

Senebiera didyma, Pers.

Sisymbrium

assoanum, Ruiz et Pav.

austriacum, Jacq.

tanacetifolium, Benth. et Hook.

Thlaspi

alpestre, Linn.

arvense, Linn.

perfoliatum, Linn.

\section{RESEDACEA.}

Reseda Luteola, Linn.

Reseda Phyteuma, Linn.

\section{CISTINEA.}

Cistus albiflorus, Hort.

Helianthemum

polifolium, Mill.

Helianthemum-contd.

Tuberaria, Willd.

vulgare, Gærtn.

\section{VIOLARIEÆ.}

Viola

alpestris, Jord.

canadensis, Linn.

canina, Linn.

Munbyana, Vill.

Viola-contd.

nana, DC,

Orphanides, Rgl.

palustris, Linn.

tricolor, Linn.

\section{CARYOPHYLLEA.}

Arenaria

balearica, Linn.

cæspitosa, Ehret.

caulescens, Hort.

graminifolia, Schrad.

verna, Linn.

Cerastium

alpinum, Linn.

chloræfolium, F. et. M.

dichotomum, Linn.

perfoliatum, Linn.

Dianthus

alpinus, Linn.

arenarius, Linn.

britannicus, Hort.

capitatus, DC.
Dianthus-contd. cæsius, $\mathrm{Sm}$. cæspitosus, Thunb. calocephalus, Boiss. Caryophyllus, Linn. caucasicus, Bbrst. corsicus, Fisch. deltoideus, Linn. plumarius, Linn. - var. 'Little Gem,' Hort. plumarius, Linn, sub. sp. serotinus, Waldst. et Kit. pungens, Linn. saxatilis, Pers. Simsii, Hort. superbus, Linn. 
Gypsophila elegans, Bieb.

Lychnis

chalcedonica, Linn.

Coronaria, Desv.

diurna, Sibth.

Flos-cueuli, Linn.

Flos-Jovis, Desv.

Lagaseæ, Nym.

pyrenaica, Berger.

Viscaria, Linn.

Sagina

- var. purpurea, Hort.

glabra, Koch.

pilifera, Fenzl.

Saponaria ocymoides, Linn.

Silene

acaulis, Linn.
Silene-contd.

alpestris, Linn.

apetala, Willd.

caucasicus, Boiss.

chloræfolia, Smith, var. swertiæfolia, Boiss.

ciliata, Pourr.

Czerei, Baumg.

italica, Pers.

muscipula, Linn.

pusilla, Waldst. et Kit.

Schafta, Gmel.

Spergula arvensis, Linn.

Stellaria

graminea, Linn.

media, Linn.

uliginosa, Linn.

\section{PORTULACEÆ.}

Calandrinia discolor, Hook.

Claytonia perfoliata, Don.

\section{HYPERICINEA.}

Hypericum

Androsæmum, Linn.

Gebleri, Ledeb.
Hypericum-contd.

hirsutum, Linn.

pulchrum, Linn.

\section{MALVACE $Æ$.}

Abutilon vitifolium, Cav.

Hibiscus pedunculatus, Thunb.

Malva

moschata, Linn., var. alba, Hort. sylvestris, Linn.
Sidalcea

candida, Gray.

malvæflora, Gray.

Murrayana, Hort.

\section{STERCULIACEÆE.}

Thomasia purpurea, Gay.

\section{LINEA.}

Linum alpinum, Linn.

Linum perenne, Linn.

\section{GERANIACEÆ.}

\section{Erodium}

malacoides, Willd.

Manescavi, Coss.
Geranium anemonæfolium, L'Hérit. armenum, Boiss. 
Geranium-contd.

dahuricum, DC.

eriostemon, Fisch.

ibericum, Cav.

Lamberti, Sweet.

lucidum, Linn.

pratense, Linn.

- var. album, Hort.

sanguineum, Linn.

- var. lancastriense, With.
Geranium-contd.

sibiricum, Linn.

subcaulescens, L'Hérit.

Impatiens

Wallichianum, Sweet.

Noli-me-tangere, Linn.

parviflora, DC.

Roylei, Walp.

Oxalis sensitiva, Linn.

Limnanthes grandiflora, $\mathrm{R}$. $\mathrm{Br}$.

\section{OCHNACEÆ.}

Ochna mozambiciensis, Klotzsch.

\section{LEGUMINOSAE.}

Acacia

armata, R. Br.

grandis, Henf.

Anthyllis

Vulneraria, Linn.

Astragalus

bicolor, Lamk.

Cicer, Linn.

glycyphyllus, Linn.

Hypoglottis, Linn.

- var. albus, Hort.

oroboides, Hornem.

ponticus, Willd.

Brachysema subcordata, Benth.

Cytisus

scoparius, Link.

Laburnum, Linn.

- var. quercifolium.

Desmodium gyrans, DC.

Genista

sagittalis, Linn.

tinctoria, Linn.

Kennedya rubicunda, Vent.

Lotus corniculatus, Linn.

Lathyrus
Aphaca, Linn.
coccineus, Mill.
cyanæus, Stev.
hirsutus, Linn.
lævigatus, Waldst, et Kit.
macrorhizus, Wimm.
niger, Wimm.
luteus, Benth et Hook.
tuberosus, Linn.
vernus, Bernh.

Lupinus

arboreus, Sims.

Cruikshanksii, Hook.

Hartwegii, Lindl.

nootkatensis, Don.

polyphyllus, Lindl.

Medicago

ciliaris, Savi.

Echinus, DC.

elegans, Jacq.

maculata, Sibth.

radiata, Linn.

Terebellum, Willd.

Melilotus

speciosa, Dur.

officinalis, Lamk.

Mimosa pudica, Linn.

Ononis rotundifolia, Linn.

Oxytropis

campestris, DC.

Hallerii, Bunge.

lapponica, Wahl.

Parochætus communis, Hamilt.

Trifolium

alpinum, Linn.

Balansæ, Boiss.

Bocconi, Savi.

pannonicum, Linn.

subterraneum, Linn.

Trigonella

Fœnum-græcum, Linn.

pinnatifida, Cav.

Ulx eeuropæus, Linn 
Vicia

Cracea, Linn.

narbonensis, Linn.

onobrychoides, Linn.

Orobus, DC.
Vicia-contd.

pyrenaica, Pourr.

Sepium, Linn.

- var. album, Hort.

villosa, Roth.

\section{ROSACEAE.}

Acæna

adscendens, Vahl.

microphylla, Hook. f.

myriophylla, Ld].

pulchella, Hort.

sarmentosa, Carmich.

Alchemilla

alpina, Linn.

- var. conjuncta, Bab.

arvensis, Lamk.

pubescens, Linn.

Cotoneaster microphylla, Wall.

Dryas Drummondii, Watson.

Eriobotrya japonica, Lindl.

Geum

coccineum, Sibth.

Grahamii, Steud.

parviflorum, Comm.

intermedium, Ehr.

rivale, Linn.

triflorum. Pursh.

urbanum, Linn.

Potentilla

argentea, Linn.
Potentilla-contd.

atrosanguinea, Lod.

Fenzlii, Lehm.

inclinata, Vill.

insignis, Royle.

kurdica, Boiss.

M'Nabiana, Hort.

nepalensis, Hook.

nevadensis, Boiss.

opaca, Linn.

pedata, Willd.

Phœbis, Hort.

recta, Linn.

- var. Hookeriana, Lehm.

rupestris, Linn.

sericea, Linn.

Schrenkiana, Hort.

Sibbaldia, Haller fil.

Rosa rugosa, Thunb.

Spiræa

digitata, Willd.

- var. glabra, Hort.

palmata, Thunb.

— var, alba, Hort.

\section{SAXIFRAGACE正.}

Boykinia aconitifolia, Nutt.

Heuchera

Arlei, Hort.

cylindrica, Dougl.

Drummondii, Hort.

laxa, Hort.

ribifolia, F. et Lallem.

Richardsoni, R. Br.

sanguinea, Eng.

spicata, Hort.

Parnassia

nubicola, Hook. fil.

Saxifraga palustris, Linn.

acanthifolia, Hort.

ægilops, Hort.

affinis, Don.
Saxifraga-contd.

Aizoon, Jacq.

- var. pectinata, Schott.

- var. notata, Schott.

- var. rosularis, Schleich.

angulosa, Schott.

Andrewsii, Harv.

bronchialis, Linn.

Bucklandii, Hort.

- var. major, Hort.

cespitosa, Linn.

capillipes, Rchb.

earinthiaca, Schott.

canaliculata, Boiss.

ceratophylla, Dryand.

corsicana, G. et G.

crustata, Vest. 
Saxifraga-contd.

- dentata, Link.

Geum, Linn.

- var. elegans, Mackay.

- var. gracilis, Mackay.

Gmelinii, Host.

granulata, Linn.

hirsuta, Linn.

- var. minor, Hort.

hirta, Don.

hypnoides, Linn.

intacta, Willd.

- var. farinosa, Hort.

irrigua, M. B.

kolenatiana, Rgl.

lasiophylla, Schott.

lingulata, Bell.

longifolia, Lap.

media, Gouan.

Melvilleii, Desf.

Saxifraga-contd.

M'Nabiana, Hort.

muscoides, Wulf.

- var. purpurea, Hort.

mutata, Linn.

nepalensis, Hort.

oppositifolia, Linn.

- var.

peltata, Torr.

portae, Stein.

Prostii, Sternb.

pulchella, Don.

repanda, Willd.

serratifolia, Mack.

Sturmiana, Schott.

taygetea, Boiss.

tenella, Wulf.

umbrosa, Linn.

valdensis, DC.

virginiensis, Michx.

\section{CRASSULACEÆ.}

Sedum

Aizoon, Linn.

annuum, Linn.

californicum, Hort.

crassipes, Wall.
Sedum-contd.

Kamtschaticum, Fisch. oppositifolium, Sims. Rhodiola, DC. trifidum, Wall.

\section{DROSERACEAE.}

Drosera

Burkeana, Pl,

capensis, Linn.
Drosera-contd.

madagascariensis, DC. spathulata, Labill.

MYRTACEÆ.

Eugenia dysentarica, Mart.

\section{LYTHRARIE $Æ$.}

Lythrum

Salicaria, Linn,

Lythrum-contd.

Salicaria, Linn.

- var, angustifolium, Hort. — - var, tomentosum, Mill. 
ONAGRARIEA.

Epilobium

brachycarpum, Presl.

boreale, Hauskn.

latifolium, Linn.

nummulariæfolium, Cunn.

- var. longipes, Hort.
Enothera

biennis, Linn.

fruticosa, Linn.

glauca, Michx.

taraxacifolia, Sweet.

\section{LOASACEÆ.}

Loasa tricolor, Lindl.

\section{CUCURBITACEÆ.}

Bryonia dioica, Linn.

Momordica Elaterium, Linn.

\section{UMBELLIFERAE.}

Aciphylla squarrosa, Forst.

Egopodium major, Hort.

Anthriscus sylvestris, Hoffm.

Archangelica decurrens, Ledeb.

Arracacia arguta, Benth. et Hook.

Astrantia

major, Linn.

montana, Hort.

Bupleurum

angulosum, Linn.

Candollei, Wall.

graminifolium, Vahl.

ranunculoides, Linn.

Conium maculatum, Linn.

Heracleum

angustifolium, $\mathrm{Sm}$.

Sphondylium, Linn.

villosum, Fisch.
Laserpitium

alatum, Linn.

aquilegiæfolium, DC.

Siler, Linn.

Ligusticum

Retzi, Vitm.

scoticum, Linn.

Magydaris panacina, DC.

Meum athamanticum, Jacq.

Myrrhis odorata, Scop.

Enanthe

crocata, Linn.

globulosa, Linn.

Jordani, Tenor.

Physospermum commutatum, Spreng.

Sanicula europæa, Linn.

Seseli gummiferum, Sm.

\section{RUBIACEA.}

Asperula

azurea, Jaub. et Spach.

lævigata, Linn.

Galium tinctoria, Linn.

Aparine, Linn. pulchellum, Hort. pusillum, Sm.
Galium-contd. rubioides, Linn. saxatile, Linn.

Pentas carnea, Benth.

Psychotria cyanococca, Hort.

Rubia angustifolia, Linn.

Sherardia arvensis, Linn. 


\section{VALERIANACEÆ.}

Centranthus ruber, DC.

Plectritis congesta, Dec.

Valeriana

officinalis, Linn.
Valeriana officinalis, Linn., var. sambucifolia, Mik.

Valerianella Morisonii, DC. olitoria, Mœench.

\section{DIPSACEA.}

Scabiosa

alpina, Linn. arvensis, Linn. caucasica, Bbrst. Columbaria, Linn. dipsacifolia, Schott. pilosiuseula, Hort. pubescens, Kit. Webbiana, Don.

\section{COMPOSITAE.}

Ammobium alatum, R. Br.

Arctium Lappa, Linn. minus, Schkr.

Arctotis revoluta, Jacq. Arnica Chamissonis, Less. Artemisia

borealis, Pall. capillacea, Hort. rupestris, Linn. Aster neglecta, Spr.

alpinus, Linn. peregrinus, Pursh. pseud-amellus, Hook. f. ramulosa, Lindl.

Vahlii, Hook. et Arn.

Bellium minutum, Linn.

\section{Buphthalmum} salicifolium, DC. speciosum, Schreb.

Calendula officinalis, Linn.

\section{Centaurea}

- var. 'Hen and Chicken.'

cinerea, Lam.

Clementei, Boiss.

dealbata, Willd.

Rhaponticum, Fisch.

Chrysanthemum

corymbosum, Linn.
Chrysanthemum--contd. macrophyllum, Waldst, et Kit. tauricum. Hort.

Willemottii, Hort.

Leucanthemum, Linn.

- var. grandiflorum, Hort.

- var. tripteris, Hort. uliginosum, Pers.

Cineraria Fischerii, Ledeb.

Cnicus

arvensis, Hoffm.

Benedictus, Linn.

Biebersteinii, Hort.

Carolorum, $\times$ Jenner.

elatus, Hort.

eriophorus, Hoffm.

lanceolatus, Hoffm.

oleraceus, Linn.

pannonicus, DC.

rigidus, Hort.

tataricus, Willd.

tuberosus, Linn.

Coreopsis tenuifolia, Ehrh.

Crepis rubra, Linn.

Dimorphotheca annua, Less.

Erigeron

acre, Linn.

alpinum, Linn.

aurantiacum, Rgl. 
Erigeron-contd.

glabellum, Nutt.

purpureum, Ait.

Royleii, DC.

speciosum, DC.

Eupatorium

cannabinum, Linn.

purpureum, Linn.

sessilifoliun, Linn.

verticillatum, Muhl.

Gaillardia

pulchella, Foug.

- var. grandiflora, Hort.

- var. maxima, Hort.

Gerbera anandria, Schultz.

Grindelia

hirsuta, Hook. et Arn.

squarrosa, Dunal.

Hieracium

aurantiacum, Linn.

Auricula, Linn.

autumnale, Hort.

elongatum, Lap.

glomeratum, Froel.

lævigatum, Linn.

murorum, Linn.

nigrescens, Willd.

pyrenaicum, Jord.
Hieracium--contd, strictum, Fries.

Helenium autumnale, Linn.

- var. pumilum, Gray.

Helianthella uniflora, Torr. et Gray.

Inula

ensifolia, Linn.

Helenium, Linn.

Hookerii, Clarke.

Leontopodium alpinum, Cass.

Liatris odoratissima, Willd.

Ligularia macrophylla, Bbrst.

Onopordon Acanthium, Linn.

Rudbeckia triloba, Linn.

Scorzonera hispanica, Linn.

Senecio

campestris, DC.

Doronicum, Linn.

Serratula concolor, DC.

coronata, Linn.

heterophylla, Desf.

quinquefolia, Bbrst.

Silybum Marianum, Gærtn.

Solidago Virgaurea, Linn.

Sonchus oleraceus, Linn.

Troximon marginatum, Nutt.

\section{STYLIDIACE E.}

\begin{tabular}{l|l} 
Stylidium adnatum, R. Br. & Stylidium fruticosum, R. Br.
\end{tabular}

\section{CAMPANULACE $Æ$.}

Campanula

alliariæfolia, Willd.

barbata, Linn.

carpathica, Linn. fil.

elegans, R, et $\mathrm{S}$.

glomerata, L.

latifolia, Linn.

- var. alba, Hort.

- var. macrantha, Fisch.

Medium, Linn.

pulla, Linn.

pumila, Curt.

rhomboidea, Linn.

rapunculoides, Linn.

rotundifolia, Linn.

- var. maxima, Hort.

sarmatica, Ker.
Campanula-contd. thyrsoidea, Linn.

turbinata, Schott.

- var. alba, Hort.

Vidallii, H. C. Wats.

Codonopis

clematidea, Schrenck.

ovata, Benth.

Cyananthus lobatus, Wall.

Jasione montana, Linn.

Phyteuma

limonifolia, Sib. et. Sm.

orbiculare, Linn.

Scheuchzeri, All.

Symphiandra

Hoffmanni, Pane.

pendula, DC. 
ERICACEÆ.

Gaultheria

carnea, Hort.

Shallon, Pursh.

Oxycoccos palustris, Pers.

Erica cinerea, Linn.

\section{MYRSINEÆ.}

Ardisia primulæfolia, Hort.

\section{PLUMBAGINEA.}

Armeria

allioides, Boiss.

bupleuroides, Hort.

Hallerii, Wallr.

Armeria-contd. maritima, Willd. latifolia, Willd.

Statice incana, Hort.

\section{PRIMULACEÆ.}

Anagallis arvensis, Linn.

Androsace lactea, Linn.

Primula-contd. japonica, Gray. mollis, Nutt. reticulata, Wall. sibirica, Jacq. sikkimensis, Hook. vulgaris, Huds. cortusoides, Linn. elatior, Jacq.

\section{GENTIANEÆ.}

Erythræa diffusa, Woods. Gentiana

asclepiadea, Linn.

- var. alba, Hort.

Kochiana, Pers. ornata, Wall.

phlogifolia, Sch.

Gentiana-contd. punctata, Linn. septemfida, Pall. - var. cordifolia, Boiss. tibetica, King. verna, Linn.

\section{POLEMONIACEA.}

Collomia coccinea, Lehm. grandiflora, Dougl.

Polemonium-contd. - var. fasciatum, Hort. flavum, Greene. humile, Willd.

Polemonium cæruleum, Linn.

\section{HYDROPHYLLACEA.}

Romanzoffia sitchensis, Cham. 


\section{BORAGINEA.}

Anchusa sempervirens, Linn.

Borago officinalis, Linn.

Cerinthe alpina, Kit.
Cynoglossum officinale, Linn.

Eritrichium californicum, Fisch.

\section{CONVOLVULACEÆ.}

Convolvulus tricolor, Linn.

\section{SOLANACEÆ.}

Atropa Belladonna, Linn.

Nicotiana Tabacum, Linn.

Scopolia lurida, Desf.

\section{SCROPHULARINEA.}

Alonzoa Warcewiczii, Rgl.

Antirrhinum

Asarina, Linn.

majus, Linn.

- var. 'Tom Thumb.'

Celsia cretica, Linn.

Collinsia

bicolor, Benth.

grandiflora, Dougl.

Digitalis purpurea, Linn.

Erinus

alpinus, Linn.

Linaria

var. hispanicus, Pers.

$$
\text { alpina, DC. }
$$

vulgaris, Mill.

Maurandia erubescens, Gray.

Mimulus

cupreus, Veitch.

luteus, Linn.

Pentstemon

Mackayanus, Knowl.

Menziesii, Hook.
Pentstemon-contd. nitidus, Dougl. procerus, Dougl. pubescens, Sol.

Ramondia pyrenaica, Rich.

Scrophularia aquatica, Linn. nodosa, Linn. vernalis, Linn. species.

Veronica

alpina, Linn. arvensis, Linn.

Bidwillii, Hook. f.

Buxbaumii, Ten.

Cataractæ, Forst.

Colensoii, Hook. f.

elliptica, Forst.

fruticulosa, Linn.

hirsuta, Hopk. saxatilis, Linn.

- var. Guthrieana, Hort.

Wulfenia carinthiaca, Jacq.

\section{GESNERACEÆ.}

Streptocarpus Rexii, Lindl.

\section{ACANTHACEÆ}

Aphelandra aurantiaca, Lindl.

Beloperone oblongata, Hort. - var. Rœzlii, Hort. 


\section{VERBENACEÆ.}

Clerodendron

Thomsonæ, Balf.
Clerodendron-contd. Thomsonæ, var. Balfourii, Hort.

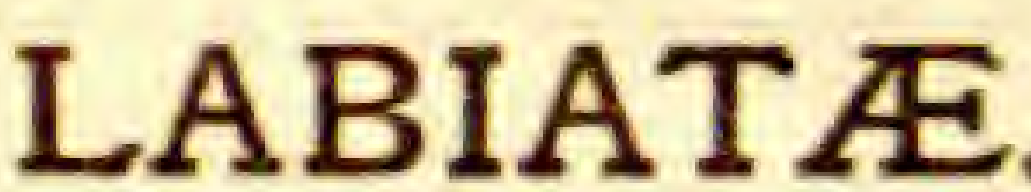

Horminum pyrenaicum, Linn.

Lamium Orvala, Linn.

Marrubium vulgare, Linn.

Melissa officinalis, Linn.

Melittis Melissophyllum, Linn.

Nepeta

altissima, Schrenck.

macrantha, Fisch.

Orthosiphon stamineus, Benth.

Phlomis fruticosa, Linn.
Physostegia virginiana, Benth. Plectranthus fruticosus, L'Hérit Salvia argentea, Linn. limbata, Meyer. pratensis, Linn. taraxacifolia, Coss, et Bal. Scutellaria galericulata, Linn. Stachys sylvatica, Linn.

Thymus Serpyllum, Fries.

\section{PLANTAGINEÆ.}
Plantago
acanthophylla, Dene.
Coronopus, Linn.
Kamtschatica, Cham.
lanceolata, Linn.
lagopus, Linn.

Plantago-contd. major, Linn. - var. monstrosa, Hort. maritima, Linn. media, Linn. montana, Lamk.

\section{CHENOPODIACE $Æ$.}

Atriplex laciniata, Linn. Spinacia oleracea, Linn.

Chenopodium Bonus-Henricus, Linn.

\section{POLYGONACEÆ.}

Fagopyrum esculentum, Linn.

Oxyria elatior, R. Br.

Polygonum

amplexicaule, Don.

sphærostachyum, Meissn.

Rheum

verticillatum, All.

collinianum, Fric.

palmatum, Linn.

- var. tanguticum, Rgl.
Rumex

acetosella, Linn.

alpinus, Linn.

compacta, Hort.

intermedius, DC.

rupestris, Le Gall.

sanguineus, Linn.

sanguisorbæfolius.

stenophyllus, Ledeb.

tingitanus, Linn.

\section{NEPENTHACE $Æ$.}

Nepenthes khasiana, Hook. f. (N. distillatoria, Grah.). 
THYMELEACE尺.

Daphne Mezereum, Linn. | Daphne Mezereum, var. album.

\section{ARISTOLOCHIACEÆ.}

Aristolochia elegans, Mast.

\section{EUPHORBIACE $Æ$.}

Euphorbia

Lathyris, Linn.

Euphorbia-contd.

Myrsinites, Linn. verrucosa, Linn.

\section{URTICACEA.}

Laportea moroides, Wedd.

Parietaria mauritanica, Dur.

Urtica-contd. palmata, Lesch. pentandra, Roxb. Ulmus montana, Sm.

Urtica grandidentata, Banks. pilulifera, Linn.

\section{SCITAMINEA.}

Costus igneus, N.E.B.

Hedychium

Gardnerianum, Wall.

Roxburghii, Wight.
Canna

patens, Rosc.

floribunda, Bche.

\section{IRIDEAE.}

Aristea cyanea, Ait.

Bobartia aurantiaca, Hort.

Crocus medius, Balbis.
Homeria collina, Vent.

Libertia grandiflora, Sweet.

Sisyrinchium mucronatum, Mich.

\section{HCEMADORACEA.}

Anigozanthus rufa, Labill.

\section{AMARYLLIDEÆ.}

Leucoium

vernum, Linn.

Narcissus

- var. carpathicum, Steud.

nobilis, Haw.
Narcissus-contd.

muticus, Gay.

pallidus-proecox, Hort.

pumilus, Haw.

Estrada, Hort. 


\section{LILIACEÆ.}

Agapanthus umbellatus, L'Hérit. Allium

Babingtonii, Boner.

Dioscoridos, Sm.

globosum, Bbrst.

- var. albidum, Hort.

M'Leanii, Baker.

MNabianum, Hort.

Moly, Linn.

triquetrum, Linn.

Victorialis, Linn.

Anthericum Liliago, Linn.

Asphodelus ramosus, Linn.

Brodiæa congesta, Sm.

Bulbinella Hookerii, Benth.

Bulbocodium vernum, Linn.

Camassia

Brownii, Hort.

esculenta, Lindl.

- var. alba, Hort.

- var. major, Hort.

Chionodoxa

Luciliæ, Boiss.

sardensis, Hort.

Chlorophytum orchidiastrum, Lindl.

Clintonia Andrewsiana, Torr.

Colchicum speciosum, Stev.

Erythronium

Dens-canis, Linn.

- var. grandiflorum, Hort.

giganteum, Lindl.

- var. roseum, Hort.

Fritillaria Meleagris, Linn.

Galtonia candicans, Dcne.
Gloriosa Plantii, Planch.

Hesperoscordon lacteum, Lindl.

Hyacinthus amethystinus, Linn.

Lilium Colchicum, Stev,

Littonia modesta, Hook.

Milla longipes, Hort.

Muscari

Argæi, Schott.

armeniacum, Baker.

atlanticum, Boiss. et Reut.

Maweanum, Hort.

Szovitsianum, Rgl.

Ornithogalum pyrenaicum, Linn.

Polygonatum multiflorum, All.

Puschkinia

libanotica, Zucc.

scilloides, Adams.

Sandersonia aurantiaca, Hook.

Scilla

amœena, Linn.

campanulata, Ait.

sibirica, Andr.

verna, Huds.

Tofieldia

carinthiaca.

palustris, Huds.

glutinosa, Pers.

Tricyrtis hirta, Hook.

Trillium grandiflorum, Salisb.

Triteleia uniflora, Lindl.

Tulipa

altaica, Pall.

pulchella, Fenzl.

Zygadenus glaueus, Nutt.

\section{JUNCACEÆ.}

Juncus

effusus, Linn., var, variegatus.

glaucus, Ehrh.

lamprocarpus, Ehrh.
Luzula

albida, D.C.

nivea, Desv.

\section{PALMA.}

Euterpe edulis, Mart.

Ptychosperma elegans, Labill.

Sabal umbraculifera, Mart.

\section{AROIDEA.}

Anthurium

leuconeurum, Schott.

Scherzerianum, Schott.
Anthurium-contd. acaule, Sweet.

Arum palæstinum, Boiss.

Symplocarpus fœetidus, Salisb. 
ALISMACEÆ.

Alisma ranunculoides, Linn.

NAIADACEA.

Aponogeton distachyum, Thunb.

\section{CYPERACEÆ.}

Carex

acuta, Linn.

- var. variegata, Hort.

arenaria, Linn.

atrata, Linn.

capillaris, Linn.

Drymingii, Hort.

echinata, Murr.

Hallerii, Vest.
Carex-contd.

flava, Linn.

Grahamii, Boott.

hordeiformis, Wahl.

lagopodioides, Schk.

panicea, Linn.

pendula, Huds.

pulicaris, Linn.

vesicaria, Linn.

\section{GRAMINEÆ.}

Agrostis

nebulosa, Boiss.

pulchella, Kth.

stolonifera, Linn.

verticillata, Vill.

Aira

vulgaris, With.

bottnica, Vahl.

cæspitosa, Linn.

flexuosa, Linn.

Alopecurus

geniculatus, Linn,

giganteus, Hort.

nigricans, Jacq.

pratensis, Linn.

Anthoxanthum gracile, Biv.

Arundo

acutifolia, Hort.

festucoides, Desf.

Avena

alpina, Kunth.

elatior, Linn.

Ludoviciana, Dur.

pratensis, Linn.

sterilis, Linn.

sativa, Linn.

Beckmannia erucæformis, Host.

Brachypodium pinnatum, Beauv.

Briza

gracilis, Hort.

maxima, Linn.
Brizopyrum siculum, Lamk.

Bromus

Krausei, Rgl.

Schraderi, Kth.

sylvatica, Pall.

unioloides, Kth.

Dactylis

glomerata, Linn.

Elymus

- var. aurea, Hort.

arenarius, Linn.

mexicanus, Cav.

sabulosus, Bbrst.

tener, Linn.

villosus, Muhlbg.

Festuca

ampla, Hort.

arundinacea, Schreb.

duriuscula, Linn.

elatior, Linn.

geniculata, Willd.

ovina, Linn.

rubra, Linn.

Scheuchzeri, Gaud.

Holcus

mollis, Linn.

Kœleria

- var. variegata, Hort.

Berytha, Boiss. 
Kœleria-contd. cristata, Pers.

Lagurus ovatus, Linn.

Lolium

ibericum.

perenne, Linn.

italicum, Braun.

Melica temulentum, Linn.

altissima, Linn.

uniflora, Retz.

Milium effusum, Linn.

Oryza sativa, Linn.

Phalaris

arundinacea, Linn.

canariensis, Linn.

cœrulescens, Desf.

trigyna, Host.

Phleum pratense, Linn.

Poa

annua, Linn.
Poa-contd.

Balfourii, Parn.

chinensis, Retz.

nemoralis, Linn.

- var. sempervirens, Hort. palustris, Roth.

sudetica, Hænke.

Polypogon monspeliensis, Desf.

Secale

anatolicum, Boiss.

cereale, Linn.

Stipa

formicarum, Delil. pennata, Linn.

Triticum caninum, Huds. Elymoides, Hornem. monococcum, Linn. violaceum, Hornem.

ISAAC BAYLEY BALFOUR, Keeper.

ROBERT LINDSAY,

Curator.

Edinburgh, February 1891. 




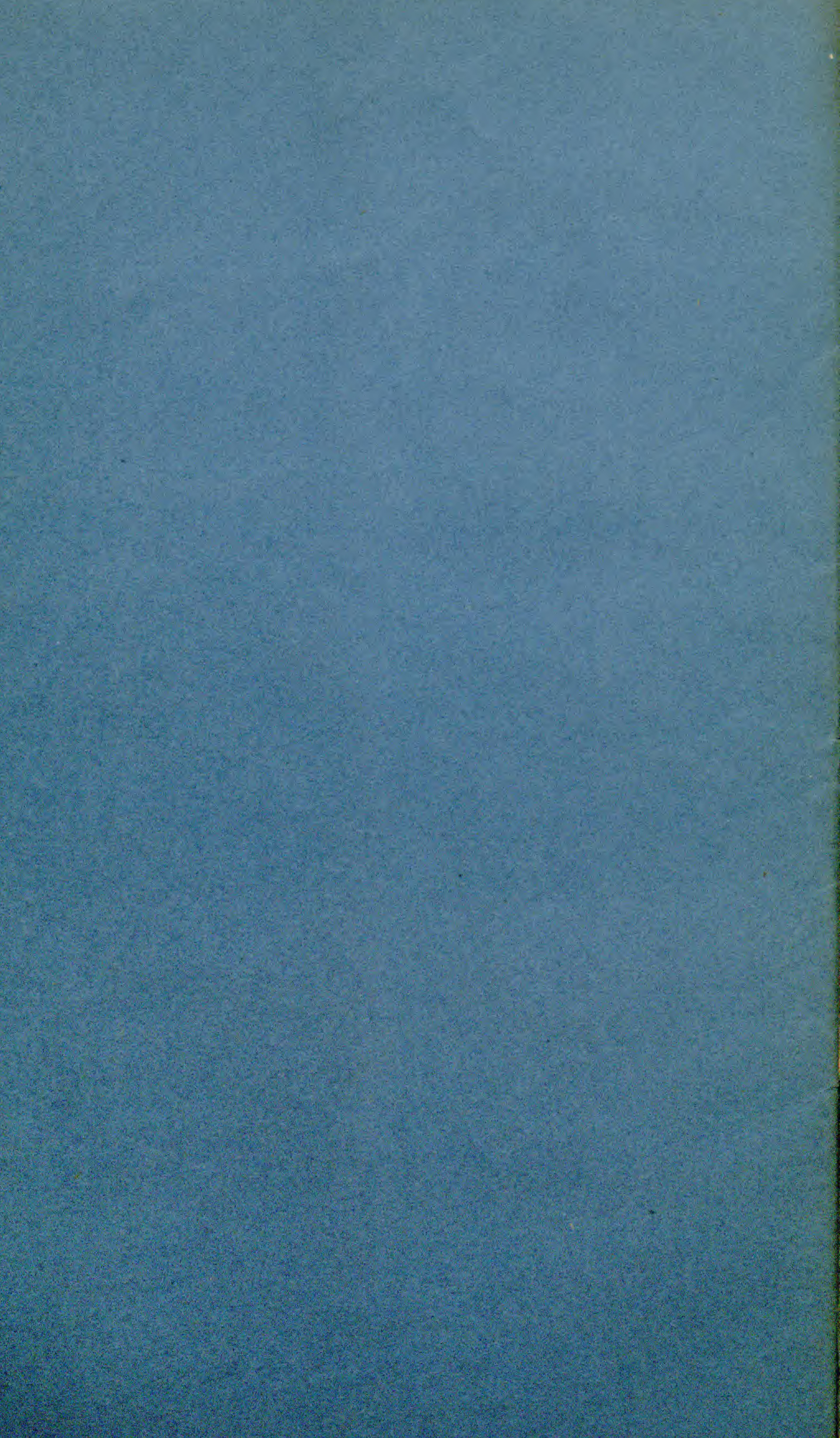




\section{ROYAL BOTANIC GARDEN, EDINBURGH}

\section{LIST OF SEEDS}

\section{COLLECTED DURING 1891.}



PRINTED FOR HER MAJESTY'S STATIONERY OFFICE, BY NELL \& COMPANY, BangerRar. 







\section{ROYAL BOTANIC GARDEN, EDINBURGH.}

\section{IIST OF SEEDS}

\section{COLLECTED DURING 1891.}

An asterisk * indicates that the quantity of seed collected is very small.

\section{RANUNCULACEÆ.}

Aconitum

longigaleatum, Hort.

Lycoctonum, Linn.

moldavicum, Bes.

Napellus, Linn.

- var.

Actæa

septentrionale, Baumg.

spicata, Linn.

Adonis

- var. rubra, Michx.

æstivalis, Linn.

pyrenaica, DC.

vernalis, Willd.

Anemone

alba, Juss.

alpina, Linn.

- var. sulphurea, DC.

decapetala, Linn.

*narcissiflora, Linn.

* _ var.

Pulsatilla, Linn.

- var. montana.

rivularis, Buchan.

Aquilegia

umbellata, Willd.

atropurpurea, Willd.

- var.

californica, var. alba, Hort.

canadensis, Linn.

chrysantha, Gray.

cœrulea, James.

flavescens, S. Watson.

olympica, Boiss.

pyrenaica, DC.

Stuartii, Hort.

*trinityensis, Hort.

viridiflora, Pall.

vulgaris, Linn.

- var. Kitaibelii, Schott.
Caltha

*biflora, DC.

palustris, Linn.

*purpurascens.

*radicans, Forst.

Cimicifuga

*americana, Michx.

racemosa, Nutt.

Clematis angustifolia, Jacq. erecta, All.

Delphinium

Ajacis, Rchb.

altissimum, Wall.

Brunonianum, Royle.

cashmirianum, Royle.

crassifolium, Schrad.,var. turkestanicum, Rgl.

formosum, Boiss. et Reut.

grandiflorum, Linn.

hybridum, Steph.

Menziesii, DC.

Nahamah, Hort.

*nudicaule, Torr. et Gray.

sp. (Yunan)

velutinum, Bertol.

Wheelerii, Hort.

*Helleborus angustifolius, Riverston, var. Hort.

Myosurus minimus, Linn.

Nigella orientalis, Linn.

Pæonia decora, Anders., var. elatior.

Ranunculus acris, Linn.

- sub-sp. sylvestris, Thuill. amplexicaulis, Linn.

auricomus, Linn.

propinquus, Meyer.

repens, Linn., alpine form.

serbicus, Vis.

${ }^{*}$ uniflorus, Hort. 
Thalictrum

lucidum, Linn.

minus, Linn.

- sub.-sp. majus, Jacq.

Schweiggeri, Spreng.

sibiricum, Ledeb.

simplex, Linn., var. Jordani, Hort.
Trollius

aconitifolius, Hort.

asiaticus, Linn.

- var. hybridus, Hort.

caucasicus, Stev.

europæus, Linn.

japonicus, Hort.

laxus, Salisb.

\section{BERBERIDEÆ.}

Diphylleia cymosa, Michx.

Podophyllum Emodi, Wall.

Mahonia glumacea, DC.

\section{PAPAVERACEÆ.}

Cathcartia villosa, Hook. fil.

Chelidonium

majus, Linn.

Corydalis

- var, laciniatum, DC.

claviculata, DC.

nobilis, Pers.

Eschscholtzia

ealifornica, Cham.

\section{Fumaria}

- var. Mandarin, Hort.

$$
\text { pallidiflora, Jord. }
$$

officinalis, Linn.

Glaucium

corniculatum, Curt.

flavum, Crantz.

Hypecoum procnmbens, Linn.

Meconopsis

cambrica, Vig.
Meconopsis-contd.

cambrica, Vig., var, orange coloured, Hort.

Papaver

aculeatum, Thunb.

alpinum, Linn.

- var.

Argemone, Linn.

bracteatum, Lindl.

Heldreichii, Boiss.

nudicaule, Linn.

orientale, Linn.

pilosum, Sibth.

pinnatifidum, Moris.

Rhœas, Linn.

rupifragum, Boiss. et Reut. somniferum, Linn.

Platystemon californicus, Benth.

Sanguinaria canadensis, Linn.

\section{CRUCIFERÆ.}

Alyssum

argenteum, Vitm.

gemonense, Linn.

* podolicum, Benth. et Hook.

* pyrenaicum, Lap.

saxatile, Linn.

- var. variegatum, Hort.

Arabis spinosum, Linn,

albida, Stev.

cenisia, Rchb.

lucida, Linn. fil.

*petræa, Lamk.
Aubrietia

deltoidea, DC.

- var. Bougainvillei, Hort.

- var. Campbellii, Hort.

- var. cœlestis, Hort.

- var. columnea, Hort.

- var. Frobellii, Hort.

- var. græca, Griseb.

- var. grandiflora, Hort.

- var. Hendersonii, Hort,

- var. olympica, Hort.

- var. Pinardii, Hort.

erubescens, Griseb. 
Barbarea

alpina, Koch.

vulgaris, R. Br.

Biscutella

ciliata, DC.

columnea, Tenor.

didyma, Linn.

raphanifolia, Poir.

Brassica

alba, Boiss.

Napus, Linn.

nigra, Koch.

olèracea, Linn.

Sinapis, Vis.

Braya alpina, Sternb.

Camelina

microcarpa, Andrz. sativa, Crantz.

Capsella

Bursa-pastoris, Moench. pauciflora, Koch.

Cardamine

*bellidifolia, Linn.

*latifolia, Vahl.

*ludoviciana, Hook.

Cheiranthus Senoneri, Held. et Sart,

Clypeola Jonthlaspi, Linn.

Cochlearia

officinalis, Linn.

- var. alpina, Wats.

saxatilis, Benth. et Hook.

Crambe maritima, Linn.

Diplotaxis erucoides, DC.

Draba

aizoides, Linn.

Aizoon, Wahl.

altaica, Bunge.

Gmelini, Adams.

incana, Linn.

*lasiocarpa, Rchb.

rupestris, R. Br.

tomentosa, Clair.

Erysimum

canescens, Roth.
Erysimum-contd.

hieracifolium, Linn.

ochroleucum, DC.

rupestre, DC.

yunanense, Franch.

Hesperis fragrans, Fisch.

Hutchinsia alpina, R. Br.

Ionopsidium acaule, Rchb.

Iberis

corifolia, Sweet.

garrexiana, All.

petræa, Jord.

saxatilis, Linn.

sempervirens, Linn.

superba, Hort.

umbellata, Linn.

Lepidium

campestre, R. Br.

sativum, Linn.

Lunaria annua, Linn.

Nasturtium pyrenaicum, R. Br.

Raphanus

caudatus, Linn.

sativus, Linn.

Schizopetalon Walkerii, Sims.

Sisymbrium

Alliaria, Scop.

austriacum, Jacq.

Loeselii, Linn.

assoanum, Ruiz et Pav.

tanacetifolium, Linn.

Teesdalia nudicaulis, R. Br.

Thlaspi

alpestre, Linn.

arvense, Linn.

brevicaule, Boiss. et Kotsch.

Vella perfoliatum, Linn.

*pseudo-cytisus, Linn.

*spinosa, Boiss.

Vesicaria

* sinuata, Poir.

*utriculata, Lam.

\section{RESEDACEÆ.}

Reseda

crystallina, Webb.

fruticulosa, Linn.

Luteola, Linn.
Reseda-contd.

odorata, Linn.

Phyteuma, Linn. 


\section{CISTINEÆ.}

Cistus laurifolius, Linn.

Helianthemum

lavandulæfolium, Pers.

polifolium, Mill.
Helianthemum-contd. vulgare, Gærtn.

- var. Garibaldi, Hort.

- var. The Bride, Hort.

\section{VIOLARIEÆ.}

Viola

alpestris, Jord.

arenaria, DC.

canadensis, Linn., var. alba.

canina, Linn.

- var, alba.

cornuta, Linn.

declinata, Waldst, et Kit.
Viola-contd.

lancifolia, Bbrst. macedonica, Boiss. et Held. occulta, Lehm.

*odorata, Linn. palustris, Linn. tricolor, Linn.

\section{CARYOPHYLLEÆ.}

Arenaria

balearica, Linn.

cæspitosa, Ehret.

gothica, Fr.

graminifolia, Schrad.

verna, Linn.

Cerastium

arvense, Linn, var. scarani, Ten. chloræfolium, F. et. M.

dichotomum, Linn.

perfoliatum, Linn.

*tomentosum, DC.

Dianthus

*alpinus, Linn.

arenarius, Linn.

barbatus, Linn.

cæspitosus, Thunb.

calocephalus, Boiss.

Caryophyllus, Linn.

caucasicus, Bbrst.

corsicus, Fisch.

deltoides, Linn.

glacialis, Hænk.

*Little Gem, Hort.

*neglectus, Loisl.

* - var.

plumarius, Linn., sub-sp. serotinus, Waldst, et Kit. petræus, Waldst. et Kit.

* * pulchellus, Pers. superbus, Linn.

tymphresteus, Held. et Sart.

Gypsophila

elegans, Bbrst.
Gypsophila-contd.

*repens, Linn.

Lychnis

cœlirosa, Desv.

chalcedonica, Linn.

coronaria, Desv.

diurna, Sibth.

Flos-cuculi, Linn.

Flos-Jovis, Desv.

purpurea, Hort.

* pyrenaica, Berger.

Sieboldii, Max.

Viscaria, Linn.

Saponaria

ocymoides, Linn.

Silene

Vaccaria, Linn.

*alpestris, Linn.

* caucasica, Boiss.

ciliata, Pourr.

Cucubalus, Wibel.

Czerei, Baumg.

gallica, Linn.

italica, Pers,

muscipula, Linn.

pendula, Linn.

- var. compacta.

* pusilla, Wald. et Kit. quinquevulnera, Linn.

Schafta, Gmel.

stellata, Ait.

Zawadskii, Herbich,

Stellaria graminea, Linn.

Wahlbergella pauciflora, DC. 


\section{PORTULACEÆ.}

Claytonia

perfoliata, Don.

* virginica, Linn.
Claytonia-contd. virginica, Linn., white var.

Monocosmia monandra, DC.

\section{TAMARISCINEÆ.}

Tamarix elegans, Spach.

\section{HYPERICINEÆ.}

Hypericum

Androsæmum, Linn.

Gebleri, Ledeb.

hirsutum, Linn.
Hypericum-contd. perforatum, Linn. pulchrum, Linn. quadrangulum, Linn., var. dubium, Leers.

\section{MALVACEÆ.}

*Abutilon vitifolium, Cav. Althæa ficifolia, Cav.

Hibiscus

diversifolius, Jacq.

pedunculatus, Thunb.

*Lavatera thuringiaca, Linn.

Malope grandiflora, Hort.

Malva

Alcea, Linn.

crispa, Linn.

Durieui, Spach.

moschata, Linn. var. alba, Hort.

Malva-contd.

nicæensis, All.

- var. Tenoreana, Kunze.

oxyloba, Boiss.

pulchella, Bernh.

sylvestris, Iinn.

- subsp. plebeia, Stev.

Palavia malvæfolia, Cav.

Sidalcea

candida, Gray.

malvæflora, Gray.

Murrayanna, Hort.

\section{LINEA.}

Linum

*alpinum, Linn.

grandiflorum, Linn., var. rubrum, Hort.
Linum-contd.

humile, Mill.

usitatissimum, Linn.

\section{GERANIACEÆ.}

Erodium

*malacoides, Willd.

Manescavi, Coss.

Geranium

anemonæfolium, L'Hérit.
Geranium-contd. armenum, Boiss.

* cinereum, Cav. dahuricum, DC. Endressii, Gray. 
Geranium-contd.

ibericum, Cav,

Lambertii, Sweet.

*lancastriense, With.

*macrorhizum, Linn.

*maculatum, Linn. pallidum, Sweet.

pratense, Linn., var. album, Hort.

*Robertianum, Linn.

sanguineum, Linn.

subcaulescens, L'Hérit.
Geranium-contd. sylvaticum, Linn. Wilfredii, Maxim.

Impatiens parviflorum, DC. Roylei, Walp.

Limnanthes grandiflora, $\mathrm{R}$. Br.

Oxalis alsinoides, Walp. tropæoloides, Hook.

Tropæolum majus, Linn.

minus, Linn., var, coccineum.

\section{RUTACEÆ.}

*Dictamnus tauricus, Hort.

Ruta graveolens, Linn. macrophylla, Hort.

\section{LEGUMINOSÆ.}

Astragalus

*alpinus, Linn.

bicolor, Lamk.

Cicer, Linn.

glycyphyllus, Linn.

himalayanus, $\mathrm{Kl}$.

oroboides, Hornem.

vicioides, Ledeb.

*Baptisia lutea, Hort.

Cytisus

hirsutus, Linn.

Laburnum, Linn.

- var. Adami, Hort.

- var. quercifolium, Hort.

scoparius, Link.

* - var. Andreanum, Hort.

- pendulus, Hort.

- præcox, Hort.

- præcox albus, Hort.

Galega officinalis, Linn.

\section{Genista}

germanica, Linn.

* hispanica, Linn.

sagittalis, Linn.

tinctoria, Linn.

- var. prostrata, Hort.

Kennedya rubicunda, Vent.

Lathyrus

angustifolius, Schkr.

Aphaca, Linn.

Clusii, Hort.

coccineus, Mill.

cyaneus, Koch.
Lathyrus-contd.

Drummondii, Hort.

flaccidus, Hort.

lævigatus, Waldst. et Kit.

latifolius, Linn.

Lowii, Hort.

luteus, Benth. et Hook.

macrorhizus, Wimm.

maritimus, Linn.

niger, Wimm.

odoratus, Linn.

sylvestris, Linn.

tenuifolius, Desf.

vernus, Benth.

Lupinus

arboreus, Sims.

Hartwegii, Lindl.

nootkaensis, Don.

perennis, Linn.

polyphyllus, Lindl.

Medicago pulchellus, Sweet.

falcata, Linn.

lupulina, linn.

murex, Willd.

sativa, Linn.

*tornata, Willd., var. minor. tuberculata, Willd.

Melilotus officinalis, Lamk.

Mimosa pudica, Linn.

Onobrychis sativa, Linn.

Ononis

repens, Linn. 
Ononis-contd.

rotundifolia, Linn.

*spinosa, Linn.

Oxytropis

campestris, DC.

*lactea, Hort.

lapponica, Wahl.

*uralensis, DC.

Scorpiurus sulcata, Linn. vermiculata, Linn.

Trifolium alpinum, Linn. Bocconi, Savi.

* badium, Schreb. diffusum, Ehrh.
Trifolium-contd.

gmellus, Pourr.

hybridum, Linn.

pannonicum, Linn., var.

* repens, Linn., var. pictum subterraneum, Linn.

Trigonella corniculata, Linn.

Ulex europæus, Linn.

Vicia

amphicarpa, Dorth.

Cracca, Linn.

onobrychoides, Linn.

Orobus, DC.

pyrenaica, Pourr.

villosa, Roth.

\section{ROSACEA.}

Acæna

*adscendens, Vahl.

microphylla, Hook. fil.

sanguisorba, Vahl.

Alchemilla

alpina, Linn, var. conjuncta, Bab.

Agrimonia agrimonioides, Linn.

Cotoneaster

Hookeriana, Hort.

Simonsii, Baker.

*thymifolia, Hort.

vulgaris, Lindl., var. pendula,

Dryas octopetala, Linn.

Geum

miniatum, Hort.

* parviflorum, Comm. montanum, Linn.

urbanum, Linn.

Potentilla

alchemilloides, Lap.

*Alfred Salter, Hort.

argentea, Linn., subsp. calabra, Ten.

arguta, Pursh.

argyrophylla, Wall.

bifurca, Linn., var. pectinata.

* chameleon, Hort.

Comarum, Nestl.

Fenzlii, Lehm.

inclinata, Vill.
Potentilla-contd.

kurdica, Boiss.

*Louis Van Houtte, Hort.

M'Nabiana, Hort.

Mooniana, Wight.

*nevadensis, Boiss.

opaca, Linn.

pedata, Willd.

* peduncularis, Don.

Phœbus, Hort.

salisburgensis, Haenke.

Schrenkianna, Regel.

sericea, Linn.

sp. (East Pyrenees.)

*Wm. Rouillard, Hort.

\section{Poterium}

dictyocarpum, Spach.

dodecandrum, Benth. et Hook.

*muricatum, Spach.

polygamum, W. K.

Sanguisorba, Linn.

Rosa rugosa, Thunb.

Rubus

corylifolius, Sm.

- var. foliis variegatis, Hort.

Pyrus latifolia, Syme.

Spiræa

Filipendula, Linn.

palmata, Thunb., var. albe, Hort.

- var. purpurea, Hort.

* gigantea. 


\section{SAXIFRAGEÆ.}

Boykinia aconitifolia, Nutt.

Heuchera

Arlei, Hort.

eylindrica, Dougl.

Drummondii, Hort.

*laxa, Hort.

ribifolia, F. et Lallem.

sanguinea, Eng.

Mitella pentandra, Hook.

Parnassia nubicola, Hook. f.

Ribes sanguineum, Pursh.

Saxifraga

affinis, Don.

aizoides, Linn,, var. aurantiaca, Hort.

Aizoon, Jacq.

- var, notata, Schott.

- var. pectinata, Schott.

- var. rosularis, Schleich.

angulosa, Schott.

*aspera, DC.

bronchialis, Linn.

Bucklandii, Hort.

- var. major, Hort.

* canaliculata, Boiss. ceratophylla, Dry. condensata, Gmel. cordifolia, Hort. corsicana, G. et G. crustata, Vest.

cymbalaria, Linn.

decipiens, Ehrh.

densa, Willd.

diversifolia, Wall.

elatior, Bbrst.

Geum, Linn.

- var. elegans, Mackay.

- var. gracilis, Mackay.

* - var. ovalifolia, Hort.

globosa.

${ }^{*}$ Gmelinii, Host.
Saxifraga-contd.

granulata, Linn.

groenlandica, Linn.

hederæfolia, Hochst.

hirsuta, Linn.

- var. minor, Hort.

Hostii, Tausch.

Huettiana, Boiss.

hibernica, Haw.

hypnoides, Linn.

hirta, Don.

intacta, Willd.

- var. farinosa, Hort.

kolenatiana, Rgl.

lingulata, Bell.

longifolia, Lap.

mutata, Linn.

nepalensis, Hort.

nervosa, Lap.

oppositifolia, Linn. var. fusca

Hort.

- var. pyrenaica, Hort.

paradoxa, Kit.

peltata, Torr.

platypetala, Sm.

Portæ, Stein.

Prostii, Sternb.

pygmæa, Haw.

pulchella, Don.

repanda, Willd.

retusa, Gouan.

Rhoeii, Schott.

rotundifolia, Linn.

serratifolia, Mack.

Stansfieldii, Hort.

Sturmiana, Schott.

tenella, WuIf.

tridentata, Don.

umbrosa, Linn.

valdensis, DC.

Tellima grandiflora, R. Br.

\section{CRASSULACEÆ.}

Sedum

acre, Linn.

Aizoon, Linn.

asiaticum, DC,

ibericum, Stev.

kamtschaticum, Fisch.

maximum, Sut. var. purpureum,

Koch.

oppositifolium, Sim.

Rhodiola, DC.
Sedum-contd.

rufescens, Ten.

* spathulatum, Waldst. et Kit.

spurium, Bbrst.

Sempervivum

Braunii, Ledeb.

flagelliformis, Fisch.

globiferum, Linn.

Pomelii, Lamotte. 


\section{DROSERACEÆ.}

Drosera capensis, Linn.

Drosera spathulata, Labill.

\section{LYTHRARIEA.}

Lythrum

hirsutum.

Salicaria, Linn.

- var. grandiflorum, Hort.
Nesæa

triflora, H.B.K.

verticillata, H.B.K.

\section{ONAGRARIEÆ.}

Epilobium

angustifolium, Linn. boreale, Hauskn.

brachycarpum, Presl.

Fleischerii, Hochst.

latifolium, Linn.

montanum, Linn.

nummulariæfolium, Cunn.

- var. longipes, Hort.

rosmarinifolium, Haenke.

sericeum, Benth.
Enothera

biennis, Linn.

fruticosa, Linn.

glauca, Michx.

- var. Fraserii.

pumila, Linn.

riparia, Nutt.

Sellowii, Link.

taraxacifolia, Sweet.

triloba, Nutt.

LOASE $Æ$.

Loasa lateritia, Gill et Hook. | Loasa tricolor, Lindl.

\section{CUCURBITACEA.}

Bryonia alba, Linn

Momordica Elaterium, Linn.

\section{UMBELLIFERA.}

Anthriscus sylvestris, Hoffm.

Archangelica decurrens, Ledeb. officinalis, Hoff.
Arracacia arguta, Benth. et Hook.

Astrantia major, Linn.

Bupleurum

Candollei, Wall. 
Bupleurum-contd.

graminifolium, Vahl.

ranunculoides, Linn.

semi-compositum, Linn.

Carum Carui, Linn.

Bulbocastanum, Koch.

Chærophyllum

aromaticum, Jacq.

aureum, Linn.

Coriandrum sativum, Linn.

Daucus pusillus, Michx.

Eryngium agavæfolium, Delar.

Ferula

assafœetida, Linn.

tingitana, Linn.
Fœniculum vulgare, Gaertn.

Heracleum

angustifolium, Sm.

barbatum, Ledb.

giganteum, Fisch.

Sphondylium, Linn.

Laserpitium crithmum, Link.

Ligusticum scoticum, Linn.

Meum athamanticum, Jacq.

Myrrhis odorata, Scop.

Enanthe

globulosa, Linn.

apiifolia, Brot.

*Sanicula europæa, Linn.

Smyrnium perfoliatum, Mill.

\section{ARALIACE尼.}

Aralia hispida, Michx.

\section{CAPRIFOLIACEÆ.}

Leycesteria formosa, Wall.

Viburnum Opulus, Linn.

\section{RUBIACEA.}

Asperula

azurea, Jaub. et Spach.

cynanchica, Linn.

lævigata, Linn.

Galium tinctoria, Linn.

Aparine, Linn.

boreale, Linn.

humifusum, Bbrst.
Galium-contd. parisiense, Linn. pusillum, Sm. rubioides, Linn. rubrum, Linn. saxatile, Linn. urecolatum. verum, Linn.

*Rubia angustifolia, Linn.

\section{VALERIANE $Æ$.}

Centranthus ruber, DC.

\section{*Valeriana}

dioica, Limn.

officinalis, Linn.

pyrenaica, Linn.

Valerianella

congesta, Lindl.

Morisonii, DC.

olitoria, Mœench.

Szovitsiana, F.M. 


\section{DIPSACEA.}

Cephalaria tatarica, Gmel,, var. minor. Dipsacus

ferox, Loisl.

Fullonum, Linn.

Scabiosa

alpina, Linn.
Seabiosa-cont.

caucasica, Bbrst.

dipsacifolia, Schott

elata, Hornem.

*prolifera, Linn.

pubescens, Kit.

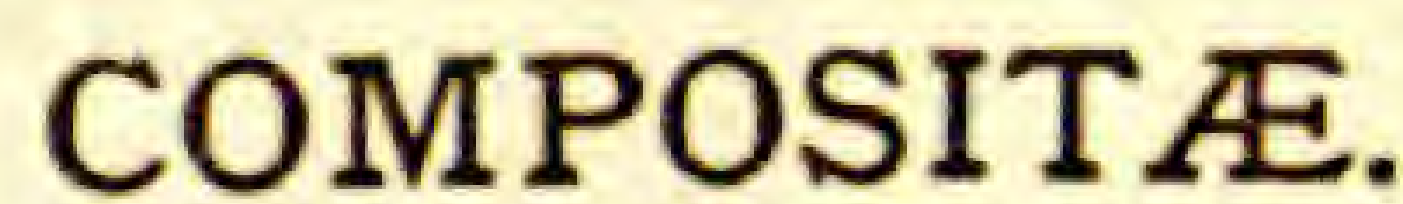

Anacyclus

pulcher, Bess.

Pyrethrum, Cass.

Arctium majus, Schk.

Andryala lanata, Linn.

Arnica

*Chamissonis, Less.

*montana, Linn.

Antennaria dioica, R. Br.

Anthemis Bourgœi, Boiss. et Reut.

Artemisia

Absinthium, Linn.

græca, Hort.

Aster neglecta, Spr.

alpinus, Linn.

- var. albus.

peregrinus, Pursh.

*Brachycome Sinclairii, Hook. fil.

Calendula

officinalis, Linn.

Carduus

- var. Hen and Chicken.

inermis, Pers.

nutans, Linn.

pannonicus, Linn. fil.

- var. divaricatus.

tataricus, Willd.

Centaurea

calcitrapoides, Linn.

cinerea, Lamk.

Clementei, Boiss.

Cyanus, Linn.

dealbata, Willd.

Debauxi, Hort.

elata, Poir.

Jacea, Linn.

pallida, Friv.

* pectinata, Linn.

scabiosa, Linn.

Chrysanthemum

corymbosum, Linn.

Roxburghii, Desf.
Chrysanthemum-contd.

segetum, Linn., var. grandiflorum, Hort.

tricolor, Hort.

tripteris, Hort.

Cnicus

purpureum, Pers.

*Biebersteinii, Hort.

elatus, Hort.

Kotschyi, Schultz.

eriophorus, Hoffm.

heterophyllus, Willd.

olaraceus, Linn.

palustris, Hoffm.

* purpureus, Hort.

rigidus, Hort.

Coreopsis Drummondii, Hort.

Crepis

aurea, Less.

- var. crocea, Fries.

blattarioides, Vill.

succisæfolia, Tausch.

Eupatorium

*ageratoides, Linn.

verticillatum, Muhl.

Gaillardia

maxima, Hort.

Gerbera

splendidissima, Hort.

anandria, Schultz.

Kunzeana, A, Br. et Ascher.

Helipterum Manglesii, Benth.

Hieracium

alpinum, Linn., var. melanocephalum, Tausch.

amplexicaule, Linn.

angulosum, Linn.

bupleuroides, Gm.

caulescens, Linn.

cerinthoides, Linn., var, molle

collinum, Fries.

compositum, Lap. 
Hieracium-contd.

cydonifolium, Lamk.

elongatum, Lap.

humile, Jaeq.

laciniatum, Monn.

nigrescens, Willd.

Pilosella $\times$ aurantiacum, Linn. pulmonarium, Sm.

rupestre, Bab.

saxatile, Jacq.

stoloniferum, Kit.

strictum, Fries.

sylvaticum, Fries.

undulatum, Ait.

vulgatum, Fries.

Hypochæris maculata, Linn.

Kaulfussia amelloides, Linn.

Lactuca alpina, Benth.

Lecntopodium alpinum, Cass.

Iiatris

pilosa, Willd.

pumila, Lodd.

spicata, Willd.
Onopordon

Acanthium, Linn.

illyrieum, Linn.

Rudbeckia

fulgida, Ait.

triloba, Linn.

Santolina alpina, Linn.

* Saussurea hypoleuca, Spreng.

Scorzonera hispanica, Linn.

Senecio Jacobæa, Linn.

Serratula

arguta, Fisch.

coronata, Linn.

quinquefolia, Bbrst.

Solidago Virga-aurea, Linn.

Sonchus

lapponicus, Linn.

palustris, Linn.

Tragopogon

crocifolius, Linn.

orientalis, Linn.

*Tussilago alpina, Linn.

\section{STYLIDIEÆ.}

Stylidium adnatum, R. Br.

Stylidium fruticosum, R. Br.

\section{CAMPANULACE $Æ$.}

Campanula

*abietina, Hort.

alliariaefolia, Willd.

barbata, Linn.

- var. alba.

Baumgartenii, Becker.

carpatica, Linn, fil.

elegans, R. et S.

glomerata, Linn.

Hendersonii, Hort.

latifolia, Linn.

- var. alba, Hort.

- var. macrantha, Fisch.

Medium, Linn.

*nitida, Ait., var. alba, Hort.

nobilis, Lindl.

pelviformis, Hort.

persicifolia, Linn.

pulchella, Salisb., var. polypetala, Hort.

pyramidalis, Linn.

- var. alba, Hort.

rhomboidalis, Linn.
Campanula-contd.

Scheuchzeri, Lodd., var. glabra, Hort.

Speculum, Linn,

tomentosa, Lam.

turbinata, Schott.

urticifolia, Schmidt.

vesula, All,

Vidallii, H. C. Wats.

Codonopsis

clematidea, Schrenk.

ovata, Benth.

Cyananthus lobatus, Wall.

Jasione

montana, Linn.

perennis, Lam.

Lobelia Wallichii, Steud.

Phyteuma

orbiculare, Linn.

Scheuchzeri, All.

Symphiandra

Hoffmanni, Panc.

pendula, DC. 


\section{ERICACEÆ.}

Dabœocia Erica

*polifolia, Don, var, nana, Hort.

cinerea, Linn., var. bicolor, Hort.

- var. spicata alba, Hort.

- var. purpurea, Hort.

* stricta, Don.

Gaultheria

carnea, Hort.

hispida, $\mathrm{R}$. Br.

Shallon, Pursh.

Rhododendron ciliatum, Hook. fil.

ferrugineum, Linn.

* - var. album, Sweet.

\section{PLUMBAGINEÆ.}

Armeria

bupleuroides, Hort.

fasciculata, Willd.

Hallerii, Wall.

juncea, Gir.

maritima, Willd., var. alba, Hort.
Armeria-contd.

Statice pubescens, Link.

globulariæfolia, Desf. Gmelini, Willd.

\section{PRIMULACEÆ.}

Androsace

coronopifolia, Andr.

lactea, Linn.

Cortusa Mathioli, Linn.

Cyclamen hederæfolium, Willd.

Dodecatheon integrifolium, Michx.

Lysimachia

*affinis, Penny.

*Leschenaultii, Duby.

*vulgaris, Linn.

Primula

*auricula, Linn.
Primula-contd.

* cortusoides, Linn.

* elatior, Jacq.

* grandis, Trautv.

*inflata, Lehm.

*intermedia, Hort.

*japonica, Gray.

*mollis, Nutt.

*sikkimensis, Hook.

*variabilis, Bast.

* vulgaris, Huds.

\section{ASCLEPIADEA.}

*Cynanchum nigrum, Linn.

\section{GENTIANEÆ.}

${ }^{*}$ Chlora perfoliata, Linn.

Gentiana

adscendens, Pall.

asclepiadea, Linn.

- var. alba, Hort.

Burserii, Lap.

Kochiana, Pers.
Gentiana-contd. phlogifolia, Sch. septemfida, Pall.

— var. cordifolia, Boiss.

tibetica, King.

verna, Linn.

Wallichii, Hort. 


\section{POLEMONIACEÆ.}

Collomia

coccinea, Lehm.

Gilia

grandiflora, Dougl.

achilleæfolia, Benth.

alba, Hort.

tricolor, Benth.

Leptosiphon androsaceus, Benth.

Polemonium

cœeruleum, Linn.

- var. album, Hort.
Polemonium-contd.

- var. bipinnatifidum, Hort.

- var. fasciatum, Hort.

flavum, Greene.

humile, Willd.

pauciflorum, Wats.

pulchellum, Bunge.

Richardsonii, Graham.

sp. (Gilgit).

\section{HYDROPHYLLACEÆ.}

Eutoca viscida, Benth.

Hydrolea spinosa, Linn.

Nemophila

atomaria, F. et Mey.

insignis, Benth.
Nemophila-contd. maculata, Benth.

*Romanzoffia sitchensis, Cham.

Whitlavia minor, Hans.

\section{BORAGINE $Æ$.}

Anchusa

incarnata, Schrad. sempervirens, Linn.

*Arnebia echioides, A. DC.

Borago officinalis, Linn.

Cerinthe alpinum, Kit.

Cynoglossum nervosum.

* Mertensia oblongifolia, G. Don.

Myosotis arvensis, Sibth. australis, R. Br.

*Symphytum asperrimum, Bbrst.

\section{CONVOLVULACEAE.}

Convolvulus

minor, Catesb.

pentapetaloides, Linn.
Convolvulus-contd. siculus, Linn. undulatus, Cav.

\section{SOLANACEÆ.}

Anisodus luridus, Link.

Atropa

Belladonna, Linn. Datura

- var. fructu-flava, Hort.

inermis, Jacq.

Leichardtii, F. et M. quercifolia, H. B. K.
Datura-contd.

Tatula, Linn.

Hyoscyamus niger, Linn.

Nicotiana rustica, Linn.

Scopolia lurida, Desf.

Solanum

Dulcamara, Linn.

nigrum, Linn. 


\section{SCROPHULARINEÆ.}

Collinsia

bicolor, Benth. grandiflora, Dougl.

Calceolaria mexicana, Benth,

Celsia cretica, Linn.

Digitalis purpurea, Linn.

Erinus

alpinus, Linn.

hispanicus, Pers.

Linaria

alpina, DC.

bipartita, Willd.

genistifolia, Mill.

vulgaris, Mill.

Mimulus

moschatus, Dougl.

roseus, Dougl.

Pentstemon

Menziesii, Hook.

nitida, Dougl.

procerus, Dougl.

speciosus, Dougl.

Ramondia pyrenaica, Rich.

Scrophularia

alata, Gill.

aquatica, Linn.

glandulifera, Clarke.

mandshurica, Regel.

nodosa, Linn.

scopolia, Hoppl.

sp. (Dr King).

trifoliata, Linn.

vernalis, Linn.

Verbascum

floccosum, Waldst. et Kit.
Verbascum-contd. Thapsus, Linn.

Veronica arvensis, Linn. austriaca, Linn.

Beccabunga, Linn. Bidwillii, Hook. fil. Buxbaumii, Linn. candida, Lodd. Colensoi, Hook. fil. corymbosa, Hort., var. variegata decussata, Ait.

fruticulosa, Linn. gentianoides, Vahl.

- var. variegata, Hort.

grandis, Fisch.

Guthriana, Hort.

hederæfolia, Linn.

Jaequinii, Baumg.

laciniata, H. K.

officinalis, Linn., var. hirsuta, Hopk.

- var. rubra, Hort.

orchidea, Crantz.

pinnata, Linn.

Ponæ, Gouan.

saxatilis, Linn.

serpyllifolia, Linn., var. humifusa, Dicks.

spicata, Linn.

-- var. alba.

- var. hybrida.

spuria, Linn.

urticifolia, Linn.

Wulfenia carinthiaca, Jacq.

\section{OROBANCHACEAE.}

*Orobanche rubra, Sm.

\section{LENTIBULARIEA.}

* Pinguicula vulgaris, Linn.

\section{GESNERACEÆ.}

*Haberlea rhodopensis, Friw, var. Streptocarpus Dunnii, Hook. f. robusta, Hort. Rhexii, Lindl.

\section{VEBBENACEA .}

Clerodendron Thomsonæ, Balf., var. Balfourii, Hort. 


\section{LABIAT $Æ$.}

*Ajuga pyramidalis, Linn.

Ballota

italica, Benth. nigra, Linn.

Brunella grandiflora, Linn. laciniata, Linn. vulgaris, Linn.

- var. hispida, Hort. Webbiana, Hort.

Calamintha patavina, Host.

Dracocephalum

nutans, Linn.

speciosum, Benth.

stamineum, Kar. et Kir.

thymiflorum, Linn.

Horminum pyrenaicum Linn.

Hyssopus officinalis, Linn.

Lamium

Galeobdolon, Crantz.

maculatum, Linn.

* Orvala, Linn.

purpureum, Linn., var. album.,

\section{Leonurus} Hort.

Cardiaca, Linn.

villosus, Desf.

Lycopus exaltatus, Linn.

Marrubium

peregrinum, Linn.

vulgare, Linn.

Mentha arvensis, Linn.

* Melissa grandiflora, Benth.

* Micromeria piperella, Benth.

Nepeta

altissima, Schrenk.
Nepeta-contd. macrantha, Fisch. pannonica, Jacq.

Origanum pulchrum, Boiss, et Heldr. vulgare, Linn.

Orthosiphon stamineus, Benth.

Phlomis

fruticosus, Linn.

Russeliana, Lag.

*Samia, Willd.

Salvia

argentea, Linn,

carduacea, Bth.

dominica, Linn.

limbata, Meyer.

nubicola, Wall.

officinalis, Linn.

pratensis, Linn.

Sibthorpii, Sibth.

taraxacifolia, Cost. et Bal.

Verbenaca, Linn.

Satureja

hortensis, Linu.

spicata, Vis.

Scutellaria

alpina, All.

altaica, Fisch.

Stachys

galericulata, Linn.

Betonica, Benth.

Teucrium

* - var. carnea.

pyrenaicum, Linn.

Scorodonia, Linn.

Thymus Serpyllum, Fries.

\section{PLANTAGINEÆ.}

\section{Plantago}

arenaria, Waldst, et Kit.

Coronopus, Linn.

gentianoides, $\mathrm{Sm}$.

Hookeriana, F. et. M.

kamtschatica, Cham.

lanceolata, Linn.
Plantago-contd. major, Linn.

- var. monstrosa, Hort.

- var. rubra, Hort.

montana, Lamk.

Psyllium, Linn

\section{AMARANTACEÆ.}

Amaranthus melancholicus, Linn. 


\section{CHENOPODIACEÆ.}

Atriplex

laciniata, Linn. hortensis, Linn. Beta

patellaris, Moq.
Beta-contd.

Rapa, Dumort.

Chenopodium

Bonus-Henricus, Linn.

Quinoa, Willd.

Spinacia oleracea, Liun.

\section{POLYGONACE $Æ$.}

Oxyria digyna, Hill.

Polygonum

Bistorta, Linn.

- var. major, Hort.

capitatum, Hamilt.

sphærostachyum, Meisn.

viviparum, Linn.

Pterostegia drymarioides, Fisch.

Rheum

collinianum, Baill.

officinale, Baill.

palmatum, Linn.

- var. tanguticum, Rgl.

sibiricum, Pall.

Rumex
Acetosella, Linn.
alpinus, Linn.
aquaticus, Linn.
compactus, Hort.
exaltatus, Hort.
intermedius, DC.
Hydrolapathum, Huds.
nepalensis, Spr.
Patientia, Linn.
rupestris, Le Gall.
sanguineus, Linn.
sanguisorbæfolius, Hort.
staticæfolia Hort.
stenophyllus, Ledeb.

\section{ARISTOLOCHIACEÆ.}

Asarum canadense, Linn.

\section{THYMELAEACEÆ.}

Daphne Mezereum, Linn.

\section{EUPHORBIACEÆ.}

Euphorbia

glaucescens, W.

Euphorbia-contd.

Hierosolymitans, Boiss.

Myrsinites, Linn.

\section{URTICACEÆ.}

Urtica

dioica, Linn.

grandidentata, Banks.

magellanica, Poir.
Urtica-contd. palmata, Lesch. pilulifera, Linn. 


\section{BETULACEÆ.}

Alnus

cordifolia, Ten.

glutinosa, Willd.
Betula alba, Linn., var. pendula

\section{SCITAMINE $Æ$.}

Canna floribunda, Bche.

Hedychium Roxburghii, Wight

\section{BROMELIACEÆ.}

Billbergia zebrina, Lindl.

\section{HAMMADORACE $Æ$.}

Anigozanthos rufa, Labill.

Peliosanthes, sp.

*Wachendorfia brevifolia, Ker

\section{IRIDEA.}

Aristea

Crocus

cyanea, Ait.

Ecklonii, Baker.

asturicus, Herb.

*Kotschyanus, Koch.

medius, Balbis.

*Phologandrus, Orphan.

vernus, All.

Homeria collina, Vent.

Iris

asiatica, Hort.

correana, Hort.

lævigata, F. et. M., var. stricta, Hort.

lurida, Soland.

* prismatica, Pursh.

Pseud-acorus, Linn.
Iris-contd.

- var. variegata, Hort.

rubicaulis, Hort.

setosa, Pall.

sibirica, Linn.

triflora, Balb.

Xiphioides, Linn.

Lapeyrousia cruenta, Baker.

Libertia

grandiflora, Sweet.

ixioides, Spreng.

Moræa bicolor, Spr.

Sisyrinchium

Bermudiana, Linn. grandiflorum, Dougl. mucronatum, Michx.

Sparaxis pendula, Ker.

Trichonema rosea, Banks.

\section{AMARYLLIDEÆ.}

*Alstrcemeria aurea, Grah.

Galanthus plicatus, Bbrst.

Hæmanthus puniceus, Linn.

Imantophyllum miniatum, Hook.

Leucoium

vernum, Linn.

Narcissus

- var. carpaticum, Steud.

*alariz, Hort.

*bicolor, Linn., var. Horsfieldii, Hort.

*Bulbocodium, Linn.
Narcissus-contd.

*calathinus, Linn.

citrinus, Baker.

Grælsii, Webb.

juncifolius, Lag.

*muticus, Gay.

nobilis, Haw.

*pallidus, Poir.

- var. præcox, Hort.

*polyanthus, Lois

* - var.

pumilus, Haw. 
LILIACEA.

Allium

ampeloprasum, Linn., var.

Babingtonii, Borr.

arenarium, Schrad.

* coeruleum, Pall.

Cyrilli, Ten.

Dioscoridos, Sm.

fistulosum, Linn.

giganteum, Rgl.

globosum, Redouté.

- var. albidum, Hort.

*karataviense, Rgl.

Kochii, Hort.

liliflorum, Zeyher.

M'Leanii, Baker.

M'Nabianum, Hort.

*narcissiflorum, Vill.

paradoxum, Don.

pulchellum, Don.

* rubens, Sch.

*serecolatum, Hort.

Victorialis, Linn.

vineale, Mill.

Anthericum

Liliago, Linn.

liliastrum, Bert.

* - var. major, Hort.

Arthropodium paniculatum, Sims.

*Asphodelus ramosus, Linn.

*Brodiæa congesta, Sm.

Bulbinella Hookerii, Benth.

Bulbocodium vernum, Linn.

Camassia

Brownii, Hort.

esculenta, Lindl. tardiflora, Hort.

Chionodoxa Lucilliæ, Boiss.

Chlorogalum Leichtlinii, Baker.

Chlorophytum orchidiastrum, Lindl.

Colchicum

*crociflorum, Andr.

speciosum, Stev.

Convallaria

majalis, Linn.

Erythronium

- var. major, Hort.

Dens-canis, Linn.

giganteum, Lindl.

grandiflorum, Hort.

Fritillaria Meleagris, Linn.

Funkia

albo-marginata, Hort.

Sieboldiana, Lodd.
*Helonias bullata, Linn.

Hyacinthus amethystinus, Linn.

Lilium

*aurantiacum, Sieb.

canadense, Linn., var. penduliflorum Hort.

colchicum, Stev.

giganteum, Wall.

* Martagon, Linn.

* - var. dalmaticum, Maly.

* monadelphum, Bbrst.

Milla laxa, Baker.

${ }^{*}$ Littonia modesta, Hook.

Muscari

Argæi, Schott.

armeniacum, Baker.

atlanticum, Boiss. et Reut.

*botryoides, Mill. var. pallidus, Hort.

racemosum, Mill.

Szovitsianum, Rgl.

Ornithogalum

Elwesii, Hort.

nutans, Linn.

Puschkinia

libanotica, Zucc., var. compacta, Hort.

Scilla scilloides, Adams.

amœna, Linn.

campanulata, var. rubra, Hort. sibirica, Andr.

verna, Huds.

*Smilacina racemosa, Desf.

Tofieldia

palustris, Huds., var. earinthiaca.

glutinosa, Pers.

Tricyrtis australis, Hort.

Trillium grandiflorum, Salisb.

Triteleia

Murrayana, Hort.

* uniflora, Lindl.

* - var.

Tulipa

altaica, Pall.

Gesneriana, Linn.

pulchella, Fenzl.

*Veratrum nigrum, Linn.

Zygadenus

* glaucus, Nutt.

- var. humilis. 


\section{COMMELINACEÆ.}

Tradescantia virginica, Linn.

Tradescantia virginica, Linn., var. alba, Hort.

\section{JUNCACEÆ.}

Juncus

*balticus, Willd.

conglomeratus, Linn.

effusus, Linn.

glaucus, Ehrh.

lamprocarpus, Ehrh.

tenuis, Willd.

Juncus-contd. trifidus, Linn.

Luzula

campestris, DC.

Forsteri, DC.

pilosa, Willd.

spicata, DC.

\section{PALMEÆ.}

Euterpe edulis, Mart.

Ptychospherma elegans, Labill

TYPHACEÆ.

Sparganium ramosum, Huds.

\section{AROIDEÆ.}

Anthurium acaule, Sweet.

Arum

Arum-contd.

tenuifolium, Linn.

palæstinum, Boiss.

Symplocarpus foetidus, Salisb.

\section{ALISMACEÆ.}

Alisma ranunculoides, Linn.

\section{CYPERACEÆ.}

Carex

*alpina, Sw.

*aquatilis, Wahl.

atrata, Linn.

binervis, $\mathrm{Sm}$.

capillaris, Linn.
Carex-contd.

Drymingii, Hort.

fulva, Good.

*Grahamii, Boott. microglochin, Wahl.

* paniculata, Linn. 
Carex-contd.

pilulifera, Linn., var. variegata, Hort.

præcox, Jacq.

* riparia, Curtis, var. variegata, Hort.

stictocarpa, Sm.
Carex-contd.

sylvatica, Huds. ustulata, Wahl.

vulpina, Linn.

Eleocharis palustris, R. Br.

Eriophorum polystachyum, Linn.

*Schoenus nigricans, Linn.

\section{GRAMINEÆ.}

Agrostis

alba, Linn., var. stolonifera, Linn.

*pulchella, Kth.

*Reuterii, Boiss.

vulgaris, With.

Aira bottnica, Vahl.

Alopecurus

alpinus, Sm.

geniculatus, Linn.

nigricans, Hornem.

pratensis, Linn., var. variegata, Hort.

Anthoxanthum

gracile, Biv.

odoratum, Linn.

Arrhenatherum avenaceum, Beauv.

Avena

alpina, Kunth.

barbata, Bbrst.

brevis, Roth.

fatua, Linn.

pratensis, Linn.

pubescens, Linn.

*sterilis, Linn.

Beckmannia erucæformis, Host.

Brachypodium pinnatum, Beauv.

Briza

gracilis, Hort.

maxima, Linn., var, rufibarbis, Hort.

Bromus

congestus, Hort.

Gussonii, Parl.

japonicus, Chumb.

Krausei, Rgl.

macrostachys, Desf.

Schraderii, Kth.

sylvatica, Pall.

unioloides, Kth.

Brizopyrum siculum, Lamk.

Cinna mexicana, Beauv.

Coix Lachryma, Linn.

Cynosurus polybracteatus, Poir.

Dactylis glomerata, Linn., var. aurea,

Hort:

Deschampsia caespitosa, Beauv. flexuosa, Trin.

Elymus

arenarius, Linn. glaucifolius, Gray.

tener, Linn.

villosus, Muhlbg.

Eragrostis peruviana, Trin.

Festuca

arundinacea, Schreb.

duriuscula, Linn.

elatior, Linn.

- var. pratensis, Huds.

Hallerii, All.

Myurus, Linn.

ovina, Linn.

- var. tenuifolia.

rubra, Linn.

Scheuchzerii, Gaud.

*thalasica, Ktz.

Holcus

lanatus, Linn. mollis, Linn.

Hordeum jubatum, Linn. vulgare, Linn.

*Knappia agrostidea, Sm.

Melica uniflora, Retz.

Milium effusum, Linn.

Molinia cœrulea, Moench, var. variegata, Hort.

Oryza sativa, Linn.

Phalaris

arundinacea, Linn.

* - var. variegata, Hort.

canariensis, Linn.

minor, Retz.

trigyna, Host. 
Phleum

*pratense, Linn.

Poa tenue, Schrad.

chinensis, Kœnig. nemoralis, Linn. - var. sempervirens, Hort. palustris, Linn. sudetica, Hænke.
Spartina cynosuroides, Willd. Stipa pennata, Linn. tenacissimum, Linn.

Triticum chinense, Trin. elymoides, Hornem. villosum, Beauv. violaceum, Hornem.

\section{CONIFERÆ.}

Cupressus macrocarpa, Hort.

*Pinus

Jeffreyii, Balf.

Koræensis, Sieb.
Taxus

baccata, Linn.

- var. fastigiata, Lindl.

canadensis, Willd., var. Washingtonia, Hort.

ISAAC BAYLEY BALFOUR,

Keeper.

Edinburgh, Febriary 1892.

\section{ROBERT LINDSAY,}

Curator of the Garden.

\section{A. D. RICHARDSON,}

Foreman in charge of the Arboretum.
ALEX. ANDERSON,

Foreman in charge of the Herbaceous Department.

Note.-Correspondents are requested to address their communications to the KEEPER.

PRINTED FOR HER MAJESTY'S STATIONERY OFFICE BY NEILL AND CO., EDINBURGH. 




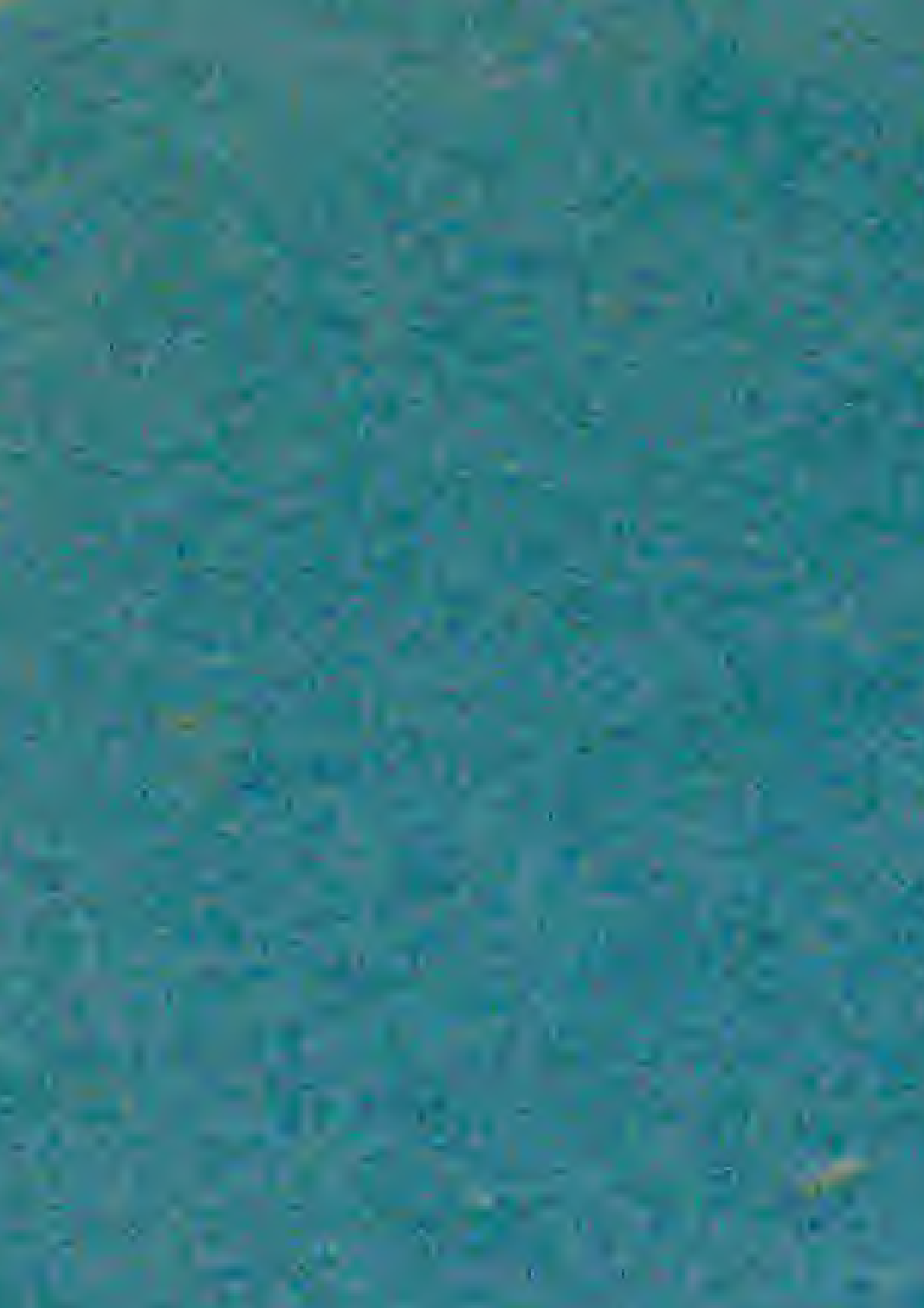

ictions

(2)

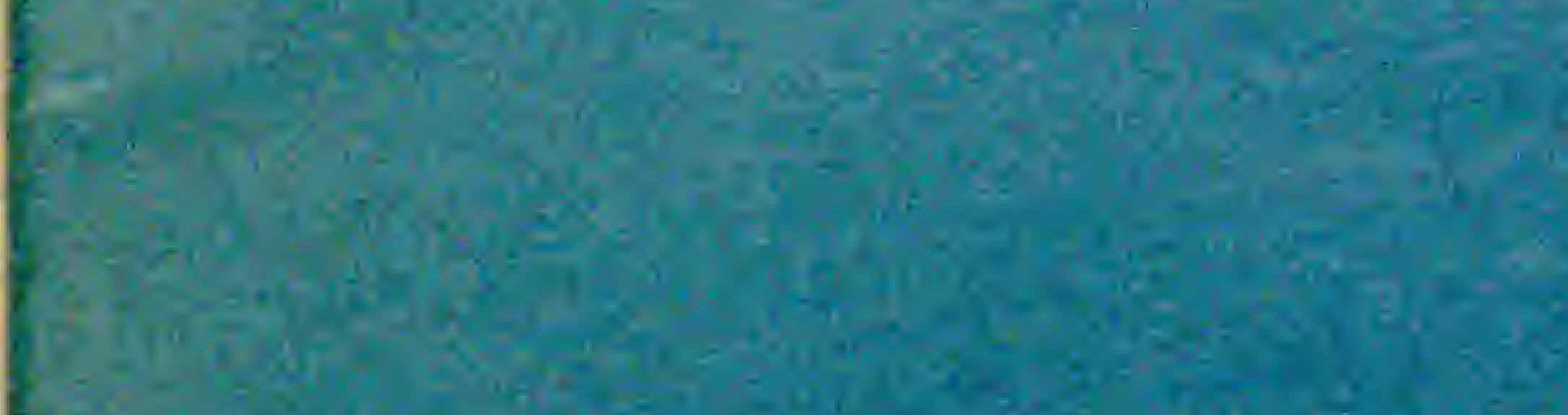

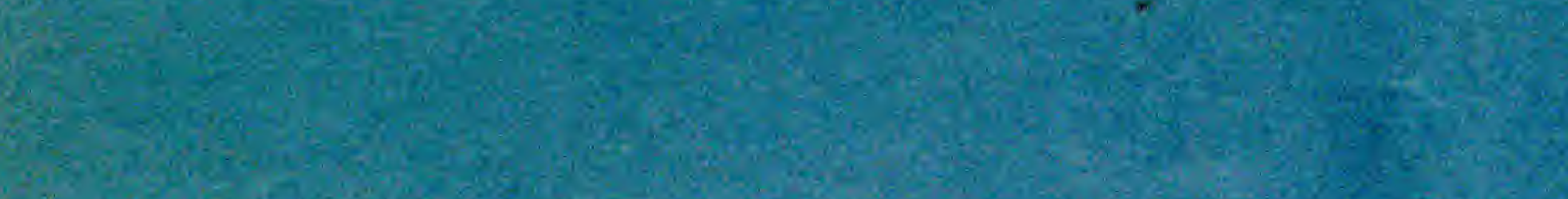

atsing

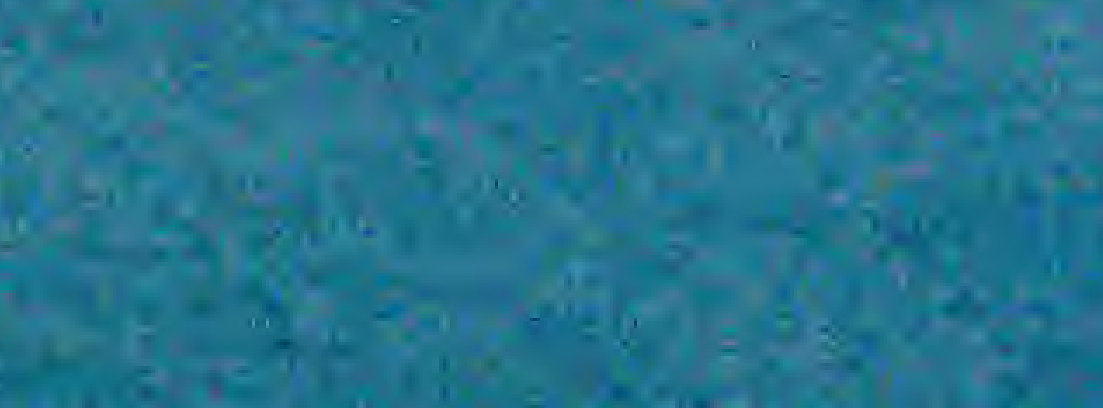

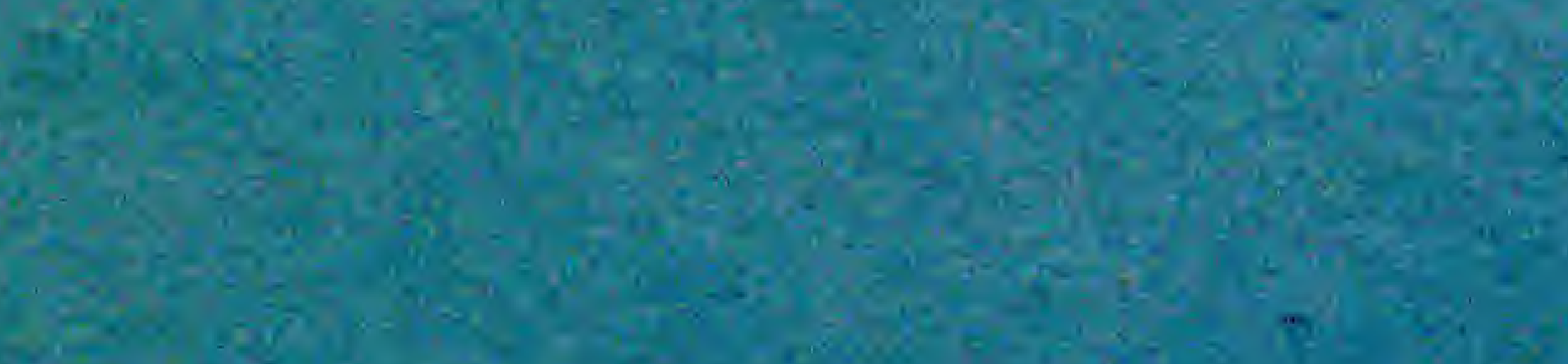

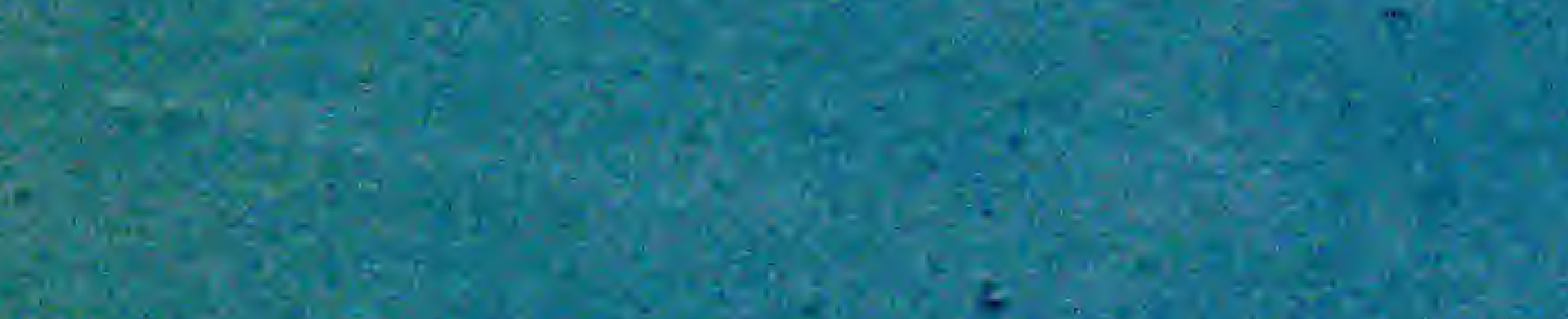

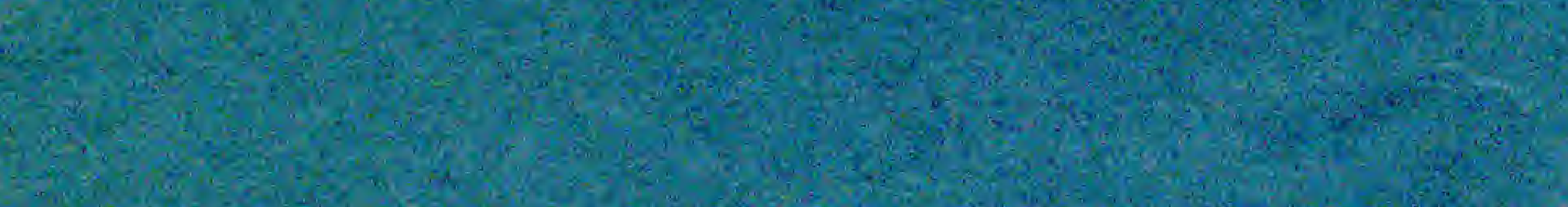

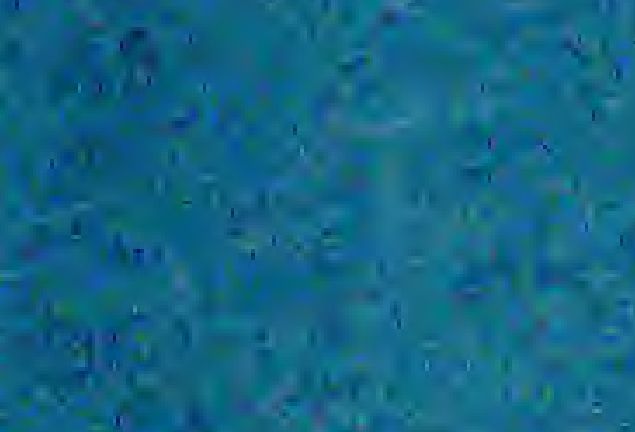

(2)

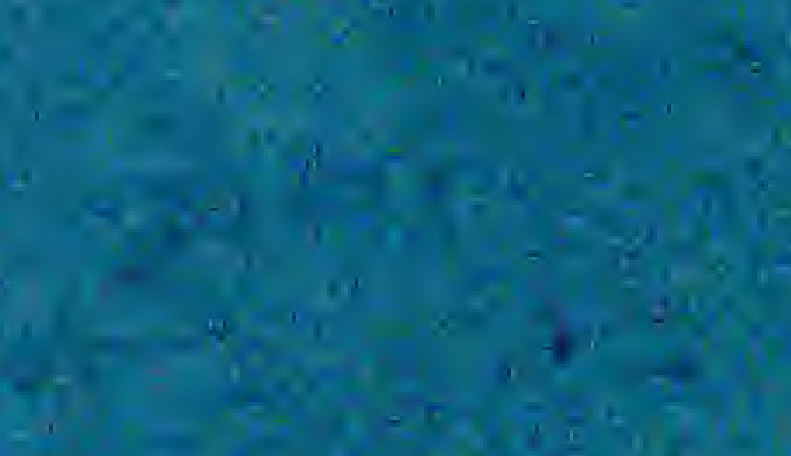

(2)

- 20

(1)

3

C.5.

(2)

(2) W

20. (5) 


\section{ROYAL BOTANIC GARDEN, EDINBURGH}

\section{LIST OF SEEDS}

COLLECTED DURING 1892 .

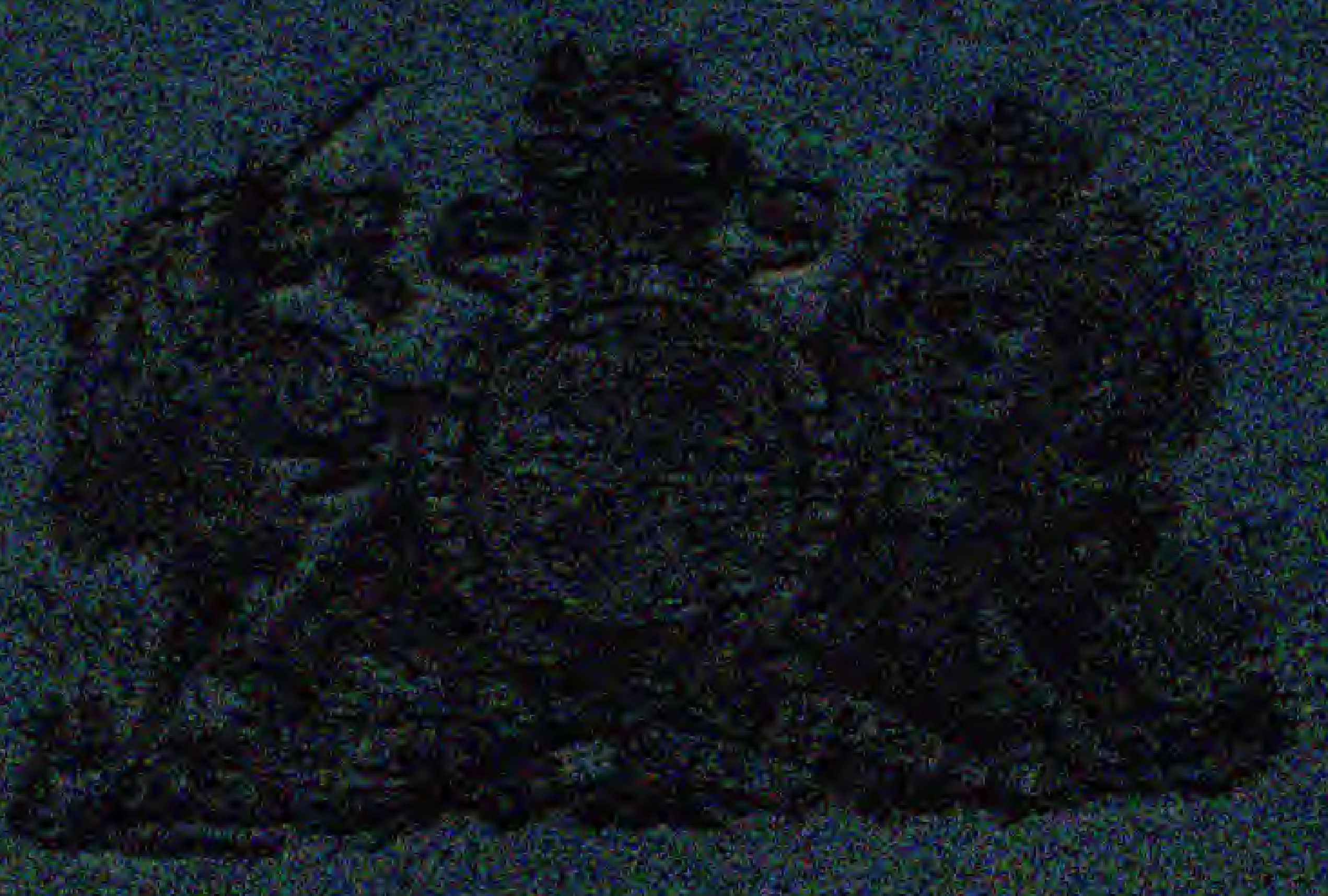

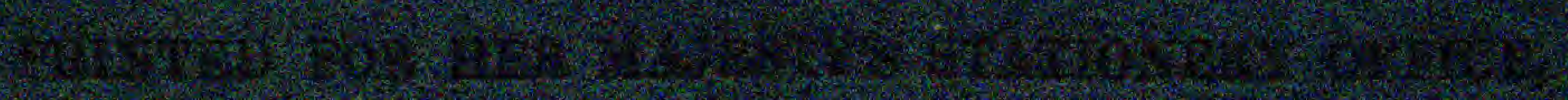




\section{ROYAL BOTANIC GARDEN, EDINBURGH.}

\section{LIST OF SEEDS}

\section{COLLECTED DURING 1892.}

An asterisk * indicates that the quantity of seed collected is very small.

\section{RANUNCULACEÆ.}

Aconitum

Anthora, Linn.

eminens, Koch.

longigaleatum, Hort.

Lycoctonum, Linn.

— var. moldavicum (A. moldavicum, Bess.).

- var. septentrionale (A. septentrionale, Baumg.).

- var. alba.

* - Napellus, Linn.

Actæa

paniculatum, Lamk.

spicata, Linn.

Adonis

- var. rubra, Michx.

flammea, Jacq.

pyrenaica, DC.

Anemone vernalis, Willd.

alpina, Linn.

- var. sulphurea, DC.

*angulosa, Lamk.

*apennina, Linn.

baikalensis, Hort.

hepatica, Linn.

*narcissiflora, Linn.

*nemorosa, Linn.

* patens, Linn.

Pulsatilla, Linn.

ranunculoides, Linn.

rivularis, Buch.-Ham.

Aquilegia sylvestris, Linn

alpina, Linn.

atropurpurea, Willd.

Buergeriana, Sieb. et Zucc.

californica, A. Gray, var. alba,
Aquilegia-contd.

canadensis, Linn.

chrysantha, Gray.

cœrulea, James.

*flavescens, S. Watson.

olympica, Boiss.

pyrenaica, DC.

Stuartii, Hort.

viridiflora, Pall.

vulgaris, Linn.

- var. Kitaibelii, Schott.

Caltha

*Whitmaniana, Hort.

palustris, Linn.

- var. biflora (C. biflora, DC.).

radicans, T. F. Forst.

Cimicifuga americana, Michx.

Clematis

angustifolia, Jacq.

* diversifolia, Gilib.

erecta, All.

${ }^{*}$ Coptis trifolia, Salisb.

Delphinium

Brunonianum, Royle.

cashmirianum, Royle.

crassifolium, Schrad.

- var. turkestanicum, Rgl.

formosum, Boiss. et Reut.

hybridum, Steph.

Nahamah, Hort.

*nudicaule, Torr. et Gray. palmatifidum, Dec.

sp. (Yunan).

Wheelerii, Hort.

*Eranthis hyemalis, Salisb.

Helleborus fœtidus, Linn.

Isopyrum fumarioides, Linn.

* Myosurus minimus, Linn.

*Nigella orientalis, Linn. 


\section{Pæonia}

coralliua, Retz.

officinalis, Retz.

Ranunculus

*aconitifolius, Linn.

*aduncus, Gren.

amplexicaulis, Linn.

auricomus, Linn.

Chius, DC.

*Flammula, Linn.

*Friesianus, Jord.

incrassatus, Guss.

Lingua, Linn.

parviflorus, Linn.

Philonotis, Retz.

propinquus, Meyer.

repens, Linn.

*Reuterianus, Boiss.

trachycarpus, Fisch. et Mey.
Thalictrum

appendiculatum, Ledeb

Cornuti, Linn.

elatum, Jacq.

glaucum, DC.

lucidum, Linn.

minus, Linn.

Schweiggeri, Spreng.

Trollius simplex, Linn, var. Jordani, Hort.

asiaticus, Linn.

- var. hybridus, Hort.

- var. intermedius.

caucasicus, Stev.

* europæus, Linn.

japonicus, Hort.

laxus, Salisb.

\section{BERBERIDEÆ.}

Diphylleia cymosa, Michx.

| Podophyllum Emodi, Wall.

\section{PAPAVERACE $Æ$.}

Cathcartia villosa, Hook. fil.

Chelidonium

majus, Linn.

- var. grandiflorum (C. grandiflorum, DC.).

- var. laciniatum (C. lacinia-

Corydalis tum, DC.).

aurea, DC.

capnoides, Pers.

nobilis, Pers.

procumbens, Hort.

glauca Pers., var. rosea, Hort.

Eschscholtzia

californica, Cham.

- var. mandarin, Hort.

crocea, Benth.

Fumaria officinalis, Linn.

Glaucium

*flavum, Crantz.

luteum, Linn.

tricolor, Linn.

*Hypecoum procumbens, Linn.

Meconopsis cambrica, Vig.

- var. aurantiaca, Hort.

Wallichii, Hook.
Papaver

*alpinum, Linn.

apulum, Ten.

*arenarium, Biebrst.

coccum, Ledeb.

dubium, Linn.

*errabundum, Jord.

pilosum, Sibth.

- var. Heldreichii, Boiss.

modestum.

Murcelli.

- var. Van Houtt.

obtusifolium, Desf.

opiiferum, Forsk.

orientale, Linn.

- var. bracteatum, Lindl.

pavonicum, C. A. Mey.

pinnatifidum, Moris.

Rhoeas, Linn.

rupifragum, Boiss. et Reut.

somniferum, Linn.

umbrosum, Hort.

*Platystemon californicus, Benth.

Sanguinaria canadensis, Linn. 


\section{CRUCIFERÆ.}

* Athionema diastrophis, Bunge.

Alyssum

*argenteum, Vitm.

* gemonense, Linn.

*montanum, Linn.

podolicum, Benth. et Hook.

*rostratum, Stev. (A. Wierzbeckii, Heuffl.).

saxatile, Linn.

- var. variegatum.

Arabis spinosum, Linn.

albida, Stev.

- var. variegata.

*alpina, Linn.

*alpestris, Schleich. (A. cenisia, Reut.).

arenosa, Scop.

blepharophylla, Hook.

*hirsuta, Scop.

*lucida, Linn. fil.

* ludoviciana, C. A. Mey. (Cardamine ludoviciana, Hook.). sagittata, DC.

Turrita, Linn.

Aubrietia

deltoidea, DC.

- var. cœlestis, Hort.

- var. columnea, Hort.

* - var. conspicua, Hort.

* - var. Frœbellii, Hort.

- var. græca, Griseb.

- var. grandiflora, Hort.

- var. Pinardii, Hort.

- var. spathulata, Hort.

* - var. tauricola, Hort.

* erubescens, Griseb.

Barbarea

vulgaris, R. Br.

Biscutella areuata, Rehb.

apula, Linn.

didyma, Linn.

— var. Columnæ (B. Columnæ, Tenor.).

- var. raphanifolia (B. raphanierigerifolia, DC. folia, Poir.).

Brassica

* lævigata, Linn.

*alba, Boiss.

brassicata (Sinapis brassicata, Linn.)

*Erucastrum, Vill.

juncea, Gern.
Brassica-contd.

ngra, Koch.

pekinensis (Sinapis pekinensis,

Linn.).

Sinapistrum, Boiss.

Braya alpina, Sternb. pinnatifidum, Koch.

Bunias orientalis, Linn.

Camelina

dentata, Pers.

fœtida, Fenzl.

sativa, Crantz.

sylvestris, Wallr.

- var. microcarpa.

Capsella Bursa-pastoris, Medic.

Cardamine

*bellidifolia, Linn.

* chenopodifolia, Juss.

*impatiens, Linn.

*latifolia, Vahl.

Cheiranthus

Allioni, Hort.

Cheiri, Linn.

Semenowii, Rgl.

Senoneri, Heldr. et Sart.

Chorispora

iberica, DC.

*tenella, DC.

Clypeola cyclodontea, Delil.

Cochlearia danica, Linn.

officinalis, Linn.

* saxatilis, Lamk.

Crambe

cordifolia, Stev.

hispanica, Linn.

maritima, Linn.

*tatarica, Jacq.

Diplotaxis

*siifolia, Kunze.

* virgata, DC.

Draba

aizodes, Linn.

Aizoon, Wahl.

altiaca, Bunge.

Gmelini, Adams.

*incana, Linn.

rupestris, R. Br.

*Eruca sativa, Lamk.

Erysimum

*canescens (Cheiranthus Bocconi, All.).

commutatum, Hort.

hieracifolium, Linn.

latifolium, Hort.

ochroleucum, DC. 
Erysimum-contd.

*Perofskianum, Fisch. et Mey. rupestre, DC.

Hesperis fragrans, Fisch. matronalis, Linn.

Iberis *tristis, Linn.

Guldenstædina, Biebrst.

hesperidifolia, Hort.

nana, All.

pectinata.

* petræa, Jord.

pinnata, Linn.

saxatilis, Linn.

* superba, Hort.

umbellata, Linn.

*Ionopsidium acaule, Reichb.

Isatis

alpina, Linn.

hebecarpa, DC.

Lepidium

crassifolium, Waldst. et Kit.

Draba, Linn.

perfoliatum, Linn.

sativum, Linn.

Smithii, Hook.

Lunaria biennis, Linn.

* Malcomia flexuosa, Sibth.
Matthiola sinuata, R. Br.

Myagrum perfoliatum, Linn.

Nasturtium

officinale, R. Br.

pyrenaicum, R. Br.

Ochthodium ægypticum, DC.

Rapistrum Linnæanum, All.

Raphanus

* caudatus, Linn.

*sativus, Linn.

*Schizopetalon Walkerii, Sims.

Senebiera

Coronopus, Poir. didyma, Pers.

Sisymbrium bursifolium, Linn.

Iris, Linn.

Sophia, Linn.

tanacetifolium, Linn.

Succovia balearica, DC.

Teesdalia nudicaulis, R. Br.

Thlaspi

alpestre, Linn.

* perfoliatum, Linn.

prostrata, Hort.

Vesicaria sylvium, Gaud.

gracilis, Hook.

* sinuata, Poir.

* utriculata, Lamk.

\section{RESEDACEA.}

Astrocarpus sesamoides, DC.

Reseda

abyssinica, Fres.

* crystallina, Webb.

fruticulosa, Linn.

Gayana, Boiss.

Jacquini, Reichb.
Reseda-contd.

*lutea, Linn.

luteola, Linn.

*myriophylla, Tenore.

Phyteuma, Linn.

ramosissima, Pour.

\section{CISTINE $\approx$.}

Helianthemum

lavendulæfolium, Pers. polifolium, Mill.

vulgare, Gærtn.
Helianthemum-contd. vulgare, Gaertn., var. Garibaldi, Hort.

- var. The Bride, Hort.

\section{VIOLARIEÆ.}

Viola

ætholica, Hort. alpestris, Jord.

* canadensis, Linn., var. alba. canina, Linn.

- var. alba.

- var. arenaria.

*Cornuti, Linn.
Viola-contd.

Cornuti, Linn. var. Hœltzeri, Hort.

flavicornis, Smith.

*macedonica, Boiss. et Held.

odorata, Linn.

Ruppii, All.

tricolor, Linn.

var. Thomas Glen. 
Arenaria

balearica, Linn.

* caulescens, Hort.

* graminifolia, Schrad. holosteoides, Biebrst. serpyllifolia, Linn.

Cerastium

*alpinum, Linn.

*arvense, Linn.

brachypetalum, Desf.

dichotomum, Linn.

Dianthus mosiacum, Frivald.

alpinus, Linn.

*arboreus, Linn.

arenarius, Linn.

barbatus, Linn.

britannicus, Heuffl.

* cæsius, Sm.

cæspitosus, Thunb.

caucasicus, Biebrst.

- seedlings.

corsicus, Fisch.

deltoides, Linn.

- var. glaucus, Hort.

*flos-cuculoides, Hort.

glacialis, Hænk.

* grandiflorus, Poir.

monspessulanus, Linn.

neglectus, Loisl.

petræus, Waldst, et Kit.

plumarius, Linn., sub-sp. serotinus, Waidst. et Kit.

proliferus, Linn.

pungens, Linn.

Simsii, Hort.

squarrosus, Biebrst.

superbus, Linn.

* sylvestris, Wulf.

Gypsophila elegans, Biebrst.

Lychnis

*alpina, Linn.

bicolor, Hort.

chalcedonica, Linn.

ccelirosa, Desv.

- var. oculata.

corsica, Loisl.

diurna, Sibth.

Flos-cuculi, Linn

Flos-jovis, Desv.

inclusa, Lehm.

laeta, Ait.

nicæensis, Hort.

* pyrenaica, Berger.

\section{CAROPHYLLE $Æ$.}

Lychnis-contd.

Sartori, Hort.

Sieboldii, Maxm.

Viscaria, Linn.

- var. purpurea, Hort.

* Polycarpon tetraphyllum, Linn. fil.

* Sagina glabra, Koch.

Linnæi, Presl, var. maxima.

* pilifera, Fenzl.

procumbens, Linn.

* Saponaria cæspitosa, DC.

cerastioides, Fisch. et Mey.

*multiflora, Hort.

Silene oxydonta, Hort.

*acaulis, Linn., var. alba. alpestris, Linn. apetala, Willd.

Argoa, Boiss.

Atocion, Murr.

caueasica, Boiss.

cerastioides, Linn.

ciliata, Pourr.

coarctata, Lag.

conoidea, Linn.

Cucubalus, Wibel.

Czerei, Baumg.

diurniflora, Kunze.

echinata, Oth.

Fortunei, Vis.

fuscata, Link.

Græfferi, Gus.

italica, Pers.

juncea, Sib.

linifolia, Willd.

longicaulis, Pourr.

Lydia, Boiss.

muscipula, Linn.

noctiflora, Linn.

Orgæa, Fisch, et Mey.

* pendula, Lehm.

Persoonii, Tod.

pteropleura, Boiss.

rubella, Linn.

Saxifraga, Linn.

Tenoreana, Guss.

undulatifolia, Moris.

Spergula

vesiculifera, Gay.

arvensis, Linn.

Tunica

*marginata

prolifera, Scop.

velutina, Guss. 
PORTULACEA.

Calandrina sericea, Hook. et Arn.

Claytonia

perfoliata, Don.

* sibirica, Linn.

virginica, Linn., var. alba.
Montia

* fontana, Linn.

Portulaca Gillesii, Hook.

\section{HYPERICINEÆ.}

\section{Hypericum}

Androsæmum, Linn.

Coris, Linn.

Gebleri, Ledeb.

hirsutum, Linn.
Hypericum-contd.

* pendulum, Linn.

pulchrum, Linn.

quadrangulum, Linn.

- var. dubium, Leers.

\section{MALVACEÆ.}

Althæa ficifolia, Cav.

Lavatera

*thuringiaca, Linn.

trimestris, Linn.

Malope grandiflora, Hort.

Malva

*Alcea, Linn.

Durieui, Spach.

mareotica, Delil.

microcarpa, Desf.

moschata, Linn.

- var. alba.

nicæensis, All.

- var. Tenoreana, Kunze.
Malva-contd.

parviflora, Linn.

peruviana, Linn.

pulchella, Bernh.

sylvestris, Linn.

- sub-sp. plebeia, Stev.

trifida, Cav.

verticillata, Linn.

Nuttalia pedata, Dicks.

Palavia flexuosa, Mart.

Sidalcea

candida, Gray.

malvæflora, Gray.

Murrayana, Hort.

\section{LINEA.}

Linum

africanum, Linn.

aquilinum, DC.

candidissimum, Van Houtte.
Linum-contd.

humile, Mill.

nervosum, Waldst. et Kit.

usitatissimum, Linn.

\section{GERANIACEÆ.}

Erodium

cicutarium, L'Hérit.

*Jacquinianum, Hort.

Geranium maritimum, Smith.

armenum, Boiss.

dahuricum, DC.

*eriostemum, Fisch.

ibericum, Cav.

Lambertii, Sweet.

lancastriense, With.
Geranium-contd.

*maculatum, Linn.

omphaloideum, Lange.

*pallidum, Sweet.

*phæum, Linn.

pratense, Linn.

- var. album.

Robertianum, Linn.

- var. album.

* rotundifolium, Linn. sanguineum, Linn. 
Geranium-contd.

sylvaticum, Linn.

*Wallichianum, Sweet.

*Wilfredii, Maxim.

Impatiens

parviflorum, DC.

Royleii, Walp.

*tricornis, Wall.
Limnanthes

alba, Hort.

Douglasii, R. Br.

* Oxalis alsinoides, Walp.

Tropæolum

*majus, Linn.

*minus, Linn., var. coccineum.

\section{RUTACEA.}

Dictamnus tauricus, Hort. Ruta

* graveolens, Linn.

Ruta-contd. macrophylla, Hort.

\section{LEGUMINOSÆ.}

Anthyllis

vulneraria, Linn.

Astragalus

- var. rubra, Hort.

*alpinus, Linn.

bicolor, Lamk.

*boeticus, Linn.

chlorostachys, Lindl.

*Cicer, Linn.

Glycyphyllos, Linn.

hypoglottis, Linn.

macropterus, DC.

*ponticus, Pall.

*Baptisia lutea, Hort.

*Cytisus Arduinii, Hort.

Genista tinctoria, Linn.

*Galega officinalis, Linn.

Hedysarum

coronarium, Linn.

obscurum, Linn.

Kennedya rubicunda, Vent.

Lathyrus

angustifolius, Schkr.

articulatus, Linn.

bithynicus, Lam.

* cirrhosus, Sering.

Clusii, Hort.

coccineus, Mill.

cyaneus, Koch.

Drummondii, Hort.

flaccidus, Hort.

*Gargoni, Parl.

inconspicuus, Hort.

incurvis, Willd.

lævigatus, Waldst. et Kit.

Lowii, Hort.

luteus, Benth. et Hook. macrorhizus, Wimm.
Lathyrus-contd.

*pseudo-aphaca, Boiss. tenuifolius, Desf. tingitanus, Linn. vernus, Bernh.

Lotus

*corniculatus, Linn. ornithopoides, Linn.

Lupinus

arboreus, Sims.

*Hartwegii, Lindl. Hilariensis, Benth. hirsutissimus, Benth. mutabilis, Sweet. nootkatensis, Biebrst. perennis, Lindl. polyphyllus, Lindl. subcarnosus, Hook. succulentus, Dougl.

Medicago catalonica, Schrank.

* ciliaris, Willd.

*disciformis, DC.

Echinus, DC.

* elegans, Linn.

lupulina, Linn.

* marginata, Willd

*Murex, Willd.

*orbicularis, Willd.

* sativa, Linn. scutellata, All.

*tentaculata, Willd.

*Terebellum, Willd. tornata, Willd.

- var. minor.

*turbinata, Willd.

Melilotus cœrulea, Lamk. 
Melilotus-contd.

italica, Lamk.

officinalis, Lamk.

speciosa, Dur.

Mimosa pudica, Linn.

Onobrychis paucidentata, Hort.

Ononis

* crotalarioides, Coss.

* geminiflora, Lagase.

*repens, Linn.

rotundifolia, Linn.

spinosa, Linn.

Oxytropis

campestris, DC.

* lactea, Hort.

*lapponica, Wahl.

uralensis, DC.

*Phaseolus multiflorus, Lam.

Pisum sativum, Linn. var.

*Scorpiurus vermiculata, Linn.

Tetragonolobus Requieni, Seb.et Maur.

Trifolium

alpinum, Linn.

alpestre, Linn.

*fragiferum, Linn.

*incarnatum, Linn.

Mollinerii, Pall.

*medium, Linn.

Perreymondi, Gren.
Trifolium-contd.

phleoides, Pourr.

pinnatifidum, Hort.

- var. geminiflorum.

pratense, Linn.

* reclinatum, Waldst. et Kit.

repens, Linn.

- var. pictum.

rubens, Linn.

subterraneum, Linn.

*trichocephalum, Biebrst.

Trigonella

*cancellata, Desf.

Fœnum-græcum, Linn.

* geminiflora, Lagasc.

* platycarpos, Linn.

Vicia

amphicarpa, Dorth.

calcarata, Desf.

Cracea, Linn.

ferruguinea, Bess.

hirsuta, Fisch.

hirsutissima, Cyrill.

lutea, Linn.

musquinex, Bosc.

Orobus, DC.

pisiformis, Linn.

pyrenaica, Pourr.

villosa, Poth.

\section{ROSACE $Æ$.}

Acæna

microphylla, Hook. fil.

myriophylla, Lindl.

Sanguisorbæ, Vahl.

- var. sarmentosa (A. sarmentosa, Carm.).

*Agrimonia nepalensis, D. Don.

- agrimonioides, Linn.)

Alchemilla alpina, Linn.

- var. conjuncta.

* - var. argentea (A. argentea, Don).

arvensis, Scop.

pubescens, Biebrst.

vulgaris, Linn.

*Dryas octopetala, Linn.

* - var. Drummondii, S. Watson.

Fragaria vesca, Linn.

Geum

- var. muricata

altaicum, Bess.

coccineum, Lindl.

Grahamii, Steud.
Geum-contd.

intermedium, Ehrh.

*latifolium, Hort.

montanum, Linn.

parviflorum, Comm.

* reptans, Linn.

rivale, Linn.

rubellum, Fisch. et Mey.

urbanum, Linn.

Potentilla

alchemilloides, Lap.

alpestris, Hall.

argentea, Linn.

*argyrophylla, Wall.

atrosanguinea, Lod.

aurea, Linn.

bifurca, Linn.

- var. pectinata.

Comarum, Nestl.

Dombeyi, Nestl.

*erecta, Hort.

Fenzlii, Lehm.

inclinata, Vill.

insignis, Royle. 
Potentilla-contd.

kurdica, Boiss.

*lupinoides, Hook.

M'Nabiana, Hort.

Mooniana, Wight.

nepalensis, Hook.

*nevadensis, Boiss.

*nivea, Linn.

norvegica, Linn.

ontopoda, Dougl.

opaca, Linn.

pedata, Willd.

* peduncularis, Don.

rupestris, Linn.

Salisburgensis, Haenck.
Potentilla-contd.

Schrenkiana, Hort.

sericea, Linn.

Sieboldii, Haller fil.

tridendata, Ait.

*Valderia, Linn.

Poterium

dictyocarpum, Spach.

dodecandrum, Benth. et Hook.

muricatum, Spach.

platylophum, Hort.

Spiræa

* polygamum, Waldst. et Kit.

Filipendula, Linn.

gigantea, Hort.

\section{SAXIFRAGEA.}

Astilbe rivularis, Buch.-Ham.

Boykinia aconitifolia, Nutt.

*Chrysosplenium oppositifolium,

Linn.

Heuchera

Arlei, Hort.

sanguinea, Eng.

* Parnassia nubicola, Wall.

Saxifraga

acanthifolia, Hort.

affinis, Don.

*Aizoon, Jaicq.

- var. minor.

* - var. notata, Schott.

* - var. pectinata, Schott.

- var. rosularis, Schleich. aspera, DC.

bronchialis, Linn.

Bucklandii, Hort.

* cesia, Linn.

canaliculata, Boiss.

* capillaris, Reichb.

*carinthiaca, Schott.

* ceratophylla, Ait.

- var. major.

*Clusii, Gouan.

condensata, Gmel.

cordifolia, Hort.

corsicana, Gren. et Godr.

* - var.

crustata, Vest.

* - var. hybrida.

* cuneifolia, Linn.

Cymbalaria, Linn.

decipiens, Ehrh.

*densa, Willd.

* dentata, Link.

*diversifolia, Wall.

elatior, Biebrst.
Saxifraga-contd.

* exarata, Vill.

Geum, Linn.

- var. elegans, Mackay.

* - var. gracilis, Mackay.

* - var. ovalifolia, Hort.

globosa, Hort.

Gmelinii, Host.

grandiflora, Hort.

granulata, Linn.

grœenlandica, Linn.

hederæfolia, Hochst.

*hibernica, Haw.

hirta, Syme.

hirsuta, Linn.

* - var. minor, Hort.

Hostii, Boiss.

hypnoides, Linn.

intacta, Willd.

- var. farinosa, Hort.

- var. minor.

*kolenatiana, Rgl.

lasiophylla, Schott.

lingulata, Bell.

longifolia, Lap.

*M'Nabiana, Hort.

*media, Gouan.

* Melvillei, Desf.

*muscoides, Wulf.

- var. purpurea Hort.

*mutata, Linn.

nepalensis, Hort.

nervosa, Lap.

oppositifolia, Linn.

* - var. aretioides, Hort..

* - var. coccinea, Hort.

- var. fusca, Hort.

- var. pyrenaica, Hort.

paradoxa, Kit. 
Saxifraga-contd.

* pedatifida, Sm. peltata, Torr.

Prostii, Sternb. pulchella, Don. repanda, Willd.

*retusa, Gouan.

* - var. bryoides.

Rhoeii, Schott. rotundifolia, Linn. Stansfieldii, Hort. stricta, Hort.
Saxifraga-contcl.

Sturmiana, Schott.

*taygetta, Boiss.

*tenella, Wulf. tridentata, Don. umbrosa, Linn.

* - var. serratifolia.

- var. variegata.

valdensis, DC.

*virginiensis, Michx.

*Tellima grandiflora, R. Br.

\section{DROSERACEÆ.}

Drosera capensis, Linn.

Drosera spathulata, Labill.

\section{CRASSULACE平.}

*Cotyledon umbilicus, Linn.

Grammanthes chloræfolia, DC

Sedum

*acre, Linn.

Aizoon, Linn.

Anacampseros, Linn.

asiaticum, DC.

*boloniense, Loisl.

* collinum, Willd.

crassipes, Wall.

* cruciatum, Desf. cyaneum, Rudolph.

*hispanicum, Linn.

*ibericum, Stev.

kamtschaticum, Fisch.

*maximum, Sut.

- var. purpureum.

oreganum, Nutt.

Rhodiola, DC.
Sedum-contd.

*rufescens, Tenore.

* spathulatum, Waldst. et Kit. spurium, Biebrst.

— var. album.

trifidum, Wall.

Sempervivum

*acuminatum, Hort.

*arenarium, Koch.

* Boutigyanum, Hort.

*Braunii, Ledeb.

*californicum, Hort.

flagelliforme, Fisch.

hirtum, Linn.

Pittonii, Schott.

*Pomelli, Lamotte.

* rupestre, Hort.

*Verlotii, Lamotte.

Wulfenii, Hopp.

\section{HALORAGEACEÆ.}

Hippurus vulgaris, Linn.

\section{MYRTACEÆ.}

Eugenia dysenterica, Mart. 
LYTHRARIEÆ.

Lythrum

hirsutum, Hort.

Salicaria, Linn.

- var. grandiflora, Hort.

- var. pubescens.
Lythrum-contd.

Nesæa tomentosum, Mill.

verticillata, H.B. et K.

\section{ONAGRARIEA.}

Epilobium

adnatum, Griseb.

*affine, Bong.

alsinifolium, Villars.

Billardierianum, Sering.

*brachycarpum, Presl.

foliosum, Hochst.

latifolium, Linn.

montanum, Linn.

nummulariæfolium, A. Cunn.

- var. longipes, Hort.

* rosmarinifolium, Haenke.

- var. Fleischeri, Hochst.

- var. sericeum, Benth.

*trigonum, Schrank.

virgatum, Lamk.

*Eucharidium grandiflorum, Fisch.
CEnothera amoena, Lehm. - var. rubicunda, Hort. biennis, Linn. campylocalyx, K. et B. concinna (Boisduvalia concinna, Spach).

*epilobiifolia, H. B. et K. fruticulosa, Linn.

insignis (Godetia insignis, Spach).

glauca, Michx.

Lindleyana, Dougl.

purpurea, Curt.

* riparia, Nutt.

Sellowii, Link.

tenella, Cav.

- var. dasycarpa.

\section{LOASEÆ.}

Loasa prostrata, Gill.

Loasa tricolor, Lindl.

\section{CUCURBITACEÆ.}

Bryonia dioica, Jacq.

I *Momordica Elaterium, Linn

\section{BEGONIACEA.}

Begonia semperflorens, Link et Otto, var. purpurea.

\section{UMBELLIFERA.}

* Aigopodium Podagaria, Linn.

Anthriscus

Cerefolium, Hoffm.

sylvestris, Hoffm.

Archangelica

officinalis, Hoffm.

- var. decurrens (A. decurrens, Ledeb.).

Arracacia arguta, Benth. et Hook.

Astrantia major, Linn.
Bifora testiculata, DC.

Bunium luteum, Hoffm.

Bupleurum angulosum, Linn. (B. Burserianum, Willd.).

*junceum, Linn.

petræum, Linn. (B. graminifolium, Vahl).

ranunculoides, Linn.

* rotundifolium, Linn,

Carum Carui, Linn. 
Chærophyllum aromaticum, Linn. aureum, Linn.

*Conium maculatum, Linn. Coriandrum mephiticum.

*Dondia Epipactis, Spr.

*Daucus marinus, Desf.

Eryngium

*alpinum, Linn. spinalba, Vill.

Ferula conspicua.

Heracleum giganteum, Fisch. - var. purpurea, Hort. Sphondylium, Linn.

Laserpitium alatum, Linn. aquilegifolium, DC.
Ligusticum scoticum, Linn.

*Lophosciadium meifolium, DC.

Magydaris panacina, DC.

Meum athamanticum, Jacq.

Molopospermum peloponnesicum.

Myrrhis odorata, Scop.

Enanthe apiifolia, Brot. crocata, Linn. globulosa, Linn. silaifolia, Heuffl.

Pastinaca Fleischmanni, Koch.

Pleurospermum austriacum, Hoffm.

*Sanicula europæa, Linn.

Seseli gummiferum, Sm.

* Siler caucasicum(Agasyllis caucasica, Spreng.).

Trinia Kitaibelii, Biebrst.

ARALIACEÆ.

*Aralia hispida, Michx.

\section{RUBIACE无,}

Asperula

cynanchica, Linn.

Galium

* lævigata, Linn.

Aparine, Linn.

*boreale, Linn.

humifusum, Bbrst.

Galium-contd.

pulchellum, Hort.

*pusillum, Sm.

rubioides, Linn.

saxatile, Linn.

*Sherardia arvensis, Linn.

\section{VALERIANEÆ.}

*Centranthus ruber, DC.

Crucianella latifolia, Linn.

Fedia cornucopia, Vahl.

Valeriana

*dioica, Linn.

montana, Linn.

* officinalis, Linn.

pyrenaica, Linn.

Valeriana-contd.

*tuberosa, Linn.

Valerianella

Auricula, DC.

carinata, Loisl.

fallax, Desf.

Morisonii, DC.

Olitoria, Moench.

\section{DIPSACEA.}

Cephalaria tatarica, Schrad.

$$
\text { - var. minor. }
$$

Dipsacus

atrata, Hort.

*Fullonum, Linn.
Knautia arvensis, Linn.

Morina

*betonicoides, Benth.

caucasica (Asterocephalus caucasicus, Spreng.). 
Morina-contd.

Coulterii, Royle.

longifolia, Wall.

Scabiosa

agrestis, Waldst. et Kit.

alpina, Linn.

dipsacifolia, Schott.

elata, Hornem.
Scabiosa-contd.

graminifolium, Linn.

Hladnickiana, Host.

pilosiuscula, Hort.

* pubescens, Kit. succisa, Linn.

sylvatica, Linn.

*Webbiana, Don.

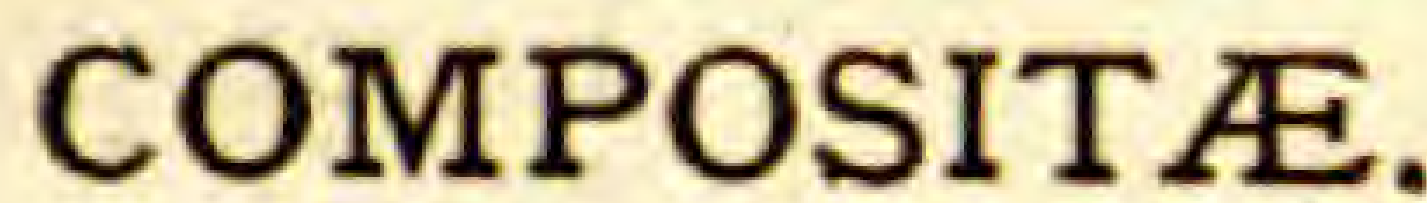

*Ainsliæa aptera, DC.

Antennaria

boreale.

dioica, Gærtn.

Anthemis

Chia, Linn.

chrysoleuca, Gray.

${ }^{*}$ Guicciardi, Heldr.

Arctium psorosperma.

Bardana, Willd.

majus, Schk.

*Arctotis aureola, Ker-Gawl.

Arnica

${ }^{*}$ Chamissonis, Less., var.

*montana, Linn.

Artemisia desertorum, Spreng. (A. neglecta, Spreng.).

græca, Hort.

rupestris, Linn.

sacrorum, Ledeb. (A. Messerschmidtiana, Bess.).

Aster

*selengensis, Bess.

alpinus, Linn.

- var. albus.

- var. speciosus.

peregrinus, Pursh.

- var. salsuginosus (A. salsuginosus, Hook.).

pseudamellus, Hook. fil.

Bellis

Vahlii, Hook. et Arn.

Bellium

rotundifolia, Boiss.

- var, coerulescens.

bellidiastrum, Desf.

minutum, Linn.

Brachycome

* goniocarpa, Sond. et Mull.

*Sinclairii, Hook. fil.

Calendula

* cristagalli, Vill.

officinalis, Linn.
Carduus

*Carolorum, Howie and Jenner. X

* crassifolius, Willd.

mexicanus, Gaertn.

nutans, Linn.

*tataricus, Linn.

*tuberosus, Linn.

Centaurea aurea, Ait. cinerea, Lamk.

crocodylium, Linn.

dealbata, Willd.

elata, Poir.

Jacea, Linn.

*melitensis, Linn.

napifolia, Linn.

pallida, Frivald.

- var. gigantea.

*phrygia, Linn.

*procumbens, Balb.

scabiosa, Linn.

solstitialis, Linn.

*verutum, Linn.

Chrysanthemum

leucanthemum, Linn.

- var. grandiflorum.

- var. tubulariflorum.

maximum, DC.

Roxburghii, Bert.

segetum, Linn., var. grandiflorum, Hort.

Tchihatcheffii, Rgl.

Cirsium

tubulariflora, Hort.

*horridum, Biebrst.

Cnicus

pannonicum, DC.

arvensis, Hoffm.

benedictus, Linn.

* eriophorus, Hoffm.

*heterophyllus, Willd.

oleraceus, Linn.

* pannonicus, Hort.

- var. divaricatus.

rigidus, Hort. 
${ }^{*}$ Cotula filicaulis (Leptinella filiCrepis caulis, Cass.)

aurea, Cass.

- var. crocea.

blattarioides, Vill.

hieracioides, Waldst. et Kit.

latifolia, Balb.

Dimorphotheca pluvialis, Linn.

Doronicum austriacum, Jacq.

Erigeron

alpinum, Linn.

aurantiacum, Rgl.

*bellidifolium, Muhl.

glabellum, Nutt.

- var. molle.

Eupatorium

purpureum, Linn.

sessilifolium, Linn.

*verticillatum, Muhl.

Gerbera Anandria, Schultz.

Gaillardia splendidissima, Hort.

Helenium ellipticum, Linn.

Helianthella uniflora, Torr. et Gray.

Helianthus occidentalis, Riddel.

Hieracium andryaloides, Vill. (Andryala lanata, Linn.).

balkanum.

chlorocephalum, Wimm.

collinum, Fries.

glomeratum, Froel.

graminifolium, Gaud.

humile, Jacq.

murorum, Linn.

nigrescens, Willd.

phlomoides, Froel.

præmorsum, Linn.

pulmonarium, $\mathrm{Sm}$.

Ruphaeum, Uecht.

saxatile, Jacq.

stoloniferum, Kit.

strictum, Frivald.

sylvaticum, Smith.

vulgatum, Fries.

*Homogyne alpina, Cass.

Hypochæris

arachnoidea, Poir.

maculata, Linn.
Hypochæris - contd.

* pinnatifida (Robertia pinnatifida, DC.)

Inula

glandulosa, Willd.

Hookerii, Clarke.

Kaulfussia

amelloides, Linn.

Mullerii, Salzm.

Lasthenia glabra, Cass.

Leontopodium alpinum, Cass.

Liatris

pilosa, Willd.

odoratissima, Willd.

spicata, Willd.

*Ligularia macrophylla, DC.

Metabasis æthnensis, DC.

Notabasis syriaca, Cav.

Onopordon Acanthium, Linn.

*Prenanthes purpurea, Linn.

Rudbeckia

frigida, Ait.

triloba, Linn.

Scorzonera hispanica, Linn.

Senecio

*alpinus (Cacalia alpina, Linn.)

crassifolius, Willd.

*Doronicum, Linn.

* erraticus, Bertol.

*lyratifolius, Rchbch.

sarracenicus, Jacq.

soongoricus, Fisch.

squalidus, Linn.

verbenæfolia, Jacq.

Serratula arguta, Fisch.

Silphium conjunctum, Willd.

Solidago coriaceus, Hort.

Sonchus

arvensis, Linn.

lapponicus, Linn.

oleraceus, Linn.

palustris, Linn.

pinnatus, Ait.

Taraxacum caucasicum, DC.

*Tragopogon floccosus, W. K.

Urospermum picroides, Desf.

Wyethia robusta, Mitt.

\section{STYLIDIEÆ.}

Stylidium adnatum, R. Br.

Stylidium fruticosum, R. Br. 


\section{CAMPANULACEÆ.}

Adenophora

*polymorpha, Ledeb. stylosa, Fisch.

Campanula alliariæfolia, Willd. americana, Linn. Baumgartenii, Becker.

*cespitosa, Scop. (C. tenella, Jord.).

carpatica, Jacq. (C. turbinata, Scott).

collina, Biebrst.

elegans, Roem. et Schult.

glomerata, Linn.

* - var. lilacina.

Grossekii, Heuffl.

latifolia, Linn.

- var. alba.

- var. macrantha(C. macrantha, Fisch.).

- - var. alba.

Medium, Linn.

pelviformis, Hort.

persicifolia, Linn. (C. vesula, All.).

* - var. alba.

punctata, Lamk. (C. nobilis, Lindl.)

rapunculoides, Linn.
Campanula-contd. rhomboidea, Murr. rotundifolia, Linn.

* - var. pluripetala.

* - var. maxima.

* sarmatica, Ker-Gawl.

Scheuchzerii, Lodd.

- var. glabra.

Trachelium, Linn. (C. urticifolia, Schmidt.)

*Zoysii, Wulf.

Codonopsis

Clematidea, Schk.

ovata, Benth.

*Cyananthus lobatus, Wall.

Jasione

montana, Linn.

perennis, Lam.

*Lobelia Wallichii, Steud.

Phyteuma orbiculare, Linn. Scheuchzerii, All.

Wahlenbergia dalmatica, A. DC.

* gracile, DC.

lobelioides, DC.

nutabunda, DC.

tenuifolia, A. DC.

undulata, DC.

\section{ERICACEÆ.}

Aretostaphylos Uva-Ursi, Spreng.

*Dabœocia polifolia, Don. * - var. alba.

Gaultheria carnea, Hort.

Erica

carnea, Linn.
Erica-contd.

cinerea, Linn., var. atropurpurea.

- var. bicolor, Hort.

- var. longaspica, Hort.

\section{PLUMBAGIEA.}

Armeria

alliacea, Roem. et Schult.

allioides, Boiss.

berlengensis, Daveau.

*boetica, Boiss., var. hirta.

canariensis, Boiss.

fasciculata, Willd.

pinifolia, Link.

plantaginea, Willd.
Armeria - contd.

pubescens, Link.

splendens, Webb.

Welwitschii, Boiss.

Plumbago micrantha, Spach.

Statice

coronata, Hort.

tatarica, Linn.

Suworowii, Rgl. 


\section{PRIMULACEÆ.}

* Anagallis arvensis, Linn.

Androsace

* carnea, Linn. (A. brigantica, Jord.).

lactea, Linn.

lactiflora (Fisch.), Hort. (A. coronopifolia, Andr.).

maxima, Linn.

Cortusa Matthioli, Linn.

Cyclamen hederæfolium, Willd.

Dodecatheon integrifolium, Michx.

*Glaux maritima, Linn.

*Hottonia palustris, Linn.

*Primula Delavayi, Franch.
Primula-contd.

* elatior, Jacq.

* - var. grandiflora.

*farinosa, Linn. grandis, Trautv. magellanica, Lehm. mollis, Mitt.

reticulata, Wall.

*suaveolens, Bertol. sikkimensis, Hook.

*variabilis, Bast.

*venusta, Host. vulgaris, Huds. Warei, Hort.

\section{MYRSINEÆ.}

Ardisia primulæfolia, Hort.

\section{ASCLEPIADEA.}

*Cynanchum nigrum, Jinn.

\section{GENTIANEÆ.}

\section{Gentiana}

*acaulis, Linn.

*Andrewsii, Griseb. asclepiadea, Linn. brevidens, Rgl. Burserii, Lapey. albiflora, Hort. Kochiana, Pers.

Gentiana-contd. phlogifolia, Sch. punctata, Linn. septemfida, Pall. - var. cordifolia. tibetica, King. verna, Linn. Wallichii, Hort.

\section{POLEMONIACEÆ.}

Collomia

coccinea, Lehm. gillioides, Benth. * grandiflora, Dougl. Gilia * linearis, Nutt.

achilleæfolia, Benth.

*alba, Hort. androsacea, Steud. capitata, Dougl. crassifolia, Benth

Gilia-contd. multicaulis, Benth.

Polemonium coeruleum, Linn. flavum, Green. *humile, Willd. lacteum, Lehm. pauciflorum, Wats. *pulchellum, Bunge. Richardsonii, Graham. villosum, Rudolph. 


\section{HYDROPHYLLACEÆ.}

Nemophila

*aurita, Lindl.

maculata, Benth.

Phacelia tanacetifolia, Benth.
Phacelia Wrangeliana (Eutoca Wrangeliana, Fisch. et Mey.).

Romanzoffia sitchensis, Cham.

*Whitlavia minor, Hans.

\section{BORAGINEÆ.}

Anchusa

arvensis, Benth.

officinalis, Linn., var. incarnata.

Cerinthe

alpina, Kit.

aspera, Roth.

*auriculata, Tenore.

retorta, Sibth, et Sm.

Cynoglossum

Dioscoridis, Vili.

nervosum, Benth.

Echium rubrum; Jacq.

Eritrichium corniculatum, Lamk.

Exarrhena Lyallii, Hook. fil.

Lindelofia spectabilis, Lehm.
Macrotomia echioides, (Arnebia echoides, A. DC.).

* Mertensia sibirica, Don.

Myosotis

*arvensis, Sibth.

*australis, R. Br.

intermedia, Link.

lingulata, Fries. palustris, With.

Pulmonaria

*mollis, Wulf.

*saccharata, Mill.

Symphytum asperrimum, Biebrst. caucasicum, Biebrst.

\section{CONVOLVULACEÆ.}

Convolvulus

minor, Catesb.

*pilosellus, Liebm.

\section{- Convolvulus-contd. siculus, Linn.}

\section{SOLANACEÆ.}

Atropa

Belladonna, Linn.

- var. fructu flava, Hort.

Cacabus quadrivalvis (Dictyocalyx

Datura

$$
\text { quadrivalvis, Hook. fil.) }
$$

gigantea, Hort.

humilis, Desf.

Stramonium, Linn.

- var. Tatula, Linn.
Hyoscyamus canariensis, Ker. niger, Linn. pictus, Bern.

Nicotiana glauca, Grah.

Solanum

*Dulcamara, Linn.

*nigrum, Linn.

villosum, Lamk.

\section{SCROPHULARINEA.}

Alonsoa

incisæfolia, Ruiz et Pav.

Wallichii, Hook, fil.

Antirrhinum majus, Linn.

*Calceolaria chelidonioides, H.B.
Celsia Arcturus, Linn.

Collinsia

*bartsiæfolia, Benth.

bicolor, Benth. 
Collinsia-contd.

*verna, Nutt.

* violacea, Nutt.

Digitalis purpurea, Linn.

Erinus

alpinus, Linn.

hispanicus, Pers.

\section{Linaria}

- var. albus.

alpina, DC.

delphinioides, Gay.

maroccana, Hook. fil.

multipunctata, Hffmsg. et Lk

purpurea, Linn.

*saphirina, Hoffm.

*striata, DC.

vulgaris, Mill.

Mimulus

cardinalis, Dougl.

* Jeffreyanus, Hort.

Pentstemon confertum, Dougl.

Hartwegii, Benth.

* Menziesii, Hook.

nitida, Dougl.

pubescens, Sol.

Ramondia pyrenaica, Rich.

Schizanthus

*lilacinus, Hort.

*pinnatus, Ruiz et Pav. violaceus, Hort.

Scrophularia

aquatica, Linn.

Ehrhartii, Stev. glandulifera, Clarke

*nodosa, Linn. scopolia, Hoppl.
Scrophularia - contd.

*sp. (Dr King).

trifoliata, Linn.

vernalis, Linn.

Verbascum

phœniceum, Linn.

Veronica

Anagallis, Linn.

Beccabunga, Linn.

Buxbaumii, Tenore.

corymbosa, Hort.

- var. variegata.

fruticulosa, Linn.

* gentianoides, Vahl.

* - var. variegata.

grandis, Fisch.

*incisa, Ait.

*Jacquinii, Baumg.

* Lyallii, Hook. fil.

maritima, Linn.

- var. alba.

officinalis, Linn.

- var. alba.

orchidea, Crantz.

pinnata, Linn.

Ponæ, Gouan.

saxatilis, Linn.

- var. Guthriana, Hort.

serpyllifolia, Linn, var. humifusum, Dicks.

* spicata, Linn.

- var. alba.

- var. hybrida.

spuria, Linn.

taurica, Willd.

*urticæfolia, Linn.

*Wulfenia carinthiaca, Jacq.

\section{OROBANCHACEA.}

*Orobanche rubra, Sm.

\section{LENTIBULARIEÆ.}

*Pinguicula vulgaris, Linn.

\section{VERBENACEÆ.}

Clerodendron Thomsonæ, Balf., var. | Verbena pulchella, Hort. (ShuttleBalfourii, Hort. worthia pulchella, Meissn.). 
LABIAT $Æ$.

*Ajuga pyramidalis, Linn.

Brunella

grandiflora, Linn.

vulgaris, Linn.

- var. hispida, Hort.

- var. Webbiana, Hort.

Dracocephalum

* grandiflorum, Linn.

nutans, Linn.

parviflorum, Nutt.

*Ruyschianum, Linn.

* stamineum, Kar. et Kit.

*Eremostachys iberica.

Horminum pyrenaicum, Linn.

Hyssopus officinalis, Linn.

Lamium

album, Linn.

Galeobdolon, Crantz.

* Orvala, Linn.

Lycopus exaltatus, Linn.

Marrubium

*peregrinum, Linn.

Melissa vulgare, Linn.

grandiflora, Linn.

* patavina, Benth.

*Melittis melissophyllum, Linn.

Mentha

*arvensis, Linn.

Nepeta

Requienii, Benth.

altissima, Schrank.

* pannonica, Jacq.

Origanum

*pulchellum, Boiss. et Heldr.
Origanum-contd.

*vulgare, Linn.

Phlomis Russelianus, Lag.

Salvia

argentea, Linn.

carduacea, Benth.

*dominicu, Linn.

*Horminum, Linn.

*limbata, Meyer.

*nubicola, Wall.

obovata, Ell.

*officinalis, Linn.

pratensis, Linn.

* Regeliana, Trautv.

taraxacifolia, Coss.

Verbenaca, Linn.

Satureia

*illyrica, Hort.

spicata, Visian.

Scutellaria

alpina, Linn.

altaica, Fisch.

*hastæfolia, Linn.

*hortensis, Linn.

*japonica, Morr. et DC.

* Stachys Betonica, Benth.

Teucrium

*pyrenaicum, Linn.

*Scorodonia, Linn.

*Thymus

azureus, Maund.

*micans, Lowe.

rotundifolium, Schrad.

Serpyllum, Linn.

\section{PLANTAGINEÆ.}

*Littorella lacustris, Linn.

Plantago

acanthiphylla, DC.

alpina, Linn.

*amplexicaulis, Cav.

arachnoidea, Schrenek.

arborescens, Poir.

argentea, Lamk.

Cornuti, Gouan.

Coronopus, Linn.

divaricata, Zucc.

eriostachya, Tenore.

gentianoides, Sm.
Plantago-contd.

kamtschatica, Cham.

Lagopus, Linn.

major, Linn.

- var. monstrosus, Hort.

- var. rubra, Hort.

maritima, Linn.

media, Linn.

montana, Lamk.

saxatilis, Biebrst.

subulata, Linn.

xorulensis, H.B.

\section{ILLECEBRACEÆ.}

Herniaria ciliata, Bab.

Herniaria glabra, Linn. 
AMARANTACEÆ.

Amaranthus

*hypochondriacus, Linn.

* - var. monstrosus.
Amaranthus-contd. mantegazzianus, Pass. melancholicus, Linn.

\section{NYCTAGINEA.}

Oxybaphus nyctagineus, Sweet.

\section{CHENOPODIACEÆ.}

Atriplex

laciniata, Linn.

nutans, Biebrst.

Beta

phalone, Desf.

Rapa, Dum.

vulgaris, Linn.

Chenopodium

aristatum, Linn.
Chenopodium-contd.

Bonus-Henricus, Linn.

ficifolium, Sm.

petiolare (Blitumpetiolare, Lnk).

*quercifolium, Schrad.

virgatum (Blitum virgatum, Linn.).

* Spinacia oleracea, Linn.

*Teloxys aristata, Moq.

\section{POLYGONACEÆ.}

Polygonum

alpinum, All.

Bistorta, Linn.

- var. major, Hort.

capitatum, Don.

sphærostachyum, Meissn.

Pterostegia drymarioides, Fisch.

Rheum

hybridum, Ait.

officinale, Baill.

palmatum, Linn.

- var. tanguticum, Rgl.

Rhaponticum, Linn.

sibiricum, Pall.
Rumex

alpinus, Linn.

- var. majus.

aquaticus, Linn.

* compactus, Hort.

Hydrolapathum, Huds.

nepalensis, Spr.

Patientia, Linn.

rupestris, Le Gall.

sanguineus, Linn.

sanguisorbæfolius, Hort.

*scutatus, Linn.

stenophyllus, Ledeb.

\section{ARISTOLOCHIACEÆE.}

Aristolochia elegans, Mast.

Asarum

canadense, Linn.
Asarum-contd. caudatum, Lindl.

\section{EUPHORBIACEA.}

Euphorbia

glaucescens, Willd.

* Kingii, Hort.
Euphorbia-contd.

Myrsinites, Linn.

*nicæensis, All. 


\section{URTICACEÆ.}

Cannabis sativa, Linn.

*Parietaria officinalis, Linn.

Urtica

*dioica, Linn.
Urtica-contd.

grandidentata, Banks. magellanica, Poir. pilulifera, Linn.

\section{ZINGIBERACEÆ.}

Canna

floribunda, Bche.

patens, Rose.
Globba Schomburghii, Hook. fil.

\section{BROMELIACE $\Subset$.}

Hoplophytum cærulescens, Morr.

\section{HÆMADORACE正}

Peliosanthes, sp.

\section{IRIDEÆ.}

\section{Aristea}

Ecklonii, Baker.

\section{Crocus}

*Imperati, Tenore.

* - var. albiflos, Herb.

*Kotschyanus, Koch. medius, Balbis.

* Newberyi, Hort.

* pulchellus, Herb.

Sieberi, Gay.

*sp. (Asia Minor).

* speciosus, Biebrst.

*tulipæfolia, Hort.

*vallicola, Herb.

vernus, All.

* - var. leucorhynchus.

- vars.

*Homeria collina, Vent.

Iris

hæmatophylla, Fisch.

Kaempferi, Sieb.
Iris-contd.

lævigata, Fisch.

- var. stricta.

lurida, Soland.

orientalis, Thunbg.

prismatica, Pursh.

rubicaulis, Hort.

setosa, Pall.

triflora, Balb.

versicolor, Linn.

- var. Kraush.

xiphioides, Ehrh.

Libertia grandiflora, Sweet.

* Moræa sinensis, Lindl.

Sisyrinchium

*Bermudianum, Linn.

*Engelmanii.

grandiflorum, Dougl.

* - var. album.

mueronatum, Michx.

Sparaxis pendula, Ker.

\section{AMARYLLIDEÆ,}

*Alstrœmeria aurea, R. Grah.

Leucoium

${ }^{*}$ Galanthus plicatus, Biebrst. vernum, Linn. 
*Leucoium vernum, Linn., var. carpathicum, Herb.

*Narcissus

Bulbocodium, Linn., var. citrinus, Baker.

*poeticus, Linn., var. majus, Hort.

* _ var. poetarum, Haw.
Narcissus-contd.

* poeticus, var. tripodalis, Salisb. (ornatus, Haw.)

Pseudo-narcissus, Linn., sub-sp. bi-color, Linn.

- sub-sp. pumilus, Haw.

- sub-sp. muticus, Gay.

- var. pallidus-præcox, Haw.

\section{LILIACEÆ.}

Agapanthus umbellatus, L'Hérit. Allium

ampeloprasum, Linn., var. Babingtonii, Borr.

arenarium, Schrad.

* carinatum, Linn.

* coeruleum, Pall.

Cyrilli, Tenore.

Dioscoridis, Sibth. et Sm.

- var. Leichtlinii.

- var. siculum (A. siculum, Ucria).

fistulosum, Linn.

* fragrans, Vent.

giganteum, $\mathrm{Rgl}$.

globosum, Biebrst.

- var. albidum, Hort.

*karataviense, Rgl.

M'Nabianum, Hort.

*multiflorum, Vill.

*narcissiflorum, Vill. neapolitanum, Cyr. (A. lilifforum, Zeyh.).

*oreophyllum, A. Meyer. polyphyllum, Kar. et Kir. paradoxum, G. Don.

* serecolatum, Hort. stipitatum, Rgl. subhirsutum, Linn. vineale, Mill.

* - var. Kochii, Hort.

*Asphodelus ramosus, Linn.

Brodiæa congesta, Sm.

Bulbine

annua, Willd.

Bulbinella semibarbatus, Spr.

Hookerii, Benth.

Liliastrum (Anthericum Liliastrum, Bert.).

- var. major, Hort.

Bulbocodium vernum, Linn.

Camassia

Brownii, Hort.

esculenta, Lindl.

- var. alba, Hort.

- var. major, Hort.
Camassia-contd.

sp.

tardiflora, Hort.

Chionodoxa

sardensis, Hort.

*Tmolusii.

Chlorogalum Leichtlinii, Baker.

Clintonia Andrewsiana, Torrey.

Colchicum

autumnale, Linn.

speciosum, Stev., var. maximum, Hort.

Convallaria

*majalis, Linn.

*Polygonatum, Linn., var. major.

Erythronium

Dens-canis, Linn.

giganteum, Lindl.

grandiflorum, Hort.

Fritillaria

Meleagris, Linn.

- var.

Helonias

bullata, Linn.

* - var. pallida.

Hyacinthus

amethystinus, Linn.

*azureus, Baker.

*romana, Linn.

*Kniphoffia caulescens.

Lilium

canadense, Linn., var. penduliflo-

rum.

* Martagon, Linn.

- var. dalmaticum, Maly.

monadelphum, Biebrst. polyphyllum, Don.

Littonia modesta, Hook.

Milla laxa, Baker.

Muscari

Argæa, Schott.

*armeniacum, Baker.

atlanticum, Boiss. et Reut.

Maweanum, Baker.

racemosum, Mill.

Szovitsianum, Rgl.

Narthecium americanum, Ker. 
Ornithogalum

*Elwesii, Hort.

*nutans, Linn.

Puschkinia

libanotica, Zucc., var. compacta, Hort.

Scilla scilloides, Adams.

amœna, Linn.

campanulata, Ait., var. rubra, Hort.

nutans, Sm.

peruviana, Linn.

sibirica, Andr.

- var. præcox.

* - var. striata.

verna, Huds.

*Smilacina racemosa, Desf.
*Streptopus roseus, Michx.

Tofieldia carinthiaca. glutinosa, Willd.

Trillium grandiflorum, Salisb.

Triteleia Murrayana, Hort. *uniflora, Lindl.

Tulipa Gesneriana, Linn., var. pulchella, Fenzl.

Veratrum album, Linn.

Zygadenus canadensis, Sch. glaucus, Nutt.

* - var. humilis.

- var. major,

\section{COMMELINACEÆ.}

${ }^{*}$ Commelina dianthifolia, DC.

*Tradescantia atropurpurea.

*elata, Lodd.
Tradescantia-contd.

* subaspera, Ker.

*virginica, Linn.

- var. alba, Hort.

\section{JUNCACEÆ.}

Juncus

balticus, Willd.

conglomeratus, Linn.

effusus, Linn.

- var. variegatus.

glaucus, Ehrh.

* lamprocarpus, Ehrh.

tenuis, Willd.
Juncas-contd.

Luzula trifidus, Linn.

*albida, DC.

* campestris, DC.

spicata, DC.

*sylvatica, Rich.

PALMÆ.

Ptychospherma elegans, Labill.

TYPHACE $Æ$.

Aponogeton distachyon, Thunbg. | Sparganium ramosum, Huds.

\section{AROIDEA.}

Arum

maculatum, Linn. palestinum, Boiss.
Arum-contd. tenuifolium, Linn. Symplocarpus foetidus, Salisb. 


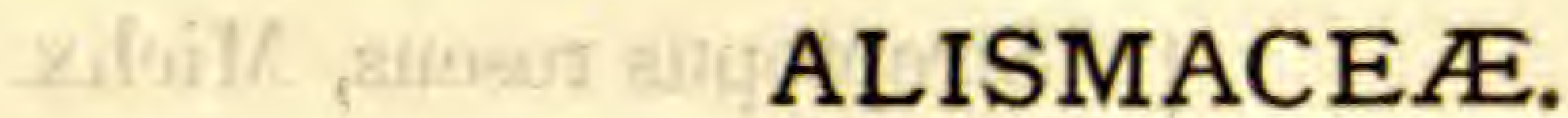

Alisma

Plantago, Linn.

- var. rubra.
Alisma-contd.

ranunculoides, Linn.

*Butomus umbellatus, Iinn.

\section{CYPERACE $Æ$.}

Carex

*axillaris, Good. (C. Boenninghausiana), Weibe.

atrata, Linn.

binervis, $\mathrm{Sm}$.

capillaris, Linn.

Drymingii, Hort.

frigida, All.

fulva, Good.

${ }^{*}$ Grahami, Boot.

*lagopodioides, Schkuhr.

- Mairii, Coss.

microglochin, Wahlenb.

*ovalis, Good.

panicea, Linn.

paniculata, Huds.

* pendula, Huds.

præcox, Jacq.
*Carex-contd.

rhyncophysa, Fisch. et Mey.

riparia, Curt.

stictocarpa, Sm.

sylvatica, Huds.

Vahlii, Schkuhr.

vulpina, Linn.

Eleocharis palustris, $\mathrm{Br}$.

*Eriophorum

*alpinum, Linn.

*vaginatum, Linn.

Kobresia caricina, Willd.

*Schœenus nigricans, Linn.

Scirpus

atrovirens, Willd.

*lacustris, Linn.

Tabernæmontani, Gmel.

\section{GRAMINEÆ.}

Agropyron villosum, Link, (Aegilops Agrostis cordata, Linn.)

alba, Linn.

- var. stolonifera, Linn.

*interrupta, Linn.

Spica-venti, Linn.

Aira vulgaris, With.

atropurpurea, Wahlenb. (Avena atropurpurea, Kunth.). præcox, Linn.

* pulchella, Tenore.

Alopecurus

alpinus, $\mathrm{Sm}$.

*arundinaceus, Poir. (A. nigricans, Hornem.) pratensis, Linn. variegatus, Hort.

Anthoxanthum

*aristatum, Boiss. gracile, Bivon. odoratum, Linn.

Arrhenatherum

*avenaceum, Beauv.
Arrhenatherum-contd.

- var. bulbosum (A. bulbosum, Fresi.).

Arundo acutiloba.

Avena

canariensis, Nees.

fatua, Linn.

pratensis, Linn. (A. alpina, Sm.).

* - var. planiculmis (A. planiculmis, Schrad.).

sempervirens, Vill.

Beckmannia erucæformis, Host.

Brachypodiun

distachyum, Beauv.

Briza sylvaticum, Beauv.

gracilis, Hort.

*maxima, Linn. minor, Linn.

Bromus

commutatus, Schrad.

japonicus, Thunb.

maximus, Desf.

mollis, Linn.

* purpurascens, Delil. 
Bromus-contd.

racemosus, Linn.

* scoparius, Desf.

sterilis, Linn.

tectorum, Linn.

unioloides, H. B. et K.

vestitus, Schrad.

Calamagrostis

epigeijos, Roth.

*laxa, Host.

Chrysopogon Gryllus (Andropogon

*Cinna Gryllus, Linn.).

*americana, Beauv.

*mexicana, Beauv.

Cynosurus

cristatus, Linn.

* - var.

echinatus, Linn.

elegans, Desf.

Dactylis glomerata, Linn.

- var. variegata.

Desmazeria sieula, Dumort.

Deschampsia cæspitosa, Beauv.

Elymus

arenarius, Linn.

* glaucus, Gray.

sabulosus, Biebrst.

tener, Linn.

villosus, Muhlb.

Festuca

arundinacea, Schreb.

*borealis, Mert., Koch.

delicatula, Lag.

duriuscula, Linn.

elatior, Linu.

Hallerii, All.

heterophylla, Haenk.

Myurus, Linn.

ovina, Linn.

- var. tenuifolia.

- var.

picta, Kit.

rubra, Linn.

sciuroides, Roth.

*thalasica, Ktz.

uniglumis.

*Gaudinia geminiflora, Gay.

Glyceria aquatica, Linn.

*Hierochloa odorata.

Holcus mollis, Linn:

Hordeum

mandschurianum, Rgl.

murinum, Linn.

trifurcatum, Ser.

vulgare, Linn.

Zeocriton, Linn.
Knappia agrostidea, Sm.

* Koeleria

brachystachys, DC.

cristata, Pers.

vallesiaca, DC.

*Lagurus ovata, Linn.

Lolium

italicum, Braun.

perenne, Linn.

- var.

scabrum, Presl.

Melica

ciliata, Linn.

magnolica, Greu. et Godr. nutans, Linn.

*uniflora, Retz.

*Milium effusum, Linn.

Molinia cœrulea, Moench.

Nardurus - var. variegata.

Poa, Boiss.

tenella, Reichb,

unilateralis, Boiss.

Oryza sativa, Linn.

Phalaris

arundinacea, Linn.

brachystachys, Link.

canariensis, Linn.

paradoxa, Linn.

* trigyna, Host.

*truncata, Guss.

Phleum

*arenarium, Linn.

asperum, Vill.

Poa

pratensis, Linn.

anceps, Forsk.

Balfourii, Parn.

cæsia, Smith.

- var. glauca.

nemoralis, Linn.

- var. sempervirens, Hort. palustris, Linn.

Polypogon

affinis, Brongn.

*interruptus, H. B.

*littoralis, Sm.

Secale

anatolicum, Boiss.

*dalmaticum, Vis.

Serrafalcus

*divaricatus, Rohd.

secalinus, Parl.

vestitus, Willd.

*Sesleria

* coerulea, Arduin.

*tenuifolia, Sehrad. 
* Setaria viridis, Beauv.

*Triodea decumbens, Beauv.

Triticum

caninum, Huds.

dicoceum, Sehrank.

elymoides, Hornem.
Triticum-contd.

pannonicum, Linn.

Spelta, Linn.

*ventricosum, Tausch. violaceum, Hornem.

\section{TREES AND SHRUBS.}

\section{BERBERIDEÆ.}

Berberis vulgaris, Linn. Mahonia

Aquifolium, Nutt.

Mahonia-contd.

glumacea, DC.

\section{CRUCIFERÆ.}

Vella pseudo-cytisus, Linn.

\section{TILIACEÆ.}

Tilia vulgaris, Hayne.

\section{RUTACEÆ.}

Ptelea trifoliata, Linn.

\section{ILICINEAE.}

Ilex

Aquifolium, Linn.

Ilex-contd. Aquifolium, Linn.

- var. camelliæfolium, Hort.

- var. fructu lutea, Hort.

\section{RHAMNEÆ.}

Rhamnus alnifolius, Linn.

| Rhamnus latifolius, L'Hérit. 
SAPINDACEA.

Acer

circinatum, Pursh.

Pseudo-platanus, Linn.

Acer Pseudo-platanus, Linn., var. purpurea, Hort.

Esculus Hippocastanum, Linn.

\section{LEGUMINOSÆ.}

Colutea arborescens, Linn.

Cytisus

biflorus, L'Hérit.

scoparius, Link.

Laburnum

alpinum, Griseb.

vulgare, Griseb.

Ulex europæus, Linn.

\section{ROSACEA.}

Cotoneaster

acuminata, Lindl.

Fontanesii, Spach.

frigida, Wall.

Hookeriana, Hort. microphylla, Wall. sikkimensis, Hort.

Simonsii, Baker.

Cratægus

Aronia, Bosc.

coccinea, Linn.

- var. acerifolia, Hort.

orientalis, Bose.

Oxyacantha, Linn.

- var. horrida, Hort.

- var. pendula, Hort.

- var. punicea, Hort.

punctata, Ait.

Mespilus (Lady Harwick's).

Neillia opulifolia, Benth. et Hook.
Prunus

Arvium, Linn.

Pattonii, Hort.

Pyrus

americana, DC.

Aria, Ehrh.

Aucuparia, Gærtn.

- var. fastigiata, Hort.

- var. fructu lutea, Hort.

baccata, Linn.

latifolia, Syme.

pinnatifida, Ehrh.

Toringa, Hort.

Rhodotypus kerrioides, Sieb. et Zucc.

Rosa

polyantha, Sieb. et Zucc.

Rubus - var. alba.

laciniatus, Willd.

(Lawton's).

nutkanus, Moc.

\section{SAXIFRAGACEÆ.}

*Escallonia Phillipiana, Hort. I Ribes sanguineum, Pursh.

अงคมี

\section{ARALIACEÆ.}

Aralia hispida, Michx. 


\section{CORNACEAE.}

Cornus alba, Linn.

| Cornus alternifolius, Linn.

\section{CAPRIFOLIAC $Æ$.}

Leycesteria formosa, Wall.

Symphoricarpus racemosus, Michx.

Sambucus

Fontainesii, Hort.

nigra, Linn.

- var. rubrocarpa, Hort.
Sambucus-contd. racemosa, Linn.

Viburnum

Lantana, Linn.

Opulus, Linn.

Weigelia amabilis, Planch.

\section{COMPOSIT $Æ$.}

Olearia Haastii, Hook. fil.

\section{ERICACEÆ.}

Azalea pontica, Linn.

Gaultheria Shallon, Pursh.

Kalmia angustifolia, Linn.

Ledum palustre, Linn.

Pieris floribunda, Benth, et Hook, fil.

Rhododendron

campanulatum, Don.

catawbiense, Michx.

caucasieum, Pall.

cinnabarinum, Hook. fil.
Rhododendron-contd. daphnoides, Hort. ferrugineum, Linn. - var. album, Hort. glaucum, Don. hirsutum, Linn. lepidotum, Wall. ponticum, Linn. punctatum, Andr.

\section{OLEACEÆ.}

Syringa vulgaris, Linn.

\section{SCROPHULARINEA.}

Veronica Traversii, Hook, fil.

\section{THYMELÆACEÆ.}

Daphne Mezereum, Lindl., var. flore albo, Hort.

\section{ELÆAGNACEÆ.}

Elæagnus odoratus, Hort, var. edulis.

\section{CUPULIFERÆ.}

Alnus

cordifolia, Lodd. glutinosa, Gærtn.
Betula

alba, Linn.

— var. pendula, Sm. 


\section{CONIFERÆ.}

Chamæcyparis

Lawsoniana, Parl.

nutkäensis, Spach.

Pinus parviflora, Sieb.

Taxus

adpressa, Knight.

baccata, Linn.

\section{ISAAC BAYLEY BALFOUR, \\ Keeper.}

EDInburgh, January 1893.

\section{ROBERT LINDSAY,}

Curator of the Garden.

A. D. RICHARDSON,

Foreman in charge of the Arboretum.
ALEX. ANDERSON,

Foreman in charge of the Herbaceous Department. 


\section{9397107}

tom int

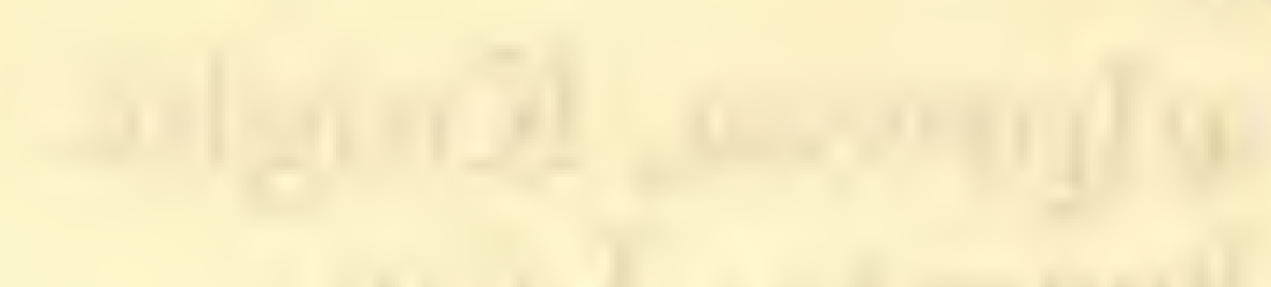

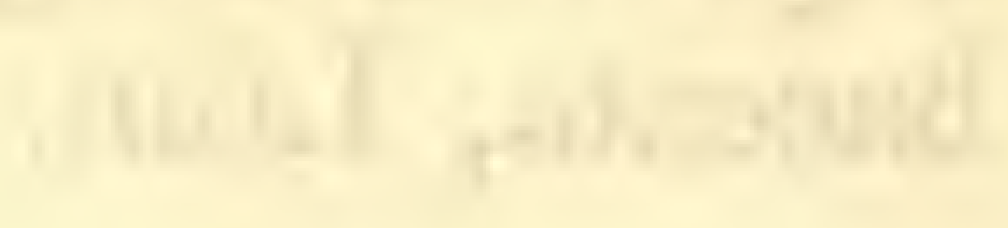

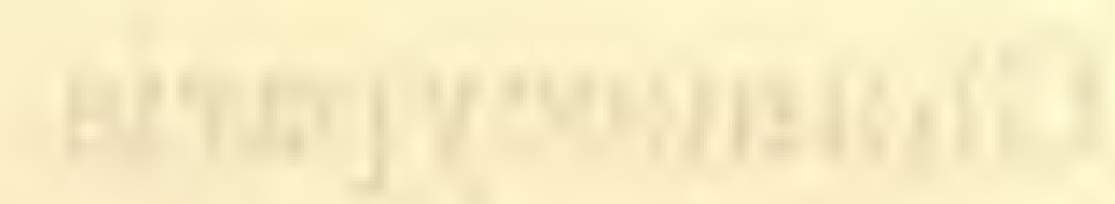

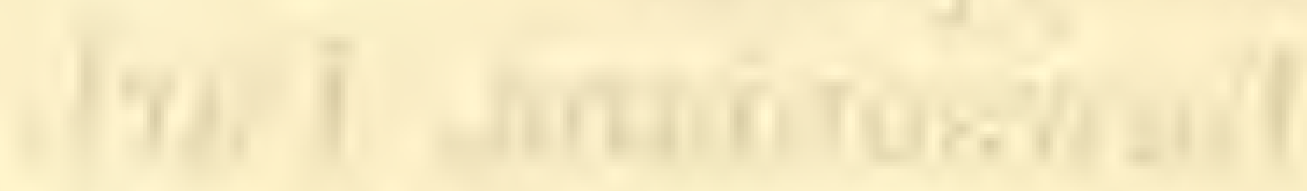

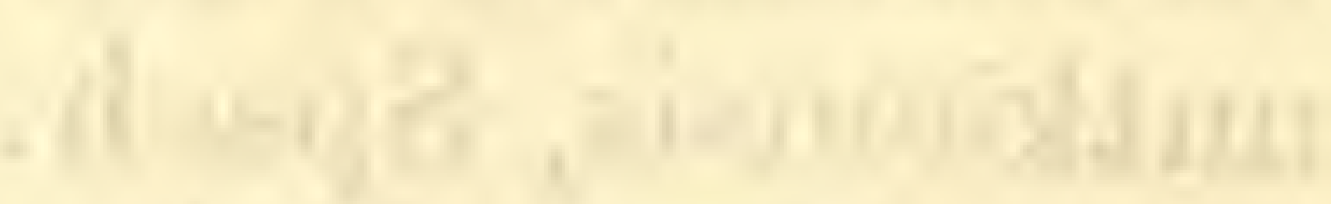

dakn fitcoliving donst

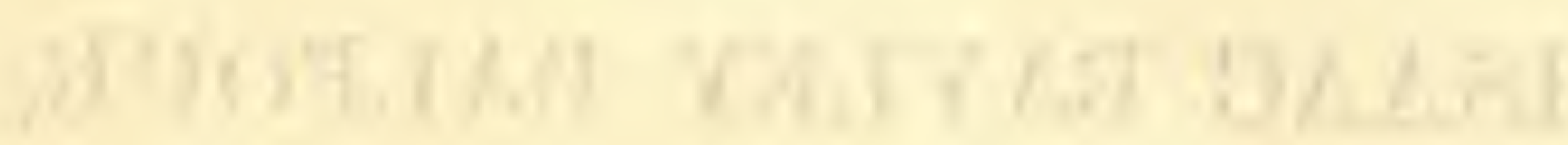 \\ jomitit}

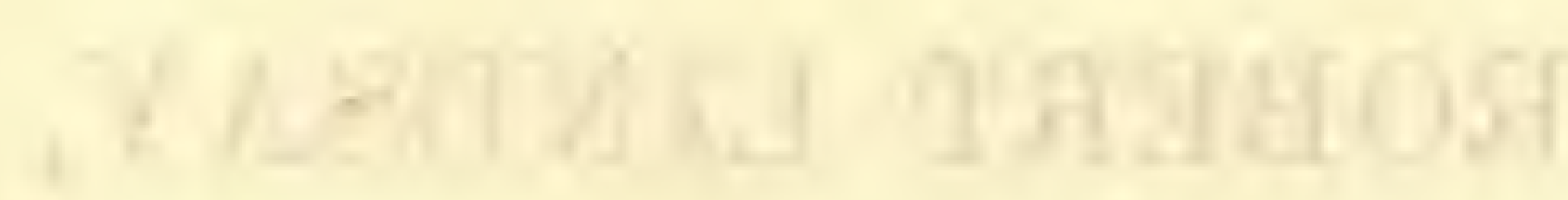

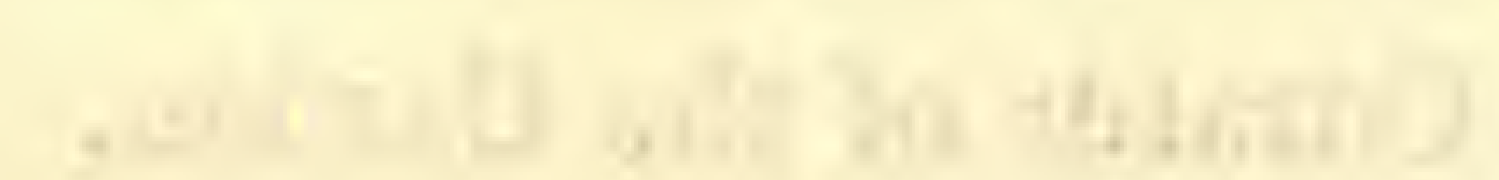

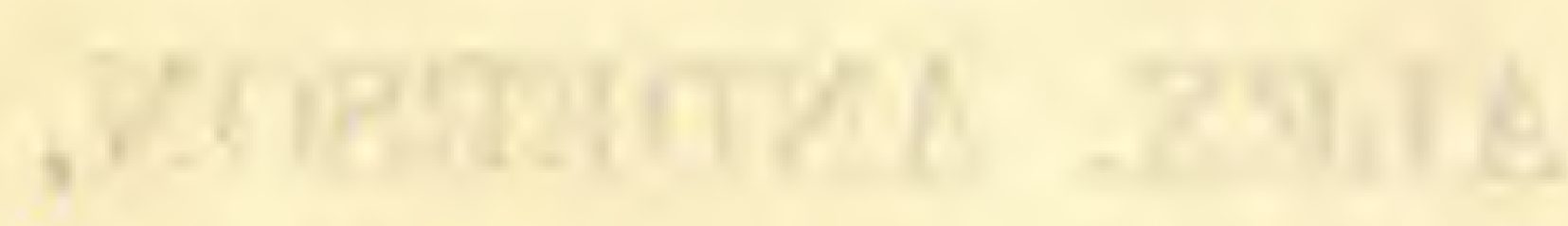

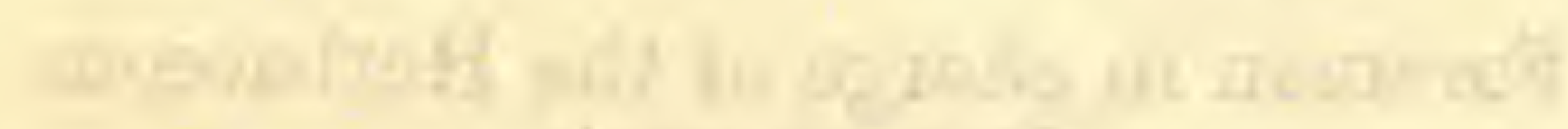

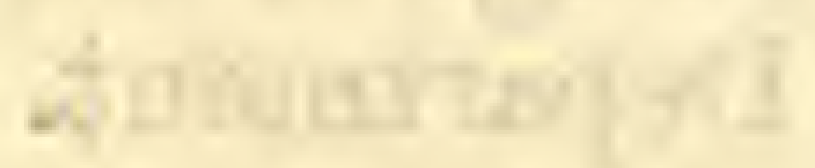

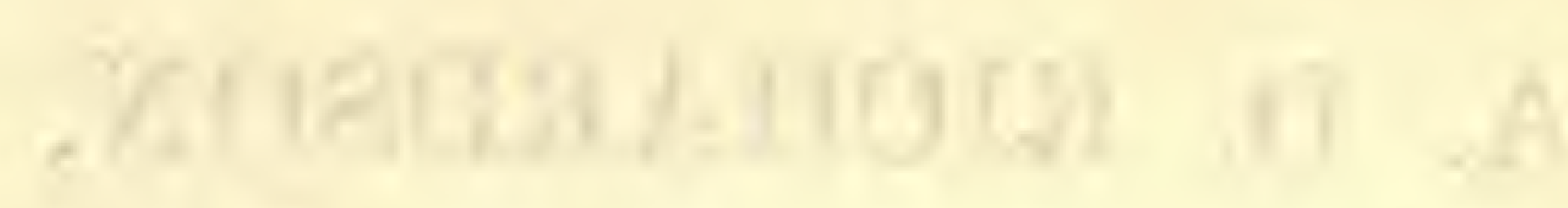

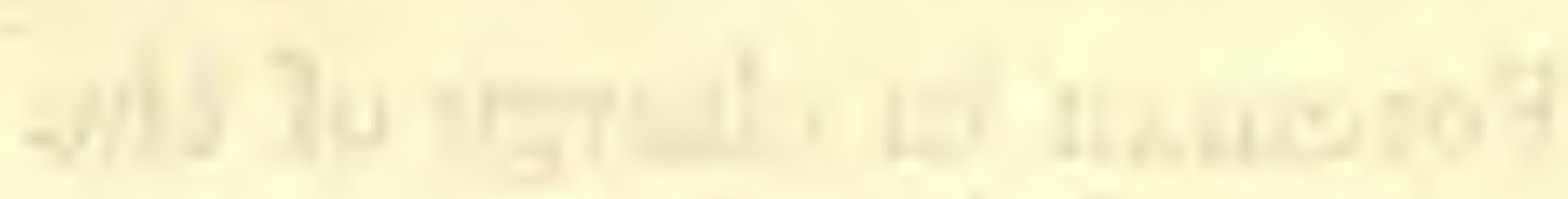

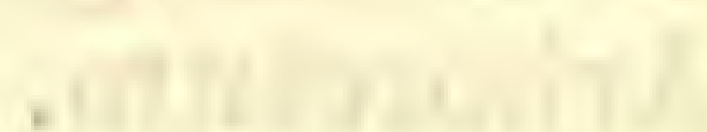






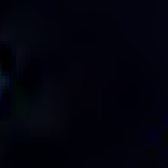





\title{
ROYAL BOTANIC GARDEN, EDINBURGH
}

\section{LIST OF SEEDS}

\author{
COLLECTED DURING 1893.
}

\section{Ma. Buk. Garituit. \\ 1894}

PRINTED FOR HER MAJESTY'S STATIONERY OFRICE,

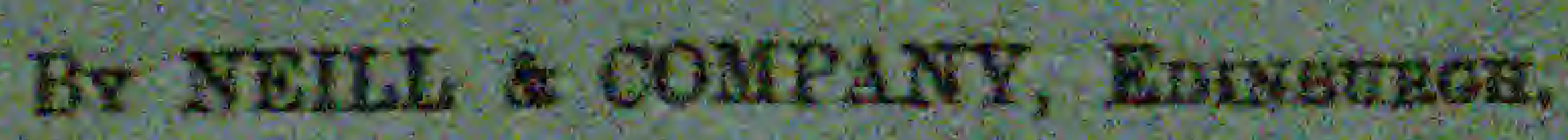

1894. 
16\%

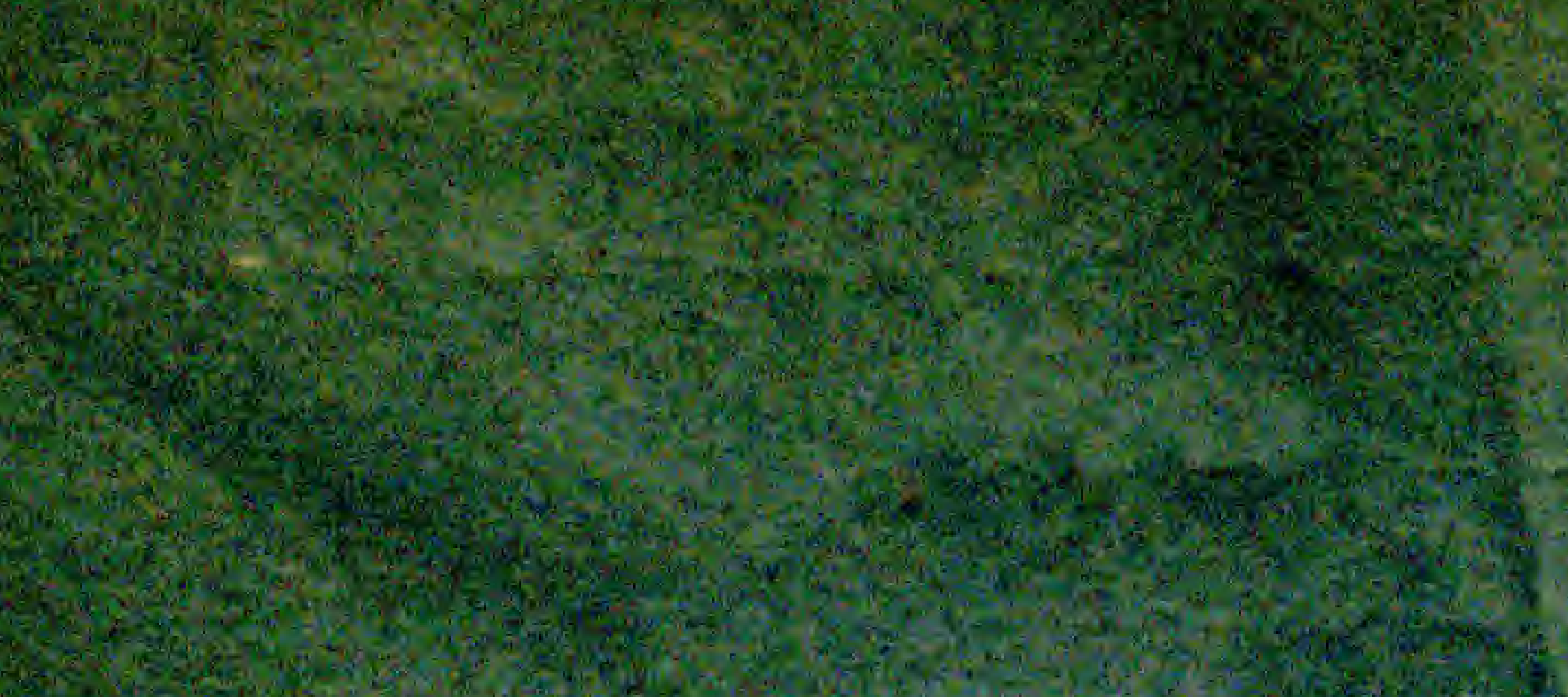

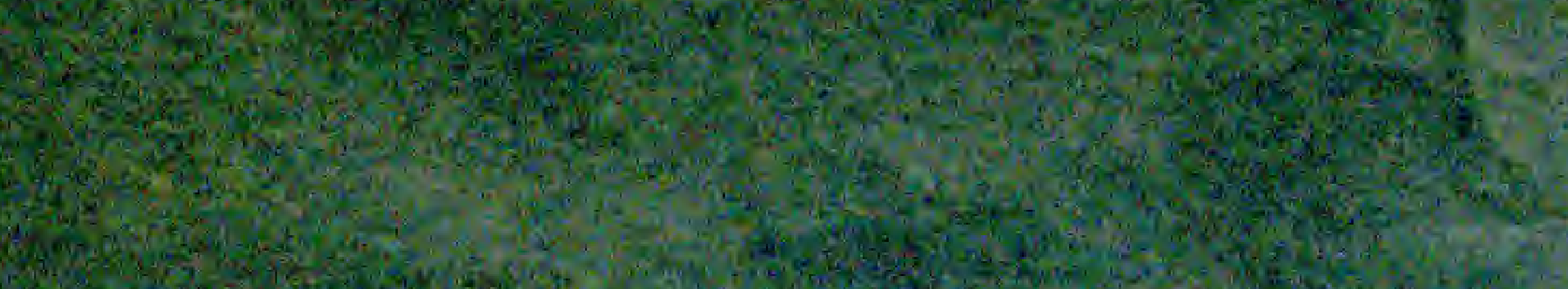

3.

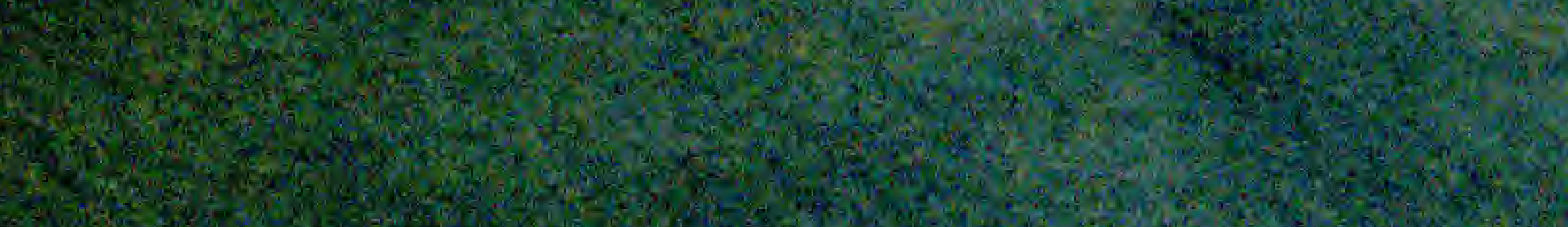

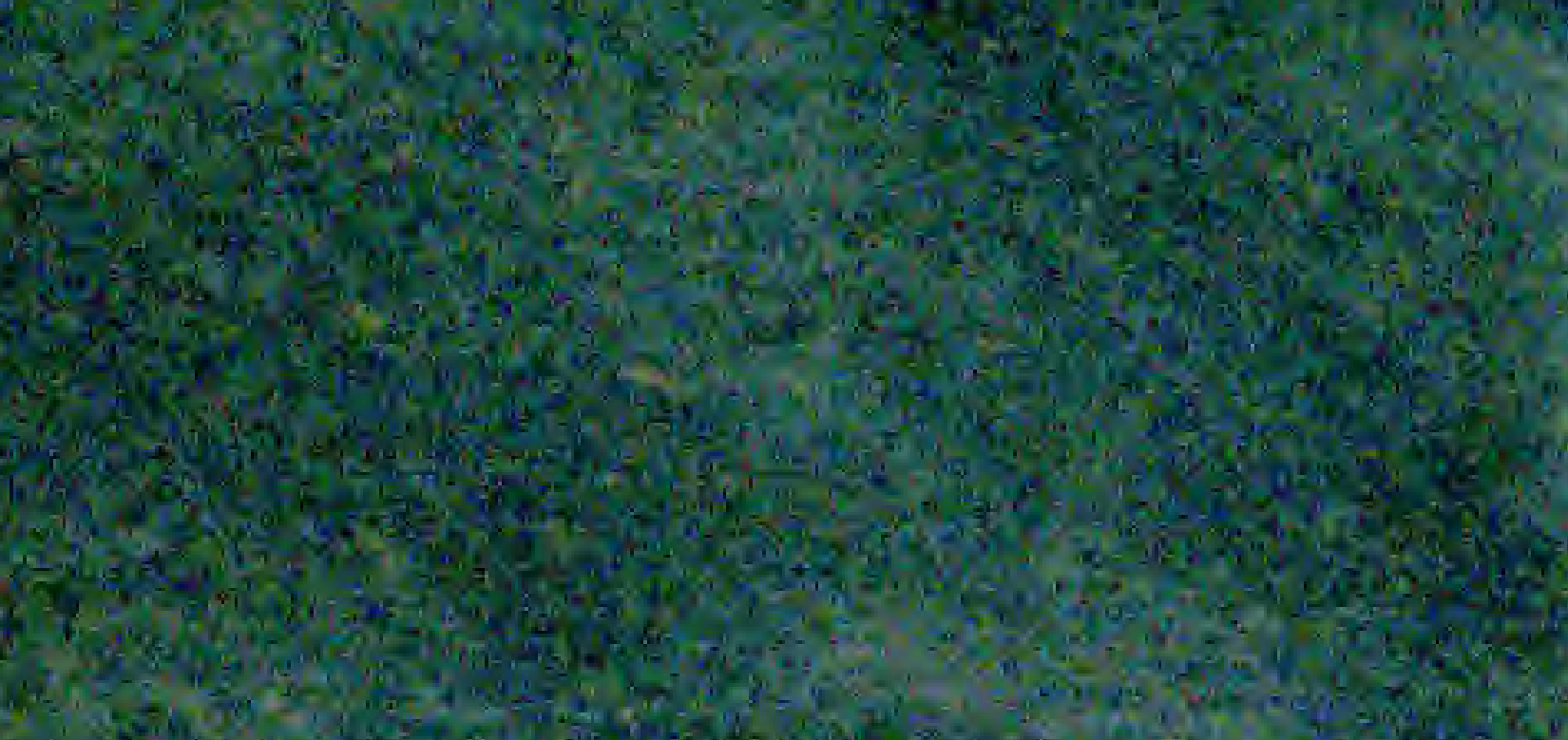

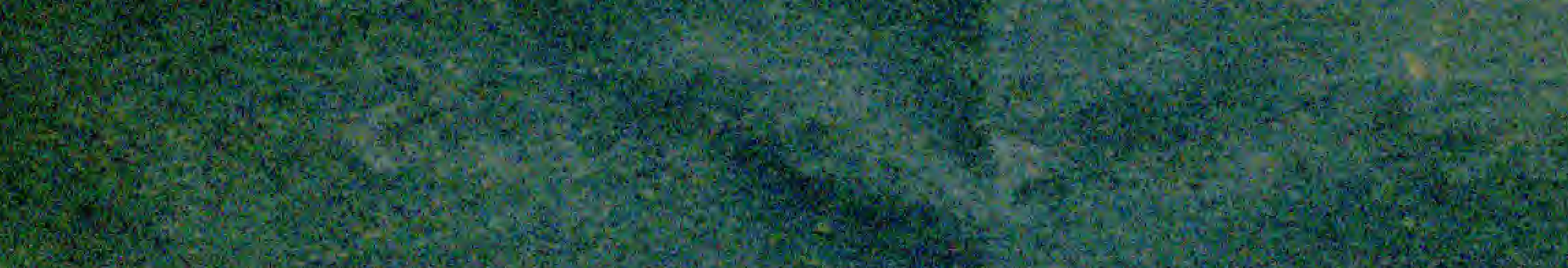

(2)

1.

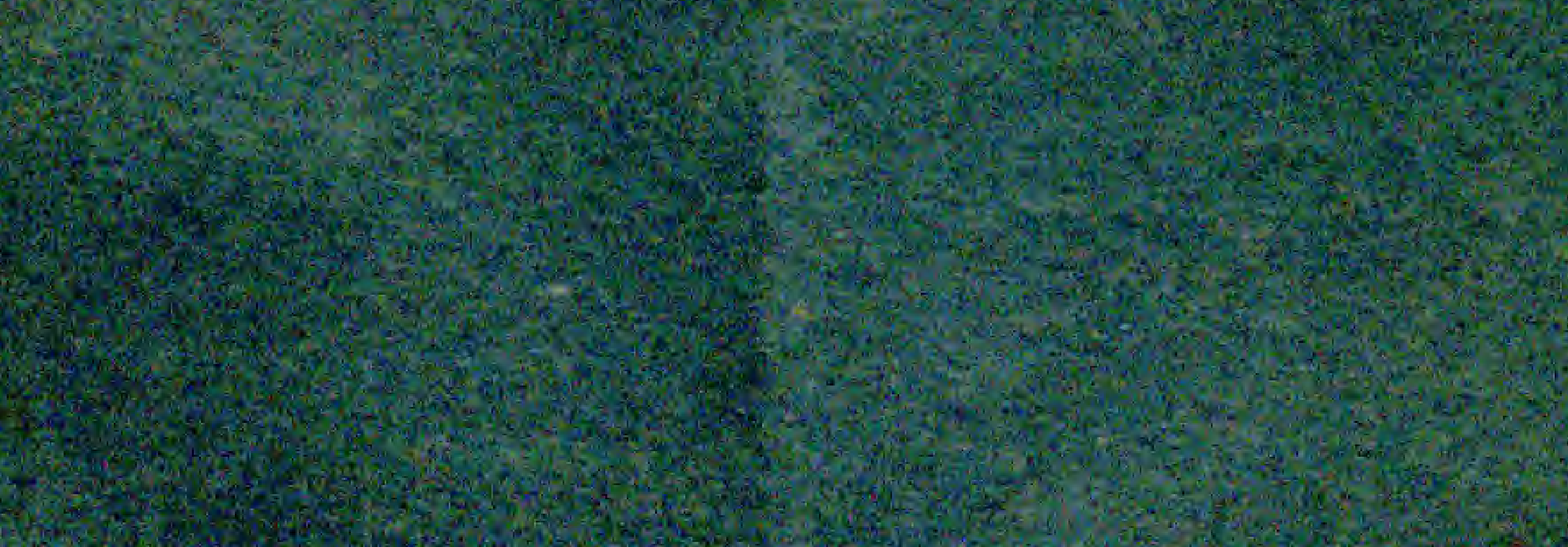

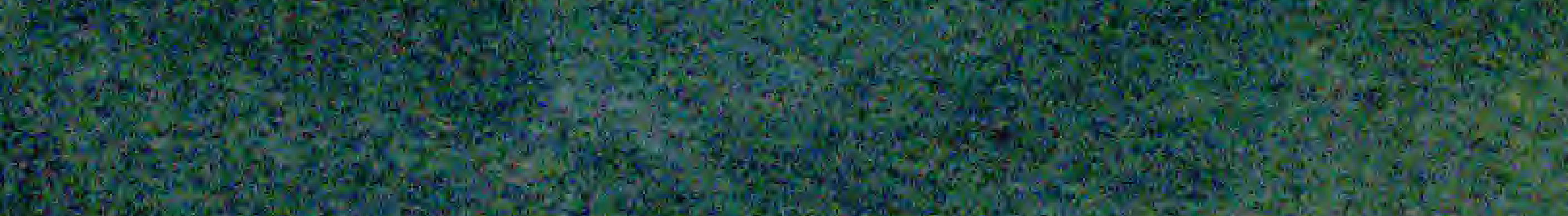


ROYAL BOTANIC GARDEN, EDINBURGH.

\title{
LIST OF SEEDS
}

\author{
COLLECTED DURING 1893.
}

\section{HERBACEOUS PLANTS.}

Acæna argentea, Ruiz. et Pav.

inermis, Hook. fil.

microphylla, Hook. fil.

myriophylla, Lindl.

Sanguisorbae, Vahl.

- var. sarmentosa.

Achillea Millefolium, Linn.

- var. roseum.

- - strictum.

nobilis, Linn.

Aconitum Anthora, Linn. eminens, Koch.

heterophyllum, Wall.

longigaleatum, Hort.

Lycoctonum, Linn.

- var. moldavicum.

- septentrionale.

- var, alba.

Actæa spicata, Linn.

- var. alba.

- - rubra, Michx.

Adenophora stylosa, Fisch.

Adonis pyrenaica, DC.

vernalis, Willd.

Athionema ovalifolium, Boiss.

Agasyllis caucasicus, Spreng.

Agrimonia agrimonioides, Linn.

Eupatoria, Linn.

nepalensis, D. Don.

Agrostis alba, Linn. var. stolonifera. nebulosa, Boiss.

vulgaris, With.

Alchemilla alpina, Linn, var. conjuncta.

montana, Willd.

pubescens, Biebrst.

vulgaris, Linn.

Alfredia cernua, Cass.
Alisma Plantago, Linn.

- - var. rubra.

ranunculoides, Linn.

Allium ampeloprasum, Linn. var. Babingtonii, Borr.

arenarium, Schrad.

carinatum, Linn.

descendens, Linn.

Dioscoridis, Sibth. et Sm.

fistulosum, Linn.

flavum, Linn. var. pulchellum.

globosum, Biebrst. var. album.

M'Leanii, Baker.

neapolitanum, Cyr.

paradoxum, G. Don.

polyphyllum, Kar. et Kit.

rubens, Schrad.

Scorodoprasum, Linn.

serecolatum, Hort.

stipitatum, Regel.

strictum, Schrad.

tenuifolium, Frivald.

vineale, Mill.

- var. Kochii.

Alopecurus arundinaceus, Poir.

lasiostachyus, Link.

pratensis, Linn.

- var. variegatus.

Althæa ficifolia, Cav.

Hohenackeri, Boiss. et Huet.

Kragujevacensis, Pancic. pallida, Waldst. et Kit.

Alyssum gemonense, Linn.

podolicum, Benth. et Hook. rostratum, Stev.

saxatilis, Linn.

spinosum, Linn.

tortuosum, Rupr 
Amarantus caudatus, Linn. - var. albiflorus. glomeratus, Otto. hypochondriacus, Linn. - var. pyramidalis. mantegazzianus, Pass. paniculatus, Linn. retroflexus, Linn.

Ampelodesma tenax, Link.

Anagallis arvensis, Linn.

Anchusa sempervirens, Linn. sikkimensis, C. B. Clarke.

Androsace lactea, Linn. lactiflora, Hort. Laggerii, Huet. septentrionale, Linn. var.

Anemone alpina, Linn.

- var. sulphurea, DC.

baikalensis, Hort. decapetala, Linn. narcissiflora, Linn. var. palmata, Linn. patens, Linn. Pulsatilla, Linn. rivularis, Buchan. umbellata, Willd.

Angelica Gingidium, Hook, fil.

Anisodus luridus, Link.

Antennaria dioica, Gaertn.

Anthemis Chia, Linn. tinctoria, Linn.

- var discoidea.

Anthericum Liliago, Linn. - var. algeriense. Liliastrum, Bert.

Anthoxanthum gracile, Bivon.

Anthriscus Cerefolium, Hoffm. sylvestris, Hoffm.

Anthyllis tetraphylla, Linn. Vulneraria, Linn.

- var. rubra, Hort.

Antirrhinum Asarina, Linn. majus, Linn. numidicum, Hort.

Aponogeton distachyon, Thunbg.

Aquilegia atropurpurea, Willd.

Burgeriana, Sieb. et Zucc. californica, A. Gray, var. alba. chrysantha, A. Gray.

cœrulea, James.

Einseleana, Fr. Schultz.

flavescens, S. Watson.
Aquilegia-contd.

glandulosa, Fisch.

- var. jucunda.

grata, Maly.

Hulkeana, Hort.

Kitaibelii, Schott.

lutea, Lamk.

pyrenaica, DC.

vervœniana, Hort.

viridiflora, Pall.

vulgaris, Linn.

Arabis alpestris, Schleich. et Reichb.

anachordica, Hort.

bellidifolia, Jacq.

fladnicensis, Hort.

japonica, Regel.

lucida, Linn. fil.

muralis, Bertol.

petræa, Lamk.

sagittata, DC.

Soyeri, Reut. et Huet.

stricta, Huds.

turrita, Linn.

Aralia hispida, Michx.

Archangelica officinalis, Hoffm.

Arctium Bardana, Willd.

majus, Schk.

Arenaria gothica, Fries.

graminifolia, Schrad.

saxatilis, Linn.

serpyllifolia, Linn.

stricta, Michx.

Argemone speciosa, Hort.

Armeria alpina, Willd.

berlingensis, Daveau.

bupleuroides, Hort.

cæspitosa, Boiss.

canariensis, Boiss.

- var. rumelica.

dianthifolia, Hort.

Duriæii, Boiss.

fasciculata, Willd.

filicaulis, Boiss.

longearistata, Boiss. et Reut.

longibracteata, Hort.

majellensis, Boiss.

maritima, Willd.

mauritanica, Wallr.

- var. alba.

pinifolia, Link.

plantaginea, Willd. 
Armeria-contd.

pubescens, Link.

splendens, Webb.

Welwitschii, Boiss.

Arnica montana, Linn.

Arracacia arguta, Benth. et Hook.

Arrhenatherum avenaceum, Beauv.

- var. bulbosum.

Thorei, Desm.

Artemisia campestris, Linn.

desertorum, Spreng.

græca, Hort.

rupestris, Linn.

sacrorum, Ledeb.

vulgaris, Linn.

Arum maculatum, Linn.

palæstinum, Boiss.

Arundo acutiloba, Hort.

Asarum europæum, Linn.

Asparagus officinalis, Linn.

Asperula arvensis, Linn.

cynanchica, Linn.

lævigata, Linn.

Asphodelus ramosus, Linn.

Aster acris, Linn.

alpinus, Linn.

- var. albus.

- - speciosus.

falcatus, Lindl.

Fortunei, Hort.

Lindleyanus, Torr. et Gray.

paniculatus, Lamk.

sibiricus, Linn.

Thomsoni, C. B. Clarke.

Astragalus bicolor, Lamk.

chlorostachys, Lindl.

Cicer, Linn.

Glycyphyllos, Linn.

lapponica, Hort.

monspessulanus, Linn.

vicioides, Ledeb.

Astrantia major, Linn.

Astrocarpus sesamoides, DC.

Atriplex patula, Linn., var. Babingtonii.

Atropa Belladonna, Linn.

- var. fructu flava, Hort.

Aubrietia deltoidea, DC.

- var. coelestis, Hort.

- columnea, Hort.

- - græca, Griseb.

- - grandiflora, Hort.

- - Pinardii, Hort.
Avena

fatua, Linn.

pratensis, Linn.

- var. planiculmis.

pubescens, Linn.

Bahia lanata, Nutt.

Ballota hispanica, Benth. italica, Benth.

Baptisia australis, R.Br.

exaltata, Sweet.

leucophæa, Nutt.

lutea, Hort.

Barbarea altissima, Hort.

arcuata, Rchb.

vulgaris, R.Br.

Bartonia aurea, Lindl.

Beckmannia erucæformis, Host.

Beta maritima, Linn.

Bidens connata, Muhl. cordifolia, Sch.

Novæ-Caledoniæ, Forst.

Biscutella cichoriifolia, Loisel. ciliata, DC.

didyma, Linn., var. columnæ. lævigata, Linn.

Blumenbachia insignis, Schrad.

Borago officinalis, Linn.

Boykinia aconitifolia, Nutt. - var. major.

Brachycome Sinclairii, Hook. fil.

Brachypodium pinnatum, Beauv.

Brassica alba, Boiss.

lanceolata, Lange.

Napus, Linn.

nigra, Koch.

Sinapistrum, Boiss.

Braya alpina, Sternb.

Briza graciìis, Hort. maxima, Linn. minor, Linn.

Brodiæa congesta, Sm.

Bromus adoensis, Hochst. congestus, Hort.

sylvatica, Pall.

tectorum, Linn.

Brunella grandiflora, Linn. hyssopifolia, Linn.

laciniata, Linn.

vulgaris, Linn. var. hispida.

- var, Webbiana, Hort. 
Bryonia dioica, Jacq.

Bulbine alooides, Willd.

Bulbinella Hookeri, Colenso.

Bunias orientalis, Linn.

Bupleurum petræum Linn. protractum, Hoffmgg., et Link. ranunculoides, Linn. rotundifolia, Linn.

Butomus umbellatus, Linn.

Calamagrostis epigeijos, Roth.

Calamintha Clinopodium, Bth.

Calandrina Andrewsii, Sweet. linearifolia, DC.

Calceolaria mexicana, Benth.

Calendula officinalis, Linn. stellata, Cav.

Caltha palustris, Linn. - var. biflora. sylvestris, Hort.

Calystegia sepium, R. Br.

Camassia Brownii, Hort. esculenta, Lindl.

- var. alba.

- - major.

tardiflora, Hort.

Camelina sativa, Crantz. sylvestris, Wallr. var. microcarpa.

Campanula abietina, Griseb. et Schrenk.

barbata, Linn.

Baumgartenii, Becker.

cæspitosa, Scop.

carpatica, Jacq.

- var. alba.

collina, Biebrst.

elegans, Roem. et Schult.

glomerata, Linn.

- var. lilacina.

Grossekii, Heuffl.

latifolia, Linn.

- var. macrantha.

- - - alba.

medium, Linn.

persicifolia, Linn.

- var. alba.

- - grandis.

pulcherrima, Schrank. et Zeyh.

punctata, Lamk.

rapunculoides, Linn.

rotundifolia, Linn.

- var. pluripetala.

sarmatica, Ker. Gawl.
Campanula-contd.

speciosa, Hornem.

Speculum, Linn.

thyrsoides, Linn.

Trachelium, Linn.

valdensis, All.

Cannabis gigantea, Hcrt. sativa, Linn.

Capsella Bursa-pastoris, Medic. procumbens, Fries.

Carbenia benedicta, Adans.

Carduus defloratus, Linn.

Carex anceps, Muhl. aquatilis, Wahl. atrata, Linn.

binervis, Sm. capillaris, Linn.

caucasica, Stev.

Drymingii, Hort.

frigida, All.

fulva, Good.

Grayii, Carey.

Mairii, Coss.

microglochin, Wahlenb.

ovalis, Good.

paniculata, Huds.

pilulifera, Linn.

riparia, Curt.

stictocarpa, Sm.

straminea, Willd.

Vahlii, Schkuhr.

vulpina, Linn.

Carum Bulbocastanum, Koch.

Cathcartia villosa, Hook. fil.

Celsia laciniata, Poir.

Centaurea alpina, Linn.

calcitrapoides, Linn.

centauroides, Linn.

cinerea, Lamk.

Clementei, Boiss.

Cyanus, Linn.

dealbata, Willd.

Debeauxi, Hort.

elata, Poir.

græeca, Boiss.

macrocephala, Muss.

oleraceus, Linn.

orientalis, Linn. var. rubra.

pallida, Frivald, var. gigantea.

pectinata, Linn.

Phrygia, Linn.

ragusina, Linn. var. candidis. sima.

Rhapontica, Linn.

quinquifolia, Hort. 
Centaurea-contd.

scabiosa, Linn.

sulphurea, Willd.

Centranthus ruber, DC.

Cephalaria tatarica, Schrad.

Cerastium Mæsiacum, Frivald.

Cerinthe alpina, Kit.

minor, Linn.

retorta, Sibth. et Sm.

Chærophyllum aromaticum, Jacq. nodosum, Crantz.

Chardinia xeranthemoides, Desf.

Cheiranthus Allionii, Hort.

Cheiri, Linn.

Senoneri, Heldr. et Sart.

Chelidonium majus, Linn.

- var. grandiflorum.

- laciniatum.

Chenopodium Bonus-Henricus, Linn.

foetidum, Schrad.

Frankemanum, F. Muell.

mitrariaceum, Hort.

Quinoa, Willd.

Chionodoxa Luciliæ, Boiss.

Chrysanthemum atratum, Linn. rotundifolium, Waldst. et Kit.

Roxburghii, Desf.

segetum, Linn. var. grandiflorum.

tricolor, Hort.

tripteris, Hort.

Chrysopogon Gryllus, Trin.

Cichorium Intybus, Linn.

Cineraria concolor, Hort. geifolia, Linn.

Cinna americana, Beauv. mexicana, Beauv.

Cirsium horridum, Biebrst. pannonicum, DC.

Cistus laurifolius, Linn.

Clarkia elegans, Lindl. pulchella, Pursh.

Claytonia perfoliata, Don. sibirica, Linn.

Clematis angustifolius, Jacq. diversifolia, Gilib. erecta, All. integrifolia, Linn.

Clintonia Andrewsiana, Torrey.

Clypeola Jonthlaspi, Linn.

Cnicus arvensis, Hoffm. atropurpureus, Hort. centauroides, Linn.
Cnicus-contd.

elatus, Hort.

eriophorus, Roth.

flavispinus, Ball.

græcus, Hort.

heterophyllus, Roth.

pannonicus, Host. var. divaricatus.

rigidus, Hort.

serrulatus, Biebrst.

Cochlearia officinalis, Linn. saxatilis, Lamk.

Codonopsis ovata, Benth.

Colchicum crociflorum, Sims.

Collomia coccinea, Lehm.

Collinsia bicolor, Benth. grandiflora, Dougl.

Commelina kamtschatica, Hort.

Convallaria majalis, Linn.

Convolvulus siculus, Linn. tricolor, Linn.

Coptis trifolia, Salisb.

Cortusa Matthioli, Linn.

Corydalis glauca, Pers. var. rosea, Hort.

nobilis, Pers.

Cotoneaster horizontalis, Decue. thymifolia, Hort.

Cotyledon Umbilicus, Linn.

Crambe maritima, Linn.

Craspedia Richea, Cass.

Cratoxylon Blancoi, Blume.

Crepis aurea, Cass. chrysantha, Frnel.

Crocus medius, Balbis. vernus, All. - var.

Crucianella stylosa, Trin.

Cyclamen hederæfolium, Willd.

Cynanchum nigrum, Linn.

Cynoglossum magellense, Tenore.

Daboecia polifolia, Don.

- var. alba.

Dactylis glomerata, Linn.

- var. aurea.

- variegata.

Datura fastuosa, Linn.

foetidum, Rafin.

inermis, Jacq.

Leichardtii, Fisch. et Mey. 


\section{Datura-contd.}

muricata, Link.

Stramonium, Lirm.

- var. Tatula, Hort.

Delphinium Ajacis, Reichb.

altissimum, Wall.

Brunonianum, Royle.

cashmirianum, Royle.

crassifolium, Schrad, var. turkestanicum.

formosum, Boiss, et Reut.

hybridum, Steph.

Maackianum, Regel.

macranthum.

Nahamah, Hort.

nudicaule, Torr. et Gray.

palmatifidum, DC.

velutinum, Bert.

Wheelerii, Hort.

Demazeria sicula, Dum.

Deschampsia cæspitosa, Beauv.

Dianthus alpinus, Linn. arborescens, Linn. arenarius, Linn.

asper, Willd.

Balbisii, Ser.

barbatus, Linn.

cæsius, Sm.

carthusianorum, Linn.

caucasicus, Biebrst.

corsicus, Fisch.

glacialis, Hænke.

grandiflorus, Poir.

hæmatocalyx, Boiss. et Heldr. microlepis, Boiss.

monspessulanus, Linn.

multipunctatus, Ser.

neglectus, Loisl.

pelviformis, Heuff.

petræus, Waldst. et Kit.

plumarius, Linn., sub. sp. serotinus, Waldst. et Kit.

polymorphus, Biebrst.

proliferus, Linn.

pungens, Linn.

Simsii, Hort.

squarrosus, Biebrst.

superbus, Linn.

superbus $\times$ barbatus.

sylvestris, Wulf.

trifasciculatus, Kit.

viscidus, Bory. et Chaub.

Dictamnus albus, Linn.

- var. ruber.

tauricus, Hort.
Digitalis lutea, Linn. purpurea, Linn.

Diplotaxis erucoides, DC. viminea, DC.

Dipsacus centaurifolia, Hort.

ferox, Linn.

Fullonum, Linn.

laciniatus, Linn.

sylvestris, Linn.

Dodecatheon integrifolia, Michx.

Draba Aizoon, Wahl. altiaca, Bunge.

alpina, Linn.

Gmelini, Adams.

incana, Linn.

rupestris, R.Br.

Dracocephalum Ruyschianum, Linn. speciosum, Benth.

Dryas octopetala, Linn.

- var. minor.

Echinops azureus, Hort. humilis, Biebrst. microcephalus, Sibth. et Sm. spinosus, Linn. strictus, Biebrst.

Echium violaceum, Linn.

Eleocharis palustris, $\mathrm{Br}$.

Elsholtzia cristata, Willd.

Elymus arenarius, Linn. condensatus, J. et C. Presl. sibiricus, Linn.

sabulosus, Biebrst. tener, Linn. villosus, Muhlb.

Emex spinosa, Camb.

Epilobium alsinifolium, Vill. angustifolium, Linn.

Dodonæi, Vill.

- var. Fleischerii.

glandulosum, Lehm.

hirsutum, Linn.

hypericifolium, Tausch.

latifolium, Linn.

microphyllum, A. Rich.

nummularifolum, R. Cunn.

obscurum, Schreb.

pedunculare, A. Cunn.

virgatum, $\mathrm{A}$. Cunn.

Eranthis hyemalis, Salisb.

Eremostachys laciniata, Bunge.

Erica cinerea, Linn. var. atropurpurea.

- var. bicolor.

vagans, Linn. var. alba minor. 
Erigeron glabellum, Nutt., var. molle. multiradiatus, Benth.

Roylei, DC.

speciosa, DC.

Erinus hispanicus, Pers., var, albus.

Eriophorum alpinum, Linn. vaginatum, Linn.

Eritrichium corniculatum, Lamk.

Erodium aconitifolium, L'Herit. Manescavi, Coss.

Eryngium agavæfolium, Delar. alpinum, Linn. amethystinum, Linn. dichotomum, Desf. falcatum, Delar. macrocalyx, Schrenk. maritimum, Linn. planum, Linn. Wrightii, A. Gray.

Erysimum canescens, Roth. cheiranthoides, Linn. hieracifolium, Linn. latifolium, Hort.

Marshallianum, Andrz. ochroleucum, DC.

Perofskianum, Fisch. et Mey.

Erythronium grandiflorum, Pursh.

Eschscholzia californica, Cham.

- var. mandarin, Hort.

- var. Douglasii, Benth.

Eupatorium cannabinum, Linn. glechonophyllum, Less. purpureum, Linn. sessilifolium, Linn.

Euphorbia Myrsinites, Linn.

Fedia cornucopia, Vald.

Ferula Assa-fœetida, Linn.

Festuca amethystina, Linn. arundinacea, Vill. duriuscula, Linn. elatior, Linn.

- var. loliacea.

Halleri, All.

heterophylla, Lamk.

indigesta, Boiss.

Myuros, Linn.

ovina, Linn.

- var. tenuifolia.

- - sylvatica.

Pancici, Hack.
Festuca-contd.

pichinchae, Hort. pulchella, Schrad. rubra, Linn. varia, Hænke.

Fritillaria Meleagris, Linn.

Funkia lancifolia, Spreng. marginata, Sieb. Sieboldii, Lindl.

Gaillardia maxima, Hort. pulchella, Fouger. splendidissima, Hort.

Galega bicolor, Boiss. et Haussk. officinalis, Linn.

Galeopsis foetida, Hort.

Galium boreale, Linn. Mollugo, Linn. parisiense, Linn. saxatile, Linn. verum, Linn.

Galtonia candicans, Decne.

Gaultheria carnea, Hort.

Genista germanica, Linn. sagittalis, Linn. tinctoria, Linn., var. prostrata.

Gentiana acaulis, Linn. - var. Kochiana, Pers. albiflora, Schur.

Andrewsii, Griseb. asclepiadea, Linn., var. alba. brevidens, Franch et Sav.

Burseri, Lapeyr. cruciata, Linn. lutea, Linn. ornata, Wall. phlogifolia, Schott et Kotschy. purpurea, Linn. Saponaria, Linn. scabra, Bunge. septemfida, Pall. - var, cordifolia, C. Koch. tibetica, King. verna, Linn. Wallichii, Hort.

Geranium armenum, Boiss, balkanum, Hort. dahuricum, DC. Lambertii, Sweet. lancastriense, With. macrorrhizum, Linn. 
Geranium-contd.

maculatum, Linn.

pratense, Linn.

- var. alba.

sanguineum, Linn.

Wallichianum, Sweet.

Wilfredi, Maxim.

Gerbera Kunzeana, A. Br. et Aschers.

Geum altaicum, Bess. coccineum, Lindl. elatum, Wall.

Grahamii, Steud. intermedium, Ehrh. Laxmanni, Gaertn. miniatum, Hort.

nutans, Hort.

parviflorum, Comm.

rivale, Linn.

strictum, Soland.

Gilia achilleæfolia, Benth. alba, Hort. macrantha, Steud, var. aurea. nivalis, Hort. parviflora, Spreng. tricolor, Benth.

Glaucium squamigerum, Kar. et Kir. tricolor, Linn.

Glyceria aquatica, Linn. maritima, Mert. et Koch.

Graphephorum arundinaceum, Aschers.

Grindelia integrifolia, DC.

Gypsophila cerastioides, D. Don. elegans, Biebrst. perfoliata, Linn.

Hablitzia tamnoides, Biebrst.

Hedysarum obscurum, Linn.

Helianthella uniflora, Torr. et Gray.

Helianthemum vulgare, Gaertn.

- var. Garibaldi, Hort.

- - The Bride, Hort.

Helianthus decapetalus, Linn. occidentalis, Riddel.

Helichrysum bracteatum, Willd.

Helleborus fœtidus, Linn.

Helonias bullata, Linn. - - var. pallida.

Hepatica triloba, Chaix.

Heracleum giganteum, Fisch. var. diversifolium, Wall. Sphondylium, Linn.

Hesperis fragrans, Fisch. tristis, Linn
Heuchera americana, Linn.

Arlei, Hort.

cylindrica, Lindl.

Drummondii, Hort.

laxa, Hort.

obtusissima, Regel et Rach.

pubescens, Pursh.

sanguinea, Engelm.

- var.

spicata, Hort.

undulata, Regel et Rach.

Hieracium amplexicaule, Linn.

andryaloides, Vill.

angulosum, Linn.

balkanum, Uechtr.

bupleuroides, C.C. Gmel.

chlorocephalum, Wimm.

collinum, Fries.

cydoniæfolium, Vill.

foliosum, Waldst. et Kit.

glomeratum, Froel.

humile, Jacq.

intybaceum, Jacq.

Lagascæ, Hort.

nigrescens, Willd.

phlomoides, Froel.

præmorsum, Linn.

pyrenaicum, Jord.

saxatile, Jacq.

strictum, Frivald.

sylvaticum, Smith.

vulgatum, Fries.

Holcus lanatus, Linn.

Homogyne alpinum, Cass.

Hordeum bulbosum, Linn. himalayense, Schult. jubatum, Linn.

Horminum pyrenaicum, Linn.

Hyacinthus amethystinus, Linn.

Hyoscyamus niger, Linn.

reticulatus, Linn.

Hypecoum grandiflorum, Benth.

Hypericum Androsæmum, Linn.

Coris, Linn.

Gebleri, Ledeb.

hirsutum, Linn.

perforatum, Linn.

pulchrum, Linn.

quadrangulum, Linn., var. dubium.

trigonum, Hort.

Hypochæris apargioides, Hook, et

Arn.

arachnoidea, Linn.

Hyssopus officinalis, Linn. 
Iberis corifolia, Sweet. semperflorens, Linn. sempervirens, Linn. superba, Hort.

Tenoreana, DC. umbellata, Linn.

Impatiens Noli-me-tangere, Linn. parviflora, DC.

Roylei, Walp. scabrida, DC.

Inula glandulosa, Willd.

Helenium, Linn.

Hookeri, Clarke.

Iris germanica, Linn., var. asiatica.

Gueldenstaedtiana, Lepech., var. olbiensis.

laevigata, Fisch.

lurida, Soland.

prismatica, Pursh.

Pseudacorus, Linn.

setosa, Pall.

sibirica, Linn,

- var. orientalis.

- - sanguinea.

- var.

versicolor, Linn.

xiphioides, Ehrh.

- var.

Isatis hebecarpa, DC.

microcarpa, F. Gay.

præcox, Ledeb.

Isopyrum fumarioides, Linn.

Jasione montana, Linn.

Juncus balticus, Willd.

conglomeratus, Linn.

effusus, Linn.

- var. lutea.

lamprocarpus, Ehrh.

supinus, Moench.

tenuis, Willd.

trifidus, Linn.

Jurinea polyclonos, DC.

Kentrophyllum leucocaulon, DC.

Knappia agrostidea, Sm.

Knautia arvensis, Linn.

Kobresia caricina, Willd.

Kœleria Barrelieri, Ten.

cristata, Pers.

laxa, Link.

valesiaca, DC.
Lactuca virosa, Linn.

Lallemantia peltata, Fisch. et Mey.

Lamarckia aurea, Moench.

Lamium Galeobdolon, Crantz.

Lappa intermedia, Reichb. f.

Lapsana grandiflora, Bbrst.

Laserpitium alatum, Linn. aquilegifolium, DC.

crithmifolium, Link.

Lathyrus angustifolius, Schkr.

Armitageanus, Westc.

cyanus, Koch.

Drummondii, Hort.

elegans, Vogel.

flaccidus, Hort.

Gargoni, Parl.

lævigatus, Waldst. et Kit.

Lowii, Hort.

maritimus, Big.

niger, Wimm.

polyanthus, Boiss, et Bl.

vernus, Bernb.

Lavatera cretica, Linn.

punctata, All.

thuringiaca, Linn.

trimestris, Linn.

Leontopodium alpinum, Cass.

Leonurus Cardiaca, Linn.

glaucescens, Bunge.

marrubiastrum, Linn.

villosa, Desf.

Lepidium crassifolium, Waldst. et Kit.

densiflorum, Schrad.

ruderale, Linn.

sativum, Linn.

Smithii, Hook.

Leptosiphon androsaceus, Benth.

Liatris odoratissima, Willd.

pilosa, Willd.

pumila, Lodd.

Libertia grandiflora, Sweet.

Ligularia macrophylla, DC.

Lilium chalcedonicum, Linn.

Limnanthes alba, Hort.

Douglasii, R.Br.

Linaria alpina, DC.

maroccana, Hook. fil.

multipunctata, Hoffmsgg. et Lk.

peloponnesiaca, B. et Heldr.

pyrenaica, DC.

vulgaris, Mill. 
Linum austriacum, Linn. capitatum, Kit. et Schult. diffusum, Schrad. et Schult. flavum, Linn. grandiflorum, Desf., var. rubrum.

Mulleri, Moris. nervosum, Waldst. et Kit. perenne, Linn. usitatissimum, Linn.

- var. album.

- - grandiflorum.

Lithospermum latifolium, Michx.

Lobelia campanuloides, Thunbg.

Lolium italicum, A. Br. lepturoides, Boiss. perenne, Linn., var. monstrosum.

Lophanthes nepetioides, Benth. rugosus, Fisch. et Mey.

Lotus corniculatus, Linn.

Lunaria biennis, Linn.

Lupinus Chamissonis, Eschsch. Douglasii, Agardh. guatemalensis, Hort. littoralis, Dougl. mutabilis, Sweet. nootkatensis, Donn. perennis, Lindl. polyphyllus, Lindl. victoria, $\mathrm{Br}$.

Luzula albida, DC. campestris, DC. Fosteri, DC. nivea, Desv. pilosa, Willd. spicata, DC. sylvatica, Rich.

Lychnis alpinus, Linn. chalcedonica, Linn.

- var. alba.

diurna, Sibth.

Flos-cuculi, Linn.

Flos-jovis, Desv. macrocarpa, Boiss. nemoralis, Heuff.

Sartori, Hort.

Sieboldii, Max. viscaria, Linn., var, alba. - var. purpurea. vespertina, Sm.

Lycopus exaltatus, Linn.

Lysimachia affinis, Bunge.
Lythrum hirsutum, Hort.

Salicarı, Linn.

- var. grandiflorum.

- - pubescens.

tomentosum, Mill.

Macrotomia echioides, Linn.

Magydaris panacina, DC.

Malcolmia maritima, R.Br.

Malope grandiflora, Hort.

Malva Durieui, Spach. mareotica, Delil. moschata, Linn. - var. alba. nicæensis, All. - var. Tenoreana. pulchella, Bernh. sylvestris, Linn. verticillatus, Linn.

Marrubium vulgare, Linn.

Matricaria Parthenium, Linn.

Meconopsis cambrica, Vig. - var. aurantiaca, Hort. nepalensis, DC. Wallichii, Hook.

Medicago ciliaris, Willd. echinus, DC.

elegans, Linn.

falcata, Linn.

lupulina, Linn.

orbicularis, Willd.

sativa, Linn.

terebellum, Willd.

Melica altissima, Linn.

Magnolii, Gren. et Godr. nutans, Linn. uniflora, Retz.

Melilotus alba, Desr. longifolius, Tenore. officinalis, Desr.

Melissa patavina, Benth.

Melittis Melissophyllum, Linn.

Mentha arvensis, Linn. canescens, Roth., Sieb. latifolia, Host. rotundifolia, Linn.

Menyanthes trifoliata, Linn. Mertensia oblongifolia, G. Don. Mesembryanthemum tricolor, Willd. Meum athamanticum, Jacq. Milla laxa, Baker.

Mimulus cardinalis, Dougl. Mœhringia muscosa, Linn. 
Molinia coerulea, Moench.

Momordica Elaterium, Linn.

Monarda Bradburyana, Beck.

Montbretia Pottsii, Baker.

Morina longifolia, Wall.

Muretia tanaicensis, Boiss.

Muscari armeniacum, Baker. atlanticum, Boiss. et Reut. Maweana, Baker. racemosa, Mill, Szovitsianum, Rgl.

Myosotis alpestris, Schmidt. arvensis, Sibth. australis, R. Br. palustris, With.

Myrrhis odorata, Scop.

Narcissus bicolor, Linn., var. Horsfieldii.

- sub-sp. muticus. incomparabilis, Mill. juncifolius, Lag. pallida, Poir.

- var. præcox. poeticus, Linn., var. tripodalis.

Nardostachys Jatamansi, DC.

Nepeta altissima, Schrank. Mussini, Biebrst. pannonica, Jacq.

Nesæa triflora, H.B.K. verticillata, H.B.K.

Nicotiana rustica, Linn.

Nigella corniculata, DC. pygmæa, Pers. sativa, Linn.

Nolana atriplicifolia, D. Don.

Nuttallia pedata, Dicks.

Enothera biennis, Linn. campylocalyx, K. et B. concinna, Spach. fruticosa, Linn. glauca, Michx. - var. Fraseri. Lindleyana, Dougl. muricata, Linn. pumila, Linn. Sellowii, Link.

Enanthe globulosa, Linn. Jordani, Ten. peucedanifolia, Poll. silaifolia, Heuffl.
Omphalodes linifolia, Moench.

Onobrychis sativus, Linn.

Ononis arvensis, Linn. crotalarioides, Coss. hircina, Jacq. repens, Linn. rotundifolia, Linn. spinosa, Linn.

Onopordon Acanthium, Linn. acaule, Linn. alexandrinum, Boiss.

Origanum humile, Poir. pallidum, Desf. pulchellum, Boiss. et Heldr. vulgare, Linn.

Ornithogalum Elwesii, Hort. pyramidale, Linn.

Oxalis corniculatus, Linn. tropæoloides, Hook.

Oxyria didyma, Hill.

Oxytropis campestris, DC. lactea, Hort. uralensis, DC.

Pæonia corallina, Retz. officinalis, Retz.

Palavia flexuosa, Mart.

Papaver alpinum, Linn. armeniacum, Lam. croceum, Ledeb. murcelli, var. Van Houtte. nudicaule, Linn. orientale, Linn.

- var. bracteatum, Lindl. persicum, Lindl. pilosum, Sibth. - var. Heldreichii, Boiss. pinnatifidum, Moris. pyrenaicum, Willd. Rhœeas, Linn. rupifragum, Boiss. - var. atlanticum, Ball. somniferum, Linn.

Parietaria diffusa, Mert. et Koch. erecta, Mert. et Koch.

Parnassia nubicola, Wall. palustris, Linn.

Pastinaca Fleischmanni, Hoffm. lucida, Linn.

Peliosanthes sp.

Pentstemon confertum, Dougl. Hartwegii, Benth. nitidum, Dougl. procerum, Dougl. 
Phacelia congesta, Hook. tanacetifolia, Benth. Wrangeliana, Fisch. et Mey.

Phalaris arundinacea, Linn. canariensis, Linn. minor, Retz. trigyna, Host.

Phaseolus multiflorus, Lam. Phlomis Russelianus, Lag. setigera, Falc.

Phlox carolina, Linn. ovata, Linn.

Phygelius capensis, Fisch. et Mey. Phyteuma betonicæfolium, Vill. campanuloides, Bieb.

Charmelii, Vill.

Halleri, All.

orbiculare, Linn.

Scheuchzeri, All.

Pinguicula vulgaris, Linn.

Pisum arvense, Linn.

Plantago acanthophylla, DC. afra, Linn.

amplexicaulis, Cav. arachnoidea, Schrenk.

argentea, Lamk.

cornuta, Gouan.

Cynops, Linn.

divaricata, Zucc.

eriostachys, Tenore.

gentianoides, Sm.

gnaphalioides, Nutt.

kamtschatica, Cham.

lanceolata, Linn.

major, Linn.

- var. monstrosa.

maritima, Linn.

media, Linn.

mexicana, Link.

montana, Lamk.

saxatilis, Biebrst.

serpentina, Lam.

subulata, Linn.

Platystemon californicum, Benth.

Pleurospermum austriacum, Hoffm.

Poa Balfourii, Parn.

cæsia, Smith, var, glauca.

chinensis, Retz.

nemoralis, Linn., var. sempervirens.

palustris, Linn.

sudetica, Hænke.

tenax, Link.

trivialis, Linn.
Podophyllum Emodi, Wall.

Polemonium cœruleum, Linn.

- var. acutifolium.

himalayanum, Baker.

humile, Willd.

pauciflorum, Wats.

pulchellum, Bunge.

Richardsonii, Graham.

sibiricum, Sweet.

Polygonum capitatum, Don. cuspidatum, Sieb. et Zucc. sphærostachyum, Meisn.

Potentilla alchemilloides, Lap. anserina, Linn. argentea, Linn. - var. calabra. argyrophylla, Wall. astracanica, Jacq. atrosanguinea, Lodd. aurea, Linn.

chameleon, Hort. cathaclines, Lehm.

Comarum, Nestl. confertiflora, Torr. crassicaulis.

Dombeyi, Nestl.

Drummondii, Lehm.

Fenzlii, Lehm. geoides, Biebrst. hirta, Linn.

Hookeriana, Lehm. insignis, Royle.

leucotricha.

M'Nabiana, Hort.

marginata.

nivea, Linn.

ontopoda, Dougl.

opaca, Linn.

Phæbis, Hort.

pedata, Willd.

Romanzoffi.

rupestris, Linn.

salisburgensis, Hænke,

Schrenkiana, Hort. stolonifera, Lehm.

Poterium muricatum, Spach. platylophum, Hort.

Prenanthes alba, Linn.

Primula auriculata, Lam. ciliata, Linn., var. Lindsayi. elatior, Jacq.

farinosa, Linn.

grandis, Trautv. 
Primula-contd.

inflata, Lehm.

intricata, Gren.

involucrata, Wall.

magellanica, Lehm.

reticulata, Wall.

rosea, Royle, var. grandiflora.

sikkimensis, Hook.

variabilis, Bart.

veris, Linn.

vulgaris, Huds.

Warei, Hort.

Psamma baltica, Roem. et Schult.

Pterestegia drymarioides, Fisch.

Pulmonaria mollis, Wulf. virginica, Linn.

Puschkinia scilloides, Adams.

Pyrethrum balsamita, Linn.

corymbosum, Willd.

macrophyllum, Willd.

Ramondia pyrenaica, Rich.

Ranunculus aconitifolius, Linn.

acris, Linn.

aduncus, Gren.

amplexicaulis, Linn.

bulbosus, Linn.

chærophyllus, Linn.

Friesianus, Jord.

fumariæfolius, Desf.

gramineus, Linn.

granatensis, Boiss.

lomatocarpus, Fisch. et Mey.

monspeliacus, Linn.

propinquus, Meyer.

repens, Linn., large fl. var.

Reuterianus, Boiss.

Raphanus, caudatus, Linn.

sativus, Linn.

Rhagadiolus edulis, Gaert.

Reseda abyssinica, Fres.

fruticulosa, Linn.

Gayana, Boiss.

Jacquinii, Reichb.

lutea, Linn.

odorata, Linn.

Phyteuma, Linn.

ramosissima, Pour.

Rheum palmatum, Linn.

- var. tanguticum, Rgl.

sibiricum, Pall.

Rhodanthe Manglesii, Lindl.

Rosa pyrenaica, Gouan.
Rudbeckia californica, Gray.

frigida, Ait.

triloba, Linn.

Rumex alpinus, Linn.

- var. majus.

aquaticus, Linn.

compactus, Hort.

crispus, Linn.

Hydrolapathum, Huds.

nepalensis, Spr.

Patientia, Linn.

rupestris, Le Gall.

sanguineus, Linn.

sanguisorbæfolius, Hort.

scutatus, Lindl.

Steudelii, Hochst.

Ruta graveolens, Linn. macrophylla, Hort.

Salvia argentea, Linn.

carduacea, Benth.

clandestina, Rchb.

dominica, Linn.

hians, Royle.

nubia, Ait.

nubicola, Wall.

officinalis, Linn.

pratensis, Linn.

Sibthorpii, Sibth. et Sm.

taraxacifolia, Coss.

Verbenaca, Linn.

Sanguinaria canadensis, Linn.

Sanguisorba media, Linn.

Sanicula europæa, Linn.

Santolina alpina, Linn.

rosmarinifolia, Linn.

Saponaria cæspitosa, DC. oxydonta, Hort.

Satureia hortensis, Linn. illyrica, Hort. spicata, Visian.

Saxifraga acanthifolia, Hort. affinis, Don.

Aizoon, Jacq., var. minor.

- notata.

- pectinata.

- - rosularis.

aretioides, Lap.

bronchialis, Linn.

Bucklandii, Hort.

cæsia, Linn.

cæspitosa, Linn., var. grandiflora. 
Saxifraga-contd.

Clusii, Gouan.

condensata, Gmel.

crustata, Vent.

Cymbalaria, Linn.

decipiens, Ehrh.

Geum, Linn.

- var. elegans, Mackay.

- - gracilis, Mackay.

globosa, Hort.

Gmelini, Host.

granulata, Linn.

grœnlandica, Linn.

hederæefolia, Hochst.

hypnoides, Linn.

-- var. hirta.

intacta, Willd.

- var. farinosa.

lasiophylla, Schott.

lingulata, Bell.

longifolia, Lap.

mutata, Linn.

nepalensis, Hort.

nivalis, Linn.

odontophylla, Wall.

oppositifolia, Linn., var. fusca.

paradoxa, Kit.

peltata, Torr.

Prostii, Sternb.

pulchella, Don.

repanda, Willd.

Rhæii, Schott.

rotundifolia, Linn.

Seguerii, Spr.

squarrosa, Sieber.

Stansfieldii, Hort.

Sturmiana, Schott.

taygetta, Boiss.

tenella, Wulf.

tridentata, Don.

umbrosa, Linn,, var. variegata.

virginiensis, Michx.

Scabiosa agrestis, Waldst. et Kit.

caucasica, Bieb.

dipsacifolia, Schott.

elata, Hornem.

graminifolium, Linn.

Haldnickiana, Host.

pilosiuscula, Hort.

succisa, Linn.

- var. alba.

sylvatica, Linn.

Schizanthus pinnatus, Ruiz et Pav. Schizopetalon Walkeri, Sims.
Schœenus nigricans, Linn.

Scilla amœena, Linn. campanulata, Ait, var. rubra. hispanica, Mill. peruviana, Linn. sibirica, Andr.

- var. præcox. verna, Huds.

Scirpus atrovirens, Willd. Holoschœenus, Linn. lacustris, Linn. Tabernæmontani, Gmel.

Scorpiurus subvillosa, Linn. vermiculata, Linn.

Scorzonera hispanica, Linn.

Scrophularia aquatica, Linn.

Ehrhartii, Stev.

elatior, Benth.

glandulifera, Clarke.

glandulosa, Waldst. et Kit.

nodosa, Linn.

sambucifolia, Linn., var.

Scopolii, Hoppe.

trifoliata, Linn.

Scutellaria albida, Linn.

alpina, Linn.

altaica, Fisch.

japonica, Morr, et DC.

Secale anatolicum, Boiss. dalmaticum, Vis.

Sedum Aizoon, Linn. altissimum, Poir.

Anacampseros, Linn.

Andersonii, D. Don.

collinum, Willd.

crassipes, Wall.

glaucum, Sm.

Hillebrandi, Fenzl.

hirsutum, All.

kamtschaticum, Fisch.

maximum, Sut., var. purpureum.

Maximowiczii, Regel.

oppositifolium, Sims.

oreganum, Nutt.

populifolium, Linn.

Rhodiola, DC.

roseum, Stev.

rupestre, Huds.

- var. elegans.

spurium, Biebrst.

trifidum, Wall.

virescens, $W$.

- var. monstrosa. 
Sempervivum

acuminatum, Hort.

- var. nigra.

arachnoideum, Linn.

Boutigyanum, Hort.

californicum, Hort.

fimbriatum, Lehm.

flagelliforme, Fisch.

Pittonii, Schott.

Reginæ Amaliæ, Held. et Sart. tectorum, Linn.

Senebiera Coronopus, Poir. didyma, Pers.

Senecio adonidifolius, Lois. alpinus, Linn.

Doronicum, Linn.

Serratula coronata, Linn. heterophylla, Desf. græca, Spreng. quinquefolia, Biebrst.

Sesleria argentea, Savi.

Setaria italica, Beauv., var. macrochæta.

verticillata, Beauv.

Shortia galacifolia, Gray.

Sidalcea candida, Gray. malvæflora, Gray. Murrayana, Hort.

Silene acaulis, Linn., var. alba. alpestris, Linn.

ciliata, Pourr.

caucasica, Boiss.

Cucubalus, Wibel.

Græfferi, Gus.

italica, Pers.

Lerchenfeldi, Baumg., var. vera.

linifolia, Willd,

mollissima, Smith.

muscipula, Linn.

neglecta, Ten., var. diffusa.

orgæa, Fisch. et Mey.

pendula, Lehm.

petræa, Waldst. et Kit.

pusilla, Waldst. et Kit.

quadridentata, Pers.

reticulata, Desf.

Saxifraga, Linn.

Schaftii, Gmel.

Silphium conjunctum, Willd.

Sisyrinchium bermudianum, Linn.

- var. anceps.

mucronatum, Michx.
Sisymbrium assoanum, Ruiz et Pav. austriacum, Jacq. Irio, Linn.

laxiflorum, Boiss.

officinale, Scop.

strictissimum, Linn.

tanacetifolium, Linn.

Sium angustifolium, Linn.

Solanum Dulcamara, Linn. gracile, Otto.

villosum, Lamk.

Solenanthus mollissimus, Lehm.

Solidago californica, Nutt.

coriacea, Hort.

nemoralis, Ait.

ohioensis, Ridd.

rigida, Linn.

salicifolia, Wall.

Virgaurea, Linn.

Sonchus lapponicus, Linn. palustris, Linn.

Sparaxis pendula, Ker.

Sparganium ramosum, Huds.

Specularia pentagonia, Linn.

Spiræa Filipendula, Iinn. palmata, Thunbg.

Stachys Betonica, Benth.

Statice coronata, Hort.

Gmelini, Willd.

tatarica, Linn.

Stipa capillata, Linn. gigantea, Lag. pennata, Linn.

Symphyandra Hoffmanii, Panc. Wanneri, Heuff.

Symphytum asperrimum, Biebrst. caucasicum, Biebrst. officinale, Linn.

- var. atropurpureum.

Symplocarpus foetidus, Salisb.

Tanacetum boreale, Fisch. vulgare, Linn.

Teesdalia nudicaulis, R.Br.

Tellima grandiflora, R.Br.

Tetragonolobus biflorus, Ser.

Teucrium Scorodonia, Linn.

Thermopsis montana, Nutt.

Thalictrum appendiculatum, Ledeb.

elatum, Jacq.

glaucum, DC.

lucidum, Linn.

minus, Linn.

- var. majus.

Schweiggeri, Spreng. 
Thalictrum-contd. sibiricum, Linn. simplex, Linn., var. Jordani, Hort.

Thlaspi alpestre, Linn. sylvium, Gaud.

Thymus micans, Low. Serpyllum, Linn.

Tofieldia palustris, Huds, var. carinthiaca. glutinosa, Willd.

Tradescantia elata, Lodd. virginica, Linn.

- var. alba, Hort.

Tragopogon orientalis, Linn.

Tricyrtis australis, Hort.

Trifolium alpinum, Linn.

Bocconi, Savi.

fragiferum, Linn.

repens, Linn., var pictum.

rubens, Linn., var.

trichocephalum, Biebrst.

Trigonella Fœnum-græcum, Linn. pinnatifida, Cav.

Trinia vulgaris, DC.

Trisetum pratense, Linn.

Triteleia Murrayana, Hort.

Triticum caninum, Huds. elymoides, Hornem. monococcum, Linn. Tumonia, Schrad. violaceum, Hornem. vulgare, Vill.

Trollius aconitifolius, Hort. altaicus, C. A. Meyer. asiaticus, Linn., var. hybridus. caucasicus, Stev. japonicus, Hort. laxus, Salisb.

Tropæolum majus, Linn. minus, Linn., var. coccinea. - var. Tom Thumb.

Tulipa altaica, Pall. Gesneriana, Linn., var. pulchella, Fenzl.

\section{Urtica magellanica, Poir.}

Valeriana officinalis, Linn. pyrenaica, Linn.

Valerianella olitoria, Moench.

Vella spinosa,' Boiss.

Veratrum album, Linn. - var. viride.
Verbascum compactum, Bieb. heteromallum, Panc.

Lagurus, Fisch. et Mey. niveum, Tenore.

Thapsus, Linn.

Veronica Allionii, Vill.

Anagallis, Linn.

arvensis, Linn.

Bidwillii, Hook, fil.

Buxbaumii, Tenore.

candida, Lodd.

Colensoi, Hook. (Kew).

- var. glauca.

corymbosa, Hort.

- var. variegata.

fruticulosa, Linn.

gentianoides, Vahl.

grandis, Fisch.

incisa, Ait.

Jacquinii, Baumg.

ligustrifolia, A. Cunn.

maritima, Linn., var. alba.

monticola, Trautv.

officinalis, Linn.

- var. hirsuta.

orchidea, Crantz.

pinnata, Linn.

Ponæ, Gouan.

prostrata, Linn, var. satureiæfolia.

saxatilis, Linn.

- var. Guthriana.

serpyllifolia, Linn.

spicata, Linn.

- var. alba.

- - hybrida.

spuria, Linn.

urticifolia, Linn.

vernicosa, Hook. fil.

Vesicaria sinuata, Poir. utriculata, Lamk.

Vicia argentea, Lap.

Cracca, Linn.

onobrychioides, Linn.

Orobus, DC.

villosa, Roth.

Vincetoxicum luteum, Sieb.

Viola canadensis, Linn., var. alba. canina, Linn., var, alba. cornuta, Linn.

declinata, Waldst. et Kit.

lancifolia, Biebrst.

macedonica, Boiss. et Held.

Munbyana.

oculata, Lehm.

Orphanidis, Boiss. 
Viola-contd.

Ruppii, All.

Wahlenbergia dalmatica, A.DC. lobelioides, DC. nutabunda, DC.

Whitlavia minor, Hans.

Wulfenia carinthiaca, Jacq.
Wyethia robusta, Mitt.

Xeranthemum annuum, Linn.

Zygadenus elegans, Pursh. glaucus, Nutt.

- var humilis.

\section{TREES AND SHRUBS.}

Acer Pseudo-platanus, Linn. - var. purpurea, Hort.

Asculus Hippocastanum, Linn. var. rubicundum, Schub.

Alnus cordifolia, Lodd. glutinosa, Gærtn.

Berberis Aquifolium, Pursh. asiatica, Roxb. Jamesoni, Lindl. nervosa, Pursh. vulgaris, Linn.

Betula alba, Linn. - var. pendula, Sm.

Caragana arborescens, Lam. Castanea vulgaris, Lam.

Colutea arborescens, Linn.

Cornus alternifolius, Linn.

Cotoneaster acuminata, Lindl. Fontanesii, Spach. frigida, Wall. Hookeriana, Hort. laxiflora, Jacq. microphylla, Wall. sikkimensis, Hort. Simonsii, Baker.

Cratægus Aronia, Bosc. coccinea, Linn. orientalis, Bieb.

Oxyacantha, Linn. - var. horrida, Hort.

- var. pendula, Hort.

- var. punicea, Hort.

Cupressus Lawsoniana, Murr. nutkäensis, Lamb.

Cytisus biflorus, L'Herit. capitatus, Jacq. scoparius, Link.
Daphne Mezereum, Linn. - var. flore albo, Hort.

Escallonia Philippiana, Engl.

Fraxinus excelsior, Linn.

Ilex Aquifolium, Linn.

- var. camelliæfolium, Hort.

- var. fructu luteo, Hort.

Laburnum alpinum, Griseb. vulgare, Griseb.

- var. Adami, Hort.

- var. autumnale, Hort.

Ledum palustre, Linn.

Leycesteria formosa, Wall.

Mespilus, Lady Hardwick's.

Neillia opulifolia, Benth. et Hook.

Olearia Haastii, Hook. fil.

Pinus parviflora, Sieb.

Piptanthus nepalensis, Swt.

Prunus Avium, Linn.

Ptelea trifoliata, Linn.

Pyrus americana, DC. Aria, Ehrh.

Aucuparia, Gærtn.

- var. fastigiata, Hort.

- var. fructu luteo, Hort.

baccata, Linn.

latifolia, Syme. pinnatifida, Ehrh.

Quercus Cerris, Linn. pedunculata, Willd.

Rhamnus alnifolius, Linn. latifolius, L'Herit. 
Rhododendron campanulatum, D. Don.

catawbiense, Michx. caucasicum, Pall. - var. album, Hort. ferrugineum, Linn. flavum, Don. glaucum, D. Don. hirsutum, Linn. ponticum, Linn.

Rosa acicularis, Lindl. rugosa, Sieb. et Zucc. .

Rubus laciniatus, Willd. Lawton's.

Sambucus nigra, Linn. - var. rubrocarpa, Hort.
Sambucus-contd. canadensis, Linn. racemosa, Linn. Symphoricarpus racemosus, Michx.

Taxus adpressa, Knight. baccata, Linn. - var. fastigiata, Loud.

Tilia vulgaris, Hayne.

Ulex europæus, Linn.

Viburnum Lantana, Linn. Opulus, Linn.

Weigelia amabilis, Planch.

Zenobia speciosa, D. Don.

\section{PLANTS UNDER GLASS.}

Achmea bromeliæfolia, Baker. Aphelandra pumila, Nichols. Archontophoenix Cunninghamii, H. Wendl. and Drude.

Aristolochia bætica, Linn.

Aristea cyanea, Soland. Eckloni, Baker.

Asclepias curassavica, Linn.

Canna floribunda, Bouché. Annei, Hort. patens, Rosc.

Celsia Arcturus, Jacq.

Desmodium gyrans, DC.

Dianella intermedia, Endl.

Didymocarpus Humboldtiana, Gardn.

Diplarrhena Moraea, Labill.

Drosera capensis, Linn. rotundifolia, Linn. spathulata, Labill.

Eugenia dysenterica, DC. Eupatorium ianthinum, Hemsl.

Gynura sarmentosa, DC.
Hibiscus pedunculatus, Linn. f.

Kennedya rubicunda, Vent.

Laportea peltata, Gaudich. Littonia modesta, Hook. Livistona chinensis R. Br.

Maurandya scandens, Pers. Mimosa pudica, Linn.

Moræa ramosa, Gaul.

Nicotiana glauca, Grah.

Oryza sativa, Linn.

Oxalis sensitiva, Linn.

Polyalthia suberosa, Benth. et Hook. f.

Primula mollis, Mett. verticillata, Forsk.

Ranunculus cortusæefolius, Willd. Rhodochiton volubile, Zucc. 
Salvia obovata, Ell.

Sarracenia Maddisoniana, Hort. Senecio cruentus, Roth.

Sida triloba, Thunb.

Skimmia japonica, Thunb.

Stylidium adnatum, R. Br. fruticosum, R. Br.
Tetranema mexicanum, Benth. Tigridia grandiflora, Salisb. Torenia Fournieri, Lind. Trichosanthes anguina, Linn. Tristania laurina, R. Br.

\title{
ISAAC BAYLEY BALFOUR, Keeper.
}

\section{ROBERT LINDSAY,}

Curator of the Garden.

A. D. RICHARDSON, Foreman in charge of the Arboretum.
ALEX. ANDERSON, Foreman in charge of the Herbaceous Department.

\author{
R. L. HARROW, \\ Foreman in charge of the \\ Glass Department.
}

EDINBURGH, January 1894. 





\section{ROYAL BOTANIC GARDEN, EDINBURGH}

\section{LIST OF SEEDS}

\section{COLLECTED DURING 1894.}

PRINTED FOR HER VAJESTYS STATTONERY OFFICE,

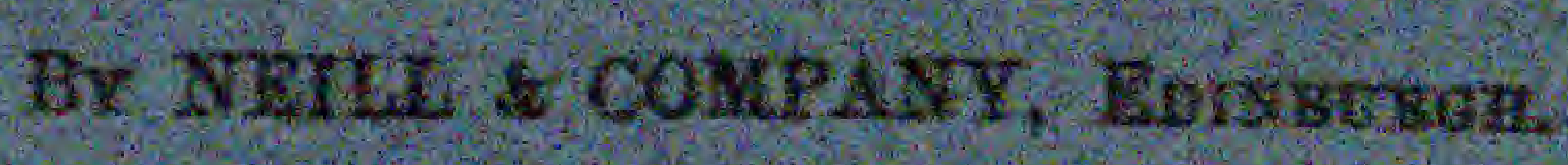
1895. 

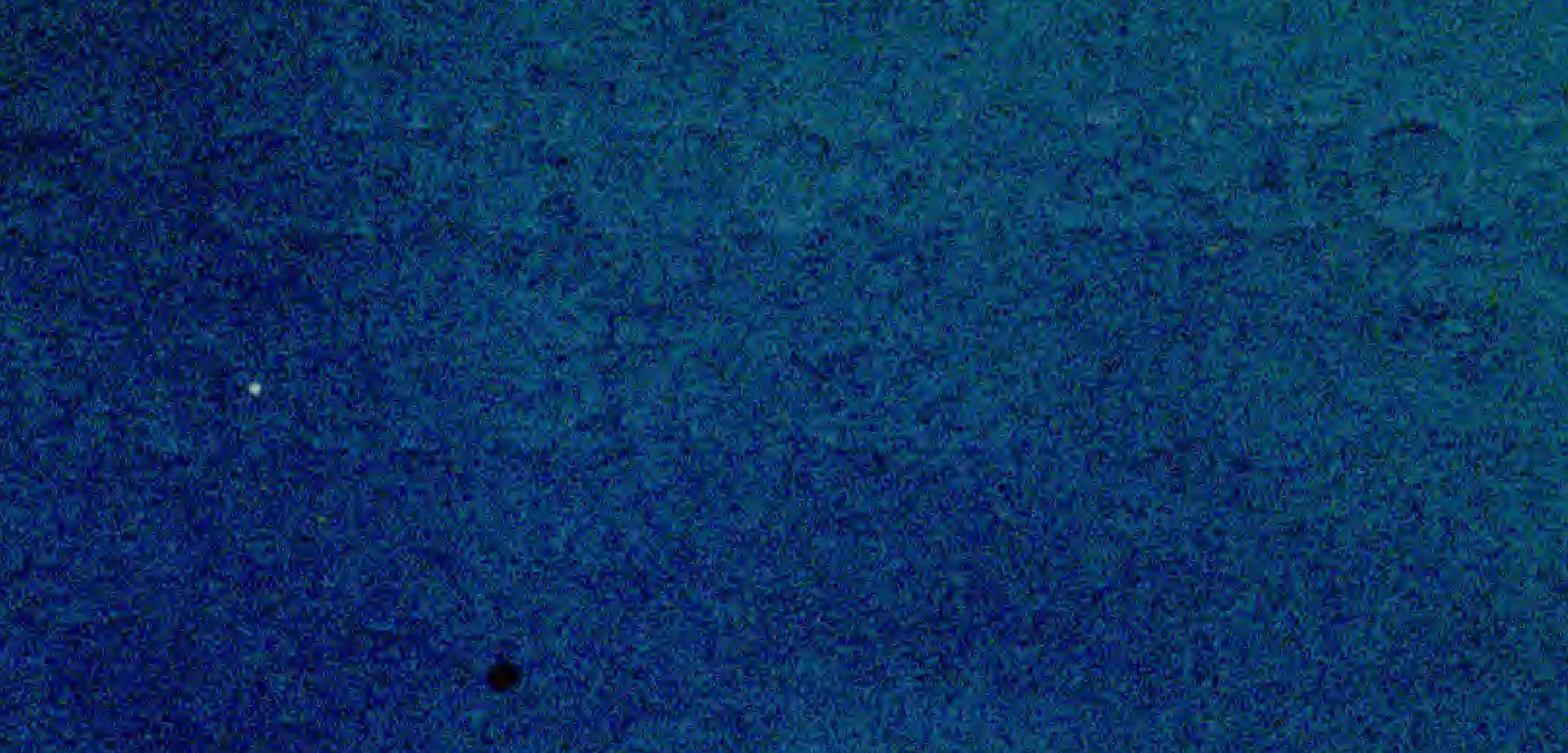

(1)

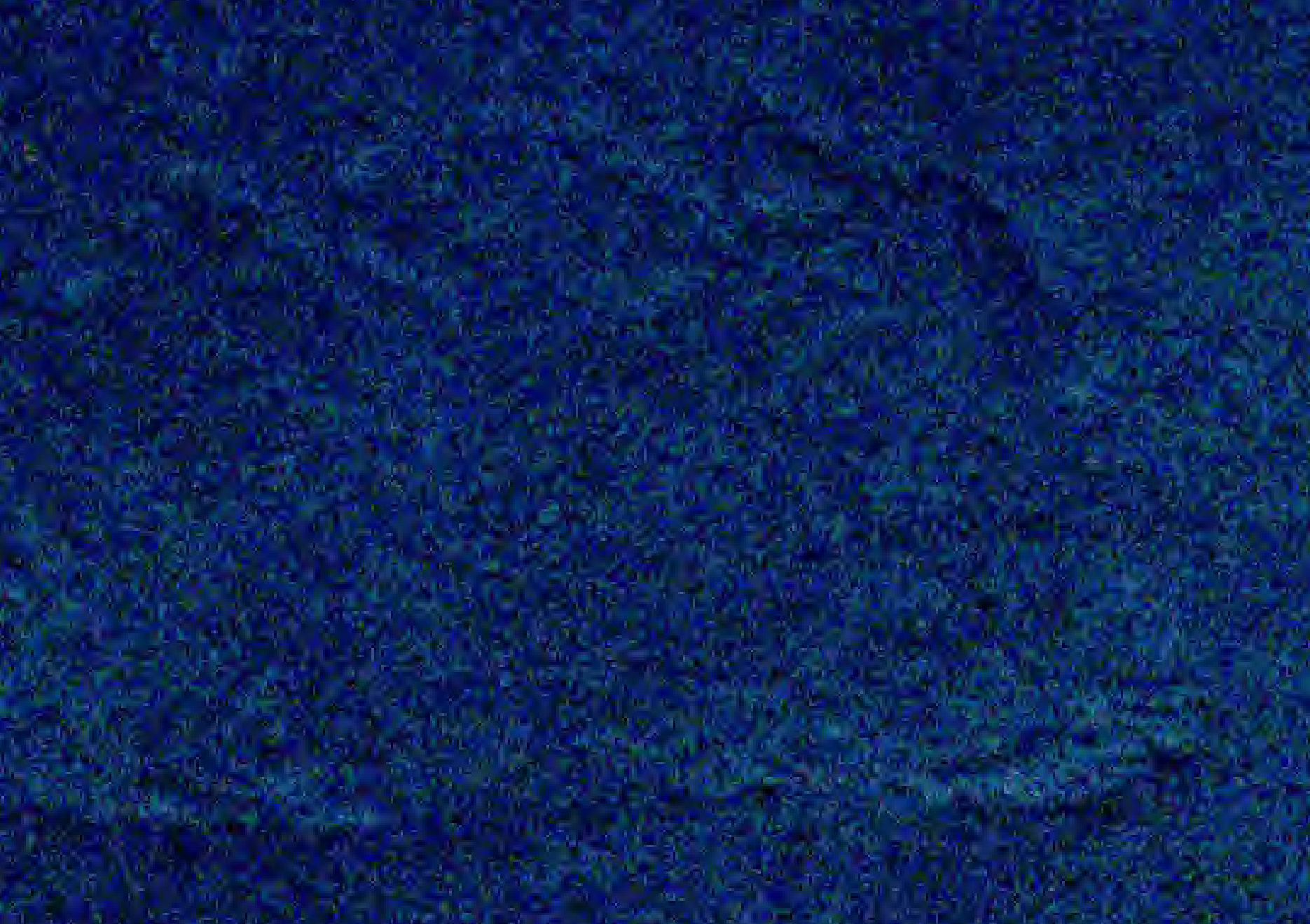




\title{
ROYAL BOTANIC GARDEN, EDINBURGH.
}

\author{
LIST OF SEEDS \\ COLLECTED DURING 1894.
}

\section{HERBACEOUS PLANTS.}

Acæna argentea, Ruiz. et Pav. inermis, Hook. fil. microphylla, Hook. fil. myriophylla, Lindl. Sanguisorbæ, Vahl. Achillea Millefolium, Liun. - var. roseum.

Aconitum Anthora, Linn. californicum, Hort. longigaleatum, Hort. Lycoctonum, Linn.

— var. album.

_- moldavicum.

- - septentrionale.

Napellus, Linn.

- var.

paniculatum, Lam.

Actæa spicata, Linn.

- var. rubra, Michx.

Adenophora latifolia, Fisch. marsupiiflora, Fisch.

stylosa, Fisch.

Adlumia cirrhosa, Rafin.

Adonis vernalis, Linn.

Athionema cordatum, Boiss.

Agrimonia agrimonoides, Linn.

Eupatoria, Linn.

Agrostis alba, Linn. var, stolonifera, Linn.

vulgaris, With.

Aira atropurpurea, Wahlenb.

Alchemilla alpina, Linn.

- var. conjuncta.

vulgaris, Linn.

Alisma Plantago, Linn.

- var. rubra.

N. $-500-1 / 95$.
Allium ampeloprasum, Linn. var. californicum, Hort. carinatum, Linn.

Dioscoridis, Sibth. et Sm.

- var. siculum.

fistulosum, Linn.

- var. catavistum.

flavum, Linn. var. pulchellum.

globosum, Redouté var. album.

karataviense, Regel.

Ledebourianum, Schult. fil.

MacLeanii, Baker.

MacNabianum, Hort.

Moly, Linn.

narcissiflorum, Vill.

neapolitanum, Cyr.

polyphyllum, Kar. et Kir.

sativum, Linn.

saxatile, Hort.

Schœnoprasum, Linn.

Scorodoprasum, Linn.

sphærocephalum, Linn.

Stellerianum, Willd.

stipitatum, Regel.

vineale, Linn.

- var. Kochii.

Alopecurus arundinaceus, Poir.

geniculatus, Linn.

pratensis, Linn.

- var. variegatus.

Alstrœmeria aurea, R. Grah.

Althæa ficifolia, Cav.

Hohenackeri, Boiss, et Huet.

Alyssum argenteum, Vitm.

Marschallianum, Andrz.

podolicum, Bess.

rostratum, Stev. 
Alyssum-contd.

saxatile, Linn.

sinuatum, Linn.

spinosum, Linn.

Amaranthus Mantegazzianus, Passer.

Ammophila baltica, Host.

Anagallis arvensis, Linn.

Anchusa italica, Retz.

officinalis, Linn., var. incaruata. sempervirens, Linn.

Androsace lactea, Linn.

lactiflora, Fisch.

sarmentosa, Wall.

Anemone alpina, Linn.

- var. sulphurea.

baicalensis, Turez.

decapetala, Arduini.

montana, Hoppe.

multifida, Poir.

narcissiflora, Linn.

- var.

palmata, Linn.

polyanthes, D. Don.

pratensis, Linn.

Pulsatilla, Linn.

rivularis, Buch. Ham.

Angelica Gingidium, Hook. fil. songorica, Regel et Schmalh.

Antennaria dioica, Gaertn. candida, Hort. plantaginea, R.Br.

Anthemis tinctoria, Linn. - var. discoidea.

Anthericum Liliago, Linn.

- var. algeriense, B. et $R$.

Anthoxanthum odoratum, Linn.

Anthriscus sylvestris, Hoffm.

Anthyllis montana, Linn.

Vulneraria, Linn., var. rubra, Hort.

Antirrhinum Asarina, Linn. majus, Linn.

- var. angustifolium.

A ponogeton distachyum, Thunb.

Aquilegia Bertolonii, Schott.

Buergeriana, Sieb. et Zucc.

californica, A. Gray, var. alba.

Eiuseleana, Fr. Schultz.

glandulosa, Fisch.

- var. jucunda.

grata, Maly.

lutea, Lamk.

pyrenaica, DC.
Aquilegia-contd. viridiflora, Pall. vulgaris, Linn.

Arabis albida, Stev. bellidifolia, Jacq. lucida, Linn. fil. muralis, Bertol. petræa, Lam. Soyeri, Reut. et Huet. Turrita, Linn.

Aralia cachemirica, Decne. hispida, Vent.

Archangelica officinalis, Hoffm.

Arctium Lappa, Linn. majus, Bernh. nemorosum, Lejeune.

Arenaria laricifolia, Linn. saxatilis, Linn. serpyllifolia, Linn. verna, Linn.

Armeria allioides, Boiss. alpina, Willd.

berlengensis, Daveau.

boetica, Boiss., var. hirta. cariensis, Boiss.

- var, rumelica.

elongata, Hoffm.

fasciculata, Willd.

filicaulis, Boiss.

longicaulis, Hort.

majellensis, Boiss.

maritima, Willd.

- var. alba.

pinifolia, Hoffmgg. et Link.

plantaginea, Willd.

splendens, Boiss.

Welwitschii, Boiss.

Arnica Chamissonis, Less.

- var.

Arracacia arguta, Benth. et Hook.

Arrhenatherum avenaceum, Beauv.

- var. bulbosum.

pallens, Link.

Artemisia Absinthium, Linn.

campestris, Linn.

capillaris, Thunb.

desertorum, Spreng.

græca, Hort.

rupestris, Linn.

sacrorum, Ledeb.

Stelleriana, Bess.

Arum palæstinum, Boiss.

Arundo acutiloba, Hort. 
Asarum canadense, Linn.

Asparagus officinalis, Linn. Asperula cynanchica, Linn. lævigata, Linn. stylosa, Boiss. tinctoria, Linn.

Asphodelus ramosus, Linn.

Aster acris, Linn. alpinus, Linn. - var. albus.

\section{- - roseus.}

- speciosus.

falcatus, Lindl. macrophyllus, Linn. paniculatus, Lam. sibiricus, Linn. sikkimensis, Hook, fil, et Thoms. stellaris, Hort. sub-hirsutus, Hort.

Astragalus bicolor, Lam. chlorostachys, Lindl. Glycyphyllos, Linn. multiflorus, Gray. vicioides, R. Grah.

Astrantia Biebersteinii, Fisch. et Mey.

major, Linn.

minor, Linn.

Atriplex laciniata, Linn.

Atropa Belladonna, Linn.

- var. fructu flava, Hort.

Aubrietia deltoidea, DC.

- var. coelestis, Hort.

- Columnae, Guss.

- - grandiflora, Hort.

Avena fatua, Linn. - Pinardi, Boiss. pratensis, Linn. sempervirens, Vill.

Ballota hispanica, Benth. rupestris, Vis.

Baptisia australis, R. Br. leucophæa, Nutt. lutea, Hort.

Barbarea altissima, Hort. arcuata, Reichb. vulgaris, R.Br.

Beckmannia erucæformis, Host. Bellis rotundifolia, Boiss. et Reut.

Bellium minutum, Linn.

Beta trigyna, Waldst, et Kit. vulgaris, Linn.
Biscutella ciliata, DC. didyma, Linn., var. Columnæ. lævigata, Linn.

Blumea Wightiana, DC.

Borago officinalis, Linn.

Buykinia aconitifolia, Nutt. var. major.

Brachycome Sinclairii, Hook. fil.

Brachypodium pinuatum, Beauv. sylvaticum, Beauv.

Brassica alba, Boiss. campestris, Linn. nigra, Koch. oleracea, Linn. Sinapistrum, Boiss.

Braya alpina, Sternb. et Hoppe.

Briza gracilis, Hort. minor, Linn.

Brodiæa congesta, Sm.

Bromus adoensis, Hochst. congestus, Hort. rigidus, Roth.

Bulbinella Hookeri.

Bunias orientalis, Linn.

Buphthalmum salicifolium, Linn.

Bupleurum petræum, Linu. ranunculoides, Linn. tenuissimum, Linn.

Butomus umbellatus, Linn.

Calamagrostis epigeins, Roth.

Calamintha alpina, Lam. Clinopodium, Benth. patavina, Host.

Calendula officinalis, Linn.

Callirhoë digitata, Nutt.

Caltha palustris, Linn., var. biflora sylvestris, Hort.

Calystegia sepium, R.Br.

Camassia Brownii, Hort. esculenta, Lindl:

- var. alba.

- major.

tardiflora, Hort.

Camelina sativa, Crantz. sylvestris, Wallr., var. macrocarpa.

Campanula alliariæfolia, Willd. americana, Linn. cæspitosa, Scop.

- var. alba. carpatica, Jacq. 
Campanula-contd.

- var. alba.

collina, Bieb.

colorata, Wall., var, tibetica.

elegans, Roem. et Schult.

glomerata, Linn.

Grossekii, Heuff.

latifolia, Linn.

- var. macrantha.

- - alba.

linifolia, Scop.

persicifolia, Linn.

- - var. alba.

pilosa, Pall.

pulchella, Hort. var. polypetala. pulcherrima, Schrank. et Zeyh. rapunculoides, Linn.

rhomboidea, Murr.

rotundifolia, Linn.

- var. maxima.

sarmatica, Ker-Gawl.

Trachelium, Linn.

Cannabis sativa, Linn.

Capsella Bursa-pastoris, Medic.

procumbens, Fries.

Carduus Carolorum $\times$, Howie et Jenner.

cernuus, Steud. nutans, Linn.

Carduncellus monspeliensium, All.

Carex aquatilis, Wahlenb.

atrata, Linn.

axillaris, Good.

binervis, $\mathrm{Sm}$.

capillaris, Linn.

Drymingii, Hort.

frigida, All.

fulva, Good.

glauca, Seop.

glutinosa, Hort,

Grayii, Carey.

paniculata, Jiun.

pendula, Huds.

pilulifera, Linn.

præcox, Jacq.

pulicaris, Linn.

rhynchophysa, Fisch. et Mey.

riparia, Curt.

rupestris, All.

saxatilis, Linn.

sylvatica, Huds.

Vahlii, Schkuhr.

vesicaria, Linn.
Carum Bulbocastanum, Koch. Carvi, Linn.

Centaurea alpina, Linn. calcitrapoides, Linn. centauroides, Linn.

cinerea, Lam.

Cyanus, Linn. dealbata, Willd.

elata, Poir.

macrocephala, Puschk.

nigra, Linn.

pallida, Frivald.

- var. gigantea.

pectinata, Linn.

phrygia, Linn.

quinquefolia, Hort.

ragusina, Linn., var. candidissima.

Rhaponticum, Linn.

rutifolia, Sibth, et Sm.

scabiosa, Linn.

Cephalaria alpina, Schrad.

corniculata, Roem. et Sch.

tatarica, Schrad.

- var. minor.

Cerastium arvense, Linn., var. grandiflorum.

dichotomum, Linn.

Cerinthe retorta, Sibth. et Sm.

Chærophyllum aromaticum, Linn.

Cheiranthus Allionii, Hort.

Cheiri, Linn.

Chelidonium majus, Linn. var. grandiflorum.

- laciniatum.

Chenopodium Bonus - Henricus, Linn.

Chionodoxa cretica, Boiss. et Heldr. gigantea, Hort.

Luciliæ, Boiss.

sardensis, Hort.

Chrysanthemum, carinatum, Schousb. coronarium, Linn.

Parthenium, Bernh.

segetum, Linn., var. grandiflorum.

tripteris, Hort.

Chrysopogon Gryllus, Trin.

Cichorium Intybus, Linn.

Cineraria concolor, Hort. geifolia, Linn.

Cimicifuga americana, Michx.

Clarkia elegans, Dougl. 
Clerkia-contd. pulchella, Pursh.

Claytonia perfoliata, Donn. sibirica, Linn.

Clematis Flammula, Linn. integrifolia, Linn. recta, Linn.

Clintonia Andrewsiana, Torr.

Cnicus atropurpureus, Hort. Biebersteinii, Hort. centauroides, Linn. elatus, Hort. eriophorus, Roth. ochroleucus, Spreng. oleraceus, Linn. pannonicus, Host.

- var. divaricatus. pratensis, Willd. rigidus, Hort.

Codonopsis ovata, Benth.

Collinsia bicolor, Benth. grandiflora, Dougl.

Collomia coccinea, Lehm.

Coluria potentilloides, R.Br.

Convolvulus siculus, Linn.

Coreopsis lanceolata, Linn.

Cortusa Matthioli, Linn.

Cotoneaster horizontalis, Deene. microphylla, Wall.

Crambe maritima, Linn.

Cratoxylon Blancoi, Blume.

Crepis crocea, Reichb. succisæfolia, Tausch.

Crocus nudiflorus, Sm.

Crucianella laxiflora, Hort.

Cryptotænia canadensis, DC.

Cyclamen europæum, Linn. hederæfolium, Ait.

Cynoglossum viridiflorum, Willd. Cynanchum glaucum, Wall. Vincetoxicum, Pers.

Daboëcia polifolia, D. Dun, - var. alba.

Dactylis glomerata, Linn., var. variegata.

Datura inermis, Jacq. Stramonium, Linn.

Delphinium Ajacis, Ledeb. altiasimum, Wall. azureum, Michx.
Delphinium-contd.

Brunonianum, Royle.

cashmerianum, Royle.

caucasicum, C. A. Mey.

cheilanthum, Fisch.

dasyanthum, Kar. et Kir.

Donkellari, Hort.

elatum, Linn.

formosum, Boiss. et Huet.

glabellum, Turez.

hybridum, Steph.

Maackianum, Regel.

Nahamah, Hort.

palmatifidum, DC.

speciosum, Bieb., var. turkestanicum.

velutinum, Bert.

Wheelerii, Hort.

Demazeria sicula, Dum.

Deschampsia cæspitosa, Beauv. flexuosa, Trin.

Dianthus alpinus, Linn. arenarius, Linn.

Armeria, Linn.

barbatus, Linn.

cresius, Sm.

carthusianorum, Linn.

Caryophyllus, Linn.

chinensis, Linn.

deltoides, Linn.

hæmatocalyx, Boiss. et Heldr.

liburnicus, Bartl. et Wendl. fil., var. Balbisii, (Ser.)

monspessulauus, Linn.

neglectus, Loisel.

petræus, Waldst. et Kit.

plumarius, Linn., sub sp. serotin.

us, Waldst. et Kit.

polymorphus, Bieb.

pungens, Linn.

Simsii, Hort.

squarrosus, Bieb.

superbus, Linn.

sylvestris, Wulf.

Dictamnus albus, Linn, var. ruber. tauricus, Hort.

Dierama ensifolium, C. Koch et Bouché.

Digitalis lutea, Linn.

ambigua, Murr.

purpurea, Linn.

Dimorphotheca pluvialis, Moench.

Dipsacus ferox, Loisel. 
Dipsacus-contd.

fullonum, Linn.

sylvestris, Mill.

Dodecatheon Meadia, Linn.

Draba Aizoon, Wahlenb.

alpina, Linn.

cuspidata, Bieb.

fladnizensis, Wulf.

incana, Linn.

repens, Bieb.

rupestris, R.Br.

siliquosa, Bieb.

Dracocephalum Ruyschiana, Linn. speciosum, Benth. thymiflorum, Linn.

Dryas octopetala, Linn.

Ecballium Elaterium, A. Rich.

Echinodorus ranunculoides, Engelm.

Echinops azureus, Hort.

microcephalus, Sibth. et Sm.

strictus, Bieb.

viscosus, DC.

Echium vulgare, Linn.

Elymus arenarius, Linn.

Caput-Medusæ, Linn.

condensatus, J. et C. Presl.

sabulosus, Bieb.

sibiricus, Linn.

villosus, Muhl.

Enkianthus himalaicus, Hook. fil. et Thoms.

Epilobium angustifolium, Linn.

- var. album.

Billardierianum, Ser.

Dodonæi, Vill.

- var. Fleischeri, (Hochst.).

latifolium, Linn.

microphyllum, A. Rich.

montanum, Linn.

nummularifolium, A. Cunn.

- var. longipes.

pedunculare, A. Cunn.

sericeum, Schum.

tetragonum, Linn.

Eremostachys laciniata, Bunge.

Erica cinerea, Linn.

- var. atropurpurea.

- bicolor.

- pallida.
Erigeron alpinus, Linn.

glabellus, Nutt. var.

multiradiatus, Benth. et Hook.

fil.

glaucus, Ker-Gawl.

speciosus, DC.

Erinus alpinus, Linn.

- var. albus.

Eriophyllum cæspitosum, Dougl.

Eryngium agavifolium, Griseb.

alpinum, Linn.

amethystinum, Linn.

bromeliæfolium, Delar.

campestre, Linn.

falcutum, Delar.

planum, Linn.

Spinalba, Vill.

Erysimum hieracifolium, Linn.

latifolium, Hort.

Perofskianum, Fisch. et Mey.

Winkleri, Hort.

yunannense, Hort.

Frythræa Massoni, Sweet.

Erythronium grandiflorum, Pursh.

Eschscholtzia californica, Cham.

- var. Mandarin, Hort.

Eupatorium ageratoides, Linn. fil. cannabinum, Linn.

glechonophyllum, Less.

purpureum, Linn.

sessilifolium, Linn.

Euphorbia Gerardiana, Jacq. Myrsinites, Linn.

Ferula Assa-fœetida, Linn.

Ferulago, Linn.

Festuca amethystina, Linn.

ampla, Hack.

arundinacea, Vill.

elatior, Linn.

- var. loliacea.

ovina, Linn.

pichinchæ, Hort:

rubra, Linn.

Funkia ovata, Spreng.

Sieboldiana, Hook.

Gaillardia aristata, Pursh. grandiflora, Hort. 
Gaillardia-contd. maxima, Hort. pulchella, Fouger.

Galium boreale, Linn. parisiense, Linn. pulchellum, Stapf. pusillum, Linn. rubioides, Linn. rubrum, Linn.

Galtonia candicans, Decne.

Gaultheria carnea, Hort. nummularioides, D. Don.

Genista sagittalis, Linn. tinctoria, Linn., var. prostrata.

Gentiana acaulis, Linn. - var. Kochiana, (Perr. et Song.)

asclepiadea, Linn.

- var. alba.

brevidens, Franch. et Sav.

Burseri, Lapeyr.

Cruciata, Linn.

phlogifolia, Schott et Kotschy.

punctata, Linn. septemfida, Pall.

- var. cordifolia, C. Koch.

tibetica, King.

Geranium armenum, Boiss.

collinum, Steph.

columbinum, Linn.

dahuricum, DC.

Endressi, J. Gay.

eriostemon, Fisch.

Grevilleanum, Wall.

ibericum, Cav.

maculatum, Linn.

pratense, Linn.

- var. alba.

rotundifolium, Linn.

sanguineum, Linn.

- var. lancastriense, Mill.

sibiricum, 'Linn.

sub-caulescens, L'Herit.

sylvaticum, Linn.

Wallichianum, D. Don.

Gerbera Kunzeana, A. Br. et Aschers.

Geum elatum, Wall.

Laxmanni, Gaertn.

miniatum, Hort.

molle, Vis. et Panc.

nutans, Hort.

parviflorum, Sm.
Geum-contd.

rivale, Linn.

strietum, Soland.

triflorum, Pursh.

Gilia achilleæfolia, Benth.

alba, Hort.

androsacea, Steud.

densiflora, Benth.

tricolor, Benth.

Globularia vulgaris, Linn.

Glyceria aquatica, Wablenb. maritima, Mert. et Koch.

Graphephorum arundinaceum, Aschers.

Gypsophila elegans, Bieb. fastigiata, Linn. repens, Linn.

Hedysarum obscurum, Linn.

Helianthella uniflora, Torr. et Gray.

Helianthemum polifolium, DC. thymifolium, Pers. Tuberaria, Mill. velutinum, Pomel. vulgare, Gaertn.

Helichrysum arenarium, Moench. rutidolepis, DC.

Heracleum giganteum, Fisch.

- var.

lanatum, Michx.

Leichtlini, Hort.

Sphondylium, Linn.

villosum, Fisch.

Hesperis fragrans, Fisch. matronalis, Linn.

Heuchera americana, Linn. cylindrica, Dougl.

Drummondii, Hort. macrophylla, Lodd. pubescens, Pursh. sanguinea, Engelm. undulata, Regel et Rich.

Hieracium amplexicaule, Linn. andryaloides, Vill. Auricula, Linn. balkanum, Uechtr. bohemicum, Fries. boreale, Fries.

buglossoides, Arv. bupleuroides, C.C. Gmel. caulescens, Hort. 
Hieracium-contd.

cerinthoides, Linn., var, molle.

collinum, Fries.

cydoniæfolium, Vill.

cymosum, Linn.

foliosum, Waldst, et Kit.

glomeratum, Froel.

humile, Jacq.

iricum, Fries.

Kalmii, Hort.

laciniatum, Hort.

montanum, Hort., var. pseudodichotomum.

nigrescens, Willd.

phlomoides, Froel.

prenanthoides, Vill.

prostratum, Poir.

rupestre, All.

scoticum, Hort.

strictum, Fries.

tridentatum, Fries.

umbellatum, Linn.

undulatum, Ait.

Hippuris vulgaris, Linn.

Holcus lanatus, Linn. mollis, Linn.

Homeria collina, Vent.

Homogyne alpina, Cass.

Hordeum bulbosum, Linn.

distichon, Linn.

jubatum, Linn.

vulgare, Linn.

Horminum pyrenaicum, Linn.

Hutchinsia alpina, R. Br.

Hyacinthus amethystiuus, Linn.

Hyoscyamus niger, Linn.

Hypericum Androsæmum, Linn.

Coris, Linn.

Gebleri, C. A. Mey.

hirsutum, Linn.

perforatum, Linn.

pulchrum, Linn.

quadrangulum, Linn.

- var. dubium.

Hypochæris glabra, Liun. maculata, Linn.

Hyssopus officinalis, Linn.

Iberis corifolia, Swret. gibraltarica, Linn., var. hybrida. sempervirens, Linn.

superbus, Hort.
Iberis-contd.

umbellata, Linn.

Impatiens Roylei, Walp.

Inula Oculus-Christi, Linn.

Iris Gueldenstaedtiana, Lepech., var. olbiensis.

Pseudacorus, Linn.

setosa, Pall.

sibirica, Linn.

- var. orientalis.

- - sanguinea.

- var.

versicolor, Linn.

xiphioides, Ehrh.

Isatis alpina, All. bastigas, Hort.

littoralis, Stev.

tinctoria, Linn.

Jasione perennis, Lam.

Juncus balticus, Willd. effusus, Linn.

- var. lutea.

- - spiralis.

glaucus, Sibth.

lamprocarpus, Ehrh.

tenuis, Willd.

Jurinea glycacantha, DC.

Kobresia caricina, Willd.

Koeleria cristata, Pers.

setacea, DC.

Lactuca Plumieri, Gren. et Godr. virosa, Linn.

Lamium Galeobdolon, Crantz. maculatum, Linn.

Orvala, Linn.

Lathyrus angustifolius, Schkr.

Aphaca, Liun.

Clusii, Hort.

cyaneus, C. Koch.

Drummondii, Hort.

Gorgoni, Parl.

lævigatus, Waldst. et Kit.

Lowii, Hort.

luteus, Munby.

macrorrhizus, Wimm.

nervosus, Lam. 
Lathyrus-contd.

niger, Berub.

tuberosus, Linn., var. tenuifolius.

vernus, Bernh.

Lavatera Olbia, Linn. thuringiaca, Linn. trimestris, Linn.

Leersia oryzoides, Sw.

Leontopodium alpinum, Cass. transylvannicum, Hort.

Leonurus Cardiaca, Linn. glaucescens, Bunge.

Lepidium crassifolium, Waldst. et Kit.

graninifolium, Linn.

hirtum, Sm. sativum, Linn.

Liatris graminifolia, Pursh. spicata, Willd.

Libertia grandiflora, Sweet.

Ligusticum scoticum, Linn.

Lilium pardalinum, Kellogg.

Linaria alpina, Mill. bipartita, Willd.

maroccana, Hook. fil.

origanifolia, DC.

sapphirina, Hoffmgg. et Link. vulgaris, Mill.

Linum alpinum, Linn. perenne, Linn.

usitatissimum, Linn.

- var. album.

- grandiflorum.

Lobelia inflata, Linn.

Lophanthus nepetoides, Benth. rugosus, Fisch. et Mey.

Lophosciadium meifolium, DC.

Lotus corniculatus, Linn. ornithopodioides, Linu.

Lunaria annua, Linn.

Lupinus Douglasii, Agardh. Hartwegii, Lindl. littoralis, Dougl. mutabilis, Sweet. nootkatensis, Donn. perennis, Linn.

Luzula albida, DC.

campestris, DC.

maxima, DC.

nivea, DC.

spicata, DC.

vernalis, DC.
Lychnis chalcedonica, Linn.

- var. alba.

Coeli-rosa, Desv.

coronaria, Desr.

dioica, linn.

- var. vespertina, Sm.

Flos-jovis, Desr.

nemoralis, Heuff.

Sartori, Hort.

Viscaria, Linn.

- var. purpurea.

Lycopus europæus, Linn.

Lysimachia ciliata, Linn. quadrifolia, Linn.

Lythrum hirsutum, Hort.

Salicaria, Linn.

- var. grandiflorum.

- - pubescens.

virgatum, Linn.

Magydaris panacina, DC.

Malcomia mari ima, R.Br.

- var. alba.

Malope trifida, Cav.

Malva moschata, Linn.

- var. alba.

nicæënsis, All.

- var. Tenoreana.

oxyloba, Boiss.

sylvestris, Linn.

trifida, Cav.

Marrubium alpinum, Hort. peregrinum, Linn. vulgare, Linn.

Matthiola incana, R. Br.

Meconopsis cambrica, Vig.

Medicago Echinus, DC. elegans, Linn. falcata, Linn.

- $x$ sativa. sativa, Linn.

Melica altissima, Linn. atropurpurea, Hort.

ciliata, Linn. nutans, Linn. uniflora, Retz.

Melilotus alba, Desr. officinalis, Lam. speciosa, Dur.

Melittis Melissophyllum, Linn.

Mentha arvensis, Linn. Requieni, Benth. 
Mentha-contd.

rotundifolia, Huds. sylvestris, Linn.

Mentzelia Lindleyi, Torr. et Gray.

Mertensia oblongifolia, G. Don. pulmonarioides, Roth.

Mesembryanthemum pyropeum, Haw.

Meum athamanticum, Jacq.

Mibora verna, Beauv.

Microglossa albescens, C. B. Clarke.

Milium paradoxum, Hort.

Mimulus luteus, Linn.

Mina sanguinea, Hort.

Molinia cærulea, Moench. - var. variegata.

Monarda Bradburiana, Beck. didyma, Linn.

Morina betonicoides, Benth. longifolia, Wall.

Muscari Argæi, Hort. armeniacum, Leichtlin.

Maweana, Baker. racemosum, Mill.

Szovitsianum, Baker.

Myosotis sylvatica, Hoffm.

Napæa dioica, Linn.

Narthecium ossifragum, Huds.

Nasturtium officinale, R.Br.

Nemesia floribunda, Lehm.

Nemophila maculata, Benth.

Nepeta camphorata, Boiss. et Heldr.

Cataria, Linn.

Mussini, Spreng.

nuda, Linn.

Nesæa verticillata, H.B.K.

Nolana atriplicifolia, D. Don. crocata, Linn.

globulosa, Linn.

Jordani, Ten.

peucedanifolia, Poll.

Enothera amœna, Lehm.

biennis, Linn.

campylocalyx, C. Koch et

Bouché.

concinna, D. Don.

glauca, Michx., var. Fraseri.

Sellowii, Link et Otto.

Onobrychis viciafolia, Scop.
Ononis hircina, Jacq. rotundifolia, Linn.

spinosa, Iinn.

Onopordon Acanthium, Linn.

Origanum Onites, Linn.

pulchrum, Boiss. et Heldr.

vulgare, Linn.

- var. aurea.

Ornithogalum narbonense, Linn.

Oxalis corniculatus, Linn.

Oxytropis campestris, DC.

lactea, Hort.

lapponica, Gaud.

Papaver caucasicum, Bieb.

lateritium, C. Koch.

Murcelli, var. Van Houtte.

nudicaule, Linn.

orientale, Linn.

- var. bracteatum (Lindl.)

pilosum, Sibth. et Sm.

- var. Heldreichii (Boiss.)

pinnatifidum, Moris.

Rhœeas, Linn.

rupifragum, Boiss, et Reut.

somniferum, Linn.

Paradisea Liliastrum, Bertol.

Parietaria officinalis, Linn.

Parnassia nubicola, Wall. palustris, Linn.

Pastinaca Fleischmanni, Hoffm. fulgida, Hort.

Peliosanthes sp.

Peltaria angustifolia, DC.

Pentstemon acuminatus, Dougl. confertus, Dougl.

diffusus, Dougl.

Hartwegii, Benth.

pubescens, Soland., var. major.

Phacelia congesta, Hook. tanacetifolia, Benth. viscida, Torr.

Phalaris arundinacea, Linn. canariensis, Linn.

Phaseolus multiflorus, Lam.

Phleum Michelii, All. tenue, Schrad.

Phlomis samia, Linn. setigera, Fale. viscosa, Poir.

Phormium tenax, Forst.

Phygelius capensis, E. Mey.

Physalis Alkekengi, Linn. 
Phyteuma campanuloides, Bieb.

Halleri, All.

Michelii, All.

orbiculare, Linn.

Scheuchzeri, All.

Picris echioides, Linn.

Pisum sativum, Linn.

Plantago acanthophylla, Decne. arachnoides, Schrenk. argentea, Chaix.

Coronopus, Linn. gentianoides, Sm. kamtschatica, Cham.

Lagopus, Linn.

lanceolata, Linn.

major, Linn.

- var. monstrosa.

maritima, Linn.

media, Linn.

mexicana, Link.

montana, Huds.

Psyllium, Linn.

saxatilis, Bieb.

subulata, Linn.

Platystemon californicus, Benth.

Plumbago micrantha, Ledeb.

Poa cæsia, Smith.

- var glauca.

chinensis, Linn.

nemoralis, Linn., var. sempervirens.

Podophyllum Emodi, Wall.

Polemonium cæruleum, Linn.

- var. acutiflorum, (Willd.)

- album, (Fisch.)

- fasciated.

flavum, Greene.

grandiflorum, Benth.

himalayanum, Baker.

humile, Willd.

lacteum, Lehm.

pauciflorum, Wats.

Polygonum alpinum, All.

Bistorta, Linn., var. major.

macrophyllum, D. Don.

pratensis, Linn.

Potentilla alchemilloides, Lapeyr.

ambigua, Cambess.

anserina, Linn.

argentea, Linn.

- var. calabra, (Ten.)

arguta, Pursh.

argyrophylla, Wall.
Potentilla-contd.

aurea, Linn.

chrysantha, Trevir.

dissecta, Pursh.

Dombeyi, Nestl.

Dr André.

hirta, Linn.

Hookeriana, Lehm.

japonica, Bl.

kurdica, Boiss. et Hohen.

leucotricha, Hort.

Louis Van Houte.

MacNabiana, $\times$ Lem.

marginata, Hort.

Meyeri, Boiss.

nevadensis, Boiss.

nivea, Linn.

norvegica, Iinn.

ontopoda, Dougl.

opaca, Linn.

palustris, Scop.

pedata, Willd.

peduncularis, D. Don.

Phæbis, Hort.

purpurea, Hook. fil., var. lutea.

recta, Nutt.

rupestris, Linn.

salisburgensis, Hænke.

Schrenkiana, Regel.

sericea, Linn.

Sibbaldi, Hall fil.

tridentata, Ait.

Poterium canadense, Benth. et Hook. fil.

dodecanthum, Hort.

officinale, Hook. fil.

polygamum, Waldst. et Kit.

Sanguisorba, Linn.

sitchense, Wats.

Primula cortusoides, Linn. elatior, Hill.

- var. intricata (Gren. et Godr.)

farinosa, Linn.

- var. magellanica, (Lehm.)

involucrata, Wall, var. Munroi. (Lindl.)

japonica, Gray.

officinalis, Jacq.

pubescens, $\times$ Jacq.

rosea, Royle, var. grandiflora.

sibirica, Jacq.

sikkimensis, Hook.

Warei, Stein. 
Prunella grandiflora, Linn. laciniata, Linn. vulgaris, Linn.

- var. hispida.

- Webbiana.

Pterostegia drymarioides, Fisch. et Mey.

Pulmonaria mollis, Wolff.

Puschkinia scilloides, Adams.

Pyrethrum Balsamita, Willd.

carneum, Bieb.

tauricum, Hort.

Ramondia pyrenaica, Rich.

- var. alba.

Ranunculus aconitifolius, Linn. acris, Linn.

— sub sp. sylvaticus, Thuill.

- var. sylvestris.

anemonefolius, DC.

bulbosus, Linn.

fumariæfolius, Desf.

Hollianus, Reichb.

nemorosus, DC.

propinquus, C. A. Mey.

repens, Linn.

- var. large fl.

Reuterianus, Boiss.

villosus, DC.

Raphanus caudatus, Linn.

sativus, Linn.

Reseda fruticulosa, Linn.

Jacquini, Reichb.

Luteola, Linn.

odorata, Linn.

Phyteuma, Linn.

ramosissima, Pourr.

undata, Linn.

Rheum hybridum, Ait.

nutans, Fisch.

officinale, Baill.

palmatum, Linn.

- var. tanguticum.

spiciforme, Royle.

undulatum, Linn., var, macrocarpum.

Rhododendron Chamæcistus, Linn.

Rudbeckia californica, Gray.

fulgida, Ait.

triloba, Linn.

Rumex Acetosella, Linn.

alpinus, Linn.
Rumex-contd.

alpinus, var. major.

aquaticus, Linn.

compactus, Hort.

cordifolins, Hornem.

crispus, Linn.

exaltatus, Hort.

Hydrolapathum, Huds.

intermedius, DC.

nepalensis, Spr.

ohtusifolius, Linn.

Patientia, Linn.

pulcher, Linn.

rupestris, Le Gall.

sanguineus, Linn.

sanguisorbæfolius, Hort.

scutatus, Linn.

stenophyllus, Ledeb.

Steudelii, Hochst.

tingitanus, Linn.

Ruta graveolens, Linn.

macrophylla, Ker.

Salvia argentea, Linn.

carduacea, Benth.

disermas, Linn.

glutinosa, Linn.

graveolens, Vahl.

hians, Royle.

lusitanica, Jacq. fil.

nubia, Ait.

pratensis, Jinn.

tiliæfolia, Vahl.

Verbenaca, Linn.

verticillata, Linu.

virgata, Ait.

viscosa, Jacq.

Sanicula europæa, Linn.

Santolina alpina, Linn.

Satureia montana, Linn., sub sp. illyrica, Host.

Saxifraga acanthifolia, Hort. ægilops, Hort.

Aizoon, Linn.

- brevifolia, Sterub.

- var. farinosa, Hort.

- - minor.

- - gracilis, Engler.

- notata (Schott).

- pectinata (Schott).

- - robusta (Schott).

bronchialis, Linn. 
Saxifraga-contd.

canaliculata, Boiss, et Reut.

crustata, Vest.

decipiens, Ehrh.

Geum, Linn., var. dentata, Link.

- - minor, Hort.

- - elegans, Mack.

- - ovalifolia, Hort.

granulata, Linn.

hederæfolia, Hochst.

hypnoides, Linn.

- var. purpurascens, Hort.

lingulata, Bell, var. cochlearis, Reichb.

- - minor, Hort.

- lantoscana, Boiss. et Reut. longifolia, Lap., var. superba, Hort.

Melvillei, Hort.

moschata, Wulf., var. pygmæa, (Haw.)

muscoides, All.

mutata, Linn.

nepalensis, Hort.

oppositifolia, Linn., var. fusca, Hort. Edin.

- var. pyrenaica, Hort.

pentadactylis, Lap.

Prostii, Sternb.

pulchella, Hort.

rotundifolia, Linn.

- var. glandulosa, Griseb.

- - repanda (Willd.)

- - taygetea, Boiss. et Heldr. squarrosa, Sieber.

Stansfieldii, Hort.

stellaris, Linn.

stricta, Hort.

tenella, Wulf.

umbrosa, Linn.

- var. variegata, Hort.

valdensis, DC.

Scabiosa agrestis, Waldst. et Kit.

arvensis, Linn.

caucasica, Bieb.

graminifolia, Linn.

Hladnikiana, Host.

magnifica.

succisa, Linn.

- var. alba.

Schizopetalon Walkeri, Sims.

Schønus nigricans, Linn.

Scilla amœna, Linn.
Scilla-contd.

campanulata, Ait., var. rubra.

nutans, $\mathrm{Sm}$.

peruviana, Linn.

sibirica, Andr.

- var. præcox, (Don).

verna, Huds.

Scirpus atrovirens, Muhl.

Holoschœnus, Linn.

lacustris, Linn.

Tabernæmontani, Gmel.

Scorpiurus vermiculata, Linn.

Scorzonera hispanica, Linn. stricta, Hornem.

Scrophularia aquatica, Linn. nodosa, Linn.

sambucifolia, Linn., var.

Scopolii, Hoppe.

trifoliata, Linn.

vernalis, Linn.

Scutellaria alpina, Linn.

altaica, Fisch.

japonica, Morr. et Decne.

peregrina, Linn.

Secale montanum, Guss., var. anatolicum, (Boiss.)

Sedum altissimum, Poir.

Anacampseros, Linn.

asiaticum, DC.

Beyrichianum, Hort.

californicum, Linn.

cyaneum, Rudolph.

dasyphyllum, Linn., var. oblongifolium.

Ewersii, Ledeb.

glaucum, Waldst. et Kit.

hirsutum, All.

kamtschaticum, Fisch.

lividum, Willd.

maximum, Sut.

- var. purpureum.

oppositifolium, Sims.

populifolium, Linn. fil.

reflexum, Linn.

rupestre, Huds.

spathulatum, Hook.

spurium, Bieb.

- var. album.

trifidum, Wall.

virescens, Willd., var. monstrosa.

Selinum Candollii, DC.

Senecio adonidifolius, Loisel.

alpinus, Linn. fil. 
Senecio-contd.

Doronicum, Linn.

Jacobæa, Linn.

Ledebourii, Hort.

Serratula coronata, Linn. quinquefolia, Bieb. tinctoria, Linn.

Seseli gummiferum, Sm.

Sherardia arvensis, Linn.

Sidalcea candida, Gray. malvæflora, Gray. Murrayana, Hort.

Silene alpestris, Jacq. caucasica, Boiss. chloræfolia, Sm., var. swertiæfolia (Boiss.)

ciliata, Pourr.

Cucubalus, Wibel.

glareosa, Jord.

italica, Pers.

linifolia, Sibth. et Sm.

muscipula, Linn.

orgæa, Fisch. et Mey.

quadridentata, DC.

Schafta, Gmel.

viridiflora, Linn.

Siler caucasicum, Spreng.

Silphium perfoliatum, Linn.

Sisymbrium assoanum, Losc.

Alliaria, Scop.

austriacum, Jacq.

strictissimum, Linn.

tanacetifolium, Linn.

Sisyrinchium bermudianum, Linn. angustifolium, Mill., var. mucronatum, (Michx.) striatum, Sm.

Smilacina racemosa, Desf.

Smyrnium perfoliatum, Mill.

Solanum Dulcamara, Linn. villosum, Lam.

Solidago californica, Nutt. canadensis, Linn. coriacea, Hort. nemoralis, Ait. ohioensis, Ridd. rigida, Liun. saligna, Hort. Virgaurea, Linn.

Sonchus palustris, Linn.

Sparganium ramosum, Huds. Specularia pentagonia, A. DC. Spiræa gigantea, Hort.
Spiræa-contd.

Filipendula, Linn.

palmata, Thunb., var. elegans.

Stachys Betonica, Benth.

- var. carnea.

lanata, Jaeq.

coccinea, Jacq.

grandiflora, Benth.

Statice coronata, Hort.

Gmelini, Willd.

tatarica, Linn.

Stipa capillata, Linn.

pennata, Linn.

tenacissima, Linn.

Symphyandra Hofmanni, Pautocs.

Symphytum asperrimum, Bieb. cordatum, Waldst. et Kit.

officinale, Linn.

tauricum, Willd.

tuberosum, Linn.

Tamus communis, Linn.

Tanacetum boreale, Fisch. vulgare, Linn.

Tellima grandiflora, R.Br. - var. virescens.

Teucrium Chamædrys, Linn. multiflorum, Linn. pyrenaicum, Linn. Scorodonia, Linn.

Thalictrum angustifolium, Jacq. asplenifolium, Hort.

Cornuti, Linn.

glaucum, Desf. minus, Linn.

- var. elatum.

- var. majus.

simplex, Linn., var. Jordani, Hort.

Thermopsis fabacea, DC. montana, Nutt.

Thlaspi alpestre, Linn. arvense, Linn. prostrata, Hort.

Thymus Serpyllum, Linn.

Tofieldia glutinosa, Pers. calyculata, Wahlenb., var. carinthiaca.

Tradescantia virginica, Linn. - var. alba.

- - atropurpurea.

Tragopogon majus, Jacq. pratense, Linn. 
Trichera sylvatica, Schrad. Tricyrtis australis, Hort. Trifolium alpinum, Linn. fragiferum, Linn. pannonicum, Linn. - var.

pratense, Linn.

repens, I.inn., var. pictum.

rubens, Linn., var.

subterraneum, Linn. trichocephalum, Bieb.

Trigonella Fœnum-græcum, Linn.

Trilisa odoratissima, Cass.

Trillium grandiflorum, Salisb.

Trinia Kitaibelii, Bieb. vulgaris, DC.

Trisetum pratense, Linn.

Triticum caninum, Huds. Humboldtii, Hort. monococcum, Linn. Spelta, Linn. violaceum, Hornem. vulgare, Vill.

Trollius altaicus, C. A. Mey. asiaticus, Linn.

- var. hybridus. caucasicus, Stev. europæus, Linn. intermedius, Hort. japonicus, Hort.

Tropæolum majus, Linn. minus, Linn.

- var. Tom Thumb.

Urtica magellanica, Poir.

Valeriana officinalis, Linn. tuberosa, Linn.

Vella spinosa, Boiss.

Verbascum Blattaria, Linn., sub-sp. virgatum, With.

Lagurus, Fisch. et Mey. ovalifolium, Donn. phœeniceum, Linn. repandum, Willd.

Veronica Allionii, Vill. Anagallis, Linn. austriaca, Linn. Bidwillii, Hook, fil.
Veronica-contd. candida, Lodd. carnosula, Hook. fil. cataractæ, Forst. Colensoi, Hook. fil. - var glauca. corymbosa, Hort. - var. variegata. fruticulosa, Linu. incisa, Ait. latifolia, Linn. ligustrifolia, A. Cunu. longifolia, Linn., var. alba. monticola, Armstr. officinalis, Linn.

- var. hirsuta. orchidea, Crantz. pinnata, Linn. prostrata, Linn., var. satureiæfolia.

saxatilis, Linn.

- var. Guthriana. serpyllifolia, Linn. spicata, Linn. spuria, Linn.

Traversii, Hook. fil.

Vesicaria utriculata, Lam.

Vicia Cracea, Linn. onobrychoides, Jinn.

Orobus, DC. villosa, Roth.

Vincetoxicum nigrum, Moench.

Viola arenaria, DC. canadensis, Linn., var. alba. canina, Linn. declinata, Waldst. et Kit. Munbyana, Hort. oculata, Lehm. odorata, Linn.

Wahlenbergia gracilis, A. DC. nutabunda A. DC.

Wulfenia Amberstiana, Benth. carinthiaca, Jacq.

Wyethia angustifolia, Nutt. mollis, Gray.

Zygadenus elegans, Pursh. glaucus, Nutt. - var. humilis. 


\section{TREES AND SHRUBS.}

Acer Pseudo-platanus, Linn.

Alnus cordifolia, Ten. glutinosa, Medic. serrulata, Willd.

Berberis asiatica, Roxb. Jamesonii, Lindl. Thunbergii, DC. vulgaris, Linn.

Betula alba, Linn.

Cotoneaster bacillaris, Wall. frigida, Wall. microphylla, Wall. nummularia, Fisch. et Mey. Simonsii, Baker.

Cratægus Azarolus, Linn. Oxyacantha, Linn. - var. fastigiata, Hurt. - - punicea, Hort. tanacetifolia, Pers. tomentosa, Linn.

Cytisus biflorus, L'Herit. capitatus, Scop. scoparius, Link.

Euonymus europæus, Linn.

Fraxinus excelsior, Linn.

Gaultheria Shallon, Pursh.

Ilex Aquifolium, Linn.
Laburnum alpinum, J. Presl. anagyroides, Medic.

- var. Adami, Hort.

Leycesteria formosa, Wall.

Olearia Haastii, Hook. fil.

Pernettya mucronata, Gaudich.

Pyrus Aria, Ehrh.

latifolia, Syme.

Rhamnus alnifolius, Linn. latifolius, L'Herit.

Rhododendron catawbiense, Michx. ferrugineum, Linn.

flavum, Don.

hirsutum, Linn.

ponticum, Linn.

Rosa acieularis, Lindl. rugosa, Sieb. et Zucc.

Rubus laciniatus, Willd.

Skimmia Fortunei, Mast.

Symphoricarpus racemosus, Michx. Syringa vulgaris, Linn.

- var. Lindleyana, Hort.

- - rubra insignis, Hort.

Ulex europæus, Linn.

Veronica Kirkii, Armstr. Traversii, Hook. fil.

\section{PLANTS UNDER GLASS.}

Acacia pulchella, R. Br. dealbata, Link.

Alonsoa incisifolia, Ruiz et Pav. Warcewiczii, Regel.

Andropogon ægyptiacus, Hort.

Archontophœenix Cunninghamii, $\mathrm{H}$. Wendl, et Drude.

Aristolochia fimbriata, Cham.

Aristea capitata, Ker-Gawl. Eckloni, Baker.

Arthropodium cirrhatum, R.Br.

Asclepias curassavica, Linn.

Asparagus crispus, Lam.
Asparagus-contel. scandens, Thunb.

Begonia Martiana, Link et Otto, var. gracilis.

Billbergia zebrina, Lindl.

Brachychilum Horsfieldii, Peters.

Canna patens, Rosc.

Capsicum annuum, Linn.

Celsia Areturus, Jacq. cretica, Linn. fil.

Chlorophytum Orchidastrum, Liudl. 
Crossandra undulæfolia, Salisb.

Datura Cornucopia, Hook.

Desmodium gyrans, DC.

Didymocarpus Humbolutiana, Gardn.

Drosera binata, Labill. capensis, Linn. spathulata, Labill.

Dyckia rariflora, Schult, fil.

Eccremocarpus scaber, Ruiz et Pav.

Eranthemum albiflorum, Hook. marmoratum, Hort. ex Regel.

Eugenia dysenterica, DC.

Exacum affine, Balf. fil.

Frucræa gigantea, Vent., var. variegata.

Garcinia Xanthochymus, Hook. fil.

Hibiscus pedunculatus, Linn. fil.

Hydrolea spinosa, Liun.

Indigofera australis, Willd.

Ipomœa umbellata, Linn.

Kennedya prostrata, R. Br., var. Marryatæ, Hort. rubicunda, Vent.

Libertia ixioides, Spreng.

Littonia modesta, Hook.

Luffa gigantea, Hort.
Maurandya scandens, Gray.

Mimosa pudica, Linn.

Momordica Charantia, Linn.

Myrtus Luma, Barn.

Ugni, Molina.

mossambicensis, Klotzsch.

Ochua multiflora, DC.

Ornithogalum tenellum, Jacq.

Passiflora filamentosa, Cav.

Philydrum lanuginosum, Banks.

Pitcairnia maidifolia, Decne.

Primula mollis, Nutt.

Richardia Rehmanni, Harrow.

Skimmia Fortunei, Mast.

Solanum Seaforthianum, Andrews.

Sollya heterophylla, Lindl.

Stylidium glandulosum, Salisb.

Tetranema mexicanum, Benth.

Tigridia Pavonia, Ker.

Tillandsia splendens, Brongu.

Torenia flava, Ham. peduncularis, Benth.

Trachelium coruleum, Linn.

Trichosanthes Anguina, Linn.

Veronica Andersoni, Hort.

Watsonia rubra, Baker.

Correspondents are requested to address all communications to the Regius Keeper, Royal Botanic Garden, Edinburgh.

EDINBURGH, January 1895. 





\section{ROYAL BOTANIC GARDEN, EDINBURGH.}

\section{LIST OF SEEDS}

COLLECTED DURING 1895.

PRINTES FOR HBR LATRSTXS STATIONERY OPHER.

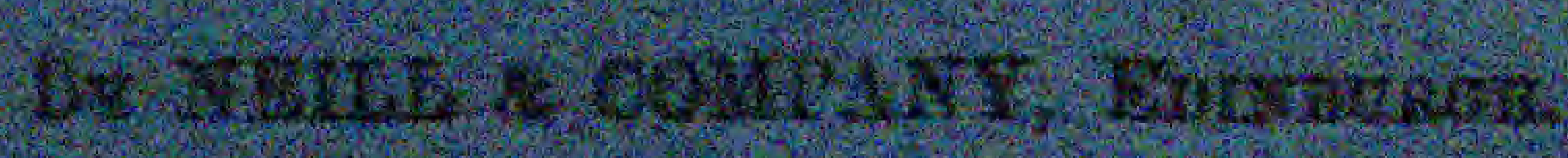
1595. 


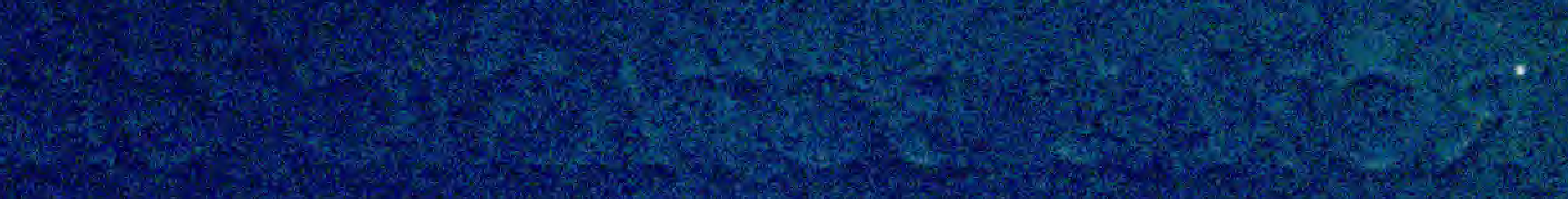

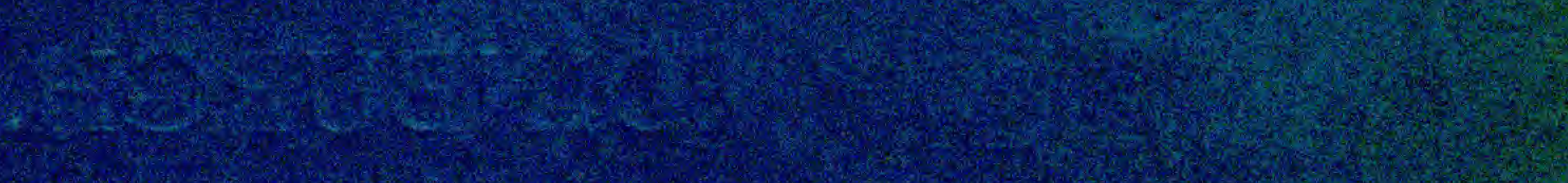

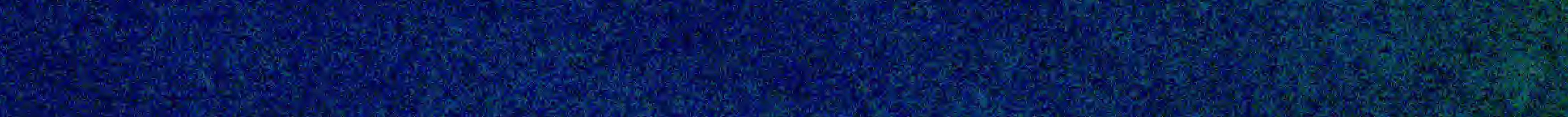

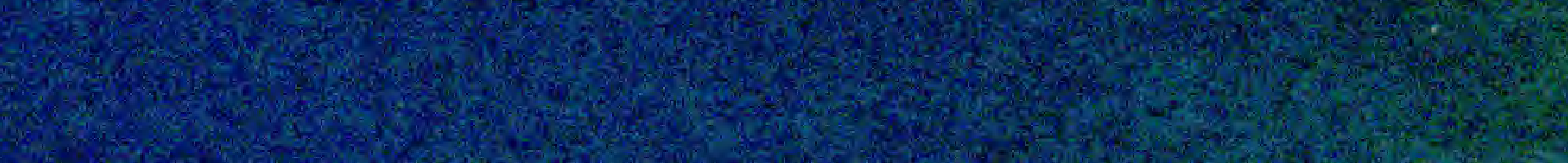
(1)

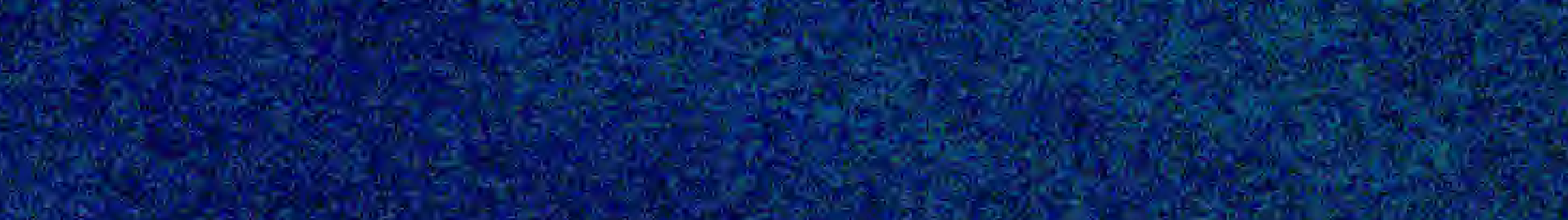

1.2. 


\title{
ROYAL BOTANIC GARDEN, EDINBURGH.
}

\section{LIST OF SEEDS}

\author{
COLLECTED DURING 1895.
}

\section{HERBACEOUS PLANTS.}

Aoæna argentea, Ruiz et Pav.

Achillea asplenifolia, Vent. Millefolium, Linn.

- var. rosea.

tomentosa, Linn.

Aconitum Anthora, Linn. culifornicum, Hort.

longigaleatum, Hort.

Lycoctonum, Linn.

- var. septentrionale.

- - album.

moldavicum, Hacq.

Napellus, Linn. paniculatum, Lam.

Actæa spicata, Linn,

Adenophora stylosa, Fisch.

Ethionema coridifolium, DC.

Agrimonia agrimonoides, Linn.

Eupatoria, Linn.

odorata, Mill.

Agropyron caninum, Beauv.

Agrostis alba, Linn., var. stolonifera (Linn.).

alpina, Scop.

vulgaris, With.

Alchemilla alpina, Linn.

conjuncta, Bab.

vulgaris, Linn.

Alisma Plantago, Linn.

Allium cæruleum, Pall.

carinatum, Linn.

cyaneum, Regel.

Dioscoridis, Sibth. et $\mathrm{Sm}$.

fistulosum, Linn.

flavum, Linn., var. pulchellum.

N, $-500-12 / 95$,
Allium-oontd.

karataviense, Regel.

Ledebourianum, Schult, f.

lineare, Linn.

macranthum, Baker.

MacNabianum, Hort.

Moly, Linn.

narcissiflorum, Vill.

neapolitauum, Cyr.

oreophilum, C. A. Mey.

polyphyllum, Kar, et Kir.

rubens, Baker.

sativum, Linn.

Schœnoprasum, Linn.

stipitatum, Regel.

subhirsutum, Linn.

Victorialis, Linn.

vineale, Linn.

- var. Kochii.

Alopecurus arundinaceus, Poir.

pratensis, Liun., var. foliis variegatis, Hort.

Alysaum gemonense, Linn.

Mœhlendorfianum, Hort.

montanum, Linn.

saxatile, Linn.

spinosum, Linn.

Amaranthus atropurpureus, Roxb.

gangeticus, Linn.

retroflexus, Linn.

Anagallis arvensis, Linn.

Anchusa officinalis, Linn,

Androsace lactea, Linn. villose, Linn.

Anemone alpina, Linn.

- var. sulphurea. 
Anemone-contd.

angulosa, Lam.

dichotoma, Linn.

narcissiflora, Linn.

- var.

nemorosa, Linn.

polyanthes, D. Don.

Pulsatilla, Linn. var. montana. rivularis, Buch.-Ham.

Anemonopsis macrophylla, Sieb. et Zucc.

Antennaria candida, Hort.

dioica, Gaertn.

- var. tomentosa, Hort.

Anthemis alpina, Linn.

santolinoides, Munby.

tinctoria, Linn.

- var. discoidea.

Anthericum Liliago, Linn.

Anthriscus sylvestris, Hoffm.

Antirrhinum majus, Linn.

Aquilegia alpina, Linn.

Buergeriana, Sieb. et Zuce.

californica, A. Gray.

- var. alba.

cærulea, James.

flavescens, S. Wats.

formosa, Fisch.

glandulosa, Fisch.

lutea, Lam.

pyrenaica, DC.

viridiflora, Pall.

vulgaris, Linn.

Arabis albida, Stev.

bellidifolia, Jacq.

incana, Roth.

lucida, Linn. f.

petræa, Lam.

Soyeri, Reut, et Huet,

Arctium Lappa, Linn. majus, Bernh.

Arenaria graminifolia, Schrad. pinifolia, Bieb. saxatilis, Linn. stricta, Michx. verna, Linn.

Armeria allioides, Boiss.

alpina, Willd.

cariensis, Boiss., var, rumelica (Boiss.).

maritima, Willd.

- var. alba.

plantaginea, Willd.
Arnica Chamissonis, Less. montana, Linn.

Arrhenatherum avenaceum, Beauv.

Artemisia campestris, Linn.

capillaris, Thunb.

desertorum, Spreng.

græca, Hort.

nitida, Bertol.

pontica, Linn.

rupestris, Linn.

sacrorum, Ledeb.

vulgaris, Linn.

Arum palæstinum, Boiss.

Asarum europæum, Linn.

Asparagus officinalis, Linn.

Asperula cynanchica, Linn.

Asphodelus ramosus, Linn.

Aster alpinus, Linn.

- var. albus.

Bellidiastrum, Scop.

ericoides, Linn, var. mollis.

macrophyllus, Linn.

paniculatus, Lam.

sibiricus, Linn.

spectabilis, Ait.

Stracheyi, Hook. f.

stellaris, Hort.

Astilbe rivularis, Buch.-Ham.

Astragalus alpinus, Linn.

bicolor, Lam.

chlorostachys, Lindl.

Cicer, Linn.

Glycyphyllos, Linn.

hypoglottis, Linn.

ponticus, Pall.

vaginatus, Pall.

vicioides, R, Grah.

Astrantia Biebersteinii, Fisch, et Mey.

carniolica, Wulf,

major, Linn.

minor, Linn.

Atriplex hortensis, Linn.

Atropa Belladonna, Linn.

Aubrietia croatica, Schott. deltoidea, DC.

- var. Columnæ (Guss.).

- - celestis.

- Richardi.

erubescens, Griseb.

Avena sempervirens, Vill. 
Baptisia australis, R. Br.

Barbarea vulgaris, R. Br. arcuata, Reichb.

Beckmannia erucæformis, Hort.

Brachypodium sylvaticum, Beauv.

Brassica alba, Boiss. nigra, Koch.

Briza maxima, Linn. minor, Linn.

Bromus adoënsis, Hochst. arduennensis, Dum. congestus, Hort. rigidus, Roth. unioloides, H. B. et K.

Bryanthus taxifolius, A. Gray.

Bulbinella Hookeri, Benth. et Hook. f.

Bunias orientalis, Linn.

Buphthalmum salicifolium, Linn. speciosum, Schreb.

Bupleurum aureum, Fisch. ranunculoides, Linn.

Calendula officinalis, Linn.

Callirhoë digitata, Nutt.

Caltha palustris, Linn,, var. biflora. sylvestris, Hort.

Camassia Brownii, Hort. esculenta, Lindl.

- var. alba.

- - major.

- - tardiflora, Hort.

Campanula alliariæfolia, Willd. americana, Linn.

carpatica, Jacq.

- var. turbinata (Schott).

collina, Bieb.

elegans, Rœm. et Schult.

glomerata, Linn.

Grossekii, Heuffl.

latifolia, Linn.

- var. macrantha (Fisch.).

- - alba.

latiloba, A. DC.

linifolia, Scop.

pelviformis, Lam.

persicifolia, var. alba grandiflora.

pulla, Linn.

punctata, Lam.

rapunculoides, Linn.

rhomboidea, Murr.
Campanula-contd.

rotundifolia, Linn.

speciosa, Pourr.

Carduus Carolorum $\times$, Howie et Jenner.

cernuus, Steud.

Carex atrata, Linn.

binervis, $\mathrm{Sm}$.

capillaris, Linn.

frigida, All.

fulva, Good.

glauea, Scop.

Grayii, Carey.

Mairii, Coss. et Germ.

microglochin, Wahlenb.

præcox, Jacq.

pulicaris, Linn.

rhyncophysa, Fisch. et Mey.

rupestris, All.

vesicaria, Linn.

Carum Carvi, Linn.

Cassandra calyculata, D. Don.

Centaurea calcitrapoides, Linn.

dealbata, Willd.

elata, Poir.

microcephala, Benth. et Hook. f.

ragusina, Linn., var. candidissima.

Scabiosa, Linn.

Centranthus ruber, DC.

Cephalaria tatarica, Schrad.

- - var. minor.

Cerastium arvense, Linn., var. grandiflorum.

dichotomum, Linn.

Cerinthe alpina, Kit.

Cheiranthus Cheiri, Linn. ochroleucus, Hall. f.

Chelidonium majus, Linn.

- var. grandiflorum.

- - laciniatum.

Chelone Lyoni, Pursh.

Chenopodium Bonus - Henricus, Linn.

Quinoa, Willd.

Chrysanthemum coronopifolium, Masf.

corymbosum, Linn.

Chrysopogon Gryllus, Trin.

Chrysosplenium alternifolium, Linn.

Cichorium Intybus, Linn.

Cimicifuga americana, Michx.

fœtida, Linn. 
Clarkia elegans, Dougl. pulchella, Pursh.

Claytonia virginica, Linn.

Clematis diversifolia, DC. Flammula, Linn. recta, Linn.

Cnicus Biebersteinii, Hort.

Casabonæ, Roth. centauroides, Linn. elatus, Hort. eriophorus, Roth. heterophyllus, Roth. horridus, Bieb. ochroleucus, Spreng. oleraceus, Linn. pannonicus, Hort., var. divaricatus.

pratensis, Willd. rigidus, Hort. serrulatus, Bieb. tartaricus, Willd.

Cochlearia saxatilis, Linn.

Codonopsis ovata, Benth.

Collinsia sparsiflora, Fisch. et Mey.

Convallaria majalis, Linn. - var. foliis strictis.

Convolvulus pentapetaloides, Linn. undulatus, Cav.

Corydalis capnoides, Wahlenb. glauca, Pursh, var. rosea. lutea, DC. nobilis, Pers.

Cotoneaster horizontalis, Deene. microphylla, Wall. thymifolia, Hort.

Cotula Filicula, Hook. f. reptans, Benth.

Crepis aurea, Reichb., var. crocea. sibirica, Linn. succisæfolia, Tausch.

Crocus asturicus, Herb. nudiflorus, Sm. - var. albus. speciosus, Bieb.

Crucianella laxiflora, Hort.

Cryptotænia canadensis, DC.

Cucubalus baccifer, Linn.

Cyananthus lobatus, Wall.

Cynanchum acutum, Linn.

Cynoglossum officinale, Linn.

Cytisus Ardoini, Fourn. nigricans, Linn.
Dactylis glomerata, Linn., var, variegata.

Datura inermis, Jacq. Tatula, Linn.

Delphinium altissimum, Wall. cashmirianum, Royle.

formosum, Boiss, et Huet.

glabellum, Turez.

hybridum, Steph.

Nahamah, Hort.

palmatifidum, DO.

speciosum, Bieb., var. turkesta icum.

velutinum, Bertol.

Deschampsia cæspitosa, Beauv.

- var. vivipara.

Deyeuxia acutiflora, Beauv.

Dianthus alpinus, Linn.

arenarius, Linn.

barbatus, Linn.

cæsius, Sm.

cæsio-caricinus $x$.

calocephalus, Boiss,

Caryophyllus, Linn.

chinensis, Linn.

deltoides, Linn.

grandiflorus, Poir.

intermedius, Boiss.

microlepis, Boise.

monspessulanus, Linn.

neglectus, Loisel., var. rosea.

petræus, Waldst, et Kit.

plumarius, Linn.

plumarius $\times$ Hellwigii, var. serotinus (Waldst, et Kit.).

Simsii, Hort.

squarrosus, Bieb.

superbus, Linn,

- var. Holtzeri (Winkler).

superbus $\times$ alpinus.

tener, Balb.

virgineus, Linn.

Wimmeri $\times$ chinensis.

Dictamnus albus, Linn.

- var. ruber.

- - tauricus.

Digitalis purpurea, Linn.

Diplotaxis erucoides, DC.

Dipsacus laciniatus, Linn.

Dodecatheon Meadia, Linn.

Doronicum grandiflorum, Lam. 
Drabs Aizoon, Wahlenb.

- var. eriocarpa.

alpina, Linn.

bruniæfolia, Stev.

cuspidata, Bieb.

fladnizensis, Wulf.

incana, Limn.

Mawii, Hook. f.

repens, Bieb.

rupestris, $\mathrm{R}$. Br.

siliquosa, Bieb.

Dracocephalum nutans, Linn. stamineum, Kar. et Kir.

Dryas Drummondii, Richerds. octopetala, Linn.

Echium plantagineum, Linn.

Elymus condensatus, J. et C. Presl. tener, Linn. $f$.

Epilobium angustifolium, Linn.

- var. album.

Dodonæi, Vill., var. Fleischeri (Hochst.).

latifolium, Linn.

nummularifolium, R, Cunn., var. longipes.

rosmarinifolium, Hænke, var. sericeum (Schum.).

tetragonum, Linn.

Eranthis hyemalis, Salisb.

Erica cinerea, Linn.

Erigeron aurantiacus, Regel.

caucasicus, Stev.

glabellus, Nntt.

- var. mollis.

multiradiatus, Benth. et Hook. f. pulchellus, DC.

Roylei, DC.

uniflorus, Linn.

Erinus alpinus, Linn.

Eriophorum alpinum, Linn.

Eryngium alpinum, Linn.

giganteum, Bieb.

glaciale, Boiss.

macrocalyx, Schrenk.

planum, Linn.

Erysimum cheiranthoides, Linn.

hieracifolium, Linn.

Perofskianum, Fisch. et Mey.

Erythronium Dens-canis, Linn.

grandiflorum, Pursh.

Eschscholzia californica, Cham.
Eupatorium ageratoides, Linn, f. cannabinun, Linn. glechonophyllum, Less. purpureum, Linn. sessilifolium, Linn.

Euphorbia amygdaloides, Linn. Gerardiana, Jacq.

Ferula Ferulago, Linn.

Festuca amethystina, Linn. arundinacea, Vill. duriuscula, Linn. elatior, Linn.

Halleri, All.

heterophylla, Lam. indigesta, Boiss. ovina, Linn.

Panciěiana, Hack. rubra, Linn. tenuis, Nym.

Fragaria californica, Cham. et Schlecht.

Fumaria officinalis, Linn.

Funkia Sieboldiana, Hook.

Gaillardia aristata, Pursh. - var maxima, Hort. pulchella, Fouger.

Galanthus nivalis, Linn. plicatus, Bieb.

Galega officinalis, Linn.

Galium boreale, Linn. pulchellum, Stapf. pusillum, Linn. saxatile, Linn,

Galtonia candicans, Decne.

Genista germanica, Linn. tinctoria, Linn.

Gentiana acaulis, Linn,, var. Kochiana (Perr. et Song.). asclepiadea, Linn.

- var. alba.

brevidens, Franch, et Sav.

Burseri, Lapeyr.

ornata, Wall.

phlogifolia, Schott et Kotschy. punetata, Linn.

septemfida, Pall., var. cordifolia (Hook, f.).

tibetica, King.

Walujewi, Regel et Schmalh. 
Geranium angulatum, Curt. balkanum, Hort.

dahuricum, DC.

Endressi, J. Gay.

Grevilleanum, Wall.

ibericum, Cav.

MacNabianum, Hort.

macrorrhizum, Linn.

maculatum, Linn.

pratense, Linn.

rotundifolium, Linn., var, album.

sanguineum, Linn.

- var. lancastriense (Mill.).

sylvaticum, Linn.

Geum chiloense, Balb., var. miniatum, Hort.

elatum, Wall.

montanum, Linn.

parviflorum, Commers.

pyrenaicum, Mill.

strictum, Soland.

triflorum, Pursh.

Gilia mierantha, Steud.

Globularia nana, Lam. vulgaris, Linn.

Gnaphalium supinum, Linn.

Graphephorum arundinaceum, Aschers.

Grindelia integrifolia, DC.

Gypsophila elegans, Bieb. perfoliata, Linn.

Hablitzia tamnoides, Bieb.

Hedysarum obscurum, Linn.

Helenium autumnale, Linn.

Helianthemum canum, Boiss.

Helianthus decapetalus, Linn. mollis, Lam.

Helichrysum rutidolepis, DC.

Helleborus niger, Linn., var. angustifolius.

Helonias bullata, Linn.

Heracleum lanatum, Michx.

Heuchera pubescens, Pursh. sanguinea, Engelm.

Hieracium alpinum, Linn. amplexicaule, Linn. argenteum, Fries. Auricula, Linn.

balkanum, Uechtr. buglossoides, Arv.
Hieracium-contd.

bupleuroides, C. C. Gmel,

cæsium, Fries:

compositum, Lapeyr.

cydoniæfolium, Vill.

eximium, Backh.

floribundum, Wimm. et Grab.

foliosum, Waldst, et Kit.

humile, Jacq.

iricum, Fries.

Marshalli, Lint.

nigreseens, Willd.

phlomoides, Froel.

Pilosella $\times$ aurantiaca,

prenanthoides, Vill.

prostratum, Poir.

pulmonarioides, Vill.

pyrenaicum, Jord.

rubescens, Gilib.

sabaudum, Linn.

saxatile, Vill.

scoticum, Hort.

stoloniflorum, Waldst. et Kit.

strictum, Fries.

tenellum, Backh.

tridentatum, Fries.

umbellatum, Linn.

vulgatum, Fries.

Hierochloe australis, Rœem. et Schult. borealis, Rœm, et Schult.

Holcus lanatus, Linn.

Homogyne alpina, Cass.

Hordeum bulbosum, Linn. jubatum, Linn.

maritimum, With.

Horminum pyrenaicum, Linn.

Hunnemannia fumariæfolia, Sweet.

Hyoscyamus albus, Linn.

Hypecoum procumbens, Linn.

Hypericum Coris, Linn.

hirsutum, Linn.

nummularium, Linn.

olympicum, Linn.

origanifolium, Willd.

perforatum, Linn.

quadrangulum, Linn.

undulatum, Schousb.

Hypochoeris glabra, Linn.

maculata, Linn.

Hyssopus officinalis, Linn.

Iberis corifolia, Sweet. 
Iberis-contd.

saxatilis, Linn.

sempervirens, Linn.

superbus, Hort.

Tenoreana, DC,

Impatiens Roylei, Walp.

Inula glandulosa, Puschk.

Hookeri, Clarke.

Oculus-Christi, Linn.

Iris Bloudowii, Ledeb.

Gueldenstaedtiana, Lepech.

kumaonensis, Wall.

missouriensis, Nutt.

Monnieri, DC.

Pseudacorus, Linn.

- var. variegata.

setosa, Pall.

sibirica, Linn.

- var. alba.

- orientalis, Baker.

- - sanguinea (Donn).

versicolor, Linn.

virescens, DC.

xiphioides, Ehrh.

- var. alba.

Isatis alpina, All.

tinctoria, Linn.

Jasione Jankae, Neilr.

Juncus balticus, Willd.

conglomeratus, Linn.

effusus, Linn.

- var. luteus.

glaucus, Sibth.

tenuis, Willd.

trifidus, Linn.

Jurinea glycacantha, DC.

Kaufmannia Semenovi, Regel. Koeleria setacea, DC.

Lagurus ovatus, Linn.

Lamium Galeobdolon, Crantz. maculatum, Linn., var. album. Orvala, Linn.

Lapsana grandiflora, Bieb.

Lathyrus Clusii, Hort. cyaneus, C. Koch. hirsutus, Linn.
Lathyrus-contd.

Lowii, Hort.

lutens, Munby.

montanus, Bernh.

nervosus, Lam.

niger, Bernh.

vernus, Bernh.

- var. flaccidus (Kit).

Lavatera mauritanica, Duch.

Leontopodium alpinum, Cass.

- var, transsylvanieum.

Leucojum æstivum, Linn.

vernum, Linn., var. earpatieum

(Herb).

Liatris spicata, Willd.

Ligusticum scoticum, Linn.

Lilium Martagon, Linn.

pardalinum, Kellogg.

- var. Robinsonianum.

Linaria alpina, Mill. anticaria, Boiss. et Reut. maroceana, Hook. f. origanifolia, DC.

sapphirina, Hoffmgg, et Link.

vulgaris, Mill.

Lindelofia spectabilis, Lehm.

Linum alpinum, Linn.

Lophosciadium meifolium, DC.

Lotus corniculatus, Linn.

Lunaria annua, Linn.

Lupinus Chamissonis, Eschseh.

Hartwegii, Lindl.

luteus, Linn.

nootkatensis, Donn.

perennis, Linn.

polyptyllus, Lindl.

Luzula albida, DC.

Forsteri, DC.

maxima, DC.

vernalis, DC.

Lychnis chalcedonica, Linn.

- var. alba.

coronaria, Desr.

- var. alba.

dioica, Linn.

- var. vespertina.

Flos-jovis, Desr.

grandiflora, Jacq., var. alba.

Sartori, Hort.

Viscaria, Linn,, var, purpurea.

Lysimachia affinis, Bunge.

barystachys, Bunge.

vulgaris, Linn. 
Lythrum hirsutum, Hort.

Salicaria, Linn.

- var. grandiflora.

- - pubescens (Sweet).

Macrotomia echioides, Boiss.

Malva crispa, Linn. nicæensis, All.

- var. Tenoreana.

moschata, Linn., var. alba.

Matthiols ineana, R. Br.

Meconopsis cambrica, Vig.

Medicago ciliaris, Krock.

falcata, Linn.

falcata $\times$ sativa.

platycarpa, Trautv.

sativa, Linn.

truncatula, Gaertn.

Melica altissima, Linn. nutans, Linn. uniflora, Retz.

Melilotus speciosa, Dur.

Melittis Melissophyllum, Linn.

Mentha arvensis, Linn.

Mertensia oblongifolia, G. Don.

Meum athamanticum, Jacq.

Mitella pentandra, Hook.

Molinia cærulea, Mønch.

Molopospermum cicutarium, DC.

Monarda didyma, Linn.

Morina betonicoides, Benth. longifolia, Wall.

Muehlenbergia mexicana, Trin.

Muretia tanaicensis, Boiss.

Muscari Argæi, Hort. armeniacum, Baker. atlanticum, Boiss. et Reut. Maweanum, Baker. racemosum, Mill. Szovitsianum, Baker.

Myosotis palustris, Lam. sylvatica, Hoffm.

Myrrhis odorata, Scop.

Napæa dioica, Linn.

Nardus stricta, Linn.

Narthecium ossifragum, Huds.

Nepeta Mussini, Spreng. nuda, Linn.

Nesæa verticillata, H.B, et K.

Nicotiana rustica, Linn.

Nigella orientalis, Linn.
Enothera amœna, Lehm.

biennis, Linn.

campylocalyx, C. Koch et

Bouché.

fruticosa, Linn.

- var. Youngei, Hort.

glauca, Michx.

- var. Fraseri (Pursh.).

Ononis arvensis, Linn, spinosa, Linn.

Onosma Visianii, Clem.

Orchis latifolia, Linn.

Origanum pulchrum, Boiss. et Heldr. vulgare, Linn.

- var. aureum.

Oxytropis campestris, DC. lactea, Hort.

lapponica, Gaud, uralensis, DC.

Pæonia officinalis, Linn., var. anemonæflora.

- var. Sabinii.

Papaver alpinum, Linn. nudicaule, Linn. orientale, Linn. persicum, Lindl. pinnatifidum, Moris. rupifragum, Boiss, et Reut. somniferum, Linn.

Parnassia nubicola, Wall. palustris, Linn.

Pentstemon acuminatus, Dougl. barbatus, Roth, var. Torreyi (Benth.).

confertus, Dougl. glaucus, R. Grah.

Phaseolus multiflorus, Willd.

Phlomis setigera, Falc. viscosa, Poir.

Physostegia virginiana, Benth.

Phyteuma orbiculare, Linn.

Scheuchzeri, All.

Sieberi, Spreng.

Picris echioides, Linn.

Plantago Lagopus, Linn.

lanceolata, Linn.

major, Linn.

maritima, Linn.

media, Linn.

montana, Huds.

Psyllium, Linn.

subulata, Linu. 
Pleurospermum austriacum, Hoffm. pulchrum, Aitch. et Hemsl.

Plumbago micranthe, Ledeb.

Poa chinensis, Linn. nemoralis, Linn.

- var. sempervirens.

Podophyllum Emodi, Wall.

Polemonium cæruleum, Linn.

- var. album (Fisch.).

- - fasciated.

flavum, Greene.

humile, Willd.

lacteum, Lehm. pauciflorum, S. Wats. reptans, Linn.

Polygonum affine, D. Don.

alpinum, All.

officinale, All.

Portulaca oleracee, Linn,

Potentilla andicola, Benth. argentea, Linn.

- var. calabra (Tenore).

arguta, Pursh.

argyrophylla, Wall.

aurea, Linn.

collina, Wibel.

dissecta, Pursh.

Dombeyi, Nestl.

Fenzlii, Lehm.

gelida, C. A. Mey.

grandiflora, Linn.

hirta, Linn.

japonica, $\mathrm{Bl}$.

Meyeri, Boiss.

nevadensis, Boiss.

nivea, Linn.

ontopoda, Dougl.

opaca, Linn.

palustris, Scop.

pyrenaica, Ram.

recta, Linn.

rupestris, Linn.

Schrenkiana, Regel.

Sibbaldi, Hall. f.

Valderia, Linn.

Poterium canadense, A. Gray.

dodecandrum, Benth. et Hook. f.

officinale, A. Gray.

polygamum, Waldst. et Kit.

Pratia macrodon, Hook. f.

Primula Auricula, Linn.

farinosa, Linn.

japonica, A. Gray, var. alba.
Primula-contd.

officinalis, Jacq.

- var, uralensis (Fisch.).

scotica, Hook.

sikkimensis, Hook.

Prunella grandiflora, Linn. vulgaris, Linn.

- var. Webbiana.

Pulmonaria arvernensis, Hort.

Puschkinia scilloides, Adams.

Pyrethrum tauricum, Hort.

Ramondia pyrenaica, Rich.

Ranunculus aconitifolius, Linn.

acris, Linn.

amplexicaulis, Linn.

auricomus, Linn.

bulbosus, Linn.

millefoliatus, Vahl.

nemorosus, DC.

parnassifolius, Linn.

pyrenæus, Linn.

repens, Linn.

- - var.

Reuterianus, Boiss.

Thora, Linn.

Reseda alba, Linn.

crystallina, Webb et Berth.

Jacquini, Reichb.

lutea, Linn.

ramosissima, Pourr.

Rheum hybridum, Murr.

officinale, Baill.

palmatum, Linn.

- var. tanghuticum.

Rhaponticum, Linn.

undulatum, Linn., var. macrocarpum.

Rudbeckia laciniata, Linn.

Rumex alpinus, Linn.

- var. major.

aquaticus, Linn.

compactus, Hort.

cordifolius, Hornem.

intermedius, DC.

nepalensis, Spreng.

obtusifolius, Linn.

rupestris, Le Gall.

sanguineus, Linn.

sanguisorbaefolius, Ho:t.

scutatus, Linn.

stenophyllus, Ledeb. 
Ruta graveolens, Linn. macrophylla, Soland.

Salvia glutinosa, Linn. hians, Royle.

limbata, C. A. Mey.

lusitanica, Jacq. fil.

officinalis, Linn.

pratensis, Linn.

taraxacifolia, Coss. et Bal.

tiliæfolia, Vahl.

verticillata, Linn.

Sanguinaria canadensis, Linn.

Saponaria cæspitosa, DC.

ocymoides, Linn.

- var. Loderi.

officinalis, Linn.

Satureia hortensis, Linn.

montana, Linn., var. illyrica (Host).

Saxifraga Aizoon, Jacq.

- var. balkana.

- - brevifolia, Sternb.

- - - farinosa, Hort.

- - Gaudinii (Brügg.).

- major.

- - minor.

- - pectinata (Schott).

- - punctata.

- - robusta (Schott).

- rotata.

aretioides, Lap., var. primulina. bronchialis, Linn.

- var, conferta.

cæsia, Linn.

cæspitosa, Linn.

cartilaginea, Willd.

cernua, Linn.

cochlearis, Reichb.

Cotyledon, Linn.

- var. pyramidalis (Lapeyr.).

crassifolia, Linn.

crustata, Vest.

- var. hybrida.

cuneifolia, Linn.

decipiens, Ehrh.

- var. grœnlandica (Linn.).

- - grandiflora, Hort.

- palmata, Panc.

diapensioides, Bell.
Saxifraga-contct.

exarata, Vill.

Geum, Linn.

- var. elegans (Mackay).

glaucescens, Reut.

Gmelini, Hort.

granulata, Linn.

- var. Russi.

hirsuta, Linn.

- var. dentata (Link).

- - minor.

Hostii, Tausch.

hypnoides, Linn.

-- var. hirta, Donn.

hybrida, Sternb., var. splendens.

intacta, Willd.

- var. farinosa.

- - minor.

lingulata, Bell.

- var. cochlearis, Reichb., var. minor Hort.

longifolia, Lap.

- var. superba, Hort.

MacNabiana, Hort.

Melvillei, Hort.

muscoides, Wulf.

mutata, Linn.

nepalensis, Hort.

oppositifolia, Linn.

- var. pyrenaica, Hort.

- crimson.

- - dark.

paradoxa, Sternb.

pedatifida, Ehrh.

peltata, Torr. et Gray.

planifolia, Lapeyr.

Portae, Engl.

pulchella, Hort.

retusa, Gouan.

- var. bryoides, Hort.

- - maritima.

Reyeri $\times$.

rotundifolia, Linn.

- var. lasiophylla (Sehott, Nym.

et Kotschy).

- - repanda (Willd.).

sancta, Griseb.

Seguieri, Spreng.

squarrosa, Sieber.

Stansfieldii, Hort.

stricta, Hort.

Sturmiana, Hort.

taygetea, Boiss. et Heldr, 
Saxifraga-contd.

tricuspidata, Rottb:

umbrosa, Linn.

- var. variegata, Hort.

virginiensis, Michx.

Scabiosa arvensis, Linn.

banatica, Waldst. et Kit.

cretica, Linn.

graminifolia, Linn.

lucida, Vill.

plumosa, Sibth. et Sm.

Succisa, Linn.

sylvatica, Linn.

Schizopetalon Walkeri, Sims.

Scilla sibirica, Andr.

- var. præcox (Donn).

Scirpus Holoschøenus, Linn.

lacustris, Linn., var. Tabernæmontani, C. C. Gmel.

sylvaticus, Linn.

Scrophularia alata, Gilib.

aquatica, Linn.

nodosa, Linn.

sambucifolia, Linn., var.

Scopolii, Hoppe.

trifoliata, Linn.

vernalis, Linn.

Scutellaria alpina, Linn.

minor, Huds.

Secale cereale, Linn., var. anatolicum (Boiss.).

Sedum Aizoon, Linn.

asiaticum, Spreng

kamtschaticum, Fisch. et Mey.

maximum, Suter.

- var. atropurpureum.

nicæënse, All.

oppositifolium, Sims.

rupestre, Linn., var. monstrosum.

stoloniferum, S. T. Gmel.

- var. album.

Telephium, Iinn.

trifidum, Wall.

Selinum Candollii, DC.

Sempervivum acuminatum, Jacquem.

- var. nigrum.

arvernense, Lecoq et Lamotte.

flagelliforme, Fisch.

glaucum, Tenore.

globiferum, Linn.

hirtum, Linn.

montanum, Linn,
Sempervivum-contd.

Pittonii, Schott, Nym. et

Kotschy.

Pomelii, Lamotte.

stenopetalum, Schnittsp. et C. B.

Lehm.

Wulfeni, Hoppe.

Senebiera Coronopus, Poir. pinnatifida, DC.

Senecio Doronicum, Liun.

Serratula coronata, Linn. heterophylla, Desf. quinquefolia, Bieb. tinctoria, Linn.

Seseli gummiferum, Pall.

Sidalcea candida, A. Gray.

Murrayana, Hort. neo-mexicana, A. Gray.

Silene alpestris, Jacq. eaucasica, Boiss. ciliata, Pourr.

Cucubalus, Wibel.

Elisabethæ, Jan.

flavescens, Waldst, et Kit.

italica, Pers.

Muscipula, Linn.

nutans, Linn.

quadridentata, Pers.

Reichenbachii, Vis.

Saxifraga, Linn.

Sisymbrium Alliaria, Scop.

strictissimum, Linn.

tanacetifolium, Linn.

Sisyrinchium angustifolium, Mill., var, mucronatum (Michx.).

bermudiana, Linn.

Sellowianum, Klatt.

striatum, Sm.

Sium erectum, Huds.

Sobolewskia clavata, Fenzl.

Solanum Dulcamara, Linn. nigrum, Linn.

Solidago californica, Nutt. canadensis, Linn.

elongata, Nutt.

latifolia, Linn.

nemoralis, Ait.

saligna, Hort.

Virgaurea, Linn.

Sonchus arvensis, Linn.

lapponicus, Froel.

oleraceus, Linn.

palustris, Linn. 
Spiræa Aruncus, Linn.

bullata, Maxim.

Filipendula, Linn.

palmata, Thunb., var. alba.

- var. elegans.

venusta, Otto et Dietr.

Stachys Alopecuros, Benth.

betonioa, Benth.

coccinea, Jacq.

Stellaria biflora, Linn,

Swertia perennis, Linn.

Symphyandra Hofmanni, Paut. pendula, A. DC.

Wanneri, Heuff.

Eymphytum asperrimum, Donn. caucasicum, Bieb.

officinale, Linn.

Symplocarpus foetidus, Nutt.

Tanacetum boreule, Fisch. vulgare, Linn.

Tellima grandiflora, R.Br.

Teucrium multiflorum, Linn.

Scorodonia, Linn.

Thalictrum angustifolium, Linn.

glaucum, Desf.

majus, Murr.

minus, Linn.

- var. acuminatum (Spreng.).

- elatum (Jacq.).

simplex, Linn., var. Jordani

(F. Schultz).

Thermopsis fabacea, DC.

montana, Nutt.

Thlaspi alpestre, Linn. arvense, Linn. prostrata, Hort.

Thymus hirsutus, Bieb.

Serpyllum, Linn.

Tofieldia calyculata, Wahlenb.

- var. carinthiaca.

Tradescantia virginiana, Linn.

- var. atropurpurea.

- - elata (Lodd.).

- major.

- - subaspera (Ker-Gawl.).

Tricyrtis australis, Hort.
Trifolium alpinum, Linn.

Bocconi, Savi.

pannonicum, Jacq.

pratense, Linn.

rubens, Linn.

trichocephalum, Bieb.

Trigonella Fœnum-græcum, Linn.

Trilisa odoratissima, Cass.

Trillium grandiflorum, Salisb.

- var. maximum.

Triticum elymoides, Hochst. monococcum, Linn. vulgare, Vill.

Trollius altaicus, C. A. Mey. americanus, Muhl. asiaticus, Linn.

- var. Fortunei, Hort.

- - intermedius.

caucasicus, Stev.

europæus, Linn.

japonicus, Miq.

Tropæolum majus, Linn.

Tulipa biflora, Pall.

Urtica magellanica, Juss.

Vaccinium Vitis-Idæa, Linn.

Valeriana montana, Linı.

Veratrum Maackii, Regel. nigrum, Linn.

Verbascum Lychnitis, Linn. phœenicoum, Linn. virgatum, Stokes.

Veronica aphylla, Linn. austriaca, Linn.

Bidwillii, Hook. f.

Chamædrys, Linn., var.variegata.

Colensoi, Hook. f.

- var. glauca.

corymbosa, Hort.

- var. variegata.

fruticulosa, Linn.

gentianoides, Vahl.

- var. variegata.

grandis, Fisch.

Guthriana, Hort.

ineisa, Ait. 
Veronica-contd.

latifolia, Linn.

Lyallii, Hook. f.

longifolia, Linn.

- var. alba.

officinalis, Linn;

orchides, Crantz,

pinnata, Linn.

Ponæ, Gouan.

saxatilis, Scop.

serpyllifolia, Lin̨n.

spicata, Linn.

- var, alba.

- hybrida (Linn.).

spuria, Linn.

Tournefortii, C. C, Gmel.

vernicosa, Hook. f.

Vesicaria utriculata, DC,

Vicia Cracca, Linn.

onobrychoides, Linn.

Orobus, DC.

pyrenaica, Pourr.

sepium, Linn.

villosa, Roth.
Vincetoxioum luteum, Hoffmgg. et Link.

nigrum, Moench.

officinale, Moench.

Viola canadensis, Linn., var, alba. canina, Linn.

cornuta, Linn.

lutea, Huds.

odorata, Linn.

tricolor, Linn.

Wyethia mollis, A. Gray. angustifolia, Nutt.

Xerophyllum asphodeloides, Nutt.

Zygadenus elegans, Purah. glaucus, Nutt. - var. humilis.

\section{PLANTS UNDER GLASS.}

Abutilon Regnelii, Miq.

Acacia pulchella, R. Br.

Archontophœnix Cunninghamii, $\mathrm{H}$.

Wendl, et Drude.

Ardisia primulæfolia, Gardn, et Champ.

Aristea capitata, Ker-Gawl. Eckloni, Baker.

Asparagus crispus, Lam. scandens, Thunb.

Begonia Frœbelii, A. DC.

Canna patens, Rosc.

Capsicum annuum, Linn.

Cedronella triphylla, Moench.

Chlorophytum Orchidastrum, Lindl.

Clerodendron splendens, G. Don.

Thomsonæ, Balf. var. Balfouriana, Hort.

Coffea arabica, Linn.

Costus igneus, N. E. Br.
Crossandra undulæfolia, Salisb.

Cyclamen hederæfolium.

Cyperus congestus, Vahl.

Cyrtanthus Huttoni, Baker.

Desmodium gyrans, DC.

Drosera Burkeana, Planch. capensis, Linn.

spathulata, Labill.

Eccremocarpus scaber, Ruiz et Pav, Eranthemum albiflorum, Hook, leuconeurum, Hort. marmoratum, Hort.

Hæmanthus puniceus, Linn.

Indigofera australis, Willd.

Isotoma longiflora, Presl, 
Kennedya rubicunda, Vent.

Mahernia glabrata, Cav.

Maurandia Barclaiana, Lindl.

Melochia corchorifolia, Linn.

Mimosa latispinosa, Lam. pudica, Linn.

Momordica Charantia, Linn.

Myrtus Luma, Barn.

Ugni, Moliua,

Orthosiphon stamineus, Benth.

Orthrosanthus multiflorus, Sweet.

Paulo-Wilhelmia speciosa, Hochst.

Petiveria alliacea, Linn.

Philydrum lanuginosum, Banks,
Primula mollis, Nutt. verticillata, Forsk.

Psychotria cyanocarpa, Seem.

Richardia Rehmannii, N. E. Br.

Ruellia biflora, Pav.

Blumei, Steud.

Scutellaria Mociniana, Benth.

Sida triloba, Cav.

Sollya heterophylla, Lindl.

Streptocarpus Saundersii, Hook.

Stylidium glandulosum, Salisb.

Tachiadenus carinatus, Griseb.

Tetranema mexicanum, Benth.

Trachelium cæruleum, Linn.

Tweedia cærulea, D. Don,

\section{TREES AND SHRUBS.}

Acer Pseudo-platanus, Linn.

Esculus carnea, Willd.

Hippocastanum, Linn.

Alnus cordifolia, Tenore. glutinosa, Medic. serrulata, Willd.

Betula alba, Linn.

Caragana arborescens, Lam.

Cotoneaster affinis, Jindl.

bacillaris, Wall.

frigida, Wall.

microphylla, Wall.

Simonsii, Baker.

Cratægus coccinea, Linn.

- var. indentata, Lodd.

mollis, Scheele.

Oxyacantha, Linn.

Cytisus biflorus, L'Herit. scoparius, Link.

Elæagnus multiflora, Thunb.

Ilex Aquifolium, Linn.
Laburnum vulgare, J. S. Presl.

Piptanthus nepalensis, Sweet.

Pyrus Aria, Linn.

Aucuparia, Linn.

- - var. fastigiata.

- - - fructu-luteo.

- _ - pendula variegata.

rotundifolia, Bechst.

Rhododendron catawbiense, Michx.

ferrugineum, Linn.

flavum, G. Don.

hirsutum, Linn.

ponticum, Linn.

Rosa anemonæflora, Fortune. pomifera, Herrm.

rubella $\mathrm{Sm} . \times$.

rugosa, Thunb.

Sambucus Fontenaysii, Carr. nigra, Linn.

- - var. rubrocarpa.

racemosa, Linn.

Viburnum Lantana, Linn.

Correspondents are requested to address all communications to the Regius Keeper, Royal Botanic Gardens, Edinburgh. 




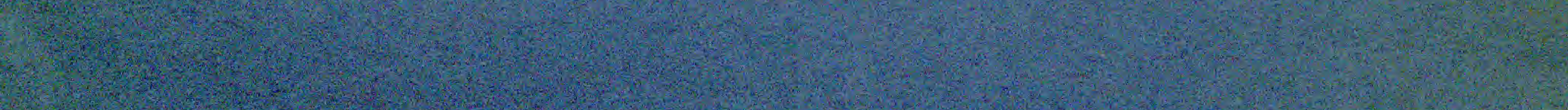

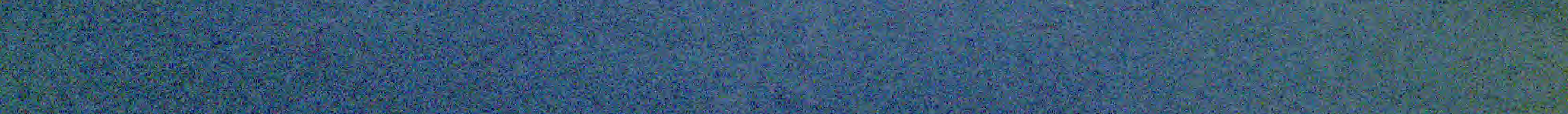

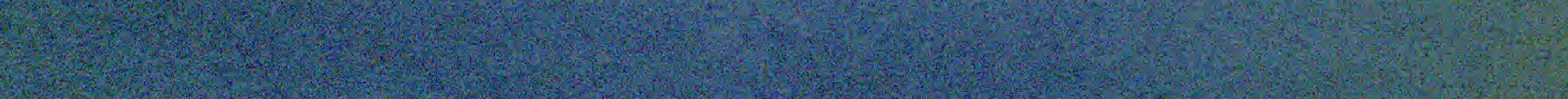

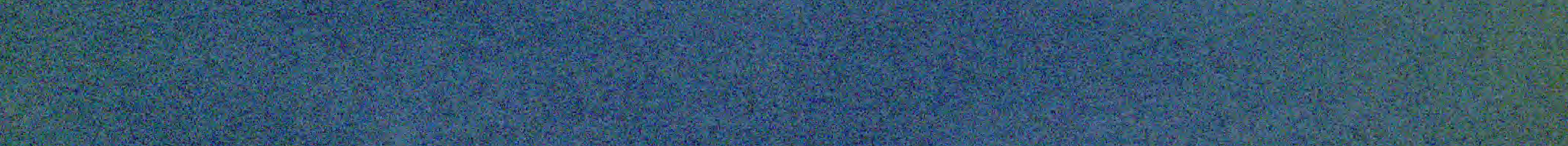
(2) (2) (2)

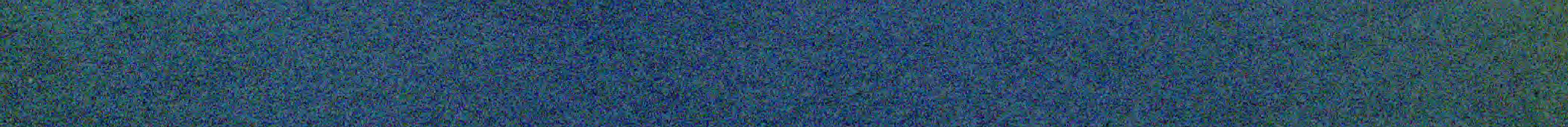

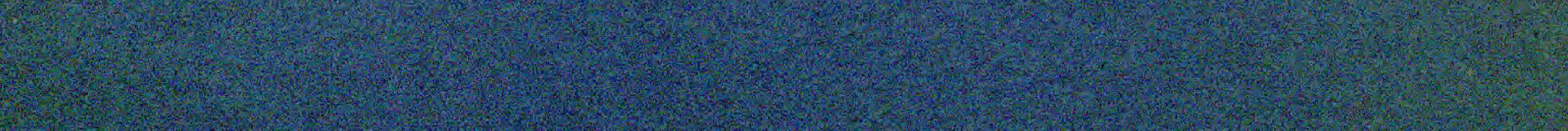

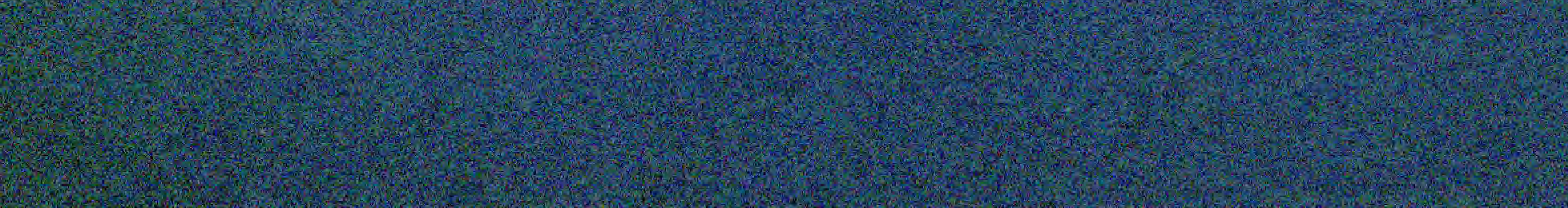




\title{
ROYAL BOTANIC GARDEN, EDINBURGH.
}

\section{LIST OF SEEDS}

\author{
COLLECTED DURING 1896.
}

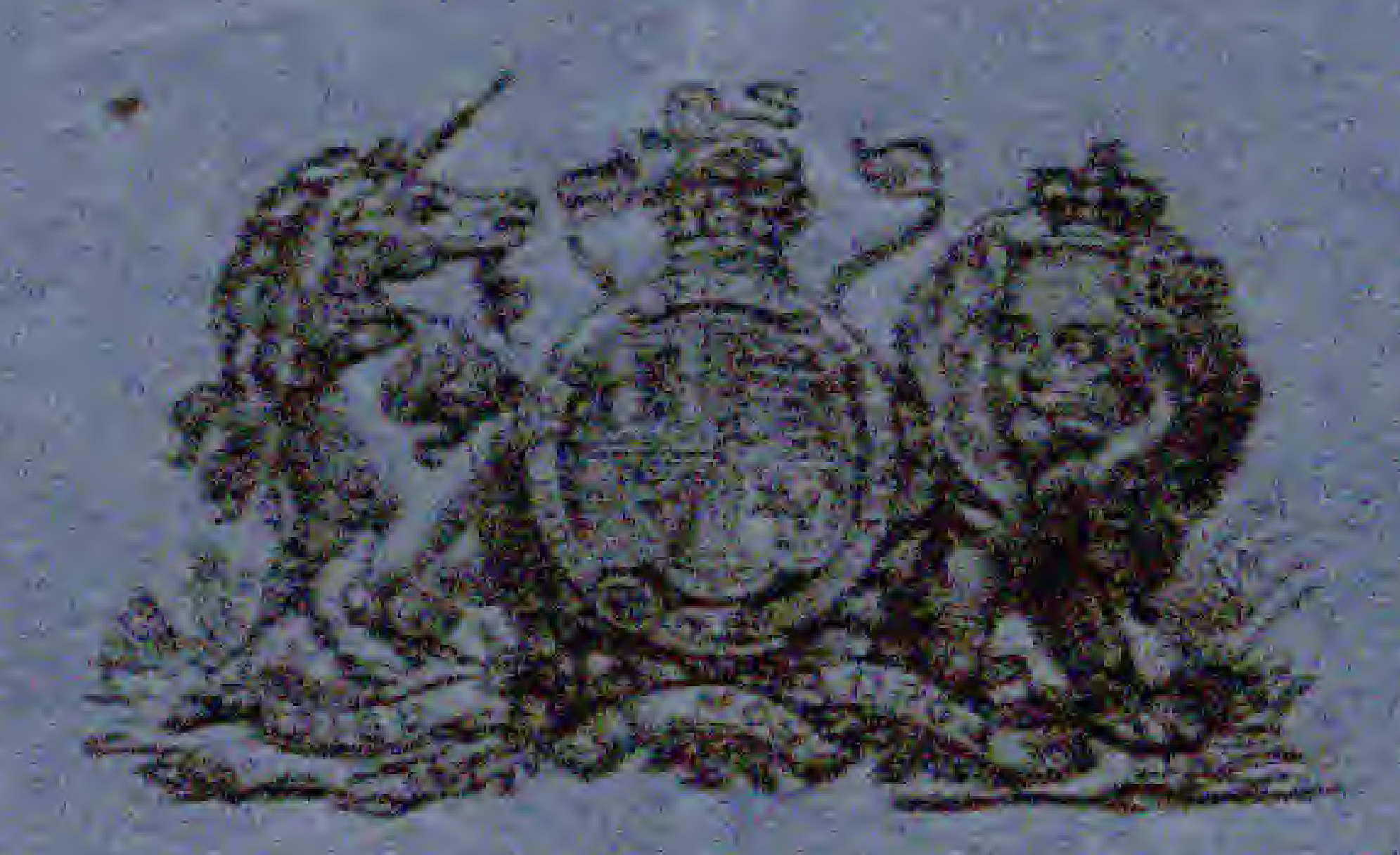
Mo. Bot. Garden 1897.

PRINTED FOR HER MAJESTYS STATIONERY OFFICE, BY NEILL \& COMPAXY, FnRTERTH. 1897. 


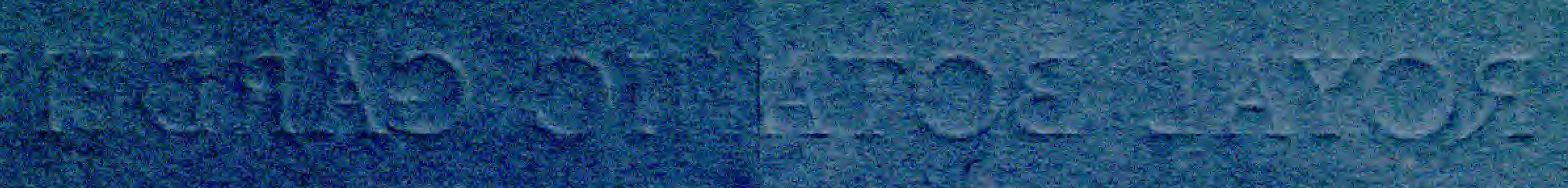

\section{6) 2105}

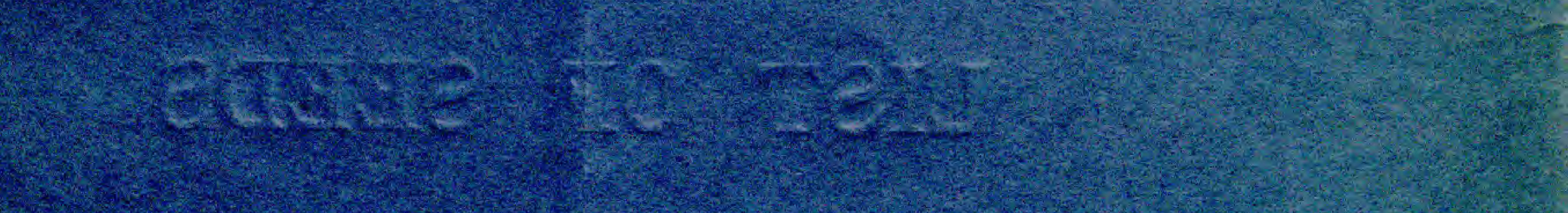

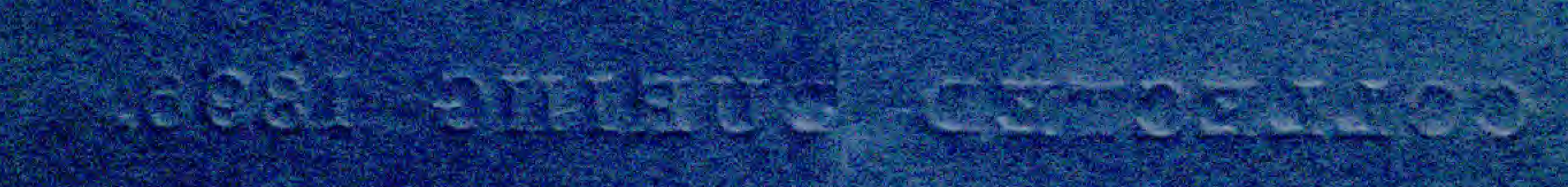

5

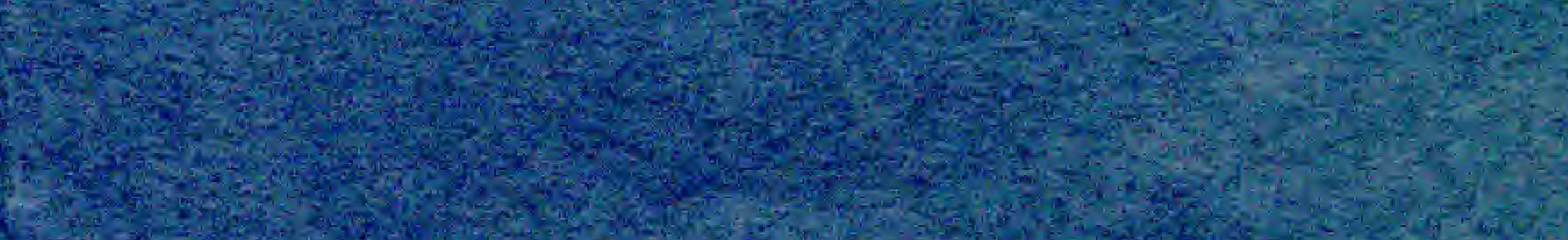

(1)

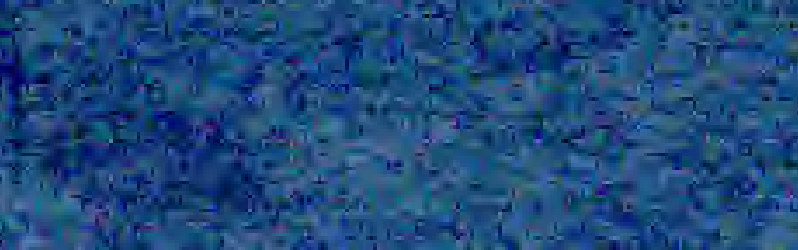

1.2.

S.

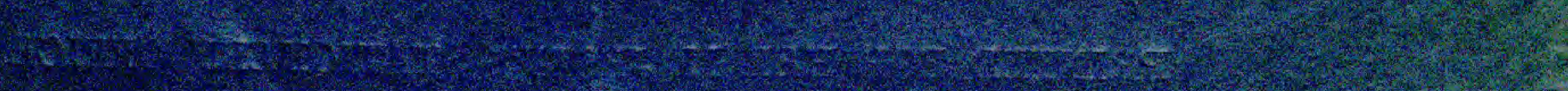

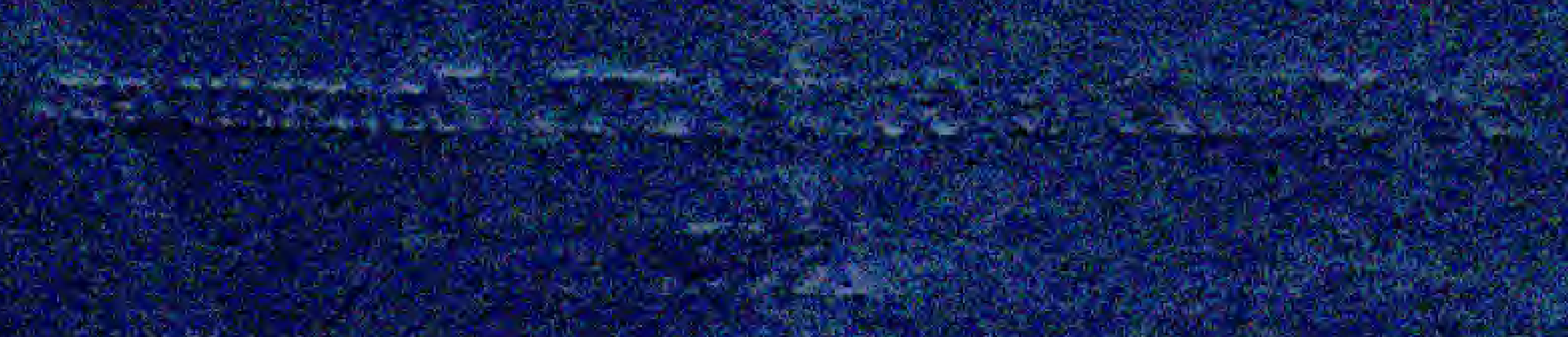




\section{ROYAL BOTANIC GARDEN, EDINBURGH.}

Mo. Bot. Garden,

1897.

\section{LIST OF SEEDS}

\section{COLLECTED DURING 1896.}

Note.-Of those marked with an asterisk*, the quantity collected is very small.

\section{HERBACE0US PLANTS.}

Acæna *argentea, Ruiz \& Pav. inermis, Hook. fil.

Sanguisorbæ, Vahl.

Acantholimon glumaceum, Boiss.

Achillea filipendulina, Lam.

leucophylla, Hort.

Millefolium, Linn.

nobilis, Linn.

Reichardtiana $\times$, G. Beck. rupestris, Huter.

*Thomasiana $\times$, Hall. fil. tomentosa, Linn. umbellata, Sibth. \& Sm. - var. argentea.

Aconitum Anthora, Linn. longigaleatum, Hort.

Lycoctonum, Linn.

- var. album.

moldavicum, Hacq.

Napellus, Linn.

Actæa spicata, Linn.

Adenophora *stylosa, Fisch.

Adonis *vernalis, Linn.

Athionema *armenum, Boiss. cordatum, Boiss.

Agrimonia Eupatoria, Linn.

Ajuga * orientalis, Linn. pyramidalis, Linn.

Alchemilla alpina, Linn.

*vulgaris, Linn.

Alisma Plantago, Linn.

Alkanna tinctoria, Tausch.

Allium arenarium.

*californicum, Hort.

* carinatum, Linn.

flavum, Linn., var, pulchellum.
Allium-contd.

globosum, Red., var. albidum.

* Karataviense, Regel.

*Ledebourianum, Schult. fil. paradoxum, G. Don. pulchellum, Don. scorzoneræfolium, DC. senescens, Linn. subhirsutum, Linn. tenuifolium, Frivald. urceolatum, Regel.

Alyssum *arachnoideum, Hort.

argenteum, Vitm.

Benthamianum, Hort., var. compactum.

gemonense, Linn.

* montanum, Linn.

petræum, Hort.

*podolicum, Bess.

saxatile, Linn.

Wierzbeckii, Heuff.

Ammobium alatum, R. Br.

Anacyclus radiatus, Loisel.

Anagallis arvensis, Linn., var. cærulea (Lam.).

linifolia, Linn.

Androsace commutata, Schlecht. maxima, Linn.

obtusifolia, All.

septentrionalis, Linn.

Anemone alba, Hort.

alpina, Linn.

- var. sulphurea.

* baicalensis, Turcz.

*blanda, Schott \& Kotschy.

Hepatica, Linn. 
Anemone-contd.

Hepatica, var. pyrenaica.

montana, Hoppe.

multifida, Poir.

*narcissiflora, Linn.

${ }^{*}$ nemorosa, Linn.

- var. *bracteata.

- - ${ }^{*}$ purpurea.

_ _ ${ }^{*}$ Robinsoniana.

*polyanthes, Don.

pratensis, Linn.

Pulsatilla, Linn.

*ranunculoides, Linn. rivularis, Buch.-Ham.

sylvestris, Linn.

*thalictroides, Linn.

Angelica *Gingidium, Hook. fil.

* songarica, Regel \& Schmalh.

Antennaria borealis, Hort.

caudata, Hort.

* dioica, Gærtn.

- var. tomentosa.

sericea, Hort.

Anthemis arabica.

carpatica, Willd.

chia, Linn.

Grisebachii, Hort.

tinctoria, Linn.

- var. discoidea (Willd.).

Anthericum Liliago, Linn.

Anthriscus sylvestris, Hoffm.

Anthyllis Vulneraria, Linn.

Antirrhinum Asarina, Linn. majus, Linn.

Aponogeton distachyum, Thunb.

Aquilegia Buergeriana, Sieb. \& Zucc. cærulea, James.

- var. lutea.

* californica, A. Gray, var. alba.

*canadensis, Linn.

chrysantha, Gray.

*formosa, Fisch.

* glandulosa, Fisch.

* - var. jucunda, Fisch. \& Lallem.

* nevadensis, Boiss. \& Reut.

*olympica, Boiss., var. Whitmanniana, Stev.

* pyrenaica, DC.

* viridiflora, Pall.

*vulgaris, Linn.

- var. alba.

Arabis albida, Stev. alpina, Linn
Arabis-contc.

* cærulea, Hænke.

*collina, Ten., var. purpurascens.

hirsuta, Scop.

lucida, Linn. fil.

- var. variegata.

Turrita, Linn.

Aralia *achemirica, Decne.

Arctium majus, Bernh.

Arenaria Cherleria, Hook, fil.

*ciliata, Linn., var. norvegica, Gunn.

*graminifolia, Schrad.

montana, Linn.

* pinifolia, Bieb.

* saxatilis, Linn.

*stricta, Michx.

*verna, Linn.

Armeria allioides, Boiss.

alpina, Willd.

boetica, Boiss., var. hirta.

elongata, Hoffm.

fasciculata, Willd.

latifolia, Willd., var. bracteata rubra.

longearistata, Boiss. \& Reut.

maritima, Willd.

- var. alba.

pubescens, Link.

Arnica Chamissonis, Less.

- var.

montana, Linu.

Arrhenatherum avenaceum, Beauv.

Artemisia campestris, Linn.

capillaris, Thunb.

desertorum, Spreng.

græca, Hort.

rupestris, Linn.

sacrorum, Ledeb.

Santoricum, Linn.

scoparia, Waldst. \& Kit.

Arum *palæstinum, Boiss.

Asparagus scaber, Brign.

Asperula cynanchica, Linn.

Aster acris, Linn.

alpinus, Linn.

- var. albus.

- - speciosus.

corymbosus, Ait.

Curtisii, Torr. \& Gray.

dumosus, Linn.

elegans, Torr. \& Gray.

ericoides, Linn. 
Aster-contd.

grandiflorus; Linn.

Lindleyanus, Torr. \& Gray.

Linosyris, Bernh.

longifolius, Lam.

macrophyllus, Linn.

mutabilis, Linn.

paniculatus, Lam.

patens, Ait.

Pattersoni, A. Gray.

patulus, Lam.

polyphyllus, Willd.

ramulosus, Hort.

sagittifolius, Wedem.

salsuginosus, Hort.

Shortii, Lindl.

sibiricus, Linn.

sikkimensis, Hook. fil. \& Thoms.

spectabilis, Ait.

stellaris, Hort.

tartaricus, Linn. fil.

tenellus, Thunb.

trinervius, Roxb., var. congestus.

turbinellus, Lindl.

Astragalus * baicalensis, Bunge.

bicolor, Lam.

chlorostachys, Lindl.

Glycyphyllos, Linn.

* ponticus, Pall.

vicioides, R. Grah.

AstrantiaBiebersteinii, Fisch. \& Mey. carniolica, Wulf.

major, Linn.

Astydamia canariensis, DC.

Atriplex nitens, Schkuhr.

Atropa Belladonna, Linn.

Aubrietia * croatica, Schott. deltoidea, DC.

- var. græca (Griseb.).

_ ${ }^{*}$ Hendersoni.

- Richardi.

Avena sempervirens, Vill.

Bæria coronaria, A. Gray.

Baptisia australis, R. Br.

Barbarea bracteosa, Guss. orthoceras, Ledeb.

vulgaris, $\mathrm{R}$. Br.

Beckmannia erue formis, Host.

Bellis perennis, Linn.

- ${ }^{*}$ crimson var.

sylvestris, Cyrill.
Bellium minutun, Linn.

Biscutella *auriculata, Linn. cichoriifolia, Linn.

*1ævigata, Linn.

Bocconia cordata, Willd.

- var. Thunbergii.

Borago officinalis, Linn.

Boykinia aconitifolia, Nutt.

Brassica campestris, Linn.

Schimperi, Boiss.

Bryonia dioica, Jacq.

Bulbine semibarbata, Haw.

Bulbinella Hookeri, Benth. \& Hk. fil.

Bulbocodium * vernum, Linn.

Bupthalmum speciosum, Schreb.

Bupleurum *aureum, Fisch.

Butomus umbellatus, Linn.

Calamintha Clinopodium, Benth.

Calendula * eriocarpa, DC.

maritima, Guss.

microphylla, Lange.

officinalis, Linn.

stellata, Car.

suffruticosa, Vahl.

Callirhoë digitata, Nutt.

Caltha palustris, Linn. radicans, T. F. Forst. sylvestris, Hort.

Camassia Brownii, Hort. esculenta, Lindl.

- var. * tardiflora.

Campanula *abietina, Griseb. \&

Schenk.

alliariæfolia, Willd.

*americana, Linn.

* carnica, Schiede.

carpatica, Jacq., var, turbinata.

- var. turbinata alba.

dichotoma, Linn.

*drabæfolia, Sibth. \& Sm.

*Elatines, Linn.

glomerata, Linn.

latifolia, Linn.

- var. *macrantha.

*linifolia, Scop.

persicifolia, Linn.

- - var. alba grandiflora.

* pulchella, var. polypetala.

punctata, Lam.

Rapunculus, Linn.

rotundifolia, Linn. 
Campanula-contd.

rotundifolia, var. Baumgartenii

(Becker).

* specularioides, Coss.

thyrsoides, Linn.

Capsella Bursa-Pastoris, Medic.

Carbenia benedicta, Adans.

Cardamine *bulbifera, R. Br.

* carnosa, Waldst. \& Kit.

Carduus Carolorum $\times$, Howie \& Jenner.

Carex atrata, Linn.

binervis, $\mathrm{Sm}$.

*distans, Linn.

* frigida, All.

leporina, Linn.

Mairii, Coss. \& Germ.

microglochin, Wahlenb.

riparia, Curt.

- var. *variegata.

saxatilis, Linn.

stricta, Good.

sylvatica, Huds.

*vaginata, Tausch.

vulpina, Linn.

Carlina acaulis, Linn.

Carthamus tinctorius, Linn.

Carum Bulbocastanum, Koch.

Catananche eandidissima, Hort.

Centaurea *americana, Nutt. calcitrapoides, Linn. centauroides, Linn. cinerea, Lam.

${ }^{*}$ Crocodylium, Linn.

${ }^{*}$ Crupina, Hort.

Cyanus, Linn.

- var. alba.

dealbata, Willd.

*depressa, Bieb.

- var. ${ }^{*}$ rosea.

* elata, Poir.

heterophylla, Hort.

*laxiseta, Hort.

macrocephala, Puschk.

Moschata, Linn., var, alba.

- var. atropurpurea.

- - cærulea.

nigra, Linn.

pectinata, Linn.

* pseudo-depressa, Hort.

Rhaponticum, Linn.

rutifolia, Sibth. \& Sm.

salmantica, Linn.
Centaurea-contd.

Scabiosa, Linn.

*Seridis, Linn.

sphærocephala, Linn.

Cephalaria corniculata, Roem. \& Schult.

tatarica, Schrad.

- var. minor.

Cerastium *alpinum, Linn.

dichotomum, Linn.

* grandiflorum, Waldst. \& Kit.

mæsiacum, Hort.

*ovatum, Hoppe.

perfoliatum, Linn.

* tomentosum, Linn.

Cerinthe *aspera, Roth.

*auriculata, Ten.

bicolor, Hort.

major, Linn.

- var. flore luteo.

retorta, Sibth. \& Sn.

Chærophyllum aureum, Linn.

hirsutum, Linn.

Charieis heterophylla, Cass.

- var, alba.

- - rosea.

Cheiranthus Cheiri, Linn.

- var. Harbinger.

Chelidonium majus, Linn.

- var, * grandiflorum (DC.).

- - laciniatum (Mill.).

Chelone *obliqua, Linn.

Chenopodium Bonus-Henricus, Linn.

Quinoa, Willd.

Chionodoxa Luciliæ, Boiss.

- var. Alleni.

- - gigantea.

- - sardensis.

Chrysanthemum atratum, Linn.

Balsamita, Linn.

coronopifolium, Masf.

corymbosum, Willd.

filiferum, Hort. Smith, Newry.

grandiflorum, Willd.

macrophyllum, Waldst. \& Kit.

paludosum, Poir.

præaltum, Vent.

segetum, Linn.

Tchihatchewii, Hort, Kew.

Chrysopogon Gryllus, Trin.

* Cimicifuga foetida, Linn.

Clarkia elegans, Dougl. 
Clarkia-contd.

pulchella, Pursh.

- var. *alba.

- - * grandiflora alba.

- - integripetala.

- - marginata.

- - nana.

- - - alba.

- nana.

- - alba.

Claytonia * sibirica, Linn.

* virginica, Linn.

Clematis diversifolia, DC.

lathroides, Hort.

recta, Linn.

Clypeola *Jonthlaspi, Linn.

Cnicus atropurpureus, Hort.

Biebersteinii, Hort.

centauroides, Linn.

elatus, Hort.

eriophorus, Roth.

* græcus, Hort.

*heterophyllus, Roth.

* Kammonianus, Hort.

* pannonicus, Host.

* pratensis, Willd.

*rigidus, Hort.

spinosissimus, Linn.

syriacus, Roth.

tartaricus, Willd.

Cochlearia officinalis, Linn. saxatilis, Linn.

Codonopsis Clematidea, Schrank. *ovata, Benth.

Collinsia bicolor, Benth., var. multicolor.

sparsiflora, Fisch. \& Mey.

*tinctoria, Hartw.

Commelina * colestis, Willd. - var. *alba.

Coptis asperiifolia, Salisb.

Coreopsis lanceolata, Linn.

Cortusa Matthiola, Linn., var. grandiflora.

Corydalis *angustifolia, DC.

* bulbosa, DC.

*lutea, DC.

nobilis, Pers.

Cotula aurea, Loefl.

Cotyledon * umbilicus, Linn.

Crambe cordifolia, Stev. hispanica, Linn.

Crepis aurea, Reichb.
Crepis-contd.

aurea, Reicbb., var. crocea.

Dioscoridis, Linn.

* rubra, Linn.

- var. alba.

*sibirica, Linn.

succisæfolia, Tausch.

Cryptotænia canadensis, DC.

Cuphea lanceolata, Ait.

- var. Zimapani.

Cynanchum acutum, Linn.

Cynoglossum microglochin, B enth. officinale, Linn.

Datura inermis, Jacq. Stramonium, Linn.

Delphinium altissimum, Wall.

cashmirianum, Royle.

elatum, Linn.

formosum, Boiss. \& Huet.

* grandiflorum, Linn.

hybridum, Steph.

Maackianum, Regel.

Nahamah, Hort.

nudicaule, Torr. \& Gray.

palmatifidum, DC.

velutinum, Bertol.

Dianthus *alpinus, Lim.

*angulatus, Royle.

arenarius, Linn.

atrorubens, All.

* cæsio-caricinus $\times$. capitatus, DC.

* Carthusianorum, Linn.

chinensis, Linn.

*Freynii, Hort. Leichtlin.

* glutinosus, Boiss. \& Heldr.

* grandiflorus, Poir.

hungaricus, Hort.

monspessulanus, Linn.

* nitidus, Waldst. \& Kit.

*pelviformis, Heuff.

* plumarius, Linn., var. serotinus

(Waldst. \& Kit.).

- var. viridis.

* squarrosus, Bieb.

* superbus, Linn.

- var. ${ }^{*}$ Hooltzeri (Winkler).

Diapensia *lapponica, Linn.

Dictamnus *albus, Linn.

- var. tauricus.

Digitalis_ambigua, Murr.

purpurea, Linn. 
Digitalis-contd.

${ }^{*}$ purpurea $\times$ ochroleuca.

Dimorphotheca *Ecklonis, pluvialis, Mœench.

Diphylleia *ymosa, Michx.

Diplotaxis viminea, DC.

Dodecatheon *Meadia, Linn. - var. *lancifolium.

Doronicum * caucasicum, Bieb. *Clusii, Tausch. draytonense, Hort. * grandiflorum, Lam. Harpur-Crewe, Hort. hirsutum, Lam. macrophyllum, Fisch. Pardalianches, Linn.

Draba Aizoon, Wahlenb. aurea, Vahl.

* cuspidata, Bieb.

* fladnizensis, Wulf. frigida, Sauter.

* glacialis, Hort.

*hirta, Linn.

incana, Linn.

* Mawii, Hook. fil.

nemorosa, Linn.

*nivalis, Hort.

pyrenaica, Linn. repens, Bieb.

* rupestris, R. Br. scandinavica, Lindblom.

* siliquosa, Bieb.

*stellata, Jacq.

*Zahlbruckneri, Host.

Dracocephalum * speciosum, Benth.

Drosera *anglica, Huds. *rotundifolia, Linn.

Dryas octopetala, Linn.

Echinacea purpurea, Monch.

Echinodorus ranunculoides, Engelm.

Echinops azureus, Hort. bannaticus, Rochel. dahuricus, Fisch. globifer, Janka. græcus, Mill. strictus, Hort.

Echium *vulgare, Linn.

Epilobium alsinifolium, Vill. angustifolium, Linn.

- var. album. coloratum, Muhl,
Epilobium-contc.

Dodonæi, Vill.

latifolium, Linn.

nummularifolium, R. Cunn., var.

longipes.

olympicum, Hort.

rosmarinifolium, Hænke, var. sericeum.

*tetragonum, Linn.

Eranthis *hyemalis, Salisb.

Erigeron acris, Linn.

alpinus, Linn.

aurantiacus, Regel.

bellidifolius, Muhl.

caucasicus, Stev.

glabellus, Nutt.

- var. mollis.

glabratus, Hoppe \& Hornsch.

glaucus, Ker-Gawl.

macranthus, Nutt.

multiradiatus, Benth. \& Hook.

fil.

Roylei, DC.

speciosus, DC.

- var. superbus, Linn.

uniflorus, Linn.

Erinus *alpinus, Linn.

Eriophila *vulgaris, DC.

Eriophorum alpinum, Linn.

Erodium * cicutarium, L'Hérit.

gruinum, Soland.

hymenodes, L'Hérit.

maritimum, Sm.

Salzmanni, Del.

Eryngium aquifolium, Cav.

campestre, Jinn.

cæruleum, Linn,

dichotomum, Desf,

giganteum, Bieb.

Erysimum *asperum, DC,, var. purpureum.

*hieracifolium, Linn.

latifolium, Hort.

Perofskianum, Fisch. \& Mey.

Erythronium * grandiflorum, Pursh.

Eupatorium ageratifolium, DC.

ageratoides, Linn.

altissimum, Linn.

glechonophyllum, Less.

purpureum, Linn.

sessilifolinm, Linn.

Euphorbia Gerardiana, Jacq.

Lathyris, Linn. 
Ferula *Ferulago, Linn.

Fumaria *macrocarpa, Parl.

Gaillardia *aristata, Pursh.

- var. grandiflora, Hort.

- - maxima, Hort.

- splendidissima.

pulchella, Fouger.

Galanthus *nivalis, Linn.

Galax *aphylla, Linn.

Galium Mollugo, Linn. rubioides, Linn.

* urceolatum, Hort.

Gamolepis annua, Less.

Gentiana *acaulis, Linn.

asclepiadea, Linn.

*Burseri, Lapeyr.

* campestris, Linn.

- var. *alba.

cruciata, Linn.

decumbens, Linn.

*excisa, Koch.

*Moorcroftiana, Wall.

*nivalis, Linn.

*phlogifolia, Schott \& Kotschy. punctata, Linn.

septemfida, Pall.

- var. * cordifolia, Hook. fil.

tibetica, King.

* verna, Linn.

*Walujewi, Regel \& Schmidt.

Geranium angulatum, Curt.

* eriostemon, Fisch.

* Grevilleanum, Wall.

* macrorhizon, Linn.

phæum, Linn.

* pratense, Linn. sanguineum, Linn. - var. *lancastriense (Mill.).

* sibiricum, Linn.

*subcaulescens, L'Hérit.

*Wallichianum, G. Don.

Geum album, J. F. Gmel. coccineum, Sibth. \& Sm.

*elatum, Wall.

montanum, Linn. nutans, Host.

parviflorum, Commers.

potentilloides, Hort.

rivale, Linn.

strictum, Soland.
Geum-contd.

*triflorum, Pursh.

urbanum, Linn.

Gilia *alba, Hort. muticus, Hort.

Glaucium persicum, DC. squamigerum, Kar. \& Kir.

Globularia *trichosantha, Fisch. \& Mey.

Gnaphalium * supinum, Linn.

Grindelia pulchella, Dunal.

Gunnera chilensis, Lam.

Gypsophila *elegans, Bieb. perfoliata, Linn.

Hablitzia tamnoides, Bieb.

Hacquetia *Epipactis, DC.

Hedysarum coronarium, Linn,, var. album.

obscurum, Linn.

Helenium autumnale, Linn.

- var. pumilum (Willd.).

Bigelovii, A. Gray.

grandicephalum, Hort., var. striatum.

Heliauthella uniflora, Torr. \& Gray.

Helianthemum * guttatum, Mill.

Helianthus decapetalus, Linn. mollis, Lam.

*nanus, Hort. occidentalis, Riddell. rigidus, Desf.

*strumosus, Linn.

Helichrysum arenarium, Mœnch. compositum, Hort. rutidilepis, DC.

Heliopsis lævis, Pers,, var. scaber. - var. superba.

Helleborus *antiquorum, A. Br.

Helonias *bullata, Linn.

- var. *pallida.

Hemerocallis * minor, Mill.

Heracleum Halleri, Hort. lanatum, Michx.

Hesperis *fragrans, Fisch.

Heuchera * hispida, Pursh. cylindrica, Dougl.

* sanguinea, Engelm.

* spicata, Hort.

*villosa, Michx. 
Hieracium alpinum, Linn. amplexicaule, Linn. andryaloides, Vill.

*argenteum, Fries. Auricula, Linn. balkanum, Uechtr. Bornmülleri, Hort. buglossoides, Arv. bupleuroides, C. C. Gmel.

* crsium, Fries. cambricum, Hort. cerinthoides, Linn. compositum, Lapeyr. cydoniæfolium, Vill.

* dianthoides, Hort.

*eximium, Backh.

foliosum, Waldst. \& Kit.

Halleri, Hort.

humile, Jacq.

intybaceum, Jacq.

iricum, Fries.

laciniatum, Hort. lasiophyllum, Koch.

lingulatum, Backh. Marshalli, Linton.

*murorum, Linn.

Neo-cerinthe, Fries. nigrescens, Willd.

*nitidum, Hort. Pilosella, Linn. Pilosella $\times$ aurantiaca, prenanthoides, Vill. prostratum, Poir. pulmonarioides, Vill. rupestre, All. sabaudum, Linn.

*saxatile, Vill. saxetanum, Vill. stoloniferum, Waldst. \& Kit. strictum, Fries, suecicum, Fries. sylvaticum, Hort. tenellum, Backh. tridentatum, Fries. undulatum, Ait. villosum, Jacq. vulgatum, Fries.

Hierochloë borealis, Roem, \& Schult. Homogyne alpina, Cass. sylvestris, Cass.

Horminum * pyrenaicum, Linn. Hutchinsia *alpina, R. Br. * pauciflora, Nym.
Hyacinthus amethystinus, Linn. romanus, Linn.

Hyoscyamus albus, Linn, niger, Linn.

Hypericum * coris, Linn. * elegans, Steph. floribundum, Dryand. *Gebleri, C. A. Mey. hirsutum, Linn. linarifolium, Vahl. montanum, Linn. origanifolium, Willd. * patulum, Thunb. pentaphyllum, Hort. perforatum, Linn. * pulchrum, Linn. quadrangulum, Linn. tetrapterum, Fries. umbellatum, Kern. undulatum, Schousb. Hypochæris maculata, Linn. radicata, Linn.

Hyssopus officinalis, Linn.

Iberis Lagascana, DC.

*panduræformis, Pour.

*Pruiti, Tineo. sempervirens, Linn. - var. *superba. umbellata, Linn, - var. nana atropurpurea.

- - carnea.

- - lilacina.

Impatiens *leptoceras, DC. Roylei, Walp. * scabrida, DC.

Inula Conyza, DC. ensifolia, Linn. glandulosa, Puschk. Helenium, Linn. Hookeri, C. B. Clarke. Oculus-Christi, Linn. speciosa, Hort.

Iris biflora, Linn. flavissima, Pall., var. Bloudowii.

* prismatica, Pursh. Pseudacorus, Linn. - var. variegata. setosa, Pall. sibirica, Linn. - var. alba. - - sanguinea (Donn.). 
Isatis alpina, All.

bastigas, Hort.

hebecarpa, Regel \& Schmalh.

tinctoria, Linn.

Juncus * biglumis, Linn.

conglomeratus, Linn.

effusus, Linn.

glaucus, Sibth.

lamprocarpus, Ehrh.

trifidus, Linn.

*triglumis, Linn.

Jurinea glycacantha, DC.

Lactuca alpina, Benth \& Hook. fil. floridana, Gærtn. virosa, Linn.

Lallemantia *iberica, Fisch. \& Mey.

Lamium album, Linn.

* Orvala, Linn.

purpureum, Linn.

Lapsana grandiflora, Bieb.

Laserpitium Nestleri, Soyer-Willem.

Lathyrus articulatus, Linn. cyaneus, C. Koch.

*Clymenum, Linn.

dumetorum.

*hirsutus, Linn.

Lowii, Hort.

luteus, Munby.

macrorrhizus, Wimm.

magellanicus, Lam.

- var. *albus.

- ${ }^{*}$ roseus.

*mauritanicus, Hort.

montanus, Bernh.

niger, Bernh.

Ochrus, DC.

*sphæricus, Retz.

tingitanus, Linn.

vernus, Linn.

- var. flaccidus.

Lavatera thuringiaca, Linn. trimestris, Linn., var. rubra.

Leontodon *alpinus, Hort. hispidus, Linn.

Leontopodium alpinum, Cass.

Leonurus capitatus, Fresen.

Lepidium Smithii, Hook.

Leptosyne Stillmanni, A. Gray.
Liatris graminifolia, Pursh. spicata, Willd.

Libertia *grandiflora, Sweet.

Ligusticum involucratum, Regel.

peleponnense, Falk. scoticum, Linn.

Lilium *pardalinum, Kellogg.

Limnanthes Douglasii, R. Br.

Linaria anticaria, Boiss.

Broussonetii, Chav.

origanifolia, DC.

Pančici, Hort.

repens, Mill.

sapphirina, Hoffmgg,

Tournefortii, Steud.

vulgaris, Mill.

Linum *alpinum, Linn. angustifolium, Huds.

usitatissimum, Linn.

- var. *album.

Lophosciadium meifolium, DC.

Lotus hispidus, Desf.

Lunaria annua, Linn.

Lupinus *affinis, Agardh.

albus, Linn.

cæruleus, Hort.

Cosentinii, Guss.

Cruckshanksii, Hook.

- var, *hybridus.

densiflorus, Benth.

Dunnettii, Hort.

elegans, H, B. \& K.

*Hartwegii, Lindl.

- var. *albus.

- - ${ }^{*}$ coelestinus.

- ${ }^{*}$ roseus.

*hirsutus, Linn, var, albus.

- var. * cæruleus.

- - * ${ }^{*}$ carneus.

insignis $x$, Lehm.

- var. nigrescens.

- ${ }^{*}$ roseus.

- - superbus.

mutabilis, Sw.

- var. * roseus.

- - *versicolor.

nootkatensis, Don.

pantelerianus, Hort.

* perennis, Linn.

polyphyllus, Lindl.

pubescens, Benth.

- var. elegans.

pulcherrimus, Hort. 
Lupinus-contd.

reticulatus, Desv.

*venustus, Hort.

- var. *tricolor.

Luzula * campestris, DC. nivea, DC.

sylvatica, DC.

Lychnis Cœli-rosea, Backh.

coronaria, Desv.

divaricata, Reichb.

Flos-jovis, Desv.

*nemoralis, Heuff.

Sartori, Hort.

Viscaria, Linn.

- var. alba.

- purpurea.

Lysimachia *affinis, Bunge.

barystachys, Bunge.

lobelioides, Wall.

vulgaris, Linn.

Lythrum alatum, Pursh,

myrtifolium, Lodd.

Salicaria, Linn.

- var. cinereum, Griseb.

- - grandiflorum.

virgatum, Linn.

Macrotomia *echioides, Boiss.

Madia * sativa, Molina.

Malcolmia maritima, R. Br.

- var. carminea splendens.

- compacta.

- - alba.

Malope trifida, Cav.

Malva Alcea, Linn.

Duriæi, Hort. Kew.

moschata, Linn., var. alba.

sylvestris, Linn.

Margyricarpus * setosus, Ruiz \& Pav.

Matthiola bicornis, DC.

*incana, R. Br. sinuata, R. Br.

Meconopsis *aculeata, Royle.

cambrica, Vig.

nepalensis, DC.

- * var.

simplicifolia, G. Don.

Wallichii, Hook.

Medicago *Decandollei, Tineo.

Echinus, DC.

hispida, Gærtn.
Medicago-contd.

*intertexta, Mill.

* lupulina, Linn.

Medeola virginiana, Linn.

Melilotus gracilis, DC.

*Halicus, Hort.

Meum athamanticum, Jacq.

Micromeria *Piperella, Benth.

Mimulus hybridus, Hort. tigrinus, Hort.

Mitella pedemontana, Hort. * pentandra, Hook.

Monarda *atropurpurea, "Hort. *didyma, Linn.

Moneses grandiflora, S. F. Gray.

Morina *betonicoides, Benth. *longifolia, Wall.

Muretia tanaicensis, Boiss.

Muscari *Argæi, Hort. *armeniacum, Baker.

* Maweanum, Baker.

*minus, Hort.

*pallens, Fisch.

* racemosum, Mill.

Myagrum perfoliatum, Linn.

Myosotis alpestris, F. W. Schmidt.

- var. *alba.

sylvatica, Hoffm.

Myrrhis odorata, Scop.

Napæa dioica, Linn.

Narcissus *juncifolius, Lag., var. rupicola (Duf.).

Nardostachys Jatamansi, DC.

Narthecium * ossifragum, Huds.

Nemesia versicolor, E. Mey.

Nepeta * Mussini, Spreng.

Nicotiana rustica, Linn.

Nigella damascena, Linn.

orientalis, Linn.

sativa, Linn.

Nonnea rosea, Link.

Nothoscordum fragrans, Kunth.

CEnanthe crocata, Linn.

globulosa, Linn.

peucedanifolia, Pollieh.

Enothera amcena, Lehm.

- var. alba.

biennis, Linn. 
Enothera-contd.

cæspitosa, Nutt.

campylocalyx, C. Koch \& Bouché.

fruticosa, Linn.

Gayana, Steud.

* glauca, Michx.

Pilgrimi, Hort.

quadrivulnera, Dougl.

*tenella, Cav.

viminea, Dougl.

Whitneyi, A. Gray.

- var. flammea.

- - *hybrida splendens.

Onobrychis viciæfolia, Scop.

Ononis spinosa, Linn.

Onopordon Acanthium, Linn.

illyricum, Linn.

Ornithogalum *Elwesii.

Orobanche * rubra.

Oxalis * corniculata, Linn., var. tropæoloides, Hort.

Oxytropis * campestris, DC.

*lapponica, Gaud.

strobilacea, Bunge.

*uralensis, DC.

Pæonia decora, Anders.

Papaver *aculeatum, Thunb.

*alpinum, Linn.

apulum, Tenore.

caucasicum, Bieb.

glaucum, Boiss. \& Haussk.

Heldreichi, Boiss.

* nudicaule, Linn.

* orientale, Linn.

- var. semi-plenum.

persicum, Lindl.

pilosum, Sibth. \& Sm.

pinnatifidum, Moris.

* rupifragum, Boiss. \& Reut.

- var. atlanticum (Ball).

Parietaria officinalis, Linn.

Parnassia *nubicola, Wall.

* ovata, Ledeb.

palustris, Linn.

Pedicularis sylvatica.

Pentstemon acuminatus, Dougl.

* confertus, Dougl.

*deustus, Dougl.

diffusus, Dougl.
Pentstemon-contd:

lævigatus, Soland, var. Digitalis

(A. Gray).

pubescens, Soland.

Richardsonii, Dougl.

Petalostemon violaceus, Michx.

Phlomis setigera, Falc. viscosa, Poir.

Phlox *hybrida $\times$, Rafin.

Phygelius capensis, E. Mey.

Phyteuma * campanuloides, Bieb.

* comosum, Linn.

*hemisphæricum, Linn.

Michelii, All.

* orbiculare, Linn.

*Scheuchzeri, All.

Sieberi, Spreng.

Picris echioides, Linn.

Picrorhiza * Kurroa, Royle.

Pinguicula *lusitanica, Linn.

Plantago kamtschatica, Hort.

lanceolata, Linn.

major, Linn., var. rubra.

maritima, Linn.

montana, Huds.

Psyllium, Linn.

saxatilis, Bieb.

subulata, Linn.

Platycodon grandiflorum, A. DC. var. album.

Platystemon * californicus, Benth.

Pleurospermum austriacum, Hoffm.

Plumbago micrantha, Ledeb.

Podophyllum *Emodi, Wall.

Polemonium *boreale, Adams. cæruleum, Linn.

- var. album.

*flavum, Greene. gracile, Willd.

humile, Willd.

Polygonum alpinum, Linn.

*Bistorta, Linn.

- var. major.

* viviparum, Linn.

Portulaca oleracea, Linn.

Potentilla alchemilloides, Lapeyr. alpestris, Hall, fil.

* argentea, Linn.

- var. Calabra (Tenore).

argyrophylla, Wall.

*aurea, Linn.

* chrysantha, Trevir. collina, Wibel. 
Potentilla-contd.

Dichtliana, Kern.

* dissecta, Pursh.

Dombeyi, Nestl.

Fenzlii, Lehm.

fragarioides, Linn.

* gelida, C. A. Mey.

* grandiflora, Linn.

Herbichii, Blocki.

hirta, Linn.

Hotzlii, Hort.

Honorati, Hort.

Macnabiana $\times$, Lem.

nepalensis, Hook.

${ }^{*}$ nevadensis, Boiss.

*nivea, Linn.

ontopoda, Dougl.

opaca, Linn.

palustris, Scop.

recta, Linn.

rupestris, Linn.

Schrenkiana, Regel.

*sericea, Linn.

Sibbaldi, Hall, fil.

*Tormentilla, Neek.

* umbrosa, Stev.

villosa, Pall.

Poterium dodecandrum, Benth. \& Hook, fil,

Pratia angulata, Hook, fil.

Prenanthes purpurea, Linn.

Primula *Auricula, Linn.

capitata, Hook.

*Clusiana, Tausch.

*cortusoides, Linn.

* denticulata, Sm.

elatior, Hill.

- var. ${ }^{*}$ carpatica (Fuss.).

*farinosa, Linn.

- var. * scotica (Hook).

* frondosa, Janka.

*intermedia $\times$, Portenschl.

*involucrata, Wall.

- var. *Munroi.

japonica, A. Gray.

- var. lilacina.

longiflora, All.

* officinalis, Jacq.

- var. Columnæ (Tenore).

- - Tommasinii (Gren. \& Godr.).

- - *uralensis.

pannonica, Hort.
Primula-contd.

*pedemontana, Thom.

Poissoni, Franch.

* Reidii, Duthie.

rosea, Royle.

- var. * grandiflora.

*sibirica, Jacq.

* sikkimensis, Hook.

* Stuarti, Wall.

var. purpurea.

*vulgaris, Huds.

- blue variety.

Prunella grandiflora, Jacq.

*vulgaris, Linn.

- var. laciniata.

- - Webbiana, Hort.

Pulicaria vulgaris, Gærtn.

Pulmonaria *arvernense, Hort.

Puschkinia scilloides, Adams.

- var. compacta.

Pyrola *media, Sw.

Ramondia *pyrenaica, Rich.

Ranunculus aconitifolius, Linn.

acris, Linn.

- var. sylvestris.

auricomus, Linn.

- var, sylvestris.

bulbosus, Linn.

cornutus, DC.

* glacialis, Linn.

- var. holosericeus.

* gracilis, Clarke.

*hederaceus, Linn.

*millefoliatus, Vahl.

*montanus, Willd.

* parnassifolius, Linn.

* pyrenæus, Linn.

repens, Linn.

Reuterianus, Boiss.

*rutæfolius, Linn.

*Thora, Linn.

Reseda alba, Linn.

Jacquinii, Rchb.

lutea, Linn.

Luteola, Linn.

Phyteuma, Linn.

ramosissima, Pourr.

undata, Hort.

Rheum Collinianum, Baill.

hybridum, Murr. 
Rheum-contd.

officinale, Baill.

palmatum, Linn.

- var. tanghuticum.

Rhaponticum, Linn.

Ribes, Linn.

Rodgersia podophylla, A. Gray.

Rodigia Kalmii, Hort.

Romulea * Columnæ, Sebast. \& Mauri.

Rudbeckia fulgida, Ait.

grandiflora, Gmel.

hirta, Linn.

laciniata, Linn.

lanceolata, Bisch.

speciosa, Wender.

triloba, Linn.

Rumex Acetosella, Linn.

alpinus, Linn.

- var. major.

aquaticus, Linn.

intermedius, DC.

nepalensis, Spreng.

sanguineus, Linn.

scutatus, Linn.

staticifolia, Hort.

stenophyllus, Ledeb.

tingitanus, Linn.

Ruta graveolens, Linn. macrophylla, Soland.

Sagina * glabra, Fenzl.

Salvia argentea, Linn. dominica, Linn.

glutinosa, Linn.

*hians, Royle.

*imbricata, Hort.

pratensis, Linn.

*Sibthorpii, Hort.

Verbenaca, Linn.

Sanguinaria * canadensis, Linn.

Sanicula europæa, Linn.

Santolina alpina, Hort.

Saponaria * Boissieri, Hort.

* cæspitosa, DC.

*calabrica, Guss.

*lutea, Linn.

*ocymoides, Linn.

- var. splendidissima.

* officinalis, Linn.

Saxifraga *aizoides, Linn.

*Aizoon, Linn.

- var. *balcana.
Saxifraga-contd.

Aizoon, var. *brevifolia.

_ _ * brevifolia farinosa.

- - ${ }^{*}$ major.

- - * pectinata (Schott).

- ${ }^{*}$ rosularis (Haw.).

*Andrewsii $\times$, Harvey.

*aretioides, Lapeyr., var. primulina.

*aspera, Linn.

*bronchialis, Linn., var. conferta.

*Burseriana, Linn.

- var. *multiflora.

*cæsia, Linn.

${ }^{*}$ crsia $\times$ Aizoon.

* cospitosa, Linn., var. hirta.

* cartilaginea, Willd.

- var. *Kolenatiana, Regel.

* citrina, Hegetschw.

* conifera, Coss. \& Dur.

crassifolia, Linn.

*decipiens, Ehrh.

- var. * gronlandica (Linn.).

- - *palmata (Panč.).

diapensioides, Bell.

- var. *tombeanensis (Boiss.).

* diversifolia, Wall.

*exarata, Vill.

- var. *aromatica.

* geranioides, Linn.

*Geum, Linn.

- var. gracilis (Mackay).

granulata, Linn.

*Hausmanni x, Kern.

*hederæfolia, Hochst.

*Hostii, Tausch.

- var. *altissima (Kern.).

- - elatior (Mert. \& Koch).

*hypnoides, Linn.

intacta, Willd., var. farinosa.

*irrigua, Bieb.

*Launcestoni, Hort.

*lingulata, Bell., var. cochlearis (Reich.).

- var. * cochlearis minor.

*longifolia, Lapeyr., var. hybrida.

- var. *major.

* MacNabiana $\times$.

*muscoides, Wulf., var. atropurpurea.

- var. *Rhei (Schott, Nym. \& Kotschy).

*oppositifolia, Linn. 
Saxifraga-contd. oppositifolia var, *pyrenaica.

- - *rimson.

* paradoxa, Sternb.

* pedatifida, Ehrb.

* pedemontana, All., var, cervicornis (Viv.).

* peltata, Torr. \& Gray.

petræa, Linn.

- var. *Blavii (G. Beck).

* porophylla, Berthol.

* Portæ x, Engelm.

*pseudo-Fosteri $x$.

*retusa, Gouan.

- var. *bryoides.

- - *maritima.

rotundifolia, Linn.

- var. *repanda (Willd.).

- - *Taygetea.

* rupestris, Hort.

* sancta, Griseb.

* Seguieri, Spreng.

* sponhemica, C, C. Gmel, var. affinis (D. Don).

*Stansfieldi $x$.

* transsylvanica, Fuss.

* umbrosa, Linn.

- var. *hirsuta (Linn.).

- - hirsuta dentata.

- - * serratifolia (Mackay).

${ }^{*}$ valdensis, DC.

Scabiosa alpina, Hort.

arvensis, Linn.

banatica, Waldst \& Kit.

brachiata, Sibth. \& Sm.

caucasica, Bieb.

centaurifolia, Hort.

*Columbaria, Linn.

fumarioides, Vis. \& Panč.

graminifolia, Linn.

lucida, Vill.

pubescens, Hort.

stylosa, Hort.

Suecisa, Linn.

- var. alba.

sylvatica, Linn.

triniæfolia, Frivald.

Schizanthus * pinnatus, Ruiz \& Pav.

Schizopetalon *Walkeri, Sims.

Scilla *amœna, Linn.

bifolia, Linn.

- var. *alba.

- ${ }^{*}$ rosea.
Scilla-contd.

bifolia, var. * taurica.

festalis, Salisb.

* patula, DC., var. major.

peruviana, Linn.

sibirica, Andr.

- var. * præcox.

Scirpus lacustris, Linn.

- var. Tabernæmontani (C. C. Gmel.).

Scorpiurus *muricata, Linn.

sulcata, Linn.

*vermiculata, Linn.

Scorzonera hispanica, Linn.

* purpurea, Linn.

* stricta, Hornem.

Scrophularia *alata, Gilib. nodosa, Linn. trifoliata, Linn,

*vernalis, Linn.

Scutellaria *alpina, Linn.

*altissima, Linn.

* japonica, Hort.

Securigera Coronilla, Linn.

Sedum Aizoon, Linn.

*Anacampseros, Linn.

asiaticum, Spreng.

* cordifolium, Baker.

*Ewersii, Ledeb.

laxiflorum, DC.

maximum, Suter.

- var. atropurpureum.

* pallidum, Bieb.

Pittonii, Hort.

* populifolium, Pall.

roseum, Scop.

* rupestre, Linn.

trifidum, Wall.

* villosum, Linn.

Selinum ${ }^{*}$ Candollii, DC.

Sempervivum *arachnoideum, Linn.

*arvernense, Lecoq \& Lamotte.

*Braunii, Funk.

* globiferum, Linn.

*Pittonii, Schott, Nym. \& Kotschy.

* Pomelii, Lamotte.

Reginæ-Amaliæ, Heldr.

*Schottii, Baker, var. acuminatum (Schott).

Senebiera Coronopus, Poir.

Senecio adonidifolius, Loisel.

*alpinus, Scop. 
Senecio-contd.

Doronicum, Linn.

japonicus, Sch. Bip.

Ledebourii, Sch. Bip.

Ligularia, Hook, fil.

paludosus, Linn.

sibiricus, Linn.

Serratula coronata, Linn.

heterophylla, Desf.

nervosa, Hort.

purpurea, Hort.

tinctoria, Linn.

Seseli gummiferum, Sm.

Sidalcea candida, A. Gray. malvæflora, A. Gray.

Murrayana, Hort.

Silene *acaulis, Linn. ægyptiaca, Linn.

alpestris, Jacq.

antirrhina, Linn.

Armeria, Linn.

- var. *alba.

* caucasica, Boiss.

* ciliata, Pourr.

cretica, Linn.

Cucubalus, Wibel.

flavescens, Waldst. \& Kit.

gallica, Linn.

- var. quinquevulnera.

italica, Pers.

maritima, With.

*multicaulis, Guss.

Muscipula, Linn.

nutans, Linn.

* pulchella, Hort.

Pseudo-atocion, Desf.

* quadridentata, Pers.

Reichenbachii, Vis.

reticulata, Desf.

rubella, Linn.

* saxifraga, Linn.

*Schafta, Gmel.

sedoides, Jacq.

squamigera, Boiss.

tatarica, Pers.

Thorei, Desf.

* viridiflora, Linn.

Silphium perfoliatum, Linn,

scaberrimum, Ell.

terebinthaceum, Jacq.

Silybium *eburneum, Coss. \& Dur.

Marianum, Gærtn.

Sisymbrium Alliaria, Scop.
Sisymbrium-contd.

*assoanum, Lose. \& Pard.

Irio, Linn.

Loselii, Linn.

strictissimum, Linn.

tanacetifolium, Linn.

Sisyrinchium angustifolium, Mill.

- var. *mucronatum (Michx.).

- - Nuttallii (Sweet).

bermudiana, Linn.

striatum, Sm.

Solanum villosum, Willd.

Solidago californica, Nutt.

canadensis, Limn.

coriacea, Hort.

elongata, Nutt.

memoralis, Ait.

ohioënsis, Riddell.

rigida, Linn.

Saligna, Hort.

stricta, Mœench.

virgaurea, Linn.

Sonchus palustris, Linn.

Specularia pentagonia, A. DC.

*speculum, A. DC.

Sphæralcea *acerifolia, Nutt.

Spiræa Aruncus, Linn.

Filipendula, Linn.

* palmata, Sternb, var. alba.

- var. elegans.

venusta, Otto \& Dietr.

Stachys Alopecurus, Benth.

Betonica, Benth.

germanica, Benth.

tuberifera, Hort.

Stellaria * graminea, Linn, var, aurea.

Swertia Chirata, Buch-Ham.

Symphyandra Hofmanni, Pant.

Symphytum *asperrimum, Donn.

*caucasicum, Bieb.

officinale, Linn.

*tauricum, Willd.

Synthyris * reniformis, Benth.

Tanacetum boreale, Fisch. vulgare, Linn.

Taraxacum officinale, Weber.

Tellima grandiflora, R. Br.

- var. virescens.

Teucrium Chamædrys, Linn.

*pyrenaicum, Linn.

Scorodonia, Linn. 
Thalictrum angustifolium, Linn. glaucum, Desf. lucidum, Hort. majus, Murr. minus, Linn.

- var, *acuminatum.

- - elatum.

rhynchocarpum, Q. Dillon \& A. Rich.

sibiricum, Linn.

* speciosum, Hort.

Thlaspi *alpestre, Linn. * prostrata, Hort.

Thymus *alpinus, Hort.

Tofieldia *ealyeulata, Wahlenb. * glutinosa, Pers.

*palustris, Huds.

Tolpis barbata, Gærtn.

- var. *alba.

- - nana compacta.

Tradescantia * virginiana, Linn.

- var. *alba.

- - *atropurpurea.

- * *lata.

- * *major.

- - *subaspera.

Tragopogon orientalis, Linn.

Tricyrtis macropoda, Miq.

Trifolium alpinum, Linn,

pannonicum, Huds. Jacq.

* pratense, Linn.

repens, Linn., var. pictum.

rubens, Linn.

trichocephalum, Bieb.

Trigonella *Fœnum-græcum, Linn.

Trillium *grandiflorum, Salisb. - var. ${ }^{*}$ maximum.

Trollius *americanus, Mubl.

*asiaticus, Linn.

- var. Fortunei, Hort.

- _ *intermedius.

* caucasicus, Stev.

europæus, Linn.

- var. *napellifolius, Roep.

*japonicus, Hort.

Typha angustifolia, Linn.

latifolia, Linn.

Ursinia anthemoides, Gærtn. Uvularia *grandiflora, Sm.
Valeriana *apula, Pourr.

dioica, Linn.

*montana, Linn.

*tuberosa, Linn.

Veratrum *album, Linn.

Verbascum Lychnitis, Linn. phlomoides, Linn.

*phøniceum, Linn.

repandum, Hort.

Veronica *Anagallis, Linn.

austriaca, Linn.

*Bidwillii, Hook, fil.

Buxbaumii, Tenore.

corymbosa, Hort.

- var. variegata

crassifolia, Zeyh.

* fruticulosa, Linn.

gentianoides, Vahl.

grandis, Fisch.

incisa, Soland.

longifolia, Linn.

- var. alba.

- _ ${ }^{*}$ subsessilis (Miq.).

*orchidea, Crantz.

pinnata, Linn.

* rupestris, Tardent. satureioides, Vis.

* saxatilis, Scop.

- var, *rosea.

serpyllifolia, Linn.

spicata, Linn.

- var. alba.

- - hybrida.

spuria, Linn.

- var. foliosa (Waldst. \& Kit.).

virginica, Linn., var. japonica.

Waldsteiniana, Hort.

Vesicaria * utriculata, DC.

Vicia * cornigera, Chaub.

Cracca, Linn,

Orobus, DC.

tetrasperma, Moench.

villosa, Roth.

Vincetoxicum nigrum, Mœench.

officinale, Mœench.

Viola aquatica, Hort.

* biflora, Linn.

canadensis, Linn.

canina, Linn.

Jooi, Janka.

lutea, Huds.

montana, Hort.

Munbyana, Boiss. \& Reut. 
Viola-contd. sciaphylla, Koch. tricolor, Linn.

Wahlenbergia procumbens, A. DC.

*Pumilio, A. DC. saxicola, A. DC.

Wulfenia carinthiaca, Jacq.
Wyethia angustifolia, Nutt. mollis, A. Gray.

Xerophyllum *asphodeloides, Nutt.

Zygadenus elegans, Pursh.

- var. glaucus.

- major.

\section{TREES AND SHRUBS.}

Acer platanoides, Linn.

Pseudo-platanus, Linn.

Esculus carnea, Willd.

Hippocastanum, Linn.

Amelanchier canadensis, Torr. \& Gray.

Alnus cordifolia, Ten.

glutinosa, Medic.

Berberis Aquifolium, Pursh.

Betula alba, Linn.

- var. pendula.

Bryanthus empetriformis, A. Gray.

Calluna vulgaris, Salisb., var. alba Serlei.

Caragana arborescens, Lam.

Cassinia fulvida, Hook, fil.

Cassandra calyculata, D. Don.

Cistus laurifolius, Linn.

Clematis Flammula, Linn.

Corylus Avellana, Linn.

Cotoneaster affinis, Lindl.

frigida, Wall.

horizontalis, Dene.

microphylla, Wall.

Nummularia, F. \& M.

thymifolia, Baker.

Cratægus mollis, Scheele. Oxyacantha, Linn.

Cytisus Ardoini, Fourn. nigricans, Linn. scoparius, Link.

- var. Andréanus.

Daböecia polifolia, D. Don, var. alba.
Daphne Mezereum, Linn.

- var. album.

Enkianthus himalaicus, Hook, f. \& Thoms.

Erica ciliaris, Linn.

cinerea, Linn.

stricta, Donn.

vagans, Linn,

- var. alba.

- - alba minor.

Forsythia * suspensa, Vahl,

Fuchsia Riccartoni, Hort.

Fraxinus excelsior, Linn.

Gaultheria procumbens, Linn.

Shallon, Pursh.

Genista germanica, Linn. tinctoria, Linn.

Hypericum Ascyron, Linn.

Ilex Aquifolium, Linn.

Jamesia *americana, Torr. \& Gray.

Laburnum alpinum, J. S. Presl. vulgare, J. S. Presl.

Ledum palustre, Linn.

Microglossa albescens, Clarke. 
Olearia Haastii, Hook, fil.

Pachystima ${ }^{*}$ Canbyi, Gray.

Pernettya mucronata, Gaudich.

Potentilla fruticosa, Linn.

Prunus Laurocerasus, Linn.

Pyrus Aria, Linn.

Aucuparia, Gærtn.

- intermedia, Ehrh.

*lobata, Nichols.

* nigra, Sargent.

rotundifolia, Bechst.

*salicifolia, Linn.

Quercus pedunculata, Ehrh.

Rhododendron catawbiense, Michx. caucasicum, Pall.

ferrugineum, Linn.

flavum, G. Don.

hirsutum, Linn.

*lepidotum, Wall.

ponticum, Linn.

Rhodotypus kerrioides, Sieb. \& Zucc.

Rosa rugosa, Thunb.

- var. alba.
Sambucus canadensis, Linn. nigra, Linn.

Skimmia Fortunei, Mast.

Symphoricarpus racemosus, Michx.

Spiræa bullata, Maxim. *japonica, Linn, fil. - var. Bumalda.

Staphylea pinnata, Linn.

Veronica amplexicaulis, Armstr.

anomala, Armstr.

*Colensoi, Hook, fil.

Cookiana, Colenso.

Kirkii, Armstr.

lobelioides, Hort.

* Lyallii, Hook, fil. macroura, Hook, fil.

*monticola, Armstr. parviflora, Vahl.

pimeleoides, Hook, fil.

salicifolia, Forst.

*Traversii, Hook, fil.

Viburnum Opulus, Linn.

Zenobia speciosa, D. Don.

\section{PLANTS UNDER GLASS.}

Abrus precatorius, Linn.

Abutilon Regnelli, Miq.

Acacia Farnesiana, Willd.

Echmea cœlestis, E. Morr.

Asparagus deflexus, Baker. scandens, Thunb.

Alonzoa Warcewiczii, Regel.

Aloe mitriformis, Mill.

Anigosanthos flavida, DC. rufa, Labill.

Anopteris glandulosa, Labill.

Aplopappus ciliatus, DC.

* Aristea capitata, Ker-Gawl. Eckloni, Baker. corymbosa.

*Aristolochia fimbriata, Cham.
Brachysema subcordatum, Benth.

Billardiera longiflora, Labill.

Barbacenia squamata, Lindl.

Campanula pyramidalis, Linn.

Canna patens, Rose.

Warszewiczii, Hort.

Chorizema diversifolia, A. DC.

Celsia cretica, Linn.

Callicarpa purpurea, Juss.

Clerodendron splendens, G. Don.

Thomsonæ Balf. v. Balfourianum.

* Costus igneus, N. E. Br.

Cytisus racemosus, Hort, 
Disa grandiflora, Linn.

Dianella nemorosa, Lam. revoluta, $\mathrm{R}$. Br.

*Drosophyllum lusitanicum, Link.

*Drosera Burkeana, Planch. * intermedia, Hayne. *ramentacea, Burch. * spathulata, Labill.

Dyckia rariflora, Schult.

*Desmodium gyrans, DC.

Echinocactus Wislizenii, Engelm. Eranthemum albiflorum, Hook.

Fagelia bituminosa, DC.

Genista pilosa, Linn.

Geissorhiza obtusata, Ker-Gawl. secunda, Ker-Gawl.

Gladiolus gracilis, Jacq.

Goodia lotifolia, Salisb.

Gomphocarpus fruticosus, R. Br.

Gynura sarmentosa, DC.

Hakea gibbosa, Cav.

Hibiscus lunariifolius, Willd. pedunculatus, Linn. fil. schizopetalus, Hook, fil. splendens, Fras.

Homeria collina, Sweet.

Indigofera australis, Willd. Incarvillea Delavayi, Franch. Ixia maculata, Linn.

Kennedya rubicunda, Vent.

Lapeyrousia corymbosa, Ker-Gawl. Lagenaria longissima, Hort. Libertia ixioides, Spreng.
Momordica Charantia, Linn.

Melia Azederach, Linn.

Myrtus Luma, Barn.

Nicotiana Tabacum, Linn.

Orchis incarnata, Linn.

Oxylobium ellipticum, R. Br. lanceolatum, Vent.

Parochœetus communis, Buch-Ham.

Passiflora cœrtlea, Linn. edulis, Sims.

Primula mollis, Nutt. prolifera, Wall. verticillata, Forsk.

Psychotria cyanocarpa, Seem.

Philydrum lanuginosum, Banks.

Richardia Rehmanni, N. E. Br. Rhodochiton volubile, Zucc.

Sarracenia Drummondii, Croom.

Senecio cruentus, DC.

Sida triloba, Cav.

Sisyrinchium californicum, Dryand.

Scilla chinensis, Benth.

Solanum Seaforthianum, Andr.

Synecanthus fibrosus, H. Wendl.

Sabal umbraculifera, Glazebrook.

Schubertia grandiflora, Mart.

Tigridia pavonia, Ker-Gawl. Thomasia purpurea, J. Gay.

Torenia Fournieri, Linden.

Veronica Hulkeana, F. Muell.

Vitis heterophylla, Thunb., var. humulifolia.

ISAAC BAYLEY BALFOUR, Regius Keeper.

A, D. RICHARDSON, Head Gardener.
R. L. HARROW, Assistant Head Gardener. 



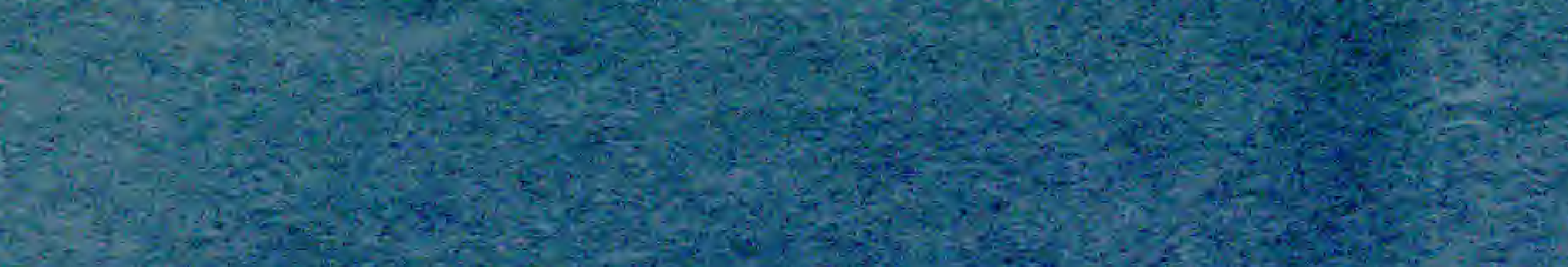

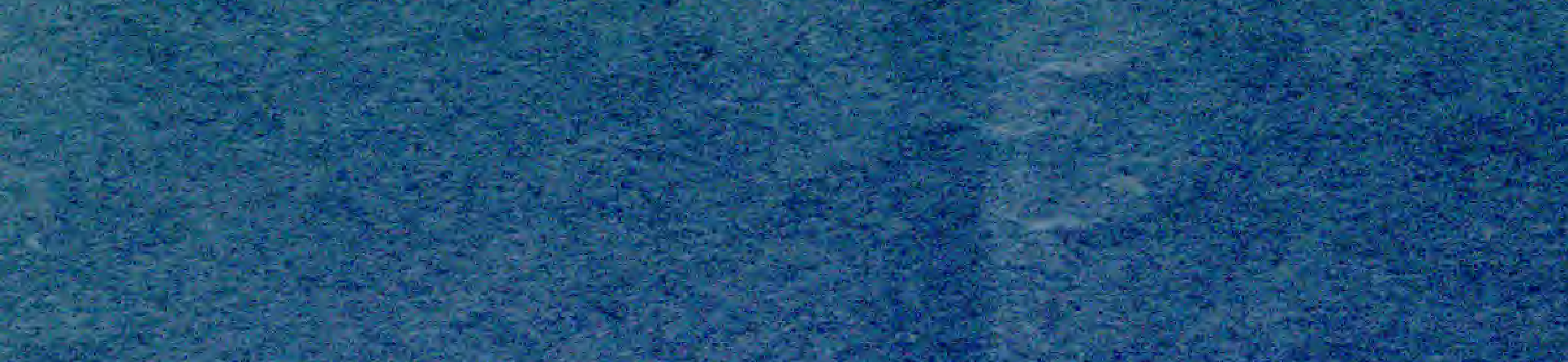

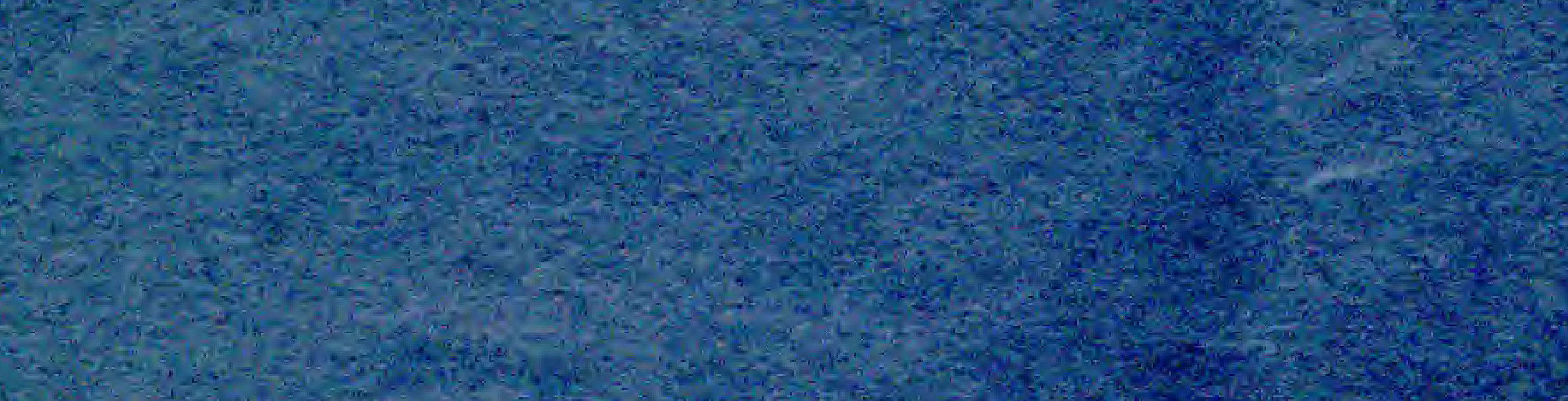

S.

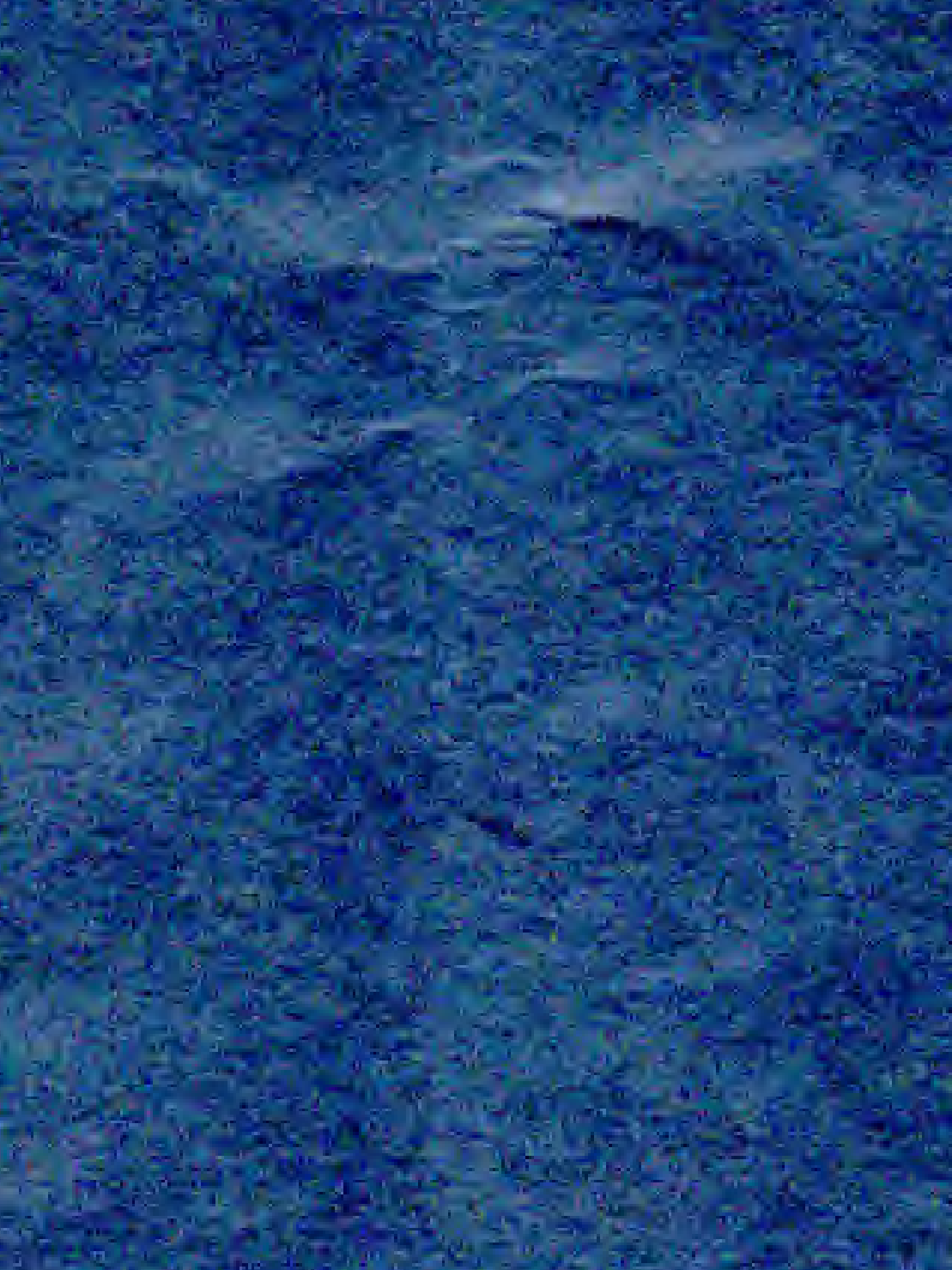

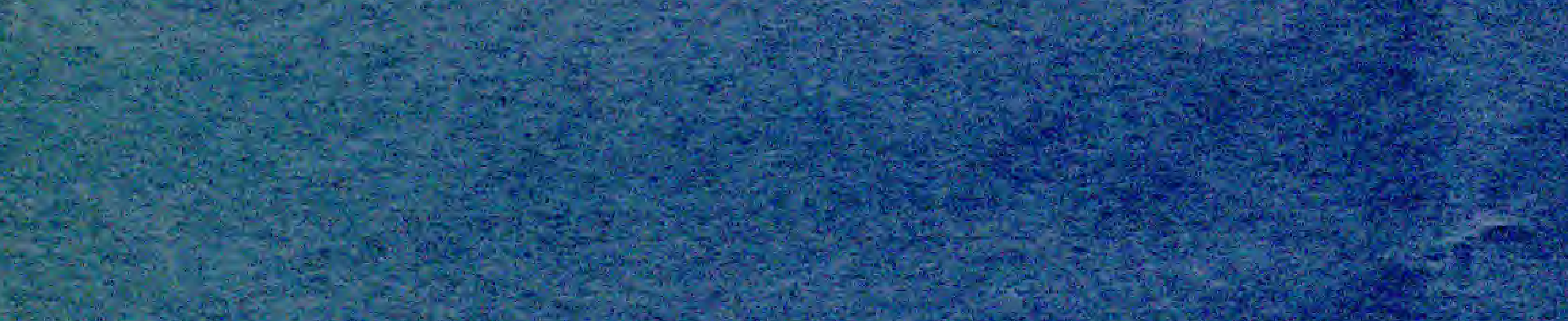

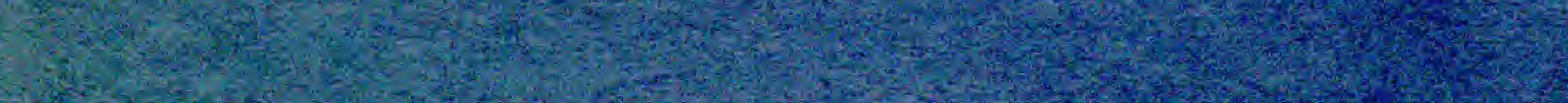

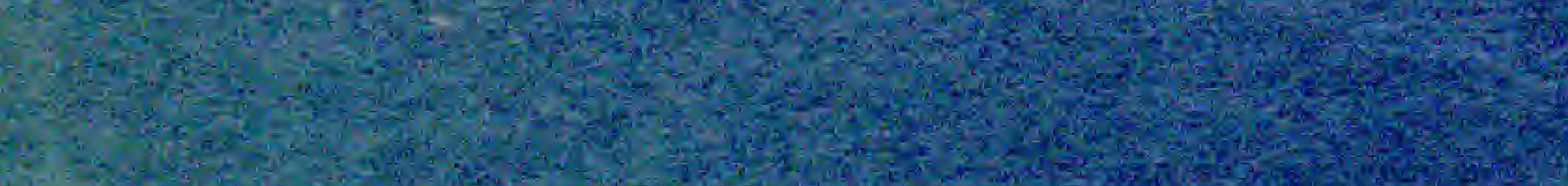

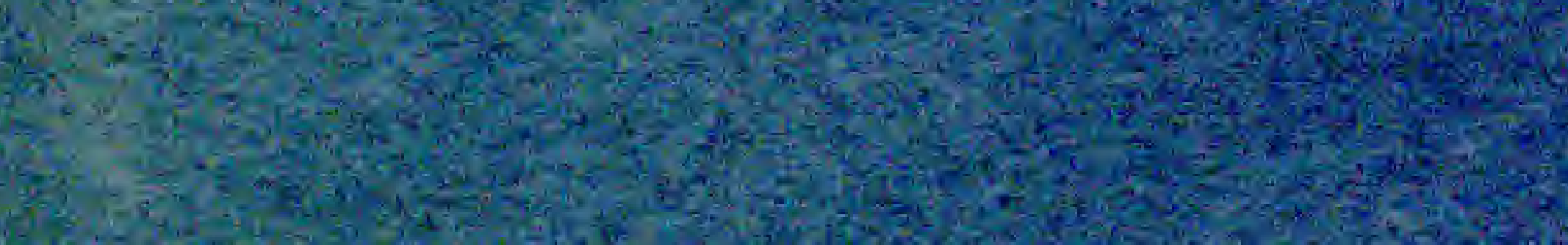

it.

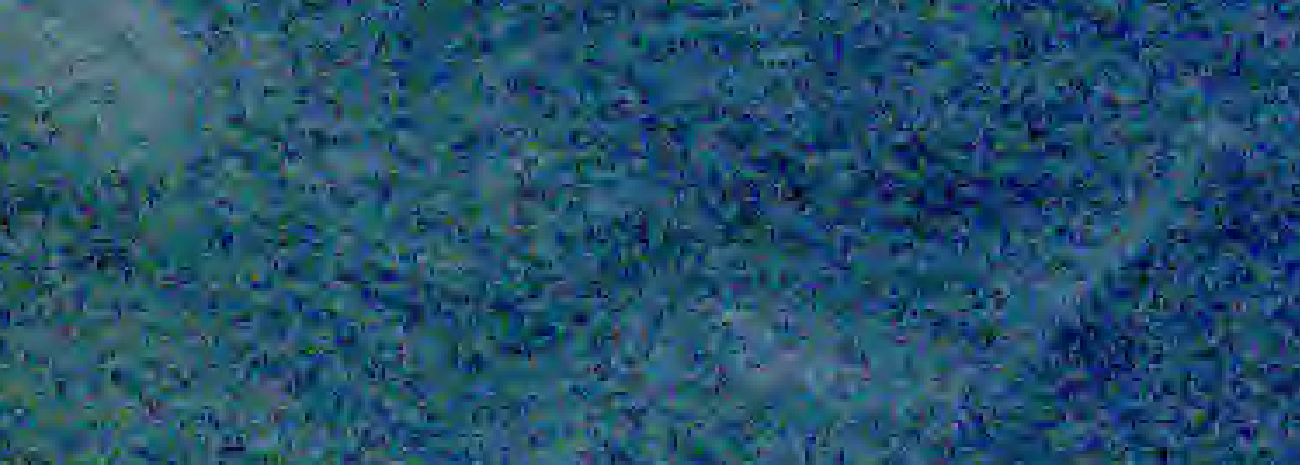

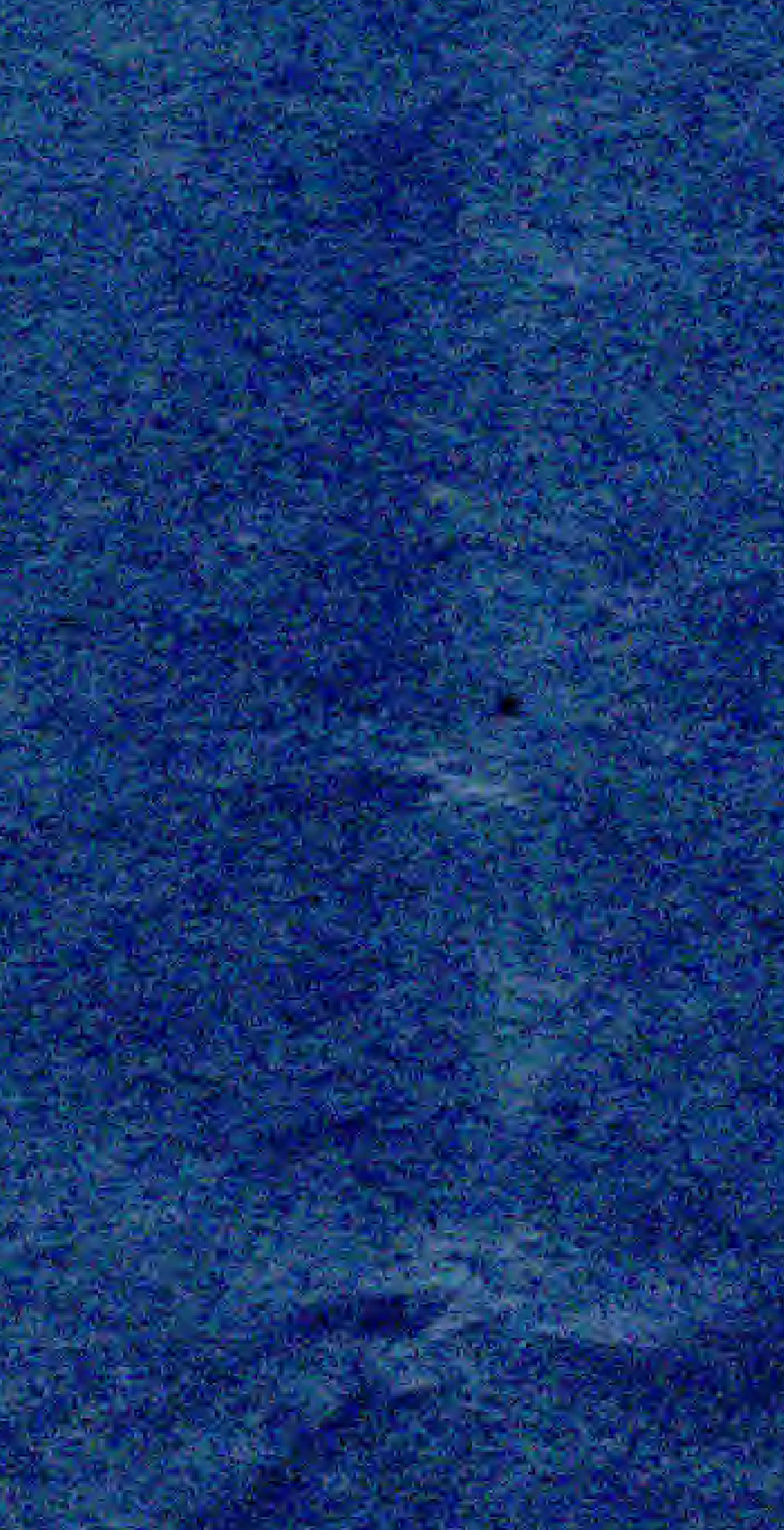

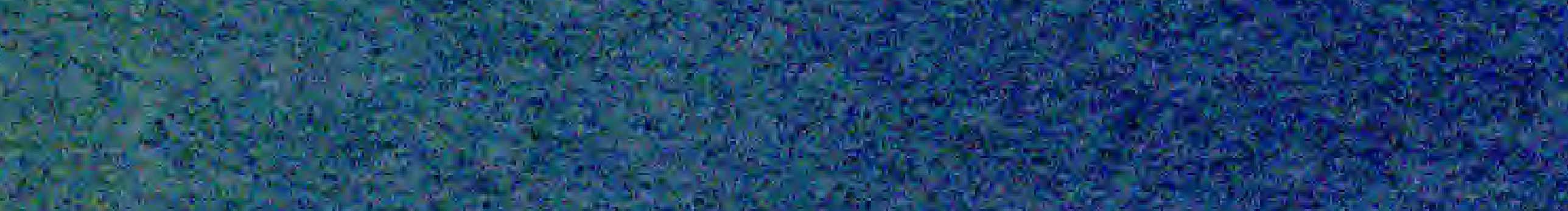

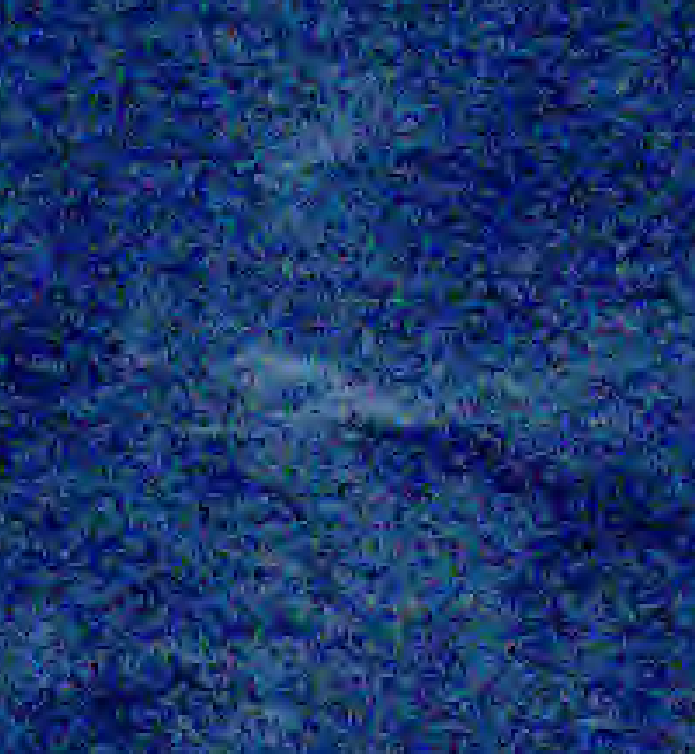

$-7$.

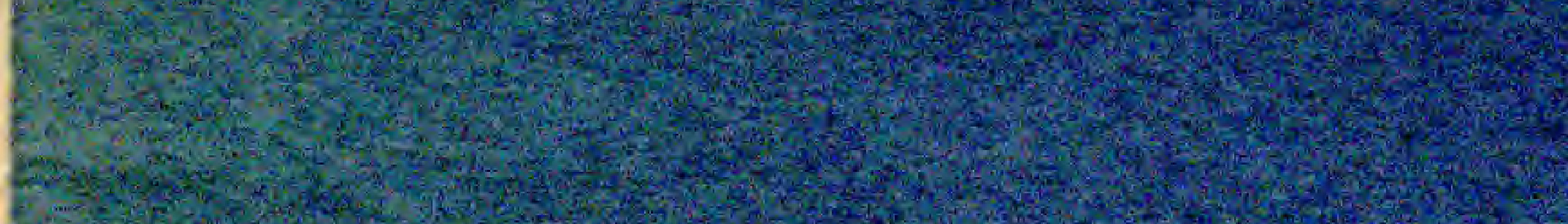

S. 



\title{
ROYAL BOTANIC GARDEN, EDINBURGH.
}

\section{LIST OF SEEDS}

\author{
COLLECTED DURING. 1897.
}

\section{GL.ASGOW:}

PRINTED FOR HER MA.IESTY'S STARUNBRY OKFICE, BV JAMES HEDDERWTOK \& SONE,

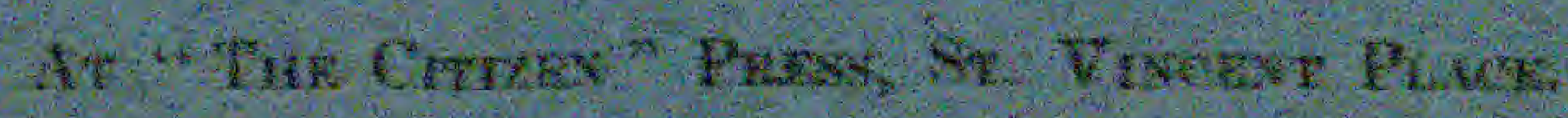




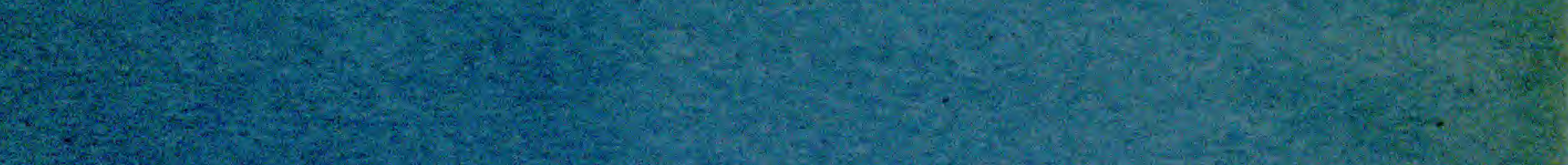

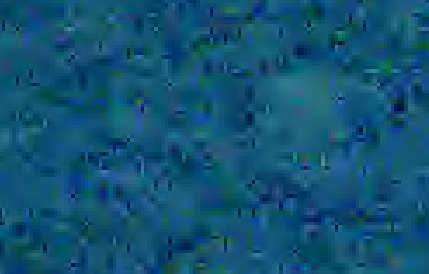




\title{
ROYAL BOTANIC GARDEN, EDINBURGH.
}

\section{LIST OF SEEDS}

\author{
COLLECTED DURING 1897.
}

Note,-Of those marked with an asterisk, ${ }^{*}$ the quantity collected is very small.

\section{HERBACEOUS PLANTS.}

Acæna inermis, Hook. fil. microphylla, Hook. f.

Acantholimon g] umaceum, Boiss.

Achillea ageratifolia, Benth. \& Hk.f. Clavennæ, Linn.

crithmifolia, Waldst. \& Kit.

Huteri, Sendt.

Jaborneggix, Halacsy.

ligustica, All.

macrophylla, Linn.

Millefolium, Linn.

- - var. rosea.

nobilis, Linn.

obscura $x$, Nees.

Reiehardtiana $\times$, G. Beck.

rupestris, Huter.

tomentosa, L.

Tournefortii, DC.

umbellata, Sibth. \& Sm.

_ - var. argentea.

Aconitum Anthora, Linn.

Lycoctonum, Linn.

moldavicum, Hacq.

Napellus, Linn.

paniculatum, Lam.

Actra spicata, Linn.

Adenophora stylosa, Fisch.

Athionemn cordatum, Boiss.

*saxatile, R. Br.

Agrimonia Eupatoria, Linn.

odorata, Mill.

Agropyron pectinatum, Beauv.

Agrostis nebulosa, Boiss. \& Reut.

Ajuga pyramidalis, Linn.

Alchemilla alpina, Linn., var. conjuncta, Bab.

vulgaris, Linn.
Allium.

fistulosum, Linn.

globosum, DC., var. albidum.

neapolitanum, Cyr.

*oreophilum, C. A. Mey.

paradoxum, G. Don.

seorzoneræfolium, DC.

senescens, Linn.

sphærocephalum, Linn.

Alonsoa *acutifolia, Ruiz \& Pav. caulialata, Ruiz \& Pav.

*Warscewiczii, Regel.

Alyssum alpestre, Linn.

argenteum, Vitm.

calycinum, Linn.

* compactum, De Not.

incanum, Linn.

*montanum, Linn. saxatile, Linn.

Amethystea cærulea, Linn.

Ammobium alatum, R. Br.

Amphoricarpus *Neumayeri, Vis.

Anagallis arvensis, Lin.

- - var. cærulea (Lam.).

Anaphalis alata, Maxim. margaritacea, Benth. \&Hook. f.

Androsace elongata, Linn.

filiformis, Retz.

lanuginosa, Wall.

_ - var. oculata.

maxima, Linn.

obtusifolia, All.

*sarmentosa, Wall. septentrionalis, Linn.

Anemone alpina, Linn.

_ _ var. sulphurea.

angulosa, Lam. 
Anemone-contd.

decapetala, Arduini.

Hepatica, Linn.

- var. alba.

- - pyrenaica.

- - - rubra.

*montana, Hoppe.

narcissiflora, Linn.

nemorosa, Linn.

*pratensis, Linn.

pulsatilla, Linn.

rivularis, Buch.-Ham.

Angelica songorica, Regel \& Schmalh.

Antennaria dioica, Gærtn., var. tomentosa.

sericea, Hort.

Anthemis cinerea, Panc.

Cota, Linn.

tinctoria, Linn.

Triumfetti, DC.

Anthyllis montana, Linn.

Vulneraria, Linn.

Antirrhinum Asarina, Linn. majus, Linn.

Apium graveolens, Linn.

A ponogeton distachyum, Thunb.

Aquilegia Buergeriana, Sieb. \& Zuce. californica, A. Gray, var, alba. chrysantha, A. Gray.

glandulosa, Fiseh.

nevadensis, Boiss, \& Reut.

olympica, Boiss.

* pyrenaica, DC.

*viridiflora, Pall.

Arabis albida, Stev.

Allionii, DC.

blepharophylla, Hook. \& Arn.

hirsuta, Scop.

lucida, Linn. fil.

Stelleri, DC.

Turrita, Linn.

Archangelica officinalis, Hoffm.

Arenaria graminifolia, Schrad., var. parviflora.

saxatilis, Linn.

stricta, Michx.

Armeria alpina, Willd.

- - var, rosea.

boetica, Boiss., var. hirta.

cariensis, Boiss.

elongata, Hoffm.

fasciculata, Willd.

latifolia, Willd.
Armeria-contd.

latifolia, Willd. var. rubra.

maritima, Willd.

— - var. alba.

plantaginea, Willd.

Welwitschii, Boiss.

Arnica Chamissonis, Less.

- - var.

montana, Linn.

Arrhenatherum avenaceum, Beauv.

Artemisia annua, Linn.

campestris, Linn.

capillaris, Thunb.

desertorum, Spreng.

discolor, Dougl.

gallica, Willd.

Stelleriana, Bess.

Arum *palrestinum, Boiss.

Asparagus officinalis, Linn.

Asperula *eynanchica, Linn. lævigata, Linn.

Asphodelus * ramosus, Linn.

Aster acris, Linn.

adscendens, Lindl.

alpinus, Linn.

- - var. albus.

- - spéciosus.

amellus, Linn, var. major.

amethystinus, Nutt.

azureus, Lindl.

canus, Waldst. \& Kit.

cordifolius, Linn.

- - var. major.

corymbosus, Ait.

*Curtisii, Torr. \& Gray.

dahuricus, Benth.

discolor, Hort.

dumosus, Linn.

elegans, Torr. \& Gray.

falcatus, Lindl.

floribundus, Nutt.

glaucus, Torr. \& Gray.

grandiflorus, Linn.

junceus, Ait.

lævis, Linn.

- - var. Arachne.

- - Pygmalion.

- - Stella.

Lindleyanus, Torr. \& Gray.

longifolius, Lam.

macrophyllus, Linn.

mutabilis, Linn.

Novæ Angliæ, Linn., var, præcox, 
Aster-contd.

Novi-Belgii, Linn., var.

Harpur Crewe.

- - - littoreus.

- - Vesta.

paniculatus, Lam.

- - var. laxus.

patens, Ait.

patulus, Lam.

ptarmicoides, Torr. \& Gray.

puniceus, Linn.

pyrenaeus, DC.

rivularis, Cass.

sagittifolius, Wedem.

salsuginosus, Hort.

Shortii, Lindl.

sibiricus, Linn.

sikkimensis, Hook. f. \& Thoms.

spectabilis, Ait.

stellaris, Hort.

tartaricus, Linn. fil.

trinervius, Roxb.

- - var, congestus.

versicolor, Willd., var. Themis.

Astilbe chinensis, Franch. \& Sav.

Astragahus bicolor, Lam.

chlorostachys, Lindl.

Glycyphyllos, Linn.

hamosus, Linn.

vicioides, R. Grah.

Astrantia Biebersteinii, Fisch. \& Mey.

carniolica, Wulf.

major, Linn.

minor, Linn.

neglecta, C. Koch. \& Bouché.

Astydamia canariensis, DC.

Atriplex hortensis, Linn.

- - var. rubra.

Atropa belladonna, Linn.

Aubrietia deltoidea, DC.

- - var. græca (Griseb.).

* _ - Hendersoni.

- - Richardi.

erubescens, Griseb.

Avena barbata, Brot.

fatua, Linn.

strigosa, Schreb.

Baptisia leucophra, Nutt.

Barbarea areuata, Reichb.

bracteosa, Guss.

vulgaris, R. Br.
Bellis rotundifolia, Boiss. \& Reut.

*sylvestris, Cyrill.

Bellium minutum, Linn.

Biscutella auriculata, Linn. ciliata, DC.

lævigata, Linn.

Borago officinalis, Linn.

Boykinia aconitifolia, Nutt.

Brachycome *Sinclairii, Hook. fil.

Brachypodium distachyum, Beauv.

Brassica campestris, Linn.

Brodiæa * Bridgesii, S. Wats. congesta, Sm.

Bromus.

mollis, Iinn.

Tacna, Steud.

Bryonia dioica, Jacq.

Bulbine annua, Willd.

Bulbinella Hookeri, Benth. \& Hk.f.

Buphthalmum salicifolium, L.

Bupleurum rotundifolium, Linn.

Calamintha umbrosa, Fisch. \& Mey.

Calendula maritima, Guss. officinalis, Linn.

stellata, Car.

suffruticosa, Vahl.

Calochortus venustus, Benth., var. citrinus.

* _ _ Eldorado.

* _ - oculatus.

Caltha *leptosepala, DC. palustris, Linn., var. biflora.

Camassia Brownii, Hort.

Cusickii, S. Wats.

esculenta, Lindl.

- - var. tardiflora.

Camelina sativa, Crantz.

Campanula alliariæfolia, Willd., var. alba.

alpina, Jacq.

crespitosa, Scop.

carpatica, Jacq.

- - var. turbinata (Schott.).

cervicaria, Linn.

elegans, Roem. \& Schult.

glomerata, Linn.

Grossekii, Heuffl.

latifolia, Linn.

- - var, alba.

- macrantha (Fisch.).

linifolia, Scop.

Medium, Linn. 
Campanula - contd. persicifolin, Linn.

- - var. alba.

- - - grandiflora.

- - - grandis.

pilosa, Pall.

pusilla, Hrenke.

Rapunculus, Linn.

rhomboidalis, Linn.

rotundifolia, Linn.

- - var. alba.

- - Baumgartenii

(Becker.).

Cannabis sativa, Linn., var. gigantea.

Capsella Bursa-pastoris, Medic.

Cardamine hirsuta, Linn.

Carduus Carolorum $\times$, Howie \& Jenner.

Carex atrata, Linn.

flava, Linn.

Mairii, Coss, \& Germ.

microglochin, Wahlenb.

muricata, Linn.

stricta, Good.

sylvatica, Huds.

*ustulata, Wahl.

Carlina acaulis, Linn.

Carum Bulbocastanum, Koch.

Catananche lutea, L.

Catheartia *villosa, Hook. fil.

Centaurea algeriensis, Dur. \& Coss. calcitrapoides, Linn.

eariensis, Boiss.

elata, Poir.

eriophora, Linn.

Jacea, Linn.

macrocephala, Puschk.

montana, Linn.

nigra, Linn.

pectinata, Linn.

Rhaponticum, Linn.

sphrerocephala, Linn.

stereophylla, Bess.

Centranthus macrosiphon, Boiss.

Cerastium arvense, Linn., var. grandiflorum.

ovatum, Hoppe.

tomentosum, Linn.

Cerinthe *alpina, Kit. major, Linn., var. flore luteo. minor, Linn.

Charieis heterophylla, Cass.
Cheiranthus Cheiri, Linn., var. Harbinger.

Chelidonium majus, Linn.

Chenopodium ambrosioides, Linn.

urbicum, Linn.

virgatum, Thumb.

Chionodoxa Lucilia, Boiss.

— - var. gigantea.

_ _ sardensis.

*__ _ T Tmolusii.

Chrysanthemum achillexfolium,DC. atratum, Linn.

coronarium, Linn.

graminifolium, Linn. grandiflorum, Willd. — - var. Wolley Dod.

macrophyllum, Waldst. \& Kit.

Parthenium, Bernh.

Tchihatchewii, Hort. Kew.

tripteris, Hort.

uliginosum, Pers.

Clarkia pulchella, Pursh.

- - var, integripetala.

Claytonia virginica, Linn.

Cnicus atropurpureus, Hort.

eriophorus, Roth.

gnaphalodes, Willd.

heterophyllus, Roth., var. albus. palustris, Willd.

pannonicus, Host., var. divaricatus.

pratensis, Willd.

spinosissimus, Linn.

Cochlearia oflicinalis, Linn.

- - var. alpina, Sweet.

saxatilis, Linn.

Codonopsis ovata, Benth.

Collinsia bicolor, Benth., var. multicolor.

sparsiflora, Fisch. \& Mey.

Collomia linearis, Nutt.

Convolvulus *tricolor, Linn.

Coreopsis grandiflora, Nutt. verticillata, Linn.

Coronilla elegans, Panč.

Corydalis glauea, Pursh.

lutea, DC.

sibirica, Pers,

Cotula aurea, Løfl.

Crambe cordifolia, Ster.

Crepis aurea, Reichb.

- var. crocea.

*hyoseridifolia, Reichb. 
Crepis-contd.

sibirica, Linn.

succisæfolia, Tausch.

Crucianella regyptiaca, Iim.

Cryptotrenia canadensis, DC.

Cuphea *lanceolata, Ait.

Cyananthus *lobatus, Wall.

Cynanchum acutum, Linn.

Cynoglossum glochidiatum, Wall. microglochin, Benth. petiolatum, A. DC.

Wallichii, G. Don.

Cynosurus echinatus, Linn.

Datura inermis, Jacq.

Stramonium, Linn.

Delphinium Ajacis, Reichb.

altissimum, Wall.

elatum, Linn.

formosum, Boiss \& Huet.

Maackianum, Regel.

*olopetalum, Boiss.

*orientale, F. Gay. palmatifidum, DC. velutinum, Bertol.

Dianthus ambiguus, Panc. banaticus, Heuff. brevicaulis, Fenzl.

*ersius, Sm.

*apitatus, DC. caucasicus, Sims. chinensis, Linn.

* cinnabarinus, Sprun. cruentus, Griseb. deltoides, Linn.

hæmatocalyx, Boiss \& Heldr. hungaricus, Hort. liburnicus, Bartl. \& Wendl. mosiacus, Vis. \& Pane.

*monspessulanus, Linn.

*neglectus, Loisel. pelviformis, Heuff.

petreus, Waldst. \& Kit. plumarius, Linn.

- - var, serotinus (Waldst do Kit.).

pubescens, Sibth. d Sm.

squarrosus, Bieb.

superbus, Linn.

_-var. Holtzeri (Winkler).

*sylvestris, Wulf.

tener, Balb.

virgineus, Linn.
Digitalis ambigua, Murr.

argyrostigma, Hort.

ferruginea, Linn., var. gigantea.

Fontanesia, Steud.

gloxinioides, Carr., var, alba.

Diphylleia cymosa, Michx.

Diplotaxis erucoides, DC.

Dipsacus laciniatus, Linn.

Dodoeatheon "Meadia, Linn., var. album.

Doronicum Clusii, Tausch.

Draytonense, Hort.

grandiflorum, Lam.

hirsutum, Lam.

macrophyllum, Fisch.

Draba aizoon, Wahlenb.

__. var. Haynaldi (Stur.).

*alpina, Linn.

aurea, Vahl.

cornuta, Hort.

cuspidata, Bieb.

fladnizensis, Wulf.

frigida, Sauter.

hirta, Linn.

hispida, Willd.

incana, Linn.

nemorosa, Linn.

rupestris, R. Br.

siliquosa, Bieb., var. glabrata. stellata, Jacq.

Drosera *longifolia, Linn.

Dryas octopetala, Linn.

Echinacea purpurea, Mench.

Echinops dahuricus, Fisch.

niveus, Wall.

speciosus, Hort.

spharocephalus, Linn.

Echinospermum Lappula, Lehm.

Elsholtzia cristata, Willd.

Epilobium alpinum, Linn. angustifolium, Linn. coloratum, Muhl, montanum, Lim. repens, Schlecht. rosmarinifolium, Hrnke. sericeum, Schum.

*Eranthis hyemalis, Salisb.

Erigeron alpinus, Linn. aurantiacus, Regel. caucasicus, Stev. glabellus, Nutt. 
Erigeron-contd.

glabellus, Nutt., var. mollis. glabratus, Hoppe \& Hornsch. glandulosus, Porter \& Coulter. grandiflorus, Hook. macranthus, Nutt.

multiradiatus, Benth. \& Hook.f. *pulchellus, DC., var. albus.

Roylei, DC.

salsuginosus, A. Gray. speciosus, DC.

- - var. superbus, Linn.

*trifidus, Schlecht. uniflorus, Linn.

Erinus *alpinus, Linn.

Erodium *Manescavi, Coss,

Eruca sativa, Mill.

Eryngium alpinum, Linn. amethystinum, Linn. aquifolium, Cav. azureum, Hort. Billardieri, Delar. cæruleum, Bieb. dichotomum, Desf. glaciale, Boiss. yuccæfolium, Michx.

Erysimum hieracifolium, Linn. pumilum, Nutt. rupestre, DC.

Erythronium *Dens-canis, Linn.

Eschscholzia californica, Cham.

Eucharidium coneinnum, Fisch. \& Mey., var. grandiflorum.

Eupatorium ageratoides, Linn. glechonophyllum, Less. purpureum, Linn. sessilifolium, Linn.

Euphorbia Myrsinites, Linn. verrucosa, Hort.

Farsetia eriocarpa, DC.

Fedia * Cornucopie, Grertn.

Festuca delicatula, Lag. Myuros, Linn. rigida, Kunth.

*Fragaria vesca, Linn., var, eflagellaris.

Funkia *lancifolia, Spreng. ovata, Spreng. Sieboldiana, Spreng.
Gaillardia aristata, Pursh., var. maxima, Hort.

Galanthus Elwesii, Hook. f.

_ var. Cassabah.

* plicatus, Bieb.

Galium erectum, Huds. tenuissimum, Bieb. tricorne, Stokes.

Gentiana asclepiadea, Linn.

- var. alba.

Burseri, Lapeyr.

decumbens, L.

*excisa, Koch. punctata, Linn. septemfida, Pall.

_- var. cordifolia, Hook, f. tibetica, King.

Geranium asphodeloides, Burm. cinereum, Cav. collinum, Steph.

*ibericum, Cav. pratense, Linn. pyrenaicum, Burm. sanguineum, Linn., var. roseum. sibiricum, Linn.

Gerbera Anandria, Sch. Bip. nivea, Sch. Bip.

Geum coccineum, Sibth. \& Sm. elatum, Wall. montanum, Linn. nutans, Hort. parviflorum, Commers. pyrenaicum, Mill. rivale, Linn. strictum, Ait.

Gilia achillexfolia, Benth. capitata, Sims. laciniata, Ruiz \& Pav. Gnaphalium supinum, Linn. Grammanthes *gentianoides, DC. Grindelia inuloides, Willd. * pulchella, Dunal.

Gypsophila perfoliata, Linn. *prostrata, Linn.

Hablitzia *tamnoides, Bieb.

Habenaria * conopsea, Benth.

Helenium autumnale, Linn.

— - var. grandiflorum (Nutt.). - - _ pumilum (Willd.).

Bigelovii, A. Gray. Hoopesii, A. Gray. tenuifolium, Nutt. 
Helianthus decapetalus, Linn. lactiflorus, Pers. multiflorus, Linn. - - var. maximus.

*orgyalis, DC. rigidus, Desf.

Heliopsis lavis, Per's. - - var. padula, Wender.

Helminthia echioides, Gritn. Helonias bullata, Linn. - - var. pallida.

Heracleum Sphondylium, Linn. Heuchera bracteata, Ser. cylindrica, Dougl. Drummondii, Hort. sanguinea, Engelm.

Hieracium alpinum, Liun. amplexicaule, Linn. argenteum, Fries.

*Auricula, Linn. buglossoides, Arv. bupleuroides, C. G. Gmel. cæsium, Fries. crinitum, Sibth. \& Sm. cymosum, Linn. eximium, Backh. foliosum, Waldst. \& Kit. humile, Jacq. incisum, Hoppe. intybaceum, Jacq. iricum, Fries. Jankæ, Uechtr. lasiophyllum, Koch. lingulatum, Backh. Marshalli, Linton. murorum, Linn. Neo-cerinthe, Fries. nitidum, Hort. pallidum, Biv. Pilosella, Linn. Pilosella $x$ aurantiacum. *prenanthoides, Vill. prostratum, Poir. pulmonarioides, Vill. rubescens, Gilib. rupestre, All. suecicum, Fries. tenellum, Backh. tridentatum, Fries. umbellatum, Linn. undulatum, Ait. valdepilosum, Vill. villosum, Jacq.
Hieracium - contd.

*vulgatum, Fries. - - var. sylvaticum (Lam.).

Hippocrepis glauca, Tenore.

Holeus lanatus, Linn.

Homogyne alpina, Cass. sylvestris, Cass.

Horminum pyrenaicum, Limn. Hyoscyamus albus, Linn. niger, Linn.

Hyoseris radiata, Linn.

Hypericum elegans, Steph. *hirsutum, Linn. japonicum, Thunb. linarifolium, Vahl. montanum, Linn. perforatum, Linn. pulchrum, Linn. quadrangulum, Linn. Richeri, Vill. tetrapterum, Fries.

Hypochæris glabra, Limn. radiata, Linn.

Hyssopus ofticinalis, Linn.

Iberis gibraltarica, Linn., var. hybrida.

Pruiti, Tineo. sempervirens, Linn. -- - var. superba. umbellata, Linn. - - var. purpurea.

Impatiens Noli-me-tangere, Linn. Roylei, Walp.

Inula Conyza, DC. crithmifolia, Linn. ensifolia, Linn. glandulosa, Puschk. grandiflora, Willd.

Helenium, Linn.

Hookeri, C. B. Clarke.

Newmannii, Hort.

Iris ensata, Thunb. lurida, Soland. nepalensis, D. Don. Pseudacorus, Linn. setosa, Pall. sibirica, Linn. - var. alba.

*tenax, Dougl.

Isatis tinctoria, Linn.

Ixolirion *Pallasii, Fisch. it Mey. 
Juneus balticus, Willd. conglomeratus, Linn. effusus, Limn.

*filiformis, Linn. glaucus, Sibth. lamprocarpus, Ehrh. tenuis, Willd.

Jurinea glycacantha, DC.

Kaufmannia Semenovi, Regel.

Lactuca alpina, Benth. \& Hook. f. floridana, Grertn.

*virosa, Linn.

Lallemantir canescens, Fisch. d Mey. Lamium album, Linn. Orvala, Linn.

Lapeyrousia juncea, Pourr. Lapsana communis, Linn. Laserpitium *Siler, Linn.

Lasthenia glabrata, Lindl.

Lathyrus Aphaca, L. cyaneus, C. Koch. hirsutus, Linn. macrorrhizus, Wimm. magellanicus, Lam. maritimus, Bigel. nervosus, Boiss. niger, Bernh. odoratus, Linn. rotundifolius, Willd. vermus, Linn.

Lavatera thuringiaca, Linn.

Layia elegans, Torr. \& Gray. glandulosa, Hook. \& Arn.

Leontodon autumnalis, Linn. hastilis, Linn. hispidus, Linn.

Leonurus capitatus, Fresen, sibiricus, Linn.

Leontopodium alpinum, Cass., var. altaicum (Spreng.). - - transylvanicum.

Leptosyne maritima, A. Gray. *Stillmanni, A. Gray.

Liatris pyenostachya, Michx.

Libertia grandiflora, Sweet.

Ligusticum involucratum, Regel. pyrenaicum, Gouan. scoticum, Linn.
Lilium canadense, Linn., var. penduliflorum (DC.).

dauricum, Ker-Gawl.

pardalinum, Kellogg.

limnanthes Douglasii, R. Br.

Linaria *bipartita, Willd., var. splendens.

capraria, Moris \& De Not.

viscida, Møench.

vulgaris, Mill.

vulgaris $\times$ repens.

Lindheimera texana, A. Gray.

Linum alpinum, Linn.

*angustifolium, Huds. grandiflorum, Desf.

perenne, Linn.

- - var. roseum.

Lithospermum * prostratum, Loisel.

Loasa *tricolor, Lindl.

Lolium multiflorum, Lam.

perenne, Linn.

tèmulentum, Linn.

Lonas inodora, Gærtn.

Lophanthus rugosus, Fisch. \& Mey.

Lotus corniculatus, Linn., var.

Delorti (Timb.).

Lunaria annua, Linn.

Lupinus angustifolius, Linn.

Chamissonis, Eschseh.

Cruckshanksii, Hook.

*Dunnettii, Hort.

elegans, H. B. \& K.

hirsutus, Linn.

insignis $\times$, Lam.

* - var. vigrescens.

luteus, Linn.

micranthus, Dougl.

nootkatensis, Don.

perennis, Linn.

* pilosus, Murr.

polyphyllus, Lindl.

- - var. albus.

Lychnis alba, Mill.

chalcedonica, Linn.

coronaria, Desv.

dioica. Linn.

Flos-jovis, Desv.

*fulgens, Fisch.

Githago, Scop.

Sartori, Hort.

Viscaria, Linn.

- var. alba.

Lysimachia affinis, Bunge.

decurrens, Forst. 
Lysimachía-contd.

lobelioides, Wall.

verticillata, Bieb.

Lythrum myrtifolium, Lodd.

Salicaria, Linn.

— - var, cinereum (Griseb.).

- - - grandiflorum.

- - - hirsutum.

virginatum, Linn.

Macrotomia echioides, Boiss.

Madia elegans, D. Don.

Malva crispa, Linn.

Duriæi, Hort. Kew.

moschata, Linn.

- - var. alba.

sylvestris, Linn.

Martubium Kotschyi,Boiss \&Hohen. leonuroides, Desr.

Matricaria inodora, Linn.

Matthiola arborescens, Sternb.

Meconopsis *aculeata, Royle. nepalensis, DC.

*simplicifolia, G. Don.

*Wallichii, Hook.

Medicago denticulata, Willd. orbicularis, All.

Pironæ, Vis.

Megacarpæa polyandra, Benth.

Melica *uniflora, Retz.

Mentha piperita, Linn. sylvestris, Linn.

Mimulus hybridus, Hort. tigrinus, Hort.

Mitella pentandra, Hook.

Morina *betonicoides, Benth. longifolia, Wall.

Muretia *anadensis, Boiss.

Muscari Argæi, Hort. armeniacum, Leichtlin. atlanticum, Boiss \& Reut. botryoides, Mill.

- - var. magna.

Maweanum, Baker.

*paradoxum, C. Koch.

Szovitsianum, Baker.

Myosotis *alpestris, F. W. Schmidt.

* - var. olympica.

azoriea, H. C. Wats.

dissitiflora, Baker., var. alba.

sylvatica, Hoffm.
Narcissus pallidus, Hort., var. * praecox.

triandrus, Linn., var, *albus.

Nardostachys *Jatamansi, DC.

Narthecium *ossifragum, Huds.

Nasturtium pyrenaicum, R. Br.

Nemesia *strumosa, Benth., var. Suttoni.

versicolor, E. Mey.

Nepeta grandiflora, Bieb. latifolia, DC.

Nessa verticillata, H. B. \& K.

Enanthe crocata, Linn.

globulosa, Linn.

Lachenalii, C. C. Gmel. pencedanifolia, Pollich. silaifolia, Bieb.

Enothera biennis, Linn. taraxacifolia, Sw., var. alba.

Onobrychis viciæfolia, Scop.

Ononis alopecuroides, Linn. spinosa, Linn.

Onopordon Acanthium, Linn. Sibthorpianum, Boiss \& Heldr. tauricum, Willd.

Orchis latifolia, Linn.

Ornithogalum *exscapum, Tenore.

Oxalis *crassipes, Urb.

*floribunda, Lehm. valdiviensis, Barn.

Oxytropis campestris, D.C. glabra, DC.

strobilacea, Bunge. uralensis, D.C.

Pæonia decora, Anders.

Panicum virgatum, Linn.

Papaver aculeatum, Thunb. alpinum, Linn., var. Juteum.

Argemone, Linn. caucasicum, Bieb. lateritium, C. Koch. orientale, Linn.

pilosum, Sibth. \& Sm, pinnatifidum, Moris. Rhoas, Linn. rupifragum, Boiss \& Reut. somniferum, Linn. 
Parnassia *vata, Ledeb.

Pentstemon acuminatus, Dougl. barbatus, Roth. confertus, Dougl. deustus, Dongl. diffusus, Dougl. gentianoides, Poir. pubescens, Soland.

Phalaris canariensis, Linu. paradoxa, Linn. tuberosa, Linn.

Phaseolus multiflor us, Willd. Mungo, Linn.

Phlomis setigera, Falc. viscosa, Poir.

Physospermum commutatum, Spreng.

Physostegia virginiara, Benth. Phyteuma campanuloides, Bieb. orbiculare, Linn.

Scheuchzeri, All.

Sieberi, Spreng.

Phytolacea decandra, Linn.

Picris echioides, Linn.

Plantago arenaria, Waldst. \& Kit.

Bellardi, All.

lanceolata, Linn.

major, Linn.

maritima, Linn.

media, Limn.

montana, Huds.

Psyllium, Linn.

Rugelii, Decne.

Platystemon californicus, Benth.

Poa cresia, Sm.

Podophyllum Emodi, Wall.

Polemonium boreale, Adams. cæruleum, Linn.

- - var. album.

gracile, Willd.

himalayanum, Baker.

humile, Willd.

lacteum, Lehm.

*Richardsoni, Grah., var, album.

Polygonatum miltiflorum, All.

Polygonum Convolvulus, Linn. mite, Schrank.

Potentilla alchemilloides, Lapeyr. alpestris, Hall. fil. andicola, Benth. argyrophylla, Wall. desertorum, Bunge. Dombeyi, Nestl. gelida, C. A. Mey.
Potentilla-contd.

geoides, Bieb.

Goldbachii, Rupr.

grandiflora, Linn.

Herbichii, Blocki.

hirta, Linn.

Macnabiana $\times$, Lem.

malacophylla, Borb.

minima, Hall. fil.

nepalensis, Hook.

nevadensis, Boiss.

ontopoda, Dougl.

palustris, Scop.

Piersii, Siegfr.

pseudochrysantha $x$.

rupestris, Linn.

Sadleri, Reichb.

Sanguisorba, Willd.

Schrenkiana, Regel.

Sibbaldi, Hall. fil.

thyriaca, Blocki.

Valderia, Linn.

villosa, Pall.

vlasicensis, Siegfi:

Poterium canadense, Linn.

Pratia *angulata, Hook. fil.

*Primula Auricula, Linn., var. grandiflora.

- _ var. "marginata.

*denticulata, Sm., var. alba.

- _ - var. cashmeriana (Carr).

elatior, Hill.

*arinosa, Linn, var, scotica (Hook).

*frondosa, Janka. grandis, Trautv.

*involucrata, Wall., var. Munroi. officinalis, Jacq.

- - var. Tommasinii.

-_ - uralensis (Fisch.).

*pubescens $\times$, Jacq.

* Reidii, Duthie.

rosea, Royle., var, grandiflora.

* Sieboldii, Morr., var. Cordelia.

sikkimensis, Hook.

*viscosa, Vill, var. nivalis, Hort.

Prismatocarpus strictus, A. DC.

Prunella grandiflora, Jacq.

- — var, alba.

vulgaris, Linn.

- var. laciniata.

Pulicaria vulgaris, Gærtn. 
Pulmonaria saccharata, Mill. Puschkinia scilloides, Adams. *Pyenanthemum lanceolatum,Pursh Pyrola *medir, Sw.

Ranunculus abortivus, Linn. aconitifolius, Linn. acris, Linn. auricomus, Linn.

*millefoliatus, Vahl.

*montanus, Willd. monspeliacus, Linn. muricatus, Linn. pyrenæus, Linn.

* - var. alba. serbicus, Vis.

*Thora, Linn.

Reseda alba, Linn. complicata, Bory. crystallina, Webb \& Berth. lutea, Linn. ramosissima, Pourr.

Rhagadiolus Hedypnois, All.

Rheum macropterum, Mart.

Rodgersia podophylla, A. Gray.

Rudbeckia amplexicaulis, Vahl. hirta, Linn. speciosa, Wender.

Rumex nepalensis, Spreng. stenophyllus, Ledeb.

Ruta graveolens, Linn. macrophylla, Soland.

Salvia Beckeri, Trautv.

Carduacea, Benth.

* chionantha, Boiss. cleistogama, De Bary \& Paul. hians, Royle.

limbata, C. A. Mey. officinalis, Linn. pratensis, Linn. pyrenaica, Linn. Regeliana, Trautv. sylvestris, Linn. Verbenaca, Jinn. verticillata, Linn. virgata, Ait.
* Samolus Valerandi, Linn.

Sanicula europæa, Linn.

Saponaria Vacearia, Linn.

Saxifraga Aizoon, Linn., var. balcana.

*__ _ brevifolia farinosa.

* - - carinthiaca (Schott.).

- - major

- _ minima.

_ - notata (Schott, Nym. \& Kotschy.).

*__ pectinata (Schott.).

*__ _ rosularis (Haw.).

*_ - Sturmiana (Schott, Nym. \& Kotschy.).

Andrewsii $x$, Harvey, var. Guthriana, Hort.

apiculata $x$, Engler.

*Boydii $x$, Dewar.

bronchialis, Linn., rar. cherlerioides (D. Don.).

- - conferta, Hort.

Bucklandii, Hort.

- - var. major.

* Burseriana, Linn.

cæsia, Linn.

crespitosa, Linn., var. hirta.

*cartilaginea, Willd. var. Kolenatiana, Regel.

Cotyledon, Linn.

var. pyramidalis (Lapeyr.). crassifolia, Linn.

crustata, Vest.

*__ var. hybrida.

cuneata, Willd.

Cymbalaria, Linn.

decipiens, Ehrh.

* - var. grcenlandica(Linn.).

- - palmata (Panč.),

*diapensioides, Bell., var. tombeanensis (Boiss.).

diversifolia, Wall.

erosa, Pursh.

exarata, Vill.

- - var. aromatica.

*Gaudini x, Brïgg.

geranioides, Linn.

Geum, Linn.

- - var. gracilis (Mackay).

* - - hirsutum.

granulata, Linn.

- - var. Russi.

*Hausmannix, Kern. 
Saxifraga-contd.

hederæfolia, Hochst.

Hostii, Tausch., var. altissima

(Kern.).

- - elatior.(Mert.\& Koch).

- - elegans.

Huetiana, Boiss.

*hybrida $\times$, Sternb., var. splendens.

hypnoides, Linn.

intacta, Willd.

- _ var. farinosa.

irrigua, Bieb.

lingulata, Bell.

- - var. cochlearis (Reich.).

- - lantoscana (Boiss. \& Reut.).

longifolia, Lapeyr., var, major.

*luteo-viridis, Schott \& Kotschy.

* MacNabiana $x$.

* MacNabiana $\times$ Hausmanii.

*media, Gouan.

Milesii, Leichtl.

*mixta, Lapeyr. muscoides, Wulf.

- - var. atropurpurea.

* - - moschata, Wulf.

—_ Rhei (Schott, Nym.

nivalis, Linn. \& Kotschy.).

ohioensis, Hort.

*aradoxa, Sternb.

patens $\times$, Kern.

pedatifida, Ehrh.

pedemontana, All., var. cervicornis (Viv.).

porophylla, Bertol.

* Portax x, Engl.

* pubescens, Pourr.

punctata, Linn.

retusa, Gouan.

- var. bryoides.

Rocheliana, Sternb., var. *coriophylla (Griesb.). rotundifolia, Linn.

* Dálomonix

*sancta, Griseb.

scardica, Griseb.

serpyllifolia, Pursh.

sponhemica, C. C. Gmel., var. affinis (D. Don.).

* squarrosa, Sieber.

Stansfieldix.
Saxifraga-contd.

*stricta, Hort.

*taygetea, Boiss. \& Heldr.

tenella, Wulf., va1. muscoides.

tenella, Wulf. $x$, sedoides, L.

*tyrolensis $x$, Кетn.

umbrosa, Linn.

- - var. hirsuta (Linn.).

— - serratifolia (Mackay).

- - variegata.

*valdensis DC.

*virginiensis, Michx.

Wallacei $x$, MeNab.

Scabiosa *caucasica, Bieb.

centauroides, Mill.

cretica, Linn.

lucida, Vill.

micrantha, Desf.

ochrolenca, Linn., var.

Webbiana (D. Don.).

pectinata, Lag.

prolifera, Linn.

pterocephala, Linn.

silenifolia, Waldst. \& Kit.

succisa, L.

- - var. alba.

trinirefolia, Frivald.

Seandix brachycarpa, Guss.

Schizopetalon *Walkeri, Sims.

Scilla *bifolia, Linn., var. rubra.

festalis, Salisb.

- - var. alba.

hispanica, Mill.

- - var. grandiflora.

patula, DC.

_- var. major.

sibirica, Andr.

*_ _ var. alba.

placox.

Scolymus hispanicus, Limn.

maculatus, Linn.

seorpinrus muricata, Linn.

vermiculata, Linn.

Scorzonera hispanica, Limm.

Scrophularia trifoliata, Linn.

Scutellaria alpina, Linn. orientalis, Linn.

Sedum acre, Linn.

- - var. elegans.

Aizoon, Linn.

album, Lim.

Anacampseros, Linn.

asiaticum, Spreng.

Beyrichianum, Turez. 
Sedum-contd.

* cæruleum, Lim.

Ewersii, Leteb.

hispanicum, Limn.

laxiflorum, DC.

maximum, Suter.

*oreganum, Nutt.

pallidum, Bieb.

rupestre, Linn.

- - var. elegans.

trifidum, Wall.

villosum, Linn.

Selinum Carvifolia, Linn.

Sempervivum *arachnoideum, Linn.

arenarium, Koch.

arvernense, Lecoq \& Lamotte.

calcareum, Jord.

ciliatum, Willd.

cinerascens, Hort.

globiferum, Linn.

hirtum, Linn.

leucanthum, Panc.

montanum, Linn.

Pittonii, Schott., Nym. \& Kotschy.

*Pomelli, Lamotte.

*Schnittspahni, Lagg.

Verloti, Lamotte.

Senebiera Coronopus, Poir.

Senecio abrotanifolius, Linn.

alpinus, Scop.

aurantiacus, Reichb.

Jacobra, Linn.

japonicus, Sch. Bip.

laxifolius, J. Buch.

Ledebourii, Sch. Bip.

paludosus, Linn.

sibiricus, Linn.

thrysoideus, $\mathrm{DO}$.

vulgaris, Linn.

Serratula coronata., Linn.

heterophylla, Desf.

*tinctoria, Linn.

Seseli gummiferum, Sm.

Setaria italica, Beauv. verticillata, Beauv.

Shortia *galacifolia, Torr. \& Gray.

Sidalcea candida, A. Gray. malveflora, A. Gray.

Sideritis lanata, Linn.

Silene alpestris, Jacq.

caucasica, Boiss.

* ciliata, Pourr.

*cretica, Linn.
Silene-contd.

cucubalus, Wibel.

flavescens, Waldst. \& Kit.

longicaulis, Pourr.

maritima, With.

*pensylvanica, Michx.

quadrifida, Linn.

Reichenbachii, Vis.

Saxifraga, Linn.

Thorei, Duf.

Silphium perfoliatum, Linn.

Sisymbrium austriacum, Jacq. officinale, Scop.

Sisyrinchum angustifolium, Mill., var. mucronatum (Michx.). bermudiana, Linn.

Solidago californica, Nutt. eanadensis, Linn.

elongata, Nutt.

flabelliformis, Wendl.

nemoralis, Ait.

ohioensis, Riddell.

stricta, Mønch.

Virgaurea, Linn.

Sonchus oleraceus, Linn. palustris, Linn.

Spirrea Aruncus, Linn.

astilboides, Carr., var. floribunda.

Filipendula, Linn.

palmata, Sternb.

Ulmaria, Linn.

Stachys Alopecuros, Benth. alpina, Linn., var. intermedia. grandiflora, Benth.

Statice Gmelini, Willd. latifolia, Sm.

Stellaria graminea, Linn., var. aurea.

Symphyandra Hofmanii, Panč. pendula, A.DC.

Symphytum asperrimum, Donn. tauricum, Willd.

Tagetes signata, Bartl., var. pumila. Tanacetum boreale, Fisch.

Meyerianum, Sch. Bip. vulgare, Linn.

Taraxacum ofticinale, Weber.

Tellima grandiflora, R. Br. var. virescens.

Teucrium Scorodonia, Linn. -.. var, cristatum, 
Thalictrum angustifolium, Linn.

*glaucum, Desf.

*minus, Linn.

- var. elatum (Jacq.).

Thlaspi alpestre, Linn. arvense, Linn.

Thymus serpyllum, I.inn.

- - var. hirsutus.

Tofieldia calyculata, Wahlenb. glutinosa, Pers.

Tolpis barbata, Gærtn.

Tradescantia virginiana, Linn.

*_ var alba.

- - - atropurpurea.

— - - elata (Lodd.).

Tragopogon longifolius, Heldr. \& Sart.

Trifolium pannonicum, Jacq. pratense, Linn. repens, Linn.

— - var. pictum.

trichocephalum, Bieb.

Trillium grandiflorum, Salisb.

Trinia vulgaris, IDC.

Triticum monococeum, Linn. polonicum, Linn. Spelta, Linn.

Tritonia Pottsii, Benth.

Trollius americanus, Muhl.

*asiaticus, Linn.

_ - var. hybridus.

_ _ - intermedius.

caucasicus, Stev.

europaeus, Linn.

- - var. napellifolius (Roep.).

Tropeolum Lobbianum, Hort. peregrinum, Linn.

Tulipa pulchella, Fenzl.

Tunica Saxifraga, Seop.

Typha angustifolia, Linn.

latifolia, Linn.

Urospermum picroides, F. W. Schmidt.

Ursinia anthemoides, Grertn. speciosa, DC.

Urtica cannabina, Linn.

dioica, Linn.

magellanica, Juss.

urens, Linn.
Valeriana dioica, Linn. tuberosa, Linn.

Valerianella vesicaria, Mœench.

Venedium *decurrens, Less.

Verbascum Blattaria, Linn.

Chaixi, Vill.

malacotrichum, Boiss. \& Heldr.

*phøniceum, Linn.

— - var, album.

Veronica alpina. Linn. austriaca, Linn., var. Prenja

(G. Beck.).

candida, Lodd.

corymbosa, Hort.

- - var. variegata.

fruticulosa, Linn.

glauca, Sibth. \& Sm.

incisa, Soland.

longifolia, Iinn.

Nummularia, Gounn.

officinalis, Linn.

orchidea, Crantz.

Ponæ, Gouan.

precox, All.

rupestris, Tardent.

spicata, Linn.

- - var. hybrida (Linn.).

spuria, Linn., var. foliosa

(Waldst. \& Kit.).

Teucrium, Linn., var, latifolia. virginica, Linn.

Vicia amphicarpa, Dorthes.

narbonensis, Linn.

oroboides, Wulf.

sepium, Limn.

Viola canina, Linn.

Jooi, Janka.

lutea, Huds.

Munbyana, Boiss. \& Reut.

odorata, Linn.

sylvestris, Lam.

tricolor, Linn.

Wahlenbergia saxicola, A. DC.

Wulfenia carinthiaca, Jacq.

Wyethia mollis, A. Gray.

Xanthium spinosum, Linn.

Xerophyllum asphodeloides, Nutt.

Zygadenus elegans, Pursh.

_ - var. glaucus (Nutt.). 


\section{TREES AND SHREBS.}

Acer Pseudo-platanus, Linn.

Alnus cordifolia, Ten. glutinosa, Merlic.

Berberis angulosa, Wall.

Aquifolium, Pursh.

Darwinii, Hook. nervosa, Pursh.

Betula alba, Linn. _ - var. pendula.

Bryanthus empetriformis, A. Gray. Buddleia intermedia, Carr.

Cassinia fulvida, Hook. fil.

Clematis *alpina, Mill.

Cotoneaster affinis, Lindl. frigida, Wall.

horizontalis, Dene.

microphylla, Wall.

Nummularia, F. \& M.

Simonsii, Baker.

thymifolia, Baker.

Cratægus coccinea, Linn. mollis, Scheele. oxyacantha, Linn.

Cytisus *Ardoini, Fourn. præcox, Hort. scoparius, Link.

- var. Andréanus.

Daboëcia polifolia, D. Don. var. alba.

Daphne Mezereum, Linn. — - var. album.

Empetrum nigrum, Lim.

Enkianthus himalaieus, Hook. fil. \& Thoms.

Erica stricta, Donn. vagans, Linn.

Fraxinus excelsior, Linn.

Genista tinctoria, Linn.
Helianthemum *anum, Boiss.

*inereum, Pers. glutinosum, Pers. vulgare, Gertn.

Hypericum Androsemum, Linn. Ascyron, Linn.

Ilex Aquifolium, Linn.

Jamesia *americana, Torr. \& Gray.

Laburnum alpinum, J. S. Presl. vulgare, J. S. Presi.

Ledum palustre, Linn.

Olearia Haastii, Hook. fil.

Pachystima *anbyi, Gray.

Pernettya mucronata, Gaudich.

Potentilla fruticosa, Linn.

Pyrus Aria, Linn.

Aucuparia, Gærtn. intermedia, Ehrh. rotundifolia, Bechst.

Quercus Cerris, Linn.

Rhododendron ferrugineum, Linn. flavum, G. Don ponticum, Lirn.

Rhodotypus *kerrioides, Sieb. \& Zuec.

Rosa alba, Linn. alpina, Linn. canina, Linn. pomifera, Herrm., var. proxima. rubiginosa, Linn. rugosa, Thunb.

- var. alba.

- bicolor.

Sambucus nigra, Linn.

Spiræa bullata, Maxim.

japonica, L. fil., var. Bumalda. Symphoricarpus racemosus, Michx, 
Veronica *amplexicaulis, Armstr. anomala, Armstr. Bidwillii, Hook. fil. *earnosula, Hook. fil. *decumbens, Armstr.

*Hectori, Hook. fil.

*Kirkii, Armstr. lohelioides, Armstr.
Veronica-contd.

*Lyallii, Hook, fil.

*monticola, Armstr. parviflora, Vahl. salieifolia, Forst. Traversii, Hook. fil.

*vernicosa, Hook. fil.

Viburnum Lantana, Linn.

\section{PLANTS UNDER GLASS.}

Archontophoenix Cunninghami, $\mathrm{H}$. Wendl. \& Drude.

Argyrolobium Andrewsianum, Steud.

Aristolochia fimbriata, Cham. Agapetes buxifolia, Nutt.

Arthropodium cirrhatum, R. Br.

Asclepias curassavica, Linn.

Asparagus Sprengeri, Regel.

Begonia Evansiana, Andr. Blandfordia grandiflora, R. Br.

Bilbergia zebrina, Lindl.

Brodiæa lactea, S. Wats.

Callicarpa purpurea, Juss. Campanula Vidali, H. C. Wats. Ceratopteris thalictrioides, Brong. Cyanotis tricolor, D. Don.

Cyrtanthus hybridus, Hort.

Drosophyllum lusitanicum, Link. Drosera Burkeana, Planch.

- capensis, Linn.

- ramentacen, Burch.

Eccremocarpus scaber, Ruiz, \& Pav. Eucomis punctata, L'Herit.

- regia, Ait.

Eurycles Cunninghami, Ait.
Francoa ramosa, D. Don.

Genista monosperma, Lam. Gelasine azurea, Herb.

Globba strobilifera, Zoll. \& Mor.

Hypoxis stellata, Linn.

Hibiscus lunariifolius, Willd.

Hydrolea spinosa, Linn.

Incarvillea Delavayii, Franch.

Kennedya prostrata, Ait., var. glabrata.

Maurandia semperflorens, Jacq.

Moræa prolongata, Hort.

Medicago arborea, Aubl.

Mussehia Wollastoni, Lowe.

Nicotiana rustica, Linn.

Nematanthus longipes, DC.

Orehis latifolia, Linn.

Opuntia mamillata, Schott, 
Passitlora capsularis, Limn.

Pentas Kermesina, Hort.

Primula floribunda. Wall.

- imperialis, Jungh.

- mollis, Nutt.

- verticillata, Forst.

Philydrum lanuginosum. Banks.

Phynchosia curibar, DC:

Stapelia mutabilis, Jacq.

- variegita, Linn, var, pallida.

- revoluta. Mass. marmorata, Jacq.
Sollya heterophylla, Lindl.

Senecio cruentus, DC.

- subscaudens, Hochst.

- grandifolius, Less.

Shortia galacifolia, Torr. \& Gray.

Salphiglossis sinuata, Ruiz. \& Pav.

Sabal umbraculifera. Mart.

Stylidium glandulosum, Salisb.

synecanthus fibrosus, H. Wendl.

Trichosanthes anguina, Lim.

Vitis heterophylla, Thunb. var. humulifolia.

Xylophylla montana, Sw.

ISAAG BAYLEY BALFOUR. Regins Keeper.

\begin{abstract}
A. D. RICHARDSON.
Head Gardener.
\end{abstract}

R. L. HARROW, Assistant Head Gardener.

Fonvereh, January I898. 




\section{ROYAL BOTANIC GARDEN, EDINBURGH.}

\section{LIST OF SEEDS \\ COLLECTED DURING 1898 .}

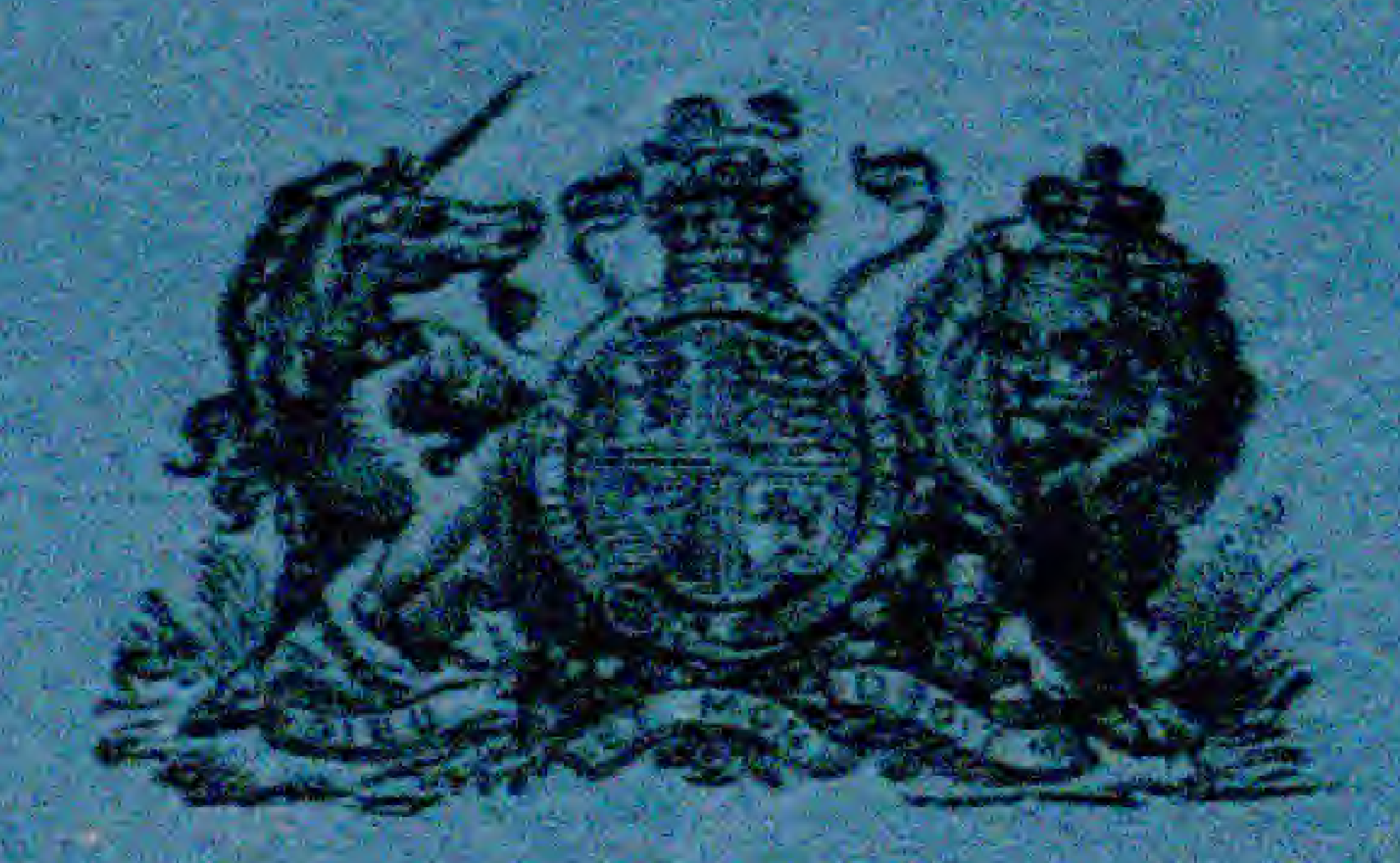

PRINTED FOR HER MAJESTYS STATIONERY OFFICE,

Br JAMES HEDDERWICK \& SONS,

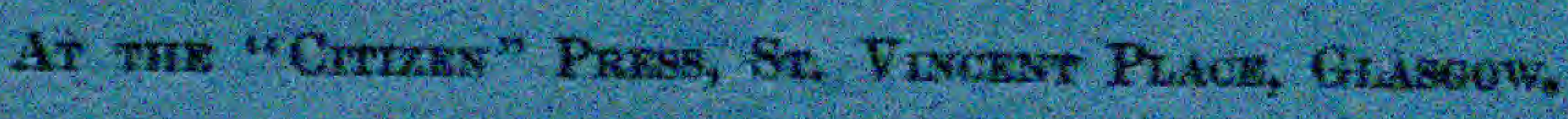
1899. 







\title{
ROYAL BOTANIC GARDEN, EDINBURGH.
}

\author{
Mo. Bots Garden, \\ LIST OF SEEDS
}

\author{
COLLECTED DURING 1898.
}

Note.-Of those marked with an asterisk*, the quantity collected is very small.

\section{HERBACEOUS PLANTS.}

Aconitum californicum, Hort.

longigaleatum, Hort.

Lycoctonum, Linn.

moldavicum, Hacq.

Napellus, Linn.

paniculatum, Lam.

Actra alba, Mill.

spicata, Linn.

Adenophora stylosa, Fisch.

Adesmia muricata, DC.

Ethionema *saxatile, R. Br.

Agrimonia Eupatoria, Linn.

Allium globosum, Red., var. albidum neapolitanum, Cyr.

*oreophilum, C. A. Mey.

*platycaule, S. Wats.

sativum, Linn.

scorzoneræfolium, DC.

urceolatum, Regel

Alyssum calyeinum, Linn.

* Moehlendorfianum, Hort.

saxatile, Linn.

Wiersbeckii, Heuff.

Androsace *elongata, Linn.

*Hausmanni, Leyb.

maxima, Linn.

*obtusifolia, All.

septentrionalis, Linn.

Anemone alpina, Linn.

- var. sulphurea

*decapetala, Arduini

*Hepatica, Linn.

- var. alba

- var. *rubra

narcissiflora, Linn.

nemorosa, Linn.
Anemone-contd.

pratensis, Linn.

rivularis, Buch.-Ham.

Angelica pachycarpa, Lange

sylvestris, Linn.

Anoda hastata, Cav.

Antennaria dioica, Gærtn.

Anthericum *Liliago, Linn.

Antirrhinum majus, Linn.

Orontium, Linn.

Apium graveolens, Linn.

Aponogeton distachyum, Thunb.

Aquilegia *alpina, Linn.

Buergeriana, Sieb, et Zucc.

californica, A. Gray, var. alba

canadensis, Linn.

chrysantha, A. Gray

formosa, Fisch.

glandulosa, Fisch.

grandiflora, Hort., var. alba

nevadensis, Boiss. et Reut.

olympica, Boiss.

vulgaris, Linn.

- *ar. alba

Arabis Allionii, DC.

alpina, Linn., var. anachoretica, Port.

*arenosa, Scop.

* ciliata, R. Br.

hirsuta, Scop.

*Holboellii, Horn., var. patula japonica, Regel. lucida, Linn. fil. serpyllifolia, Vill.

Turrita, Linn. 
Arenaria fasciculata, Gouan graminifolia, Schrad. - var. parviflora

*laricifolia, Linn.

Arnica Chamissonis, Less.

Argemone mexicana, Linn. ochroleuca, Sweet

Artemisia Pichardsoniana, Hook.

Asparagus officinalis, Linn.

Aster japonicus, Less. linariæfolius, Linn. pyrenæus, DC.

Astilbe *chinensis, Franch. et Sav.

Astragalus bicolor, Lam. Glycyphyllos, Linn. hamosus, Linn. reflexistipulus, Miq.

*vaginatus, Pall.

Astrantia Biebersteinii, Fisch. et Mey.

carniolica, Wulf.

major, Linn.

neglecta, C. Koch et Bouche

Atropa Belladonna, Linn.

Aubrietia deltoidea, DC., var. græca, (Griseb.)

- var. grandiflora

- var. taurica

erubescens, Griseb.

Avena sterilis, Linn.

Bidens grandiflora, Balb.

Biscutella lævigata, Linn.

Blumenbachia insignis, Schrad.

Brassica juncea, Coss.

Briza minor, Linn.

Bromus breviaristatus, Buckl.

Kalmii, A. Gray

Tacna, Steud.

Bryonia dioica, Jacq.

Bulbinella Hookeri, Benth. et Hook. f.

Bulbocodium vernum, Linn.

Bupleurum angulosum, Linn.

Calamintha grandiflora, Moench

umbrosa, Fisch, et Mey.

Calendula arvensis, Linn.

Calla palustris, Linn.

Callipeltis Cucullaria, Stev.

Callirhoe digitata, Nutt.
Calochortus *venustus, Benth., var.

Eldorado

Camassia Brownii, Hort.

Cusickii, S. Wats.

esculenta, Lindl.

- var. tardiflora

Camelina sativa, Crantz

Campanula alliariæfolia, Willd.

carpatica, Jacq.

- var. turbinata, (Schott)

glomerata, Linn.

Grossekii, Heuff.

Medium, Linn.

*persicifolia, Linn.

- var. grandiflora

rapunculoides, Linn.

Rapunculus, Linn.

rhomboidalis, Linn.

rotundifolia, Linn.

- var. alba

sibirica, Linn.

— var. divergens, (Willd.)

subpyrenaica, Timb.

Trachelium, Linn.

- var. aggregata

Cardamine *bellidifolia, Linn.

hirsuta, Linn.

resedæfolia, linn.

Carex atrata, Linn.

*Buchanani, Berggr.

Carum Petroselinum, Benth. et Hook. f.

Centaurea calcitrapoides, Linn. macrocephala, Puschk. nigra, Linn.

ragusina, Linn,, var. candidissima

Centranthus *ruber, DC.

Cephalaria alpina, Schrad. radiata, Griseb. et Schenk tatarica, Schrad.

Cerastium Biebersteinii, DC. pennsylvanicum, Hort.

Chærophyllum aureum, Linn.

Cheiranthus Cheiri, Linn.

Chelidonium majus, Linn.

- var. laciniatum, (Mill)

Chiondoxa Luciliæ, Boiss.

- var. gigantea

- var. sardensis

Chrysanthemum caucasicum, Pers. macrophyllum, Waldst. et Kit.

Cimicifuga americana, Michx.

*japonica, Miq. 
Cnicus Biebersteinii, Hort.

Casabonæ, Roth

heterophyllus, Roth

pannonicus, Host, var. divaricatus

Cochlearia saxatilis, Linn.

Colchicum *montanum, Linn.

*speciosum, Stev.

Collomia linearis, Nutt.

Conium maculatum, Linn.

Convolvulus tricolor, Linn.

Coronilla elegans, Panč.

Cortusa Matthioli, Linn., var. grandiflora

Corydalis *glauca, Pursh

Cousinia Hystrix, C. A. Mey.

Crupina vulgaris, Cass.

Cynanchum acutum, Linn.

Cynoglossum microglochin, Benth.

Dahlia variabilis, Desf.

Datura Tatula, Linn.

- var. inermis

Delphinium altissimum, Wall.

cashmirianum, Royle

consolida, Linn.

elatum, Linn.

formosum, Boiss. et Huet.

Maackianum, Regel.

occidentale, S. Wats.

speciosum, Bieb., var. turkestanicum

truncatum, Hort.

Dianthus angulatus, Royle

ambiguus, Panč.

*arenarius, Linn.

atrorubens, All.

banaticus, Heuffl.

caucasicus, Sims

chinensis, Linn.

deltoides, Linn.

grandiflorus, Poir.

Helwiggii, $x$

*neglectus, Loisel.

*nitidus, Waldst. et Kit.

plumarius, Linn., var. serotinus, (Waldst. et Kit.)

pubescens, Sibth. et Sm.

squarrosus, Bieb.

Diapensia *lapponica, Linn.

Dictamnus albus, Linn., var. tauricus
Diphylleia cymosa, Michx.

Digitalis media, Roth purpurea, Linn.

Diplotaxis erucoides, DC.

Dipsacus laciniatus, Linn.

Doronicum grandiflorum, Lam.

Draba Aizoon, Wahlenb. arabisans, Michx.

aurea, Vahl

cornuta, Hort.

cuspidata, Bieb.

frigida, Sauter

incana, Linn.

* _ var. hebecarpa

*longirostra, Schott, Nym. et Kotschy.

rupestris, $\mathrm{R}$. Br.

scandinavica, Lindblom

siliquosa, Bieb.

- var. glabrata

stellata, Jacq.

Dracocephalum heterophyllum, Benth.

speciosum, Benth.

Elsholtzia cristata, Willd.

Elymus virginicus, Linn.

Epilobium montanum, Linn.

*obcordatum, A. Gray

rosmarinifolium, Haenke

tetragonum, Linn.

Erigeron aurantiacus, Regel glabellus, Nutt.

- var. mollis

multiradiatus, Benth. et Hook.f.

Roylei, DC.

speciosus, DC.

trifidus, Schlecht.

Erinus *alpinus, Linn.

Erodium carvifolium, Boiss. serotinum, Stev.

Eruca sativa, Mill.

Eryngium alpinum, Linn.

Erysimum rupestre, DC.

Eschscholzia californica, Cham.

Eucharidium concinnum, Fisch, et Mey., var. grandiflorum

Eupatorium glechonophyllum, Less. purpureum, Linn.

Euphorbia Lathyris, Linn. verrucosa, Hort. 
Funkia lancifolia, Spreng., var. albomarginata

Sieboldiana, Spreng.

Galanthus Elwesii, Hook. f.

- var. unguicularis

Galium *purpureum, Linn. pusillum, Linn.

saxatile, Linn.

Gentiana asclepiadea, Linn.

- var. alba

acaulis, Linn.

Burseri, Lapeyr.

cruciata, Linn.

*decumbens, Linn.

Kesselringii, Regel

lutea, Linn.

punctata, Linn.

Saponaria, Linn.

septemfida, Pall., var. cordifolia (Hook.)

tibetica, King.

*verna, Linn.

Geranium albiflorum, Ledeb.

cinereum, Cav.

ibericum, Cav.

pratense, Linn.

sylvaticum, Linn.

Gerbera nivea, Sch. Bip.

Geum coccineum, Sibth. et Sm. elatum, Wall.

pyrenaicum, Mill.

strictum, Soland.

Gilia capitata, Sims multicaulis, Benth.

Glaucium corniculatum, Curt.

Fischeri, Bernh.

flavum, Crantz

Hedysarum flexuosum, Linn.

Heliophylla amplexicaulis, Linn. f.

Heracleum villosum, Fisch.

Hesperis *violacea, Boiss.

Heuchera Drummondii, Hort. hispida, Pursh.

*laxa, Hort.

sanguinea, Engelm.

Hieracium alpinum, Linn.

Bornmullerii, Hort.

bupleuroides, C. C. Gmel.

crinitum, Sibth. et Sm.

cymosum, Linn.
Hieracium - contd.

foliosum, Waldst. et Kit.

iricum, Fries

Jankae, Uechtr.

laciniatum, Hort.

lingulatum, Backh.

Marshalli, Linton

Neo-cerinthe, Fries

Pilosella, Linn.

prenanthoides, Vill.

pulmonarioides, Vill.

sabaudum, Linn.

scoticum, Hort.

Homogyne alpina, Cass.

sylvestris, Cass.

Hordeum maritimum, With.

Hyoscyamus niger, Linn.

Hypericum Androsæmum, Linn.

hirsutum, Linn.

montanum, Linn.

orientale, Linn.

perforatum, Linn.

pyramidatum, Dryand.

quadrangulum, Linn.

Richeri, Vill., var. Burseri (Spach)

tetrapterum, Fries

Hypochæris maculata, Linn.

Hyssopus officinalis, Linn.

Iberis amara, Linn.

intermedia, Guersent

semperflorens, Linn.

sempervirens, Linn.

-- var. superba

umbellata, Linn.

- var. purpurea

Impatiens Roylei, Walp.

Inula glandulosa, Puschk.

Hookeri, C. B. Clarke

Iris biflora, Linn.

lurida, Soland.

nepalensis, D. Don

setosa, Pall.

sibirica, Linn.

- var. alba

- var. flexuosa

- var. orientalis

tenax, Dougl.

versicolor, Linn.

xiphioides, Ehrh.

Isatis tinctoria, Linn.

Isopyrum fumarioides, Linn. 
Kniphofia Tuckii, Baker

Lactuca alpina, Benth. et Hook. f.

*virosa, Linn.

Lallemantia peltata, Fisch. et Mey.

Lamium album, Linn.

Laserpitium Siler, Linn.

Lathyrus articulatus, Linn.

Clymenum, Linn.

Drummondii, Hort.

latifolius, Linn.

macrorrhizus, Wimm.

magellanicus, Lam.

nervosus, Boiss.

odoratus, Linn.

Ochrus, DC.

rotundifolius, Willd.

tingitanus, Linn.

vernus, Linn.

Leonurus Cardiaca, Linn.

Lepidium tenuicaule, T. Kirk

Leucojum vernum, Linn., var. carpaticum, Herb.

Libertia grandiflora, Sweet

Ligusticum ferulaceum, All.

Lilium canadense, Linn., var. penduliflorum (DC.)

Humboldtii, Roezl et Leicht., var. magnificum, Hort. Purdy

Leichtlini, Hook. f.

Martagon, Linn,, var, dalmaticum (Vis.)

pardalinum, Kellogg

tigrinum, Ker-Gawl,, var. splendens (Baker)

Linaria *bipartita, Willd., var. splendens

dalmatica, Mill.

vulgaris, Mill.

Linum campanulatum, Linn.

perenne, Linn.

Lopezia racemosa, Cav.

Lophanthus rugosus, Fisch, et Mey.

Lotus major, Scop.

Lunaria annua, Linn.

Lupinus Cruckshanksii, Hook.

Dunnettii, Hort.

elegans, H. B. et $\mathbf{K}$.

*hirsutus, Linn.

insignis $\times$, Lem.

micranthus, Dougl.

nootkatensis, Don
Lupinus-contd.

perennis, Linn.

polyphyllus, Lindl.

- var. albus

Lychnis alba, Mill.

chalcedonian, Linn., var. carnea

coronaria, Desv.

Flos-jovis, Desv.

Githago, Scop.

Viscaria, Linn.

- var. alba

Lysimachia lobelioides, Wall. punctata, Linn.

Lythrum Salicaria, Linn.

- var. grandiflorum

virgatum, Linn.

Madia sativa, Molina

Malcolmia *africana, R. Br.

Malva sylvestris, Linn. thuringiaca, $\mathrm{V}$ is.

Marrubium leonuroides, Desv,

Matthiola arborescens, Sternb. bicornis, DC.

Meconopsis *aculeata, Royle nepalensis, DC. simplicifolia, G. Don

Medicago carstiensis, Jacq. denticulata, Willd. orbicularis, All. intertexta, Mill.

Megacaryon armenum, Boiss.

Melilotus indica, All.

Meum athamanticum, Jacq.

Morina longifolia, Wall.

Muscari armeniacum, Leichtlin

*atlanticum, Boiss, et Reut.

Maweanum, Baker polyanthum, Boiss.

*racemosum, Mill.

Szovitsianum, Baker

Myosotis *australis, $\mathrm{R}$. Br.

*Traversii, Hook. f.

Welwitschii, Boiss, et Reut.

Napra dioica, Linn.

Nemesia strumosa, Benth. - - var. Suttoni

Nepeta linearis, Royle

Mussini, Spreng.

nuda, Linn.

Sibthorpii, Benth. 
Neslia paniculata, Desv,

Nicandra physaloides, Gaertn.

Nigella sativa, Linn.

Oenanthe crocata, Linn. pimpinelloides, Linn.

Oenothera pumila, Linn.

*rosea, Ait.

Onopordon Acanthium, Linn.

Sibthorpianum, Boiss. et Heldr.

Ononis hircina, Jacq.

*Natrix, Linn. spinosa, Linn.

Origanum vulgare, Jinn.

Oxalis "corniculatus, Linn., var. tropæoloides, Hort.

Oxytropis glabra, DC. strobilacea, Bunge

Papaver Apulum, Tenore lateritium, C. Koch monanthum, Trautv. orientale, Linn. - var. bracteatum (Lindl.)

*pilosum, Sibth. et Sm. rupifragum, Boiss. et Reut.

Parnassia palustris, Linn.

Peltaria alliacea, Jacq.

Pentstemon acuminatus, Dougl.

barbatus, Roth

deustus, Dougl.

ovatus, Dougl.

pubescens, Soland.

Perezia multiflora, Less.

Peucedanum coriaceum, Reichb.

Phaseolus * Mungo, Linn.

Phormium tenax, Forst.

Physalis peruviana, Linn.

Phyteuma Balbisii, A. DC. canescens, Waldst. et Kit.

Halleri, All.

hemisphæricum, Linn.

*laxiflorum, Hort.

Michelli, All., var. angustifolia orbiculare, Linn.

Scheuchzeri, All.

serratum, Viv.

Sieberi, Spreng.

Pisum elatius, Bieb.

Plantago lanceolata, Linn.

major, Linn.

maritima, Linn.
Plantago-contd.

montana, Huds.

Psyllium, Linn.

Rugelii, Deene.

subulata, Linn.

Platystemon californicus, Benth.

Polemonium cæruleum, Linn.

- var. album

- var. dissectum (Reichb.)

- var. monstrosum

himalayanum, Baker

mexicanum, Cerv.

*pauciflorum, S. Wats.

Polygonatum multiflorum, All.

Potentilla andicola, Benth.

apennina, Tenore

argyrophylla, Wall.

Buschani, Hort.

chinensis, Ser.

Dombeyi, Nestl.

gelida, C. A. Mey.

glandulosa, Lindl.

grandiflora, Linn.

Hopwoodiana $\times$, Sweet

Macnabiana $\times$, Lem.

malacophylla, Borb.

nevadensis, Boiss.

norvegica, Linn.

opaca, Linn.

palustris, Scop.

recta, Linn,, var, palmata

rupestris, Linn.

sericea, Linn.

Skovitzii, Hort.

Vlasicensis, Siegfr.

Prenanthes *purpurea, Linn.

Primula *apennina, Tenore

*Arctotis $\times$, Kern.

auricula, Linn., var. monacensis

*capitata, Hook.

elatior, Hill

- var. carpatica (Fuss.)

farinosa, Linn.

- var. magellanica (Lehm.)

- var. *scotica (Hook.)

frondosa, Janka

japonica, A. Gray

grandis, Trautv.

involucrata, Wall.

- var. Munroi

*longiflora, All.

officinalis, Jacq.

- var. *Tommasinii (Gren. et Godr.) 
Primula officinalis-contd. - var. *uralensis (Fisch.)

Peyritschii $\times$, Stein

Poissoni, Franch.

pubescens $\times$, Jacq.

*Reidii, Duthie

sikkimensis, Hook.

*venusta $\times$, Host

viscosa, Vill.

- var, nivalis, Hort.

Prunella grandiflora, Jacq. vulgaris, Linn.

- var. alba

- var. Webbiana

- var. major

Puschkinia scilloides, Adams

- var. compacta

Pyrola *media, Sw.

Ramondia *pyrenaica, Rụch.

— var. *alba

Ranunculus aconitifolius, Linn.

*lanuginosus, Linn.

millefoliatus, Vahl

*pyrenæus, Linn.

velutinus, Tenore

Reseda lutea, Iinn.

Rheum Collinianum, Baill.

leucorhizum, Pall.

Rhaponticum, Linn.

Ribes, Linn.

tataricum, Linn. f.

Rumex acutus, Linn.

Ruta graveolens, Linn. macrophylla, Soland.

Salvia austriaca, Jacq.

*Carduacea, Benth. glutinosa, Linn.

sylvestris, Linn.

- var. alba

taraxacifolia, Coss. et Bal.

verticillata, Linn.

virgata, Ait.

Sanicula europæa, Linn.

Saxifraga *Aizoon, Linn., var. minima

- var, rosularis (Haw.)

- var. Sturmiana, Schott, Nym. et Kotschy

*Andrewsiix, Harvey, var. Guthrieana, Hort.
Saxifraga-contd.

*cartilaginea, Willd.

Cotyledon, Linn.

- var. pyramidalis, Lapeyr.

crustata, Vest., var. hybrida

- var. exarata, Vill.

Hostii, Tausch., var. elatior (Mert. et Koch)

*hybrida $\times$, Sternb.

intacta, Willd.

- var. farinosa

lingulata, Bell, var. cochlearis (Reichb.)

luteo-viridis, Schott et Kotschy

*MacNabiana $\times$, Hort.

*media, Gouan

*nivalis, Linn.

*paradoxa, Sternb.

rotundifolia, Linn.

umbrosa, Linn., var. Ogilveana, Hort.

Scabiosa *aucasica, Bieb.

- var. *alba

prolifera, Linn.

sylvatica, Linn.

Scilla bifolia, Linn.

* - var. rubra

- var. taurica

festalis, Salisb.

- var. *alba

*hispanica, Mill., var. grandiflora patula, DC.

- var. *major

*peruviana, Linn.

Scorpiurus muricata, Linn.

vermiculata, Linn.

Scorzonera stricta, Hornem.

Scrophularia Kotschyana, Benth.

$$
\text { nodosa, Linn. }
$$

Scutellaria altissima, Linn.

Selinum *Candollii, DC.

Sempervivum leucanthum, Panč.

Senecio argunensis, Turcz.

Doronicum, Linn.

sibiricum, Linn.

Serratula coronata, Linn.

heterophylla, Desf.

tinctoria, Linn.

Sidalcea malvæflora, A. Gray

Sideritis scordioides, Linn.

Silene alpestris, Jacq.

Armeria, Linn.

ciliata, Pourr.

colorata, Poir, 
Silene-contd.

conoidea, Linn.

Czerei, Baumg.

gallica, Linn.

maritima, With.

Muscipula, Linn.

nutans, Linn.

*odontopetala, Fenzl

Saxifraga, Linn.

Sendtneri, Boiss.

stylosa, Bunge

Thorei, Duf.

Sisymbrium Sophia, Linn.

Sisyrinchium angustifolium, Mill.

- var. anceps (Cav.)

- var. mucronatum (Michx.)

Solanum Dulcamara, Linn. nigrum, Linn.

Solidago serotina, Ait.

Virgaurea, Linn.

Sonchus oleraceus, Linn.

Spiræa Filipendula, Linn.

Humboldtii, Hort.

palmata, Sternb.

- var. alba

Ulmaria, Linn.

Stachys recta, Linn.

Statice Gougetiana, Girard

Symphyandra pendula, A. DC.

Symphytum asperrimum, Donn *pictum, Hort.

Synthyris *reniformis, Benth.

Tellima grandiflora, R. Br.

Thalictrum majus, Murr.

Thlaspi arvense, Linn.

Tofieldia calyculata, Wahlenb.

Tricyrtis *macropoda, Miq.

Trifolium alpinum, Linn.

glomeratum, Linn.

pannonicnm, Jacq.

pratense, Linn.

repens, Linn.

- var. pictum

striatum, Linn.

Trigonella Fœenum-græcum, Linn.

Trillium grandiflorum, Salisb.

Trilisa *odoratissima, Cass.

Triticum bicorne, Forsk.

monococeum, Linn.

ovatum, Gren. et Godr.
Trollius asiaticus, Linn.

- var. hybridus

caucasicus, Stev.

europæus, Linn.

- var. napellifolius (Roep.)

americanus, Muhl.

Tropæolum majus, Linn.

mexicanum, Linn.

* peregrinum, Linn.

Tulipa pulchella, Fenzl

Tunica prolifera, Scop.

Typha angustifolia, Linn.

latifolia, Linn.

Valeriana tuberosa, Linn.

Venidium *decurrens, Less.

Veratrum Maackii, Regel

Verbascum longifolium, Tenore

Thapsus, Linn.

Veronica austriaca, Linn.

Bidwillii, Hook. f.

crassifolia, Zeyh.

fruticulosa, Linn.

gentianoides, Vahl

- var. variegata

glauca, Sibth. et Sm.

grandis, Fisch.

incisa, Soland.

officinalis, Linn.

Vicia Cracca, Linn.

dumetorum, Linn. sylvatica, Linn.

Vincetoxicum fuscatum, Reichb. nigrum, Moench officinale, Moench

Viola *anadensis, Linn., var. alba canina, Linn.

- var. alba

macedonica, Boiss. et Heldr.

persicifolia, Roth

primulæfolia, Linn.

sciaphylla, Koch

sylvestris, Lam.

Xanthium strumarium, Linn.

Zygadenus elegans, Pursh

- var. glaueus (Nutt.)

- var. major 


\section{TREES AND SHRUBS.}

Acer Pseudo-platanus, Linn.

Alnus cordifolia, Ten. glutinosa, Medic.

Berberis angulosa, Wall. Aquifolium, Pursh nervosa, Pursh vulgaris, Linn.

Betula alba, Linn. - var. pendula, Hort.

Buddleia intermedia, Carr.

Caragana *arborescens, Lam. Clematis Vitalba, Linn.

Colutea istria, Mill.

Cotoneaster buxifolia, Wall. horizontalis, Decne.

Simonsii, Baker

Cratægus coccinea, Linn. - var. indentata, Lodd. mollis, Scheele

*Pyracantha, Pers.

Cytisus capitatus, Jacq. scoparius, Link - var. Andreanus

Daphne Mezereum, Linn. - var. album

Gaultheria *procumbens, Linn. *Shallon, Pursh

Hydrangea paniculata, Sieb. et Zuce.

Ilex Aquifolium, Linn.

Laburnum alpinum, J. S. Presl vulgare, J. S. Presl

Lonicera tomentella, Hook. f. et Thoms.

Lupinus arboreus, Linn.
Notospartium * Carmichaeliæ, Hook. f.

Olearia Haastii, Hook. f.

Pernettya mucronata, Gaudich. Phygelius capensis, E. Mey. Piptanthus nepalensis, Sweet Prunus *acida, Borkh., var. semperflorens

Avium, Linn.

Pyrus americana, DC.

Aria, Linn.

Aucuparia, Gaertn. intermedia, Ehrh.

rotundifolia, Bechst.

Quercus Cerris, Linn. sessiliflora, Salisb.

Rhamnus catharticus, Linn. Purshianus, DC.

Rhododendron ferrugineum, Linn. flavum, G. Don hirsutum, Linn. - var. variegatum ponticum, Linn.

Rhodotypus kerrioides, Sieb. et Zucc. Rosa rugosa, Thunb. - var. alba

Sambucus canadensis, Linn. nigra, Linn.

Skimmia Fortunei, Masters

Spirza Douglasii, Hook. Lindleyana, Wall.

Symphoricarpus racemosus, Michx. Syringa Emodi, Wall., var. variegata

Taxus baccata, Linn. 
Veronica *amplexicaulis, Armstr.

*anomala, Armstr.

*Balfouriana, Hook. f.

Benthamiana, Hook. f.

*Cookiana, Colens.

*decumbens, Armstr. imperialis $\times$, Hort. Kirkii, Armstr.
Veronica-contd.

*Lavaudiana, Raoul

lobelioides, Armstr.

*Lyallii, Hook, f.

*parviflora, Vahl

*pimelioides, Hook. f.

salicifolia, Forst.

Traversii, Hook. f.

\section{PLANTS UNDER GLASS.}

Acacia prismatica, Hoffingg.

Agave *maculata, Regel

Adenocarpus *anagyrus, Spreng.

Acokanthera spectabilis, Hook.

Aloe Thraskii, Baker

Alonsoa linearis, Ruiz et Pav.

Armeria latifolia, Willd.

Astrocaryum mexicanum, Liebm.

Begonia Dregej, Otto et Dietr.

*Verschaffeltii $\times$, Regel

*Evansiana, Andr.

Bomarea Carderi, Mast.

*Lehmanni, Baker

Boenninghausenia albiflora, Reichb.

Cassinia leptophylla, R. Br.

Celosia cristata, Linn.

Camellia *Thea, Link

Ceratopetalum *gummiferum, Sm.

Clematis indivisa, Willd.

*Viorna, Linn.

Cleome graveolens, Rafin.

Cobrea scandens, Cav.

Cotyledon *reticulatus, Thunb.

Coffea *arabica, Linn.

Coccocypselum discolor, Van Houtte

Costus igneus, N. E. Br.

Cyphomandra betacea, Sendt.

Drosera capensis, Linn.

*ramentacea, Pursh

*spathulata, Labill.
Drosera-contd.

*hilaris, Cham. et Schlecht

*Burkeana, Planch.

Dionæa *muscipula, Ellis

Gossypium arboreum, Linn. neglectum, Tod.

Grammanthes gentianoides, DC.

Heeria *rosea, Triana

Hibiscus lunariifolius, Willd. pedunculatus, Linn.

Humea elegans, Sm.

Hypericum *balearicum, Linn.

Hypoxis *stellata, Linn.

Leptospermum scoparium, Forst.

Luffa ægyptiaca, Mill.

Mimosa marginata, Lindl.

Mesembryanthemum *roseum, Willd. curviflorum, Haw.

*lepidum, Haw. noctiflorum, Linn.

*micans, Linn.

formosum, Haw. cordifolium, Linn.

Myrtus communis, Linn. Ugni, Molina.

Nesæa myrtifolia, Desf.

Nicotiana Tabacum, Linn. 
Oxypetalum cœruleum, Decne.

Ornithogalum *tenellum, Jacq.

Philydrum lanuginosum, Banks

Primula mollis, Nutt.

Ricinus communis, Linn.

Rivina humilis, Linn.

Rhynchosia caribæa, DC.
Stapelia marmorata, Jacq. Senecio * grandifolius, Less.

Siphocampylus *lanceolatus, Regel Sandersonia *aurantiaca, Hook.

Solanum *aviculare, Forst.

Sempervivum annuum, C. Sm.

Senecio cruentus, DC.

Tetranema *mexicana, Benth.

Villamilla octandra, Hook. f.

\section{ISAAC BAYLEY BALFOUR, Regius Keeper.}
A. D. RICHARDSON,
R. L. HARROW,
Head Gardener.
Assistant Head Gardener.

Edinbergh, January 1899. 









\title{
NOTES
}

FROM THE

ROYAL BOTANIC GARDEN,

\author{
E D I N B U R GH.
}

NOVEMBER 1900.

CONTENTS,

List of Seeds collected in the Royal Botanic Garden, Edinburgh, during the Year 1900, _ _ -

Page.

$\times$ Lis Edinburgh, durit

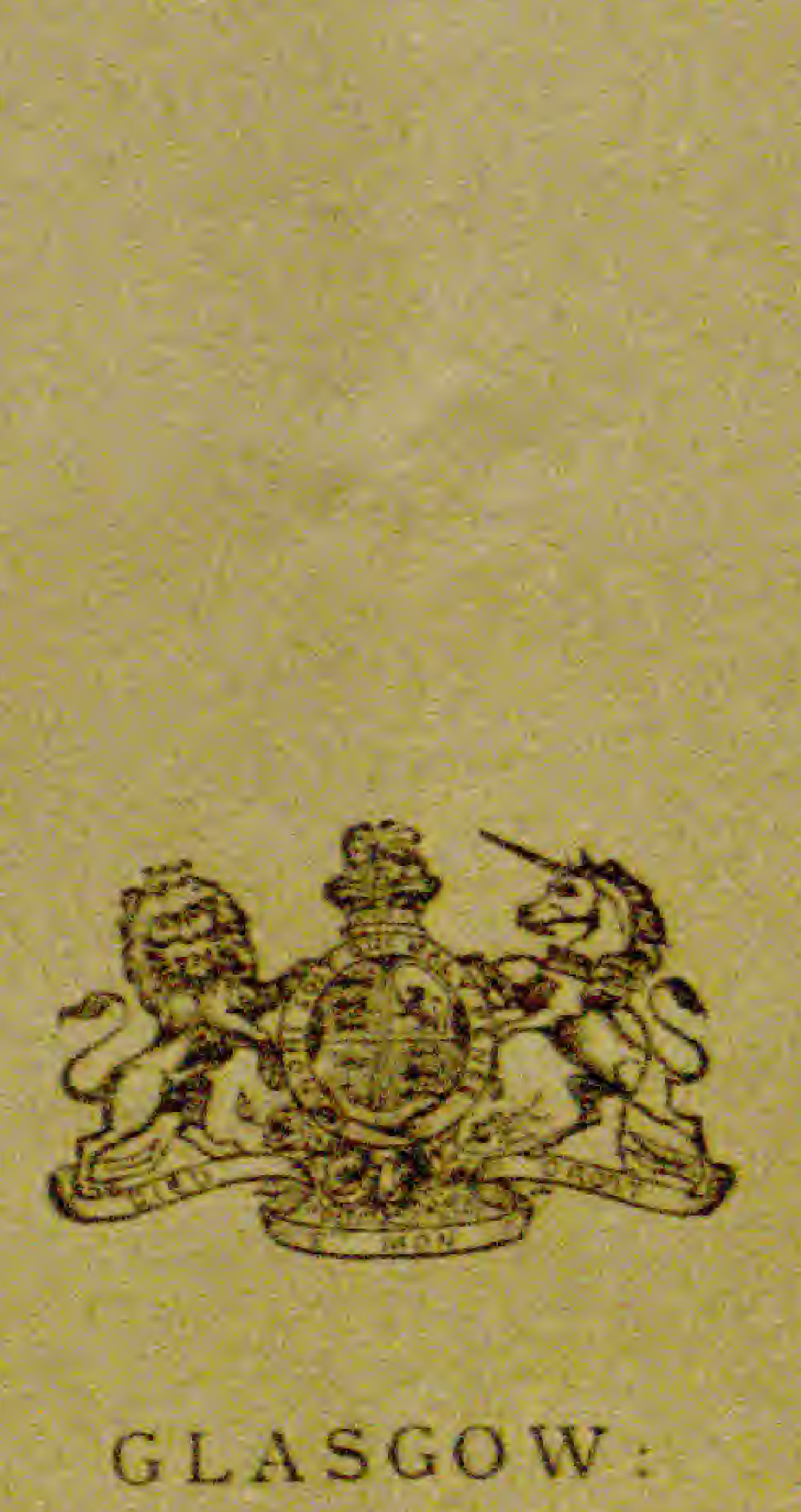

25

PRINTED FOR HER MAJESTY'S STATIONERY OFFICE BY JAMES HEDDERWICK \& SONS, AT THE "CITIZEN" PRESS, ST. VINCENT PLACE. SOLD AT THE GARDEN,

And to be purchased, either directly or through any Bookseller, from JOHN MENZIES \& CO., ROSE STREET, l.DINBURGH, and go West Nile Street, Glasgow: 


\section{Mo. Bots Garden,}

\section{List of Seeds Collected in the Royal Botanic Garden, Edinburgh, during the Year 1900.}

The following is a list of plants cultivated in the Royal Botanic Garden, Edinburgh, from which ripened seeds have been collected during the year I9oo. The quantity of seed obtained from some of the species is of limited amount. The seeds are available for exchange, but they are not for sale :-

\section{HERBACEOUS PLANTS.}

\section{Acantholimon}

glumaceum, Boiss.

\section{Achillea}

Jaborneggi $\times$, Halacsy.

\section{Aciphylla}

squarrosa, Forst.

\section{Aconitum}

Anthora, Linn.

\section{Actæa}

alba, Mill.

spicata, Linn.

\section{Adenophora}

stylosa, Fisch.

\section{Ethionema}

cappadocicum, Spreng.

cordatum, Boiss.
Agrimonia

Eupatoria, Linn.

Alonsoa

caulialata, Ruiz et Pav.

\section{Alstrœmeria}

chilensis, Lem.

\section{Alyssum}

calycinum, Linn.

creticum, Linn.

libycum, Coss.

Moehlendorfianum, Hort. montanum, Linn. saxatile, Linn.

\section{Androsace}

elongata, Linn., var. nana.

filiformis, Retz.

lactea, Linn.

maxima, Linn. 


\section{Anemone}

alpina, Linn.

— var. sulphurea.

Halleri, All.

obtusiloba, D. Don.

polyanthes, $D$. Don.

pratensis, Linn.

rivularis, Buch.-Ham.

sulphurea, Linn.

sylvestris, Linn.

\section{Anthericum}

Liliago, Linn.

— var. algeriense.

\section{Aquilegia}

Bertołonii, Schott.

formosa, Fisch.

\section{Arabis}

albida, Stev .

Allionii, $D C$.

alpina, Linn.

- var. anachoretica, Port.

blepharophylla, Hook. et Arn. Breweri, S. Wats.

lucida, Linn. fil.

\section{Arenaria}

austriaca, Jacq.

\section{Arum}

maculatum, Linn.

palæstinum, Boiss.

\section{Asarum}

canadense, Linn.

caudatum, Lindl.

europæum, Linn.

grandiflorum, Klotzsch.

\section{Aster}

alpinus, $\operatorname{Linn}$.

— var. altaicus.

- var. ruber.

\section{Astilbe}

chinensis, Franch. et Sav.

Thunbergii, Miq.

\section{Astragalus}

danicus, Retz.

Glyciphyllus, Linn.

\section{Astrantia}

carniolica, Wulf.

major, Linn.

\section{Athamanta}

Matthioli, Wulf.

\section{Aubrietia}

deltoidea, $D C$, var. Columnæ (Guss.).

\section{Avena}

sterilis, Linn.

\section{Barbarea}

arcuata, Reichb.

præcox, R. Br.

vulgaris, $R$. $B r$.

\section{Bottionea}

thysanthoides, Colla.

\section{Brassica}

Cheiranthos, Vill.

\section{Bryonia}

dioica, Jacq.

\section{Bulbinella}

Hookeri, Benth. et Hook. fil.

\section{Calochortus}

venustus, Benth. var. citrinus.

- var. Eldorado.

- var. oculatus.

- var. pictus. 


\section{Camelina}

sativa, Crantz.

sylvestris, Wallr.

\section{Campanula}

barbata, Linn.

glomerata, Linn.

Hendersoni, Hort.

lactiflora, Bieb.

latifolia, Linn., var. macrantha (Fisch.)

linifolia, Scop.

persicifolia, Linn.

- var. alba.

- var. Backhousei.

- var. grandiflora.

rapunculoides, Linn.

rotundifolia, Linn.

— var. alba.

Trachelium, Linn., var. aggregata.

- var. flore pleno.

\section{Cardamine}

bulbiflora, R. Br.

\section{Carlina}

corymbosa, Linn.

\section{Cerastium}

perfoliatum, Linn.

\section{Chærophyllum}

aromaticum, Linn.

\section{Cheiranthus}

Cheiri, Linn.

\section{Chelidonium}

majus, Linn.

- var. laciniatum (Mill.).

\section{Chrysanthemum}

anserinæfolium, Hausskn. et Born.

ceratophylloides, All.

maximum, Ramond.

\section{Cochlearia}

officinalis, Linn.

\section{Codonopsis}

rotundifolia, Royle.

\section{Collomia}

grandiflora, Doug.

\section{Coronilla}

elegans, Panc.

\section{Corydalis}

glauca, Pursh.

\section{Delphinium}

crassicaule, Ledeb.

elatum, Linn., var. glabra.

grandiflorum, Linn.

hybridum, Steph.

speciosum, Bieb., var. turkestanicum.

truncatum, Hort.

velutinum, Bertol.

\section{Dianthus}

cæsius, Sm.

calocephalus, Boiss.

chinensis, Linn.

deltoides, Linn.

glacialis, Haenke., var. gelidus.

Hellwigii $\times$, Borb.

moesiacus, Vis. et Panc.

superbus, Linn.

\section{Dictamnus}

albus, Linn.

\section{Digitalis}

ambigua, Murr.

lutea, Linn.

purpurea, Linn. 


\section{Draba}

arabisans, Michx.

aurea, $V a h l$.

carinthiaca, Hoppe, var. Traunsteineri (Hoppe).

fladnizensis, Wulf.

hispida, Willd.

incana, Linn.

- var. hebecarpa.

Kotschyi, Stur.

lactea, Adams.

longirostra, Schott, Nym. et Kotschy.

rupestris, $R$. $\mathrm{Br}$.

siliquosa, Bieb.

\section{Epilobium}

angustifolium, Linn.

Hectori, Hausskn.

luteum, Pursh.

parviflorum, Schreb.

rosmarinifolium, Haenke.

\section{Erigeron}

glabellus, Nutt.

macranthus, Nutt.

multiradiatus, Benth. et Hook. fil.

Roylei, $D C$.

speciosus, $D C$.

\section{Erinus}

alpinus, Linn.

\section{Erodium}

curvifolium, Boiss, et Reut.

ciconium, Willd.

\section{Eryngium}

cæruleum, Bicb.

\section{Erysimum}

thyrsoideum, Boiss.

\section{Eschscholzia}

californica, Cham.

- var. alba.

\section{Eupatorium}

cannabinum, Linn.

\section{Fritillaria}

aurea, Schott.

camtschatcensis, Ker-Gaw

\section{Funkia}

Sieboldiana, Hook.

\section{Gaillardia}

aristata, Pursh., var. grandiflora, Hort.

\section{Gentiana}

asclepiadea, Linn.

punctata, Linn.

Saponaria, Linn.

verna, Linn.

\section{Geranium}

asphodeloides, Burm. fil.

cinereum, Cav.

Endressi, Gay.

sanguineum, Linn.

- var. lancastriense (Mill.).

- var. roseum.

sibiricum, Linn.

subcaulescens, L'Herit.

\section{Gerbera}

nivea, Sch. Bip.

\section{Geum}

montanum, Linn.

nutans, Hort. Par.

parviflorum, Commers.

pyrenaicum, Mill.

rubellum, Fisch. et Mey 


\section{Globularia}

trichosantha, Fisch, et Mey.

\section{Gypsophila}

acutifolia, Fisch.

dubia, Willd.

\section{Helleborus}

antiquorum, $A$. Br., var. roseus. colchicus, Regel.

\section{Hesperis}

matronalis, Linn.

\section{Heuchera}

bracteata, Ser.

Drummondii, Hort. macrophylla, Lodd.

sanguinea, Engelm.

\section{Hieracium}

argenteum, Fries.

iricum, Fries.

rigidum, Hartm.

scoticum, Hort.

tridentatum, Fries.

villosum, Jacq.

\section{Homogyne}

alpina, Cass.

\section{Hyacinthus}

romanus, $\operatorname{Linn}$.

\section{Hypericum}

pulchrum, Linn.

Richeri, Vill., var. Burseri (Spach).

\section{Iberis}

intermedia, Guersent. semperflorens, Linn. sempervirens, Linn.

- var. superba. umbellata, Linn.

\section{Impatiens}

Noli-tangere, Linn.

\section{Incarvillea}

Delavayi, Bureau et Franch.

\section{Inula}

ensifolia, Linn.

Helenium, Linn.

Iris

sibirica, Linn.

— var. alba.

- var. flexuosa.

tenax, Dougl.

\section{Isatis}

tinctoria, Linn.

\section{Jasione}

montana, Linn.

\section{Kniphofia}

Tuckii, Baker.

\section{Lactuca}

muralis, E. Mey.

\section{Lathyrus}

canescens, Gren. et Godr. macrorrhizus, Wimm. magellanicus, Lam. montanus, Bernh.

\section{Leontopodium}

alpinum, Cass.

\section{Lepidium}

Menziesii, $D C$.

\section{Leucojum}

vernum, Linn, var. carpaticum (Herb.). 


\section{Ligusticum}

scoticum, Linn.

\section{Lilium}

Washingtonianum, Kellogg, var. purpureum.

\section{Linaria}

maroccana, Hook. fil.

\section{Lindelofia}

spectabilis, Lehm.

\section{Linum}

alpinum, Linn.

perenne, Linn.

\section{Lotus}

corniculatus, Linn.

\section{Lunaria}

annua, Linn.

\section{Lupinus}

micranthus, Dougl.

rivularis, Dougl.

\section{Lychnis}

divaricata, Reichb.

Flos-jovis, Desr.

Githago, Scop.

pyrenaica, Berger.

Viscaria, Linn.

- var. splendens.

\section{Malva}

Alcea, Linn.

borealis, Wallm.

crispa, Linn.

\section{Mandragora}

officinarum, $\operatorname{Linn}$.

\section{Meconopsis}

cambrica, Vig.

Wallichii, Hook.

- var. fusco-purpurea.

\section{Medicago}

truncatula, Gaertn.

\section{Melilotus}

elegans, Salzm.

Mesembryanthemum

pomeridianum, Linn.

\section{Mitella}

pentandra, Hook.

\section{Morina}

longifolia, Wall.

\section{Muscari}

Argæi, Hort.

armeniacum, Leichtlin.

botryoides, Mill.

Maweanum, Baker.

moschatum, Willd.

\section{Ochthodium}

ægypticum, $D C$.

\section{Enothera}

biennis, Linn.

\section{Onobrychis}

sativa, Lam.

\section{Ononis}

arvensis, Linn.

\section{Orchis}

mascula, Linn.

\section{Oxytropis}

glabra, $D C$.

lapponica, Gaud.

strobilacea, Bunge. 


\section{Papaver}

alpinum, Linn.

Heldreichii, Boiss.

nudicaule, Linn., var. miniatum. pilosum, Sibth. et Sm.

somniferum, Linn.

\section{Paradisea}

Liliastrum, Bertol.

\section{Pedicularis}

palustris, Linn.

— var. alba.

\section{Pentstemon}

confertus, Dougl.

deustus, Dougl.

diffusus, Dougl.

glaucus, R. Grah., var, stenope-

talus.

ovatus, Dougl.

\section{Phlomis}

setigera, Falc.

\section{Phyteuma}

orbiculare, Linn.

Scheuchzeri, All.

Sieberi, Spreng.

spicatum, Linn.

\section{Picrorhiza}

Kurroa, Royle.

\section{Pisum}

sativum, Linn.

\section{Plantago}

Raoulii; Decne.

\section{Platycodon}

grandiflorum, $A . D C$, var. Mariesii.
Podopyllum

Emodi, Wall.

\section{Polemonium}

cæruleum, Linn.

- var. album.

- var. monstrosum.

himalayanum, Baker.

\section{Polygonum}

viviparum, $\operatorname{Linn}$.

\section{Potentilla}

alchemilloides, Lapeyr.

alpestris, Hall. fil.

malacophylla, Borb.

Menziesii $\times$, Paxt.

Sibbaldia, Hall. fil.

\section{Poterium}

canadense, A. Gray.

muricatum, Spach.

officinale, A. Gray.

\section{Primula}

Auricula, Linn., var. monacensis.

calycina, Duby

capitata, Hook.

carniolica, Jacq.

elatior, Hill, var. carpatica (Fuss.).

farinosa, Linn.

frondosa, Janka.

involucrata, Wall., var. Munroi.

sikkimensis, Hook.

viscosa, Vill., var. decora, Sims.

- var. nivalis, Hort.

\section{Prunella}

grandiflora, Jacq.

vulgaris, Linn.

- var. alba. 


\section{Pyrola}

media, Sw.

rotundifolia, Linn.

\section{Ramondia}

pyrenaica, Rich.

serbica, Panc.

- var. Nathalize (Panc. et Petrov.)

\section{Ranunculus}

acris, Linn.

bulbosus, Linn., var. fasciatus, Chius, $D C$.

\section{Reseda}

complicata, Bory.

Luteola, Linn.

\section{Rheum}

Rhaponticum, Linn.

\section{Sanicula}

europæa, Linn.

\section{Saxifraga}

Aizoon, Linn., var. balcana, Hort.

- carinthiaca (Schott).

- Malyi 'Schott, Nym. et Kotschy).

- minima.

- notata (Schoth, Nym. et Kotschy).

- pectinata (Schott).

- punctata.

- rosularis, Schl.

- Sturmiana (Schott, Nym. et Kotschy).

aspera, Linn. bronchialis, Linn.

- var. cherlerioides (D. Don).

Bucklandi, Hort., var. major.

Burseriana, Linn., var. multiflora.

crustata, Vest, var. hybrida.

decipiens, Ehrh.

- var. palmata (Panc.).

exarata, Vill.

granulata, Linn.

Hostii, Tausch.

- var. altissima (Kern.).

- var. elatior (Mert. et Koch).

hypnoides, Linn.

intacta, Willd.

— var. farinosa.

leucanthemifolia, Michx.

lingulata, Bell, var. australis (Moric.).

longifolia, Lapeyr.

mutata, Linn.

oppositifolia, Linn.

- var. alba.

paradoxa, Sternb.

pedatifida, Ehrh.

Portæ $\times$, Engl.

pseudo-sancta, Janka.

retusa, Gouan, var. bryoides.

- maritima.

rotundifolia, Linn,

sancta, Griseb.

stellaris, Linn.

tenella, Wulf.

tyrolensis, Kern.

umbrosa, Linn., var. Ogilveana

Hort.

- var. serratifolia (Mackay).

\section{Scabiosa}

caucasica, Bieb.

- var. alba. 
Scilla

bifolia, Linn., var. præcox.

festalis, Salisb.

- var. alba.

hispanica, Mill.,var. grandiflora. patula, $D C$.

- var. major.

\section{Scrophularia}

nodosa, Linn.

\section{Scutellaria}

alpina, Linn.

\section{Sedum}

album, Linn.

Anacampseros, Linn.

asiaticum, Spreng.

stoloniferum, S. T. Gmel.

\section{Sempervivum}

arvernense, Lecoq et Lamotte.

glaucum, Tenore.

Pomellii, Lamotte.

Schottii, Baker, var. acuminatum (Schott).

\section{Sidalcea}

candida, A. Gray.

malvæflora, A. Gray.

\section{Silene}

alpestris, Jacq.

Armeria, Linn.

caucasica, Boiss.

colorata, Poir.

Cucubalus, Wibel.

Drummondii, Hook.

italica, Pers.

muscipula, Linn.

nicæënsis, All.

quadridentata, Pers.

rhynchocarpa, Boiss.

Saxifraga, Linn.
Schafta, Gmel.

Sendtneri, Boiss.

Thorei, Duf.

vallesia, Linn.

verecunda, $S$. Wats.

\section{Silphium}

terebinthinaceum, Jacq.

\section{Sisymbrium}

Thalianum, J. Gay.

\section{Sisyrinchium}

angustifolium, Mill.

\section{Smilacina}

racemosa, Desf.

\section{Sobolewskya}

clavata, Fensl, var. cilicica.

\section{Solanum}

Dulcamara, Linn., var. alba.

\section{Solidago}

elongata, Nutt.

multiradiata, Ait., var. scopu lorum.

Virgaurea, Linn.

\section{Spergula}

arvensis, Linn.

\section{Spiræa}

astilboides, Carr.

Filipendula, Linn.

Ulmaria, Linn.

\section{Synthyris}

reniformis, Benth.

\section{Thalictrum}

angustifolium, Linn.

calabricum, Spreng. 
Thalictrum-continued.

glaucum, Desf. minus, Linn. simplex, Linn.

\section{Thermopsis}

fabacea, $D C$.

montana, Nutt.

\section{Thlaspi}

arvense, Linn.

\section{Tofieldia}

calyculata, Wahlenb.

\section{Trifolium}

agrarium, Linn.

alpinum, Linn.

pannonicum, Jacq.

striatum, Linn.

\section{Trillium}

erythrocarpum, Curt.

grandiflorum, Salisb.

ovatum, Pursh.

stylosum, Nutt.

\section{Tritonia}

rosea, Klatt.

\section{Trollius}

altaicus, C. A. Mey.

asiaticus, Linn., var. giganteus. europæus, Linn.

— var. pumilus albus.

patulus, Salisb., var. albus.

\section{Tunica}

stricta, Fisch. et Mey. velutina, Fisch. et Mey.

\section{Typha}

angustifolia, Linn.

latifolia, Linn.

\section{Veronica}

fruticulosa, Linn.

Guthriana $\times$, Hort.

longifolia, Linn.

Lyallii, Hook. fil.

satureioides, $V i$ s.

saxatilis, Scop.

\section{Vicia}

Cracca, Linn.

sylvatica, Linn.

unijuga, A."Braun.

villosa, Roth.

\section{Viola}

canadensis, Linn, var. alba.

cornuta, Linn.

macedonica, Boiss. et Heldr.

odorata, Linn., var. lutea.

persicifolia, Roth.

polychroma, Kern.

primulæfolia, Linn.

sylvestris, Lam.

\section{Waldsteinia}

trifolia, Koch.

\section{Wulfenia}

carinthiaca, Jacq. 


\section{TREES AND SHRUBS.}

Acer

Pseudo-platanus, Linn.

\section{Alnus}

cordifolia, Ten.

glutinosa, Medic.

incana, Medic.

\section{Amelanchier}

canadensis, Torr. et Gray.

\section{Arctostaphylos}

Uva-ursi, Spreng.

\section{Berberis}

angulosa, Wall.

Aquifolium, Pursh.

\section{Betula}

alba, Linn.

- var. pendula, Hort.

\section{Caragana}

arborescens, Lam.

\section{Clematis}

Vitalba, Linn.

\section{Colutea}

istria, Mill.

\section{Cornus}

alba, Linn.

\section{Cotoneaster}

frigida, Wall.

horizontalis, Decne

microphylla, Wall.

Simonsii, Baker.

\section{Cratægus}

mollis, Scheele.

Oxyacantha, Linn.

- var. pendula, Lodd.

\section{Cytisus}

nigricans, Linn.

scoparius, Link.

- var. Andreanus.

\section{Daphne}

Mezereum, Linn.

- var. album.

\section{Dryas}

octopetala, Linn.

\section{Genista}

sagittalis, $\operatorname{Linn.}$

\section{Helianthemum}

ægyptiacum, Mill.

ledifolium, Mill. umbellatum, Mill.

vulgare, Gaerin.

- var. mutabile.

- var. roseum.

- var. venustum.

Ilex

Aquifolium, $\operatorname{Linn}$.

- var. aurea angustifolia.

- var. camelliæfolia.

- var. flavescens.

- var. fructu luteo.

- var. Hodginsii.

- var. maderensis. 


\section{Laburnum}

alpinum, J. S. Presl.

vulgare, J. S. Presl.

\section{Ledum}

latifolium, Ait.

palustre, Linn.

\section{Nuttallia}

cerasiformis, Torr. et Gray.

\section{Olearia}

Haastii, Hook. fil.

\section{Pernettya}

mucronata, Gaudich.

\section{Prunus}

Avium, Linn.

\section{Pyrus}

\section{Aria, Linn.}

Aucuparia, Gaertn.

nivalis, Jacq.

rotundifolia, Bechst.

\section{Quercus}

Cerris, Linn.

pedunculata, Ehrh.

sessiliflora, Salisb.

\section{Rhamnus}

catharticus, Linn.

Frangula, Linn.

\section{Rhododendron}

catawbiense, Michx.

caucasicum, Pall.

ferrugineum, Linn.

- var, album, Szocet.

flavum, $G$. Don.

hirsutum, Linn.
Rhododendron-continued.

hirsutum, var. album.

- var. variegatum.

ponticum, Linn.

\section{Rhodotypos}

kerrioides, Sieb. et Zucc.

\section{Rosa}

rugosa, Thunb.

- var. alba.

\section{Sambucus}

canadensis, Ltnn.

nigra, Linn.

- var. fructu albo.

\section{Skimmia}

Fortunei, Mast.

\section{Symphoricarpus}

racemosus, Michx.

\section{Taxus}

baccata, Linn.

- var. Dovastoni, Carr.

- var. fastigiata, Loud.

\section{Thymus}

Chamædrys, Fries, var: comosus (Heuff.).

Tilia

vulgaris, Hayne.

\section{Veronica}

anomala, Armstr.

Bidwillii, Hook. fil.

Colensoi, Hook. fil., var. glauca.

monticola, Armstr.

pinguifolia, Hook. fil.

vernicosa, Hook. fil., var. pur purea.

\section{Viburnum}

Lantana, Linn.

Opulus, Linn. 


\section{PLANTS UNDER GLASS.}

\section{Acokanthera}

spectabile, Hook. fil.

Actinostemma

biglandulosum, Hemsl.

\section{Ardisia}

crenata, $R o x b$

\section{Aristolochia}

brasiliensis, Mart.

fimbriata, Cham.

\section{Asparagus}

scandens, Thunb.

\section{Begonia}

coccinea, Hook.

Dregei, Otto et Dietr.

Knowsleyana, Hort.

manicata, Cels.

nitida, Dry.

Verschaffeltii, Hort.

\section{Billardiera}

longiflora, Labill.

\section{Billbergia}

pallidiflora, Liebm.

\section{Bomarea}

multiflora, Mirb.

\section{Brachychilum}

Horsfieldii, Baker.

\section{Brodiæa}

lactea, S. Wats.
Camellia

theifera, Griff.

\section{Cassia}

corymbosa, Lam.

\section{Chlorophytum}

orchidastrum, Lindl

\section{Clethra}

arborea, Ait.

\section{Cobæa}

scandens, Cav.

\section{Coffea}

arabica, Linn.

\section{Costus}

igneus, $N . E . B r$.

\section{Cotyledon}

Desmetiana, Hemsl.

\section{Crossandra}

undulæfolia, Salisb.

\section{Cyclanthera}

explodens, Naud.

\section{Darlingtonia}

californica, Torr.

\section{Dracæna}

phrynioides, Hook. fil

\section{Drosophyllum}

lusitanicum, Link. 


\section{Drosera}

capensis, Linn.

filiformis, Rafin.

peltata, $S m$.

rotundifolia, Linn.

spathulata, Labill.

\section{Eucalyptus}

ficifolia, F. Muell.

urnigera, Hook. fil.

\section{Ficus}

diversifolia, Blume.

\section{Fuchsia}

procumbens, R. Cunn.

\section{Gazania}

pygmæa, Sond.

\section{Genista}

monosperma, Lam.

\section{Gossypium}

arboreum, Linn.

herbaceum, Linn.

neglectum, Tod.

\section{Heeria}

rosea, Triana.

\section{Hedychium}

Gardnerianum, Rosc.

\section{Hibiscus}

esculentus, Linn.

lunariifolius, Willd.

Manihot, Linn.

pedunculatus, Linn.

\section{Humea}

elegans, $S m$.

\section{Hydrolea}

spinosa, Linn.

\section{Kalanchoe}

crenata, Haw.

thyrsiflora, Harv.

\section{Kennedya}

prostrata, R. Br.

\section{Lycopersicum}

Humboldtii, Dun.

racemigerum, Lange.

\section{Maurandia}

Barclayana, Lindl.

\section{Melothria}

cucumerina, Naud.

Mesembryanthemum

blandum, Haze.

curviflorum, Haw.

elegans, Jacq.

micans, Linn.

polyanthon, Haw.

roseum, Willd.

\section{Mimosa}

marginata, Lindl.

pudica, Linn.

\section{Mimulus}

glutinosus, Wendl.

\section{Momordica}

Charantia, Linn.

cochinchinensis, Spreng.

\section{Musschia}

Wollastoni, Lozere.

\section{Myrtus}

communis, Linn.

Luma, Barn.

Ugni, Mol. 


\section{Nephthytis}

liberica, N. E. Br.

\section{Nicotiana}

sylvestris.

\section{Ochna}

Kirkii, Oliv.

mossambicensis, Klotzsch.

\section{Ornithogalum}

lacteum, Jacq.

\section{Orthosanthus}

multiflorus, Sweet.

Oryza

sativa, Linn.

\section{Oxypetalum}

cæruleum, Decne.

\section{Passiflora}

edulis, Sims.

quadrangularis, Linn.

\section{Pelargonium}

ternatum, Linn.

\section{Pentas}

carnea, Benth., var. kermesina, Hort.

\section{Phyllanthus}

montanus, Sze.

\section{Pinguicula}

lusitanica, Linn.

\section{Polyalthia}

suberosa, Benth. et Hook. fil.

\section{Primula}

floribunda, Wall.

moliis, Nutt.

verticillata, Forsk.

\section{Psychotria}

micrantha, Hiern.

\section{Ricinus}

communis, Linn.

\section{Rivina}

humilis, Linn.

\section{Sarracenia}

illustrata, Hort.

\section{Senecio}

macroglossus, $D C$.

\section{Solanum}

Seaforthianum, Andrews.

\section{Tacsonia}

mixta, Juss.

\section{Tetranema}

mexicana, Benth.

\section{Tillandsia}

splendens, Brong $n$.

\section{Torenia}

flava, Buch,-Ham.

Fournieri, Linden.

\section{Turnera}

ulmifolia, Linn.

\section{Villamilla}

octandra, Hook. fil.

\section{Vitis}

heterophylla, Thunb., var. humulifolia, Hort. 





\title{
NOTES \\ FROM THE \\ ROYAL BOTANIC GARDEN, E D IN B URGH.
}

\author{
JANUARY 1902.
}

CONTENTS.

List of Seeds collected in the Royal Botanic Garden, Edinburgh, during the Year 1901, - - _ - 1

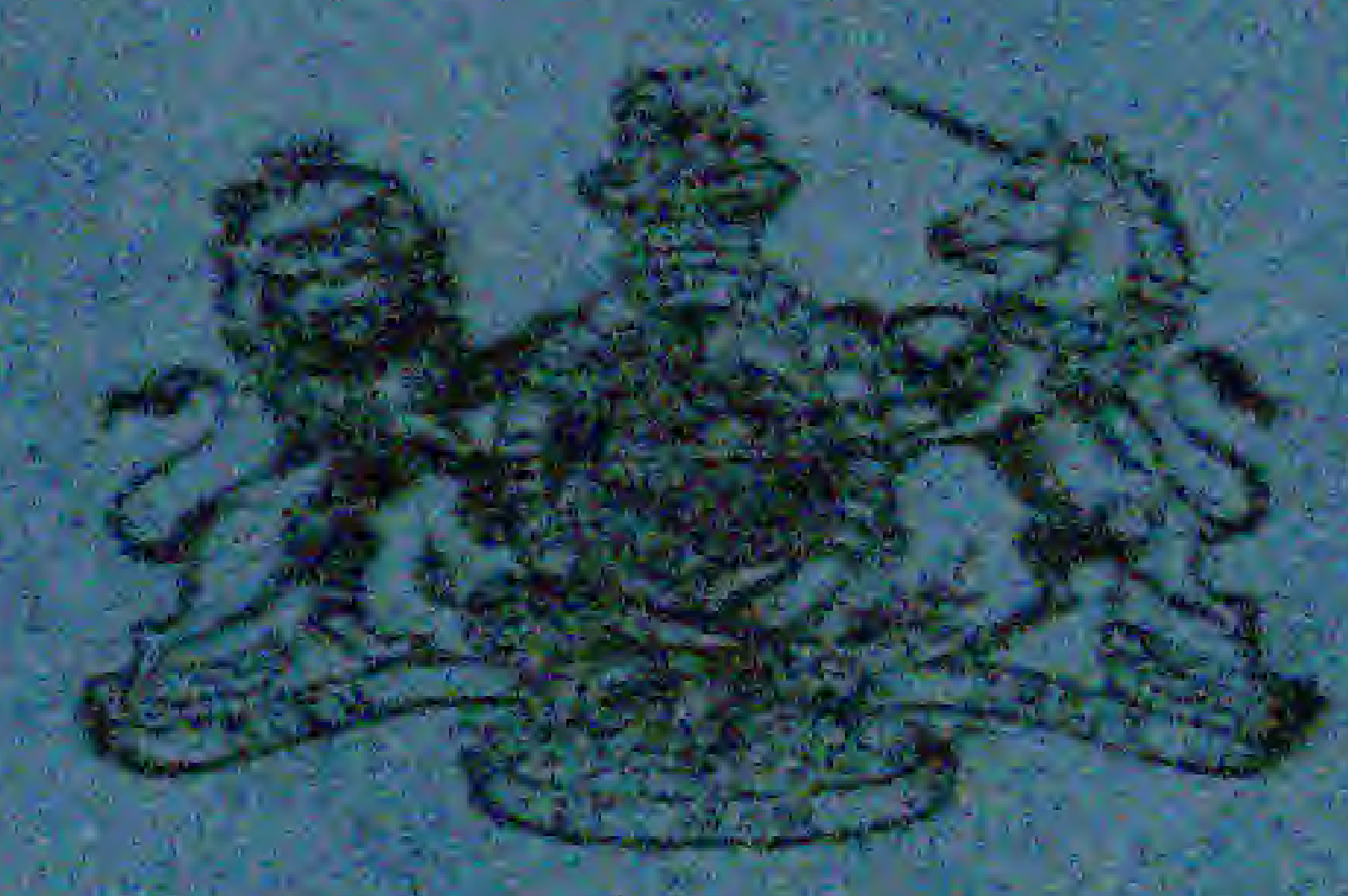

GLA SGOW

PRINTED FOR HIS MAJESTY'S STATIONERY OFFICE BY JAMES HEDUER WICK \& SONS. AT "THE CTHZKN" RRESS, ST, VINCENT PLACE: SOLD AT THE GARDEN,

And to be purchased either dinectly or throush a hy Bookseller, from OLIVER \& BOTD, BDERHURGr.

[PRICE MINEPENCE] 
W P. 2.

(1) 3.t.

(1)

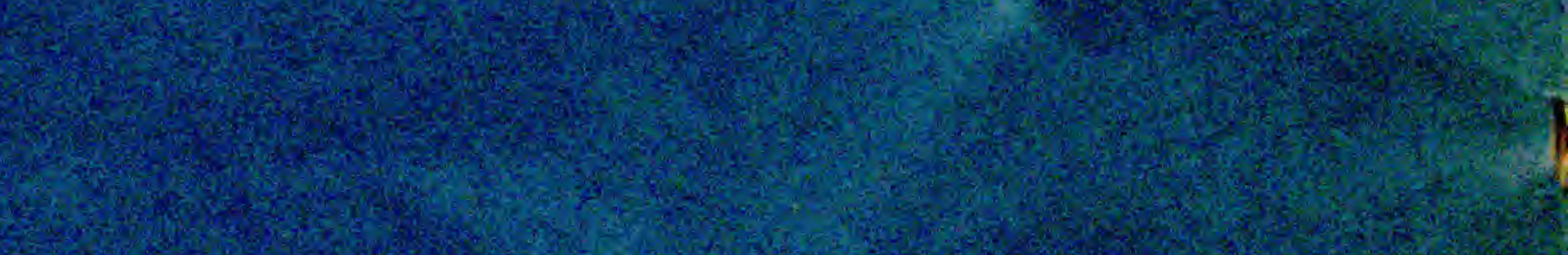

(2)

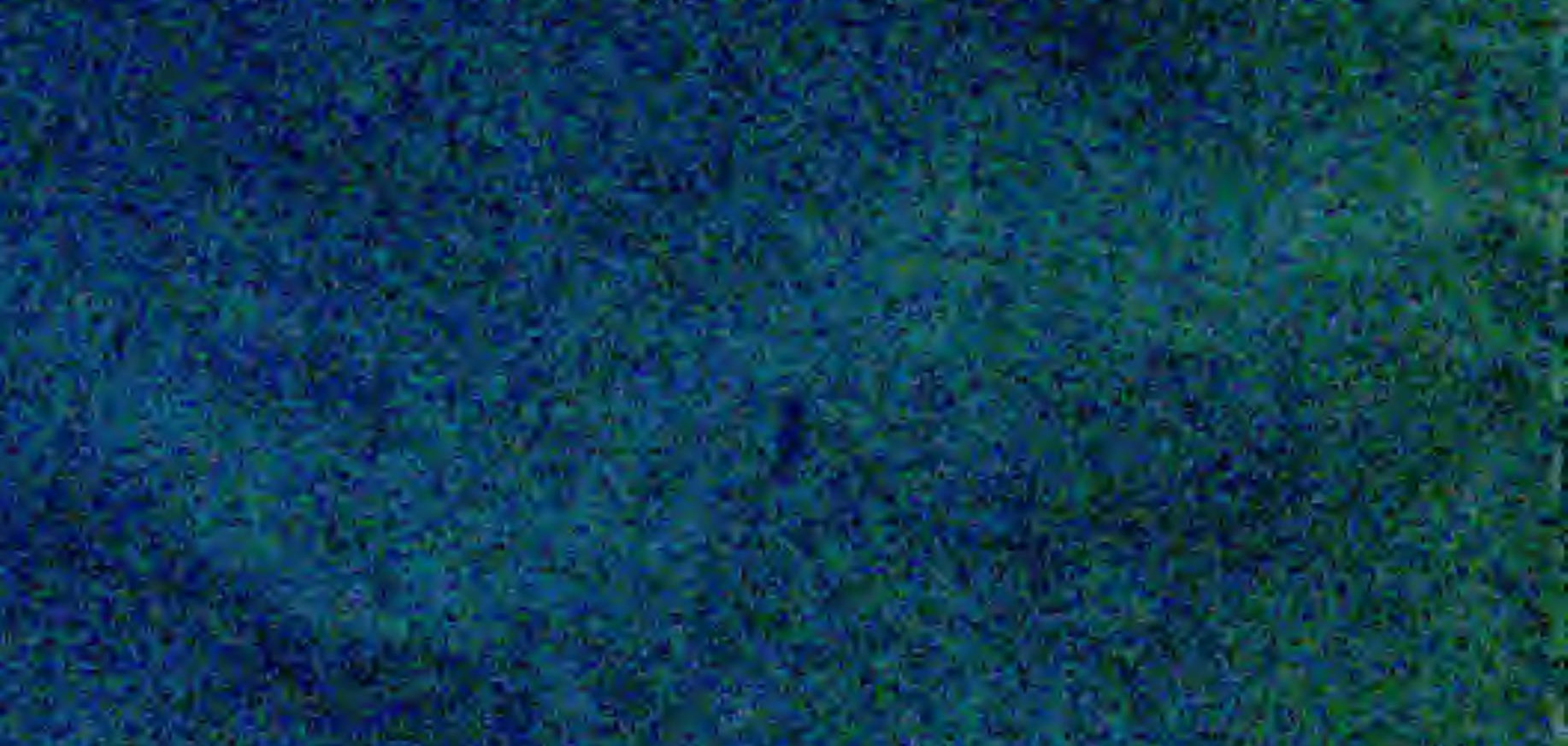

Sit. 


\section{List of Seeds Collected in the Royal Botanic Garden, Edinburgh, during the Year I90I.}

The following is a list of plants cultivated in the Royal Botanic Garden, Edinburgh, from which ripened seeds have been collected during the year I90I. The quantity of seed obtained from some of the species is of limited amount. The seeds are available for exchange, but they are not for sale :-

\section{HERBACEOUS PLANTS.}

\section{Acacia}

Farnesiana, Willd.

grandis, Henfr.

suaveolens, W illd.

\section{Acaena}

argentea, Ruiz et Pav.

Novæ-Zelandiæ, T. Kirk.

pinnatifida, Ruis et Pav.

\section{Acanthostachys}

strobilacea, Link, Klotssch et Otto.

\section{Achillea}

filipendulina, Lam.

grandiflora, Bieb.

Millefolium, Linn.

rupestris, Huter.

\section{Aconitum}

Lycoctonum, Linn.

moldavicum, Hacq.

Napellus, Linn.

paniculatum, Lam.

pyrenaicum, Hort.

reclinatum, A. Gray.

truncatum, Rafin.

\section{Actæa}

alba, Mill.

spicata, Linn.

- var. rubra, Ait.

\section{Adenophora}

stylosa, Fisch.

verticillata, Fisch. 


\section{Aechmea}

bromeliæfolia, Baker, var. pulchra.

candida, E. Morr.

Makoyana, Hort. Makoy.

Weilbachii, Dietr., var. leodiensis.

xiphophylla, Baker.

\section{Agave}

attenuata, Salm-Dyck.

\section{Aglaonema}

commutatum, Schott.

\section{Agrimonia}

agrimonioides, Linn.

Eupatoria, Linn.

odorata, Mill.

\section{Alchemilla}

alpina, Linn., var. conjuncta (Bab.).

\section{Alisma}

Plantago, Linn.

\section{Allium}

carinatum, Linn.

fistulosum, Linn.

giganteum, Regel.

globosum, Red., var. albidum.

karataviense, Regel.

odorum, Linn.

polyphyllum, Kar, et Kir.

sativum, Linn.

Schcenoprasum, Linn.

senescens, Linn.

sphærocephalum, Linn., var. descendens (Linn.).

\section{Alnus}

cordifolia, Tenore.

glutinosa, Medic.

Aloe

mitriformis, Mill., var. spinulosa (Salm-Dyck).

\section{Alstrœmeria}

chilensis, Lem.

revoluta, Ruiz et Pav.

\section{Althæa}

kurdica, Schlecht.

\section{Alyssum}

idæum, Boiss. \& Heldr.

montanum, Linn.

saxatile, Linn.

\section{Ammobium}

alatum, R. Br.

\section{Anchusa}

altissima, Desf.

\section{Androsace}

arachnoidea, Scholt.

lactea, Linn.

lanuginosa, Wall.

macrantha, Boiss. \& Huet.

septentrionalis, Linn.

\section{Anemone}

alpina, Linn.

- var. sulphurea.

narcissiflora, Linn.

pratensis, Linn.

rivularis, Buch.-Ham.

sulphurea, Linn.

\section{Antennaria}

dioica, Gaertn, var. tomentosa, Horl.

\section{Anthericum}

Liliago, Linn. 


\section{Anthyllis}

montana, Linn.

\section{Antirrhinum}

Asarina, Linn.

latifolium, Mill.

\section{Aquilegia}

canadensis, Linn.

chrysantha, A. Gray.

Skinneri, Hook.

vulgaris, Linn.

\section{Arabis}

Allionii, $D C$.

alpina, Linn.

- var. anachoretica, Port.

blepharophylla, Hook, et Arn.

hirsuta, Scop.

nivalis, Hort.

\section{Araujia}

sericifera, Brot.

\section{Arctium}

Lappa, Linn.

minus, Bernh.

\section{Arctotis}

stœechadifolia, Berg.

\section{Arenaria}

juniperina, Linn.

Rosani, Tenore.

setacea, Thuill.

\section{Aristolochia}

elegans, Mast.

\section{Arnica}

amplexicaulis, Nutt.

Chamissonis, Less.

\section{Arnoseris}

pusilla, Gaertn.

\section{Arracacia}

arguta, Benth, et Hook, f.

\section{Artemisia}

capillaris, Thunb.

discolor, Dougl.

tanacetifolia, Linn.

\section{Asclepias}

curassavica, Linn.

\section{Asperula}

cynanchica, Linn.

lævigata, Linn.

orientalis, Boiss, et Hohen.

tinctoria, Linn.

\section{Aster}

alpinus, Linn.

- var. albus.

- var. ruber.

mutabilis, Linn.

patens, Ait.

Pattersoni, A. Gray.

sagittifolius, Wedem.

turbinellus, Lindl.

\section{Astragalus}

alpinus, Linn.

bicolor, Lam.

Cicer, Linn.

falcatus, Lam.

galegiformis, Linn.

Glyciphyllus, Linn.

hypoglottis, Linn.

purpureus, Lam.

reflexistipulus, Miq.

sinicus, Linn.

vaginatus, Pall.

\section{Astrantia}

gracilis, Bartl.

major, Linn.

- var. intermedia.

neglecta, C. Koch et Bouché. 


\section{Athamanta}

Matthioli, Wulf.

\section{Atropa}

Belladonna, Linn.

\section{Aubrietia}

croatica, Scholt.

deltoidea, $D C$.

- var. grandiflora.

- var. taurica.

- var. olympica.

\section{Avena}

sterilis, Linn.

\section{Babiana}

plicata, Ker.

\section{Bellis}

sylvestris, Cyrill.

\section{Bertolonia}

maculata, $D C$.

\section{Beta}

vulgaris, Linn.

Betula

alba, Linn., var. pendula.

\section{Billbergia}

decora-nutans $\times$, Baker.

pyramid alis Lindl.

thyrsoidea, Mart., var. longifolia (C. Koch et Bouché). zebrina, Lindl.

\section{Bomarea}

patacocensis, Herb.

\section{Brachychilum}

Horsfieldii, Baker.

\section{Brassica}

juncea, Coss.

\section{Brodiæa}

lactea, S. Wats.

\section{Bryonia}

dioica, Jacq.

\section{Bulbinella}

Hookeri, Benth, et Hook f.

\section{Buphthalmum}

speciosum, Schreb.

\section{Bupleurum}

Candollii, Wall.

\section{Butomus}

umbellatus, Linn.

\section{Calamintha}

officinalis, Manch.

\section{Calandrinia}

Menziesii, Torr, et Gray.

\section{Calendula}

arvensis, Linn.

\section{Callicarpa}

purpurea, Juss.

\section{Callirhoe}

digitata, Nutt.

\section{Callistemon}

linearis, $D C$. speciosus, $D C$.

\section{Calochortus}

clavatus, $S$. Wats.

venustus, $B e n t h$.

- var. Eldorado.

- var. oculatus. 


\section{Camassia}

Cusickii, S. Wats.

esculenta, Lindl.

- var. alba.

\section{Campanula}

alliariæfolia, Willd.

barbata, Linn.

cæspitosa, Scop.

carpatica, Jacq.

- var. turbinata (Scholt).

expansa, Frivald.

Grossekii, Heuffl.

lactiflora, Bieb.

latifolia, Linn.

- var. macrantha (Fisch.)

- var. macrantha alba.

linifolia, Scop.

Medium, Linn., var, alba.

mirabilis, Alboff.

pyramidalis, Linn.

rapunculoides, Linn.

Rapunculus, Linn.

rotundifolia, Linn.

— var. alba.

- var. Baumgartenii (Becker).

Scheuchzeri, Vill.

Trachelium, Linn.

versicolor, Sibth. et $\mathrm{Sm}$.

\section{Capsicum}

annuum, Linn.

longum, Hort.

\section{Cardamine}

bellidifolia, Linn.

\section{Carduus}

acanthoides, Limn.

\section{Carex}

atrata, Linn.

irrigua, $S m$.

japonica, Hort.

muricata, Linn.

punctata, Gaud.

Vahlii, Schkuhr.

\section{Celosia}

cristata, Linn., var, nana.

\section{Centaurea}

alpina, Linn.

atropurpurea, Waldst. et Kit.

var. calocephala.

axillaris, Willd.

cariensis, Boiss.

glastifolia, Linn.

Jacea, Linn.

nigra, Linn.

nigrescens, Willd.

pectinata, Linn.

rutifolia, Sibth, et Sm.

salmantica, Linn.

sphærocephala, Linn.

\section{Cephalaria}

tatarica, Schrad.

- var. minor.

\section{Cerastium}

arvense, Linn., var. grandiflorum.

ovatum, Hoppe.

purpurascens, Adams.

tomentosum, Linn.

\section{Cerinthe}

alpina, Kit.

retorta, Sibth. et $S m$.

\section{Cestrum}

aurantiacum, Lindl. 


\section{Cheiranthus}

Senoneri, Heldr, et Sart.

\section{Chelidonium}

majus, Linn.

- var. laciniatum (Mill.).

\section{Chlorophytum}

Orchidastrum, Lindl.

\section{Chrysanthemum}

arcticum, Linn.

Balsamita, Linn.

ceratophylloides, All.

cinerariæfolium, Vis.

coronopifolium, Masf., var. hybridum.

Leucanthemum, Linn. maximum, Ramond. segetum, Linn.

\section{Cimicifuga}

racemosa, Nutt.

\section{Cistus}

monspeliensis, Linn.

\section{Clematis}

heracleæfolia, $D C$.

\section{Clerodendron}

splendens, G. Don.

\section{Clianthus}

puniceus, Banks et Sol.

\section{Clitoria}

Ternatea, Linn.

\section{Cnicus}

Casabonæ Roth.

Diacantha, Desf. eriophorus, Roth.

pannonicus,

pratensis, Willd.

tartaricus, Willd.

\section{Cobæa}

scandens, Cav.

Cochlearia

glastifolia, Linn.

Codonopsis

ovata, Benth.

rotundifolia, Royle.

\section{Colutea}

istria, Mill.

melanocalyx, Boiss.

\section{Combretum}

purpureum, Vahl.

\section{Convallaria}

majalis, Linn.

\section{Coronilla}

elegans, Panc.

montana, Scop.

vaginalis, Lam.

varia, $\operatorname{Linn}$.

\section{Cortusa}

Matthioli, Linn.

- var. grandiflora.

\section{Corydalis}

glauca, Pursh.

\section{Costus}

Afer, Ker.

igneus, N. E. Brown.

\section{Cotoneaster}

Nummularia, Fisch, et Mey.

\section{Cousinia}

Host, var. Hystrix, C. A. Mey.

\section{Cratagus}

mollis, Scheele. 


\section{Crocosmia}

aurea, Planch.

\section{Crocus}

pulchellus, Herb.

\section{Croton}

ciliato-glanduliferus, Ort.

\section{Cynoglossum}

officinale, Linn.

- var. alba.

pictum, Ait.

\section{Cytisus}

nigricans, Linn.

scoparius, Link.

\section{Dahlia}

Merckii, Lehm.

\section{Danae}

Laurus, Medic.

\section{Daucus}

Carota, Linn.

Gingidium, Linn.

\section{Delphinium}

altissimum, Wall.

azureum, Michx.

Brunonianum, Royle.

californicum, Torr. et Gray.

cashmirianum, Royle.

elatum, Linn.

flexuosum, Rafin.

formosum, Boiss, et Huet.

grandiflorum, Linn., var. album.

- var. chinense.

nudicaule, Torr. et Gray.

palmatifidum, $D C$.

scopulorum, A. Gray.

speciosum, Bieb., var.glabratum. - var. turkestanicum.

\section{Desmodium}

gyrans, $D C$.

\section{Dianthus}

alpinus, Linn.

Armeria, Linn.

atrorubens, All.

banaticus, Heuffl.

cæsius, $S m$.

chinensis, Linn.

cinnabarinus, Sprun.

deltoides, Linn.

pelviformis, Heufl.

Requienii, Gren, et Godr.

superbus, Linn.

tymphresteus, Heldr. et Sart.

\section{Dictamnus}

albus, Linn.

- var. tauricus.

\section{Diervilla}

sessilifolia, Buckl.

\section{Digitalis}

ambigua, Murr.

purpurea, Linn.

\section{Dionysia}

aretioides, Boiss.

\section{Diplotaxis}

erucoides, $D C$.

\section{Dipsacus}

atratus, Hook. $f$. et Thoms.

japonıcus, Miq.

\section{Dodecatheon}

Meadia, Linn., var. album.

—, var. integrifolium (Michx.). 


\section{Draba}

Aizoon, Wahlenb. arabisans, Michx. aurea, $V a h l$. cuspidata, Bieb. fladnizensis, Wulf. hirta, Linn. incana, Linn. - var. borealis. rupestris, $R$. $B r$. scandinavica, Lindblom.

\section{Dracocephalum}

peregrinum, Linn. Ruprechti, Regel. speciosum, Benth.

\section{Drosera}

longifolia, Linn.

spathulata, Labill.

\section{Drosophyllum}

lusitanicum, Link.

\section{Dryas}

Drummondii, Richards. octopetala, Linn.

\section{Epilobium}

alpinum, Linn. angustifolium, Linn. Billardierianum, Ser.

Dodonæi, Vill. Lamyi, Schultz. luteum, Pursh. parviflorum, Schreb. repens, Schlecht. rosmarinifolium, Hcenke. - var. sericeum.

\section{Eremurus}

himalaicus, Baker, robustus, Regel.

\section{Erigeron}

glabellus, Nutt.

- var. mollis.

macranthus, Nutt.

Roylei, $D C$.

speciosus, $D C$.

- var. superbus.

Villarsii, Bell.

\section{Erinus}

alpinus, $\operatorname{Lin} n$.

\section{Erophila}

vulgaris, $D C$.

\section{Eryngium}

alpinum, Linn.

Bourgati, Gouan.

dichotomum, Desf.

Oliverianum, Delar. var. superbum.

planum, Linn.

spinalba, Vill.

\section{Erysimum}

asperum, $D C$.

Perofskianum, Fisch. et Mey

\section{Escallonia}

exoniensis, Hort.

pterocladon, Hook.

rubra, Pers.

\section{Eschscholzia}

californica, Cham,

\section{Eucalyptus}

ficifolia, F. Muell.

\section{Eucharidium}

Breweri, A. Gray.

\section{Euphorbia}

Gerardiana, Jacq. 


\section{Evodia}

elegans, Hort.

\section{Fraxinus}

excelsior, Linn.

\section{Fritillaria}

camtschatcensis, Ker-Gawl. latifolia, Willd., var. lutea.

Meleagris, Linn.

\section{Fuchsia}

procumbens, R. Cunn.

\section{Funkia}

lancifolia, Spreng.

Sieboldiana, Hook.

\section{Galega}

officinalis, Linn.

- var. alba.

orientalis, Lam.

\section{Galium}

coronatum, Sibth, et Sm. saccharatum, All.

\section{Galtonia}

candicans, Decsne.

\section{Gasteria}

disticha, Haw., var. angustifolia, Baker.

\section{Gentiana}

asclepiadea, Linn.

Saponaria, Linn.

septemfida, Pall., var. cordifolia, Hook, $f$. tibetica, King. verna, Linn.

\section{Geonoma}

pumila, Wendl.

\section{Geranium}

argenteum, Linn.

Grevilleanum, Wall.

ibericum, Cav.

- var. platypetalum (Fisch. et Mey.).

phæum, Linn.

pratense, Linn.

- var. album.

rhoticum, Hort.

roseum, L'Herit.

sanguineum, Linn.

- var. lancastriense (Mill.).

\section{Gerbera}

Jamesoni, Bolus.

nivea, $S c h$. Bip.

\section{Geum}

bulgaricum, Panc.

intermedium $\times$, Ehrh.

montanum, Linn.

- var. aurantiacum.

\section{Glaucium}

flavum, Crants.

- var. fulvum, (Sm.).

\section{Globularia}

vulgaris, Linn.

\section{Gossypium}

herbaceum, Linn.

\section{Grevillea}

glabrata, Meissn.

\section{Grindelia}

squarrosa, Dunat.

\section{Gypsophila}

acutifolia, Fisch.

elegans, Bieb. 


\section{Haberlea}

rhodopensis, Frivald.

\section{Hedysarum}

boreale, Nutt.

microcalyx, Baker.

obscurum, Linn.

\section{Helenium}

Hoopesii, A. Gray.

\section{Helianthemum}

ledifolium, Mill.

Tuberaria, Mill.

umbellatum, Mill.

vulgare, Gaertn., var. mutabile roseum.

- var. rhodanthum.

- var. roseum.

- var. venustum.

\section{Heliopsis}

lævis, Pers.

padula, Wender.

\section{Hesperis}

matronalis, Linn.

\section{Heuchera}

Drummondi, Hort.

glabra, Willd.

macrophylla, Lodd.

sanguinea, Engelm.

\section{Hibiscus}

gossypinus, Thunb.

Huegelii, Endl.

Manihot, Linn.

\section{Hieracium}

bupleuroides, C. C. Gmel.

cæsium, Fries.

cambricum, F.J. Hanb.

crinitum, Sibth, et Sm. foliosum, Waldst. et Kit.

gymnocephalum, Griseb.

lasiophyllum, Koch.

lingulatum, Backh.

rupestre, All.

sabaudum, Linn.

scoticum, F. I. Hanb.

tridentatum, Fries.

\section{Hyacinthus}

romanus, Linn.

\section{Hydrolea}

spinosa, Linn.

\section{Hypericum}

Androsæmum, Linn.

Ascyron, Linn.

crenulatum, Boiss.

elodeoides, Choisy.

hirsutum, Linn.

montanum, Linn.

perforatum, Linn.

pulchrum, Linn.

tetrapterum, Fries.

\section{Hypochoeris}

radiata, Linn.

uniflora, Vill.

\section{Hyssopus}

officinalis, Linn.

\section{Iberis}

intermedia, Guersent.

sempervirens, Linn., var. commutata (Schott et Kotschy.).

- var. Garrexiana (All.).

- var. superba.

Tenoreana, $D C$.

umbellata, Linn. 
Ilex

Aquifolium, $\operatorname{Linn}$.

\section{Impatiens}

cuspidata, Wight.

Noli-tangere, Linn. scabrida, $D C$.

\section{Incarvillea}

Delavayi, Bureau et Franch.

\section{Inula}

glandulosa, Puschk.

Helenium, Linn.

Hookeri, Clarke.

Iris

Cengialtii, Ambrosi.

Pseudacorus, Linn., var. variegata.

sibirica, Linn.

- var. alba.

- var. flexuosa (Murr.).

virginica, Linn .

xiphioides, Ehrh.

\section{Isatis}

tinctoria, Linn.

\section{Itea}

virginica, Linn.

\section{Jasione}

montana, Linn.

\section{Juncus}

effusus, Linn., var. spiralis (Hort.).

\section{Jurinea}

glycacantha, $D C$.

\section{Kalanchoe}

flammea, Stapf.

\section{Kedrostis}

africana, $\operatorname{Cog} n$.

\section{Kniphofia}

Tuckii, Baker.

\section{Laburnum}

vulgare, J. S. Presl.

—var. austriacum.

\section{Lathyrus}

hirsutus, Linn.

latifolius, Linn.

- var. albus.

luteus, Munby.

- var. aureus.

macrorrhizus, Wimm.

maritimus, Bigel.

montanus, Bernh.

rotundifolius, Willd.

sylvestris, Willd.

tingitanus, Linn.

vernus, $\operatorname{Linn}$.

- var. azureus.

\section{Leontodon}

hispidum, Linn.

\section{Leontopodium}

alpinum, Cass.

-var. altaicum (Spreng.).

\section{Leonurus}

Cardiaca, Linn.

\section{Liatris}

spicata, Willd.

\section{Lilium}

pardalinum, Kellogg.

Parryi, S. Wats.

tigrinum, Ker-Gawl., var. sinense.

- var. splendens (Baker). 


\section{Linaria}

vulgaris, Mill.

\section{Linum}

austriacum, Linn.

- var. album.

hologynum, Reichb.

Lewisii, Pursh.

narbonense, Linn.

perenne, $\operatorname{Linn}$.

\section{Lophosciadium}

meifolium, $D C$.

\section{Lupinus}

aboreus, Sims.

- var. violaceus.

nootkatensis, Don.

polyphyllus, Lindl., var. albus. rivularis, Dougl.

\section{Lychnis}

alba, Mill.

alpina, Linn.

chalcedonica, Linn.

Flos-cuculi, Linn.

Flos-jovis, Desr.

Haageana $\times$, Lem. montana, S. Wats.

Viscaria, Linn.

\section{Lythrum}

myrtifolium, Lodd.

Salicaria, Linn., var. hirsutum.

\section{Malva}

Alcea, Linn.

sylvestris, Linn.

- var. variegata.

\section{Malvastrum}

lateritium, Nichols.

\section{Meconopsis}

aculeata, Royle.

cambrica, Vig.

Wallichii, Hook.

- var, fusco-purpurea.

\section{Medicago}

hispida, Gaertn.

-sativa, Linn.

strumaria, Hort.

\section{Melilotus}

dentata, Pers.

elegans, Salsm.

officinalis, Lam.

\section{Mimosa}

marginata, Lindl.

\section{Mimulus}

cardinalis, Dougl.

\section{Mirabilis}

Jalapa, Linn.

\section{Momordica}

Charantia, Linn.

cochinchinensis, Spreng.

\section{Morina}

longifolia, Wall.

persica, Linn.

\section{Muscari}

Argæi, Hort.

armeniacum, Baker.

botryoides, Mill.

moschatum, Willd.

\section{Myosotis}

arvensis, Lam.

dissitiflora, Baker.

\section{Myrrhis}

odorata, Scop. 


\section{Narcissus}

Bulbocodium, Linn., var. citrinus, Baker.

\section{Nepeta}

Mussini, Spreng.

Nepetella, Linn. nuda, Linn.

Sibthorpii, Benth.

\section{Nephthytis}

liberica, $N$. E. brown.

\section{Nigella}

hispanica, Linn., var. atropurpurea.

-, var. alba.

\section{Noccæa}

alpina, Reichb.

stylosa, Reichb.

\section{Enanthe}

pimpinelloides, Linn.

\section{Enothera}

odorata, Jacq.

\section{Olearia}

Haastii, Hook. f.

\section{Onobrychis}

viciæfolia, Scop.

\section{Ononis}

hircina, Jacq.

Natrix, Linn.

spinosa, Linn.

\section{Ornithogalum}

tenellum, Jacq.

\section{Oryza}

sativa, Linn.

\section{Oxalis}

corniculata, Linn,

- var, tropæoloides, Hort. stricta, Linn.

\section{Oxypetalum}

coeruleum, Decne.

\section{Oxytropis}

glabra, $D C$.

strobilacea, Bunge.

\section{Pæonia}

corallina, Rets.

officinalis, Linn. var. anemonæflora.

\section{Panicum}

Crus-galli, Linn.

\section{Papaver}

alpinum, Linn.

glaucum, Boiss. et Haussk.

Heldreichii, Boiss.

lævigatum, Bieb.

nudicaule, Linn.

pavoninum, Mey.

pilosum, Sibth, et $S m$.

\section{Passiflora}

quadrangularis, Linn.

edulis, Sims.

\section{Pentstemon}

barbatus, Roth.

- var. Torreyi (Benth.).

confertus, Dougl.

deustus, Dougl.

diffusus, Dongl.

gentianoides, Poir.

humilis, Nutt.

ovatus, Dougl.

Richardsonii, Dougl. 


\section{Perezia}

multiflora, Less.

\section{Peucedanum}

coriaceum, Reichb.f.

officinale, Linn.

\section{Phacelia}

tanacetifolia, Benth.

\section{Philydrum}

lanuginosum, Banks.

\section{Phlomis}

cashmeriana, Royle.

\section{Phyteuma}

hemisphæricum, Linn.

orbiculare, Linn.

Scheuchzeri, All.

Sieberi, Spreng.

\section{Pimpinella}

magna, Linn.

\section{Pitcairnia}

maidifolia, Decsne.

pulverulenta, Ruiz et Pav.

rubiginosa, Baker.

\section{Plantago}

Coronopus, Linn.

Raoulii, Decsne.

\section{Platystemon}

californicus, Benth.

\section{Polemonium}

cœruleum, Linn.

- var. album.

confertum, A. Gray, var. melitum, A. Gray.

himalayanum, Baker.

lacteum, Lehm.

pauciflorum, $S$ Wats.

Richardsoni, Grah.

\section{Polyalthia}

suberosa, Benth. et Hook. f.

\section{Polygonum}

amplexicaule, D. Don.

viviparum, Linn.

Weyrichii, F. Schmidt.

\section{Potentilla}

argyrophylla, Wall.

dissecta, Pursh.

Friedrichseni, Hort.

glandulosa, Lindl.

japonica, Blume.

malacophylla, Borb.

mollis, Panc.

nevadensis, Boiss.

ontopoda, Dougl.

podolica, Blocki.

procumbens, Sibth.

pseudo-obscura, Blocki.

recta, Linn., var. sulphurea.

rupestris, Linn., var. grandiceps.

Sanguisorba, Willd.

sericea, Linn.

Sibbaldi, Hall. f.

Thurberi, A. Gray.

tridentata, Soland.

umbrosa, Stev.

vlasicensis, Siegfr.

\section{Poterium}

dodecandrum, Benth. et Hook.f.

\section{Primula}

apennina, Widm.

Auricula, Linn., var. bellunensis

(Venzo).

- var. monacensis. 
Primula-continued.

carniolica, Jacq.

elatior, Hill, var. carpathica (Fuss.).

farinosa, Linn.

floribunda, Wall.

Forbesi, Franch.

frondosa, Janka.

involucrata, Wall., var. Munroi (Lindl.).

japonica, A. Gray, var. lilacina.

longiflora, All.

mollis, Nutt.

Poissoni, Franch.

sibirica, Jacq.

sikkimensis, Hook. $f$.

verticillata, Forsk.

\section{Prunella}

grandiflora, Jacq.

- var. alba.

- var. pinnatifida.

vulgaris, Linn.

- var. alba.

\section{Pyrus}

rotundifolia, Bechst.

\section{Ramondia}

pyrenaica, Rich.

serbica, Panc.

- var. Nathalize (Panc. et Petrov.).

\section{Ranunculus}

bulbosus, Linn.

cuneatus, Boiss.

lanuginosus, Linn.

montanus, Willd.

\section{Reseda}

complicata, Bory.

lutea, Linn.

\section{Rheum}

hybridum, Murr.

Rhaponticum, Linn.

Ribes, Linn.

\section{Rhodochiton}

volubile, Zucc.

\section{Rhododendron}

Nuttallii, Booth.

\section{Rhynchosia}

caribæa, $D C$.

\section{Rodgersia}

podophylla, A. Gray.

\section{Rondeletia}

odorata, Jacq.

\section{Ruellia}

tuberosa, Linn.

\section{Rumex}

occidentalis, S. Wats.

salicifolius, Weinm.

stenophyllus, Ledeb.

\section{Ruta}

macrophylla, Soland.

\section{Sabal}

Blackburniana, Glazeb.

\section{Salvia}

glutinosa, Linn.

hians, Royle.

nutans, Linn.

pratensis, Linn.

sylvestris, Linn., var. alba. 


\section{Samolus}

Valerandi, Linn.

\section{Sanicula}

europæa, Linn.

\section{Saponaria}

ocymoides, Linn.

\section{Saussurea}

alpina $D C$.

serrata, $D C$.

\section{Saxifraga}

Aizoon, Linn.

aretioides, Lapeyr.

aspera, Linn.

bronchialis, Linn.

cæsia, Linn.

cæspitosa, Linn.

canaliculata, Boiss, et Reut.

Cotyledon, Linn.

cuneifolia, Linn.

geranioides, Linn.

Geum, Linn., var. ovalifolium. granulata, Linn.

Hostii, Tausch.

hypnoides, Linn.

- var. variegata.

integrifolia, Hook.

lingulata, Bell.

luteo-viridis, Schotl et Kotschy.

muscoides, Wulf., var. atropurpurea.

mutata, Linn.

nivalis, Linn.

pedatifida, Ehrh.

planifolia, Lapeyr.

pseudo-sancta, Janka.

retusa, Gouan.

rotundifolia, Linn.

stellaris, Linn.

Stracheyi, Hook. f. et Thoms.
Saxifraga-continued.

tenella, Wulf.

umbrosa, Linn., var. Ogilvieana, Hort.

- var. serratifolia (Mackay).

\section{Scabiosa}

amœena, Jacq.

caucasica, Bieb.

- var. alba.

gramuntia, Linn.

lucida, Vill.

montana, Bieb.

Succisa, Linn.

sylvatica, Linn.

\section{Schismatoglottis}

- Roebelini, Hort.

\section{Schizanthus}

pinnatus, Ruiz et Pav.

\section{Scilla}

festalis, Salisb.

- var. carnea.

- var. compacta.

hispanica, Mill.

peruviana, Linn.

\section{Scirpus}

aucklandicus, Boeck.

\section{Scolymus}

grandiflorus, Desf.

\section{Scrophularia}

alata, Gilib.

nodosa, Linn.

vernalis, Linn.

\section{Scutellaria}

alpina, Linn.

altissima, Linn. 


\section{Sedum}

album, Linn.

Anacampseros, Linn.

asiaticum, Spreng.

hybridum, Linn.

oppositifolium, Sims.

roseum, Scop.

stoloniferum, S. T. Gmel.

\section{Sempervivum}

hirtum, Linn.

Pittonii, Schott, Nym. et Kotschy. Reginæ-Amaliæ, Heldr.

tectorum, Linn.

annuum, C. Sm.

\section{Senecio}

Doria, Linn.

Doronicum, Linn.

Fuchsii, C. C. Gmel.

Greyii, Hook.f.

Hodgsoni, Hort. Kew.

\section{Serratula}

heterophylla, Desf.

tinctoria, Linn.

\section{Sesbania}

ægyptiaca, Poir.

\section{Sidalcea}

candida, A. Gray.

malvæflora, A. Gray.

\section{Silene}

alpestris, Jacq.

caucasica, Boiss.

Cucubalus, Wibel.

italica, Pers.

maritima, With.

nicæënsis, All.

nocteolens, $W e b b$ et Berth.

Pseudo-atocion, Desf.

quadridentata, Pers.
Silene-continued.

rhynchocarpa, Boiss.

Saxifraga, Linn.

Schafta, Gmel.

Schweinfurthii, Rohrb.

stylo a, Bunge.

viridiflora, Linn.

Siler

trilobum, Crants.

\section{Silphium}

trifoliatum, Linn.

\section{Sisymbrium}

austriacum, Jacq.

strictissimum, Linn.

tanacetifolium, Linn.

\section{Sisyrinchium}

angustifolium, Mill., var. mucronatum (Michx.). convolutum, Nocca.

\section{Smilacina}

racemosa, Desf.

\section{Solanum}

auriculatum, Ait.

Dulcamara, Linn, var. alba.

hybridum, Jacq., var. Hendersonii.

pyracanthum, Jacq.

Seaforthianum, Andr.

\section{Solidago}

multiradiata, Ait., var. scopulorum,

odora, Ait.

rigida, Linn.

Virgaurea, Linn.

\section{Spergula}

arvensis, Limn, 


\section{Spiræa}

compacta, Hort., var. coccinea. Filipendula, Linn. palmata, Thunb, var, alba. tomentosa, Linn., var. alba.

\section{Stachys}

alpina, Linn., var. intermedia. Betonica, Benth. grandiflora, Benth.

\section{Stachytarpheta} indica, Vahl.

\section{Stenanthium}

angustifolium, Kunth.

\section{Succowia}

balearica, Medic.

\section{Symphoricarpus}

racemosus, Michx.

\section{Tacca}

cristata, Jack.

\section{Tacsonia}

exoniensis $\times$ Hort.

Van-Volxemii, Hook.

\section{Tanacetum}

argenteum, Willd.

vulgare, Linn.

\section{Tecoma}

Smithii, Hort.

\section{Tetranema}

mexicana, Benth.

\section{Teucrium}

Botrys, Linn.

pyrenaicum, Linn.

Scorodonia, Linn.

\section{Thalictrum}

alpinum, Linn.

calabricum, Spreng.

glaucum, Desf.

laserpitiifolium, Willd.

minus, Linn.

simplex, Linn.

\section{Thermopsis}

fabacea, $D C$.

lanceolata, R. Br.

\section{Thlaspi}

rotundifolium, Gaud.

\section{Tillandsia}

corallina, C. Koch.

splendens, Brong.

\section{Tofieldia}

glutinosa, Pers.

palustris, Huds.

\section{Trachelium}

cœeruleum, Linn.

\section{Tradescantia}

virginiana, Linn., var. alba.

\section{Trautvetteria}

palmata, Fisch. et Mey.

\section{Trifolium}

agrarium, Linn.

alpinum, Linn.

ambiguum, Bieb.

arvense, Linn.

minus, $S m$.

pannonicum, Jacq.

repens, Linn.

- var. pictum.

rubens, $\operatorname{Linn}$.

\section{Trilisa}

odoratissima, Cass. 


\section{Tristania}

laurina, $R, B r$.

\section{Trollius}

altaicus, Mey.

asiaticus, Linn.

- var. aurantiacus.

europæus, Linn.

-var. pumilus albus.

Ledebourii, $S p r$.

\section{Tulipa}

Didieri, Jord.

\section{Valeriana}

tuberosa, Linn.

\section{Verbascum}

longifolium, Tenore.

malacotrichum, Boiss, et Heldr.

pyramidatum, Bieb.

virgatum, With.

Wiedemannianum, Fisch. et Mey.

\section{Verbena}

caroliniana, Michx.

\section{Veronica}

alpina, Linn.

austriaca, Limn.

- var. pinnatifida.

Bidwillii, Hook. $f$.

buxifolia, Benth.

candida, Lodd.

Colensoi, Hook. $f$.

—var. glauca.

crassifolia, Zeyh.

decumbens, Armstr.

grandis, Fisch.

Guthriana $\times$, Hort.
Veronica - continued.

neglecta, Hort.

pinnata, Linn.

Ponæ, Gouan.

salicifolia, Forst.

saxatilis, Scop., var, rosea.

\section{Viburnum}

Opulus, Linn.

\section{Vicia}

monanthos, Desv.

Orobus, $D C$.

oroboides, Wulf.

sylvatica, Linn.

unijuga, $A$. Braun.

villosa, Roth.

\section{Vincetoxicum}

nigrum, Moench.

officinale, Moench.

\section{Viola}

canadensis, Limn.

- var. alba.

canina, Linn.

cornuta, Linn.

macedonica, Boiss. et Heldr.

persicifolia, Roth.

pinnata, Linn.

primulifolia, Linn.

rostrata, Mulll.

striata, Ait.

sylvestris, Lam.

\section{Xerophyllum}

asphodeloides, Nutt.

\section{Zygadenus}

elegans, Pursh. 


OFFICIAL COPY.

\title{
NOTES
}

\author{
FROW THE
}

\section{ROYAL BOTANIC GARDEN, E D I N B U R G H.}

\author{
JANUARY 1903.
}

\section{CONTENTS.}

List of Seeds collected in the Royal Botanic Garden, Edinburgh, during the Year 1902, . _ _ _ - 1

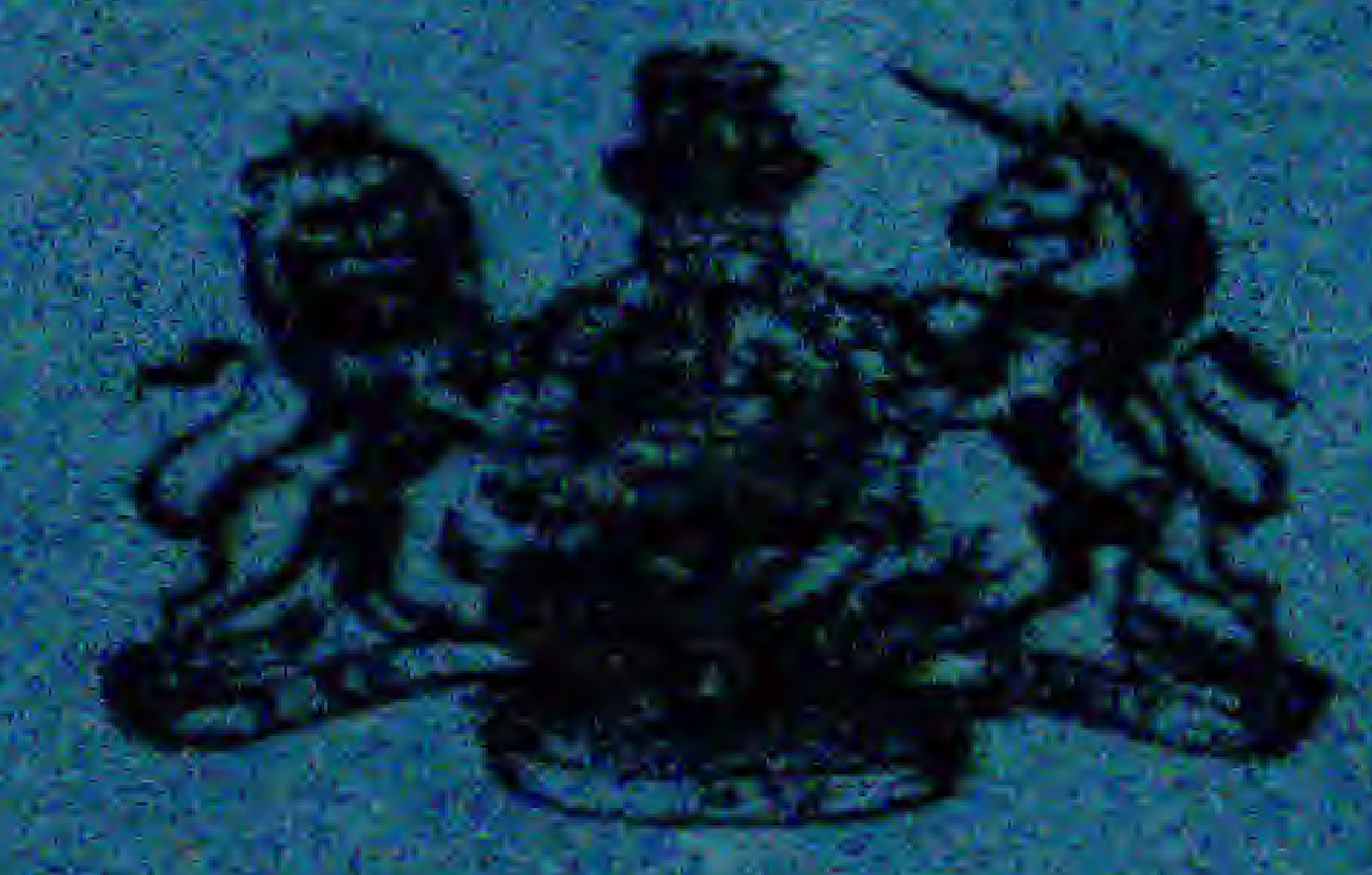

\section{GLASGOW:}

PRINTED FOR HIS MAJESTYS STATIONERY OFFICE

BY JAMES HEDDER WICK \& SONS, AT "THE CITZMN" RRESS, ST VINCENT PLACE:

SOLD AT TAE GARDEN.

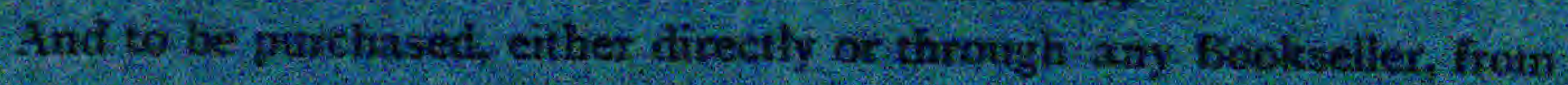

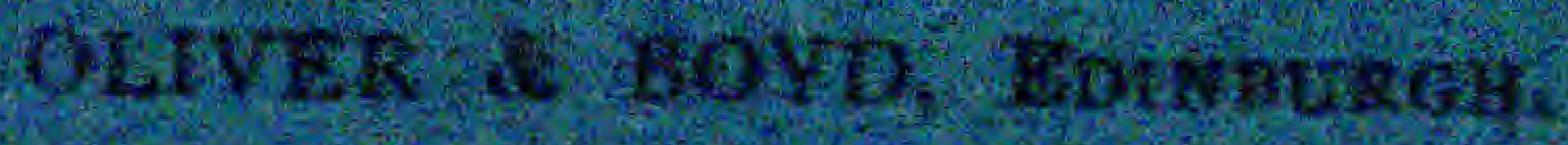

[PRICE NINEPENCE] 


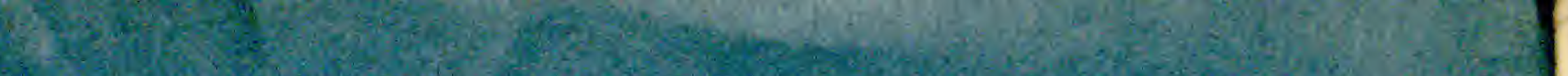




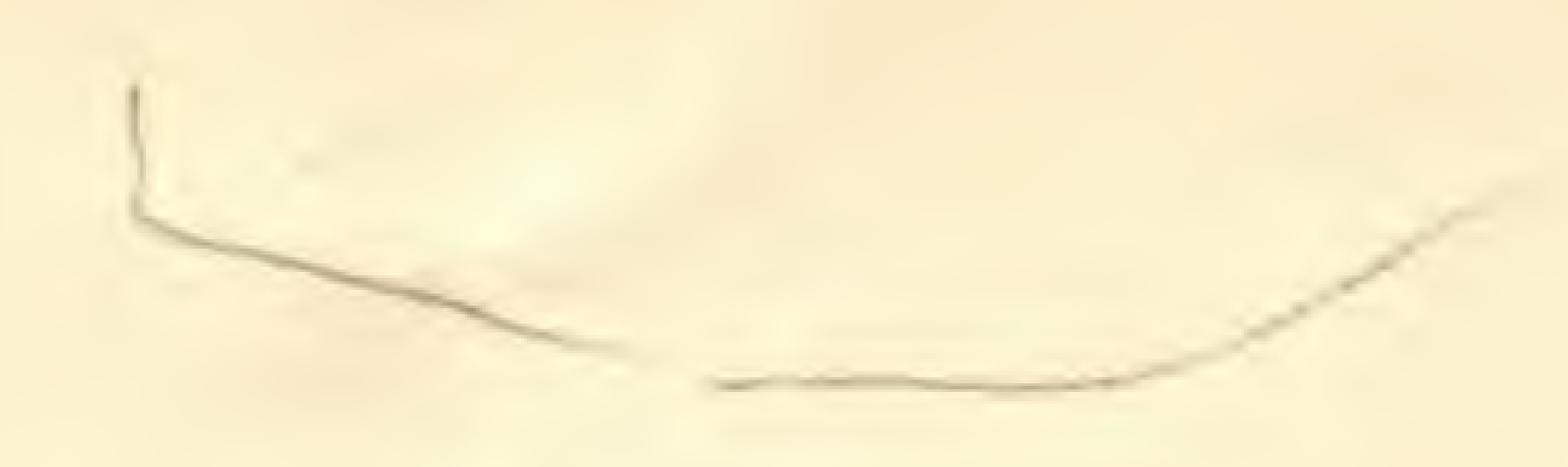

\section{List of Seeds Collected in the Royal Botanic Garden, Edinburgh, during the Year 1902.}

The following is a list of plants cultivated in the Royal Botanic Garden, Edinburgh, from which ripened seeds have been collected during the year 1902. The quantity of seed obtained from some of the species is of limited amount. The seeds are available for exchange, but they are not for sale :-

\section{PLANTS IN THE OPEN.}

\section{Acæna}

Novæ-Zelandiæ, T. Kirk.

Sanguisorbæ, Vahl.

Acer

opulifolium, Vill., var. obtusatum.

\section{Aconitum}

Napellus, Linn.

pyrenaicum, Hort.

\section{Actæa}

spicata, Linn.

- var. rubra.

\section{Adenophora}

stylosa, Fisch.

\section{Adonis}

vernalis, Linn.

\section{Ægopodium}

Podagraria, Linn.

\section{Æthionema}

saxatile, $R$. $B r$.

\section{Æthusa}

Cynapium, Linn.

\section{Allium}

carinatum, Linn.

fistulosum, Linn.

margaritaceum, Sibth, et $S m$.

Moly, Linn.

neapolitanum, $C y r$.

odorum, Linn.

oreophilum, C. A. Mey.

sativum, Linn.

Schœnoprasum, Linn.

- var. sibiricum.

vineale, Linn.

\section{Alnus}

cordifolia, Tenore.

glutinosa, Medic.

- var. rubrinervia, Hort.

japonica, Sieb. et Zucc.

serrulata, Willd. 
Alstroemeria

aurantiaca, Don.

\section{Alyssum}

creticum, Linn.

montanum, Linn.

umbellatum, Desv.

\section{Androsace}

lactiflora, Fisch.

\section{Anemone}

alpina, Linn.

rivularis, Buch.-Ham.

sylvestris, Linn.

\section{Anthericum}

Liliago, Lirn.

\section{Anthriscus}

sylvestris, Hoffm.

\section{Antirrhinum}

Asarina, Linn.

\section{Aquilegia}

chrysantha, A. Gray.

\section{Arabis}

alpina, Linn.

- var. anachoretica, Port.

blepharophylla, Hook, et Arn. hirsuta, Scop.

\section{Arctium}

Lappa, Linn.

\section{Arenaria}

gracilis, Kit.

graminifolia, Schrad.

juniperina, Linn.

peploides, Linn.

\section{Arnica}

amplexicaulis, Nutt. montana, Linn.

\section{Arracacia}

arguta, Benth. et Hook. f.

Aster

Tripolium, Linn.

\section{Astragalus}

alopecuroides, Linn.

Glyciphyllos, Linn,

danicus, Linn., var. albus.

xiphocarpus, Benth.

\section{Astrantia}

gracilis, Bartl.

helleborifolia, Salisb.

major, Linn.

- var. intermedia.

neglecta, C. Koch et Bouché.

\section{Atriplex}

Babingtonii, Woods.

\section{Atropa}

Belladonna, Linn.

\section{Aubrietia}

deltoidea, $D C$.

erubescens, Griseb.

\section{Barbarea}

vulgaris, $R . B r$.

\section{Berberis}

angulosa, Wall.

\section{Betula}

alba, Linn. 
Brassica

campestris, Linn.

juncea, Coss.

\section{Brodiæa}

Douglasii, S. Wats.

\section{Bryonia}

dioica, Jaìq.

\section{Bulbinella}

Hookeri, Benth. et Hook. f.

\section{Bupleurum}

Candollei, Wall.

\section{Callirhoe}

digitata, Nutt.

\section{Campanula}

Cervicaria, Linn.

glomerata, Linn.

linifolia, Scop.

mirabilis, Alboff.

persicifolia, Linn., var. alba.

pulla, Linn.

rotundifolia, Linn.

\section{Carbenia}

benedicta, Adans.

\section{Carduus}

acanthoides, Linn.

\section{Cenia}

turbinata, Pers.

\section{Centaurea}

macrocephala, Puschle.

nervosa, Willd.

pectinata, Linn.

Rhaponticum, Linn.
Cephalaria

tatarica, Schrad.

- var. minor.

\section{Cerastium}

alpinum, Linn.

tomentosum, Linn.

\section{Chærophyllum}

aromaticum, Linn.

aureum, Linn.

\section{Chelidonium}

majus, Linn.

- var. laciniatum.

\section{Chenopodium}

Bonus-Henricus, Linn.

\section{Chrysanthemum}

Leucanthemum, Iinn.

segetum, Linn.

\section{Cistus}

laxus, Hort.

monspeliensis, Linn

\section{Clematis}

Fremonti, S. Wats.

\section{Cnicus}

canus, Roth.

eriophorus, Roth.

tartaricus, Willd.

\section{Cochlearia}

glastifolia, Limn.

\section{Collinsia}

bicolor, Benth.

\section{Collomia}

grandiflora, Doug!. 


\section{Conium}

maculatum, Linn.

\section{Cotoneaster}

frigida, Wall.

$\checkmark$ Nummularia, Fisch, et Mey. rotundifolia, Wall.

Simonsii, Baker.

\section{Cotyledon}

Umbilicus, Linn.

\section{Crepis}

sibirica, Linn.

\section{Cytisus}

biflorus, L'Hérit.

canescens, Hort.

schipkaensis, Hort.

scoparius, Link.

- var. albus.

- var. Andreanus.

\section{Dahlia}

Merckii, Lehm.

\section{Delphinium}

altissimum, Wall.

cardinale, Hook.

hybridum, Steph.

Maackianum, Regel.

nudicaule, Torr. et Gray.

occidentale, S. Wats.

speciosum, Bieb., var. turkestanicum.

\section{Dianthus}

Armeria, Linn.

barbatus, Linn.

cæsius, Sm.

ciliatus, Guss.
Dianthus-continued.

deltoides, Linn.

petræus, Waldst. et Kit., var. spiculifolius.

plumarius, Linn.

pubescens, Sibth. et Sm.

squarrosus, Bieb.

\section{Digitalis}

purpurea, Linn.

\section{Dodecatheon}

Meadia, Linn., var. album.

\section{Draba}

Aizoon, Wahlenb.

arabisans, Michx.

aurea, $\mathrm{Vahl}$.

bruniæfolia, Stev.

cuspidata, Bieb.

hirta, Linn.

incana, Linn.

Loiseleurii, Boiss.

\section{Enkianthus}

japonicus, Hook. $f$.

\section{Epilobium}

angustifolium, Linn.

Dodonæi, Vill.

Lamyi, Schultz.

parviflorum, Schreb.

\section{Erigeron}

glabellus, Nutt., var, mollis.

\section{Erodium}

Botrys, Bertol.

\section{Eryngium}

alpinum, Linn.

Bourgati, Gouan.

Oliverianum, Delar. 


\section{Erysimum}

rupestre, $D C$.

thyrsoideum, Boiss.

\section{Eschscholzia}

cæspitosa, Benth.

californica, Cham.

\section{Euonymus}

europæus, Linn.

\section{Fraxinus}

excelsior, Linn.

\section{Funkia}

Sieboldiana, Hook.

\section{Galega}

orientalis, Lam.

\section{Galium}

saccharatum, All.

\section{Genista}

anglica, Linn.

hispanica, Linn.

præcox, Hort.

sagittalis, Linn.

\section{Gentiana}

asclepiadea, Linn.

Cruciata, Linn.

linearis, Froel.

Saponaria, Linn.

septemfida, Pall.

tibetica, King.

\section{Geranium}

pratense, Linn.

Robertianum, Linn.

sanguineum, Linn.

- var. lancastriense.

sylvaticum, Linn., var. album.

\section{Gerbera}

nivea, Sch. Bip.

\section{Geum}

Heldreichii, Hort.

hispidum, Fries.

japonicum, Thunb.

montanum, Linn., var. aurantiacum.

strictum, Ait.

\section{Gilia}

dichotoma, Benth.

minima, A. Gray, var.

coerulea.

tricolor, Benth.

\section{Hedysarum}

microcalyx, Baker.

neglectum, Ledeb.

\section{Helianthemum}

canum, Boiss.

umbellatum, Mill.

\section{Heracleum}

asperum, Bieb.

Wallichii, $D C$.

\section{Heuchera}

americana, Linn.

bracteata, Ser.

cylindrica, Dougl.

Drummondi, Hort.

\section{Hieracium}

bupleuroides, C. C. Gmel. cambricum, F.J. Hanb.

foliosum, Waldst. et Kit. gymnocephalum, Griseb.

\section{Hydrangea}

vestita, Wall. 


\section{Hydrastis}

canadensis, Linn.

\section{Hyoscyamus}

niger, Linn.

\section{Hypericum}

Androsæmum, Linn.

Ascyron, Linn.

elodeoides, Choisy.

erectum, Thunb.

hirsutum, Linn.

rhodopeum, Friv.

tetrapterum, Fries.

\section{Hypochœris}

radicata, Linn.

\section{Iberis}

sempervirens, Linn.

\section{Ilex}

Aquifolium, Linn.

Iris

Guldenstaedtiana, Lepech.

longipetala, Herb.

sibirica, Linn.

\section{Isatis}

tinctoria, Linn.

\section{Jasione}

montana, Linn.

\section{Jurinea}

glycacantha, $D C$.

\section{Kniphofia}

Tuckii, Baker.
Laburnum

alpinum, J. S. Presl.

- var. roseum.

vulgare, J. S. Presl, var, foliis aureis.

\section{Lagurus}

ovatus, Linn.

\section{Lathyrus}

Clymenum, Linn.

macrorrhizus, Wimm.

maritimus, Bigel.

montanus, Bernh.

splendens, Kellogg.

\section{Lavatera}

trimestris, Linn.

\section{Layia}

elegans, Torr. et Gray.

\section{Leontodon}

hispidus, Linn.

\section{Leontopodium}

alpinum, Cass, var. himalayanum.

\section{Leptosyne}

Stillmannii, A. Gray.

\section{Leycesteria}

formosa, Wall.

\section{Ligusticum}

Thomsoni, C. B. Clarke.

\section{Limnanthes}

Douglasii, R. Br.

\section{Linaria}

maroccana, Hook. $f$.

repens, Mill.

saxatilis, Hoffmgg. et Link.

triornithophora, Willd.

vulgaris, Mill. 
Linum

capitatum, Kit.

usitatissimum, Linn.

\section{Lophosciadium}

meifolium, $D C$.

Lotus

siliquosus, Linn.

\section{Lupinus}

arboreus, Linn.

- var. violaceus.

micranthus, Dougl.

nootkatensis, Donn.

polyphyllus, Lindl.

rivularis, Dougl.

\section{Lychnis}

alba, Mill.

Flos-jovis, Desr. montana, S. Wats.

Viscaria, Linn.

\section{Malva}

sylvestris, Linn.

\section{Matricaria}

Tchihatchewii, Hort. Kew.

\section{Meconopsis}

cambrica, Vig.

Wallichii, Hook, var. fuscopurpurea.

\section{Medicago}

carstiensis, Wulf.

Echinus, $D C$.

falcata, Linn.

hispida, Gaertn., var. apiculata. lupulina, $\operatorname{Linn}$.

sativa, Linn.

scutellata, All.

\section{Meum}

athamanticum, Jacq.

\section{Mimulus}

Muteus, Linn.

\section{Molopospermum}

cicutarium, $D C$.

\section{Monolepis}

trifida, Schrad.

\section{Moræa}

iridioides, Linn.

\section{Morina}

longifolia, Wall.

\section{Muscari}

Argæi, Hort.

armeniacum, Baker.

Maweanum, Baker.

\section{Myrrhis}

odorata, Scop.

\section{Nepeta}

nuda, Linn.

spicata, Benth.

\section{Nicotiana}

rustica, Linn.

\section{Enothera}

amœena, Lehm.

\section{Onobrychis}

viciæfolia, Scop.

\section{Ononis}

arvensis, Linn.

albo-roseum, Fisch. et Mey.

\section{Oxytropis}

glabra, $D C$.

lapponica, Gaud. 


\section{Pæonia}

corallina, Retz

\section{Papaver}

orientale, Linn.,var, bracteatum. pilosum, Sibth. et $S m$. rupifragum, Boiss, et Reut. somniferum, Linn.

\section{Pentstemon}

barbatus, Roth. confertus, Dougl. diffusus, Dougl. gentianoides, Poir.

\section{Perezia}

multiflora, Less.

\section{Phormium}

tenax, Linn.f.

\section{Phyteuma}

orbiculare, Linn.

Scheuchzeri, All.

Sieberi, Spreng.

\section{Pimpinella}

magna, Linn.

Saxifraga, Linn.

\section{Pisum}

sativum, Linn.

\section{Platystemon}

californicus, Benth.

\section{Polemonium}

boreale, Adams.

cæruleum, Linn.

himalayanum, Baker.

mexicanum, Cerv.

pauciflorum, S. Wats.

\section{Polygonum}

baldschuanicum, Regel.

Convolvulus, Linn.

Weyrichii, F. Schmidt.

\section{Potentilla}

ambigua, Jacq.

andicola, Benth.

apennina, Tenore.

argentea, Linn., var. calabra.

bifurca, Linn.

chinensis, Ser.

chrysantha, Trevir.

desertorum, Bunge.

Dombeyi, Nestl.

glandulosa, Lindl.

Hopwoodiana, Sweet.

Macnabiana $\times$, Lem.

multifida, Linn.

nepalensis, Hook.

nivea, Linn.

norvegica, Linn.

opaca, Linn.

palustris, $S c o p$.

pensylvanica, Linn.

recta, Linn.

rupestris, Linn.

semilaciniata, Hort.

vladnizensis, Sieg $f r$.

\section{Poterium}

canadense, A. Gray.

diandrum, Hook. $f$.

\section{Prenanthes}

purpurea, Linn.

\section{Primula}

apennina, Wid.

Balbisii, Lehm., var. bellunensis.

elatior, Hill.

japonica, A. Gray, var. lilacina. 
Primula-continued. mollis, Nutt.

officinalis, Jacq.

sikkimensis, Hook. $f$.

\section{Pulicaria}

vulgaris, Gaertn.

\section{Ramondia}

pyrenaica, Rich.

\section{Ranunculus}

acris, Linn.

brutius, Tenore.

Flammula, Linn.

lanuginosus, Linn.

ophioglossifolius, Vill.

sceleratus, Linn.

Thora, Linn.

\section{Raphanus}

Raphanistrum, Linn.

\section{Rosa}

rugosa, Thunb.

\section{Rumex}

maritimus, Linn.

\section{Salvia}

hians, Royle.

pratensis, Linn.

\section{Saponaria}

ocymoides, Linn.

\section{Saxifraga}

Aizoon, Jacq .

aretioides, Lapeyr.

cresia, Linn.

cæspitosa, Linn.

Cymbalaria, Linn.

decipiens, Ehrh.
Saxifraga-continued.

granulata, Linn.

Haworthii, Hort.

Hostii, Tausch.

Huetiana, Boiss.

hypnoides, Linn.

Mertensiana, Bongard.

nivalis, Linn.

pedatifida, Ehrh.

pubescens, Pourr.

Rocheliana, Sternb., var. coriophylla.

squarrosa, Sieber.

umbrosa, Linn., var. serratifolia (Mackay).

\section{Scabiosa}

caucasica, Bieb.

\section{Scorpiurus}

muricata, Linn.

\section{Senecio}

Doronicum, Linn.

Fuchsii, C. C. Gmel.

\section{Silene}

alpestris, Jacq.

caucasica, Boiss.

Cucubalus, Wibel.

Czerei, Boiss.

italica, Pers.

maritima, With.

nocteolens, Webb et Berth.

quadridentata, Pers.

rhynchocarpa, Boiss.

Saxifraga, Linn.

stylosa, Bunge.

Thorei, Duf.

verecunda, $S$. Wats.

Zawadzkii, Herbich.

\section{Sisyrinchium}

angustifolium, Mill., var. mucronatum (Michx.). 
Smyrnium

Olusatrum, Linn.

\section{Solidago}

$\checkmark$ Virgaurea, Linn.

\section{Spiræa}

Aruncus, Linn.

palmata, Thunb.

\section{Stachys}

recta, Linn.

\section{Stenanthium}

angustifolium, Kunth.

\section{Succowia}

balearica, Medic.

\section{Symphoricarpus}

racemosus, Michx.

\section{Synthyris}

reniformis, Benth.

\section{Taxus}

baccata, Linn.

\section{Thalictrum}

glaucum, Desf.

majus, Jacq.

minus, Linn.

simplex, Linn.

\section{Thermopsis}

fabacea, $D C$.

lanceolata, R. $B$ r.

\section{Tofieldia}

calyculata, Wahlenb.

Trifolium

alpinum, Ltnn.

pannonicum, Linn.

striatum, Linn.

\section{Trollius}

altaicus, Mey.

\section{Tunica}

prolifera, $S c o p$.

\section{Valeriana}

tuberosa, Linn.

\section{Veratrum}

album, Linn.

\section{Veronica}

officinalis, Linn.

\section{Vicia}

Cracca, Wulf.

oroboides, Wulf.

Orobus, $D C$.

pyrenaica, Pourr.

sylvatica, Linn.

villosa, Roth.

\section{Viola}

cornuta, Linn.

hirta, Linn.

sylvestris, Lam.

\section{Wulfenia}

carinthiaca, Jacq. 


\section{PLANTS UNDER GLASS.}

\section{Acacia}

Farnesiana, Willd.

grandis, Henfr.

juncifolia, Benth.

neriifolia, A. Cunn.

suaveolens, Willd.

\section{Acanthostachys}

strobilacea, Link, Klotzsch et Otto.

\section{Æchmea}

Ubromeliæfolia, Baker.

$<$ var. pulchrum.

candida, E. Morr.

corulescens, Baker.

4 Makoyana, Hort. Makoy.

Weilbachii, Dietr., var.

Teodiensis.

xiphophylla, Baker.

\section{Agave}

attenuata, Salm-Dyck. xalapensis, Roesl.

\section{Aglaonema}

commutatum, Scholt.

\section{Aloe}

mitriformis, Mill., var. spinulosa (Salm-Dyck).

striata, Haw.

\section{Araujia}

sericifera, Brot.

\section{Aristolochia}

brasiliensis, Mart, et Zucc.

elegans, Mast.

$\checkmark$ fimbriata, Cham.

\section{Asclepias}

curassavica, Linn.

\section{Astrocaryum}

mexicanum, Liebm.

\section{Begonia}

Dregei, Otto et Dietr.

semperflorens, Link et Otto.

Verschaffeltii $\times$, Regel.

Weltoniensis $\times$, Hort.

\section{Bertolonia}

$\checkmark$ maculata, $D C$.

\section{Billardiera}

longiflora, Labill.

\section{Billbergia}

pallidiflora, Liebm.

thyrsoidea, Mart., var. longifolia.

zebrina, Lindl.

\section{Brachychilum}

Horsfieldii, Baker.

\section{Brodiæa}

lactea, S. Wats.

\section{Callicarpa}

purpurea, Juss.

\section{Callistemon}

linearis, $D C$.

speciosus, $D C$.

\section{Carex}

$\checkmark$ japonica, Thunb. 


\section{Cassia}

corymbosa, Lam.

\section{Cedronella}

triphylla, Moench.

\section{Chamæedorea}

Sartorii, Liebm.

\section{Chlorophytum}

Orchidastrum, Lindl.

\section{Cleome}

gigantea, Linn.

\section{Clerodendron}

splendens, G. Don.

\section{Clianthus}

puniceus, Banks et Sol.

\section{Clitoria}

Ternatea, Linn.

- var. alba.

\section{Cobæa}

scandens, Cav.

\section{Coffea}

arabica, Linn.

bengalensis, Roxb.

\section{Costus}

Iafer, Ker-Gawl.

igneus, N. E. Br.

\section{Crossandra}

undulæfolia, Salisb.

\section{Cytisus}

candicans, Lam.

\section{Desmodium}

gyrans, $D C$.

\section{Didymosperma}

porphyrocarpon, H. Wendl. et Drude.

\section{Dolichos}

Lablab, Linn.

\section{Drosera}

1 spathulata, Labill.

\section{Drosophyllum}

$\checkmark$ lusitanicum, Link.

\section{Eccremocarpus}

scaber, Ruiz et Pav.

\section{Eriostemon}

buxifolius, $\mathrm{Sm}$.

\section{Erythrochiton}

brasiliensis, Nees.

\section{Eucalyptus}

ficifolia, F. Muell.

incrassata, Labill.

\section{Eucomis}

punctata L'Hérit.

\section{Eupatorium}

Weinmannianum, Regel.

\section{Evodia}

elegans, Hort.

\section{Fuchsia}

$\checkmark$ procumbens, R. Cunn.

\section{Gasteria}

disticha, Haw., var. angustifolia, Baker. 


\section{Geonoma}

L pumila, Wendl.

Gerbera

Jamesoni, Bohus.

\section{Gloriosa}

superba, $\operatorname{Linn}$.

\section{Goodia}

lotifolia, Salisb.

\section{Gossypium}

herbaceum, Lam.

\section{Grevillea}

glabrata, Meissn.

\section{Guizotia}

abyssinica, Cass.

\section{Hedychium}

Gardnerianum, Rosc.

\section{Hibiscus}

gossypinus, Thunb.

Manihot, Linn.

pedunculatus, Linn.

Trionum, Linn., var. major.

\section{Humea}

elegans, $S m$.

\section{Hydrolea}

spinosa, Linn.

\section{Indigofera}

atropurpurea, Buch.-Ham. australis, Willd.

\section{Ipomœa \\ Bona-Nox, Linn.}

\section{Kalanchoe}

crenata, Haw.

flammea, Stapf.

thyrsiflora, Harv.

Welwitschii, Britten.

\section{Kennedya}

prostrata, R. Br.

— var. Marryatæ.

\section{Kunzea}

parvifolia, Schau.

\section{Lantana}

Camara, Linn.

\section{Leptospermum}

ericoides, $A$. Rich.

scoparium, Forst.

\section{Loasa}

lateritia, Gill., var. aurantiaca, Hort.

\section{Luffa}

ægyptiaca, Mill.

\section{Lycopersicum}

esculentum, Mill.

\section{Maurandia}

Barclayana, Lindl.

\section{Melothria}

cucumerina, Naud.

\section{Mesembryanthemum}

curviflorum, Haw.

digitiforme, Thunb.

lepidum, Haw.

sanguineum, Hort.

violaceum, $D C$. 


\section{Mimosa}

marginata, Lindl.

\section{Mimulus}

longiflorus, Hort.

\section{Momordica}

Charantia, Linn.

cochinchinensis, Spreng.

\section{Moræa}

prolongata, Hort.

\section{Musschia}

Wollastoni, Lozere.

\section{Myrica}

æthiopica, Limn.

\section{Myrtus}

communis, Linn.

Luma, Barn.

Ugni, Mol.

\section{Nephthytis}

liberica, N.E. Br.

\section{Nicotiana}

sylvestris, Hort.

\section{Ornithogalum}

tenellum, Jacq.

\section{Oryza}

latifolia, Desv.

sativa, Linn.

\section{Oxypetalum}

coruleum, Disne.

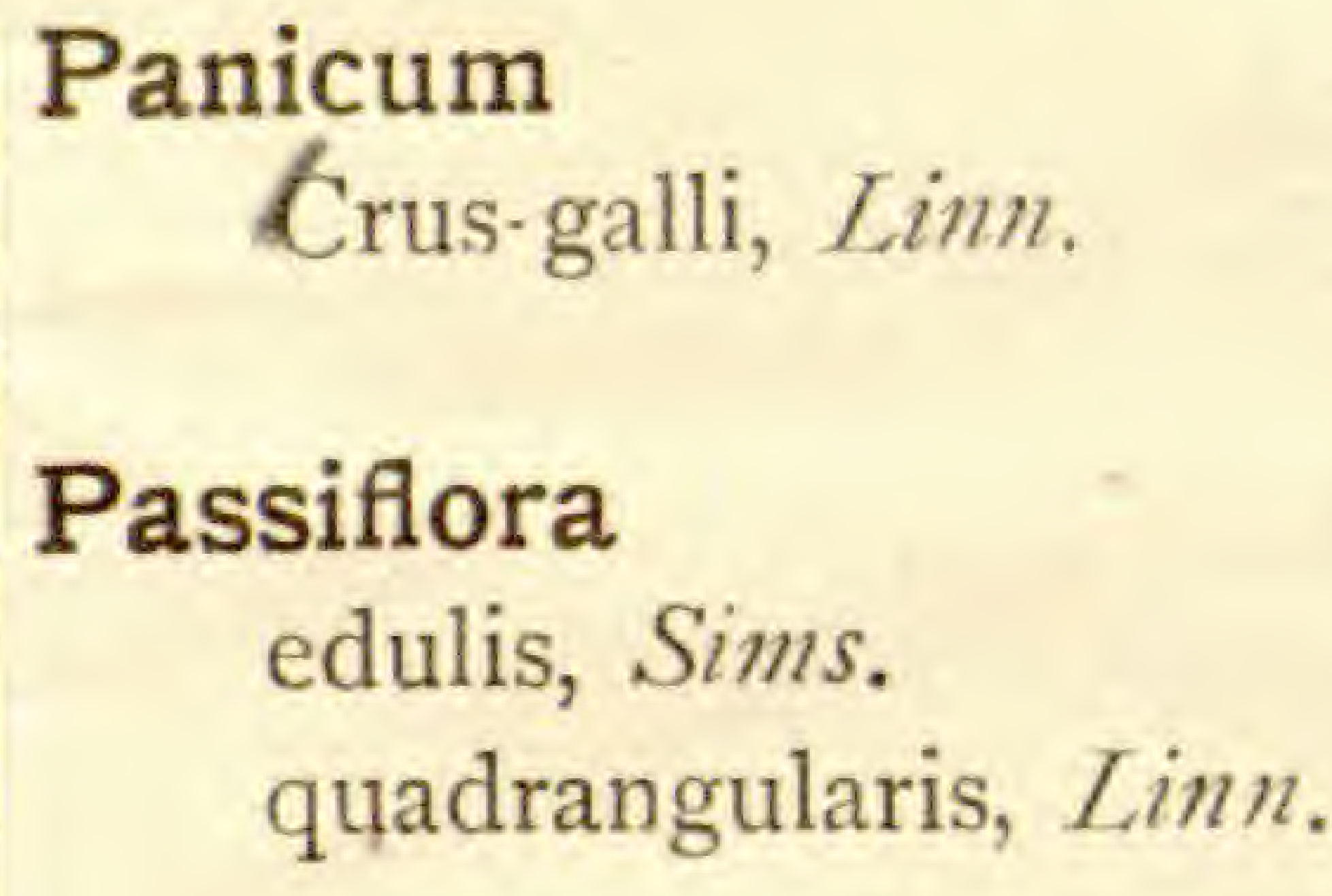

Crus-galli, Linn.

\section{Passiflora}

edulis, Sims.

quadrangularis, Linn.

\section{Pelargonium}

ternatum, Linn.

\section{Pitcairnia}

amaryllidifolia, Hort. latifolia, Soland. maidifolia, Desne. pulverulenta, Ruiz et Pav.

\section{Polyalthia}

suberosa, Benth. et Hook. f.

\section{Primula}

japonica, A. Gray, var. lilacina. mollis, Nutt.

verticillata, Forsk.

\section{Prostanthera}

lasianthos, Labill.

\section{Rhodochiton}

volubile, Zucc.

\section{Rhododendron}

Nuttallii, Booth.

\section{Rhynchosia}

caribæa, $D C$.

\section{Rivina}

humilis, Linn.

\section{Ruellia}

fuberasa, Linn. 
Sabal

Blackburniana, Glazebr.

\section{Saxifraga}

Cotyledon, Linn., var. pyrami dalis.

Macnabiana, Hort.

\section{Schismatoglottis}

Rœbelini, Hort.

\section{Sempervivum}

annuum, $C$. Sm.

tectorum, Linn.

\section{Solanum}

auriculatum, Ait.

coagulans, Hort.

fastigiatum, Willd.

hybridum, Jacq.

jasminoides, Paxt.

nigrum, Linn.

pyracanthum, Jacq.

Seaforthianum, Andr.

Stachytarpheta

indica, Vahl.

\section{Tacca}

cristata, Jack.

\section{Tacsonia}

Cexoniensis $x$, Hort.

mollissima, H.B.K.

$\measuredangle$ Van, Volxemii, Hook.

\section{Tecoma}

Smithii, Hort.

\section{Tetranema}

mexicana, Benth.

\section{Thrinax}

parviflora, Sze.

\section{Tillandsia}

$\angle$ aurea, Hort.

corallina, $K$. Koch.

splendens, Brong.

\section{Trachelium}

cœruleum, Linn.

\section{Tristania}

laurina, $R . B r$.

\section{Villamilla}

purpurascens, Hort.

\section{Vitis}

Cheterophylla, Thunb., var. humulifolia. 



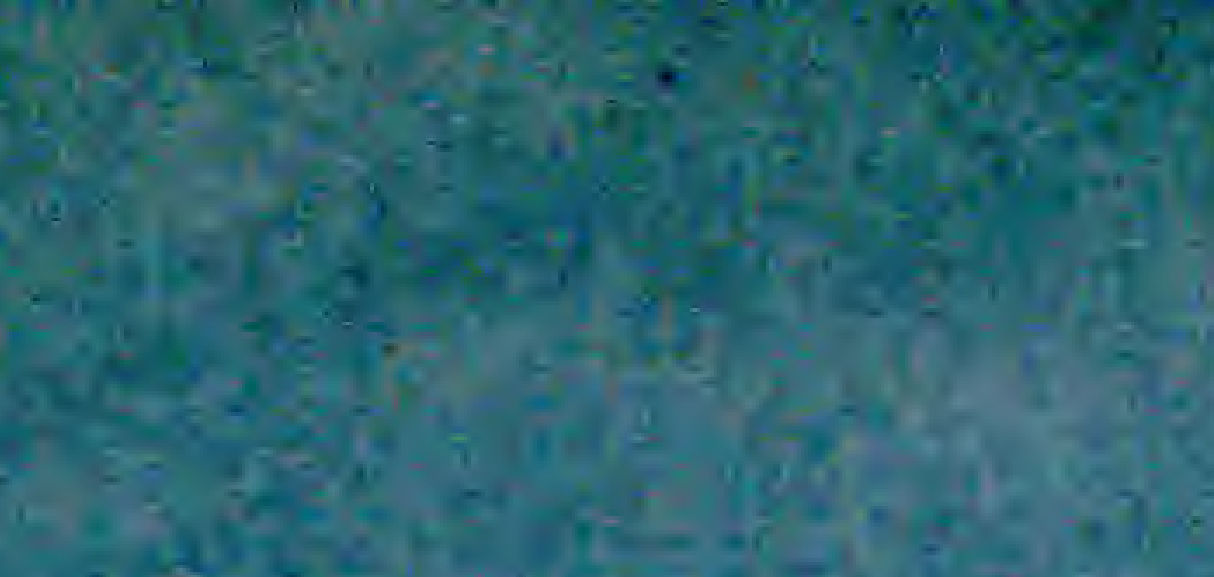



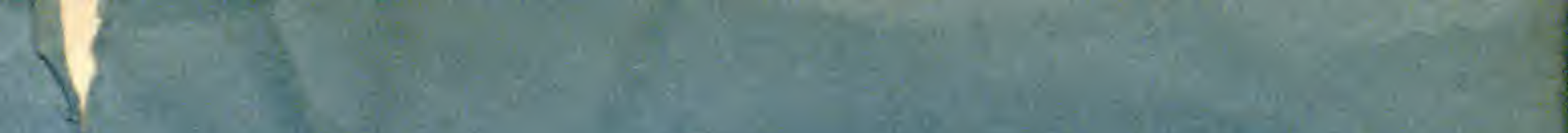
OFFICIAL COPY.

\title{
NOTES
}

FROM THE

\section{ROYAL BOTANIC GARDEN,}

\section{EDIN BURGH.}

\section{JANUARY 1904.}

\author{
CONTENTS.
}

List of Seeds collected in the Royal Botanic Garden, Page. Edinburgh, during the Year 1903, _ _ _ - xili

\section{GL. ASGOW:}

PRINTED FOR UIS MAJESTY'S STATIONERY OHFICL BY JAMES HEDOER WICK \& SONS. AT " rm CHIZES" PRESS, ST, VANCENT PLACE.

\section{SOLD AT THE GARDEN,}

And ta be porchased, erher firectly of thinough any Dookselle, from OLIVER it BOYD DOMNURCE. 


\section{List of Seeds Collected in the Royal Botanic}

\section{Garden, Edinburgh, during the Year 1903.}

The following is a list of plants cultivated in the Royal Botanic Garden, Edinburgh, from which ripened seeds have been collected during the year 1903. The quantity of seed obtained from some of the species is of limited amount. The seeds are available for exchange, but they are not for sale :-

\section{PLANTS IN THE OPEN.}

\section{Acæna}

argentea, Ruiz et Pav.

glabra, J. Buch.

inermis, Hook. $f$.

microphylla, Hook. $f$.

myriophylla, Lindl.

Sanguisorbæ, Vahl.

Acer

circinatum, Pursh.

platanoides, Linn.

Pseudo-platanus, Linn.

- var. purpureum.

\section{Achillea}

Millefolium, Linn.

\section{Aconitum}

Lycoctonum, Linn.

longigaleatum, Hort.

Napellus, Linn.

paniculatum, Lam.

reclinatum, A. Gray.

variegatum, Linn.

\section{Actæa}

spicata, Linn.

- var. rubra.

\section{Adenophora}

polymorpha, Ledeb.

stylosa, Fisch.

\section{Adenostyles}

alpina, Kern.

\section{Adonis}

autumnalis, Linn.

vernalis, Linn.

\section{Ethionema}

cordatum, Boiss.

grandiflorum, Boiss. et Hohen.

\section{Agrimonia}

Eupatoria, Linn. 


\section{Agropyron}

acutum, Roem. et Schult. dasyanthum, Ledeb. tenerum, Vasey.

\section{Alchemilla}

alpina, Linn., var. conjuncta (Bab.).

\section{Alisma}

Plantago, Linn.

\section{Allium}

cardiostemon, Fisch. et Mey. carinatum, Linn. cernuum, Roth. fistulosum, Linn. flavum, Linn. giganteum, Regel. kansuense, Regel. karataviense, Regel. macranthum, Baker. mutabile, Michx. margaritaceum, Sibth. et Sm. odorum, Linn. oleraceum, Linn. oreophilum, C. A. Mey. Ostrowskianum, Regel. rubellum, Bieb. Schœnoprasum, Linn. senescens, Linn.

\section{Alnus}

cordifolia, Tenore. glutinosa, Medic. - var. rubrinervia. incana, Moench. japonica, Sieb. et Zucc. serrulata, Willd. viridis, $D C$.

\section{Alopecurus}

geniculatus, Linn.

\section{Alstroemeria}

aurantiaca, Don.

\section{Althæa}

rosea, Cav.

\section{Alyssum}

argenteum, Vitm.

condensatum, Boiss, et

Hausskn.

creticum, Linn.

idæum, Boiss, et Heldr. saxatile, Linn.

- var. sulphurea.

\section{Amelanchier}

oligocarpa, Roem.

\section{Anchusa}

altissima, Desf.

italica, Retz.

officinalis, Linn.

\section{Androsace}

lactea, Linn.

lactiflora, Fisch.

lanuginosa, Wall.

sarmentosa, Wall.

septentrionalis, Linn.

\section{Anemone}

alpina, Linn.

- var. sulphurea.

baicalensis, Turcs.

multifida, Poir.

rivularis, Buch.-Ham.

sylvestris, Linn.

vitifolia, Buch.-Ham.

\section{Anthericum}

Liliago, Linn. 


\section{Antirrhinum}

Asarina, Linn.

majus, Linn.

Orontium, Linn.

\section{Apera}

interrupta, Beauv.

Spica-Venti, Beauv.

\section{Aphyllanthes}

monspeliensis, Linn.

\section{Aquilegia}

Einseliana, F. Schultz.

pyrenaica, $D C$.

\section{Arabis}

Allionii, $D C$.

alpina, Linn.

- var. anachoretica.

muralis, Bertol., var rosea.

petræa, Lam.

Turczaninowii, Ledeb.

\section{Arenaria}

balearica, Linn.

erythrosepala, Boiss.

foliosa, Royle.

Fendleri, A. Gray.

- var. diffusa.

gothica, Fries.

gracilis, Kit.

graminifolia, Schrad.

gypsophiloides, Linn.

Holostea, Bieb.

juniperina, Linn.

laricifolia, Linn.

lateriflora, Linn.

pinifolia, Bieb.

tetraquetra, Linn.

trinervia, Linn.

verna, Linn.

\section{Arnica}

Chamissonis, Less.

montana, Linn.

\section{Arracacia}

arguta, Benth. et Hook. f.

\section{Arrhenatherum}

avenaceum, Beauv.

\section{Artemisia}

tanacetifolia, Linn.

\section{Arum}

palæstinum, Boiss.

\section{Asperula}

ciliata, Rochel.

lævigata, Linn.

orientalis, Boiss. et Hohen.

tinctoria, Linn.

\section{Aster}

floribundus, Nutt.

leucanthemus, Rafin.

patens, Ait.

sibiricus, Linn.

\section{Astragalus}

Cicer, Linn.

danicus, Rets., var. alba.

falcatus, Lam.

Glyciphyllos, Linn.

monspessulanus, Linn.

purpureus, Lam.

sinicus, Linn.

vaginatus, Pall.

\section{Astrantia}

helleborifolia, Salisb.

major, Linn.

- var. intermedia.

neglecta, C. Koch et Bouché. 


\section{Astydamia}

canariensis, $D C$.

\section{Athamanta}

Matthioli, Wulf.

\section{Atriplex}

Babingtonii, Woods.

patula, Linn.

\section{Atropa}

Belladonna, Linn.

\section{Aubrietia}

croatica, Schott, Nyman et Kotschy.

deltoidea, $D C$, var, cilicica.

\section{Baccharis}

patagonica, Hook, et Arn.

\section{Ballota}

nigra, Linn.

\section{Barbarea}

præcox, R. Br.

vulgaris, $R . B r$.

\section{Berberis}

angulosa, Wall.

Aquifolium, Pursh.

virescens, Hook. f.

vulgaris, Linn.

\section{Betula}

alba, Linn. var. pubescens.

Ermani, Cham.

\section{Biscutella}

cichoriifolia, Loisel.

\section{Brassica}

campestris, Linn.

\section{Bravoa}

geminiflora, Lex.

\section{Briza}

maxima, Linn.

\section{Brodiæa}

Douglasii, S. Wats.

\section{Bromus}

inermis, Leyss.

unioloides, $H$.B. et $K$.

\section{Bruckenthalia}

spiculifolia, Reichb.

\section{Bryanthus}

empetriformis, A. Gray.

\section{Bryonia}

dioica, Jacq.

\section{Buddleia}

intermedia, Carr.

variabilis, Hemsl.

\section{Bulbinella}

Hookeri, Benth, et Hook, f.

\section{Bupleurum}

Candollei, Wall.

longifolium, Linn.

rotundifolium, Linn.

\section{Butomus}

umbellatus, Limn. 


\section{Calamintha}

officinalis, Moench.

patavina, Host.

\section{Calendula}

arvensis, Linn.

officinalis, Linn.

\section{Callirhoe}

digitata, Nutt.

\section{Camassia}

Cusickii, S. Wats.

\section{Campanula}

alliariæfolia, Willd.

barbata, Linn.

carpatica, Jacq., var. turbinata.

Grossekii, Heuff.

isophylla, Moretti, var. alba.

lactifiora, Bieb.

latifolia, Linn., var. macrantha. michauxioides, Boiss.

mirabilis, Alboff.

persicifolia, Linn.

- var. alba.

- var. grandiflora.

planiflora, Lam.

pulla, Linn.

pusilla, Haenke.

- var. alba.

rapunculoides, Linn.

rotundifolia, Linn.

Trachelium, Linn.

Vidalii, $H$. C. Wats.

\section{Carbenia}

benedicta, Adans.

\section{Cardamine}

Impatiens, Linn.

latifolia, Vahl.

\section{Carex}

aquatilis, Wahlenb.

arenaria, Linn.

binervis, $\mathrm{Sm}$.

capillaris, Linn.

depauperata, Good.

dioica, Linn.

distans, Linn.

extensa, Good., var. pumila.

flava, Linn.

- var. Oederi.

glauca, $S c o p$.

hirta, Linn.

hordeistichos, Vill.

irrigua, $S m$.

lævigata, $\mathrm{Sm}$.

leporina, Linn.

Mairii, Coss, et Germ.

microglochin, Wahlenb.

muricata, Linn.

pallescens, Linn.

paludosa, Good.

panicea, Linn.

pulicaris, Linn.

punctata, Gaud.

riparia, Curt.

sparganioides, Muhl.

strigosa, Huds.

sylvatica, Huds.

teretiuscula, Good.

vaginata, Tausch.

vulgaris, Fries.

\section{Carmichaelia}

flagelliformis, Colenso.

\section{Cassandra}

calyculata, D. Don.

\section{Catananche}

bicolor, Hort. 


\section{Cathcartia}

villosa, Hook. $f$.

\section{Celsia}

pontica, Boiss.

\section{Centaurea}

Cyanus, Linn., var. minor.

macrocephala, Puschk.

pectinata, Linn.

Rhaponticum, Linn.

ruthenica, Lam.

Scabiosa, Linn.

stereophylla, Bess.

\section{Centranthus}

macrosiphon, Boiss.

\section{Cephalaria}

alpina, Schrad.

radiata, Griseb. et Schenk.

tatarica, Schrad.

— var. minor.

\section{Cerastium}

alpinum, Linn.

arvense, Linn., var.grandiflorum.

Boissieri, Gren.

ovatum, Hoppe.

tomentosum, Linn.

\section{Chærophyllum}

aureum, Linn.

\section{Cheiranthus}

Cheiri, Linn.

Senoneri, Heldr. et Sart.

\section{Chelidonium}

majus, Linn.

— var. laciniatum.

\section{Chelone}

Lyoni, Pursh.

\section{Chenopodium}

Bonus-Henricus, Linn.

capitatum, Aschers.

\section{Chrysanthemum}

arcticum, Linn.

caucasicum, Pers.

coronopifolium, Vill., var.

hybridum.

Leucanthemum, Linn. maximum, Ramond. montanum, Linn. segetum, Linn.

\section{Cimicifuga}

elata, Nutt.

racemosa, Nutt.

\section{Cistus}

laurifolius, Linn.

\section{Claytonia}

virginica, Linn.

\section{Clematis}

alpina, Mill., var. alba.

paniculata, Thunb.

\section{Cnicus}

centauroides, Linn.

eriophorus, Roth.

græcus, Hort.

heterophyllus, Roth.

pannonicus, Host, var. divari-

catus.

pratensis, Willd.

tauricus, Hort. 


\section{Cochlearia}

alpina, Wats.

glastifolia, Linn.

\section{Codonopsis}

ovata, Benth.

rotundifolia, Royle.

\section{Collomia}

coccinea, Lehm.

grandiflora, Dougl.

\section{Colutea}

istria, Mill.

\section{Coreopsis}

coronata, Hook.

\section{Coronilla}

montana, Scop.

varia, $\operatorname{Linn}$.

\section{Corydalis}

glauca, Pursh.

lutea, $D C$.

racemosa, Pers.

\section{Cotoneaster}

acuminata, Lindl.

buxifolia, Wall.

frigida, Wall.

horizontalis, Decne.

microphylla, Wall.

Nummularia, Fisch. et Mey.

Simonsii, Baker.

\section{Crambe}

tatarica, Hort.

\section{Cratægus}

Azarolus, Linn.

coccinea, Linn.

mollis, Scheele.

Oxyacantha, Limn.

- sub-sp. monogyna, Jacq.

- var. pendula.

punctata, Jacq.

- tanacetifolia, Pers.

tomentosa, Linn.

\section{Crepis}

sibirica, Linn.

\section{Crocus}

speciosus, Bieb.

\section{Crucianella}

angustifolia, $\operatorname{Linn}$.

\section{Cupressus}

Lawsoniana, A. Murr.

\section{Cynoglossum}

microglochin, Benth.

officinale, $\operatorname{Linn}$.

Wallichii, G. Don.

\section{Cytisus}

albus, Linn.

Ardoini, Fourn.

biflorus, L'Hérit.

hirsutus, Linn.

leucanthus, Waldst. et Kit.

præcox, Hort.

scoparius, Link.

- var. Andreanus.

\section{Daboecia}

polifolia, D. Don.

- var. alba.

- var. bicolor. 


\section{Delphinium}

caucasicum, C. A. Mey.

flexuosum, Rafin.

formosum, Boiss, et Huet.

grandiflorum, Linn., var. album.

hybridum, Steph.

Maackianum, Regel.

nudicaule, Torr. et Gray. speciosum, Bieb., var. turkes- ' tanicum.

tatsienense, Franch.

truncatum, Hort.

\section{Deschampsia}

cæspitosa, Beauv.

\section{Dianthus}

alpinus, $\operatorname{Linn}$.

Armeria, Linn.

brevicaulis, Fenzl.

ciliatus, Guss.

deltoides, Linn.

— var. albus.

monspessulanus, Linn.

neglectus, Loisel.

pallens, Sibth. et Sm.

Requienii, Gren. et Godr.

Sternbergii, Sibth.

subacaulis, Vill.

trifasciculatus, Kit.

\section{Digitalis}

purpurea, Linn.

\section{Dimorphotheca}

pluvialis, Moench.

\section{Diphylleia}

cymosa, Michx.

\section{Diplotaxis}

erucoides, $D C$.

\section{Dipsacus}

laciniatus, Linn.

pilosus, Linn.

sylvestris, Mill.

\section{Dodecatheon}

Meadia, Linn.

- var. album.

\section{Draba}

aizoides, Linn.

arabisans, Michx.

aurea, $V a h l$.

cornuta, Hort.

fladnizensis, Wulf.

hirta, Linn.

incana, Linn.

Mawii, Hook.f.

rupestris, $R$. $B r$.

siliquosa, Bieb.

\section{Dracocephalum}

Moldavica, Linn.

speciosum, Benth.

\section{Drosera}

intermedia, Hayne.

longifolia, Hayne.

rotundifolia, Linn.

\section{Dryas}

Drummondii, Richards.

octopetala, Linn.

\section{Echinops}

Ritro, Linn.

spinosus, Linn.

\section{Echium}

plantagineum, Linn.

rosulatum, Lange. 


\section{Eleocharis}

palustris, R. Br.

\section{Elymus}

sibiricus, Linn.

\section{Embelia}

robusta, Roxb.

\section{Epilobium}

alpinum, Linn.

alsinifolium, Vill.

angustifolium, Linn.

Dodonæi, Vill.

Hectori, Hausskn.

montanum, Linn.

rosmarinifolium, Haenke.

- var. sericeum.

\section{Erica}

ciliaris, Linn.

cinerea, Linn.

- var. alba.

stricta, Donn.

\section{Erigeron}

salsuginosus, A. Gray.

\section{Erinus}

alpinus, Linn.

- var. carmineus.

\section{Erodium}

chelidonifolium, Hort. malachoides, Willd. moschatum, L'Hérit.

\section{Erophila}

vulgaris, $D C$.

\section{Eryngium}

alpinum, Linn.

Bourgati, Gouan.

Sanguisorba, Cham. et Schlecht.

\section{Erythræa}

Massoni, Sweet.

\section{Erythronium}

grandiflorum, Pursh, var. roseum.

\section{Eschscholzia}

californica, Cham.

\section{Eupatorium}

ageratoides, Linn.

cannabinum, Linn.

japonicum, Thunb., var.

saccharinum.

Lindleyanum, $D C$.

purpureum, Linn.

\section{Euphorbia}

Myrsinites, Linn.

trapezoidalis, Boiss.

\section{Filago}

germanica, Linn.

\section{Fœniculum}

giganteum, Hort.

\section{Fragaria}

vesca, Linn, var. muricata.

\section{Fraxinus}

excelsior, Linn.

\section{Fuchsia}

Riccartoni, Hort. 


\section{Fumaria}

officinalis, $\operatorname{Linn}$.

\section{Galanthus}

Elwesii, Hook. $f$.

\section{Galax}

aphylla, Linn.

\section{Galega}

officinalis, Linn.

orientalis, Lam.

\section{Galium}

boreale, Linn.

lithospermifolium, Fisch.

Mollugo, Linn.

saccharatum, All.

saxatile, Linn.

\section{Galtonia}

candicans, Decne.

\section{Gaultheria}

procumbens, Linn.

Shallon, Pursh.

\section{Genista}

hispanica, Linn.

sagittalis, Linn.

tinctoria, Linn.

\section{Gentiana}

asclepiadea, Linn.

- var. alba.

Cruciata, Linn.

decumbens, Linn.

punctata, Linn.

Saponaria, Linn.

septemfida, Pall.

tibetica, King.

verna, Linn.

\section{Geranium}

cinereum, Cav.

Fremontii, Torr.

macrorrhizum, Linn.

phæum, Linn.

polyanthes, Edgew. et Hook.f.

roseum, L'Hérit.

sanguineum, Linn.

- var. lancastriense.

sylvaticum, Linn.

\section{Gerbera}

Kunzeana, $A$. Br. et Aschers.

nivea, Sch. Bip.

\section{Geum}

Borissi, Hort.

bulgaricum, Panc.

elatum, Wall.

Eweni, Hort.

Heldreichii, Hort.

hispidum, Fries.

intermedium, Ehrh.

Laxmanni, Gaertn.

montanum, Linn.

rivale, Linn.

\section{Gilia}

dichotoma, Benth.

\section{Gillenia}

stipulacea, Nutt.

\section{Globularia}

Alypum, Linn.

\section{Graphephorum}

arundinaceum, Aschers.

\section{Gunnera}

chilensis, Lam. 


\section{Gypsophila}

dubia, Willd.

prostrata, Linn.

repens, $\operatorname{Linn}$.

- var. monstrosa.

\section{Habenaria}

intacta, Benth.

\section{Haberlea}

rhodopensis, Frivald.

\section{Hebenstreitia}

comosa, Hochst.

\section{Helianthemum}

Fumana, Mill.

umbellatum, Mill.

vulgare, Gaertn.

\section{Helichrysum}

diosmæfolium, Sweet.

\section{Heliopsis}

padula, Wender.

scabra, Dun., var. Pitcheriana.

\section{Helipterum}

Manglesii, F. Muell., var. maculatum.

\section{Helleborus}

punctatus, Hort.

\section{Helonias}

bullata, Linn.

\section{Heracleum}

Wallichii, $D C$.

\section{Hesperis}

matronalis, Linn.

\section{Heuchera}

sanguinea, Engelm.

\section{Hibiscus}

Trionum, Linn.

\section{Hieracium}

Bornmülleri, Freyn.

bupleuroides, C. C. Gmel.

cerinthoides, Linn.

gymnocephalum, Griseb.

Jankæ, Uechtrit:.

Pilosella, Linn.

- var. aurantiaca.

prenanthoides, Vill.

pulmonarioides, Vill.

tridentatum, Fries.

saxatile, Vill.

scoticum, F. J. Hanb.

\section{Hierochloe}

borealis, Roem. et Schult.

\section{Hordeum}

jubatum, Linn.

\section{Horminum}

pyrenaicum, Linn.

- var. album.

\section{Hutchinsia}

petræa, Ait.

\section{Hyacinthus}

pumilus, Hort.

\section{Hydrangea}

vestita, Wall.

\section{Hymenanthera}

crassifolia, Hook. f. 


\section{Hyoscyamus}

niger, Linn.

\section{Hypericum}

Androsæmum, Linn.

elegans, Steph.

hirsutum, Linn.

perforatum, Linn.

pulchrum, Linn.

quadrangulum, Linn.

Richeri, Vaill.

tetrapterum, Fries.

undulatum, Schousb.

\section{Hypochœris}

uniflora, Vill.

\section{Hyssopus}

officinalis, Linn.

\section{Iberis}

carnosa, Willd.

corifolia, Sweet.

Jordani, Boiss.

Lagascana, $D C$.

sempervirens, Linn.

- var. superba.

\section{Ilex}

Aquifolium, Linn.

\section{Incarvillea}

Delavayi, Bureau et Franch.

\section{Inula}

ensifolia, Linn.

Helenium, Linn.

\section{Ionopsidium}

acaule, Reichb.
Iris

humilis, Bieb.

mongolica, Hort.

sibirica, Linn.

Watsoni, Hort.

\section{Isatis}

tinctoria, Linn.

\section{Isopyrum}

thalictroides, Linn.

\section{Jasione}

montana, Linn.

\section{Juncus}

effusus, $\operatorname{Linn}$.

- var. conglomeratus.

filiformis, Linn.

glaucus, Sibth.

trifidus, Linn.

\section{Kalmia}

angustifolia, Linn.

\section{Kniphofia}

pauciflora, Baker.

Tuckii, Baker.

\section{Laburnum}

alpinum, J. S. Presl.

vulgare, J. S. Presl.

- var. austriacum.

\section{Lactuca}

Plumieri, Gren. et Godr.

\section{Lagurus}

ovatus, Linn. 


\section{Larix}

europæa, $D C$.

\section{Lathyrus}

luteus, Baker. maritimus, Bigel.

montanus, Bernh.

odoratus, Linn.

vernus, Bernh., var. flaccidus.

\section{Layia}

gaillardioides, Hook. et $A r n$.

\section{Leontodon}

hispidus, Linn.

\section{Leontopodium}

alpinum, Cass.

- var. altaicum.

\section{Leonurus}

Cardiaca, Limn.

\section{Leycesteria}

formosa, Wall.

\section{Liatris}

spicata, Willd.

\section{Ligustrum}

medium, Franch. et Sav.

\section{Limnanthes}

Douglasii, R. Br.

\section{Linaria}

alpina, Mill.

bipartita, Willd., var. splendida. maroccana, Hook. f., var. alba. origanifolia, $D C$.

Pančici, Hort.

purpurea, Mill.

repens, Mill.

\section{Lindelofia}

spectabilis, Lehm.

\section{Linum}

perenne, Linn.

\section{Lithospermum}

prostratum, Loisel.

\section{Lobelia}

syphilitica, Linn.

\section{Lonicera}

alpigena, Linn.

angustifolia, Wall.

bella, Zabel.

mündeniensis, Rehder.

muscaviensis, Rehder.

pyrenaica, Linn.

tatarica, Linn.

- var. alba grandiflora.

- var. discolor.

- var. flore rosea.

\section{Lotus}

corniculatus, Limn.

\section{Lunaria}

annua, Lim.

- var, alba.

\section{Lupinus}

arboreus, Linn.

- var. violaceus.

nootkatensis, Donn.

polyphyllus, Lindl.

- var. albus.

\section{Lychnis}

alba, Mill.

alpina, Linn.

coronaria, Desr. 
Lychnis-continued.

Flos-cuculi, Linn.

Lagascæ, Hook. $f$.

Viscaria, Linn.

— var. splendens.

\section{Lythrum}

Salicaria, Linn.

- var. grandiflora. virgatum, Linn.

\section{Malva}

sylvestris, Linn.

\section{Margyricarpus}

setosus, Ruiz et Pav.

\section{Matricaria}

inodora, Limn.

- var. plenissimum.

oreades, Boiss.

\section{Matthiola}

bicornis, $D C$.

sinuata, $R$. $B r$, var. glabra albiflora.

\section{Meconopsis}

cambrica, Vig.

Wallichii, Hook.

- var. fusco-purpurea.

\section{Medicago}

carstiensis, Wulf.

Echinus, $D C$.

hispida, Gaertn.

\section{Melica}

nutaris, Linn.

uniflora, Rets.

\section{Melilotus}

officinalis, Lam.

\section{Melittis}

Melissophyllum, Linn.

\section{Meum}

athamanticum, Jacq.

\section{Microglossa}

albescens, C. B. Clarke.

\section{Milium}

effusum, Linn.

Molopospermum

cicutarium, $D C$.

\section{Montia}

fontana, Linn.

\section{Morina}

longifolia, Wall.

\section{Muscari}

armeniacum, Baker.

botryoides, Mill.

elegans, Hort.

Massyanum, Hort.

Maweanum, Baker.

\section{Myosotis}

arvensis, Lam.

dissitiflora, Baker.

stricta, Link.

\section{Myrrhis}

odorata, Scop.

\section{Narcissus}

Bulbocodium, Linn., var. citrinus. 
Nasturtium

officinale, R. Br.

\section{Neillia}

thrysiflora, D. Don.

\section{Nepeta}

Cataria, Linn.

kokamirica, Regel.

linearis, Royle.

longiflora, Vent.

Mussini, Spreng.

nuda, Linn.

Sibthorpii, Benth.

\section{Nicotiana}

rustica, Linn.

\section{Noccæa}

alpina, Reichb.

\section{Enanthe}

peucedanifolia, Pollich.

pimpinelloides, Linn.

\section{Enothera}

fruticosa, Linn., var. Youngei.

\section{Olearia}

Haastii, Hook. $f$.

ilicifolia, Hook. $f$.

stellulata, $D C$.

\section{Omphalodes}

linifolia, Moench.

\section{Onobrychis}

viciæfolia, Scop.

\section{Ononis}

Natrix, Linn.

\section{Onopordon}

Acanthium, Linn.

\section{Onosma}

albo-roseum, Fisch, et Mey.

\section{Origanum}

vulgare, Linn.

\section{Osteospermum}

moniliferum, Linn.

\section{Oxalis}

corniculata, Linn.

- var. tropæoloides.

\section{Oxyria}

digyna, Hill.

\section{Oxytropis}

campestris, $D C$.

lapponica, Gaud.

strobilacea, Bunge.

sulphurea, Fisch.

\section{Papaver}

Heldreichii, Boiss.

lateritium, $C$. Koch.

monanthum, Trautv.

nudicaule, Linn.

orientale, Linn.,var. bracteatum.

pilosum, Sibth. et $S m$.

rupifragum, Boiss. et Reut.

somniferum, Linn.

\section{Parnassia}

palustris, Linn.

\section{Parrya}

Menziesii, Hort. 


\section{Patrinia}

scabiosæfolia, Link.

\section{Pentstemon}

barbatus, Roth.

confertus, Dougl.

- var. cœruleo-purpureus.

deustus, Dongl.

glaber, Pursh.

gracilis, Nutt.

Menziesii, Hook, var. Newberryi.

Richardsonii, Dougl. virgatus, A. Gray.

\section{Perezia}

multiflora, Less.

\section{Peucedanum}

coriaceum, Reichb.f. officinale, Linn.

\section{Phacelia}

viscida, Torr.

\section{Phaseolus}

multiflorus, Willd.

\section{Phlomis}

setigera, Falc.

\section{Physalis}

Alkekengi, Linn.

\section{Phyteuma}

canescens, Waldst, et Kit. humile, Schleich. orbiculare, Linn.

Scheuchzeri, All.

Sieberi, Spreng.

\section{Picrorhiza}

Kurroa, Royle.

\section{Pimpinella}

magna, Linn.

\section{Pinguicula}

grandiflora, Lam.

vulgaris, Linn.

\section{Pinus}

Pinaster, Soland.

sylvestris, Linn.

\section{Plantago}

alpina, Cav.

arenaria, Waldst. et Kit.

argentea, Chaix.

carinata, Schrad.

Coronopus, Linn.

lanceolata, Linn.

major, Linn., var. monstrosa.

- var. rubra.

maritima, Linn.

Raoulii, Decne.

\section{Platystemon}

californicus, Benth.

\section{Podophyllum}

Emodi, Wall.

\section{Polemonium}

cæruleum, Linn .

confertum, A. Gray, var. mellitum.

flavo-cæruleum, Hort. gracile, Willd.

himalayanum, Baker. mexicanum, Cerv. 


\section{Polygonum}

Bistorta, Linn., var. major. sphærostachyum, Meissn. vaccinifolium, Wall.

\section{Potentilla}

alchemilloides, Lapeyr.

alpestris, Hall. $f$.

arguta, Pursh.

dissecta, Pursh.

glandulosa, Lindl.

Mooniana, Wight.

nepalensis, Hook.

nitida, Linn., var. alba.

nivea, Linn.

norvegica, Linn.

procumbens, Sibth.

pseudo-obscura, Hort.

reptans, Linn.

Römeri, Hort.

rupestris, Linn.

sericea, Linn.

Sibbaldia, Hall. f.

Tormentilla, Neck.

tridentata, Soland.

villosa, Pall.

vladnizensis, Sieg fr.

\section{Primula}

apennina, Wid.

Auricula, Linn.

- var. marginata.

denticulata, Sm., var. cashmiriana.

- var. rosea.

elatior, Hill, var. carpatica.

farinosa, Limn.

frondosa, Janka.

involucrata, Wall.

japonica, A. Gray, var. lilacina.

officinalis, Jacq.

sikkimensis, Hook. f.
Primula-continued.

Stuartii, Wall, var. purpurea.

venusta $\times$, Host.

viscosa, Vill.

- var. ciliata.

- var. nivalis.

\section{Prunus}

Avium, Linn.

Laurocerasus, Linn.

\section{Ptelea}

trifoliata, Linn., var. aurea.

- var. variegata.

\section{Pyrus}

Aria, Linn.

alpina, Willd., var. superaria.

Aucuparia, Gaertn.

baccata, Linn.

rotundifolia, Bechst.

\section{Ramondia}

pyrenaica, Rich.

serbica, Panc.

\section{Ranunculus}

aconitifolius, Linn.

acris, Linn.

— var. Steveni.

brutius, Tenore.

Lingua, Linn.

ophioglossifolius, Vill

repens, Linn.

Thora, Linn.

\section{Reseda}

lutea, Linn.

\section{Rhamnus}

Frangula, Linn. 


\section{Rheum}

leucorrhizum, Pall.

Rhaponticum, Linn.

Ribes, Linn.

tataricum, Linn. $f$.

\section{Rhododendron}

catawbiense, Mich $x$.

caucasicum, Pall.

cinnabarinum, Hook. $f$.

ferrugineum, Linn.

flavum, G. Don.

glaucum, Hook. $f$.

ponticum, Linn.

Thomsoni, Hook.f.

\section{Ribes}

sanguineum, Pursh, var. albidum, Paxt.

- var. malvaceum, S. Wats.

\section{Rosa}

acicularis, Lindl.

alpina, Linn.

hibernica, Sm.

laxa, Retz.

rubiginosa, Linn.

rugosa, Thunb.

— var. alba.

sericea, Lindl.

spinosissima, Linn.

- var. rubra.

\section{Rubia}

tinctorum, Linn.

\section{Rumex}

alpinus, Limn.

flexuosus, Soland.

nepalensis, Spreng.

\section{Ruta}

graveolens, Linn.

\section{Sagina}

Linnæi, Presl.

\section{Salvia}

argentea, Linn.

hians, Royle.

nutans, Linn.

pratensis, Linn.

sylvestris, Linn.

- var. alba.

Verbenaca, Linn.

\section{Sambucus}

canadensis, Limn.

nigra, Linn.

- var. fructu albo.

\section{Sanicula}

europæa, Linn.

\section{Saponaria}

bellidifolia, $\mathrm{Sm}$.

peregrina $\times$, Hort.

pulvinaris, Boiss.

Wienmanniana, Hort.

\section{Saxifraga}

aizoides, Linn.

Aizoon, Jacq., var. balcana.

- var. notata.

- var. pectinata.

- var. rosularis.

- var. Sturmiana.

aspera, Linn.

bronchialis, Linn.

- var. cherlerioides.

Bucklandi, Hort.

cæsia, Linn.

canaliculata, Boiss. et Reut.

cartilaginea, Willd.

cochlearis, Reichb., var. minor. 
Saxifraga-continued.

Cotyledon, Limn., var. pyramidalis

cuneifolia, Limn.

- var. capillipes.

erosa, Pursh.

geranioides, Limn.

gibraltarica, Boiss, et Reut.

granulata, Limn.

Hostii, Tausch.

- var. elatior.

hypnoides, Linn.

intacta, Willd.

- var. farinosa.

Kingii, Hort.

lingulata, Bell, var. australis.

- var. lantoscana.

longifolia, Lapeyr.

- var. hybrida.

Mertensiana, Bongard.

muscoides, Wulf.,var.moschata.

- var. purpurea.

mutata, Linn.

nivalis, Linn.

pedatifida, Ehrh.

pentadactylis, Lapeyr.

retusa, Gouan, var. maritima.

Reyeri $\times$, Hort.

rotundifolia, Linn., var. angulosa.

Schraderi, Sternb. squarrosa, Sieber. umbrosa, Linn., var. hirsuta.

- var. Ogilvieana.

— var. serratifolia.

\section{Scabiosa}

caucasica, Bieb., var, alba.

centauroides, Mill.

longifolia, Waldst. et Kit.

lucida, Vill.

sylvatica, Linn!

\section{Scilla}

festalis, Salisb.

hispanica, Mill.

peruviana, Limn.

\section{Scirpus}

sylvaticus, Linn.

\section{Scleranthus}

annuus, Linn.

\section{Scolymus}

grandiflorus, Desf.

\section{Scrophularia}

alata, Gitib.

nodosa, Linn.

\section{Scutellaria}

altissima, Linn.

hirta, Sibth. et Sm.

\section{Securigera}

Coronilla, Linn.

\section{Sedum}

Aizoon, Linn.

Alberti, Regel.

Anacampseros, Linn.

asiaticum, Spreng.

laxiflorum, $D C$.

maximum, Suter.

populifolium, Pall.

roseum, $S c o p$.

stoloniferum, S. T. Gmel.

Telephium, Linn.

trifidum, Wall.

\section{Sempervivum}

arachnoideum, Linn.

globiferum, Linn.

Schnittspahni, Lagg.

tectorum, Linn.

violaceum, Hort. 
xxxii List of Seeds Collected during I9O3.

\section{Senecio}

aquaticus, Hill.

Doronicum, Linn.

viscosus, Linn.

\section{Serratula}

coronata, Lmn.

Gmelini, Ledeb.

heterophylla, Desf.

\section{Sidalcea}

candida, A. Gray.

malvæflora, A. Gray.

oregana, A. Gray.

\section{Silene}

alpestris, Jacq.

Armeria, Linn.

caucasica, Boiss.

chromodonta, Boiss, et Reut.

colorata, Poir.

compacta, Fisch.

Cucubalus, Wibel.

italica, Pers.

maritima, With.

nutans, Linn.

rhynchocarpa, Boiss.

rupestris, Linn.

Saxifraga, Linn.

Schafta, Gmel.

stylosa, Bunge.

Thorei, Duf.

Zawadzkii, Herbich.

\section{Sisymbrium}

Assoanum, Loscos et Pardo. strictissimum, Linn. tanacetifolium, Linn.

\section{Sisyrinchium}

angustifolium, Mill.

- var. mucronatum.
Sisyrinchium-continued.

Bermudianum, Linn.

grandiflorum, Dougl., var. album.

\section{Skimmia}

japonica, Thunb.

\section{Solanum}

Dulcamara, Linn.

\section{Solidago}

elongata, Nutt.

Shortii, Torr. et Gray.

Virgaurea, Linn.

\section{Spartina}

polystachya, Willd.

\section{Specularia}

hybrida, $A . D C$.

pentagonia, $A . D C$.

Speculum, A. DC.

\section{Spergula}

arvensis, Linn.

\section{Spiræa}

Aruncus, Linn.

bullata, Maxim.

palmata, Thunb.

\section{Stachys}

Betonica, Benth,

lanata, Jacq.

recta, Linn.

\section{Statice}

altaica, Hort.

\section{Stellaria}

biflora, Linn.

graminea, Linn.

Holostea, Linn. 


\section{Stenanthium}

angustifolium, Kunth.

\section{Symphoricarpus}

racemosus, Michx.

\section{Symphyandra}

Hofmanni, Pant.

\section{Synthyris}

reniformis, Benth.

\section{Syringa}

Emodi, Wall.

\section{Tanacetum}

vulgare, Linn.

\section{Taxus}

baccata, Linn.

-- var. Dovastoni, Carr.

- var. fastigiata, Loud.

- var. pyramidalis.

- var. Washingtoni.

\section{Tellima}

grandiflora, R. Br.

\section{Teucrium}

montanum, Linn.

Scorodonia, Linn.

\section{Thalictrum}

corynellum, $D C$.

glaucum, Desf.

laserpitifolium, Willd.

majus, Jacq.

minus, Linn., var. elatum.

simplex, Lim

\section{Thermopsis}

fabacea, $D C$.

\section{Thlaspi}

perfoliatum, Lmn.

\section{Thymus}

Serpyllum, Linn.

- var. coccineus.

- var. micans.

\section{Tilia}

vulgaris, Hayne.

\section{Tofieldia}

carinthiaca, Hort.

palustris, Huds.

\section{Tradescantia}

virginiana, Linn., var. alba.

- var. atropurpurea.

- var. elata.

\section{Trautvetteria}

palmata, Fisch. et Mey.

\section{Trifolium}

alpinum, Linn.

\section{Trillium}

grandiflorum, Salnsb, var. roseum.

\section{Triosteum}

perfoliatum, Linn.

\section{Triticum}

Spelta, Linn.

\section{Tritonia}

rosea, Klath. 


\section{Trollius}

altaicus, Mey. asiaticus, Linn. - var. giganteus. caucasicus, Stev. europæus, Linn.

\section{Tropæolum}

majus, Linn.

\section{Tulipa}

Didieri, Jord., var. alba. pulchella, Fenzl.

sylvestris, Linn.

\section{Tunica}

prolifera, Scop.

Saxifraga, Scop.

\section{Uniola}

latifolia, Michx.

\section{Vaccinium}

padifolium, Sm.

\section{Verbascum}

Blattaria, Linn.

Lagurus, Fisch. et Mey.

longifolium, Tenore.

Lychnitis, Linn.

phceniceum, Linn.

\section{Veronica}

alpina, Linn.

Anagallis, Limn.

aphylla, Linn.

austriaca, $\operatorname{Linn}$.

- var. pinnatifida.

Balfouriana, Hook. $f$.

Bidwillii, Hook. f.

carnosula, Hook. f. .
Veronica-continued.

Catarractæ, Forst.

Chamædrys, Linn.

corymbosa, Hort.

decumbens, Armstr.

imperialis $\times$, Hort.

incisa, Ait.

Kirkii, Armstr.

longifolia, Linn., var. alba.

Lyallii, Hook. f.

monticola, Armstr.

officinalis, Linn.

- var. hirsuta.

parviflora, Vahl, var. angustifolia, Hook. $f$.

pinguifolia, Hook. f., var. decumbens.

pinnata, Linn.

saxatilis, Scop.

T'eucrium, Linn.

virginica, Linn.

\section{Viburnum}

cotinifolium, D. Don.

Lantana, Linn.

\section{Vicia}

Cracca, Linn.

fulgens, Battand. et Trabut.

oroboides, Wulf.

Orobus, $D C$.

pyrenaica, Pourr.

unijuga, A. Braun.

\section{Vincetoxicum}

officinale, Moench.

\section{Viola}

canina, Linn.

cornuta, Linn.

lutea, Huds.

olympica, Boiss.

persicifolia, Roth. 
List of Seeds Collected during igo3. xxxv

\section{Wahlenbergia}

dinarica, Hort.

gracilis, Schrad.

Kitaibelii, A. DC,

\section{Wulfenia}

carinthiaca, Jacq.

\section{Xerophyllum}

asphodeloides, Nutt.

\section{Zenobia}

speciosa, D. Don, var. pulverulenta.

\section{Zygadenus}

elegans, Pursh.

\section{PLANTS UNDER GLASS.}

\section{Acacia}

calamifolia, Sweet.

decurrens, Willd.

Farnesiana, Willd.

grandis, Henfr.

juncifolia, Benth.

melanoxylon, R. Br.

\section{Acokanthera}

spectabilis, Hook.f.

\section{Echmea}

bromeliæfolia, Baker.

- var. pulchra.

candida, E. Morr.

cærulescens, Baker.

\section{Agave}

maculata, Regel.

\section{Aglaonema}

commutatum, Scholt.

\section{Albizzia}

lophantha, Benth.
Aloe

mitriformis, Mull.,var. spinulosa (Salm-Dyck).

- var. flavispina (Haw.).

obscura, Mill., var. picta

(Thunb.).

\section{Alonsoa}

Iinifolia, Roesl.

Warscewiczii, Regel.

\section{Aphelandra}

aurantiaca, Iindl, var. Roezlii.

\section{Araujia}

sericifera, Brot.

\section{Ardisia}

crenata, Sims.

mamillata, Hance.

\section{Aristolochia}

brasiliensis, Mart.

elegans, Mast.

fimbriata, Cham. 
xxxvi List of Seeds Collected during igO3.

\section{Asclepias}

curassavica, Linn.

\section{Astrocaryum}

mexicanum, Liebm.

\section{Asystasia}

bella (Harv.).

\section{Babiana}

plicata, Ker.

\section{Begonia}

Bruantii $\times$, Hort.

dichotoma, Jacq.

Dregei, $O$. et $D$.

gracilis, H.B. et $K$.

kewensis $\times$, Hort.

Martiana, $L$. et $O$.

nitida, Dry.

semperflorens, $L$. et $O$.

ulmifolia, Willd.

Verschaffeltii $\times$, Hort.

weltoniensis $\times$, Hort.

\section{Billardiera}

longiflora, Labill.

\section{Billbergia}

decora, Poepp.

decoro-nutans, Baker.

pallidiflora, Liebm.

pyramidalis, Lindl.

zebrina, Lindl.

\section{Biophytum}

sensitivum, $D C$.

\section{Bowiea}

volubilis, Harz.

\section{Brachychilum}

Horsfieldii, Baker.

\section{Callicarpa}

purpurea, Juss.

\section{Callistemon}

linearis, $D C$.

salignus, $D C$.

speciosus, $D C$.

\section{Campanula}

pyramidalis, Linn.

- var. alba.

Vidalii, H. C. Wats.

\section{Capparis}

flexuosa, Blume.

\section{Capsicum}

annuum, Linn.

\section{Cassia}

corymbosa, Lam.

\section{Casuarina}

distyla, Vent.

stricta, Ait.

\section{Cedronella}

triphylla, Moench.

\section{Celosia}

cristata, Linn., var. nana.

\section{Celsia}

Arcturus, Jacq.

cretica, Linn.

\section{Cestrum}

aurantiacum, Lindl.

Newellii (Veitch). 


\section{Chlorophytum}

orchidastrum, Lindl.

\section{Cleome}

grandis, Hort.

\section{Clerodendron}

fallax, Lindl.

Thomsonæ, Balf. .

\section{Clianthus}

puniceus, Banks et Sol.

\section{Cobæa}

scandens, Cav.

\section{Coffea}

arabica, Linn.

bengalensis, Roxb.

\section{Costus}

afer, Ker.

igneus, $N . E . B r$.

\section{Cotyledon}

ventricosa, Burm

\section{Crossandra}

undulæfolia, Salisb.

\section{Cyanotis}

somaliensis, C. B. Clarke.

\section{Cyclanthera}

explodens, Naud.

\section{Cytisus}

candicans, Lam.

\section{Dalechampia}

Roezliana, Muell.

\section{Dermatobotrys}

Saundersii, Bolus.

\section{Dillwynia}

floribunda, $\mathrm{Sm}$.

\section{Dolichos}

Lablab, Linn.

\section{Drosera}

hilaris, Cham. et Schlecht.

\section{Eccremocarpus}

scaber, Ruiz et Pav.

\section{Epiphyllum}

Gaertneri, Hook. $f$.

\section{Erythrochiton}

brasiliensis, Nees.

\section{Eucalyptus}

amygdalina, Labill.

incrassata, Labill.

piperita, $S m$.

\section{Eucomis}

punctata, L'Hérit.

\section{Eupatorium}

Weinmannianum, Regel.

\section{Euphorbia}

splendens, Boj.

\section{Evodia}

elegans, Hort.

formosa, Hort. 
xxxviii List of Seeds Collected DURing igo3.

\section{Ficus}

anacardiifolia, Kunth et Bouché.

pyriformis, Hook. et $A \mathrm{rn}$.

\section{Francoa}

appendiculata, Cav.

\section{Fuchsia}

procumbens, R. Cunn.

\section{Furcræa}

tuberosa, Ait. (bulbils).

\section{Gasteria}

maculata, Haw.

verrucosa, Haw.

\section{Geonoma}

pumila, Wendl.

\section{Gloriosa}

superba, Linn.

\section{Goodia}

lotifolia, Salisb.

\section{Gossypium}

herbaceum, Linn.

\section{Guizotia}

abyssinica, Cass.

\section{Hakea}

acicularis, Knight.

\section{Hedychium}

Gardnerianum, Rosc.

\section{Hibiscus}

gossypinus, Thunb.

Huegelii, Endl.

Manihot, Linn.

pedunculatus, Limn.

Trionum, Linn., var. major.

\section{Hydrolea}

spinosa, Linn.

\section{Hypoxis}

Krebsii, Fisch.

\section{Impatiens}

capensis, Meer.

cuspidata, Wight et Arn., var. arthritica.

\section{Indigofera}

atropurpurea, Buch.-Ham.

australis, Willd.

\section{Kalanchoe}

Cassiopeia, Hort.

crenata, Haw.

flammea, Stapf.

Kirkii, N. E. Br.

\section{Kedrostis}

africana, $\operatorname{Cog} n$.

\section{Kennedya}

prostrata, R. Br.

\section{Lachnostoma}

maritimum, Nichols.

\section{Lantana}

Camara, Linn. 
Leea

sambucina, Willd.

\section{Leptospermum}

arachnoideum, $\mathrm{Sm}$. scoparium, Forst.

\section{Loasa}

lateritia, Gill., var. aurantiaca, Hort.

\section{Luffa}

ægyptiaca, Mill.

\section{Lycopersicum}

esculentum, Mill.

\section{Malvastrum}

grossulariæfolium, Garcke.

\section{Maurandia}

Barclayana, Lindl.

\section{Melia}

arguta, $D C$.

Azedarach, Linn.

\section{Mesembryanthemum}

aurantiacum, Haw.

curviflorum, Haw.

lepidum, Haze.

micans, Linn.

serrulatum, Haze.

tumidulum, Haw.

vaginatum, Lam.

\section{Momordica}

Charantia, Linn.

cochinchinensis, Spreng.

\section{Monstera}

deliciosa, Liebm.

\section{Moræa}

prolongata, Hort.

\section{Musschia}

Wollastoni, Lowe.

\section{Myrica}

æthiopica, Linn.

\section{Myrtus}

communis, Linn.

Luma, Barn.

Ugni, Mol.

\section{Nephthytis}

liberica, N.E. Br.

\section{Opuntia}

nigricans, Haw.

\section{Oxypetalum}

cœruleum, Decne.

\section{Passiflora}

edulis, Sims.

quadrangularis, Linn.

\section{Pavonia}

spinifex, Cav.

\section{Pentas}

carnea, Benth., var. kermesina.

\section{Phaseolus}

vulgaris, Linn.

\section{Philydrum}

lanuginosum, Banks. 


\section{Pitcairnia}

Altensteinii, Lem.

latifolia, Soland.

maidifolia, Decne.

pulverulenta, Ruiz et Pav.

rubiginosa, Baker.

\section{Polyalthia}

suberosa, Benth, et Hook.f.

\section{Primula}

mollis, Nutt.

verticillata, Forsk.

\section{Prostanthera}

lasianthos, Labill.

\section{Rhododendron}

arboreum, $S m$.

Nuttallii, Booth.

\section{Rhynchosia}

caribæa, $D C$.

\section{Ricinus}

communis, Linn.

\section{Rivina}

aurantiaca, Warsc.

humilis, Linn.

tinctoria, Hamil.

\section{Rondeletia}

odorata, Jacq.

\section{Ruellia}

amcena, Nees.

tuberosa, Linn.

\section{Ruta}

bracteosa, $D C$.
Sabal

Blackburniana, Glazebr.

\section{Saxifraga}

Macnabiana, Hort.

\section{Schizanthus}

pinnatus, Ruiz et Pav.

\section{Sedum}

Sieboldii, Hort.

\section{Sempervivum}

annuum, $C$. Sin.

tectorum, Linn.

\section{Senecio}

grandifolius, Less.

macroglossus, $D C$.

\section{Solanum}

auriculatum, Ait.

coagulans, Hort.

fastigiatum, Willd.

Hendersoni, Hort.

jasminoides, Paxt.

nigrum, Linn.

pyracanthum, Jacq.

Seaforthianum, Andr.

\section{Spathiphyllum}

floribundum, N.E. Br.

\section{Stachytarpheta}

indica, Vahl.

\section{Stylidium}

adnatum, $R$. $B r$.

\section{Tacca}

cristata, Jack. 


\section{Talinum}

patens, Willd.

triangulare, Willd.

Tetranema

mexicana, Benth.

\section{Teucrium}

Marum, Linn.

\section{Thrinax}

parviflora, Sze.

\section{Tibouchina}

semidecandra, $\operatorname{Cog} n$.

\section{Tradescantia}

discolor, Hort.

\section{Trachelium}

cæruleum, Linn.

\section{Trichosanthes}

Anguina, Linn.

Tristania

nereifolia, R. Br.

\section{Villamilla}

octandra, Hook. f.

purpurascens, Hort.

\section{Vellozia}

candida, Mikan.

\section{Veronica}

Andersonii $\times$, Hort., var. variegata.

\section{Vitis}

heterophylla, Thunb., var. humulifolia. 




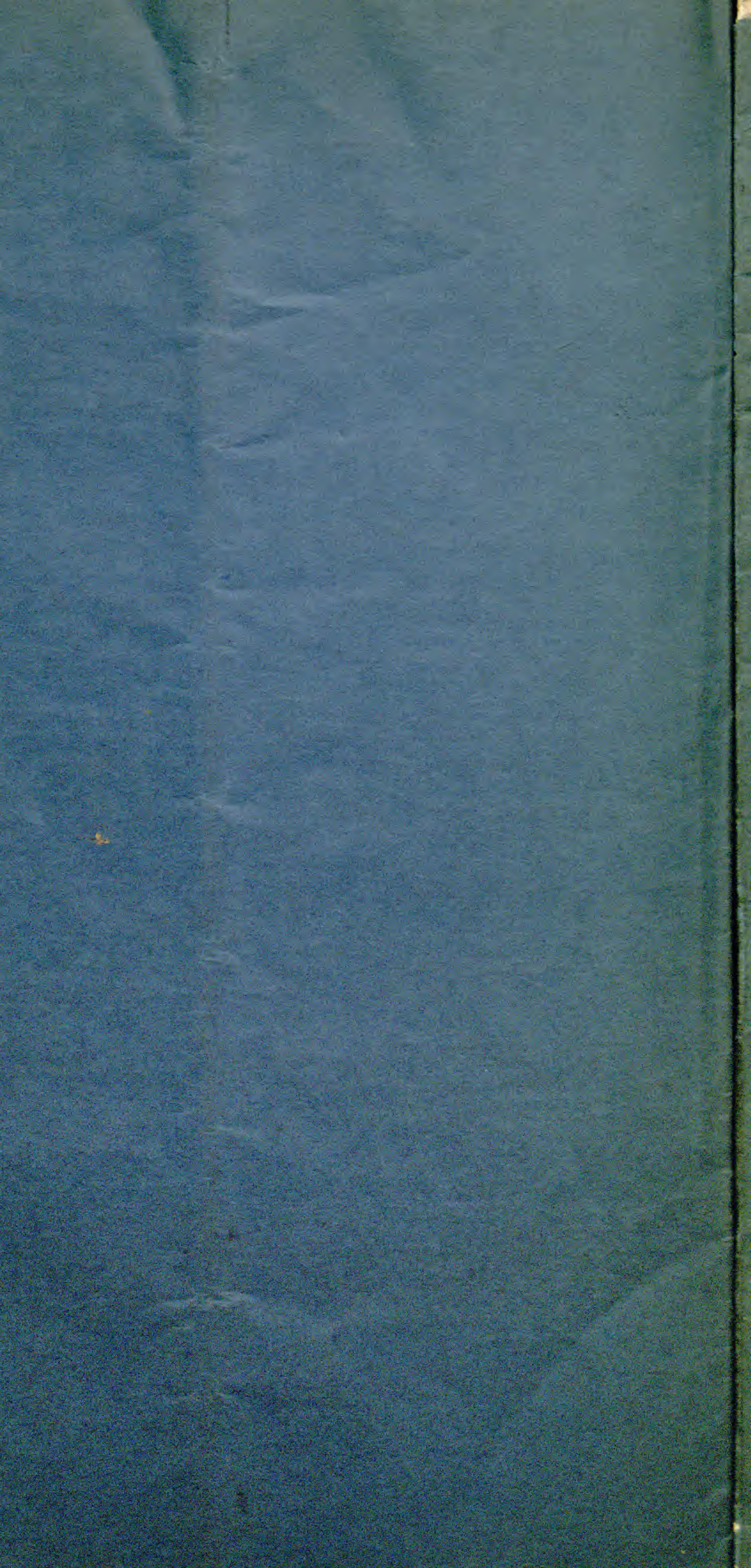




\section{NOTES}

FROM THE

\section{ROYAL BOTANIC GARDEN,}

\section{EDIN B URGH.}

\section{DECEMBER 1904.}

\section{CONTENTS.}

List of Seeds collected in the Royal Botanic Carden, Page. Edinburgh, during the Year 1904, _ _ _ _ xliii

GLASGOW:

PRINTED FOR HIS MLAESTY'S STATIONERY OFFICE

BY JAMES HEDUERWICK \& SORS, AT "THE CITLEA" PRESS, ST. VMCENT PLACE.

\section{SOLD AT THE CARDEN:}

And to be porchased, either ditectly or throggh any Rooksellen, frath OLIVER \& BOVD, BDIWLENCW:

[PRICE NINEPENCE.]

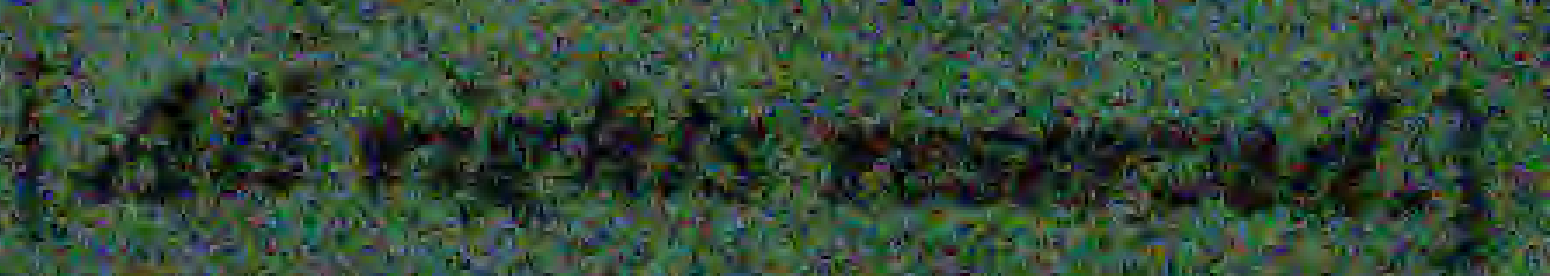

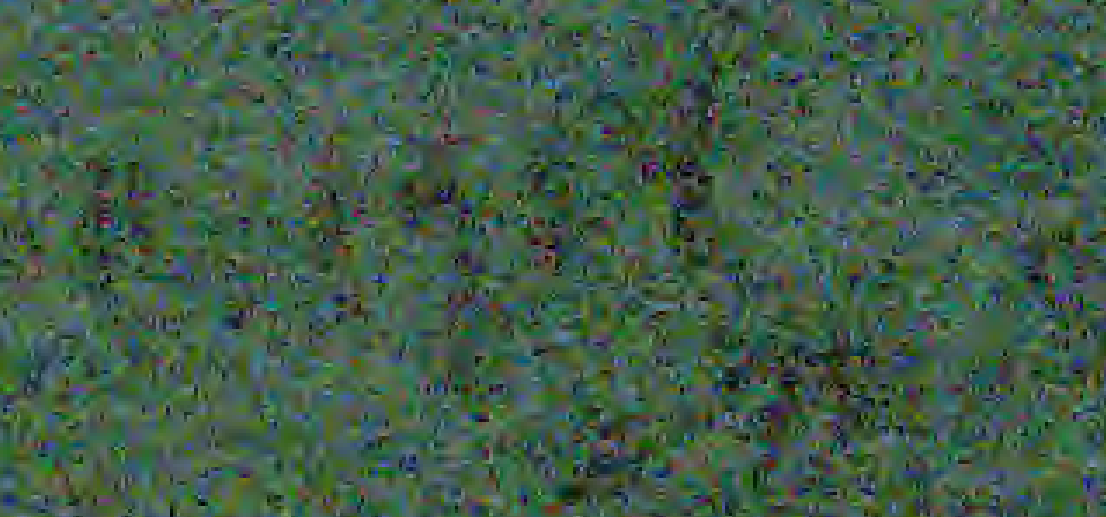




\section{List of Seeds Collected in the Royal Botanic Garden, Edinburgh, during the Year 1904.}

The following is a list of plants cultivated in the Royal Botanic Garden, Edinburgh, from which ripened seeds have been collected during the year 1904. The quantity of seed obtained from some of the species is of limited amount. The seeds are available for exchange, but they are not for sale :-

\section{PLANTS IN THE OPEN.}

\section{Acæna}

argentea, Ruiz et Pav. glabra, J. Buch. inermis, Hook.f. microphylla, Hook, f. myriophylla, Lindl. ovalifolia, Ruiz et Pav. Sanguisorbæ, $V a h l$.

Acer

circinatum, Pursh platanoides, Linn. Pseudo-platanus, Linn. - var. purpureum. rufinerve, Sieb, et Zucc.

\section{Aconitum}

Lycoctonum, Linn. longigaleatum, Hort. Napellus, Linn. paniculatum, Lam. reclinatum, A. Gray uncinatum, Linn. variegatum, Linn.

\section{Actæa}

spicata, Linn.

- var. rubra.

\section{Adenophora}

polymorpha, Ledeb.

Potanini, Batalin

stylosa, Fisch.

\section{Adonis}

autumnalis, Linn.

vernalis, Linn.

\section{ÆEthionema}

cordatum, Boiss.

grandiflorum, Boiss. et Hohen.

\section{Agrimonia}

agrimonioides, Linn.

Eupatoria, Linn.

\section{Agrostis}

nigra, With. 
xliv

List of Seeds Collected during 1904.

\section{Agropyron}

acutum, Roem. et Schult.

dasyanthum, Ledeb.

tenerum, Vasey

\section{Ajuga}

genevensis, Linn.

\section{Alchemilla}

alpina, Linn., var, conjuncta (Bab.).

\section{Alisma}

Plantago, Linn.

\section{Allium}

cardiostemon, Fisch, et Mey. carinatum, Linn. cernuum, Roth

fistulosum, Linn.

flavum, Linn.

giganteum, Regel

kansuense, Regel

karataviense, Regel

macranthum, Baker

Moly, Linn.

mutabile, Michx.

margaritaceum, Sibth, et Sm.

odorum, Linn.

oleraceum, Linn.

oreophilum, C. A. Mey.

Ostrowskianum, Regel

polyphyllum, Kar. et Kir.

pulchellum, G. Don

rubellutr, Bieb.

Schœenoprasum, Linn.

senescens, Linn.

subvillosum, Salzm.

urceolatum, Regel
Alnus

cordifolia, Tenore

glutinosa, Medic.

- var. rubrinervia.

incana, Moench.

japonica, Sieb. et Zucc.

viridis, $D C$.

\section{Alopecurus}

geniculatus, Linn.

pratensis, Linn.

\section{Alstroemeria}

aurantiaca, Don

revoluta, Ruiz et Pav.

\section{Althæa}

rosea, Cav.

\section{Alyssum}

argenteum, Vitm.

condensatum, Boiss, et Hausskn.

creticum, Linn.

idæum, Boiss, et Heldr.

montanum, Linn.

saxatile, Linn.

- var. sulphurea.

serpyllifolium, Desf.

Wierzbickii, Heuffl.

\section{Amelanchier}

oligocarpa, Roem.

\section{Ammobium}

alatum, $R$. $B$ r.

\section{Anagallis}

arvensis, Linn. 


\section{Anchusa}

altissima, Desf.

capensis, Thunb.

italica, Retz.

officinalis, Linn.

sempervirens, Linn.

\section{Androsace}

foliosa, Duby

lactea, Linn.

lactiflora, Fisch.

lanuginosa, Wall.

sarmentosa, Wall.

septentrionalis, Linn.

\section{Anemone}

alpina, Linn.

- var. sulphurea.

baicalensis, Turcz.

cylindrica, A. Gray

multifida, Poir.

pratensis, Linn.

rivularis, Buch.-Ham.

sylvestris, Linn.

vitifolia, Buch.-Ham.

\section{Anthemis}

Biebersteiniana, Koch

\section{Anthericum}

Liliago, Linn.

\section{Anthoxanthum}

odoratum, Linn.

\section{Anthyllis}

sericea, Lag.

Vulneraria, Linn., var. Dillenii.

\section{Antirrhinum}

Asarina, Linn.

majus, Linn.

Orontium, Linn.

\section{Apera}

arundinacea, Hook. $f$.

interrupta, Beauv.

Spica-Venti, Beauv.

\section{Aphyllanthes}

monspeliensis, Linn.

\section{Aquilegia}

canadensis, Linn.

Einseliana, F. Schultz

pyrenaica, $D C$.

\section{Arabis}

albida, Stev.

Allionii, $D C$.

alpina, Linn.

- var. anachoretica.

bellidifolia, Jacq.

hirsuta, Scop.

muralis, Bertol., var. rosea.

petræa, Lam.

Turczaninowii, Ledeb.

\section{Arenaria}

balearica, Linn.

erythrosepala, Boiss.

foliosa, Royle

Fendleri, A. Gray

- var. diffusa.

gothica, Fries

gracilis, Kit.

graminifolia, Schrad.

gypsophiloides, Linn.

Holostea, Bieb.

juniperina, Linn.

laricifolia, Linn.

lateriflora, Linn.

pinifolia, Bieb.

tetraquetra, Limn.

trinervia, Linn.

verna, Linn. 


\section{Armeria}

maritima, Willd.

\section{Argemone}

mexicana, Linn.

\section{Arnica}

amplexicauiis, Nutt.

Chamissonis, Less.

montana, Linn.

\section{Arracacia}

arguta, Benth, et Hook. f.

\section{Arrhenatherum}

avenaceum, Beauv.

\section{Asperula}

ciliata, Rochel

lævigata, Linn.

orientalis, Boiss. et Hohen.

tinctoria, Linn.

\section{Asphodeline}

Balansæ, J. Gay

\section{Asphodelus}

albus, Willd.

\section{Aster}

floribundus, Nutt.

himalaicus, $C . B$. Clarke

leucanthemus, Rafin.

Novæ-Angliæ, Linn.

patens, Ait.

puniceus, Linn.

Radula, Ait.

sagittifolius, Willd.

sibiricus, Linn.

tataricus, Linn. f.

\section{Astragalus}

Cicer, Linn

danicus, Retz., var. alba.

falcatus, Lam.

Glyciphyllos, Linn.

monspessulanus, Linn.

purpureus, Lam.

sinicus, Linn.

vaginatus, Pall.

\section{Astrantia}

carniolica, Wulf.

helleborifolia, Salisb.

major, Linn.

- var. intermedia.

neglecta, C. Koch et Bouché

\section{Athamanta}

Matthioli, Wulf.

\section{Atriplex}

Babingtonii, Woods patula, Linn.

\section{Atropa}

Belladonna, Linn.

\section{Aubrietia}

Columnæ, Guss.

croatica, Schott, Nyman et Kotschy

deltoidea, $D C$, var, cilicica.

\section{Avena}

sterilis, Linn.

\section{Baccharis}

patagonica, Hook. et Arn.

\section{Ballota}

nigra, Linn. 
List of Seeds Collected during i904. xlvii

\section{Barbarea}

præcox, R. Br.

vulgaris, $R, B r$.

\section{Bellis}

annua, $\operatorname{Linn}$.

\section{Berberis}

angulosa, Wall.

Aquifolium, Pursh

aristata, $D C$.

virescens, Hook. $f$.

vulgaris, Linn.

Wallichiana, $D C$.

\section{Beta}

trigyna, Waldst. et Kit.

vulgaris, Linn.

\section{Betula}

alba, Linn., var. pubescens.

Ermani, Cham.

\section{Bidens}

grandiflora, Balb.

\section{Biscutella}

cichoriifolia, Loisel.

\section{Boltonia}

incisa, Benth.

\section{Boykinia}

aconitifolia, Nutt.

\section{Borago}

laxiflora, Willd.

officinalis, Linn.

\section{Brachycome}

iberidifolia, Benth.

\section{Brassica}

oleracea, Linn.

\section{Bravoa}

geminiflora, Lex.

\section{Briza}

maxima, Linn.

\section{Brodiæa}

Douglasii, S. Wats.

\section{Bromus}

ciliatus, $\operatorname{Linn}$.

inermis, Leyss.

Tacna, Steud.

unioloides, H.B. et $K$.

\section{Bruckenthalia}

spiculifolia, Reichb.

\section{Bryanthus}

empetriformis, A. Gray

taxifolius, A. Gray

\section{Bryonia}

dioica, Jacq.

\section{Buddleia}

Hemsleyana, Hort.

intermedia, Carr.

variabilis, Hemsl.

\section{Bulbinella}

Hookeri, Benth. et Hook. f.

\section{Buphthalmum}

salicifolium, Linn.

speciosum, Schreb.

\section{Bupleurum}

Candollei, Wall.

longifolium, Linn.

rotundifolium, Linn. 


\section{Butomus}

umbellatus, Linn.

\section{Calamintha}

alpina, Lam.

patavina, Host

\section{Calendula}

arvensis, Linn.

officinalis, Linn.

\section{Callirhoë}

digitata, Nutt.

\section{Camassia}

Cusickii, S. Wats.

esculenta, Lindl.

\section{Campanula}

alliariæfolia, Willd.

barbata, Linn.

cæspitosa, Scop.

carpatica, Jacq., var. turbinata.

Grossekii, Heuff.

lactiflora, Bieb.

latifolia, Linn., var. macrantha. linifolia, Scop.

michauxioides, Boiss.

mirabilis, Alboff

persicifolia, Linn.

- var. alba.

- var. Moerheimi.

pulcherrima, Schrank et Zeyh. pulla, Linn.

punctata, Lam., var. alba.

pusilla, Haenke

- var. alba.

rapunculoides, Linn.

rotundifolia, Linn.

- var. Baumgartenii.

sibirica, Linn., var. divergens.
Campanula-continued.

thyrsoides, Linn.

Trachelium, Linn.

Vidalii, H. C. Wats.

Waldsteiniana, Roem. et Schult.

\section{Carbenia}

benedicta, Adans.

\section{Cardamine}

Impatiens, Linn.

latifolia, $V a h l$

\section{Carduus}

acanthoides, Linn.

defloratus, Linn.

nutans, Linn.

\section{Carex}

aquatilis, Wahlenb.

arenaria, Linn.

atrata, Linn.

binervis, $S m$.

capillaris, Linn.

depauperata, Good.

dioica, Linn.

distans, Linn.

disticha, Huds.

extensa, Good., var. pumila.

flava, Linn.

- var. Oederi.

- var. viridula.

glauca, $S c o p$.

Grahami, Boott

hirta, Linn.

hordeistichos, Vill.

Hornschuchiana, Hoppe

inourva, Lightf.

irrigua, $S m$.

lævigata, Sm.

leporina, Linn.

Mairii, Coss. et Germ. 
List of Seeds Collected duRing ig04. xlix

Carex-continued.

microglochin, Wahlenb.

muricata, Linn.

pallescens, Linn.

paludosa, Good.

panicea, Linn.

pulicaris, Linn.

punctata. Gaud.

rariflora, $\mathrm{Sm}$.

riparia, Curt.

saxatilis, Limn.

sparganioides, Muhl.

strigosa, Huds.

sylvatica, Huds.

teretiuscula, Good.

tomentosa, Linn.

vaginata, Tausch

vulgaris, Fries

\section{Carmichaelia}

flagelliformis, Colenso

\section{Caryopteris}

Mastacanthus, Schauer

\section{Catananche}

bicolor, Hort.

cærulea, Linn.

\section{Cathcartia}

villosa, Hook. $f$.

\section{Celsia}

cretica, Linn. $f$.

pontica, Boiss.

\section{Centaurea}

Cyanus, Linn., var. minor.

macrocephala, Puschk.

pectinata, Linn.

Rhaponticum, Linn.

ruthenica, Lam.

Scabiosa, Linn.

stereophylla, Bess.

\section{Centranthus}

macrosiphon, Boiss.

ruber, $D C$.

\section{Cephalaria}

alpina, Schrad.

radiata, Griseb. et Schenk

tatarica, Schrad.

- var. minor.

\section{Cerastium}

alpinum, Linn.

arvense, Linn., var.grandiflorum.

Boissieri, Gren.

ovatum, Hoppe

tomentosum, Linn.

\section{Chærophyllum}

aureum, Linn.

\section{Charieis}

heterophylla, Cass.

\section{Cheiranthus}

Cheiri, Linn.

mutabilis, L'Hérit.

Senoneri, Heldr. et-Sart.

\section{Chelidonium}

Franchetianum, Prain

majus, Linn.

- var. laciniatum.

\section{Chelone}

Lyoni, Pursh

\section{Chenopodium}

Bonus-Henricus, Linn.

capitatum, Aschers. 


\section{Chrysanthemum}

arcticum, Linn. caucasicum, Pers. coronopifolium, Vill., var. hybridum.

Leucanthemum, Linn. maximum, Ramond montanum, Linn.

Parthenium, Bernh. segetum, Linn.

\section{Chrysopsis}

villosa, $D C$, var. Rutteri.

\section{Cimicifuga}

elata, Nutt.

racemosa, $\mathrm{Nutt}$.

\section{Cistus}

laurifolius, Linn.

\section{Claytonia}

virginica, $\operatorname{Linn}$.

\section{Clematis}

alpina, Mill., var. alba.

orientalis, Linn.

\section{Cneorum}

tricoccum, Linn.

\section{Cnicus}

centauroides, Linn.

eriophorus, Roth

Falconeri, Hook. f.

græcus, Hort.

heterophyllus, Roth

— var. albus.

pannonicus, Host, var. divaricatus.

pratensis, Willd.

tauricus, Hort.

\section{Cochlearia}

glastifolia, Linn.

\section{Codonopsis}

ovata, Benth.

rotundifolia, Royle

\section{Collinsia}

bicolor, Benth.

\section{Collomia}

coccinea, Lehm.

grandiflora, Dougl.

\section{Colutea}

istria, Mill.

\section{Convallaria}

majalis, Linn.

\section{Convolvulus}

tenuissimus, Sibth. et Sm.

\section{Coptis}

trifolia, Salisb.

\section{Coreopsis}

coronata, Hook.

\section{Coriaria}

myrtifolia, Linn.

\section{Cornucopiæ}

cucullatum, Linn.

\section{Cornus}

alba, Linn.

\section{Coronilla}

montana, Scop.

varia, Linn. 
Cortusa

pubens, Schott, Nyman et Kotschy

villosa, Hort.

\section{Corydalis}

glauca, Pursh

lutea, $D C$.

racemosa, Pers.

\section{Cotoneaster}

acuminata, Lindl,

affinis, Lindl.

bacillaris, Wall.

buxifolia, Wall.

frigida, Wall.

horizontalis, Decne.

microphylla, Wall.

Nummularia, Fisch. et Mey.

rotundifolia, Wall.

Simonsii, Baker

thymifolia, Baker

\section{Cotyledon}

Aizoon, Benth. et Hook.f.

\section{Crambe}

tatarica, Hort.

\section{Cratægus}

Azarolus, Linn.

coccinea, Linn.

Douglasii, Lindl.

mollis, Scheele

Oxyacantha, Linn.

- sub-sp. monogyna, Jacq.

- var. pendula.

punctata, Jacq.

Pyracantha, Pers.

tanacetifolia, Pers.

tomentosa, Linn.

\section{Crepis}

paludosa, Moench

robertioides, Boiss.

sibirica, Linn.

\section{Crocus}

speciosus, Bieb.

\section{Crucianella}

angustifolia, Linn.

\section{Cupressus}

Lawsoniana, A. Murr.

\section{Cynoglossum}

microglochin, Benth.

officinale, Linn.

Wallichii, G. Don

\section{Cytisus}

albus, Linn.

Ardoini, Fourn.

biflorus, L'Hérit.

hirsutus, Linn.

leucanthus, Waldst, et Kit.

purgans, Boiss.

scoparius, Link

- var. Andreanus.

syriacus, Boiss.

\section{Daboecia}

polifolia, D. Don

\section{Datura}

Stramonium, Linn.

\section{Daphne}

Mezereum, Linn.

- var. flore albo. 


\section{Delphinium}

cashmirianum, Royle

caucasicum, C.A. Mey.

flexuosum, Rafin.

formosum, Boiss, et Huet

grandiflorum, Linn., var. album.

hybridum, Steph.

Maackianum, Regel

nudicaule, Torr. et Gray

speciosum, Bieb., var. turkestanicum.

tatsienense, Franch.

truncatum, Hort.

\section{Dianthus}

alpinus, $\operatorname{Linn}$.

Armeria, Linn.

brevicaulis, Fenzl

cæsius, $S m$.

carthusianorum, Linn.

ciliatus, Guss.

cruentus, Griseb.

deltoides, Linn.

- var. albus.

fragrans, Bieb.

Freynii, Vandas

gallicus, Pers.

monspessulanus, Linn.

neglectus, Loisel.

Noeanus, Boiss.

pallens, Sibth. et $S m$.

petræus, Waldst, et Kit.

- var. spiculifolius.

pubescens, Sibth. et $S m$.

Requienii, Gren. et Godr.

squarrosus, Bieb.

Sternbergii, Sibth.

subacaulis, Vill.

trifasciculatus, Kit.

\section{Dictamnus}

albus, Linn., var. purpureus.

\section{Digitalis}

lutea, Linn.

purpurea, Linn.

\section{Dierama}

ensifolium, C. Koch et Bouché

pulcherrimum, Baker

\section{Diervilla}

florida, Sieb. et Zucc.

japonica, $D C$.

Lonicera, Mill.

sessilifolia, Buckl.

\section{Dimorphotheca}

pluvialis, Moench

\section{Diphylleia}

cymosa, Michx.

\section{Dodecatheon}

Meadia, Linn.

— var. album.

\section{Draba}

aizoides, Linn.

Aizoon, Wahlenb.

arabisans, Michx.

Athoa, Boiss.

aurea, $\mathrm{Vahl}$

cornuta, Hort.

cuspidata, Bieb.

fladnizensis, Wulf.

grandiflora, Franch.

hirta, Linn.

incana, Linn.

Kotschyi, Stur

Mawii, Hook. f.

rupestris, $R$. $B r$.

siliquosa, Bieb.

tomentosa, Wahlenb.

- var. frigida. 


\section{Dracocephalum}

Moldavica, Linn.

speciosum, Benth.

\section{Drosera}

intermedia, Hayne

longifolia, Hayne

rotundifolia, Linn.

\section{Drypis}

spinosa, Linn.

\section{Echinodorus}

ranunculoides, Engelm.

\section{Echinops}

Ritro, Linn.

\section{Echium}

bifrons, $D C$.

plantagineum, Linn. rosulatum, Lange

vulgare, Linn.

\section{Elsholtzia}

cristata, Willd.

\section{Elymus}

condensatus, $J$. et $C$. Presl giganteus, Vahl

sibiricus, Linn.

\section{Embelia}

robusta, Roxb.

\section{Emex}

spinosa, Campd.

\section{Emilia}

flammea, Cass.

\section{Enkianthus}

himalaicus, Hook. f. et Thoms.

\section{Epilobium}

alpinum, Linn.

alsinifolium, Vill.

angustifolium, Linn.

Dodonæi, Vill.

glabellum, Forst.

haloragifolium, A. Cunn.

Hectori, Hausskn.

montanum, Linn.

nummularifolium, $R$. Cunn.

pedicellare, Presl

rosmarinifolium, Haenke

- var. sericeum.

\section{Eremurus}

himalaicus, Baker

\section{Erica}

ciliaris, Linn.

cinerea, Linn.

- var alba.

stricta, Donn

Tetralix, Linn.

\section{Erinus}

alpinus, Linn.

- var, albus.

- var. carmineus.

\section{Eriogonum}

racemosum, Nutt.

\section{Erodium}

chelidonifolium, Hort.

Manescavi, Coss.

moschatum, L'Hérit.

\section{Erophila}

vulgaris, $D C$. 


\section{Eryngium}

agavifolium, Griseb.

alpinum, Linn.

Bourgati, Gouan

giganteum, Bieb.

Oliverianum, Delar.

Sanguisorba, Cham. et Schlecht.

\section{Erysimum}

asperum, $D C$.

Perofskianum, Fisch. et Mey.

\section{Erythræa}

linarifolia, Pers.

Massoni, Sweet

\section{Erythronium}

grandiflorum, Pursh var. roseum.

\section{Escallonia}

exoniensis, Hort.

macrantha, Hook. et Arn.

Philippiana, Masters

pterocladon, Hook.

punctata, $D C$.

\section{Eschscholzia}

californica, Cham.

\section{Euphorbia}

Myrsinites, Linn.

trapezoidalis, Boiss.

\section{Evax}

asterisciflora, Pers.

\section{Fagopyrum}

esculentum, Moench

\section{Farsetia}

clypeata, R. Br.

\section{Felicia}

fragilis, Cass.

\section{Festuca}

bromoides, Linn.

duriuscula, Linn.

elatior, Linn .

Halleri, All.

ovina, Linn.

- var. tenuifolia.

rigida, Kunth

\section{Filago}

germanica, Linn.

\section{Fœniculum}

giganteum, Hort.

\section{Fragaria}

vesca, Linn., var. muricata.

\section{Fraxinus}

excelsior, Linn.

\section{Fuchsia}

Riccartoni, Hort.

\section{Fumaria}

officinalis, Linn., var. muralis.

\section{Funkia}

Sieboldiana, Hook.

\section{Galanthus}

Elwesii, Hook. f.

\section{Galax}

aphylla, Linn.

\section{Galega}

officinalis, Linn.

orientalis, Lam. 


\section{Galinsoga}

parviflora, Cav.

\section{Galium}

boreale, Linn.

lithospermifolium, Fisch.

Mollugo, Linn.

saccharatum, All.

saxatile, Linn.

\section{Galtonia}

candicans, Decne.

\section{Gaudinia}

fragilis, Beauv.

\section{Gaultheria}

procumbens, Linn.

Shallon, Pursh

trichophylla, Royle

\section{Gaura}

Lindheimeri, Engelm. et Gray

\section{Genista}

anglica, Linn.

cinerea, $D C$.

hispanica, Linn.

pilosa, Linn.

radiata, $S c o p$.

sagittalis, Linn.

tinctoria, Linn.

\section{Gentiana}

asclepiadea, Linn.

- var. alba.

Cruciata, Linn.

decumbens, Linn.

linearis, Froel.

macrophylla, Pall.

pannonica, Scop.
Gentiana-continued.

phlogifolia, Schott et Kotschy punctata, Linn.

Saponaria, Linn. septemfida, Pall.

tibetica, King

verna, $\operatorname{Linn}$.

\section{Geranium}

anemonæfolium, L'Hérit.

cinereum, Cav.

Fremontii, Torr.

incisum, Nutt.

macrorrhizum, Linn.

phæum, Linn.

polyanthes, Edgew, et Hook.f.

pratense, Linn.

sanguineum, Linn.

- var. lancastriense.

striatum, Linn.

sylvaticum, Linn.

Wilfordi, Maxim.

\section{Gerbera}

Kunzeana, A. Br. et Aschers. nivea, Sch. Bip.

\section{Geum}

Borissi, Hort.

elatum, Wall.

Eweni, Hort.

Heldreichii, Hort.

hispidum, Fries

intermedium, Ehrh.

Laxmanni, Gaertn.

montanum, Linn.

pyrenaicum, Mill.

rhæticum, Brügg.

rivale, Linn.

urbanum, Linn. 
Gilia

androsacea, Steud.

dichotoma, Benth.

tricolor, Benth.

\section{Gillenia}

stipulacea, Nutt.

\section{Glaucium}

flavum, Crantz

\section{Goodyera}

repens, $R$. $B r$.

\section{Graphephorum}

arundinaceum, Aschers.

\section{Grindelia}

squarrosa, Dun.

\section{Gunnera}

chilensis, Lam.

\section{Gypsophila}

dubia, Willd.

prostrata, Linn.

repens, Linn.

- var. monstrosa.

\section{Haberlea}

rhodopensis, Frivald.

\section{Haplocarpa}

Leichtlinii, N. E. Br.

\section{Hedysarum}

sibiricum, Poir.
Helianthemum

canum, Boiss.

Fumana, Mill.

polifolium, $D C$.

umbellatum, Mill.

vulgare, Gaertn.

\section{Helianthus}

annuus, $\operatorname{Linn}$

\section{Heliopsis}

padula, Wender.

scabra, Dun., var. Pitcheriana.

\section{Helipterum}

Manglesii, F. Muell., var. maculatum.

\section{Helleborus}

punctatus, Hort.

\section{Helonias}

bullata, Linn.

\section{Hemerocallis}

minor, Mill.

\section{Heracleım}

asperum, Bieb.

Wallichii, $D C$.

\section{Hesperis}

matronalis, Linn.

\section{Heuchera}

sanguinea, Engelm.

\section{Hibiscus}

Trionum, Linn. 


\section{Hieracium}

alpinum, Linn.

Bornmülleri, Freyn

bupleuroides, C. C. Gmel.

cerinthoides, Linn.

gymnocephalum, Griseb.

incisum, Hoppe

Jankæ, Uechtritz

Pilosella, Linn.

- var. aurantiacum.

prenanthoides, Vill.

pulmonarioides, Vill.

tridentatum, Fries

saxatile, Vill.

scoticum, F. J. Hanb.

villosum, Jacq.

\section{Hierochloë}

borealis, Roem. et Schult.

\section{Hippophae}

rhamnoides, Linn.

\section{Hordeum}

bulbosum, Linn.

jubatum, Linn.

\section{Horminum}

pyrenaicum, Linn.

- var. album.

\section{Hunnemannia}

fumariæfolia, Szeet

\section{Hutchinsia}

petræa, Ait.

\section{Hyacinthus}

amethystinus, $\operatorname{Lin}$.

dubius, Guss.

pumilus, Hort.

\section{Hydrangea}

vestita, Wall.

\section{Hymenanthera}

crassifolia, Hook. f.

\section{Hyoscyamus}

niger, Linn.

\section{Hypericum}

Androsæmum, Linn.

calycinum, Linn.

delphicum, Boiss, et Heldr.

Hookerianum, Wight et Arn.

Kotschyanum, Boiss.

polyphyllum, Boiss.

pulchrum, Linn.

Richeri, Vaill.

\section{Hypochœris}

maculata, Linn.

uniflora, Vill.

\section{Hyssopus}

officinalis, Linn.

\section{Iberis}

carnosa, Willd. corifolia, Szueet

Jordani, Boiss.

Lagascana, $D C$.

semperflorens, Linn.

sempervirens, Linn.

- var. superba.

\section{Ilex}

Aquifolium, Linn.

\section{Incarvillea}

Delavayi, Bureau et Franch. 


\section{Inula}

ensifolia, Linn.

Helenium, Linn.

\section{Ionopsidium}

acaule, Reichb.

\section{Iris}

humilis, Bieb.

mongolica, Hort.

prismatica, Pursh

sibirica, Linn.

- var. acuta.

Watsoni, Hort.

\section{Isatis}

glauca, Auch. tinctoria, Linn. undulata, Auch.

\section{Jasione}

Jankæ, Neilr. montana, Linn.

\section{Juncus}

effusus, Linn.

- var. conglomeratus.

filiformis, Linn.

glaucus, Sibth.

trifidus, Linn.

\section{Kalmia}

angustifolia, Linn.

\section{Kniphofia}

pauciflora, Baker

Tuckii, Baker

\section{Laburnum}

alpinum, J. S. Presl

vulgare, J. S. Presl

— var. austriacum.

\section{Lactuca}

Plumieri, Gren. et Godr. virosa, Linn.

\section{Lagurus}

ovatus, Linn.

\section{Larix}

europæa $D C$.

\section{Lasthenia}

glabrata, Lindl.

\section{Lathyrus}

latifolius, $\operatorname{Linn}$.

luteus, Baker

maritimus, Bigel.

montanus, Bernh.

sylvestris, Linn.

venosus, $M u h l$.

vernus, Bernh., var. flaccidus.

\section{Lavandula}

multifida, Linn.

Layia

elegans, Torr. et Gray

\section{Leiophyllum}

buxifolium, Ell.

\section{Leontodon}

hispidus, Linn.

\section{Leontopodium}

alpinum, Cass.

— var. altaicum. 


\section{Leonurus}

Cardiaca, Limn.

\section{Leycesteria}

formosa, Wall.

\section{Ligustrum}

medium, Franch. et Sav.

\section{Lilium}

Grayi, S, Wats.

\section{Limnanthes}

Douglasii, R. Br.

\section{Linaria}

alpina, Mill.

bipartita, Willd., var. splendida. maroccana, Hook. f., var. alba. origanifolia, $D C$.

Pancici, Hort.

purpurea, Mill.

repens, Mill.

triornithophora, Willd.

\section{Lindelofia}

spectabilis, $L e h \mathrm{~m}$.

\section{Linum}

austriacum, Linn.

grandiflorum, Desf.

perenne, Linn.

— var. album.

\section{Lithospermum}

prostratum, Loisel.

\section{Lobelia}

syphilitica, Linn.

\section{Lonas}

inodora, Gaertn.

\section{Lonicera}

alpigena, Linn.

angustifolia, Wall.

bella, Zabel

muscaviensis, Rehder

Periclymenum, Linn.

pyrenaica, Linn.

tatarica, Linn.

- var. alba grandiflora.

- var. discolor.

- var. flore rosea.

\section{Lotus}

corniculatus, Linn.

ornithopodioides, Linn.

\section{Lunaria}

annua, Linn.

- var. alba.

\section{Lupinus}

arboreus, Linn.

- var. violaceus.

nootkatensis, Donn

parviflorus, Nutt.

polyphyllus, Lindl.

- var. albus.

\section{Luzula}

albida, $D C$.

nivea, $D C$.

sylvatica, $D C$.

\section{Lychnis}

alba, Mill.

alpina, Linn.

chalcedonica, Linn.

coronaria, Desr.

dioica, Linn.

Drummondi, S. Wats.

Flos-cuculi, Linn.

Flos-Jovis, Desr. 
Lychnis-continued.

Lagascæ, Hook. $f$.

macrocarpa, Boiss, et Reut.

nigrescens, Edgew.

Viscaria, Linn.

- var. splendens.

\section{Lythrum}

Salicaria, Linn.

- var. cinereum.

- var. grandiflorum.

- var. hirsutum.

virgatum, Linn.

\section{Malope}

trifida, Cav.

\section{Malva}

sylvestris, Linn.

\section{Margyricarpus}

setosus, Ruiz et Pav.

\section{Marrubium}

vulgare, Linn.

\section{Matricaria}

inodora, Linn.

- var. plenissima.

oreades, Boiss.

\section{Matthiola}

bicornis, $D C$.

sinuata, $R . B r$, var. glabra albiflora.

\section{Meconopsis}

cambrica, $V i g$.

grandis, Prain

Wallichii, Hook.

- var. fusco-purpurea.

\section{Medicago}

carstiensis, Wulf.

Echinus, $D C$.

hispida, Gaertn.

sativa, Linn.

\section{Melica}

altissima, Linn.

ciliata, Linn.

nutans, Linn.

uniflora, Retz.

\section{Melilotus}

officinalis, Lam.

\section{Melittis}

Melissophyllum, Linn.

\section{Mentzelia}

Lindleyi, Torr. et Gray

\section{Meum}

athamanticum, Jacq.

\section{Microg-1ossa}

albescens, C. B. Clarke

\section{Milium}

effusum, Linn.

\section{Mimulus}

cardinalis, Dougl.

luteus, Linn.

\section{Mirabilis}

Jalapa, Linn.

\section{Mitella}

diphylla, Linn.

pentandra, Hook.

\section{Molopospermum}

cicutarium, $D C$. 
Montia

fontana, Linn.

\section{Morina}

longifolia, Wall.

\section{Muscari}

armeniacum, Baker

botryoides, Mill.

elegans, Hort.

Massyanum, Hort.

Maweanum, Baker

racemosum, Mill.

\section{Myosotis}

alpestris, F. W. Schmidt

antarctica, Hook. $f$.

arvensis, Lam.

cæspitosa, Schultz

dissitiflora, Baker

stricta, Link

\section{Myricaria}

germanica, Desv.

\section{Myrrhis}

odorata, Scop.

\section{Narcissus}

Bulbocodium, Linn., var. citrinus.

\section{Nasturtium}

officinale, R. Br.

\section{Neillia}

opulifolia, Benth. et Hook. f.

\section{Nemesia}

strumosa, Benth.

\section{Nemophila}

insignis, Benth.

Menziesii, Hook. et Arn.

\section{Nepeta}

Cataria, Linn.

concolor, Boiss. et Heldr.

kokamirica, Regel

linearis, Royle

longiflora, Vent.

Mussini, Spreng.

nuda, Linn.

Sibthorpii, Benth.

\section{Nicotiana}

rustica, Linn.

\section{Nigella}

hispanica, Linn., var. atropurpurea.

\section{Noccæa}

alpina, Reichb.

stylosa, Reichb.

\section{Nolana}

atriplicifolia, D. Don

\section{Nothoscordum}

striatum, Kunth

\section{Enanthe}

peucedanifolia, Pollich

pimpinelloides, Linn.

\section{Enothera}

fruticosa, Linn., var. Youngei.

pumila, Linn.

\section{Olearia}

Haastii, Hook. $f$.

ilicifolia, Hook. $f$.

macrodonta, Baker

stellulata, $D C$. 


\section{Omphalodes}

linifolia, Moench

\section{Onobrychis}

viciæfolia, Scop.

\section{Ononis}

Natrix, Linn.

\section{Onopordon}

Acanthium, Linn.

\section{Onosma}

albo-roseum, Fisch, et Mey. bracteata, Wall.

\section{Ophiopogon}

Jaburan, Lodd.

\section{Origanum}

vulgare, Linn.

\section{Osteospermum}

moniliferum, Linn.

\section{Oxalis}

corniculata, Linn.

- var. tropæoloides.

\section{Oxybaphus}

nyctagineus, Sweet

\section{Oxyria}

digyna, Hill

\section{Oxytropis}

campestris, $D C$. lapponica, Gaud. strobilacea, Bunge sulphurea, Fisch.

\section{Papaver}

Heldreichii, Boiss,

lateritium, $C$. Koch

libanoticum, Boiss.

monanthum, Trautv.

nudicaule, Linn.

orientale, Linn.,var. bracteatum.

pilosum, Sibth. et Sm.

rupifragum, Boiss, et Reut.

somniferum, Linn.

\section{Paradisea}

Liliastrum, Bertol.

\section{Parnassia}

caroliniana, Michx.

palustris, Linn.

\section{Parochetus}

communis, Buch.-Ham.

\section{Parrya}

Menziesii, Hort.

\section{Pentstemon}

barbatus, Roth

confertus, Dougl.

- var. cœeruleo-purpureus.

deustus, Dougt.

glaber, Pursh

gracilis, Nutt.

Menziesii, Hook., var. Newberryi.

pumilus, $N u t t$.

Richardsonii, Dougl. virgatus, A. Gray

Wrightii, Hook.

\section{Perezia}

multiflora, Less.

\section{Pernettya}

mucronata, Gaudich. 


\section{Peucedanum}

graveolens, Benth. et Hook. f. officinale, Linn.

verticillare, Spreng.

\section{Phacelia}

tanacetifolia, Benth.

viscida, Torr.

Whitlavia, A. Gray

\section{Phaseolus}

multiflorus, Willd.

\section{Philadelphus}

coronarius, Linn.

Gordonianus, Lindl.

grandiflorus, Willd.

\section{Phlomis}

Samia, Linn.

setigera, Falc.

\section{Phormium}

tenax, Forst.

\section{Photinia}

variabilis, Hemsl.

\section{Phygelius}

capensis, E. Mey.

\section{Physalis}

Alkekengi, Linn.

\section{Phyteuma}

canescens, Waldst. et Kit.

lobelioides, Willd.

orbiculare, Linn.

Scheuchzeri, All.

Sieberi, Spreng.

\section{Picris}

hieracioides, Linn.

\section{Picrorhiza}

Kurroa, Royle

\section{Pimpinella}

magna, Linn.

\section{Pinguicula}

grandiflora, Lam.

vulgaris, Linn.

\section{Pinus}

Pinaster, Soland.

sylvestris, Linn.

\section{Plantago}

alpina, Cav.

arenaria, Waldst. et Kit.

argentea, Chaix

carinata, Schrad.

Coronopus, Linn.

lanceolata, Linn.

major, Linn., var. monstrosa.

- var. rubra.

maritima, Linn.

Raoulii, Decne.

\section{Platystemon}

californicus, Benth.

Poa

pratensis, Linn.

\section{Podophyllum}

Emodi, Wall.

\section{Polemonium}

cæruleum, Linn.

flavo-cæruleum, Hort.

gracile, Willd.

himalayanum, Baker

mexicanum, Cerv. 


\section{Polycarpæa}

Teneriffæ, Lam.

\section{Polygonatum}

multiflorum, All.

\section{Polygonum}

affine, $D$. Don

amplexicaule, D. Don

Bistorta, Linn., var. major. sphærostachyum, Meissn. vaccinifolium, Wall.

Weyrichii, F. Schmidt

\section{Potentilla}

alpestris, Hall.f.

arguta, Pursh

dissecta, Pursh

glandulosa, Lindl.

Leschenaultiana, Ser.

Mooniana, Wight

nepalensis, Hook.

nitida, Linn., var. alba.

nivea, $\operatorname{Linn}$.

norvegica, Linn.

palustris, Scop.

procumbens, Sibth.

pseudo-obscura, Hort.

reptans, Linn.

Römeri, Hort.

rupestris, Linn.

sericea, Linn.

Sibbaldia, Hall.f.

Tormentilla, Neck.

tridentata, Soland.

villosa, Pall.

vladnizensis, Siegfr.

\section{Pratia}

angulata, Hook. f.

begonifolia, Lindl.

\section{Prenanthes}

purpurea, Linn.

\section{Primula}

apennina, Wid.

Auricula, Linn.

- var. marginata.

capitata, Hook. $f$.

denticulata, Sm., var. cashmiriana.

- var. rosea.

elatior, Hill, var. carpatica.

frondosa, Janka

involucrata, Wall.

japonica, A. Gray, var. lilacina.

luteola, Rupr.

officinalis, Jacq.

sikkimensis, Hook. $f$.

Stuartii, Wall., var. purpurea.

venusta $\times$, Host

viscosa, Vill.

- var. ciliata.

- var. nivalis.

\section{Prunella}

grandiflora, Jacq.

vulgaris, Linn.

\section{Prunus}

Laurocerasus, Linn.

\section{Ptelea}

trifoliata, Linn., var. aurea.

\section{Pyrus}

Aria, Linn.

alpina, Willd., var. superaria.

Aucuparia, Gaertn.

rotundifolia, Bechst.

Torminalis, $D C$. 


\section{Ramondia}

pyrenaica, Rich.

serbica, Panc., var. Nathaliæ.

\section{Ranunculus}

aconitifolius, Linn.

acris, Linn.

- var. Steveni.

auricomus, Linn.

brutius, Tenore

Lingua, Linn.

repens, Linn.

sceleratus, $\operatorname{Linn}$.

Thora, Linn.

\section{Raphanus}

sativus, Limn., var. caudatus.

\section{Reseda}

complicata, Bory

lutea, $\operatorname{Linn}$.

\section{Rhamnus}

cathartica, Linn.

Frangula, Linn.

\section{Rheum}

hybridum, Murr.

leucorrhizum, Pall.

Rhaponticum, Linn.

Ribes, Linn.

tataricum, Linn. $f$.

\section{Rhododendron}

campanulatum, D. Don

catawbiense, Michx.

caucasicum, Pall.

ciliatum, Hook. f.

cinnabarinum, Hook.f.

ferrugineum, Linn.

flavum, G. Don

glaucum, Hook. $f$.
Rhododendron-continued.

hirsutum, Linn., var. album.

kamtchaticum, Pall.

lepidotum, Wall.

niveum, Hook. f.

ponticum, Linn.

Thomsoni, Hook.f.

\section{Ribes}

sanguineum, Pursh

- var. malvaceum, $S$. Wats.

\section{Rœmeria}

violacea, Medic.

\section{Rosa}

acicularis, Lindl.

alpina, Linn.

blanda, Ait.

brachyphylla, Hort.

hibernica, Sm.

laxa, Retz.

rubiginosa, $\operatorname{Linn}$.

rugosa, Thunb.

- var. alba.

sericea, Lindl.

spinosissima, Linn.

\section{Rubus}

nitidus, Weihe et Nees

odoratus, Linn.

phoenicolasius, Maxim.

\section{Rumex}

alpinus, Linn.

flexuosus, Soland.

nepalensis, Spreng.

\section{Ruta}

graveolens, Linn. 


\section{Salvia}

argentea, Linn.

austriaca, Jacq.

cyanescens, Boiss. et Bal.

grandiflora, Etling.

glutinosa, Linn.

hians, Royle

nutans, Linn.

pratensis, Linn.

rubrifolia, Boiss.

Sclarea, Linn.

sylvestris, Linn.

- var. alba.

Verbenaca, Linn.

\section{Sambucus}

canadensis, Linn.

nigra, Linn.

racemosa, Linn.

\section{Samolus}

Valerandi, Linn.

\section{Sanicula}

europæa, Linn.

\section{Sanvitalia}

procumbens, Lam.

\section{Saponaria}

bellidifolia, Sm.

calabrica, Guss.

ocymoides, Linn.

peregrina $\times$, Hort.

\section{Saracha}

Jaltomata, Schlecht.

\section{Satureia}

montana, Linn.

\section{Saxifraga}

aizoides, Linn.

Aizoon, Jacq., var. balcana.

- var. carinthiaca (Schotz)

- var. notata.

- var. pectinata.

- var. rosularis.

— var. Sturmiana.

aspera, Linn.

bronchialis, Linn.

- var. cherlerioides.

Bucklandi, Hort.

cæsia, Linn.

canaliculata, Boiss. et Reut.

cartilaginea, Willd.

cochlearis, Reuchb., var. minor.

Cotyledon, Linn,, var. pyramidalis.

cuneifolia, Linn.

- var. capillipes.

- var. subintegra.

decipiens, Ehrh.

erosa, Pursh

Gaudini ×, Brïgg.

geranioides, Linn.

gibraltarica, Boiss, et Reut.

granulata, Linn.

Hirculus, Linn.

Hostii, Tausch

- var. altissima.

-nar. elatior.

hypnoides, Linn.

intacta, Willd.

- var. farinosa.

Kingii, Hort.

lingulata, Bell., var. australis.

- var. lantoscana.

longifolia, Lapeyr.

var. hybrida.

Mertensiana, Bongard

montaoniensis $\times$, Kerner

muscoides, Wulf.,var.moschata. 


\section{List of Seeds Collected DURING I904. Ixvii}

Saxifraga-continued.

, muscoides, Wulf, var. purpurea. mutata, Linn.

nervosa, Miégiv.

nivalis, Linn.

pedatifida, Ehrh.

pentadactylis, Lapeyr.

Portæ $\times$, Engl.

retusa, Gouan, var. maritima.

Reyeri $\times$, Hort.

Rocheliana, Stemb., var. coriophylla

rotundifolia, Linn., var. angulosa.

sancta, Griseb.

Schraderi, Sternb.

squarrosa, Sieber

tenella, Wulf.

umbrosa, Linn., var. hirsuta.

- var. Ogilvieana.

- var. serratifolia.

\section{Scabiosa}

caucasica, Bieb., var. alba.

centauroides, Mill.

longifolia, Waldst. et Kit.

lucida, Vill.

Succisa, Linn.

- var. alba.

sylvatica, $\operatorname{Linn}$.

\section{Schizanthus}

pinnatus, Ruiz et Pav.

\section{Schizopetalon}

Walkeri, Sims

\section{Scilla}

festalis, Salisb.

hispanica, Mill.

peruviana, Linn.

\section{Scirpus}

sylvaticus, Limn.

\section{Scleranthus}

annuus, Linn.

\section{Scolymus}

grandiflorus, Dest.

\section{Scorzonera}

hispanica, Linn.

\section{Scrophularia}

alata, Gilib.

nodosa, Limn.

\section{Scutellaria}

alpina, Linn.

altissima, Linn.

hirta, Sibth. et Sm.

\section{Securigera}

Coronilla, Ltmn.

\section{Sedum}

Aizoon, Linn.

Alberti, Regel

album, Linn.

altissimum, Poir.

Anacampseros, Linn.

asiaticum, Spreng.

Beyrichianum, Turcs.

dasyphyllum, Linn.

euphorbioides, Schlecht.

Ewersii, Ledeb.

hispanicum, Linn.

involucratum, Bieb.

laxiflorum, $D C$.

maximum, Suter

oreganum, Nutt.

populifolium, Pall.

reflexum, Linn.

roseum, Scop.

stoloniferum, S. T. Gmel.

Telephium, Linn.

trifidum, Wall. 


\section{Sempervivum}

arachnoideum, Linn.

fimbriatum, Schnittsp. et C. B. Lehm.

globiferum, Linn. patens, Griseb. et Schenk

Schnittspahni, Lagg.

soboliferum, Sims

tectorum, Linn.

violaceum, Hort.

\section{Senebiera}

pinnatifida, $D C$.

\section{Senecio}

Doronicum, Linn.

\section{Serratula}

Gmelini, Ledeb.

heterophylla, Desf.

\section{Seseli}

Libanotis, Koch

\section{Sidalcea}

candida, A. Gray

malvæflora, A. Gray

oregana, A. Gray

\section{Sideritis}

scordioides, Linn.

\section{Silene}

auriculata, Sibth. et Sm.

alpestris, Jacq.

Armeria, Linn.

caucasica, Boiss.

chromodonta, Boiss, et Reut.

colorata, Poir.

compacta, Fisch.

Cucubalus, Wibel

Fabaria, Sibth, et Sm.
Silene-continued.

italica, Pers.

maritima, With.

rhynchocarpa, Boiss.

quadridentata, Pers.

Reichenbachii, Vis.

rupestris, Linn.

Saxifraga, Linn.

Schafta, Gmel.

stylosa, Bunge

Thorei, Duf.

vallesia, $\operatorname{Linn}$.

Zawadzkii, Herbich

\section{Silybum}

Marianum, Gaertn.

\section{Sisymbrium}

Alliaria, Scop.

Assoanum, Loscos et Pardo strictissimum, Linn. "

tanacetifolium, Linn.

\section{Sisyrinchium}

angustifolium, Mill.

- var. mucronatum.

- var. Nuttallii.

Bermudiana, Linn.

californicum, Dryand.

grandiflorum, Dougl., var. album.

\section{Skimmia}

japonica, Thunb.

\section{Solanum}

Dulcamara, Linn.

\section{Solidago}

elongata, Nutt.

Shortii, Torr. et Gray

Virgaurea, Linn. 


\section{Spartina}

polystachya, Willd.

\section{Specularia}

hybrida, $A . D C$.

pentagonia, $A . D C$.

Speculum, $A . D C$.

\section{Spergula}

arvensis, Linn.

\section{Spinacia}

oleracea, $\operatorname{Linn}$.

\section{Spiræa}

Aruncus, Linn.

bullata, Maxim.

palmata, Thunb.

\section{Stachys}

alpina, Linn.

- var. intermedia.

Betonica, Benth.

lanata, Jacq.

recta, Linn.

\section{Statice}

altaica, Hort.

\section{Stellaria}

biflora, Linn.

graminea, Linn.

\section{Stenanthium}

angustifolium, Kunth

\section{Stokesia}

cyanea, L'Hérit.

\section{Succowia}

balearica, Medic.

\section{Symphoricarpus}

racemosus, Michx.

\section{Symphyandra}

Hofmanni, Pant.

\section{Synthyris}

reniformis, Benth.

\section{Syringa}

Emodi, Wall.

\section{Tagetes}

lucida, Cav.

signata, Bartl.

\section{Tanacetum}

vulgare, Linn.

\section{Tanakæa}

radicans, Franch.

\section{Taxus}

baccata, Linn.

- var. Dovastoni, Carr.

- var. fastigiata, Loud.

- var. pyramidalis.

- var. Washingtoni.

\section{Telephium}

Imperati, Linn.

\section{Tellima}

grandiflora, R. Br.

\section{Teucrium}

montanum, Linn.

Polium, Linn.

Scorodonia, Linn.

\section{Tetragonia}

expansa, Murr. 


\section{Thalictrum}

corynellum, $D C$.

glaucum, Desf.

laserpitifolium, Willd.

majus, Jacq.

minus, Linn., var. elatum.

simplex, Linn.

\section{Thermopsis}

fabacea, $D C$.

montana, Nutt.

\section{Thlaspi}

perfoliatum, Linn.

\section{Thymus}

Serpyllum, Linn.

- var. coccineus.

- var. micans.

\section{Tilia}

vulgaris, Hayne

\section{Tofieldią}

carinthiaca, Hort.

palustris, Huds.

\section{Tolpis}

barbata, Gaertn.

\section{Tradescantia}

virginiana, Linn., var. alba.

- var, atropurpurea.

- var. elata.

\section{Trautvetteria}

palmata, Fisch. et Mey.

\section{Trifolium}

alpinum, Linn.

pannonicum, Jacq.
Trigonella

corniculata, Linn.

Trilisa

odoratissima, Cass.

Trillium

stylosum, Nutt.

\section{Triosteum}

perfoliatum, Linn.

\section{Trisetum}

flavescens, Beauv.

\section{Triticum}

Spelta, Linn.

\section{Tritonia}

rosea, Klatt

\section{Trollius}

altaicus, Mey.

asiaticus, Linn.

- var. giganteus.

caucasicus, Stev.

europæus, Linn.

\section{Tropæolum}

majus, Linn.

\section{Tulipa}

Didieri, Jord., var. alba.

pulchella, Fenzl

sylvestris, Linn.

\section{Tunica}

prolifera, Scop.

Saxifraga, Scop.

\section{Ulex}

europæus, Linn. 


\section{Ulmus}

montana, With.

\section{Uniola}

latifolia, Michx.

\section{Urospermum}

Dalechampii, F. W. Schmidt picroides, Desf.

\section{Ursinia}

speciosa, $D C$.

\section{Urtica}

pilulifera, Linn.

\section{Vaccinium}

padifolium, $S m$.

\section{Venidium}

decurrens, Less.

\section{Veratrum}

album, Linn.

\section{Verbascum}

Blattaria, Linn.

Lagurus, Fisch. et Mey.

longifolium, Tenore

Lychnitis, Linn.

phlomoides, Linn.

phœniceum, Linn.

\section{Veronica}

alpina, Linn.

Anagallis, Linn.

aphylla, Linn.

austriaca, Linn.

- var. pinnatifida.

- var. Prenja.

Balfouriana, Hook. f.
Veronica-continued.

Bidwillii, Hook. f.

candida, Lodd.

carnosula, Hook. f.

Catarractæ, Forst.

Chamædrys, Linn.

corymbosa, Hort.

decumbens, Armstr.

formosa, R. Br.

fruticulosa, Linn., var.

cæspitosa

imperialis $\times$, Hort.

Kirkii, Armstr.

longifolia, Linn., var. alba.

Lyallii, Hook. f.

monticola, Armstr.

officinalis, Linn.

- var. hirsuta.

parviflora, Vahl, var, angustifolia, Hook. f.

pinguifolia, Hook. f., var. decumbens.

pinnata, Linn.

Ponæ, Gouan

saxatilis, Scop.

Teucrium, Linn.

virginica, Linn.

\section{Viburnum}

cotinifolium, D. Don

Lantana, Linn.

Opulus, Linn.

\section{Vicia}

bithynica, Linn.

Cracca, Linn.

oroboides, Wulf.

Orobus, $D C$.

pyrenaica, Pourr.

sativa, Linn.

unijuga, A. Braun 
Ixxii List of Seeds Collected DURING I9O4.

\section{Vincetoxicum}

officinale, Moench

\section{Viola}

blanda, Willd.

canina, Linn.

cornuta, Linn.

hirta, Linn.

lutea, Huds.

odorata, Linn.

olympica, Boiss.

sylvestris, Lam.

\section{Wahlenbergia}

dalmatica, $A . D C$.

dinarica, Hort.

Kitaibelii, $A$. $D C$.

tenuifolia, $A . D C$.

\section{Withania}

somnifera, Dun.

Wulfenia

carinthiaca, Jacq.

\section{Xerophyllum}

asphodeloides, Nutt.

\section{Zaluzianskya}

selaginoides, Walp.

\section{Zenobia}

speciosa, D. Don, var. pulverulenta.

\section{Ziziphora}

taurica, Bieb.

\section{Zygadenus}

elegans, Pursh 


\section{PLANTS UNDER GLASS.}

\section{Acacia}

calamifolia, Sweet

decurrens, Willd.

Drummondii, Benth.

Farnesiana, Willd.

grandis, Henfr.

juncifolia, Benth.

melanoxylon, $R . B r$.

verticillata, Willd.

\section{Acokanthera}

spectabilis, Hook.f.

\section{Æchmea}

bromeliæfolia, Baker

- var. pulchra.

candida, E. Morr.

cærulescens, Baker

glomerata, Hook.

\section{Agave}

densiflora, Hook.

\section{Aglaonema}

commutatum, Scholt

\section{Albizzia}

lophantha, Benth.

\section{Aloe}

mitriformis, Mill., var. spinulosa (Salm-Dyck).

- var. flavispina (Haze.).

obscura, Mill., var. picta

(Thunb.).

\section{Alonsoa}

linifolia, Roesl

Warscewiczii, Regel

Anopteris

glandulosus, Labill.

Aphelandra

aurantiaca, Iindl., var. Roezlii.

\section{Araujia}

sericifera, Brot.

\section{Ardisia}

mamillata, Hance

\section{Aristolochia}

brasiliensis, Mart.

elegans, Mast.

fimbriata, Cham.

\section{Astrocaryum}

mexicanum, Liebm.

\section{Asystasia}

bella (Harr.).

\section{Begonia}

Bruantii $\times$, Hort.

Davisii, Veitch

dichotoma, Jacq.

Dregei, $O$, et $D$.

manicata, Cels

nitida, Dryand.

semperflorens, $L$. et $O$. 
Ixxiv List of Seeds Collected DURING I904.

Begonia-continued.

ulmifolia, Willd.

Verschaffeltii $\times$, Hort.

weltoniensis $\times$, Hort.

\section{Billardiera}

longiflora, Labill.

\section{Billbergia}

decora, Poepp.

pyramidalis, Lindl.

zebrina, Lindl.

\section{Biophytum}

sensitivum, $D C$.

\section{Bowiea}

volubilis, Harv.

\section{Brachychilum}

Horsfieldii, Baker

\section{Brodiæa}

hyacinthina, Baker, var. lactea.

\section{Calliandra}

hæmatocephala, Hassk.

Callicarpa

purpurea, Juss.

\section{Callistemon}

linearis, $D C$.

speciosus, $D C$.

\section{Camellia}

theifera, Griff.

\section{Campanula}

pyramidalis, Linn.

Vidalii, H. C. Wats,

\section{Capparis}

flexuosa, Blume

\section{Capsicum}

annuum, Linn.

\section{Carica}

candamarcensis, Hook. f.

\section{Carmichælia}

australis, R. Br.

\section{Cassia}

corymbosa, Lam.

\section{Casuarina}

stricta, Ait.

\section{Cedronella}

triphylla, Moench

\section{Celosia}

cristata, Linn., var. nana.

\section{Celsia}

cretica, Lmn.

\section{Cestrum}

aurantiacum, Lindl,

Newellii (Veitch)

\section{Chlorophytum}

Orchidastrum, Lindl.

\section{Cleome}

grandis, Hort.

spinosa, Jacq.

\section{Clerodendron}

fallax, Lindl.

Thomsonæ, Balf. 


\section{List of Seeds Collected DURING I904. Ixxv}

\section{Clianthus}

puniceus, Banks et Sol.

Clitoria

Ternatea, Linn.

\section{Cobæa}

scandens, Cav.

\section{Coffea}

arabica, Linn.

bengalensis, Roxb.

\section{Costus}

afer, Ker

igneus, $N . E . B r$.

\section{Crassula}

tetragona, $\operatorname{Linn}$.

Crossandra

undulæfolia, Salisb.

\section{Cyanotis}

somaliensis, C. B. Clarke

\section{Cyclanthera}

explodens, Naud.

\section{Cyphomandra}

betacea, Sendt.

\section{Cytisus}

candicans, Lam.

\section{Dalechampia}

Roezliana, Muell.

\section{Dermatobotrys}

Saundersii, Bolus

\section{Desmodium}

gyrans, $D C$.

\section{Dillwynia}

floribunda, $S m$.

\section{Dolichos}

Lablab, Linn.

\section{Drosera}

capensis, Linn.

\section{Drosophyllum}

lusitanicum, $\operatorname{Link}$

\section{Eccremocarpus}

scaber, Ruiz et Pav.

\section{Epiphyllum}

Gaertneri, Hook. f.

\section{Erythrochiton}

brasiliensis, Nees

\section{Eucalyptus}

amygdalina, Labill.

incrassata, Labill.

piperita, $S m$.

\section{Eucomis}

amaryllidifolia, Baker

bicolor, Baker

punctata, L'Hérit.

- var. striata.

\section{Eupatorium}

Weinmannianum, Regel

\section{Euphorbia}

splendens, Boj.

\section{Evodia}

elegans, Hort.

formosa, Hort. 
Ixxvi List of Seeds Collected during I9O4.

\section{Ficus}

pyriformis, Hook. et Arn.

\section{Francoa}

appendiculata, $\mathrm{Cav}$.

\section{Fuchsia}

procumbens, R. Cunn.

\section{Genista}

monosperma, Lam.

\section{Geonoma}

pumila, Wendl.

\section{Gloriosa}

superba, Linn.

\section{Goodia}

lotifolia, Salisb.

\section{Gossypium}

herbaceum, Linn.

\section{Guizotia}

abyssinica, Cass.

\section{Hakea}

suaveolens, R. Br.

\section{Hedychium}

Gardnerianum, Rosc.

\section{Heliotropium}

peruvianum, Linn.

\section{Hibiscus}

esculentus, Linn.

Huegelii, Endl.

Manihot, Linn.

pedunculatus, Linn.

vitifolius, Linn.

\section{Humea}

elegans, Sm.

\section{Hypericum}

japonicum, Thunb.

\section{Hypoxis}

Krebsii, Fisch.

\section{Impatiens}

capensis, Meer.

cuspidata, Wight et Arn., var. arthritica.

\section{Indigofera}

atropurpurea, Buch.-Ham.

australis, Willd.

\section{Jatropha}

podagrica, Hook.

\section{Kalanchoe}

Cassiopeia, Hort.

flammea, Stapf

grandiflora, Wight et Arn.

Kirkii, N. E. Br.

\section{Kedrostis}

africana, $\operatorname{Cog} n$.

\section{Kennedya}

prostrata, R. Br.

\section{Lachnostoma}

maritimum, Nichols.

\section{Lantana}

Camara, Linn. 
List of Seeds Collected during I904. Ixxvii

Leea

sambucina, Willd.

Leptospermum

arachnoideum, $S m$. scoparium, Forst.

Loasa

lateritia, Gill., var. aurantiaca, Hort.

Luffa

ægyptiaca, Mill.

Mallotus

japonicus, Muell. Arg.

\section{Malpighia}

glabra, Linn.

\section{Malvastrum}

grossulariæfolium, Garcke

\section{Maurandia}

Barclayana, Lindl.

Melia

arguta, $D C$.

Azedarach, Linn.

\section{Mesembryanthemum}

aurantiacum, Haw.

curviflorum, Haw .

lepidum, Haze.

micans, Linn.

roseum, Willd.

serrulatum, Haw.

tumidulum, Haze.

vaginatum, Lam.

\section{Momordica}

Charantia, Linn.

cochinchinensis, Spreng.

\section{Monstera}

deliciosa, Liebm.

\section{Moræa}

prolongata, Hort.

\section{Musschia}

Wollastoni, Lowe

\section{Myrica}

æthiopica, Linn.

\section{Myrsine}

africana, $\operatorname{Linn}$.

\section{Myrtus}

communis, Linn.

Luma, Barn.

Ugni, Mol.

\section{Nephthytis}

liberica, $N$. E. Br.

\section{Nerine}

flexuosa, Herb.

\section{Ochna}

multiflora, $D C$.

\section{Opuntia}

nigricans, Haw.

\section{Oxypetalum}

cœeruleum, Decne. 


\section{Passiflora}

quadrangularis, Linn.

\section{Pavonia}

spinifex, Cav.

\section{Pentas}

carnea, Benth., var. kermesina.

\section{Phaseolus}

vulgaris, Linn.

\section{Philydrum}

lanuginosum, Banks

\section{Physalis}

peruviana, Linn.

\section{Pitcairnia}

Altensteinii, Lem.

Andreana, Linden

latifolia, Soland.

maidifolia, Decne.

\section{Polyalthia}

suberosa, Benth. et Hook. $f$.

\section{Pontaderia}

cordata, Linn.

\section{Primula}

mollis, Nutt.

verticillata, Forsk.

\section{Prostanthera}

lasianthos, Labill.

\section{Psychotria}

micrantha, Hiern

\section{Rhodochiton}

volubile, Zucc.

\section{Rhododendron}

arboreum, $S m$.

Nuttallii, Booth

\section{Rhynchosia}

caribæa, $D C$.

\section{Ricinus}

communis, Linn.

\section{Rivina}

aurantiaca, Warsc.

humilis, Linn.

tinctoria, Hamil.

\section{Rondeletia}

odorata, Jacq.

\section{Ruellia}

amcena, Nees

rosea, Hemsl.

tuberosa, Linn.

\section{Ruta}

bracteosa, $D C$.

\section{Sabal}

Blackburniana, Glazebr.

\section{Schizanthus}

pinnatus, Ruiz et Pav.

\section{Sedum}

Sieboldii, Hort.

\section{Sempervivum}

annuum, C. Sm.

glaucum, Tenore

tabuliforme, Haw. 


\section{Senecio}

grandifolius, Less.

macroglossus, $D C$.

\section{Solanum}

auriculatum, Ait.

coagulans, Hort.

fastigiatum, Willd.

Hendersoni, Hort.

jasminoides, Paxt.

nigrum, Linn.

pyracanthum, Jacq.

Seaforthianum, Andr.

\section{Sparaxis}

bulbifera, Ker

\section{Stachytarpheta}

indica, $\mathrm{Vahl}$

\section{Stylidium}

adnatum, $R$. $B r$.

\section{Tacca}

cristata, Jack

\section{Tacsonia}

mollissima, H. B. K.

Van-Volxemii, Hook.

\section{Talinum}

patens, Willd.

triangulare, Willd.
Tetranema

mexicana, Benth.

Teucrium

Marum, Linn.

\section{Tillandsia}

macropetala, Hort.

Regina, Vell.

splendens, Brongn.

\section{Trachelium}

cæruleum, Linn.

\section{Trichosanthes}

Anguina, Linn.

\section{Tristania}

laurina, $R . B r$.

nereifolia, $R$. $B r$.

\section{Turnera}

ulmifolia, Linn.

\section{Villamilla}

octandra, Hook. f.

purpurascens, Hort.

\section{Vellozia}

elegans, Talbot

\section{Vitis}

heterophylla, Thunb., var. humulifolia. 





\section{NOTES}

FROM THE

\section{ROYAL BOTANIC GARDEN, E D I N B U R G H.}

DECEMBER 1905.

\section{CONTENTS.}

List of Seeds collected in the Royal Botanic Garden, Edinburgh, during the Year 1905, _ _ _ - Ixxxi

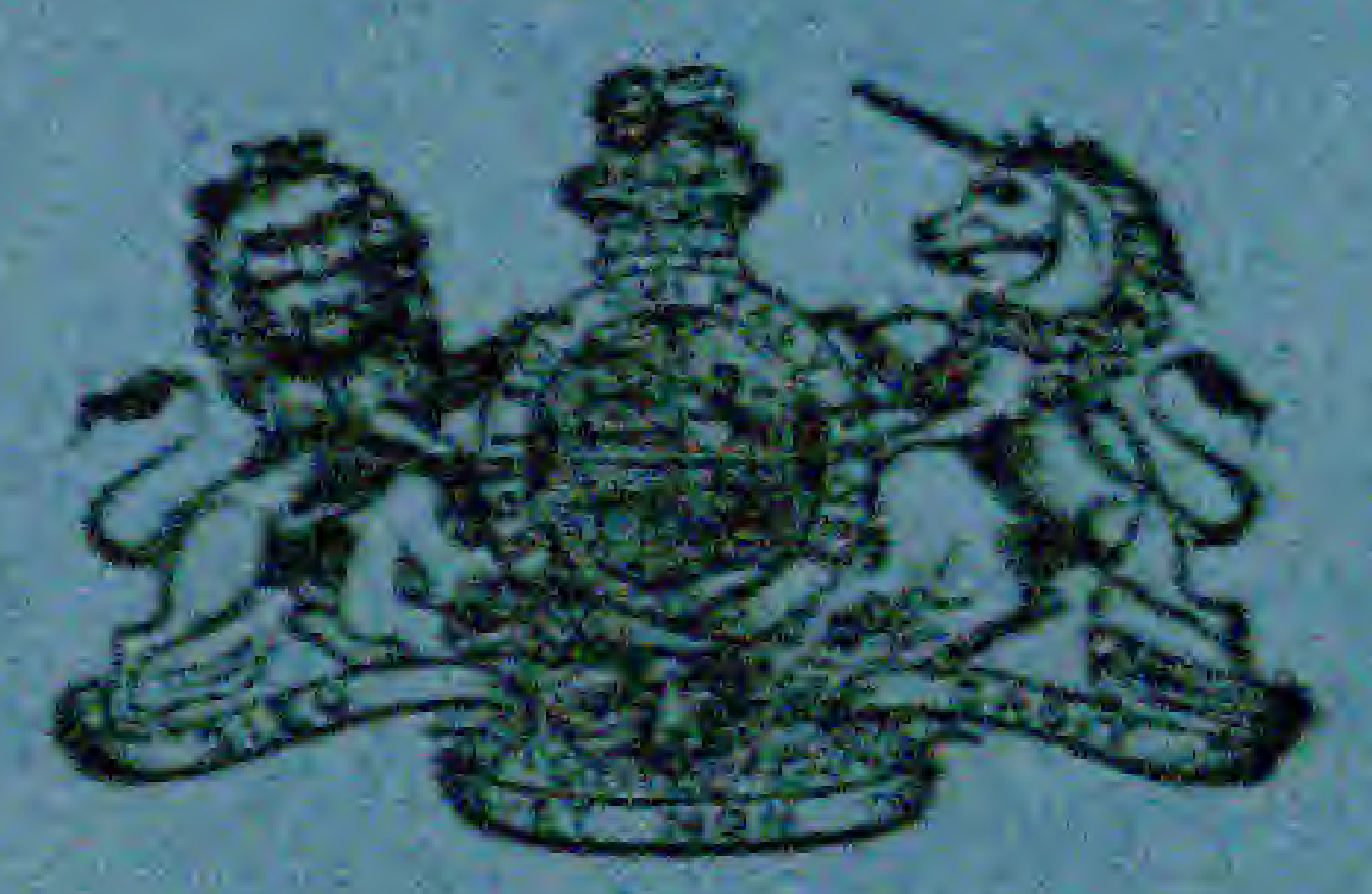

GLASGOW:

PRINTED FOR HIS MAJESTY'S STATIONERY OFFICE BY JAMES HEDDERWICK \& SONS LTD., AT "THE CITIZEN" PRESS, ST. VINCENT PLACE.

SOLD AT THE GARDEN,

And to be purchased, either directly or through any Bookseller, from OLIVER \& BOYD, EDINEURGH. 



\section{List of Seeds Collected in the Royal Botanic Garden, Edinburgh, during the Year 1905.}

The following is a list of plants cultivated in the Royal Botanic Garden, Edinburgh, from which ripened seeds have been collected during the year 1905. The quantity of seed obtained from some of the species is of limited amount. The seeds are available for exchange, but they are not for sale :-

\section{PLANTS IN THE OPEN.}

\section{Acæna}

argentea, Ruiz et Pav.

glabra, J. Buch.

inermis, Hook. $f$.

microphylla, Hook. f.

myriophylla, Lindl.

ovalifolia, Ruis et Pav.

Sanguisorbæ, $V a h l$

\section{Acanthus}

Schottii, Hort.

\section{Acer}

circinnatum, Pursh

monspessulanum, Linn.

platanoides, Linn.

Pseudo-platanus, Linn.

- var. purpureum.

\section{Aconitum}

Fischeri, Reichb.

longigaleatum, Hort.

Lycoctonum, Linn.

Napellus, Linn.

paniculatum, Lam.
Aconitum-iontinuea.

reclinatum, A. Gray

uncinatum, Linn.

variegatum, $\operatorname{Linn}$.

Acroglochin

chenopodioides, Schrad.

\section{Actæa}

alba, Mill.

spicata, Linn.

- var. rubra.

\section{Actinella}

scaposa, Nutt.

\section{Actinomeris}

helianthoides, Nutt.

\section{Adenocarpus}

intermedius, $D C$.

\section{Adenophora}

polymorpha, Ledeb.

Potanini, Batalin

stylosa, Fisch. 
Ixxxii List of SeEdS Collected DURING I905.

\section{Adlumia}

cirrhosa, Rafin.

\section{Adonis}

autumnalis, Linn.

vernalis, Linn.

\section{Assculus}

Hippocastanum, Linn.

\section{Ethionema}

armenum, Boiss.

cordatum, Boiss.

grandiflorum, Boiss. et Hohen.

\section{Agrimonia}

agrimonioides, Linn.

Eupatoria, Linn.

\section{Agropyron}

acutum, Roem. et Schult.

dasyanthum, Ledeb.

tenerum, Vasey

\section{Agrostis}

nigra, With.

\section{Ajuga}

genevensis, Linn.

pyramidalis, Linn.

\section{Alchemilla}

alpina, Linn., var. conjuncta

$(B a b$.$) .$

arvensis, $S c o p$.

\section{Alisma}

Plantago, Linn.

\section{Alkanna}

lutea, $A$. $D C$.
Allium

cardiostemon, Fisch, et Mey.

carinatum, Linn.

cernuum, Roth

fistulosum, Linn.

flavum, $\operatorname{Lin} n$.

giganteum, Regel

kansuense, Regel

karataviense, Regel

macranthum, Baker

margaritaceum, Sibth. et $S m$.

Moly, Linn.

mutabile, Michx.

odorum, Linn.

oleraceum, Linn.

oreophilum, C. A. Mey.

orientale, Boiss

Ostrowskianum, Regel

polyphyllum, Kar. et Kir.

pulchellum, G. Don

rubellurn, Bieb.

sativum, Linn.

scabriflorum, Boiss.

Schœenoprasum, Linn.

Schuberti, Zucc.

senescens, Linn.

subvillosum, Salzm.

urceolatum, Regel

vineale, $\operatorname{Linn}$.

viviparum, Kar, et Kir.

\section{Alnus}

cordifolia, Tenore

glutinosa, Medic.

- var. rubrinervia.

incana, Moench

japonica, Sieb. et Zucc.

orientalis, Decne.

rugosa, $C$. Koch

subcordata, C. A. Mey.

viridis, $D C$. 


\section{List of Seeds Collected during 1905. 1xxxiii}

\section{Alonsoa}

caulialata, Ruiz et Pav.

\section{Alopecurus}

geniculatus, Linn.

pratensis, Linn.

\section{Alstroemeria}

aurantiaca, Don

revoluta, Ruiz et Pav.

\section{Althæa}

rosea, Cav.

\section{Alyssum}

argenteum, Vitm.

condensatum, Boiss, et

Hausskn.

creticum, Linn.

idæum, Boiss, et Heldr.

montanum, Linn.

saxatile, Linn.

- var. sulphurea.

serpyllifolium, Desf.

spinosum, Linn.

Wierzbickii, Heuff.

\section{Amethystea}

cærulea, Linn.

\section{Ammobium}

alatum, $R . B r$.

\section{Anagallis}

arvensis, Linn.

\section{Anchusa}

capensis, Thunb.

italica, Retz.

officinalis, Linn.

sempervirens, Linn.
Androsace

carnea, Linn.

lactea, Linn.

lactiflora, Fisch.

Laggeri, Huet

lanuginosa, Wall.

sarmentosa, Wall.

septentrionalis, Linn.

\section{Anemone}

alpina, Linn.

- var. sulphurea.

baicalensis, Turcs.

cylindrica, A. Gray

decapetala, Arduini

multifida, Poir.

polyanthes, D. Don

pratensis, Linn.

riviilaris, Buch.-Ham.

sylvestris, Linn.

virginiana, Linn.

vitifolia, Buch.-Ham.

\section{Anthemis}

Biebersteiniana, C. Koch

ptarmicæformis, $C$. Kock

tinctoria, Linn.

\section{Anthericum}

Liliago, Linn.

\section{Anthoxanthum}

odoratum, Linn.

\section{Anthyllis}

sericea, Lag.

Vulneraria, Limn., var. Dillenii.

\section{Antirrhinum}

Asarina, Linn.

majus, Linn.

Orontium, Linn. 
Ixxxiv List of Seeds Collected DURING 1905.

Apera

arundinacea, Hook. $f$.

interrupta, Beauv.

Spica-Venti, Beauv.

\section{Aphyllanthes}

monspeliensis, Linn.

\section{Apium}

graveolens, Linn.

\section{Aquilegia}

cærulea, James

californica, A. Gray, var. alba. canadensis, Linn.

Einseliana, F. Schultz

pyrenaica, $D C$.

Skinneri, Hook.

vulgaris, Linn.

-var. flore pleno.

\section{Arabis}

albida, Stev.

Allionii, $D C$.

alpina, Linn.

- var. anachoretica.

bellidifolia, Jacq.

hirsuta, Scop.

muralis, Bertol., var. rosea.

petræa, Lam.

Turczaninowii, Ledeb.

\section{Archangelica}

officinalis, Hoffm.

\section{Arctotis}

stœechadifolia, Berg.

\section{Arenaria}

balearica, Linn.

ciliata, Linn.

erythrosepala, Boiss.

Fendleri, A. Gray

- var. diffusa.
Arenaria-continued.

foliosa, Royle

gothica, Fries

gracilis, Kit.

graminifolia, Schrad.

gypsophiloides, Linn.

Holostea, Bieb.

juniperina, Linn.

laricifolia, Linn.

pinifolia, Bieb.

purpurascens, Ramond

tetraquetra, Linn.

trinervia, Linn.

verna, Linn.

\section{Argemone}

mexicana, Linn.

\section{Armeria}

maritima, Willd.

\section{Arnica}

amplexicaulis, Nutt.

Chamissonis, Less.

montana, Linn.

\section{Arracacia}

arguta, Benth. et Hook. J.

\section{Arrhenatherum}

avenaceum, Beauv.

\section{Asperula}

ciliata, Rochel

lævigata, Linn.

longiflora, Waldst. et Kit.

odorata, Linn.

orientalis, Boiss. et Hohen.

tinctoria, Linn.

\section{Asphodeline}

Balansæ, J. Gay

Dammeriana, Siehe

liburnica, Reichb. 
List of Seeds Collected duRing ig05. Ixxxv

Asphodelus

albus, Willd.

\section{Aster}

diplostephioides, Benth. et Hook $f$.

floribundus, Nutt.

Fremonti, A. Gray

himalaicus, $C, B$. Clarke

incisus, Fisch.

lævis, Linn, var. decorus.

leucanthemus, Rafin.

macrophyllus, Linn.

Novæ-Angliæ, Linn .

paniculatus, Lam.

patens, Ait.

puniceus, Linn.

pyrenæus, $D C$.

Radula, Ait.

sagittifolius, Willd.

sibiricus, Linn.

tataricus, Linn. $f$.

\section{Astilbe}

chinensis, Maxim., var.

Davidii, Franch.

\section{Astragalus}

Cicer, Linn.

danicus, Rets., var. alba.

falcatus, Lam.

Glyciphyllos, Linn.

monspessulanus, Linn.

purpureus, Lam.

sinicus, Linn.

vaginatus, Pall.

\section{Astrantia}

carniolica, Wulf.

helleborifolia, Salisb.

major, Linn.

- var. intermedia.

neglecta, $C$. Koch et Bouche

\section{Athamanta}

Matthioli, Wulf.

\section{Atriplex}

Babingtonii, Woods

hortensis, Linn., var. purpurea. patula, Linn.

\section{Atropa}

Belladonna, Linn.

\section{Aubrietia}

Culumnæ, Guss.

croatica, Schott, Nyman et

Kotschy

deltoidea, $D C_{.}$, var. cilicica.

\section{Avena}

pratensis, Linn.

sterilis, Linn.

\section{Axyris}

amaranthoides, Linn.

\section{Baccharis}

patagonica, Hook, et $\mathrm{Am}$.

\section{Ballota}

nigra, Linn.

\section{Barbarea}

præcox, R. Br.

vulgaris, $R$. $B r$.

\section{Bellis}

annua, Linn.

perennis, Linn., var, fistulosa. rotundifolia, Boiss. et Reut, var cærulescens. 
lxxxvi List of Seeds Collected during 1905.

\section{Berberis}

angulosa, Wall.

Aquifolium, Pursh

aristata, $D C$.

caucasica, Hort.

cretica, Linn.

Darwinii, Hook.

nepalensis, Spreng.

stenophylla, Moore

virescens, Hook. $f$.

vulgaris, Linn.

Wallichiana, $D C$.

\section{Berkheya}

purpurea, Benth. et Hook. f.

Radula, Hort. Kew.

\section{Beta}

trigyna, Waldst. et Kit.

vulgaris, Linn.

\section{Betula}

alba, Linn., var. pubescens.

Ermani, Cham.

\section{Bidens}

grandiflora, Balb.

\section{Biscutella}

cichoriifolia, Loisel.

lævigata, Linn.

\section{Bocconia}

microcarpa, Maxim.

\section{Boltonia}

incisa, Benth.

\section{Borago}

laxiflora, Willd.

officinalis, Linn.

\section{Boykinia}

aconitifolia, Nutt.

rotundifolia, Parry

\section{Brachycome}

iberidifolia, Benth.

\section{Brassica}

Erucastrum, Linn.

oleracea, Linn.

\section{Bravoa}

geminiflora, Lex.

\section{Briza}

maxima, Linn.

\section{Brodiæa}

Douglasii, S. Wats.

\section{Bromus}

ciliatus, Linn.

inermis, Leyss.

Tacna, Steud.

unioloides, $H . B$. et $K$.

\section{Bruckenthalia}

spiculifolia, Reichb.

\section{Bryanthus}

empetriformis, A. Gray

taxifolius, A. Gray

\section{Bryonia}

dioica, Jacq.

\section{Buddleia}

Hemsleyana, Hort.

intermedia, Carr.

variabilis, Hemsl.

—var. Veitchiana.

\section{Bulbinella}

Hookeri, Benth. et Hook. f. 
List of Seeds Collected DURING 1905. 1xxxvii

\section{Buphthalmum}

salicifolium, Linn.

speciosum, Schreb.

\section{Bupleurum}

Candollei, Wall.

longifolium, Linn.

rotundifolium, Linn.

stellatum, Linn.

\section{Butomus}

umbellatus, Linn.

\section{Calamintha}

alpina, Lam.

Clinopodium, Benth.

grandiflora, Moench

patavina, Host

\section{Calceolaria}

chelidonioides, $H$. $B$. et $K$. plantaginea, $\mathrm{Sm}$.

\section{Calendula}

arvensis, Linn.

officinalis, Linn.

\section{Callirhoe}

digitata, Nutt.

\section{Callistephus}

hortensis, Cass.

\section{Calochortus}

venustus, Dougl.

\section{Camassia}

Cusickii, S. Wats. esculenta, Lindl.

- var. alba.

\section{Campanula}

alliariæfolia, Willd.

alpina, Jacq .

barbata, Linn.

cæspitosa, Scop.

carnica, Schiede

carpatica, Jacq., var. turbinata.

glomerata, Linn.

Grossekii, Heuffl.

lactiflora, Bieb.

- var. alba.

- var. macrantha.

- var. Van Houttei.

latifolia, Linn.

libanotica, A. DC.

linifolia, Scop.

michauxioides, Boiss.

mirabilis, Alboff

persicifolia, Linn.

- var. alba.

- var. Moerheimi.

planiflora, Lam., var. alba.

pulla, Linn.

punctata, Lam., var, alba.

pusilla, Hrenke

- var. alba.

pyramidalis, Linn., var. alba.

rapunculoides, Linn.

rhomboidalis, Linn.

rotundifolia, Linn.

sibirica, Linn., var. divergens.

thyrsoides, Linn.

Trachelium, Linn.

versicolor, Sibth. et $\mathrm{Sm}$.

Vidalii, H. C Wats.

Waldsteinian Roem. et Schult

\section{Caragana}

arborescens, Lam.

\section{Carbenia}

benedicta, Adans. 


\section{Cardamine}

Impatiens, Linn.

latifolia, $\mathrm{Vahl}$

\section{Carduus}

acanthoides, Linn.

defloratus, Linn.

nutans, Linn.

\section{Carex}

aquatilis, Wahlenb.

arenaria, Linn.

atrata, Linn.

binervis, $S m$.

capillaris, Linn.

curta, Good,, var.

robustior, Blytt

depauperata, Good.

dioica, Linn.

distans, Linn.

disticha, Huds.

divulsa, Good.

echinata, Murr.

extensa, Good., var. pumila.

flava, Linn.

- var. Oederi.

- var. viridula.

frigida, All.

glauca, $S c o p$.

Grahami, Boott

Grayii, Carey

hirta, Linn.

hordeistichos, Vill.

Hornschuchiana, Hoppe

incurva, Lightf.

irrigua, $S m$.

lævigata, $\mathrm{Sm}$.

laxiflora, Lam.

Leersii, F. Schult.

leporina, Linn.

Mairii, Coss, et Germ.

microglochin, Wahlenb.
Carex-continued.

muricata, Linn.

ornithopoda, Willd.

pallescens, Linn.

paludosa, Good.

panicea, Linn.

paniculata, Linn.

Pseudo-cyperus, Linn.

pulicaris, Linn.

punctata, Gaud.

rariflora, $\mathrm{Sm}$.

remota, Linn.

rigida, Good.

riparia, Curt.

saxatilis, Linn.

sparganioides, Muhl.

stricta, Good.

strigosa, Huds.

sylvatica, Huds.

teretiuscula, Good.

tomentosa, Linn.

vaginata, Tausch

Vahlii, Schkuhr

Vilmorini, Mottet

vulgaris, Fries

\section{Carmichaelia}

flagelliformis, Colenso

\section{Carpoceras}

cilicicum, Boiss, et Kurz

\section{Carrichtera}

Vella, $D C$.

\section{Carthamus}

tinctorius, Linn.

\section{Carum}

Petroselinum, Benth, et Hook.J.

\section{Cassandra}

calyculata, $D$. Don 


\section{Catananche}

bicolor, Hort.

cærulea, Linn.

\section{Cathcartia}

villosa, Hook. f.

\section{Cedronella}

triphylla, Moench

\section{Celastrus}

scandens, Linn.

\section{Celmisia}

spectabilis, Hook. f.

\section{Celsia}

cretica, Linn. f.

pontica, Boiss.

\section{Centaurea}

alpina, Linn.

Clementei, Boiss.

Cyanus, Linn.

- var. minor.

macrocephala, Puschk.

nigra, Linn.

pectinata, Linn.

pseudo-collina, Schur

Rhaponticum, Linn.

ruthenica, Lam.

rutifolia, Sibth, et $S m$.

Scabiosa, Linn.

sphærocephala, Linn.

stereophylla, Bess.

uniflora, Linn.

\section{Centranthus}

macrosiphon, Boiss. ruber, $D C$.

\section{Cephalaria}

alpina, Schrad.

radiata, Griseb. et Schenk

tatarica, Schrad.

- var. minor.

\section{Cerastium}

alpinum, Linn.

arvense, Linn., var.grandiflorum.

Boissieri, Gren.

ovatum, Hoppe

tomentosum, Linn.

\section{Chærophyllum}

aureum, Linn.

\section{Chamælirium}

carolinianum, Willd.

\section{Charieis}

heterophylla, Cass.

\section{Cheiranthus}

Cheiri, Linn.

mutabilis, L'Hérit.

Senoneri, Heldr. et Sart.

\section{Chelidonium}

Franchetianum, Prain

majus, Linn.

- var. laciniatum.

\section{Chelone}

Lyoni, Pursh

\section{Chenopodium}

Bonus-Henricus, Linn. capitatum, Aschers.

\section{Chlorogalum}

pomeridianum, Kunth 


\section{Chrysanthemum}

arcticum, Linn.

Balsamita, Linn.

caucasicum, Pers.

coccineum, Willd.

coronopifolium, Vill., var. hybridum.

Leucanthemum, Linn.

maximum, Ramond

montanum, Linn.

Parthenium, Bernh.

segetum, Linn.

\section{Chrysopsis}

villosa, $D C$., var. Rutteri.

\section{Cichorium}

Intybus, Linn.

\section{Cimicifuga}

elata, Nutt.

racemosa, Nutt.

\section{Cistus}

hirsutus, Lam.

laurifolius, Linn.

purpureus, Linn.

villosus, Linn., var. undulata.

\section{Claytonia}

virginica, Linn.

\section{Clematis}

alpina, Mill., var. alba.

erecta, Linn.

heracleæfolia, $D C$.

montana, Buch.-Ham.

orientalis, Linn.

paniculata, Thunb.

songorica, Bunge

\section{Cneorum}

tricoccum, Linn.

\section{Cnicus}

centauroides, Linn.

conspicuus, Hemsl.

Diacantha, Desf

eriophorus, Roth

Falconeri, Hook.f.

græcus, Hort.

heterophyllus, Roth

- var. albus.

pannonicus, Host, var. divaricatus.

pratensis, Willd.

tartaricus, Willd.

tauricus, Hort.

\section{Cochlearia}

glastifolia, Linn.

saxatilis, Linn.

\section{Codonopsis}

ovata, Benth.

rotundifolia, Royle

\section{Collinsia}

bicolor, Benth.

\section{Collomia}

coccinea, Lehm.

grandiflora, Dougl.

\section{Colutea}

arborescens, Linn.

cruenta, Ait.

istria, Mill.

longialata, Koehne.

media, Willd.

\section{Conium}

maculatum, Linn.

\section{Conringia}

orientalis, Dum. 


\section{Convallaria}

majalis, Linn.

\section{Convolvulus}

tenuissimus, Sibth. et Sm.

\section{Coptis}

trifolia, Salisb.

\section{Coreopsis}

coronata, Hook.

\section{Coriaria}

myrtifolia, Linn.

\section{Cornucopiæ}

cucullatum, Linn.

\section{Cornus}

alba, $\operatorname{Linn}$.

\section{Coronilla}

montana, Scop. varia, Linn.

\section{Cortusa}

Matthioli, Linn.

pubens, Schott, Nyman et Kotschy

villosa, Hort.

\section{Corydalis}

glauca, Pursh

lutea, $D C$.

racemosa, Pers.

\section{Cotoneaster}

acuminata, Lindl.

affinis, Lindl.

bacillaris, Wall.

buxifolia, Wall.

frigida, Wall.
Cotoneaster-continued.

horizontalis, Decne.

microphylla, Wall.

Nummularia, Fisch. et Mey.

rotundifolia, Wall.

Simonsii, Baker

thymifolia, Baker

\section{Cotyledon}

Aizoon, Benth. et Hook. f.

chrysanthus, Benth. et Hook. f.

\section{Crambe}

cordifolia, Stev.

\section{Cratægus}

Azarolus, Linn.

Carrierei, Vauvel

coccinea, Linn.

Douglasii, Lindl.

intricata, Lange

Oxyacantha, Linn.

- sub-sp. monogyna, Jacq.

- var. pinnatiloba.

- sub-sp. oxyacanthoides, Thuill.

Pyracantha, Pers.

tanacetifolia, Pers,

\section{Crepis}

paludosa, Moench

robertioides, Boiss.

sibirica, Linn.

\section{Crocus}

speciosus, Bieb.

\section{Crucianella}

angustifolia, Linn.

\section{Cupressus}

Lawsoniana, A. Murr.

macrocarpa, Hartw. 


\section{Cyananthus}

lobatus, Wall.

\section{Cyclamen}

hederæfolium, Ait., var. album.

\section{Cynara}

Cardunculus, Linn.

\section{Cynoglossum}

microglochin, Benth.

officinale, Linn.

Wallichii, G. Don

\section{Cytisus}

albus, Linn.

Ardoini, Fourn.

austriacus, Linn.

biflorus, L'Hérit.

canescens, $J$. et $C$. Presl

hirsutus, Linn.

leucanthus, Waldst, et Kit.

purgans, Boiss.

purpureus, Scop.

scoparius, Link

- var. Andreanus.

\section{Daboecia}

polifolia, D. Don

\section{Dahlia}

Merckii, Lehm.

\section{Daphne}

alpina, Linn.

Mezereum, Linn.

- var. flore albo.

\section{Datura}

Stramonium, Linn.

\section{Daucus}

Carota, Linn.

\section{Delphinium}

Ajacis, Reichb.

azureum, Michx.

cashmirianum, Royle

caucasicum, C. A. Mey.

dictyocarpum, $D \dot{C}$.

flexuosum, Rafin.

formosum, Boiss. et Huet

grandiflorum, Linn., var. album.

hybridum, Steph.

Maackianum, Regel

nudicaule, Torr. et Gray

pictum, Willd.

speciosum, Bieb., var. turkes-

tanicum.

tatsienense, Franch.

truncatum, Hort.

\section{Deschampsia}

cæspitosa, Beauv.

\section{Dianthus}

alpinus, Linn.

Armeria, Linn.

brevicaulis, Fenzl

cæsius, $\mathrm{Sm}$.

calocephalus, Boiss.

carthusianorum, Linn.

ciliatus, Guss.

cruentus, Griseb.

deltoides, Linn.

- var. albus.

fragrans, Bieb.

Freynii, Vandas

monspessulanus, Linn.

neglectus, Loisel.

Noëanus, Boiss.

pallens, Sibth. et Sm.

petræus, Waldst. et Kit.

- var. spiculifolius.

pubescens, Sibth, et Sm.

Requienii, Gren, et Godr. 
Dianthus-continued.

squarrosus, Bieb.

Sternbergii, Sibth.

strictus, Sibth. et $S m$.

subacaulis, Vill.

superbus, Linn.

trifasciculatus, Kit.

\section{Dictamnus}

albus, Linn., var. purpureus.

\section{Dierama}

ensifolium, C. Koch et Bouche pulcherrimum. Baker

\section{Diervilla}

florida, Sieb, et Zucc.

- var. alba.

japonica, $D C$.

Lonicera, Mill.

sessilifolia, Buckl.

\section{Digitalis}

ambigua, Murr.

lutea, Linn.

purpurea, Linn.

\section{Dimorphotheca}

aurantiaca, $D C$.

pluvialis, Moench

\section{Dipcadi}

serotinum, Medic.

\section{Diphylleia}

cymosa, Michx.

\section{Dipsacus}

sylvestris, Mill.

\section{Dodecatheon}

Meadia, Linn.

- var. album.

\section{Dorycnium}

suffruticosum, Vill.

\section{Draba}

aizoides, Linn.

Aizoon, Wahlenb.

arabisans, Michx.

Athoa, Boiss.

aurea, Vahl

bryoides, $D C$.

cornuta, Hort.

cuspidata, Bieb.

fladnizensis, Wulf. grandiflora, Franch.

hirta, Linn.

incana, Linn.

Kotschyi, Stur

Mawii, Hook. f. natolica, Boiss.

rupestris, $R$. $B r$.

siliquosa, Bieb.

streptocarpa, A. Gray

tomentosa, Wahlenb.

- var. frigida.

\section{Dracocephalum}

Moldavica, Linn.

Ruyschiana, Linn.

- var. japonicum.

speciosum, Benth.

\section{Drosera}

intermedia, Hayne longifolia, Hayne rotundifolia, Linn.

\section{Dryas}

octopetala, Linn. 


\section{Eccremocarpus}

scaber, Ruis et Pav.

\section{Echinodorus}

ranunculoides, Engelm.

\section{Echinops}

bannaticus, Rochel niveus, Wall.

Ritro, Linn.

\section{Echinospermum}

heteracanthunı, Ledeb.

Lappula, Lehm.

\section{Echium}

bifrons, $D C$.

plantagineum, Linn.

rosulatum, Lange

vulgare, Linn.

\section{Eleocharis}

palustris, $R$. $B r$.

\section{Elsholtzia}

cristata, Willd.

\section{Elymus}

condensatus, $J$. et $C$. Presl giganteus, $V a h l$

sibiricus, Linn.

\section{Emex}

spinosa, Campd.

\section{Emilia}

flammea, Cass.

\section{Encelia}

calva, A. Gray

\section{Enkianthus}

himalaicus, Hook. f. et Thoms.

\section{Epilobium}

alpinum, Linn.

alsinifolium, Vill.

angustifolium, Linn.

- var. album.

Billardierianum, Ser.

Dodonæi, Vill.

glabellum, Forst.

haloragifolium, A. Cunn.

Hectori, Hausskn.

Lamyi, Schultz

montanum, Linn.

nummularifolium, R. Cunn.

pedicellare, Presl

rosmarinifolium, Haenke

- var. sericeum.

\section{Eremurus}

Bungei, Baker

himalaicus, Baker

robustus, Regel

\section{Erica}

ciliaris, Linn.

cinerea, Linn.

- var. alba.

- var. coccinea.

- var. pallida.

stricta, Donn

Tetralix, Linn.

Watsoni, $D C$.

\section{Erigeron}

multiradiatus, Benth, et Hook. $f$. salsuginosus, A. Gray

Villarsii, Bell.

\section{Erinus}

alpinus, Linn.

- var. albus.

- var. carmineus.

\section{Eriogonum}

racemosum, Nutt. 


\section{Erodium}

chelidonifolium, Hort.

gruinum, Soland. guttatum, Willd.

Manescavi, Coss. moschatum, L'Hérit.

\section{Erophila}

vulgaris, $D C$.

\section{Eryngium}

agavifolium, Griseb.

alpinum, Linn.

amethystinum, Linn.

Bourgati, Gouan

giganteum, Bieb.

Oliverianum, Delar.

Sanguisorba, Cham, et Schlecht.

\section{Erysimum}

asperum, $D C$.

ochroleucum, $D C$, var.

helveticum.

Perofskianum, Fisch. et Mey.

\section{Erythræa}

elodes, Roem. et Schult.

linarifolia, Pers.

Massoni, Szweet

\section{Erythronium}

grandiflorum, Pursh, var. roseum.

\section{Escallonia}

exoniensis, Hort.

langleyensis, Hort.

macrantha, Hook, et Arn.

Philippiana, Mast.

pterocladon, Hook.

punctata, $D C$.

rubra, Pers.

\section{Eschscholzia}

californica, Cham.

\section{Eupatorium}

cannabinum, Linn.

Kirilowii, Turcz.

purpureum, Linn.

\section{Euphorbia}

Myrsinites, Linn.

trapezoidalis, Boiss.

\section{Evax}

asterisciflora, Pers.

\section{Fabiana}

imbricata, Ruiz et Pav.

\section{Fagopyrum}

esculentum, Moench

\section{Farsetia}

clypeata, R. Br.

\section{Felicia}

fragilis, Cass.

\section{Ferula}

communis, Linn.

\section{Festuca}

bromoides, Linn.

capillifolia,Dufour

duriuscula, Linn.

elatior, Linn.

Halleri, All.

heterophylla, Lam.

ovina, Linn.

- var. tenuifolia.

rigida, Kunth

rubra, Linn. 


\section{Filago}

germanica, Linn.

\section{Fœniculum}

giganteum, Hort.

\section{Fragaria}

indica, Andr.

vesca, Linn., var. muricata.

\section{Fraxinus}

excelsior, Linn.

\section{Fritillaria}

lutea, Mill.

messanensis, Rafin.

\section{Fuchsia}

Riccartoni, Hort.

\section{Fumaria}

officinalis, Linn., var. muralis.

\section{Funkia}

lancifolia, Spreng.

Sieboldiana, Hook.

\section{Galactites}

tomentosa, Moench

\section{Galanthus}

Elwesii, Hook. $f$.

\section{Galax}

aphylla, Linn.

\section{Galega}

officinalis, Linn.

- var. carnea plena. orientalis, Lam.

\section{Galinsoga}

parviflora, Cav.
Galium

boreale, Linn.

lithospermifolium, Fisch.

Mollugo, Linn.

saccharatum, All.

saxatile, Linn.

\section{Galtonia}

candicans, Decne.

\section{Gaudinia}

fragilis, Beauv.

\section{Gaultheria}

nummularioides, $D$. Don

procumbens, Linn.

Shallon, Pursh

trichophylla, Royle

\section{Gaura}

Lindheimeri, Engelm. et Gray

\section{Genista}

anglica, Linn.

cinerea, $D C$.

hispanica, Linn.

pilosa, Linn.

radiata, $S c o p$.

sagittalis, Linn.

tinctoria, Linn.

- var. elatior.

\section{Gentiana}

acaulis, Linn.

asclepiadea, Linn.

- var. alba.

brevidens, Franch. et Sav.

Cruciata, Linn.

decumbens, Linn.

linearis, Froel.

macrophylla, Pall.

pannonica, Scop. 
Gentiana-contimued.

phlogifolia, Schott et Kotschy

punctata, Linn.

Saponaria, Linn.

scabra, Bunge

septemfida, Pall.

straminea, Maxim.

tibetica, King

verna, Linn.

\section{Geranium}

anemonæfolium, L'Hérit.

cinereum, Cav.

Endressi, J. Gay

Fremontii, Torr.

incisum, Nutt.

macrorrhizum, Linn.

phæum, Linn.

polyanthes, Edgew. et Hook.f.

pratense, Linn.

pyrenaicum, Linn.

- var. album.

Richardsonii, Fisch. et Trautv.

- var. album.

Robertianum, Linn.

- var. album.

sanguineum, Linn.

- var. lancastriense.

striatum, Linn.

subcaulescens, L'Hérit.

sylvaticum, Linn.

Wilfordi, Maxim.

\section{Gerbera}

Kunzeana, $A . B r$. et Aschers. nivea, Sch. Bip.

\section{Geum}

Borissi, Hort.

chiloense, Balb.

elatum, Wall.
Geum-continuea.

Eweni, Hort.

Heldreichii, Hort.

hispidum, Fries

intermedium, Ehrh.

Laxmanni, Gaertn.

montanum, Linn.

pyrenaicum, Mill.

rhæticum, Brïgg.

rivale, $\operatorname{Linn}$.

urbanum, Linn.

\section{Gilia}

androsacea, Steud.

dichotoma, Benth.

tricolor, Benth.

\section{Gillenia}

stipulacea, Nutt.

\section{Gladiolus}

atroviolaceus, Boiss.

segetum, Ker-Gawl.

\section{Glaucium}

flavum, Crantz

leptopodum, Maxim.

\section{Goodyera}

repens, $R . B r$.

\section{Graphephorum}

arundinaceum, Aschers.

\section{Grindelia}

squarrosa, Dun.

\section{Gunnera}

chilensis, Lam. 


\section{Gypsophila}

dubia, Willd.

paniculata, Linn.

prostrata, Linn.

repens, Linn.

- var. monstrosa.

\section{Haberlea}

rhodopensis, Frivald.

\section{Hacquetia}

Epipactis, $D C$.

\section{Haplocarpha}

Leichtlinii, N.E. Br.

\section{Hebenstretia}

comosa, Hochst.

\section{Hedysarum}

coronarium, Linn.

sibiricum, Poir.

\section{Helenium}

autumnale, $\operatorname{Lin} n$.

\section{Helianthemum}

canum, Boiss.

Fumana, Mill.

polifolium, $D C$.

Tuberaria, Mill.

umbellatum, Mill.

vulgare, Gaertn.

\section{Helianthus}

annuus, $\operatorname{Linn}$

pumilus, Nutt.

\section{Heliophila}

amplexicaulis, Linn.

laevis, Pers.

\section{Heliopsis}

padula, Wender.

scabra, Dun., var. Pitcheriana

\section{Helipterum}

Humboldtianum, $D C$.

Manglesii, F. Muell., var. maculatum.

\section{Helleborus}

punctatus, Hort.

\section{Helonias}

bullata, Linn.

\section{Hemerocallis}

Dumortierii, Morr.

minor, Mill.

\section{Heracleum}

asperum, Bieb.

Wallichii, $D C$.

\section{Hesperis}

matronalis, Linn.

tristis, Linn.

\section{Heuchera}

cylindrica, Dougl.

sanguinea, Engelm.

\section{Hibiscus}

Trionum, Linn.

\section{Hieracium}

alpinum, $\operatorname{Linn}$.

Auricula, Linn.

balkanum, Uechtritz

Bornmülleri, Freyn

bupleuroides, C. C. Gmel.

cerinthoides, Linn.

foliosum, Waldst. et Kit. 
List of Seeds Collected DURING I905. xcix

Hieracium-continued.

gymnocephalum, Griseb.

humile, Jacq .

incisum, Hoppe

iricum, Fries

Jankæ, Uechtritz

norvegicum, Fries

pannosum, Boiss.

Pilosella, Linn.

- var. aurantiacum.

prenanthoides, Vill.

pulmonarioides, Vill.

rupestre, All.

saxatile, Vill.

scoticum, F.J. Hanb.

tridentatum, Fries

umbellatum, Linn.

undulatum, Ait.

villosum, Jacq.

vulgatum, Fries

\section{Hierochloe}

borealis, Roem. et Schult.

\section{Hippocrepis}

unisiliquosa, Linn.

\section{Hippophaë}

rhamnoides, Linn.

\section{Homeria}

collina, Vent.

\section{Hordeum}

bulbosum, Linn.

jubatum, Linn.

\section{Horminum}

pyrenaicum, Linn.

- var. album.

\section{Hunnemannia}

fumariæfolia, Sreet

\section{Hutchinsia}

petræa, Ait.

\section{Hyacinthus}

amethystinus, Linn.

dubius, Guss.

pumilus, Hort.

romanus, Linn.

\section{Hydrangea}

vestita, Wall.

\section{Hymenanthera}

crassifolia, Hook.f.

\section{Hyoscyamus}

niger, Linn.

\section{Hypericum}

Androsæmum, Linn.

calycinum, Linn.

delphicum, Boiss, et Heldr.

Hookerianum, Wight et Arn.

inodorum, Mill.

Kotschyanum, Boiss.

perforatum, Linn.

polyphyllum, Boiss.

pulchrum, Linn.

Richeri, Vaill.

tetrapterum, Fries

\section{Hypochceris}

maculata, Linn.

radicata, Linn.

uniflora, Vill.

\section{Hyssopus}

officinalis, Linn. 


\section{Iberis}

carnosa, Willd.

corifolia, Stueet

Jordani, Boiss.

Lagascana, $D C$.

semperflorens, Linn.

sempervirens, Linn.

- var. superba.

\section{Ilex}

Aquifolium, Linn.

\section{Incarvillea}

Delavayi, Bureau et Franch.

\section{Inula}

ensifolia, Linn.

grandiflora, Willd.

Helenium, Linn.

Royleana, $D C$.

\section{Ionopsidium}

acaule, Reichb.

\section{Iris}

aurea, Lindl.

Guldenstaedtiana, Lepech.

humilis, Bieb.

lævigata, Fisch.

Milesii, Foster

mongolica, Hort.

nepalensis, $D$. Don

prismatica, Pursh

Pseudacorus, Linn., var. acoroides.

sibirica, Linn.

- var. acuta.

- var. orientalis.

sordida, Willd

Straussii, Hort.

Watsoni, Hort.

\section{Isatis}

glauca, Auch.

tinctoria, Linn.

undulata, Auch.

\section{Jasione}

montana, Linn.

perennis, Lam.

\section{Juncus}

effusus, Linn.

- var. conglomeratus.

- var. spiralis.

filiformis, Linn.

glaucus, Sibth.

trifidus, Linn.

\section{Jurinea}

glycacantha, $D C$.

\section{Kalmia}

angustifolia, Linn.

\section{Kniphofia}

pauciflora, Baker

Tuckii, Baker

\section{Kochia}

scoparia, Schrad.

\section{Krynitzkia}

barbigera, A. Gray

\section{Laburnum}

alpinum, J. S. Presl

vulgare, J. S. Presl

... var. austriacum.

- var. roseum. 
Lactuca

Plumieri, Gren. et Godr. virosa, Linn.

\section{Lagurus}

ovatus, Linn.

\section{Lamarkia}

aurea, Moench

\section{Lamium}

album, Linn.

maculatum, Linn.

Orvala, Linn.

\section{Larix}

europæa, $D C$.

\section{Lasthenia}

glabrata, Lindl.

\section{Lathyrus}

articulatus, Linn.

grandiflorus, Sibth, et Sm.

latifolius, Linn.

luteus, Baker

maritimus, Bigel.

montanus, Bernh.

pubescens, Hook. è Arn.

rotundifolius, Willd.

sativus, Linn.

sylvestris, Linn.

undulatus, Boiss.

venosus, $M u h l$.

vernus, Bernh., var. flaccidus.

\section{Lavandula}

multifida, Linn.

Spica, Cav.

Stœehas, Linn.

vera, $D C$.

\section{Layia}

elegans, Torr. et Gray

\section{Ledum}

latifolium, Ait.

\section{Leiophyllum}

buxifolium, Ell.

\section{Leontodon}

hispidus, Linn.

\section{Leontopodium}

alpinum, Cass.

- var. altaicum.

- var. sibiricum.

japonicum, Miq.

\section{Leonurus}

Cardiaca, Linn.

- var. villosus.

sibiricus, Linn.

\section{Lepachys}

columnaris, Torr. et Gray

\section{Leptosyne}

maritima, A. Gray

\section{Leucojum}

æstivum, Linn.

\section{Leycesteria}

formosa, Wall.

\section{Liatris}

spicata, Willd.

\section{Libertia}

ormosa, Grah

xioides, Sprens 


\section{Ligustrum}

acuminatum, Hort.

macrocarpum, Hort.

medium, Franch. et Sav.

vulgare, Linn.

- var, italicum.

\section{Lilium}

dauricum, Ker-Gawl.

Grayi, S. Wats.

Martagon, Linn.

- var. album.

pyrenaicum, Gouan

umbellatum, Hort., var. maculatum.

\section{Limnanthes}

Douglasii, R. $\mathrm{Br}$.

\section{Linaria}

alpina, Mill.

bipartita, Willd., var. splendida. maroccana, Hook. f., var. alba. origanifolia, $D C$.

Pančici, Hort.

purpurea, Mill.

repens, Mill.

triornithophora, Willd.

tristis, Mill.

\section{Lindelofia}

spectabilis, Lehm.

\section{Linum}

austriacum, Linn.

grandiflorum, Desf.

perenne, Linn.

- var. album.

\section{Lithospermum}

prostratum, Loisel.

\section{Loasa}

lateritia, Gill.

\section{Lobelia}

syphilitica, Linn.

\section{Lonas}

inodora, Gaertn.

\section{Lonicera}

alpigena, Linn.

angustifolia, Wall.

bella, Zabel, var. albida.

- var, atro-rosea.

- var. rosea.

Morrowi, A. Gray

mündeniensis, Rehder

muscaviensis, Rehder

orientalis, Lam.

Periclymenum, Linn.

pyrenaica, Linn.

tatarica, Linn.

- var. alba grandiflora.

- var. discolor.

- var. flore roseo.

\section{Lotus}

corniculatus, Linn.

ornithopodioides, Linn.

\section{Lunaria}

annua, Linn.

- var. alba.

\section{Lupinus}

arboreus, Linn.

- var. violaceus.

nootkatensis, Donn

parviflorus, Nutt.

polyphyllus, Lindl.

- var. albus.

\section{Luzula}

albida, $D C$.

campestris, $D C$.

nivea, $D C$.

sylvatica, $D C$. 


\section{Lychnis}

alba, Mill.

alpina, Linn.

chalcedonica, Linn.

coronaria, Desr.

- var. alba.

dioica, Linn.

Flos-cuculi, Linn.

Flos-Jovis, Desr.

Lagascæ, Hook. f.

macrocarpa, Boiss, et Reut.

nigrescens, Edgew.

pyrenaica, Berger

Viscaria, Linn.

- var. splendens.

\section{Lythrum}

Salicaria, Linn.

- var. cinereum.

- var. grandiflorum.

- var, hirsutum.

virgatum, Linn.

\section{Maianthemum}

Convallaria, Weber

\section{Malcomia}

maritima, R. Br.

\section{Malope}

trifida, Cav.

\section{Malva}

Alcea, Linn.

sylvestris, Linn.

\section{Margyricarpus}

setosus, Ruiz et Pav.

\section{Marrubium}

vulgare, Linn.

\section{Matricaria}

inodora, Linn.

- var. plenissima.

oreades, Boiss.

\section{Matthiola}

bicornis, $D C$.

sinuata, $R$. $B r$, var. glabra albiflora.

\section{Meconopsis}

aculeata, Royle

cambrica, Vig.

grandis, Prain

integrifolia, Franch.

Wallichii, Hook.

- var. fusco-purpurea.

\section{Medicago}

carstiensis, Wulf.

Echinus, $D C$.

falcata, Linn.

hispida, Gaertn.

sativa, Linn.

\section{Melica}

altissima, $\operatorname{Linn}$.

ciliata, Linn.

nutars, Linn.

uniflora, Retz.

\section{Melilotus}

alba, Desr.

officinalis, Lam.

\section{Melittis}

Melissophyllum, Lim:

\section{Mentzelia}

Lindleyi, Torr. et Gray

\section{Menziesia}

globularis, Salisb. 
Mesembryanthemum

crystallinum, Linn.

\section{Meum}

athamanticum, Jacq.

\section{Microglossa}

albescens, $C$. B. Clarke

\section{Milium}

effusum, Linn.

\section{Mimulus}

cardinalis, Dougl.

luteus, Linn.

\section{Mirabilis}

Jalapa, Linn.

\section{Mitella}

diphylla, Linn.

pentandra, Hook.

\section{Molopospermum}

cicutarium, $D C$.

\section{Montia}

fontana, $\operatorname{Linn}$.

\section{Moricandia}

arvensis, $D C$,

\section{Morina}

longifolia, Wall.

\section{Muscari}

armeniacum, Baker

botryoides, Mill.

comosum, Mill.

elegans, Hort.

latifolium, J. Kirk

Massyanum, Hort.

Maweanum, Baker

racemosum, Mill.

\section{Myosotis}

alpestris, F. W. Schmidt

antarctica, Hook. $f$.

arvensis, Lam.

cæspitosa, Schultr

dissitiflora, Baker

stricta, Link

sylvatica, Hoffm.

\section{Myricaria}

germanica, Desv.

\section{Myrrhis}

odorata, Scop.

\section{Narcissus}

Bulbocodium, Linn., var. citrinus.

\section{Nardus}

stricta, Linn.

\section{Nasturtium}

officinale, R. Br.

\section{Neillia}

amurensis, Benth. et Hook. f. opulifolia, Benth, et Hook. f.

thyrsiflora, D. Don

\section{Nemesia}

strumosa, Benth.

\section{Nemophila}

insignis, Benth.

Menziesii, Hook. et Arn.

\section{Nepeta}

Cataria, Linn.

concolor, Boiss. et Heldr.

distans, Royle

kokamirica, Regel

linearis, Royle 
Nepeta-continued.

longiflora, Vent.

Mussini, Spreng.

nuda, Linn.

Sibthorpii, Benth.

Neslia

paniculata, Desv.

\section{Nicandra}

physaloides, Gaertn.

\section{Nicotiana}

rustica, Linn.

\section{Nigella}

hispanica, Linn., var. atropurpurea.

\section{Noccæa}

alpina, Reichb.

- var. Auerswaldii.

stylosa, Reichb.

\section{Nolana}

atriplicifolia, D. Don

\section{Nothoscordum}

striatum, Kunth

\section{Enanthe}

crocata, Linn.

peucedanifolia, Pollich

pimpinelloides, Linn.

\section{Enothera}

biennis, Linn.

fruticosa, Linn., var. Youngei. pumila, Linn.

\section{Olearia}

Haastii, Hook. $f$.

ilicifolia, Hook. $f$. macrodonta, Baker stellulata, $D C$.

\section{Omphalodes}

linifolia, Moench

\section{Onobrychis}

viciæfolia, Scop.

\section{Ononis}

arvensis, Linn.

rotundifolia, Linn.

\section{Onopordon}

Acanthium, Linn.

arabicum, Linn.

\section{Onosma}

albo-roseum, Fisch. et Miy.

bracteatum, Wall.

\section{Ophiopogon}

Jaburan, Lodd.

\section{Orchis}

foliosa, Soland.

maculata, Linn.

\section{Origanum}

vulgare, Linn.

- var. album

\section{Osteospermum}

moniliferum, Linn.

\section{Oxalis}

Acetosella, Linn.

corniculata, Linn.

- var. tropæoloides.

Deppei, Lodd.

\section{Oxybaphus}

nyctagineus, Sweet

\section{Uxyria}

digyna, Hill

- var, pluridens. 


\section{Oxytropis}

baicalensis, Pall.

campestris, $D C$.

lapponica, Gaud.

sordida, Pers.

strobilacea, Bunge

sulphurea, Fisch.

\section{Palaua}

dissecta, Benth.

\section{Papaver}

alpinum, Linn.

Heldreichii, Boiss.

lateritium, $C$. Koch

libanoticum, Boiss.

monanthum, Trautv.

nudicaule, Linn.

orientale, Linn.,var. bracteatum.

pilosum, Sibth. et $S m$.

rupifragum, Boiss. et Reut.

somniferum, Linn.

\section{Paradisea}

Liliastrum, Bertol.

\section{Parietaria}

officinalis, Linn.

\section{Parnassia}

caroliniana, Michx.

palustris, Linn.

\section{Parochetus}

communis, Buch.-Ham.

\section{Parrya}

Menziesii, Hort.

\section{Pentstemon}

barbatus, Roth

confertus, Dougl.

- var. cœruleo-purpureus.
Pentstemon-continued.

deustus, Dougl.

glaber, Pursh, var. cyananthus.

glaucus, R. Grah.

gracilis, Nutt.

humilis, Nutt.

Menziesii, Hook., var. New-

berryi.

Richardsonii, Dougl.

secundiflorus, Benth.

virgatus, $A$. Gray

Wrightii, Hook.

\section{Perezia}

multiflora, Less.

\section{Pernettya}

mucronata, Gaudich.

\section{Peucedanum}

graveolens, Benth. et Hook. f.

officinale, Linn.

sativum, Benth. et Hook. f.

verticillare, Spreng.

\section{Phacelia}

tanacetifolia, Benth.

viscida, Torr.

Whitlavia, A. Gray

\section{Phaseolus}

multiflorus, Willd.

\section{Philadelphus}

coronarius, Linn.

- var. Satsumi, Maxim.

- var. tomentosus, Hook. $f$. et Thoms.

Gordonianus, Lindl.

grandiflorus, Willd.

Lemoinei, Hort.

- var. erectus. 


\section{Phlomis}

agraria, Bunge

cashmiriana, Royle

fruticosa, Linn.

Samia, Linn.

setigera, Falc.

tuberosa, Linn.

\section{Phormium}

tenax, Forst.

\section{Photinia}

variabilis, Hemsl.

\section{Phuopsis}

stylosa, Benth. et Hook. f.

\section{Phygelius}

capensis, E. Mey.

\section{Physalis}

Alkekengi, Linn.

\section{Phyteuma}

canescens, Waldst. et Kit.

lobelioides, Willd.

Michelii, All.

nigrum, $F$. $W$. Schmidt

orbiculare, Linn.

Scheuchzeri, All.

Sibthorpianum, Roem. et Schult.

Sieberi, Spreng.

\section{Picris}

hieracioides, Linn.

\section{Picrorhiza}

Kurroa, Royle

\section{Pimpinella}

magna, Linn.

\section{Pinguicula}

grandiflora, Lam. vulgaris, Linn.
Pinus

Pinaster, Soland.

sylvestris, Linn.

\section{Pisum}

sativum, $\operatorname{Linn}$.

\section{Plantago}

alpina, Cav.

arenaria, Waldst. et Kit.

argentea, Chaix

carinata, Schrad.

Coronopus, Linn.

lanceolata, $\operatorname{Linn.}$

major, Linn., var. monstrosa.

- var. rubra.

maritima, Linn.

montana, Huds.

Raoulii, Decne.

\section{Platycodon}

grandiflorum, A.DC., var.

Mariesii album.

\section{Platystemon}

californicus, Benth.

\section{Plectranthus}

glaucocalyx, Maxim.

Poa

chinensis, Linn

pratensis, Linn.

trivialis, Linn.

\section{Podophyllum}

Emodi, Wall.

\section{Polemonium}

cæruleum, Linn.

flavo-cæruleum, Hort.

gracile, Willd.

himalayanum, Baker

mexicanum, Cerv. 
cviii List of Seeds Collected DURING 1905.

\section{Polycarpæa}

Teneriffæ, Lam.

\section{Polygonatum}

multiflorum, All.

\section{Polygonum}

affine, $D$. Don

amplexicaule, D. Don

baldschuanicum, Regel

Bistorta, Linn., var. major.

capitatum, Buch-Ham.

cilinode, Michx.

Convolvulus, Linn.

sphærostachyum, Meissn.

vaccinifolium, Wall.

viviparum, Linn.

Weyrichii, F. Schmidt

\section{Potentilla}

alpestris, Hall. f.

ambigua, Jacq.

andicola, Benth.

apennina, Tenore

arguta, Pursh

argyrophylla, Wall.

dissecta, Pursh

Friedrichseni, Späth

fruticosa, Linn.

glandulosa, Lindl.

Hippiana, Lehm.

Leschenaultiana, Ser.

mollissima, Lehm.

Mooniana, Wight

nepalensis, Hook.

nevadensis, Boiss.

nitida, Linn., var. alba.

nivea, Linn.

norvegica, Linn.

palustris, Scop.

procumbens, Sibth.

pseudo-obscura, Hort.

recta, Linn., var. laciniata.
Potentilla-continued.

reptans, Linn.

Römeri, Hort.

rupestris, Linn.

sanguisorbifolia, F. O. Wolf

semilaciniata, Hort.

sericea, Linn.

Sibbaldia, Hall. $f$.

tanacetifolia, Willd.

Tormentilla, Neck.

tridentata, Soland.

villosa, Pall.

vladnizensis, Siegfr.

\section{Poterium}

spinosum, Linn.

\section{Pratia}

angulata, Hook. $f$.

begonifolia, Lindl.

\section{Prenanthes}

purpurea, Linn.

\section{Primula}

apennina, Wid.

Auricula, Linn.

- var. marginata.

capitata, Hook. $f$.

denticulata, $S m$., var, cashmiriana.

- var. rosea.

elatior, Hill, var. carpatica.

frondosa, Janka

grandis, Trautv.

involucrata, Wall.

japonica, A. Gray

- var, lilacina.

latifolia, Lapeyr.

luteola, Rupr.

officinalis, Jacq.

Parryi, A. Gray

Reidii, Duthie 
Primula-continuea.

sikkimensis, Hook. f.

Stuartii, Wall., var. purpurea.

venusta $\times$, Host

viscosa, Vill.

- var. ciliata.

- var. nivalis.

\section{Prunella}

grandiflora, Jacq.

laciniata, Linn.

vulgaris, Linn.

\section{Prunus}

Avium, Linn.

Laurocerasus, Linn.

mollis, Walp.

\section{Ptelea}

trifoliata, Linn.

- var. aurea.

\section{Pyrus}

Aria, Linn.

Aucuparia, Gaertn.

germanica, Hook. f.

melanocarpa, $K$. Koch

rotundifolia, Bechst.

\section{Ramondia}

pyrenaica, Rich.

- var. alba.

serbica, Panc., var. Nathaliæ.

\section{Ranunculus}

aconitifolius, Linn.

acris, Linn.

- var. Steveni.

auricomus, Linn.

brutius, Tenore

bulbosus, Linn.

Lingua, Linn.
Ranunculus-continued.

repens, Linn.

sceleratus, Linn.

Thora, Linn.

\section{Raphanus}

sativus, Linn., var. caudatus.

\section{Reseda}

alba, Linn.

complicata, Bory

glauca, Linn.

lutea, Linn.

\section{Rhamnus}

cathartica, Linn.

Frangula, Linn.

\section{Rheum}

hybridum, Murr.

leucorrhizum, Pall.

Rhaponticum, Linn.

Ribes, Linn.

tataricum, Linn. $f$.

\section{Rhododendron}

campanulatum, D. Don

campylocarpum, Hook. $f$.

caucasicum, Pall.

ciliatum, Hook. f.

cinnabarinum, Hook. $f$.

ferrugineum, Linn.

flavum, G. Don

glaucum, Hook. $f$.

hirsutum, Linn.

- var. album.

kamtchaticum, Pall.

lepidotum, Wall.

niveum, Hook.f.

ponticum, Linn.

\section{Rhodotypos}

kerrioides, Sieb. et Zucc. 


\section{Ribes}

sanguineum, Pursh

- var. malvaceum, S. Wats.

\section{Rodgersia}

podophylla, $A$. Gray

\section{Roemeria}

violacea, Medic.

\section{Rosa}

acicularis, Lindl.

alpina, Linn.

blanda, Ait.

brachyphylla, Hort.

hibernica, $\mathrm{Sm}$.

laxa, Retz.

pisocarpa, A. Gray

reclinata, $R e d$.

rubiginosa, Linn.

rugosa, Thunb.

- var. alba.

sericea, Lindl.

spinosissima, Linn.

- var. altaica.

- var. rubra.

\section{Rubus}

cæsius, Linn.

fruticosus, Linn.

laciniatus, Willd.

nitidus, Weihe et Nees

odoratus, Linn.

phcenicolasius, Maxim.

\section{Rudbeckia}

laciniata, Linn.

triloba, Linn.

\section{Rumex}

alpinus, Linn.

crispus, Linn.

flexuosus, Soland.

Hydrolapathum, Huds.
Rumex-continued.

nepalensis, Spreng.

occidentalis, S. Wats.

stenophyllus, Ledeb.

\section{Ruta}

graveolens, Linn.

montana, Mill.

\section{Salsola}

Kali, Linn., var. Tragus.

\section{Salvia}

argentea, Linn.

austriaca, Jacq.

cyanescens, Boiss. et Bal.

glutinosa, Linn.

grandiflora, Etling.

Horminum, Linn.

- var. rubra.

patens, Cav.

pratensis, Linn.

rubrifolia, Boiss.

Sclarea, Linn.

sylvestris, Linn,

- var. alba.

verbascifolia, Bieb.

Verbenaca, Linn.

\section{Sambucus}

canadensis, Linn.

mexicana, Presl

nigra, Linn.

racemosa, Linn.

\section{Samolus}

Valerandi, Linn.

\section{Sanicula}

europæa, Linn.

\section{Sanvitalia}

procumbens, Lam. 


\section{Saponaria}

bellidifolia, $\mathrm{Sm}$.

cæspitosa, $D C$.

calabrica, Guss.

lutea, Linn.

ocymoides, Linn.

peregrina $\times$, Hort.

\section{Saracha}

Jaltomata, Schlecht.

\section{Satureia}

montana, Linn.

\section{Saussurea}

alpina, $D C$.

discolor, $D C$.

\section{Saxifraga}

aizoides, Linn.

Aizoon, Jacq., var. balcana.

- var. carinthiaca (Schott)

- var. Malyi.

- var. minimum.

- var. notata.

- var. pectinata.

- var. rosularis.

- var. Sturmiana.

aspera, $\operatorname{Linn}$.

bronchialis, Linn.

- var. cherlerioides.

Bucklandi, Hort.

cæsia, Linn.

canaliculata, Boiss, et Reut.

cartilaginea, Willd.

cochlearis, Reichb., var. minor.

Cotyledon, Linn., var. pyramidalis.

cuneifolia, Linn.

- var. capillipes.

- var. infundibulum.

- var. multicaulis.

- var. subintegra.
Saxifraga-continued.

decipiens, Ehrh.

- var. grœenlandica.

erosa, Pursh

Gaudini $\times$, Brügg.

geranioides, Linn.

Geum, Linn., var. ovalifolia.

gibraltarica, Boiss, et Reut.

granulata, Linn.

Hirculus, Linn.

Hostii, Tausch

- var. altissima.

- var. elatior.

Huetiana, Boiss.

hypnoides, Linn.

intacta, Willd.

- var. farinosa.

Kingii, Hort.

lingulata, Bell., var. australis.

- var. lantoscana.

longifolia, Lapeyr.

- var. hybrida.

Mertensiana, Bongard

Milesii, Leichtl.

montavoniensis $\times$, Kerner

muscoides, Wulf., var. mos. chata.

- var. atropurpurea.

muscoides, Wulf., var. purpurea.

mutata, Linn.

nervosa, Miégev.

nivalis, Linn.

oppositifolia, Linn.

- var. alba.

pedatifida, Ehrh.

pentadactylis, Lapeyr.

Portæ $\times$, Engl.

punctata, Linn.

retusa, Gouan, var. maritima.

Reyeri $\times$, Hort.

Rocheliana, Sternb., var. coriophylla. 


\section{Saxifraga-continued.}

rotundifolia, Limn., var.

angulosa.

sancta, Griseb.

Schraderi, Sternb.

squarrosa, Sieber

stellaris, Linn.

tenella, Wulf.

umbrosa, Linn., var. hirsuta.

- var. Ogilvieana.

- var. serratifolia.

Zeleborii, Schott

\section{Scabiosa}

caucasica, Bieb., var. alba.

centauroides, Mill.

longifolia, Waldst. et Kit.

lucida, Vill.

ochroleuca, Linn., var.

Webbiana.

palaestina, Linn.

prolifera, Linn.

Pterocephala, Linn.

silenifolia, Waldst. et Kit.

Succisa, Linn.

- var. alba.

sylvatica, Linn.

\section{Schizanthus}

pinnatus, Ruiz et Pav.

\section{Schizopetalon}

Walkeri, Sims

\section{Scilla}

festalis, Salisb.

hispanica, Mill.

peruviana, Linn.

\section{Scirpus}

aucklandicus, Boeck.

Holoschœnus, Linn.

sylvaticus, $\operatorname{Linn}$.

\section{Scleranthus}

annuus, Linn.

Scolymus

grandiflorus, Desf.

\section{Scorpiurus}

vermiculata, $\operatorname{Linn}$.

\section{Scorzonera}

hispanica, Linn.

\section{Scrophularia}

alata, Gilib.

dentata, Royle

Herminii, Hoffmgg. et Link nodosa, Linn.

vernalis, Linn.

\section{Scutellaria}

albida, Linn.

alpina, Linn.

altissima, Linn.

hirta, Sibth. et Sm.

\section{Securigera}

Coronilla, Linn.

\section{Sedum}

Aizoon, Linn,

Alberti, Regel

album, Linn.

altissimum, Poir.

Anacampseros, Linn.

arboreum, Mast.

asiaticum, Spreng.

Beyrichianum, Turcz.

dasyphyllum, Linn.

dentatum, $D C$.

euphorbioides, Schlecht.

Ewersii, Ledeb.

hispanicum, Linn.

hybridum, Linn.

involucratum, Bieb. 
Sedum-continued.

kamtschaticum, Fisch, et Mey. laxiflorum, $D C$.

maximum, Suter

oreganum, $N u t t$.

populifolium, Pall.

reflexum, Linn.

roseum, $S c o p$.

rupestre, Linn.

sexangulare, Linn.

stoloniferum, S. T. Gmel.

Telephium, Linn.

trifidum, Wall.

\section{Sempervivum}

anomalum, Hort.

arachnoideum, Linn.

arenarium, Koch

cinerascens, Hort.

fimbriatum, Schnittsp. et

C. B. Lehm.

globiferum, $\operatorname{Linn}$.

hirtum, Linn.

patens, Griseb. et Schenk

Schnittspahni, Lagg.

soboliferum, Sims

spinulosum, Lagg.

tectorum, Linn.

violaceum, Hort.

\section{Senebiera}

Coronopus, Poir.

pinnatifida, $D C$ :

\section{Senecio}

clivorum, Maxim.

Doronicum, Linn.

laxifolius, J. Buch.

sarracenicus, Linn.

\section{Serratula}

coronata, Linn.

Gmelini, Ledeb.

heterophylla, Desf.
Seseli

Libanotis, Koch

\section{Setaria}

macrostachya, $H$. $B$. et $K$.

\section{Sherardia}

arvensis, Linn.

\section{Sidalcea}

candida, A. Gray

Listeri, Hort.

malvæflora, A. Gray

oregana, A. Gray

spicata, Greene

\section{Sideritis}

scordioides, Linn.

\section{Silene}

acaulis, $\operatorname{Linn}$.

alpestris, Jacq.

Armeria, Linn.

Asterias, Griseb.

auriculata, Sibth, et S $m$.

caucasica, Boiss.

chromodonta, Boiss. et Reut.

colorata, Poir.

compacta, Fisch.

Cucubalus, Wibel

Fabaria, Sibth. et Sm.

Friwaldskyana, Hampe

italica, Pers.

longipetala, Vent.

maritima, With.

multicaulis, Guss.

rhynchocarpa, Boiss.

quadridentata, Pers.

Reichenbachii, Vis.

rupestris, Linn.

Saxifraga, Linn.

Schafta, Gmel.

stylosa, Bunge 
Silene-continued.

Thorei, Duf.

vallesia, Linn.

Zawadzkii, Herbich

\section{Silybum}

Marianum, Gaertn.

\section{Sisymbrium}

Alliaria, $S c o p$.

Assoanum, Loscos et Pardo strictissimum, Linn.

tanacetifolium, Linn.

\section{Sisyrinchium}

angustifolium, Mill.

- var. mucronatum.

- var. Nuttallii.

Bermudiana, Linn.

californicum, Dryand.

grandiflorum, Dougl., var. album.

striatum, $S m$.

\section{Skimmia}

japonica, Thunb.

Laureola, Hook. $f$.

\section{Smyrnium}

rotundifolium, Mill.

\section{Solanum}

Dulcamara, Linn.

\section{Solenomelus}

chilensis, Miers

\section{Solidago}

canadensis, Linn.

elongata, Nutt.

odora, Ait.

Shortii, Torr. et Gray

Virgaurea, Linn.

\section{Sonchus}

palustris, Linn.

\section{Spartina}

polystachya, Willd.

\section{Specularia}

hybrida, $A . D C$.

pentagonia, $A . D C$.

Speculum, A. DC.

\section{Spergula}

arvensis, Linn.

\section{Spinacia}

oleracea, Linn.

\section{Spiræa}

Aruncus, Linn.

astilboides, T. Moore

bullata, Maxim.

camtschatica, Pall.

discolor, Pursh.

Douglasi, Hook.

Humboldtii, Hort.

Jindleyana, Wall.

Nobleana, Hook.

palmata, Thunb.

salicifolia, Linn.

\section{Spraguea}

umbellata, Torr.

\section{Stachys}

alpina, Linn.

- var. intermedia.

Betonica, Benth.

densiflora, Benth.

grandiflora, Benth.

lanata, Jacq .

recta, Linn.

\section{Statice}

altaica, Hort. 
Stellaria

biflora, Linn.

graminea, Linn.

\section{Stenanthium}

angustifolium, Kunth

\section{Stipa}

Aristella, Linn.

capillata, Linn.

papposa, Delile.

\section{Stokesia}

cyanea, L'Hérit.

\section{Succowia}

balearica, Medic.

\section{Symphoricarpus}

racemosus, Michx.

\section{Symphyandra}

Hofmanni, Pant.

\section{Symphytum}

cordatum, Waldst, et Kit. pictum, Hort.

\section{Synthyris}

reniformis, Benth.

\section{Syringa}

Emodi, Wall.

vulgaris, Linn.

- var. alba.

\section{Tagetes}

lucida, Cav.

signata, Bartl.

\section{Tanacetum}

vulgare, Linn.

\section{Tanakæa}

radicans, Franch.

\section{Taxus}

baccata, Linn.

- var. adpressa.

- var. Dovastoni, Carr.

- var. fastigiata, Loud.

- var. pyramidalis.

- var. Washingtoni.

\section{Telephium}

Imperati, Linn.

\section{Tellima}

grandiflora, R. Br.

\section{Teucrium}

flavum, Linn.

lucidum, Linn.

montanum, Linn.

Polium, Linn.

pyrenaicum, Linn.

Scorodonia, Linn.

\section{Tetragonia}

expansa, Murr.

\section{Thalictrum}

calabricum, Spreng.

corynellum, $D C$.

Fendleri, Engelm.

glaucum, Desf.

laserpitifolium, Willd.

majus, Jacq.

minus, Linn., var. elatum.

- var. flexuosum.

simplex, Linn.

tuberosum, Linn.

\section{Thaspium}

aureum, Nutt.

\section{Thermopsis}

fabacea, $D C$.

montana, Nutt. 


\section{Thlaspi}

perfoliatum, Linn.

\section{Thymus}

Serpyllum, Linn.

- var. coccineus.

- var. micans.

vulgaris, Linn.

\section{Tilia}

cordata, Mill.

vulgaris, Hayne

\section{Tofieldia}

carinthiaca, Hort.

palustris, Huds.

\section{Tolpis}

barbata, Gaertn.

\section{Tradescantia}

virginiana, Linn.

-- var. alba.

- var. atropurpurea.

- var. elata.

\section{Trautvetteria}

palmata, Fisch. et Mey.

\section{Tridax}

bicolor, A. Gray

\section{Trifolium}

alpinum, Linn.

ambiguum, Bieb.

fragiferum, Linn.

medium, Linn.

pannonicum, Jacg.

pratense, Linn.

\section{Trigonella}

corniculata, Linn.

Fœnum-græcum, Linn.

\section{Trilisa}

odoratissima, Cass.

\section{Trillium}

stylosum, Nutt.

\section{Triosteum}

perfoliatum, Linn.

\section{Trisetum}

flavescens, Beauv.

\section{Triticum}

Spelta, Linn.

\section{Tritonia}

rosea, Klatt

\section{Trollius}

altaicus, Mey. asiaticus, Lmn.

- var. giganteus.

caucasicus, Stev.

europæus, Linn. patulus, Salisb.

\section{Tropæolum}

aduncum, $\mathrm{Sm}$. majus, Linn.

\section{Tulipa}

Billietiana, Jord.

Didieri, Jord., var. alba.

pulchella, Fenzl

sylvestris, Linn.

\section{Tunica}

prolifera, $S c o p$.

Saxifraga, $S c o p$.

\section{Ulex}

europæus, Linn.

\section{Ulmus}

montana, With. 


\section{Uniola}

latifolia, Michx.

\section{Urospermum}

Dalechampii, F. W. Schmidt picroides, Desf.

\section{Ursinia}

speciosa, $D C$.

\section{Urtica}

cannabina, $\operatorname{Linn}$.

pilulifera, Linn.

\section{Vaccinium}

padifolium, Sm.

\section{Valeriana}

montana, Linn.

\section{Valerianella}

carinata, Loisel.

rimosa, Bast.

\section{Vancouveria}

hexandra, Morr. et Decne.

\section{Venidium}

decurrens, Less.

\section{Verbascum}

Blattaria, Linn.

Chaixi, Vill.

Lagurus, Fisch, et Mey.

longifolium, Tenore, var.

pannosum.

Lychnitis, Linn.

phlomoides, Linn.

phœniceum, Linn.

\section{Veronica}

alpina, Linn.

Anagallis, Linn.

aphylla, Linn.

austriaca, Linn.
Veronica-continued.

austriaca, var. pinnatifida.

- var. Prenja.

Balfouriana, Hook. $f$.

bellidioides, Linn.

Bidwillii, Hook. f.

buxifolia, Benth.

candida, Lodd.

carnosula, Hook. f.

Catarractæ, Forst.

Chamædrys, Linn.

Colensoi, Hook. f.

corymbosa, Hort.

decumbens, Armstr.

formosa, R. Br.

fruticulosa, Linn., var.

cæspitosa.

Godfreyana, Decne.

imperialis $\times$, Hort.

Kirkii, Armstr.

longifolia, Linn.

- var. alba.

Lyallii, Hook. f.

monticola, Armstr.

Nummularia, Gouan

officinalis, Linn.

- var. hirsuta.

parviflora, Vahl, var. angusti-

folia, Hook. $f$.

pimeleoides, Hook. $f$.

pinguifolia, Hook. f., var. decumbens.

pinnata, Linn.

Ponæ, Gouan

salicifolia, Forst.

satureioides, Vis.

saxatilis, Scop.

spuria, Linn.

subsessilis, Hort., var. rosea.

T'eucrium, Linn.

Traversii, Hook. f.

virginica, Linn. 
cxviii List of Seeds Collected during 1905.

\section{Viburnum}

cotinifolium, D. Don

Lantana, Linn.

Opulus, Linn.

\section{Vicia}

bithynica, Linn.

Cracca, Linn.

oroboides, Wulf.

Orobus, $D C$.

pyrenaica, Pourr.

sativa, Linn.

sepium, Linn.

sylvatica, $\operatorname{Linn}$.

unijuga, A. Braun

\section{Vincetoxicum}

fuscatum, Reichb. f.

nigrum, Moench

officinale, Moench

\section{Viola}

blanda, Willd.

canina, Linn.

cornuta, Linn.

hederacea, Labill.

hirta, Linn.

lutea, Huds.

odorata, Linn.

olympica, Boiss.

palustris, Linn.

polychroma, Kern.

primulæfolia, Linn.

sylvestris, Lam.
Wahlenbergia

dalmatica, A. $D C$.

dinarica, Hort.

Kitaibelii, $A, D C$.

serpyllifolia, G. Beck

tenuifolia, $A$. $D C$.

\section{Withania}

somnifera, Dun.

\section{Wulfenia}

carinthiaca, Jacq.

\section{Wyethia}

mollis, A. Gray

\section{Xanthocephalum}

gymnospermoides, Benth. et Hook. $f$.

\section{Xerophyllum}

asphodeloides, Nutt.

\section{Zaluzianskya}

selaginoides, Walp.

\section{Zenobia}

speciosa, D. Don, var. pulverulenta.

\section{Ziziphora}

taurica, Breb.

\section{Zygadenus}

elegans, Pursh 


\section{PLANTS UNDER GLASS.}

\section{Acacia}

armata, R. Br. calamifolia, Sweet dealbata, Link decurrens, Willd. extensa, Lindl. Farnesiana, Willd. grandis, Henfr. juncifolia, Benth. melanoxylon, $R$. Br. neriifolia, A. Cunn. pycnantha, Benth.

\section{Echmea}

bromeliæfolia, Baker cærulescens, Baker candida, E. Morr. glomerata, Hook. Skinneri, Baker

\section{Agapanthus}

umbellatus, L' Hérit.

\section{Agave}

densiflora, Hook.

\section{Aglaonema}

commutatum, Schott

\section{Albizzia}

lophantha, Benth.

\section{Aloe}

mitriformis, Mill,,var. flavispina (Salm-Dyck).

- var. spinulosa (Haw.) obscura, var. picta (Thunb.) Serra, $D C$. striata, Haw.

\section{Alonsoa}

linifolia, Roesl

\section{Amasonia}

calycina, Hook.f.

Amorphophallus

bulbifer, Blume

\section{Anthurium}

acaule, Schott

\section{Araujia}

sericifera, Brot.

\section{Ardisia}

crenata, Roxb.

- mamillata, Hance

\section{Arenaria}

Cephalotes, Bieb.

\section{Argyreia}

speciosa, Boj.

\section{Aristea}

Eckloni, Baker

\section{Ar istolochia}

elegans, Mast.

fimbriata, Cham

\section{Asparagus}

comorensis, Baker

plumosus, Baker, var. tenuissimus, Hort.

\section{Astrocaryum}

Ayri, Mart.

mexicanum, Liebm. 


\section{Asystasia}

bella, Harv.

\section{Bæometra}

columellaris, Salisb.

\section{Barleria}

cristata, Linn.

\section{Begonia}

dichotoma, Jacq.

Dregei, $O$. et $D$.

Froebelii, $A, D C$.

malabarica, Lam.

Martiana, Link et Otto

peltata, $A . D C$.

sanguinea, Raddi

ulmifolia, Willd.

Verschaffelti $\times$, Hort.

\section{Bignonia}

purpurea, Lodd.

\section{Billardiera}

longifolia, Labill.

\section{Billbergia}

decora, Poepp.

pallidiflora, Liebm.

zebrina, Lindl.

\section{Biophytum}

sensitivum, $D C$.

\section{Bomarea}

Carderi, Mast.

Lehmanni, Bakc?

\section{Bowiea}

volubilis, Harv.

\section{Brachychilum}

Horsfieldii, Baker

\section{Brodiæa}

hyacinthina, Baker, var. lactea.

ixioides, Sims, var. splendens.

\section{Calceolaria}

fuchsiæfolia, Hort.

\section{Callicarpa}

purpurea, Juss.

\section{Callistemon}

linearis, $D C$.

salignus, Szveet

speciosus, $D C$.

\section{Calostemma}

purpureum, R. $B$ r.

\section{Campanula}

pyramidalis, Linn.

Vidalii, $H$. C. Wats.

\section{Cantua}

buxifolia, Lam.

\section{Capsicum}

annuum, Linn.

\section{Carmichaelia}

australis, R. Br.

Enysii, T. Kirk

\section{Cassia}

corymbosa, Lam.

\section{Casuarina}

stricta, Ait. .

\section{Celosia}

cristata, Linn.

\section{Cestrum}

Newellii, Veitch

Smithii, Hort. Bull 
Chlorophytum

orchidastrum, Lindl.

\section{Cleome}

grandis, Hort.

\section{Clerodendron}

fallax, Lindl.

Thomsonæ, Balf.

\section{Clianthus}

puniceus, Banks

- var. alba.

\section{Clitoria}

Ternatea, Linn.

- var. alba, G. Don.

\section{Cobæa}

macrostemma, Pav.

scandens, Cav.

\section{Corallobotrys}

acuminata, Hook. f.

\section{Corchorus}

capsularis, Linn.

\section{Costus}

afer, Ker-Gawl

igneus, $N . E . B r$.

speciosus, $\mathrm{Sm}$.

\section{Cotyledon}

nodulosa, Baker

\section{Crassula}

tetragona, Linn.

\section{Crinum}

giganteum, Andr.

\section{Crossandra}

undulæfolia, Salisb.

\section{Cyclanthera}

explodens, Naud.
Cyphomanđira

betacea, Sendt.

\section{Cytisus}

candicans, Lam.

\section{Dalechampia}

Roezliana, Muell.

\section{Dendrocalamus}

strictus, Nees

\section{Dermatobotrys}

Saundersiæ, Bolus

\section{Dianella}

cærulea, Sims

\section{Dioscorea}

sativa, L. (Tuber)

\section{Dodonæa}

viscosa, Jacq.

\section{Dolichos}

Lablab, Linn.

\section{Dorstenia}

Contrajerva, Linn.

Drakena, Linn.

Mannii, Hook.f.

nervosa, Desv.

\section{Dracæna}

surculosa, Lindl.

\section{Drosera}

hilaris, Cham.

\section{Drosophyllum}

lusitanicum, Link

\section{Dumasia}

villosa, $D C$. 
cxxii List of Seeds Collected DURing 1905.

Eccremocarpus

scaber, Ruiz et Pav.

\section{Epiphyllum}

Gaertneri, Hook. $f$.

\section{Eriostemon}

buxifolius, $S m$.

\section{Erythrochiton}

brasiliensis, Nees

\section{Eucalyptus}

amygdalina, Labill.

incrassata, Labill.

\section{Eucomis}

bicolor, Baker nana, L'Hérit.

punctata, L'Hérit.

- var. striata, Don

\section{Eugenia}

myrtifolia, Roxb.

\section{Eupatorium}

Weinmannianum, Regel

\section{Euphorbia}

dentata, Michx.

Regis-jubæ, Webb et Berth. splendens, Boj.

\section{Evodia}

elegans, Hort.

formosa, Hort.

\section{Ficus}

pyriformis, Hook, et $\mathrm{Arn}$.

\section{Francoa}

sonchifolia, Cav.

\section{Fuchsia}

parviflora, Lindl.

procumbens, R. Cunn.

\section{Genista}

monosperma, Lam.

\section{Geonoma}

pumila, Wendl.

\section{Gloriosa}

superba, Linn.

\section{Grevillea}

Caleyi, R. Br.

\section{Guizotia}

abyssinica, Cass.

\section{Hakea}

acicularis, Knight

\section{Hedychium}

Gardnerianum, Rosc.

\section{Heliotropium}

peruvianum, Linn.

\section{Hibiscus}

esculentus, Linn.

Huegelii, Endl.

Manihot, Linn.

pedunculatus, Linn.

splendens, Fras.

vitifolius, Linn.

\section{Humea}

elegans, $S m$.

\section{Hydrolea}

spinosa, Linn.

\section{Hymenanthera}

dentata, R. Br. 
Hymenocallis

littoralis, Salisb.

Hypericum

japonicum, Thunb.

\section{Hypoxis}

erecta, Linn.

Krebsii, Fisch.

Rooperii, T. Moore

villosa, Linn.

\section{Impatiens}

cuspidata, Wight et Arn.

- var. arthritica, Hook. f.

\section{Indigofera}

australis, Willd.

\section{Ipomœa}

Bona-nox, Linn.

Learii, Paxt.

purpurea, Roth

Quamoclit, Linn.

\section{Ixiolirion}

montanum, Herb.

Ixora

coccinea, Linn.

\section{Kalanchoe}

crenata, Haw.

flammea, Stapf

grandiflora, Wight et Arn.

Kirkii, $N . E . B r$.

\section{Kedrostis}

africana, $\operatorname{Cog} n$.

\section{Kennedya}

prostrata, R. Br.

- var. glabrata, Lindl.

\section{Kunzea}

parvifolia, Schau.

\section{Lachnostoma}

maritimum, Nichols.

\section{Lantana}

Camara, Linn.

\section{Laportea}

gigas, Wedd.

\section{Leptodermis}

lanceolatus, Wall.

\section{Leptospermum}

arachnoideum, $\mathrm{Sm}$.

scoparium, Forst.

\section{Leucopogon}

lanceolatus, $R$. $B r$.

\section{Libertia}

ixioides, Spreng.

\section{Littonia}

modesta, Hook.

\section{Lobelia}

tenuior, $R . B r$.

\section{Luffa}

ægyptiaca, Mill.

\section{Mallotus}

japonicus, Muell.

\section{Malpighia} glabra, Linn.

\section{Malvastrum}

grossulariæfolium, Garcke 
cxxiv List of Seeds Collected during 1905.

\section{Mapania}

pandanæfolia, Hort. Sander

\section{Maurandia}

Barclayana, Lindl.

\section{Melia}

arguta, $D C$.

Azedarach, Linn.

\section{Melianthus}

major, Limn.

\section{Mesembryanthemum}

curvilorum, Haw.

lepidum, Haw.

micans, Linn.

muricatum, Hazw.

roseum, Willd.

serrulatum, Hawe.

tricolorum, Haze,

tumidulum, Haw.

vaginatum, Lam.

\section{Mimosa}

marginata, Lindl. pudica, Linn.

\section{Momordica}

Charantia, Linn. cochinchinensis, Spreng.

\section{Moræa}

prolongata, Hort.

spathacea, Ker-Gazel.

\section{Musschia}

Wollastoni, Lowe

\section{Myrica}

æthiopica, Linn.
Myrtus

communis, Linn.

Luma, Barn.

Ugni, Mol.

Nephthytis

liberica, N.E. Br.

picturata, $N, E . B r$.

Nertera

depressa, Banks

Nicotiana

rustica, Linn.

Ochna

multiflora, $D C$.

\section{Ocimum}

viride, Willd.

Ononis

reclinata, Linn.

Opuntia

nigricans, Haze.

Ornithogalum

tenellum, Jacq.

Orthrosanthus

multiflorus, Szveet

Oryza

sativa, Hochst.

- var. atropurpurea.

Oxypetalum

cæruleum, Decne.

\section{Passiflora}

edulis, Sims

quadrangularis, Linn. 


\section{Pavonia}

spinifex, Cav.

\section{Pentas}

carnea, Benth.

\section{Phyllanthus}

parvifolius, Buch.-Ham.

\section{Physalis}

peruviana, Linn.

\section{Pimelea}

ferruginea, Labill.

\section{Pitcairnia}

Altensteinii, Lem.

Andreana, Linden

aphelandræflora, Verschaff.

latifolia, Soland.

maidifolia, Decne.

\section{Platytheca}

galioides, Steets

\section{Pleuropetalum}

costaricense, Hort.

\section{Polyalthia}

suberosa, Benth. et Hook.f.

\section{Pontederia}

cordata, Linn.

\section{Primula}

floribunda, Wall.

mollis, Nutt.

verticillata, Forsk.

\section{Prostanthera}

lasianthos, Labill.

\section{Psychotria}

micrantha, Hiem

\section{Quesnelia}

Wittmackiana, Baker

\section{Rhodochiton}

volubile, Zucc.

\section{Rhododendron}

arboreum, $S m$.

grande, Wight

Nuttallii, Booth

\section{Rhynchosia}

caribæa, $D C$.

\section{Ricinus}

communis, Linn.

- var. zanzibarensis.

\section{Rivina}

aurantiaca, Warsc.

tinctoria, Hamil.

\section{Rondeletia}

odorata, Jacq.

\section{Ruellia}

amcena, Nees

rosea, Hemsl.

tuberosa, Linn.

\section{Ruta}

bracteosa, $D C$.

Sabal

Blackburniana, Glazebr.

\section{Sagittaria}

longirostra, Hort.

\section{Sandersonia}

aurantiaca, Hook. 


\section{Sedum}

Sieboldii, Hort.

\section{Sempervivum}

annuum, $C . S m$.

glaucum, Tenore

tabuliforme, Haze.

\section{Senecio}

grandifolius, Less.

macroglossus, $D C$.

\section{Sida}

acuta, Burm.

veronicæfolia, Lam.

\section{Solanum}

auriculatum, Ait. coagulans, Hort. fastigiatum, Willd. Hendersoni, Hort. jasminoides, Paxt. nigrum, Linn.

pyracanthum, Jacq. venustum, Kunth

\section{Sparaxis}

bulbifera, Ker.Gawl.

\section{Stachytarpheta} indica, Vahl.

\section{Statice}

echioides, Linn.

\section{Stylidium}

adnatum, R.Br.

\section{Tacca}

cristata, Jack

viridis, Hemsl.

\section{Tacsonia}

Van-Volxemii, Hook.

\section{Talinum}

patens, Willd.

triangulare, Willd.

\section{Tecoma}

Smithii, Hort.

\section{Tetranema}

mexicana, Benth.

\section{Teucrium}

Marum, Linn.

\section{Thoracostachyum}

bancanum, Kurs

\section{Tillandsia}

hieroglyphica, Baker

Lubbersii, Baker

macropetala, Hort

Regina, Vell.

splendens, Brongn.

\section{Trachelium}

cæruleum, Linn.

\section{Tradescantia}

discolor, L'Hérit.

\section{Tristania}

neriifolia, R. $B$ r.

\section{Turnera}

ulmifolia, Linn.

\section{Vellozia}

elegans, Talbot

Vitis

heterophylla, Thunb, var. humulifolia, Hook. $f$.

\section{Wurmbea}

spicata, Hort. 



and 